徸

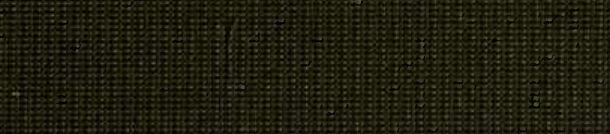




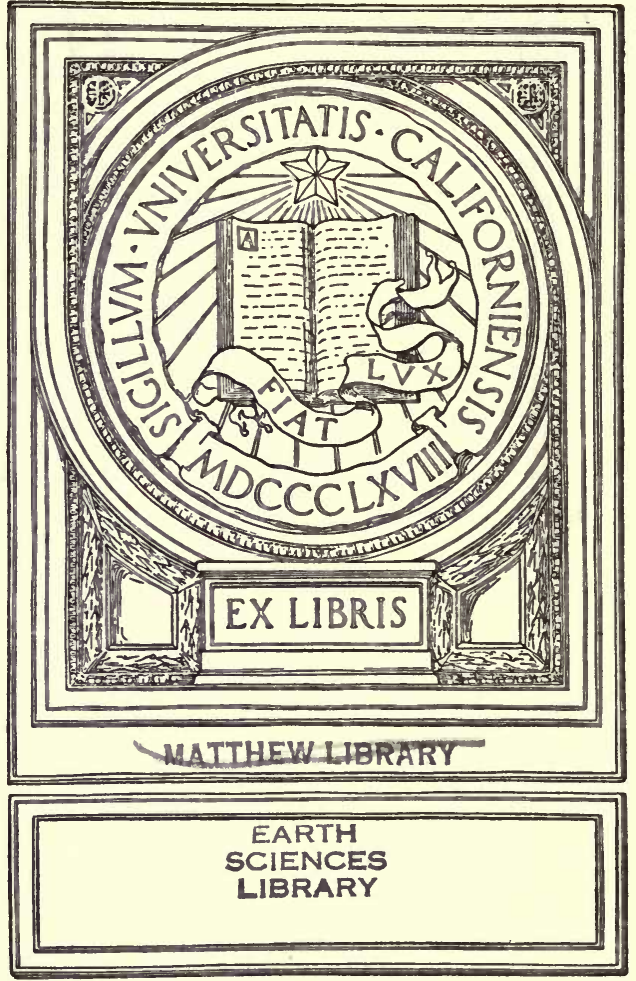


Ralph Ele, fr. ' 32 2420 Minge Rood, Barkly. 



\section{OUTLINES OF}

\section{CHORDATE DEVELOPMENT}

BY

WILLIAM E. KELLICOTT

PROFESSOR OF BIOLOGY IN GOUCHER COLLEGE

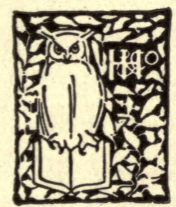

NEW YORK

HENRY HOLT AND COMPANY 


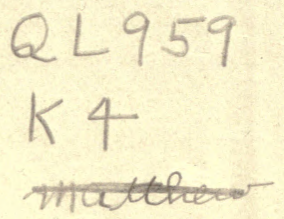

EARTY

SCIENCES

LIBP* -

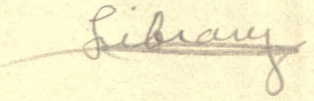

\section{MATTHEW LIBRARY}

Copyright, 1913,

$\mathrm{BY}$

HENRY HOLT AND COMPANY

August, 1927

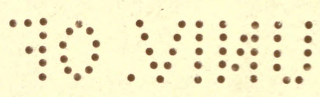

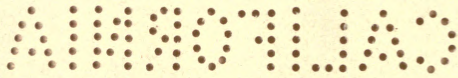




\section{PREFACE}

In these "Outlines" the student is introduced to the study of Chordate development through the embryological history of Amphioxus. Whether or not Amphioxus represents a truly primitive type of development, it affords, in simple diagrammatic style, the essentials of early Chordate ontogeny. In many respects the later phases of its history are highly modified, but this need be no objection to its use as an introductory type, since it may serve immediately to put the student upon his guard against a too exact phylogenetic interpretation of embryological facts.

Following this is a rather full account of the development of the frog, a form that represents, better than any other single type, what we may regard as the general type of Chordate development. The chapters on the chick are relatively briefer and emphasis is laid upon the embryonic membranes, and upon the early phases of development, which represent the most frequent modifications of the type of Chordate development, modifications correlated with the presence of the large yolk accumulation of the Sauropsid ovum.

It is believed that the chapters on the frog and chick have been written in such a way that either form may be omitted, in a brief course of study. Or in case the study of the early development of the frog is desired as a comparative introduction to the study of the embryology of the chick, Chapter III, on the organogeny of the frog, may be omitted without serious interruption of the continuity of such a course.

The final chapter on the Mammal includes only those phases of development that are of chief interest to the general student, namely, the earlier stages in the formation of the embryo, the establishment of its relation with the maternal organism, the development of the embryonic membranes and appendages, and the development of the external form of the human embryo. 
Several authors and publishers have very kindly permitted the use of illustrations or clichés from their works. Especial acknowledgement is made to Professor F. R. Lillie for permission to use a considerable number of the illustrations in his "Development of the Chick" (Henry Holt and Co.). I am also indebted to Professor C. S. Minot and P. Blakiston's Son and Co., for several clichés from their "Laboratory Text-book of Embryology"; to Professor J. Playfair McMurrich and P. Blakiston's Son and Co., for clichés from their "Development of the Human Body"; to Messrs. Longmans Green and Co., for clichés from "Quain's Anatomy"; to Messrs. G. P. Putnam and Co., for permission to redraw, with some modifications in most instances, certain illustrations in Marshall's "Vertebrate Embryology"; to Professor T. H. Morgan and The Macmillan Company, for clichés from their Development" of the Frog's Egg"; and to Herr Gustav Fischer and the respective authors, for clichés from O. Hertwig's "Handbuch der vergleichenden und experimentellen Entwickelungslehre der Wirbeltiere"; O. Hertwig's "Lehrbuch der Entwickelungsgeschichte des Menschen und der Wirbeltiere"; and H. E. Ziegler's "Lehrbuch der vergleichenden Entwickelungsgeschichte der niederen Wirbeltiere." In all cases the cuts thus borrowed are separately acknowledged in the figure legends. I desire also to express my debt to the authorities of the Johns Hopkins University, for library facilities generously afforded.

July, 1913.

W. E. K. 


\section{CONTENTS}

CHAPTER I

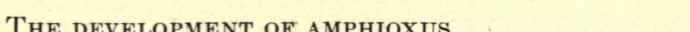

CHAPTER II

THE EARLY DEVELOPMENT OF THE FROG . . . . . . . . . . . . . . 62

CHAPTER III

The later development of the Frog: ORganogeny . . . . . . . . 126

CHAPTER IV

The EARLY DeVelopment of the Chick: THE EMbryonic Membranes AND APPENDAGES

\section{CHAPTER V}

The LATER Development of THE CHICK: ORganogeny . . . . . . . 301

\section{CHAPTER VI}

The early development of the mammal. The mammalian embryONIC MEMBRANES AND APPENDAGES . . . . . . . . . . . . 368 INDEX . . . . . . . . . . . . . . . . . 455 



\title{
OUTLINES OF CHORDATE DEVELOPMENT
}

\author{
CHAPTER I
}

\section{THE DEVELOPMENT OF AMPHIOXUS}

INTRODUCTION ........................ 2

I. THE GERM CELLS AND THEIR PRODUCTION . . . . . 4

II. THE EMBRYONIC PERIOD. . . . . . . . . . . . . . . . 9

A. From Fertilization to the Time of Hatching . . . 9

1. Fertilization . . . . . . . . . . . . . . . . . . 9

2. Cleavage .................. 11

3. Gastrulation . . . . . . . . . 15

4. The Formation of the Central Nervous System . . . . 20

5. The Formation of the Notochord . . . . . . . . . . 23

6. The Formation of the Mesoderm and Enterocals . . . 24

B. From Hatching to the Formation of the Mouth . 27

1. The Central Nervous System . . . . . . . . . . . 27

2. The Notochord . . . . . . . . . . . . . . . . 28

3. The Mesodermal Somites and Coelom . . . . . . . . 29

4. The Enteron and Its Appendages. . . . . . . . . . 33

III. THE LARVAL PERIOD . . . . . . . . . . . . . . . . . 37

1. The Central Nervous System . . . . . . . . . . . 37

2. The Gill Slits . . . . . . . . . . . . . . . . . . 39

3. The Club-shaped Gland . . . . . . . . . . . . . . 43

4. The Endostyle . . . . . . . . . . . . . . . . 43

5. The Mouth and Associated Structures . . . . . . . 44

6. The Preoral Pit and Its Derivatives . . . . . . . . 44

7. The Blood-vessels . . . . . . . . . . . . . . . . 46

8. The Atrium . . . . . . . . . . . . . . . . . . 46

9. Larval Asymmetry. . . . . . . . . . . . . . . . 49

10. The Mesodermal Somites . . . . . . . . . . . . 51

11. The Nephridia . . . . . . . . . . . . . . . . . . . . . . 53

12. The Larva at the Critical Stage. . . . . . . . . . . 54

IV. THE ADOLESCENT PERIOD ... . . . . . . . . 55 
"As an introduction to the study of embryology, and as an indispensable aid to a reasonable appreciation of the value of embryological facts, the life-history of Amphioxus provides an object which . . . is perhaps unrivalled. It is alike useful in a text-book of human embryology, and in one of invertebrate zoology." (Willey, "Amphioxus," etc., p. 104.)

Morphologically Amphioxus (Branchiostoma lanceolatum) is recognized as one of the important types of Chordata, for it illustrates in simple form the essential characteristics of this large group, most of the members of which are extremely complex. And in its mode of development, no less than in its structure, Amphioxus serves as a key to the more complicated conditions of the Craniate groups. The anatomical and embryological simplicity of this creature is commonly regarded as an indication of true primitiveness, although the morphologist recognizes, as the embryologist must also, that the simplicity of primitiveness is here obscured, in many respects, by conditions which are obviously special adaptations or secondary alterations of primary arrangements.

Many of the simple embryological characteristics of this form are correlated with the freedom of the egg from a large yolkmass. This is equivalent to saying that the accumulation of yolk in the eggs of most Chordates is a secondary character, and is, to a considerable extent, the cause of many of those modifications of the course of development which lead to unusual conditions, proving difficult of comparison with other developmental types.

The egg of Amphioxus is small, and the deutoplasm, small in amount, is scattered through its substance. In correlation with this, cleavage is total and quite regular in its course, leading to the formation of fairly typical blastula and gastrula. The organ-rudiments are all formed first as simple epithelial structures, whose origins and fates are easily followed, for each part is sharply outlined and remains clearly demarcated through the course of its development. The embryo is free swimming and externally ciliated. In all these respects Amphioxus illustrates primitive Chordate characteristics. Most of the second- 
ary modifications of development appear comparatively late in its course, and many of them are obviously correlated with rather unusual habits and activities.

The embryological history of Amphioxus is described here, then, not only because of the morphological importance of this form, but because of its embryological primitiveness and the diagrammatic simplicity of its early stages, which will aid in understanding the complicated development of the other Chordates described. Here we have Chordate development reduced to its simplest terms. A fair knowledge of the anatomy of Amphioxus is presupposed.

The whole developmental history of Amphioxus falls quite naturally into four general periods.

I. Production of the Germ Cells.-This includes the formation of the germ cells, oö- and spermatogenesis, and spawning.

II. Embryonic Period.-This extends from fertilization to the opening of the mouth. This phase of development is very rapid: at normal seasonal temperatures of the water it may occupy only thirty-six hours. The entire period is conveniently subdivided into two.

A. Before Hatching.-This includes fertilization, cleavage, gastrulation, and the first mapping out of the embryo. The conclusion of this period is marked by escape from the egg membranes-eight hours or more.

B. After Hatching.-Here the chief systems and organs of the embryo become definitely laid down. The embryo is free swimming (pelagic) - twenty-eight hours or more. The actual duration of these and the other periods depends chiefly upon temperature; it may be nearly doubled in the cooler waters of an early season.

III. Larval Period.-From the opening of the mouth to the cessation of pelagic life and the assumption of a burrowing habit. Here development is slower and consists largely in the elaboration of the parts marked out during the preceding period-about three months. At the conclusion of this period 


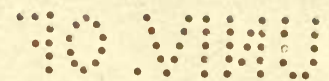

$\therefore: \therefore:$ OUTHg IFFS OF.CHORDATE DEVELOPMENT

the larva is about $3.5^{\circ} \mathrm{mm}$. long and has reached a condition known as the "critical stage."

IV. Adolescent Period.-From the critical stage to sexual maturity. This is marked by histological differentiation and the gradual appearance of adult characteristics. Germ cells are first brought to maturity in specimens about $2 \mathrm{~cm}$. in length, the age of which is doubtful. Growth to full size probably involves several years and covers several spawning periods.

\section{THE GERM CELLS AND THEIR PRODUCTION}

We may first describe the germ cells as they are extruded from the body.

The eggs of Amphioxus are spherical and only 0.10 to 0.13 $\mathrm{mm}$. in diameter. Among the Chordates smaller eggs than these are found only in some Mammals. The egg (Fig. 1) is surrounded by two membranes, a thin outer, or vitelline membrane, which at this time is usually separated by a space from the surface of the egg, and a thick inner, or perivitelline membrane, which at the time of laying is more or less fluid and closely adherent to the surface of the egg. The egg is richly supplied with deutoplasm in the form of very numerous small granules. These are not uniformly distributed through the cytoplasm for there are two definite regions comparatively free from deutoplasm, namely, a narrow superficial layer, and an irregular conical region toward one side of the ègg. Strictly speaking the egg of Amphioxus is thus telolecithal, but the deutoplasmic center is not toward the vegetal pole of the egg but toward one side, below the equator. The precise location of this yolk-free area is important for it establishes in the egg a definite bilateral symmetry which is also that of the future embryo. The animal pole is marked by the apex of this clear area and by the point at which the first polar body is given off. The first polar body has already been formed at the time the egg is laid, but since it lies outside the vitelline membrane it is ordinarily lost. At this time the animal pole is marked, however, by the position of the egg nucleus which is just in the equatorial 
plate of the second polar division. The arched base of the clear protoplasmic region lies about at the level of the equator, and the whole space lies eccentrically toward that side of the egg which, from later development, is known to be anterior or
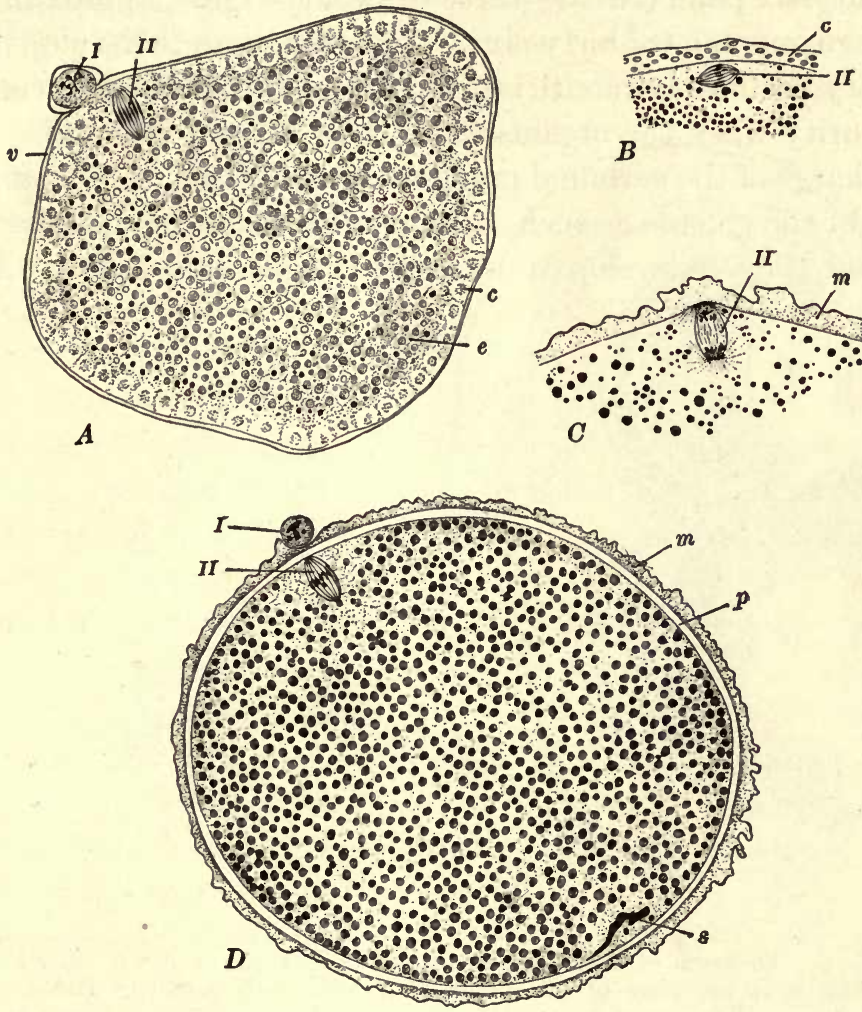

Fig. 1.-The egg of Amphioxus. $C$, after Cerfontaine, others after Sobotta. $A$. Ovarian egg showing cortical plasm. B. Cortical layer forming a membrane on the suface of the egg, within the vitelline membrane. C. Egg membrane fully formed but still attached to surface of egg. $D$. Extruded, fertilized egg. Membrane fully formed and beginning to leave the egg. $c$, Cortical layer; $e$, endoplasm; $m$, egg membrane, externally vitelline, internally a product of the exoplasm; $p$, perivitelline space; $s$, spermatozoön; $v$, vitelline membrane; $I$, first polar body; $I I$, second polar spindle.

antero-dorsal. A diagram of a sagittal section of the egg is shown in Fig. 2, in the position usually assumed by the egg in the water.

The spermatozoa are very small (15-20 micra in length); the 
rather spherical head is about 1 micron in diameter-about the size of a deutoplasm granule.

The gonads are metameric organs distributed through the middle and posterior pharyngeal region. There are about twenty-six pairs (twenty-three to twenty-eight), approximately in segments ten to thirty-six. They first begin to develop in the embryo before the mouth is opened, and apparently first come to maturity when the organisms are 2 to $2.5 \mathrm{~cm}$. in length. The discharge of the germinal products involves the nearly complete loss of the gonads as such, so that after each annual spawning period they redevelop from small rudiments. Details of the

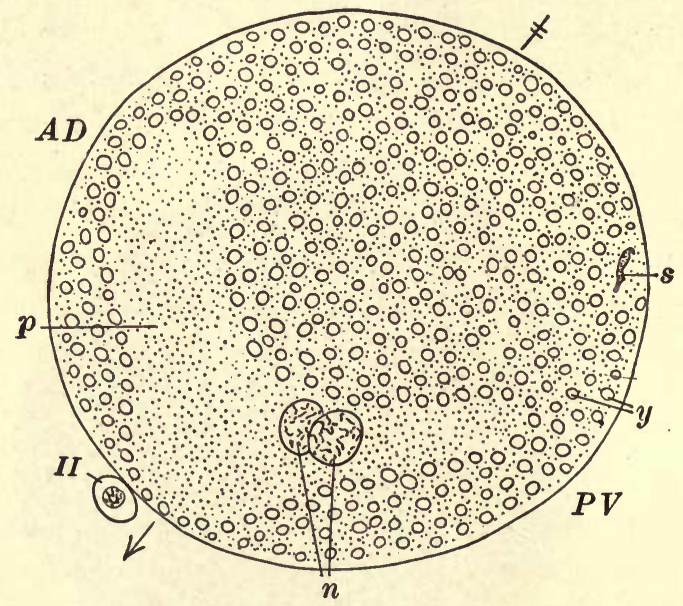

FIG. 2.-Diagram of a median sagittal section through the fertilized egg of Amphioxus at the stage of the fusion of the egg and sperm pronuclei. After Cerfontaine. The arrow marks the direction of the chief or polar axis. $A D$, Antero-dorsal region; $n$, egg and sperm pronuclei; $p$, region relatively free from deutoplasm; $P V$, postero-ventral region; $s$, remains of tail of spermatozoön; $y$, yolk or deutoplasmic bodies; $I I$, second polar body.

development of the gonads will be described later. The partially grown ovary, which the testis resembles in essentials, is a large spheroid mass, projecting from the postero-ventral margin of the segment into the atrium, covered of course by atrial epithelium. Fig. 3 represents in diagrammatic form a section through the half grown ovary in the so-called medusoid or mushroom stage. An abundant vascular supply is derived 
from branches of the posterior cardinal veins, which divide the germinal epithelium into dorsal and ventral portions. Inside the gonad is a space known as the primary ovarian or perigonadial cavity, in the outer wall of which the definitive germ cells are developed. Outside the gonad, and separating it from the atrial epithelium and body wall proper, is a secondary ovarian cavity or gonocœl. In the wall of the gonocœl, just above and below the stalk of attachment through which the blood-vessels pass, are thickened patches in the atrial wall of the gonocœl-the dorsal and ventral cicatrices. During the later stages of their formation the germ cells -oögonia or spermatogonia; enlarge so considerably as nearly to obliterate, by compression, these cavities and even to cause the gonads to encroach very considerably upon the atrial cavity.

Toward the end of the growth period the nucleus, which contains a large nucleolus, passes through the synizesis stage and

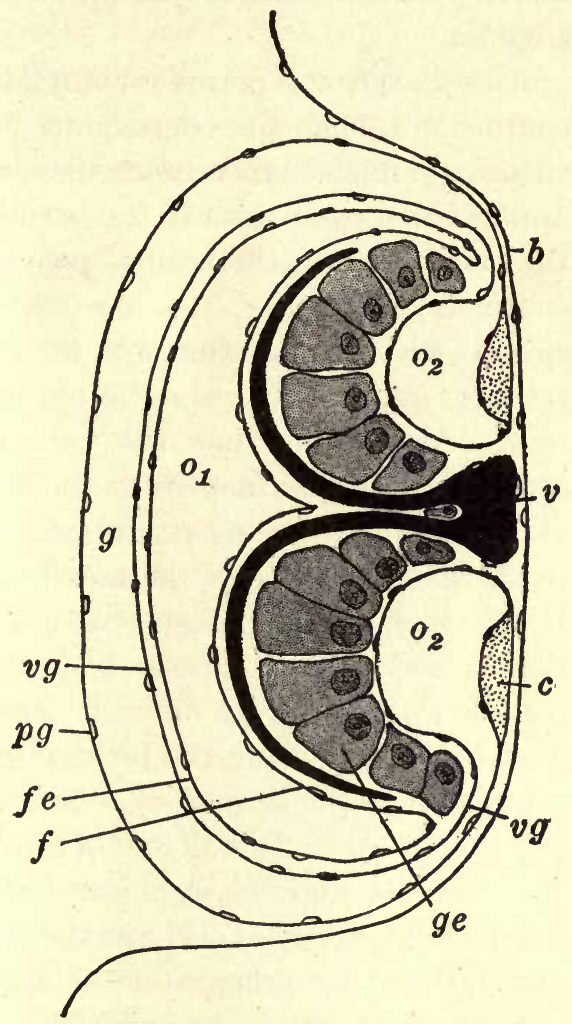

Frg. 3.-Diagram of a section through the gonad of Amphioxus. After Cerfon. taine. b, Peribranchial (atrial) epithelium; $c$, cicatrix; $f$, true follicular epithelium; $f e$, external layer of follicular epithelium; $g$, gonocœl; ge, germinal epithelium; $o_{1}$, primary ovarian cavity; $o_{2}$, secondary ovarian cavity; $p g$, parietal layer of gonocœel; $v$, cardinal vein; $v g$, visceral layer of gonocœl. the deutoplasm granules, whose formation began toward the inner side of the oögonia when these were only about $0.05 \mathrm{~mm}$. in diameter, crowd the entire cell body except in the perinuclear 
region and a narrow peripheral region which is occupied by several rows of small clearer vacuoles. It is doubtful whether there is a true cellular egg follicle: a thin layer of cells forming the wall of the primary ovarian cavity forms an ovarian follicle which folds in and covers almost completely the superficial oögonia.

After the growth period is completed the process of maturation commences while the oögonia are still within the ovary. The chromatic nuclear membrane disappears and the whole nuclear apparatus moves toward the atrial (i.e., attached) surface of the cell, marking the animal pole and establishing visibly the symmetry of the egg. As it reaches the surface a typical polar spindle with asters forms, and the chromatin forms into twelve tetrads, indicating a somatic chromosome number of twentyfour. About this time the very thin vitelline membrane is formed on the surface from the substance of the egg proper (Fig. 1), and as the first polar body is cut off it pushes this before it, so that a portion of the membrane is cut off with the polar body which may no longer remain attached to the egg. Immediately after the first polar body is given off the second polar spindle forms, but the division halts in the mesophase (Fig. 1). Just before or during the formation of the first polar body the inner wall of the secondary ovarian cavity is ruptured by contraction of the body wall and of muscle fibers in the thin walls of the gonoccel, and the eggs are partly forced into the gonocœl. All of these processes occur as the animals emerge from the sand and swim about preparatory to spawning.

Spawning extends through the greater part of spring and summer in the warmer waters, and always occurs at a definite time of day-about sundown, or from five to seven in the evening. Strong contractions of the general body musculature rupture the cicatrices and force the eggs from the gonocœl into the atrial cavity, and thence they are carried by bodily contractions and the respiratory current out through the atriopore into the free sea water.

Contact with the sea water brings about the formation of a thick mucilaginous second or inner membrane over the surface 
of the egg. The substance of this, the perivitelline membrane, seems to be derived from the material of the peripheral layers of vacuoles in the cytoplasm; these flow together forming a homogeneous superficial layer quite distinct from the underlying protoplasm (Fig. 1). The membrane is at first rather fluid but in contact with the water it soon begins to toughen.

In this condition the eggs are ready for fertilization. The formation of the spermatozoa is not so fully known but it seems to occur in a manner parallel to the formation of the ova.

\section{THE EMBRYONIC PERIOD}

\section{A. From Fertilization to the Time of Hatching}

\section{Fertilization}

Eggs and sperm are discharged into the water at about the same time and immediately each egg becomes surrounded by a crowd of the spermatozoa. The perivitelline membrane has been formed first in the region of the animal pole and at this time is gradually extending thence toward the vegetal pole. In this region then it remains longest in a more fluid condition and it is at this vegetal pole that the sperm usually gain entrance to the egg, after having penetrated first the thin vitelline membrane and traversed the perivitelline space. No micropyle has been discovered and polyspermy seems to be very frequent. It is not surely known whether the entire sperm or only the head usually enters the egg substance. Immediately after the entrance of a spermatozoön the entire perivitelline membrane is formed, toughens, leaves the surface of the egg, and comes into contact with or even partially fuses with the vitelline membrane (Fig. $1, D$ ) obliterating the original perivitelline space but leaving a wide secondary perivitelline space between itself and the egg.

Entrance of the sperm affords also the stimulus to the completion of maturation of the egg nucleus, which at this time is still in the mesophase of the second polar division. While the sperm, lying just inside the egg, is reconstituting a 
normal nucleus together with sperm centrosome and aster, the second polar body is formed and the mature egg nucleus established. The second polar body forms about fifteen minutes after entrance of the sperm. The division of the sperm centrosome and aster, the approach and fusion of the egg and sperm nuclei, and the constitution of the first cleavage nucleus, are in no way unusual (Fig. 4).

The first cleavage spindle takes up a position definitely related to the symmetry of the egg, lying horizontally, slightly

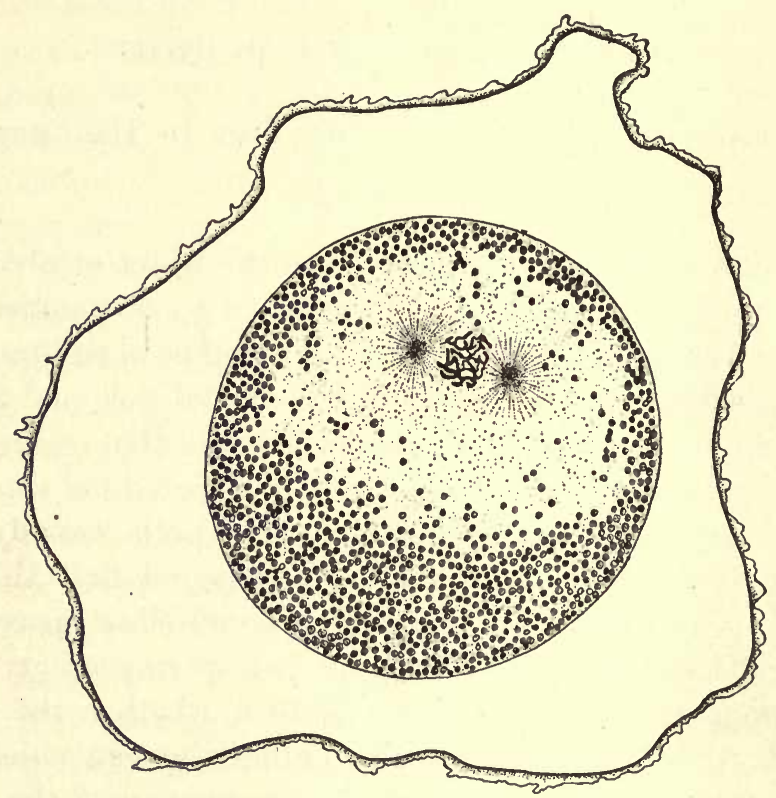

Fig. 4.-Prophase of first cleavage figure in Amphioxus. After Sobotta. Inner and outer membranes fused and separated from the egg by a wide space called the perivitelline space.

nearer the animal pole, and at right angles to the median plane. The relation of this symmetry of the egg to that of the future embryo is shown in Figs. 2 and 7, $A$. The median plane of symmetry is determined by the points of polar body formation and sperm entrance, and the eccentric position of the clear protoplasmic region. This last is displaced toward the anterodorsal region of the future embryo: one result of this is the 
greater freedom from yolk of this region of the embryo, and this has an important bearing upon subsequent development. The egg axis is at an angle of roughly $30^{\circ}$ to the antero-posterior axis of the embryo: that is, the animal pole of the egg becomes antero-ventral, the vegetal pole postero-dorsal. It is quite probable that this bilateral symmetry, and certain that the polarity of the egg, is determined while it is still within the ovary; it gains visible expression through the assumption of a peripheral position by the primary oöcyte nucleus and the eccentricity of the accompanying clear protoplasmic region. The egg of Amphioxus seems to resemble that of the Echinoderm rather than those of most other forms, in that the direction of this eccentricity of the nucleus, i.e., animal pole, is toward the region of attachment in the ovarian epithelium.

\section{Cleavage}

The cleavage of Amphioxus is total and unequal and, though subject to much variation, it is in many eggs quite regular. It is generally believed that the Amphioxus type of cleavage is primitive among Chordates; and that the cleavage modes of Craniates are to be derived from it, the chief cause of modification being the accumulation of yolk. The first division of the egg occurs about an hour after entrance of the sperm, the second about an hour after the first, and subsequent divisions about every fifteen or twenty minutes. The plane of the first cleavage as indicated by the position of the first cleavage spindle, is median (cleavage furrow meridional), dividing the egg into exactly similar right and left halves, which become the right and left halves of the embryo. The second cleavage furrow is also meridional and the plane at right angles to the first. Cleavage becomes unequal with this division for the two cells are divided into two smaller anterodorsal, and two larger postero-ventral cells, symmetrically arranged on each side of the median plane (Fig. 5, $A$ ).

The third cleavage is at right angles to the first two and the furrow equatorial, or rather latitudinal for again the division 
is slightly unequal and each of the four cells forms a smaller cell toward the animal pole, and a larger toward the vegetal.
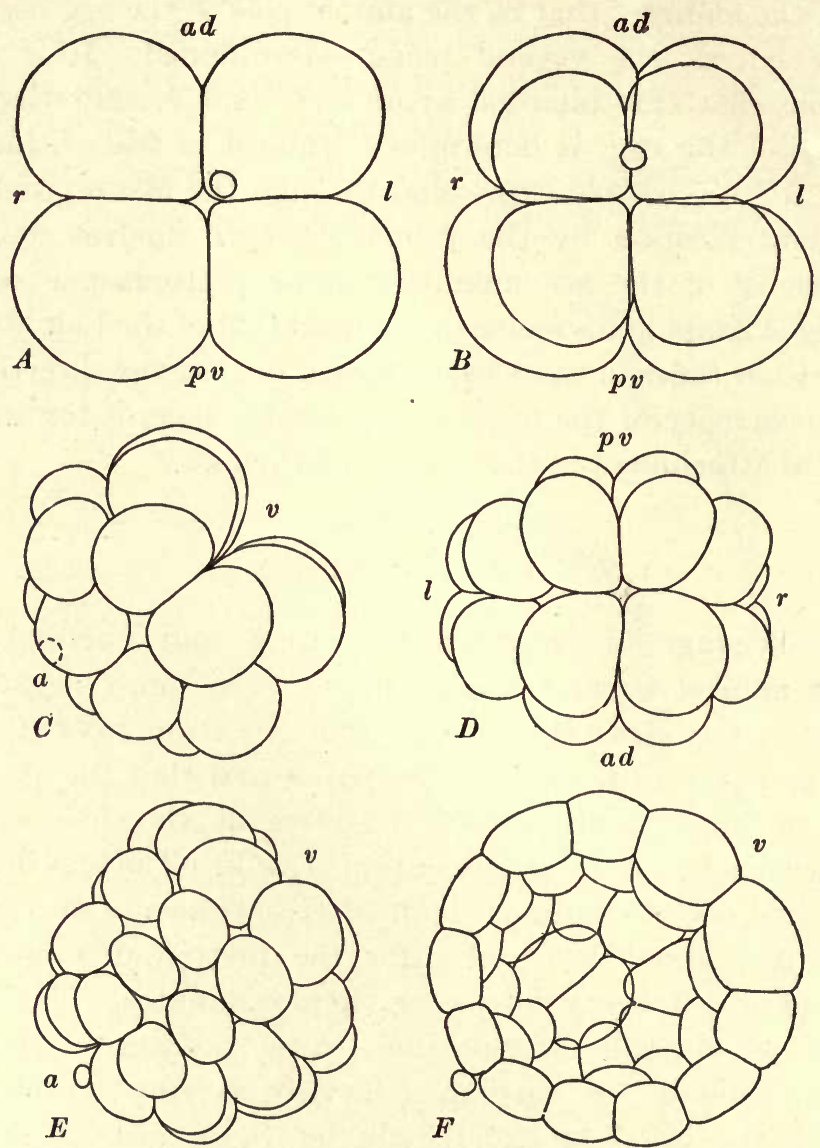

FIG. 5.-Cleavage in Amphioxus. After Cerfontaine. A. Four-cell stage viewed from animal pole. The two antero-dorsal cells are the smaller. $B$. Eight-cell stage viewed from animal pole showing the four sizes of the cells. $C$. Sixteen cells viewed from the left side. D. Thirty-two cells viewed from vegetal pole. E. Thirty-two passing into sixty-four cells, viewed from the anterodorsal region. $F$. Optical section of right half of young blastula. About 128 cells. $a$, Animal pole; $a d$, antero-dorsal; $l$, left; $p v$, postero-ventral; $r$, right; $v$, vegetal pole.

Thus in the eight-cell stage (Fig. 5, B) we have four pairs of cells of four sizes; the four micromeres of the animal pole 
consist of two smaller anterior, and two larger ventral cells, and the four macromeres of the vegetal pole, of two smaller dorsal, and two larger posterior cells, the smaller macromeres being larger than the larger micromeres. These relative inequalities in the quadrants, as well as their bilateral symmetry, continue throughout cleavage.

Strict synchronism of cleavage is lost in passing from four to eight cells, the smaller cells commencing their division before the larger. In the early stages the intervals between successive cleavages are such that, in spite of lack of synchronism, it is still possible to speak of sixteen- and thirty-two-cell stages, but after this the numerical progression is no longer regular.

Were the cleavage strictly regular the eight cells would form sixteen by meridional cleavages passing symmetrically through the entire egg, but as a matter of fact, during their formation the spindles of the eight blastomeres change their position so that the cleavage planes of the micromeres are nearly perpendicular to the median plane, while those of the macromeres are rather parallel with this plane. As a result the sixteen-cell stage (Fig. 5, C) consists of eight micromeres arranged in two rows of four cells each, parallel with the median plane and becoming arched in the same direction, and of eight macromeres in two rows of four cells each extending across the median plane, i.e., from right to left, and arched in this direction. The relation between the groups of micro- and macromeres is much like that of the two hands when partly closed and one in the palm of the other. The thirty-twocell stage (Fig. 5, D) is formed by the horizontal (latitudinal) divisions of each of the sixteen, forming eight meridional rows of four cells each. After thirty-two cells (Fig. 5, E) the divisions become irregular in their appearance. To summarize:

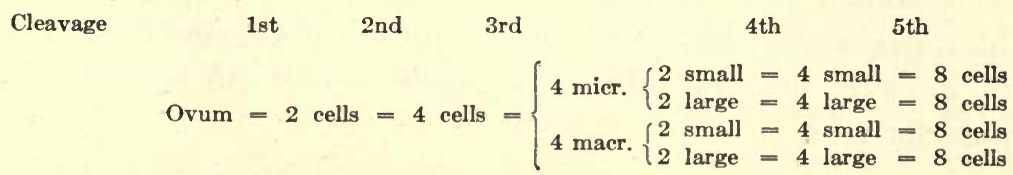


This typical arrangement of cleavage planes is by no means invariable. Variations are indeed very frequent but the cells are usually arranged according to definite plans. In many cases the cleavage pattern is nearly radial, and in others the blastomeres may shift more or less giving the appearance of a spiral cleavage. In some eggs the micromeres of the sixteen-cell stage may not all divide similarly but four may divide vertically and four horizontally. These variations in cleavage do not affect subsequent processes.

As a consequence of the early blastomeres remaining well rounded a central space is formed among them. Virtually present in the four-cell stage, this space becomes real at eight cells, and as the cells multiply and gradually lose their rounded form they push away from the center of the mass leaving a definite space within, at first open at the poles (cleavage pores). This is the beginning of the segmentation cavity or blastocel. By the time thirty-two cells are formed the poles close over and soon the blastocœl is entirely closed. From about sixtyfour cells on (Fig. 5, $E, F$ ) the blastomeres lose their rounded outline and become flattened and closely packed in a simple epithelial layer. This arrangement of the blastomeres may be taken as the beginning of the blastula stage, which may be considered fully established when the number of cells reaches one hundred and twenty-eight. The hollow spherical blastula of this type (Fig. 5, F) is called a cœloblastula, and it is commonly regarded as the most primitive type of blastula. In Amphioxus this is bilaterally symmetrical and the epithelial wall is of varying thickness on account of the varying sizes of the cells. The cells are relatively free from deutoplasm, and therefore smaller, in the anterior region, richer in deutoplasm, and therefore larger, in the posterior region, and uniformly graded in size and deutoplasmic content between these regions. The animal pole remains antero-ventral (Fig. 7, B). The blastocœl is very large and, on account of the varying thickness of its wall, may be said to be slightly eccentric toward the anterior side. 


\section{Gastrulation}

By the time about two hundred and fifty-six cells have been formed in the blastula, or about four hours after fertilization, the process of gastrulation is commenced, by which the single walled blastula is to be converted into a double walled gastrula. We should notice in advance that the gastrula of Amphioxus is formed by the three processes of invagination, involution, and epiboly, and so is not typical of Chordates generally, in which the double walled or two layered condition results more extensively from delamination, that is, by tangential divisions in the wall of the blastula, together with some involution and epiboly.

The first indication of gastrulation is the flattening of the vegetal pole of the blastula (Fig. 6, A), which is soon followed by the appearance of a slight infold on the antero-dorsal aspect, at about the level of the equator (Fig. 6, $B$ ). This infolding or invagination soon extends around the sides of the blastula, and finally the whole flattened vegetal or posterodorsal region becomes folded down into the segmentation cavity (Fig. 6, C). The process of infolding continues in a more advanced stage in the antero-dorsal region where it commenced, and here the folded layer first comes into contact with the inner surface of the cells of the animal pole so as to obliterate the blastocœl there, at a time when this remains quite widely open elsewhere. We may now speak of the invaginating and non-invaginating layers as endoderm (hypoblast) and ectoderm (epiblast) respectively, and of the whole structure as the gastrula (Fig. 6, E). While the infolding of the endoderm leads to the obliteration of the blastocœl, it leads to the formation of another cavity which is lined completely with cells of one kind-endoderm. This cavity, which can be recognized as beginning with the earliest infolding of the blastula wall, is the archenteron or primitive gut cavity, which is to give rise to the chief cavities of the later embryo. In many Chordates this cavity is virtual rather than real on account of being filled with yolk-cells. The gastrula of Amphioxus is often 

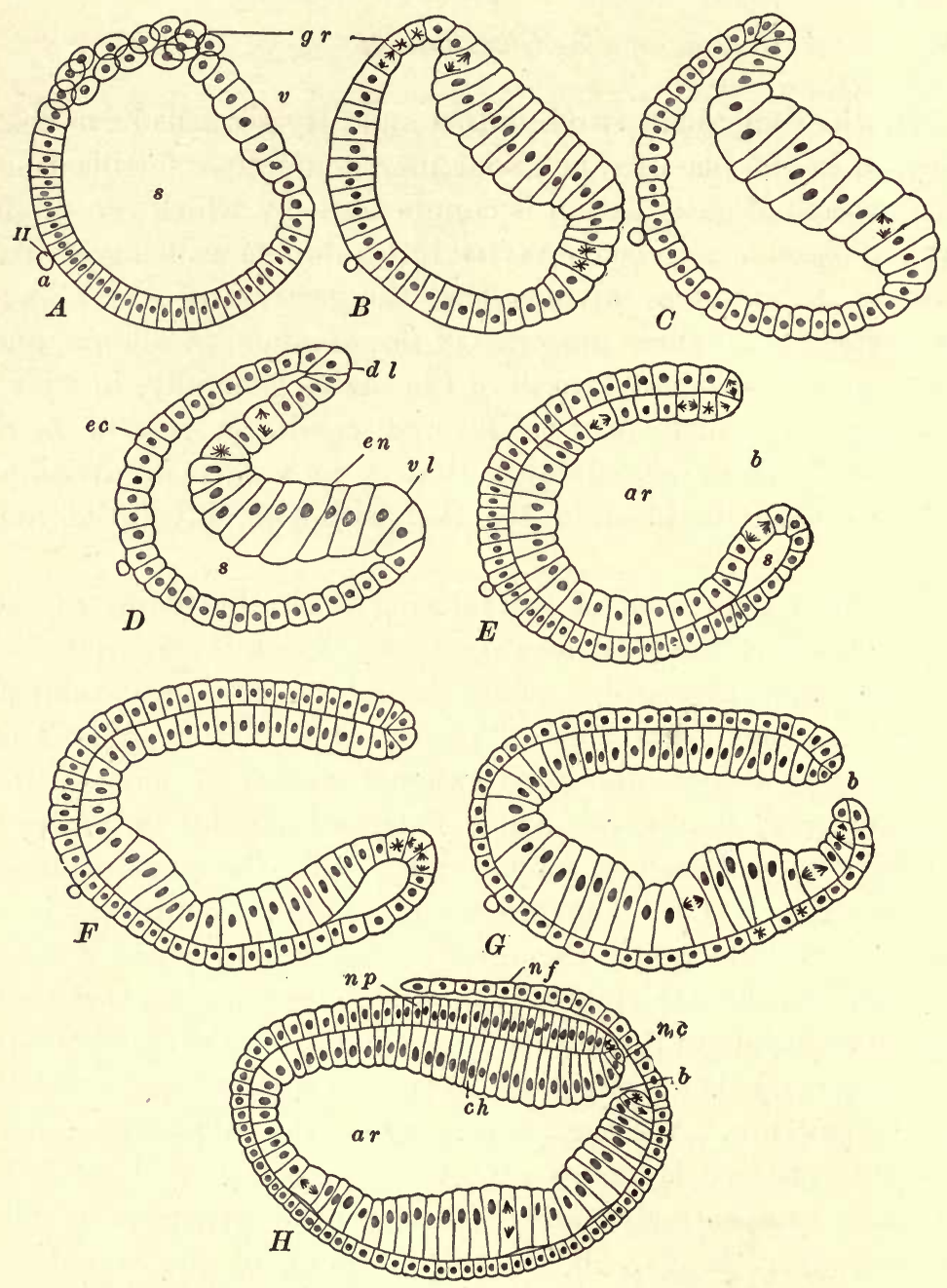

Fig. 6.-Gastrulation in Amphioxus. After Cerfontaine. A. Blastula showing flattening of the vegetal pole and the rapid proliferation of cells in the posterodorsal region (germ ring). $B$. Flattening more pronounced; mitoses in cells of germ ring. $C$. Commencement of the infolding (invagination) of the cells of the vegetal pole. $D$. Continued infolding, and inflection, or involution, of ectoderm cells in the dorsal lip of the blastopore. The blastocœl becoming obliterated and the archenteron being established. $E$. Invagination complete. Continued involution in the dorsal lip of blastopore. Mitoses in germ ring. $F$. Constriction of blastopore and commencement of elongation of the gastrula. Remnants of blastocœl in ventral lip of blastopore. $G$. Gastrulation completed. Continued elongation, and narrowing of blastopore. $H$. Neurenteric canal established by overgrowth of neural folds. Continued mitosis in germ ring. 
termed a cologastrula to indicate the fact that the archenteron is an actual widely open cavity.

While cell multiplication continues in all parts of the embryo during gastrulation, immediately after gastrulation begins, a center of very rapid cell division appears just anterior to the invaginating region on the dorsal side, and extending laterally around the margin of the blastopore; on the ventral side of
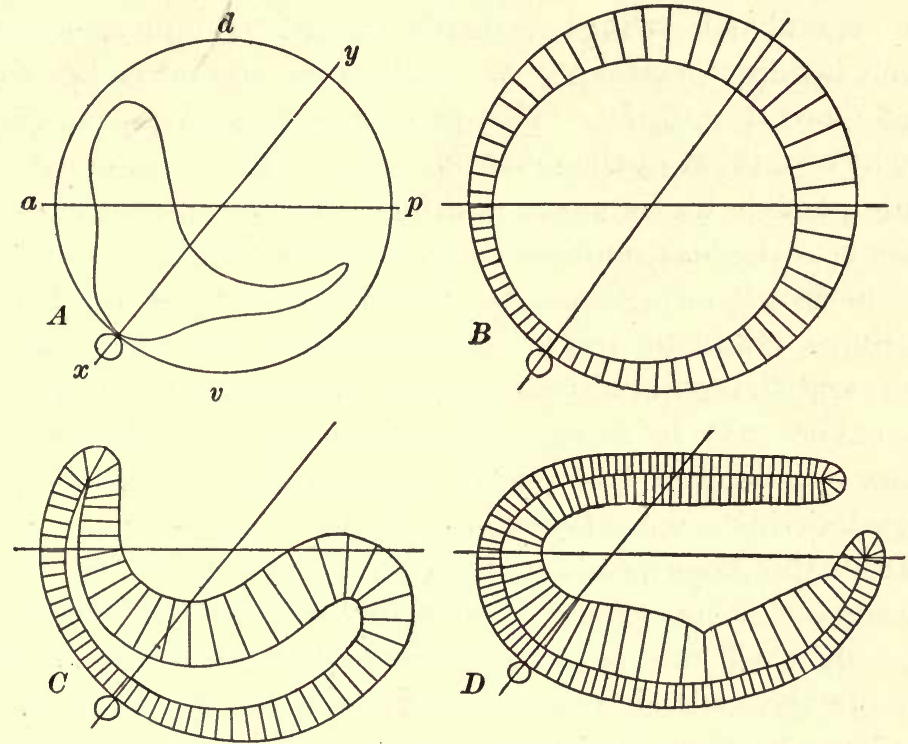

FIG. 7.-Diagrams illustrating the relations between the adult axes and the axes of the egg and early stages. After Cerfontaine. $A$. Fertilized egg. $B$. Fully formed blastula. C. Gastrulation begun. $D$. Fully formed gastrula. Note posterior elongation. $a-p$, Antero-posterior axis of adult; $d, v$, dorsal and ventral surfaces of adult; $x-y$, chief egg axis ( $x$, animal pole, $y$, vegetal pole).

the blastopore this region is less clearly marked, although distinguishable. This band of rapidly proliferating cells is to be identified as the germ ring, an embryonic region of greater importance in the other Chordates. The increase of cells at this point is so rapid that it disturbs, for a time, the simple

$a$, Animal pole; $a r$, archenteron; $b$, blastoporal opening; $c h$, rudiment of notochord; $d l$, dorsal lip of blastopore; $e c$, ectoderm; en, endoderm; $g r$, germ ring; $n c$, neuenteric canal; $n f$, neural fold; $n p$, neural plate; $s$, blastocœl or segmentation cavity; $v$, vegetal pole; $r l$, ventral lip of blastopore; $I l$, second polar body. 
epithelial arrangement of the cell layers (Fig. 6, $A$ ) and complicates somewhat the otherwise simple process of gastrulation. The transitional region between ectoderm and endoderm is the blastopore. The region of rapid cell multiplication, i.e., the germ ring, is, therefore, described as located in the lip of the blastopore, chiefly toward the dorsal side (Fig. 6, $B-G$ ).

After the cells within the dorsal lip of the blastopore have partly folded in, the flattened plate of large endoderm cells of the vegetal pole swings gradually inward without undergoing much bending or arching, as if hinged at the lower lip of the blastopore (Fig. 6, D). This process involves a much greater extent of motion in the dorsal margin of this plate, and this is made possible by the rapid multiplication of cells in the dorsal margin of the blastopore, a sheet of which is left behind as the endoderm cells swing inward. The obliteration of the blastocoel continues gradually toward the ventral margin of the blastopore, and though finally completed, for a long time a trace of the cavity may be seen in the ventral lip. Occasionally the entire blastocœl may be obliterated completely in a very early stage but this is not a typical condition.

From this description of invagination it is evident that the endodermal or inner layer cells are really of two kinds, first, the large deutoplasmic cells of the vegetal pole of the blastula forming the ventral region of the endoderm, and second, the smaller cells from the dorsal region of the transitional zone between the animal and vegetal poles of the blastula, which come first to lie in the dorsal lip of the blastopore, and then by rapid multiplication bud off a sheet of cells forming in general the dorsal region of the endoderm. This group of more active cells really lies just outside the margin of the blastopore so that the cells contributed by it to the endoderm are really turned in or inflected after their formation and a very brief existence as ectodermal cells (Fig. 6, D, E). This inflection (involution) of the marginal cells is of importance in comparing the gastrula of Amphioxus with that of other forms.

But the cells formed by divisions in the dorsal blastopora! lip (germ ring) are by no means all added to the endoderm layer. 
They contribute also to the ectoderm, and thus cause a steady extension of the dorsal lip posteriorly; that is, gastrulation is accomplished in part by epiboly, or the growth and extension backward of part of the blastoporal region. So that while at first the invaginated blastula is only little more than hemispherical in form, it soon begins to elongate, and this elongation extends chiefly posteriorly and is accomplished mainly by the rapid posterior elongation of the dorsal margin of the blastopore (Fig. 6, $\mathrm{F}-\mathrm{H}$ ).

When the invaginating phase of gastrulation is completed (Fig. 6, E) the archenteron has a nearly hemispherical form and is widely open in the dorsal or postero-dorsal direction. In Amphioxus the margin of the archenteric opening coincides with the blastopore, though we have seen that in general the blastopore should be regarded as that region where the ectoderm and endoderm are continuous, whether this borders an opening or not. As the dorsal margin of the blastopore extends backward the diameter of its opening decreases, that is, the archenteron becomes a more nearly enclosed cavity, considerably elongated posteriorly. As the blastopore closes gradually the direction of its opening becomes less dorsal and more posterior (Fig. 6, $F, G$ ). Toward the end of gastrulation the sides as well as the dorsal region of the blastoporal margin grow backward, and finally the ventral region shares in the process so that the last stages in the narrowing of the blastopore are accomplished by epiboly on every side. The result of this is the formation in the endoderm, just within the blastopore, of a band of cells, narrow below and widening laterally, which have been formed differently from the remainder of the ventral and lateral endoderm, and like the endoderm forming dorsally. This has an important bearing upon the development of later structures.

At the close of gastrulation the embryo has the form shown in Fig. 6, G. The gastrula is bilaterally symmetrical, quite elongated antero-posteriorly, flattened dorsally, rounded ventrally as well as anteriorly, while at the postero-dorsal aspect the archenteron opens directly to the outside by a narrow blastoporal aperture. The ectoderm forms a fairly uniform layer of super- 
ficial cells, and during gastrulation these have developed motile cilia, almost flagelliform, so that the gastrula is slowly rotated within the egg membranes which still envelop it. The endoderm cells lining the archenteron are somewhat unlike and of three distinct kinds, according to their origin. First there are the deutoplasmic cells, which are the deseendants of the original vegetal pole cells of the blastula, forming the greater part of the floor of the archenteron; second, cells derived from the dorsal margin of the blastopore which have been added to the endoderm through epiboly and inflection, forming a band along the roof of the archenteron; and third, around the blastopore, a rim of cells narrow ventrally but wider dorsally, formed also through epiboly and inflection, from the ventral and lateral margins of the blastopore. Thus the gastrula of Amphioxus, although superficially resembling the simple type of invaginate gastrula, such as that of many Cœlenterates, in reality is not like that, for here epiboly plays an important part in its formation. This is a leading characteristic of the gastrulas of the Chordates in general, and it is important to recognize in Amphioxus this method of gastrulation in its simplest and probably most primitive form. If one but imagines that in Fig. 6, $F$, the endoderm cells derived from the vegetal pole are multiplied and filled with a great mass of yolk, the result will be not widely unlike Fig. 32, $E$, of a section through the gastrula of the frog.

Development up to this stage has been so rapid that the completion of gastrulation occurs only six to seven hours after fertilization. During the brief period between this stage and the escape of the embryo from the egg membranes, elongation continues slowly, chiefly through the rapid multiplication of cells in a sort of "growth zone" around the blastopore. And before the close of this period certain important structures are marked out: these are, the central nervous system, the notochord, and the mesoderm and cœlom.

\section{The Formation of the Central Nervous System}

Along the dorsal flattened surface of the gastrula a median strip of ectoderm cells becomes delimited from the adjacent cells 

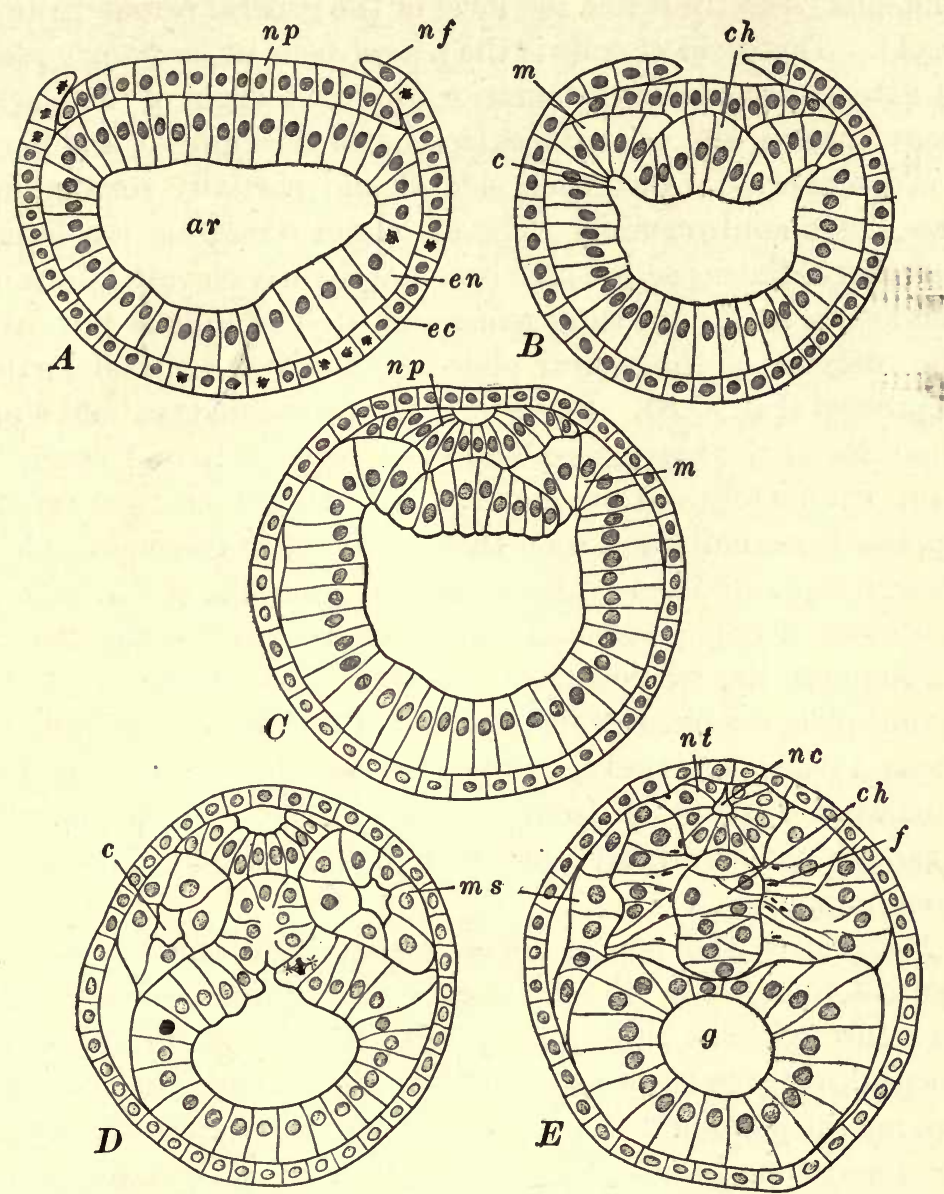

Fig. 8.- Transverse sections through young embryos of Amphioxus, showing formation of nerve cord, notochord, and mesoderm. After Cerfontaine. $A$. Commencement of the growth of the superficial ectoderm (neural folds) above the neural plate (medullary plate). B. Continued growth of the ectoderm over the neural plate. Differentiation of the notochord, and first indications of mesoderm and enterocœlic cavities. $C$. Section through middle of larva with two somites. Neural plate folding into a tube. D. Section through first pair of mesodermal somites, now completely constricted off. $E$. Section through middle of larva with nine pairs of somites. Neural plate folded into a tube. Notochord completely separated. In the inner cells of the somites, muscle fibrillæ are forming (compare Fig. 10). ar, archenteron; $c$, enterocœl; $c h$, notochord; $e c$, ectoderm; $e n$, endoderm; $f$, muscle fibrillæ; $g$, gut cavity; $m$, unsegmented mesoderm fold; $m s$, mesodermal somite: $n c$, neurocœl; $n f$, neural iold; $n p$; neural plate; $n t$, neural tube. 
and sinks slightly below the level of the general ectoderm (Fig. $8, A)$. This strip of cells is the neural plate or medullary plate. It extends from near the anterior (dorsal) margin of the blastopore forward, almost to the extremity of the embryo, and backward a short distance each side of and partially surrounding the blastoporal opening. The ectoderm bordering the lateral margins of the depressed area becomes slightly elevated, forming the neural ridges or neural folds, and these gradually flow over the margins of the neural plate as this becomes still farther depressed (Fig. 8, B). Posteriorly the neural folds extend along the sides of the blastopore, and even posteriorly and ventrally to it, completely surrounding it. The neural folds then rapidly approach medially and soon they cover over the neural plate, though separated from it by a shallow space (Fig. 8, $C$ ). These processes of depression and roofing over do not occur at once throughout the whole dorsal surface. The depression of the neural plate commences just in front of the blastopore and proceeds thence anteriorly, while the neural folds appear first somewhat further in front of the blastopore and usually, although not invariably, fuse over the neural plate first in the same region.

Posteriorly the neural folds arising from the lateral and ventral margins of the blastopore, in fusing roof over this structure without closing it, so that the blastopore no longer opens directly to the outside but into the narrow space between the neural plate and the superficial ectoderm layer formed by the fused neural folds (Fig. 6, $H$ ). In front of the point where the folds first meet, this space remains widely open upon the surface of the embryo. This opening to the cutside is called the neuropore, and as the fusion of the neural folds extends rapidly forward the neuropore is carried along toward the anterior end (Fig. 9, A). By the time of hatching it may be found at almost any point between the middle of the embryo and the anterior margin of the first somite (see below). In this latter region the neuropore remains as a definite opening until the middle of the larval period. There is some variation in different embryos as regards the region of the first fusion of 
the neural folds. In some individuals, and these have usually, and erroneously, been described as the more typical, the folds meet first over the blastopore and gradually fuse thence anteriorly. The more frequent relation seems to be that just described, and it is important to recognize that this agrees with the method of closure of the neural folds in practically all of the Craniates, where they meet and fuse first in the middle or anterior to the middle of the embryo, and then fuse in each direction from that region. Amphioxus differs, however, from all other Chordates in that the margins of the neural plate do not remain connected with the neural folds for a time, and are not elevated and closed into a tube at the same time the neural folds close together: the folding of the neural plate into a tube will be described presently.

\section{The Formation of the Notochord}

The chorda develops more slowly than the nervous system and by the time of hatching has hardly more than commenced its formation. The rudiment of the notochord is a median strip of endoderm, six to nine cells wide, forming the roof of the archenteron and lying consequently just beneath the neural plate and in contact with its lower surface (Fig. 8, $B$ ). The depression of the neural plate depresses also the flattened dorsal wall of the archenteron, so that the chorda rudiment pushes down into the archenteric cavity and appears in section concavely arched. The chorda cells are those inflected at the dorsal margin of the blastopore together with their descendants. While the gastrula or embryo is elongating the rudiment of the notochord divides posteriorly, passing a short distance around each side of the blastopore, and terminates in a growth zone similar to that concerned in the extension of the neural plate but lying just inside instead of outside the blastoporal rim (Fig. 9, B). At the time of hatching the rudiment of the chorda still remains as a flat plate of cells directly continuous, laterally and anteriorly, with the endoderm lining the remainder of the archenteron. 


\section{The Formation of Mesoderm and Enterocoels}

In the gastrula the rudiments of the middle germ layer are found in a pair of longitudinal mesoderm bands of cells iying either side of the chorda in the dorso-lateral regions of the endodermal archenteric wall. Posteriorly these bands diverge and pass either side of the blastoporal opening nearly or quite to its ventral side. The extent of the mesoderm, as of the neural plate and notochord, is increased chiefly by the addition of cells from this blastoporal region (germ ring). At the close of gastrulation therefore we can distinguish two general regions of mesoderm; first, that lying either side of the anterior part of the chorda, formed from the inflected dorso-lateral margins of the blastopore and known as gastral mesoderm, and second, that formed from the lateral and ventro-lateral margins of the blastopore, known as peristomial mesoderm. The gastral mesoderm is the earlier formed and remains limited to the anterior region, while the peristomial mesoderm forms later and during a long period after gastrulation is completed, and really constitutes all the mesoderm posterior to the very limited, and anterior gastral mesoderm. At the close of gastrulation the only difference between the two kinds of mesoderm is that of time and place of origin for they are directly continuous and not visibly differentiated from one another.

The depression of the notochordal plate, consequent upon the formation of the nerve cord, results in a rather sharp folding longitudinally of the dorso-lateral mesoderm bands, and the formation from the archenteric cavity there of a pair of longitudinal grooves (Fig. 8, B). These grooves are the first indications of the enterocolic cavities and their walls are to be spoken of as the mesoderm folds. The enterocœlic grooves extend nearly the entire length of the archenteron and, though at first shallow, rapidly deepen, particularly in the anterior region. The mesoderm folds soon become sharply differentiated from the adjoining chorda and endoderm (Fig. $8, C)$, and very early their continuity becomes interrupted by the appearance of paired transverse folds dropping down from 
their dorsal walls. The first pair of the transverse folds appears a short distance back from the anterior ends of the
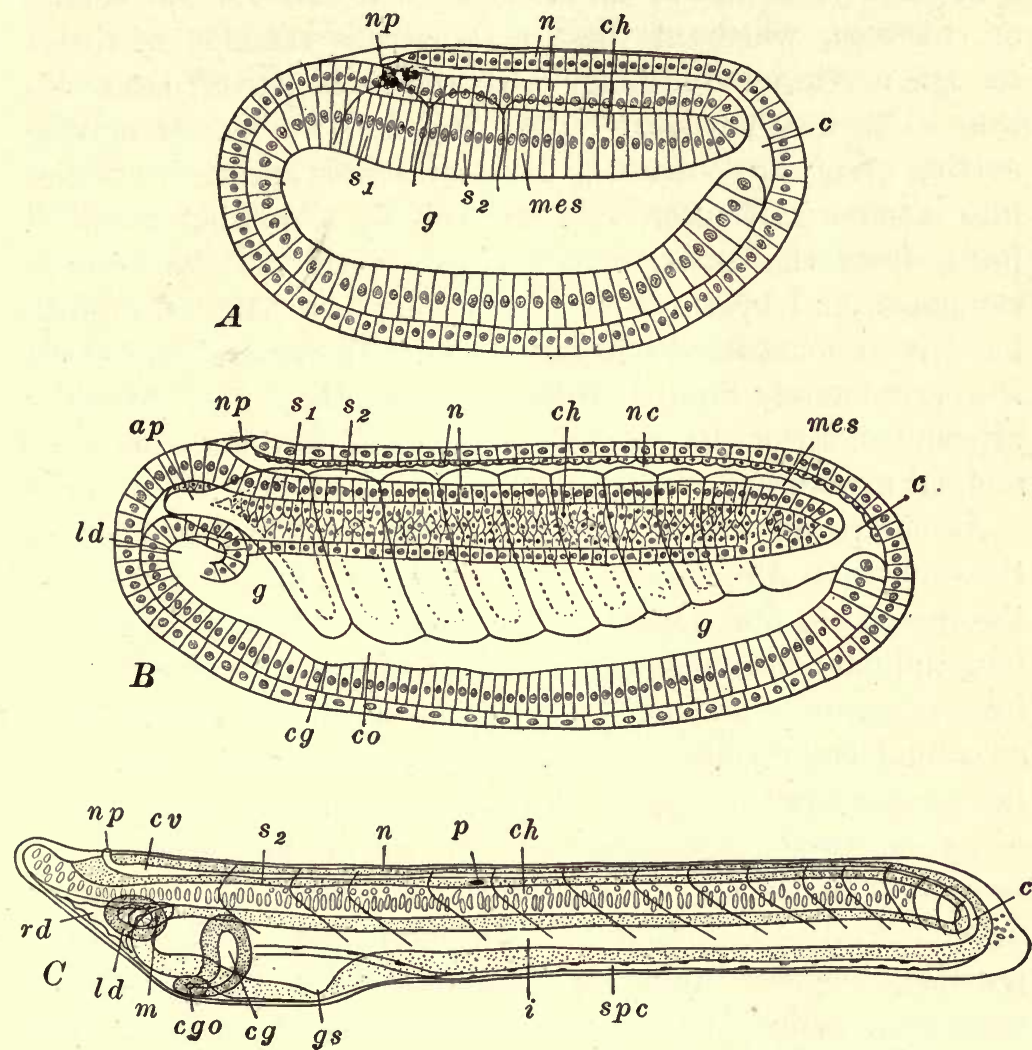

Fig. 9.-Optical sections of young embryos of Amphioxus. After Hatschek. The cilia are omitted. $A$. Two-somite stage, approximately at the time of hatching, showing relation of neuropore and neurenteric canal. $B$. Ninesomite stage, showing origin of anterior gut diverticula. C. Fifteen-somite stage. End of the embryonic period. $a p$, Anterior process of first somite; $c$, neurenteric canal; $c h$, notochord (or its rudiment, in $A$ ); $c g$, club-shaped gland (or its rudiment, in $B$ ); $c g o$, external opening of club-shaped gland; $c o$, cœlomic cavity of somite; $c v$, cerebral vesicle; $g$, gut cavity (enteron, mesenteron); $g s$, rudiment of first gill slit; $i$, intestine; $l d$, left anterior gut diverticulum (preoral pit in $C$ ); $m$, mouth; mes, unsegmented mesoderm; $n$, nerve cord (or its rudiment, in $A$ ); $n p$, neuropore; $p$, pigment spot in nerve cord; $r d$, right anterior gut diverticulum (preoral head cavity in $C$ ); $s_{1}, s_{2}$, first and second mesodermal somites; $s p c$, splanchnocœl (body cavity).

mesoderm folds and soon cuts off this region, forming thus the first pair of mesodermal somites, as they are called. The 
somites are at first nearly spherical or cubical, box-like divisions, each containing a portion of the original archenteric space now to be known as the enterocoelic cavity of the somite or enterocœl, which in these early stages remains in direct though narrowed continuity with the archenteron; later this connection becomes entirely lost (Fig. 8, D). This transverse cutting up of the continuous mesoderm folds and their cavities into somites and enterocœls proceeds, in somewhat modified form, from the anterior region posteriorly, as the embryo elongates, and by the time of hatching two pairs of somites have been constricted off (Fig. 9, $A$ ). The gastral mesoderm is approximately limited to the region of these first two pairs of somites, all of the posterior remainder forming from peristomial mesoderm.

As compared with the higher Chordates the formation of the somites in Amphioxus begins very early, for in those forms the mesoderm first separates from the endoderm as a pair of longitudinal bands which later and only in part become divided into segments or somites. The formation of the somites here, as actual enterocœlic diverticula from the archenteron, has been considered of great theoretic importance on account of its supposed primitiveness, for in all of the Craniates the mesoderm bands are at first solid and later develop a cavity never directly continuous with the archenteron. It is quite possible, however, that the formation of solid peristomial mesoderm bands is the primary arrangement and that the connection between the mesoderm cavity and the archenteron, which we shall see is limited to the somites of gastral mesoderm, is in reality a secondary condition.

The first phase of embryonic development is terminated about eight to fifteen hours after fertilization by the escape of the embryo from the egg membranes, within which it has been enclosed. During the remainder of the embryonic period it swims freely near the surface by means of the covering of long ectodermal cilia. We may conveniently summarize the characters of the embryo at the time of hatching as follows (Fig. 9, A). The embryo is in general cylindrical with a flat- 
tened dorsal surface; length nearly twice the diameter, and the diameter about equal to that of the egg. External surface completely ciliated. Blastopore completely roofed, so that the only external opening is the neuropore, opposite the anterior margin of the first somite. Mesoderm folds are formed throughout and two pairs of somites are constricted off anteriorly. Notochord delimited but not developed. Archenteron open to the outside only by way of the roofed over blastopore, through the space between neural plate and superficial ectoderm, to neuropore. All organs and parts are still formed of a single layer of epithelially arranged cells.

During the remaining twenty-eight to sixty hours of the embryonic period the chief developmental processes consist in the elaboration of the rudiments already established rather than the mapping out of new organs.

\section{B. The Embryonic Period, from Hatching to the Forma- TION OF THE Mouth}

\section{The Central Nervous System}

If not already accomplished, the neural plate is now rapidly roofed over and the neuropore is carried to the anterior margin of the first somite, where it remains throughout the larval period. Next the neural plate is converted into a tubular structure, the neural tube, through the depression of its median region and the accompanying rolling up and together of its margins (Fig. 8). As in other Chordates this process begins in the region of the first somite and extends thence posteriorly and anteriorly. The original space between the neural plate and the covering ectoderm becomes the cavity of the neural tube or neurocœl (Fig. 9, B). The cilia of these originally superficial cells now line the neurocol and continue to beat toward the posterior end of the tube. In the region of the blastopore the neural plate remains in connection with the continuous ectoderm and endoderm of that region, so that the neurocœel does not close posteriorly, but leads directly into the opening of the blastopore and so to the archenteron. This 
passage from the neurocœl to the archenteric cavity by way of the blastoporal opening, is the neurenteric canal. This canal remains open throughout the embryonic period, until after the mouth opening is formed, so that this, in connection with the neurocœl and neuropore, forms the only path by which the archenteron is in connection with the exterior (Fig. 9, $A, B, C$ ). The beating of the cilia lining the neurocœl probably keeps up an interchange of fluids between enteron and the outside.

Opposite the anterior margin of the first somite the medullary tube is somewhat enlarged (Fig. 9, C), while opposite its posterior margin, and, indeed, throughout the remainder of the tube, it becomes somewhat narrowed by the elongation of that part of the embryo, and a so-called brain region is thus marked out. About the close of this period, pigment spots begin to appear in the neural tube opposite the fifth pair of somites (Fig. 9, C): these are the first indications of the development of sense organs and are doubtless photo-receptors. The large cranial pigment spot in the anterior wall of the brain appears about the close of the embryonic period.

\section{The Notochord}

The establishment of the chorda from the plate of endoderm cells lying between the mesoderm folds, commences in the region between the first and second somites (Fig. 8). Here the plate becomes arched, its lateral halves folding together ventrally. The folding extends posteriorly and anteriorly from this region, and soon the folded plate is formed into a solid strand lying below the neural tube, between the mesodermal somites (Fig. 8, E). Posteriorly the chorda extends to the anterior wall of the neurenteric canal, and anteriorly it slowly forms to the very extremity of the embryo, and therefore in advance of the somites and neural tube (Fig. 9, $B, C)$. The extension of the chorda in front of the brain is a peculiarity of Amphioxus, for in all other Chordates it forms only to the region of the mid-brain. The chorda cells from the two sides grow across the mid-line, interlocking and later 
extending from each side completely across the entire chorda. By the time nine or ten somites are formed the notochord becomes completely cut off from the endoderm layer, and the two margins of the endoderm which were originally along the outer margins of the mesoderm folds, come together in the mid-line beneath the notochord, enclosing what remains of the archenteron, as the definitive gut cavity or enteron (mesenteron) (Fig. 8, E). Finally vacuoles appear in the notochord and, increasing in size, lead to the obliteration of the cellular structure. The nuclei are displaced dorsally and ventrally and a typical "notochordal tissue" is formed before the close of the embryonic period (Fig. 9, $C$ ).

\section{The Mesodermal Somites and Calom}

After hatching, mesodermal somites posterior to the two then present continue to form successively in the elongating mesoderm folds. The cavities of the first two pairs do not become completely separated from the archenteron until six to eight pairs have been formed and constricted off, and their enterocœels remain as definite spaces throughout their development. In the somites just posterior to the first two pairs the enterocoels close for a time just after the somites are formed, and in the more posterior ones, even before the mesoderm is quite cut off from the endoderm. In these somites cavities reappear later, and the enterocœls may be said to be virtually if not actually present throughout, for the cells composing their walls remain definitely arranged about a central point in the regions of the enterocœls (Fig. 10). Still farther posteriorly the mesoderm folds develop as solid masses without any cavities whatever at any time during their formation, but the condition in the anterior region demonstrates that Amphioxus is a true enterocolomate organism. By the time fourteen pairs of somites have been formed the mesoderm folds cut off completely from the archenteric wall, and the remainder of the mesoderm is formed directly from the proliferating cell region around the neurenteric canal (blastopore), without ever having been included as a part 
of the endodermal wall of the archenteron. At the close of the embryonic period (Fig. 9, C) fourteen or fifteen pairs of somites have been formed, and posterior to these remain short undivided regions of mesoderm from which additional somites will be formed during the larval period. These somites are of course not all in the same stage of development, but passing along the

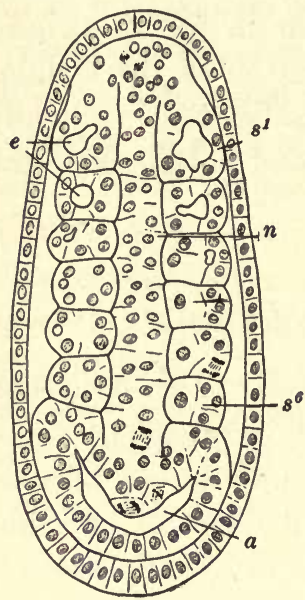

Fig. 10.-Frontal section through Amphioxus larva with six pairs of mesodermal somites, at the level of the notochord and somites. After Cerfontaine. $a$, Archenteron; $e$, enterocœl; $n$, notochord; $s^{1}, s^{6}$, first and sixth mesodermal somites. series in the anterior direction they represent successively more advanced conditions. Advance is indicated by increase in the size of the cavity of the somite, thinning of the wall, and the ventral and somewhat posterior extension of the somite between the wall of the gut and the superficial ectoderm (Fig. 8, E). By the end of the embryonic period the more anterior pairs are already bent into the <-form characteristic of the adult. The first pair of somites send forward unusual extensions from the antero-dorsal region along each side of the notochord (Fig. 9, B): it is possible, though hardly likely, that these indicate the former presence of an additional pair of somites in this region: it seems more likely that the condition is the result of the anterior prolongation of the notochord beyond the proper region of the first somite. The walls of these extensions later go through the same developmental history as those of a typical somite.

By the time five or six pairs of somites are formed they begin to show that bilateral alternation which is so characteristic of the adult (Fig. 10). Only the first and the upper part of the second pairs of somites lie exactly opposite. In the other pairs the left member comes to lie in advance of the right, and farther posteriorly the segments of the two sides exactly alternate. It is also noticeable that the left side develops slightly in advance 
of the right, e.g., when there are eight somites on the left there are but seven on the right.

The enlargement and ventral elongation of the somites mentioned above is accompanied by a differentiation of their walls. That part of the somite lying just beneath and in contact with the ectoderm becomes thinner and forms what is known as the parietal wall of the somite, or the somatic mesoderm (Fig. 8, E); the region in contact with the wall of the gut or enteron also becomes thinner and forms the visceral wall or splanchnic mesoderm: while the more restricted portion bordering the chorda thickens by the horizontal flattening and antero-posterior elongation of the cells through the extent of the somite. This last region of the somite is called the myotome, while the somatic and splanchnic regions together are termed the lateral plate. In the myotome the cells begin, as early as the nine-somite stage, to differentiate as muscle cells (Fig. 8, E) forming a muscular epithelium. At the close of the embryonic period the more anterior myotomes are capable of muscular contractions.

The thinning of the parietal and visceral walls of the somite and their downward extension, considerably enlarge the enclosed cavity and carry it down around the sides of the enteron. The cavities of the somites now become recognizable as the beginnings of the colomic space. The enlargement of the myotome partially obliterates the cavity of the somite in the dorsal region, the small cavity remaining being termed the myoccel. The larger cavity of the lateral plate is then distinguished from the myocœl by the term splanchnocoel. Before the close of the embryonic period the ventral walls of the more anterior somites push completely around the enteron and meet in the midventral region, separating the enteron from contact with the ectoderm. Presently the median walls of the splanchnocols for the most part disappear, and the splanchnocœls of each pair of somites fuse more or less completely. Remnants of these median partitions below the enteron appear to remain as the rudiments of the subintestinal vein and branchial artery. Finally the somite becomes divided by a horizontal partition between the lateral plate and myotome, and the corresponding 
cavities are completely separated. By the close of the embryonic period the anterior and posterior walls of the somites in the region of the splanchnocœls also break through, leaving these cavities continuous antero-posteriorly as well as transversely. The unsegmented cavity thus formed is the colomic cavity of

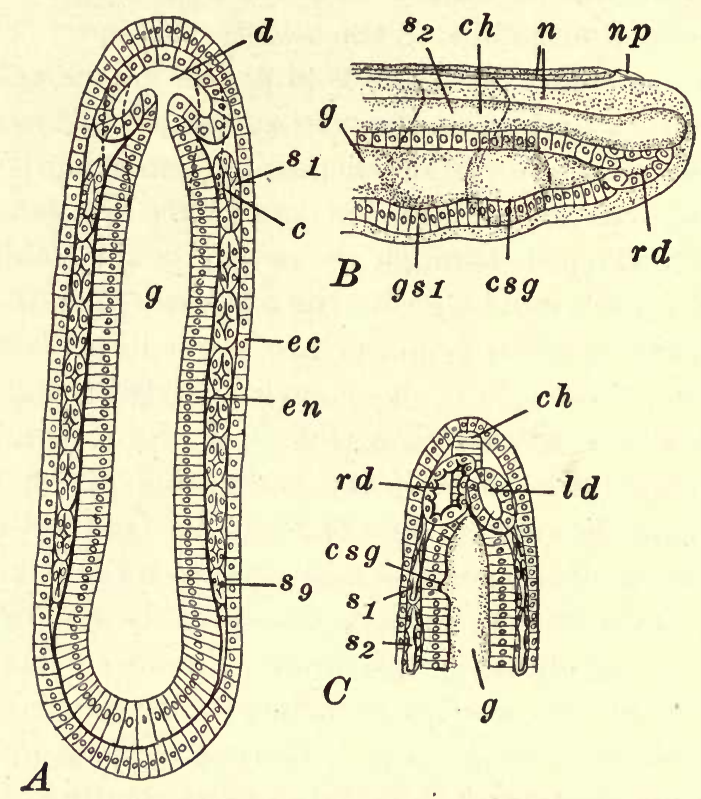

FIG. 11.-Sections through young Amphioxus embryos showing the origin of the anterior gut diverticula. After Hatschek. The cilia are omitted. $A$. Frontal section through embryo with nine pairs of somites. (See Fig. 9, B). The dotted line marks the course of the gut wall ventral to the level of the section. $B$. Optical sagittal section through anterior end of embryo with thirteen pairs of somites, showing position of right anterior gut diverticulum. $C$. Same in ventral view. $c$, Cœlomic cavity of somite; $c h$, notochord; $c s g$, rudiment of club-shaped gland; $d$, rudiment of anterior gut diverticula; $e c$, ectoderm; en, endoderm; $g$, gut cavity (enteron, mesenteron); gs1, rudiment of first gill slit; $l d$, left anterior gut diverticulum; $n$, nerve cord; $n p$, neuropore; $r d$, right anterior gut diverticulum; $s_{1}, s_{2}, s_{9}$, first, second, and ninth mesodermal somites.

the embryo and larva which is seen to be a true syncolom (Fig. 19, A).

There remain to be described, in connection with the development of the mesoderm, a pair of outgrowths from the anterior end of the enteron in front of the first pair of mesodermal somites. These cavities are known as the anterior gut diverticula 
and have been commonly regarded as endodermal derivatives of peculiar character, but although their exact homologies remain rather obscure it seems clear that they are essentially a pair of mesodermal somites which develop late, on account of the delayed forward extension of the whole anterior tip of the body. The anterior gut diverticula first appear in the embryo with seven pairs of somites, as a pair of narrow anterodorsal evaginations from an expanded anterior region of the enteron (Figs. 9, $B ; 11, A, B$ ). These push forward much like the somites, and quickly pinch off from the enteron as a pair of closed sacs (Fig. 11, C).

Although symmetrical in their origin they differ greatly in their subsequent development. In the embryo with ten pairs of somites the right diverticulum begins to grow forward and to enlarge while its wall becomes very thin. Soon it extends across the mid-line and finally, as a median structure, it occupies the whole of the space below the chorda and from the enteron forward to the tip of the embryo. It remains wholly in front of the first somite and forms what is known as the preoral cavity or head cavity (Fig. 9, C). The left anterior gut diverticulum differs widely from its antimere. It remains quite small and unmodified throughout the embryonic period, shortly after which it acquires an opening to the surface in the left side of the head below the level of the chorda, and is then known as the preoral pit (Fig. 9, $C$ ). Its history is quite complicated and will be considered later.

\section{The Enteron and its Appendages}

After the wall of the archenteric cavity has lost successively the mesoblast folds, notochord, and anterior gut diverticula, the endodermal tube remains as the wall of the enteron or mesenteron. This gives rise to the epithelial lining of the alimentary canal and related structures. After the time of hatching the enteron elongates rapidly and, except at its anterior extremity, narrows very markedly, differentiating in this way the posterior stomach-intestine from the wide anterior pharyngeal region 
opposite the first two somites (Fig. 9, C). Throughout the embryonic period, as described above, the only opening to the exterior is by way of the neurenteric canal, neurocœl, and neuropore, the essential relations among which do not change during this period. We may describe briefly the chief structures arising in connection with the enteron during the embryonic period.

\section{A. THE CLUB-SHAPED GLAND AND ENDOSTYLE}

In the floor of the enteron, just back of the anterior gut diverticula, and therefore opposite the anterior margin of the first somite, a transverse groove appears about the time nine or ten somites are present (Figs. 9, B; 11). During the latter part of the embryonic period this groove constricts off as a narrow tube, separate from and below the gut, and acquires an opening at its left end to the exterior in the region below the preoral pit, while the right end dilates into a conspicuous sac, closed at this time (Fig. 9, $C$ ).

Lying just in front of the grooved rudiment of the club-shaped gland, is a strip of high ciliated cells which seems to be the first indication of the endostyle. The later development of the gland and endostyle is described in connection with the history of the larval period.

\section{B. THE MOUTH}

The mouth, first gill slit, and anus develop almost simultaneously, toward the close of the embryonic period. At the age of fourteen somites a large patch of ectoderm on the left side of the head, along the margin of the first somite, thickens considerably and with this thickened area the adjacent wall of the enteron fuses. This fused region then becomes perforated by a small opening (Fig. 9, $C$ ), which rapidly enlarges and becomes the relatively enormous mouth opening bordered with elongated cilia (Fig. 12). 


\section{THE FIRST GILL SLIT}

At the same time the mouth is forming, the endoderm pushes out ventrally, opposite the second somite, and fuses with another thickened patch of ectoderm (Fig. 9, C). Perforation follows and forms the first gill slit which rapidly enlarges and moves up on the right side of the head, nearly opposite
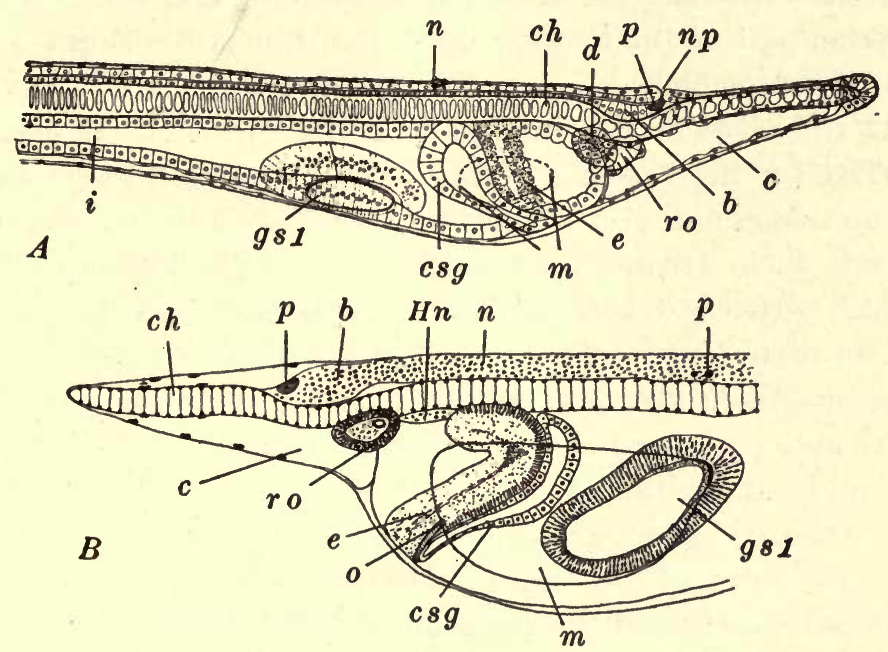

FIG. 12.-Anterior ends of Amphioxus larvæ, in optical section. A. One gill slit stage, from right side. After Hatschek. $B$. Four gill slit stage, from left side. After Lankester and Willey (mouth added). $b$, Brain region; $c$, cœlom (preoral head cavity); $c h$, notochord; $c s g$, club-shaped gland; $d$, dorsal portion of preoral pit (later forming the groove of Hatschek and Hatschek's nephridium); $e$, rudiment of endostyle; gs 1 , first gill slit; $H n$, Hatschek's nephridium; $i$, intestine; $m$, mouth; $n$, nerve cord; $n p$, neuropore; $o$, external opening of clubshaped gland; $p$, pigment; ro, Räderorgan; $v$, cerebral vesicle.

the mouth (Fig. 12, $A$ ). It remains smaller than the mouth but is similarly bordered with long cilia.

\section{THE ANUS}

Very shortly after the mouth and first gill slit are formed the wall of the narrow enteron, immediately below the neurenteric canal, fuses with the ectoderm and the region is perforated as the anus. At first this lies in the mid-line but later is displaced toward the left, by the development of the provisional caudal 
fin. Upon the formation of the anus the intestine is separated from the neurenteric canal and this structure closes and graduually moves posteriorly, away from the anal region, as the tail region grows out; but for some time the tip of the nerve cord remains curved around toward the region of its original connection with the enteron.

About the time the mouth and anus open, the alimentary tract becomes ciliated and a small diverticulum forms in the anterior part of the stomach-intestine; this is the rudiment of the cæcum or liver.

At the close of the embryonic period, which is arbitrarily marked by the opening of the mouth, the embryo is about $1.0 \mathrm{~mm}$. long and very slender (Fig. 9, C). Probably its actual bulk is little greater than that of the egg. The embryo is almost perfectly transparent, and swims about with the elongated ectodermal cilia, accompanied by irregular muscular contractions when strongly stimulated. The anterior end is dilated and prolonged forward into a rostrum, containing the preoral head cavity and supported by the elongated notochord. The external openings are, the mouth and first gill slit on opposite sides of the head, above these the small median neuropore, and near the posterior end the small ventral anus. Posteriorly the tail is expanded into a provisional caudal fin formed of elongated ectodermal cells. There are about fifteen pairs of somites in every stage of development, from the completely undifferentiated condition of the posterior pairs of the series, to the quite completely differentiated anterior pairs. Anteriorly the nervous system shows the rudiment of the brain; posteriorly it is no longer connected with the enteron on account of the closure of the neurenteric canal. The notochord is completely established. In addition to the mouth and first gill slit the enteron has formed the anterior gut diverticula and clubshaped gland, all of which have become separate structures and commenced their differentiation. In spite of its rather complex structure all parts of the embryo are still of epithelial character, though the epithelia now show some degree of elementary cellular differentiation. 


\section{THE LARVAL PERIOD}

The duration of this period is roughly about three months, during which the larva is free swimming but descends to deep water. Development is very slow and consists largely in the elaboration and modification or multiplication of structures indicated at its beginning. Although up to this time development has been simple and typical (primitive) in general, now the anterior portion of the larva becomes highly modified through the development of characters not found in the Craniata. These are, chiefly, the extensive asymmetry of the pharyngeal and associated structures, and the development of the atrium or peribranchial cavity-both to be regarded as special adaptations to the habits of the larva. The close of the larval period is marked by an extensive metamorphosis which consists largely in a "symmetrization" of the anterior end. At the close of this period the larva is said to be at the "critical stage."

\section{The Central Nervous System}

Very soon after hatching the walls of the spinal cord thicken, constricting the neurocœl, and becoming differentiated into three regions. These are $(a)$, a thin epithelial layer-the ependyma, lining the neurocœl, (b), dorso-lateral and lateral columns of nerve-cell bodies (gray substance) connecting respectively with the dorsal and ventral spinal nerves, and (c) ventral columns composed of nerve fibers (white substance).

In the brain region the walls become comparatively thin and the cavity dilates considerably (Fig. 13). At first the brain lies beneath the epidermis, and around the neuropore its wall is directly continuous with the superficial ectoderm. When the dorsal fin appears in the mid-line the neuropore is pushed to one side, usually the left. Gradually the brain sinks away from the epidermis, drawing down the neuropore into a funnel-shaped depression, and at the same time drawing out the antero-dorsal wall of the brain into a conical elevation, 
the two remaining connected by a very small tube. Not until after metamorphosis does this opening become entirely closed and the neuropore then remains as a ciliated funnel known as the olfactory pit, which retains an innervation from the elevated region of the brain and becomes sensory in character, the assumption being that it functions as an olfactory
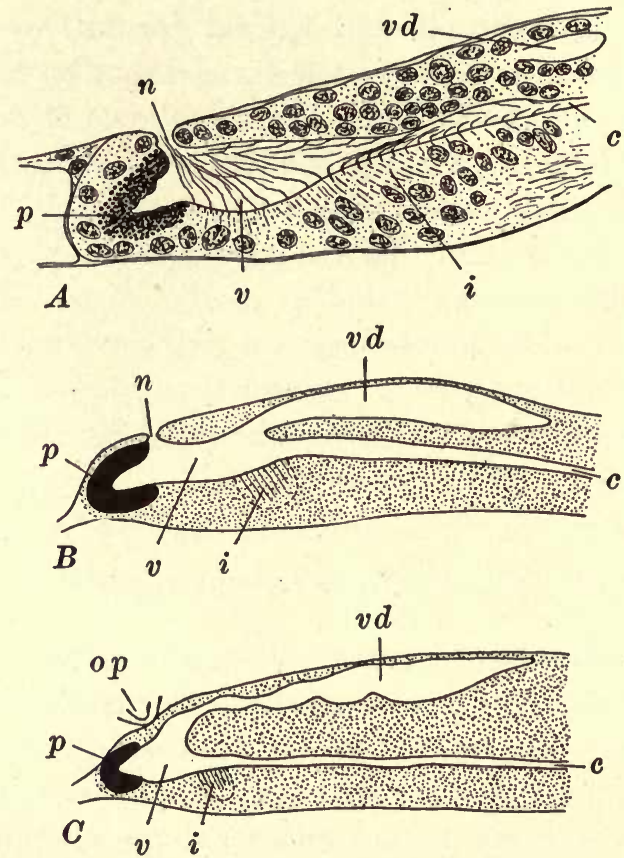

Fig. 13.-Median sagittal sections through the brain of Amphioxus. After Boeke. A. Of a larva with seven primary gill slits. $B$. Diagram of a section through the brain of a larva of $2.25 \mathrm{~mm}$., with five pairs of primary gill slits, $\times$ 933. C. Same of a young specimen of $10 \mathrm{~mm}$. $\times 233 ; c$, Central canal of cord; $i$, infundibulum; $n$, neuropore; $p$, cerebral pigment; $o p$, olfactory pit; $v$, cerebral vesicle; $v d$, postero-dorsal extension of cerebral vesicle.

organ. This antero-dorsal outgrowth of the brain may correspond with the lobus olfactorius impar which marks the morphologically anterior end of the brain of the Craniates. Just in front of and below this is the large cranial pigment spot (Fig. 13) in the general region from which the olfactory lobes grow out in higher forms. Postero-dorsally the brain wall is 
thickened by an accumulation of ganglion cells, and in its posterior part two important structures appear. Just in front of the opening of the cavity of the cord the ependymal cells elongate forming a small median pad which is regarded as the infundibulum (Fig. 13). Posterior to the cerebral vesicle proper is an extensive dorsal diverticulum of the neurocœl: this has been compared with the $I V$ ventricle of Craniates.

The simple character of the brain of Amphioxus is obviously correlated with the general lack of special sense organs, particularly with the absence of the important optic and auditory organs, and the feebly developed olfactory apparatus.

\section{The Gill Slits}

We may describe first the general morphological arrangements of the developing gill slits: the development of the first gill slit has already been mentioned.

Although finally symmetrical and paired organs the gill slits of the right and left sides develop independently, those of the left side first. These appear successively in the midventral line, posterior to the first gill slit, and as they form they shift upward, on the right side of the pharynx (Fig. 14). These are called the primary gill slits, and when twelve to fifteen, typically fourteen, have appeared, their formation ceases. At this time the more anterior slits are large ovoid openings lying well up on the right side; posteriorly they diminish in size and lie toward the mid-line. In all these early stages they are metameric and correspond with the somites, a correspondence which is entirely lost later on. When the full number of fourteen primary gill slits has been formed, the secondary gill slits, or those of the proper right side, appear, also on the right side of the pharynx and dorsal to the primary series. Their first indication is a longitudinal ridge, in which appear six thickenings alternating with the primary gill slits in the region between the third and ninth of these (Fig: $14, B$ ).

The relations of these parts of the pharynx will become clear 
if it be imagined that the formative centers located in the most anterior region of the pharynx have been twisted out of their normal positions, so that the morphological mid-ventral
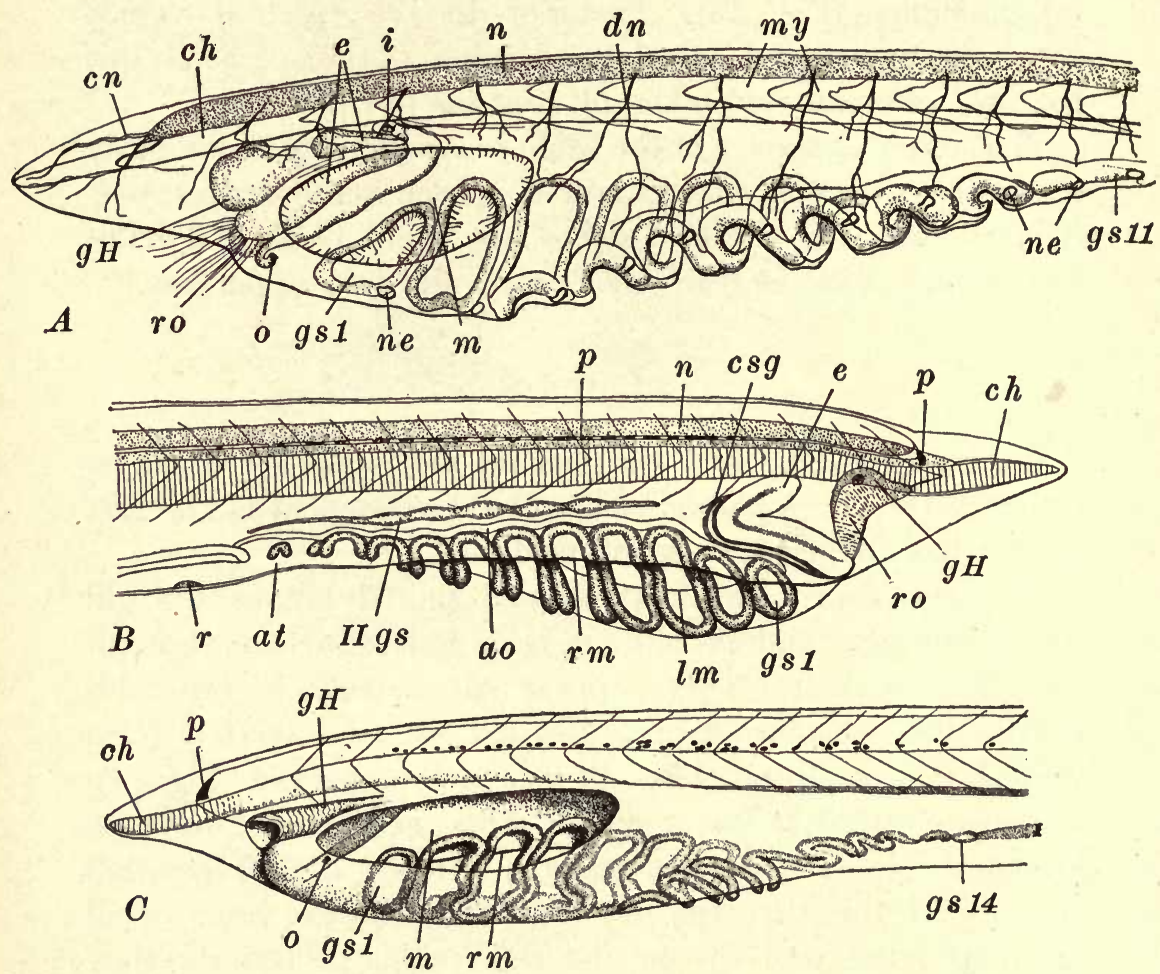

FIG. 14.-Anterior ends of Amphioxus larvæ. A. Larva with eleven primary gill slits, from left side. After Goodrich. B. Larva with eleven primary gill slits, from right side showing metapleural fold and secondary gill slit rudiments. After Willey. $C$. Larva with fourteen primary gill slits, from left side. After Lankester and Willey. ao, Ventral aorta (branchial artery); at, atrial cavity; $c h$, notochord; $c n$, cranial nerve; $c s g$, club-shaped gland; $d n$, dorsal spinal nerve; $e$, endostyle; $g H$, groove of Hatschek; $g s 1, g s 11$, gs14, first, eleventh, and fourteenth primary gill slits; II gs, rudiments of secondary gill slits; $i$, internal opening of club-shaped gland; $l m$, lower margin of mouth; $m$, mouth; $m y$, myotomes; $n$, nerve cord; ne, nephridium; $o$, external opening of club-shaped gland; $p$, pigment in nerve cord; $r$, renal cells in floor of atrial cavity; $r m$, edge of right metapleural fold; ro, Räderorgan.

line becomes displaced to the topographical right side, and structures morphologically of the right and left sides appear, respectively, dorsally and ventrally on the right side alone. 
The rudiments of the secondary gill slits enlarge and become perforated, and then an additional perforation appears at each end of the series making eight in all. As these enlarge, chiefly in the ventral direction, the primary gill slits below them are moved down to the ventral side, and then over on the left side of the pharynx to their proper position (Figs. 14, $C ; 15$ ). As a result of this migration the dorsal and ventral margins of the primary gill slits are reversed, and it is evident that the morphological ventral margins of these actually appeared first to lie dorsally. As this migration is going on, first the secondary, then the primary gill slits become divided by a downward extension of a tongue-like process from the dorsal wall of the slit which finally reaches the ventral side, divides the originally simple opening into two, and forms the tongue bar of the completed structure of the adult (Fig. 15). These tongue bars appear in all the secondary slits except the first, and in all of the primary slits except the first two and a variable number of the last. As the primary gill slits approach their final position the most anterior disappears completely (Fig. 15) and soon after, the last five also disappear, reducing the number in the primary series to eight, the same as the number originally appearing in the secondary series. This is the arrangement of the gill slits at the critical stage, the symmetrization of these structures being one of the important phases of metamorphosis. After the reduction of the gill slits to eight symmetrical pairs, arranged metamerically, additional pairs form slowly, posterior to the primary and secondary. These are the tertiary gill slits. They are not metameric in their appearance and they displace anteriorly the primary and secondary slits, so that the whole gill slit series loses its metameric arrangement.

Regarding the actual details of the formation of the gill slits little is known except in certain of the secondary series, or those of the right side proper. In these, and presumably in most of the primary series also, true gill pouches seem to be formed first, purely of endodermal cells. These are drawn out ventro-laterally into small tubes closed by a very thin 
superficial layer of transparent ectoderm cells. The tongue bar grows down like a stalactite from the roof of the gill pouch, and just before it reaches the ventral side of the pouch the ectoderm is perforated opposite the tongue bar. But by this time the atrial cavity has formed in this region, as will be described later, so that the gill slits never open directly to the

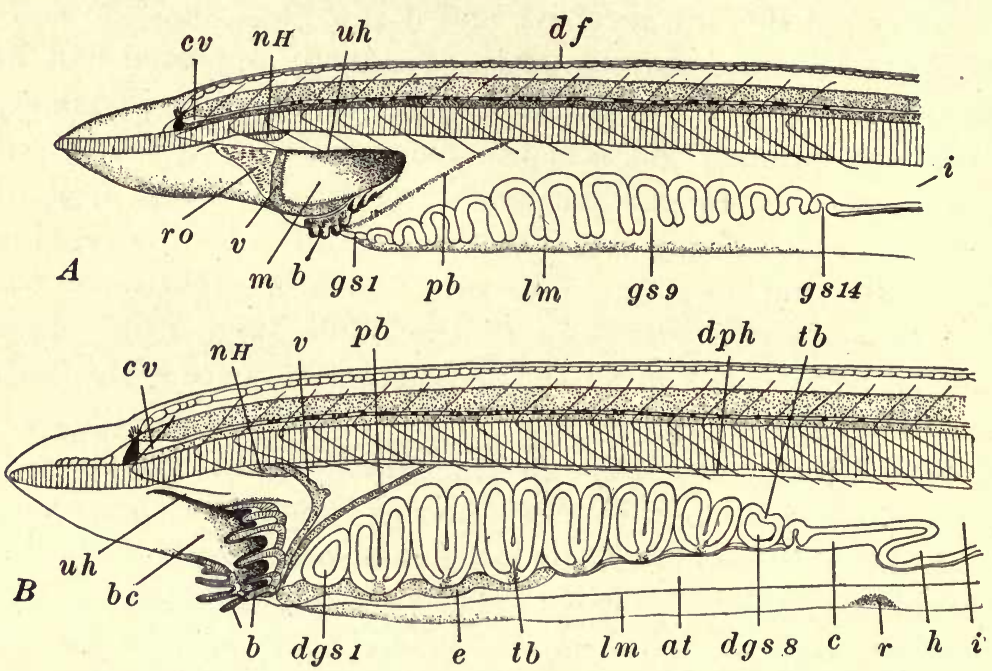

Fig. 15.-Anterior ends of Amphioxus larvæ showing the migration of the mouth, formation of tongue bars, reduction in primary gill slits, etc. After Willey. A. Larva with fourteen primary gill slits nearly in their definitive position on the left side. Oral hood in process of formation. B. Larva with nine primary, and eight secondary gill slits. Mouth nearly in its definitive anterior position and oral hood nearly completed. This larva has nearly reached the "critical stage." at, Atrial cavity; $b$, buccal (oral) cirri; $b c$, buccal cavity (cavity of oral hood); $c$, cœlom; $c v$, cerebral vesicle; $d f$, dorsal fin chambers; $d g s 1, d g s 8$, first and eighth definitive gill slits (second and ninth of the primary series); $d p h$, dorsal wall of pharynx (region of epipharyngeal groove); $e$, endostyle; gs $1, g s 9$, $g s 14$, first, ninth, and fourteenth primary gill slits; $h$, hepatic cæcum; $i$, intestine; $l m$, lower margin of left metapleural fold; $m$, mouth; $n H$, Hatschek's nephridium; $p b$, peripharyngeal band; $r$, renal papilla in floor of atrial cavity; ro, Räderorgan. $t b$, tongue bar; $u h$, upper margin of oral hood; $v$, velum.

outside; the more anterior primary gill slits apparently form exceptions to the usual arrangement in opening directly for a time (Fig. 17). As the external openings of the pouches enlarge, the tongue bars move to the surface and if it is true that they are completely covered with endoderm, the atrial 
cavity on which they border must therefore to this extent be lined with endoderm (Legros).

\section{The Club-shaped Gland}

The club-shaped gland lies far forward in the pharyngeal region, and does not share in the shifting of the gill slits and other derivatives of the pharynx with which it is at this time connected only at a single point. During the early larval period its dilated right end narrows and acquires an opening into the pharynx (Fig. 14, A). This "gland" now is in the form of a narrow tube, opening at the right end into the pharynx and at the left to the outside. It is of doubtful significance, both functional and morphological; it has been compared with a gill slit and may represent the antimere of the first primary, which is otherwise entirely lacking. It soon atrophies like this gill slit and disappears without leaving any trace (Figs. 14, 15).

\section{The Endostyle}

This appeared as a transverse, ciliated thickening, in the floor of the pharynx in front of the club-shaped gland. As a thickening of the pharyngeal wall the endostyle is involved in the general torsion of this region and passes over to the right side (Fig. 12). There its middle region draws back above the primary gill slits, i.e., in the morphological mid-ventral line, converting the transverse band into a $>$-shaped structure with the apex directed posteriorly (Fig. 14). It continues to extend backward, between the rows of primary and secondary gill slits, while its limbs draw together coming into contact and forming a double strip of cells, which in part become differentiated as glandular cells. The endostyle is finally carried back into a median ventral position by the time of the critical stage (Fig. 15, $B$ ).

From the anterior end of the endostyle two narrow ciliated bands-the rudiments of the peripharyngeal bands (Fig. 15, 
$B)$, pass around the pharyngeal wall to the dorsal side, and there extend backward as a pair of ciliated bands which later fuse into a single median structure-the epipharyngeal or hyperbranchial groove.

\section{The Mouth and Associated Structures}

This opening on the left side of the head continues to enlarge during the early part of the larval period, and finally extends from a point considerably in front of the first gill slit to the region of the fifth gill slit (Fig. 14). When the secondary slits begin to develop and the pharynx rotates back to a normal position, the mouth gradually shifts its position in a horizontal plane, moving around to the anterior end: it finally reaches an anterior median position at about the critical stage (Fig. 15). As the mouth migrates its margin extends inward as the velum, like that of Ammocotes, finally reducing the mouth opening to a small aperture. Later this becomes fringed with outgrowths from the free margin of the velumthe velar tentacles (Figs. 15, 16). From the base of the velum the oral cirri grow out. These are first indicated about the time the secondary gill slits appear, and by the time of the critical stage they are well developed and their cartilaginous supports have become differentiated. At the same time the velum begins to form, folds of the integument, first above and then below the mouth, grow out, the latter passing forward toward the region of the snout. These folds are the rudiments of the oral hood, and as the mouth passes forward they enlarge and shift so as to form the left and right sides respectively of the oral hood. The right fold becomes continuous with the extremity of the dorsal fin as this turns the anterior end of the larva (Figs. 15, 16).

\section{The Preoral Pit and Its Derivatives}

The preoral pit, the formation of which from the left anterior gut diverticulum has been described, was left as a small sac 
(Fig. 9, C), opening upon the surface of the left side of the head, in front of the mouth. When the rudimentary folds of the oral hood appear, the dorsal fold develops just above the opening of the preoral pit, which thereafter remains as a struc-

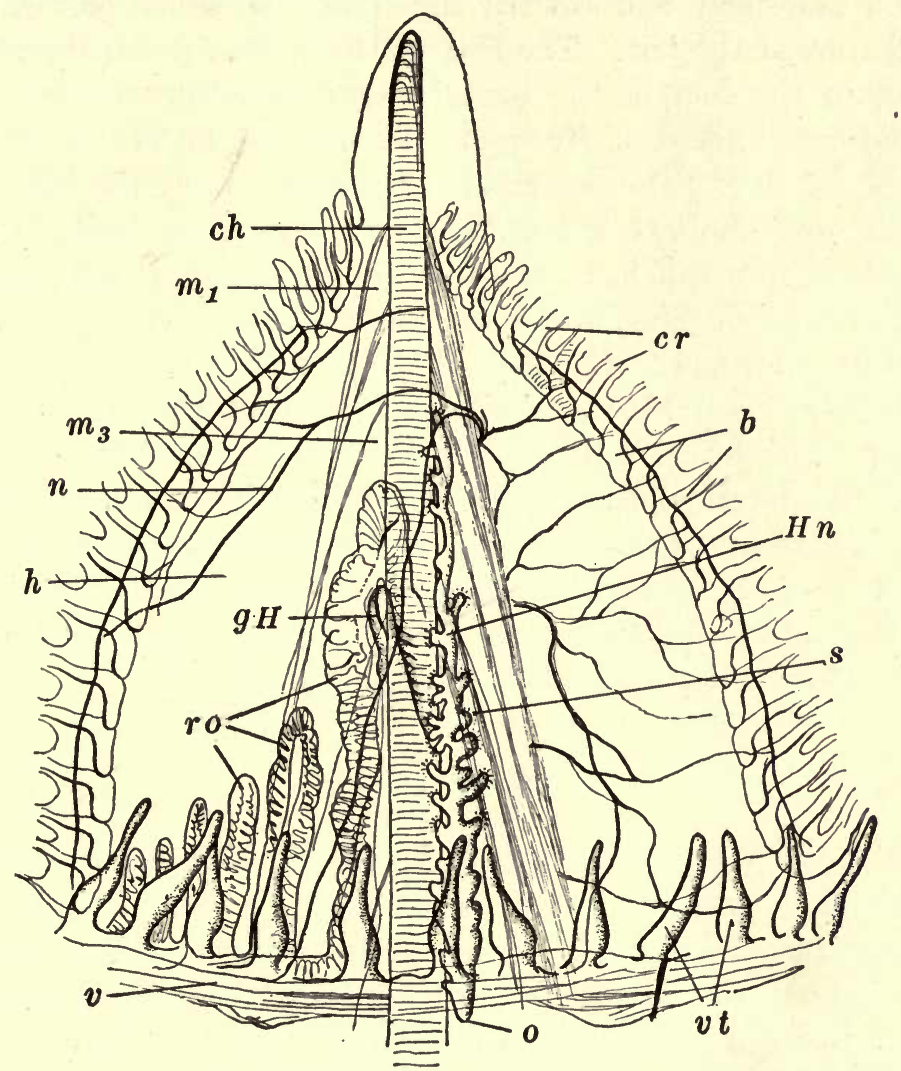

Fig. 16.-Ventral view of anterior end of adult Amphioxus. The buccal cavity has been cut open along the mid-ventral line and spread out. After Goodrich. $\quad b$, Buccal skeleton; $c h$, notochord; $c r$, cirri; $g H$, groove of Hatschek; $h$, oral hood, $H n$, Hatschek's nephridium; $m_{1}, m_{3}$, first and third myotomes; $n$, nerve coming from left side; $o$, pharyngeal opening of Hatschek's nephridium. $r o$, Räderorgan; $s$, solenocytes; $v$, velum; $v t$, velar tentacles.

ture chiefly related with the buccal cavity. Early in the larval period the preoral pit enlarges and then divides into a dorsal and a ventral portion. The ventral or stomodceal portion, as it is called, enlarges very considerably, moves to the surface, and 
spreads out over what is by that time the partly formed oral hood. It forms here the irregularly lobed ciliated patch of cells called the "Räderorgan" (Figs. 12, 15, 16). The dorsal portion of the preoral pit again divides into two parts, one lying anteriorly and toward the right, the other posteriorly and toward the left. The first of these remains on the right side, in the roof of the buccal cavity, and forms there the glandular "groove of Hatschek" leading to the oral aperture (Figs. 14, 16). The second part remains toward the left side and draws out into a long fine tube, extending back to the pharynx, into which, by the time the larva has six gill slits, it acquires an opening above the mouth, and loses its external opening (Figs. 12, 15, 16). This part is sometimes called "Hatschek's nephridium": its final structure is that of a greatly enlarged nephridium (Fig. 16). There seems to be little doubt that the dorsal division of the preoral pit is homologous with the hypophysis of the Craniates, and with the neural gland and its duct of the Tunicates. Some would restrict this homology to the anterior portion (groove of Hatschek) alone, others to the posterior part alone (Hatschek's nephridium).

\section{The Blood-vessels}

The ventral blood-vessel which was formed from cells of somewhat uncertain origin between the lower ends of the lateral plates as they approached the ventral side, is formed throughout the pharyngeal region during the larval period, lying between the primary and secondary gill slits (Fig 14, B). It first begins to show contractions about the time the first gill slit is perforated.

\section{The Atrium}

The presence of the atrium is one of the two chief characteristics wherein Amphioxus is essentially unlike other Chordates, for a similar structure is found only among the Urochorda. It is to be recognized in Amphioxus as a special adaptation to 
the burrowing habit, serving to protect the delicate and complicated pharynx. The enclosure of the branchial region by the atrium occurs early during the larval period. About the time six or seven primary gill slits are present, a pair of low folds appears in the middle of the body region, along the ventral surface. These are the socalled metapleural folds, at first merely thickenings of the ectoderm, but later hollow ridges of considerable size; their primary cavities are to be regarded as cœlomic in origin. The metapleural folds lie close together and gradually extend forward, the right fold proceeding rapidly in advance of the left and passing far up on the right side of the pharynx, above the primary gill slits, which it reaches about the time ten are formed (Fig. 17, $A$ ). Later the left fold reaches the gill-slit region, diverges widely from the right fold, and passes to the opposite side of the secondary gill slits, lying at first in the topographical ventral region (morphological left side) (Figs. 14, $B ; 17$ ). The metapleural folds enlarge and new cavities of lymphatic character appear within them, which encroach upon the
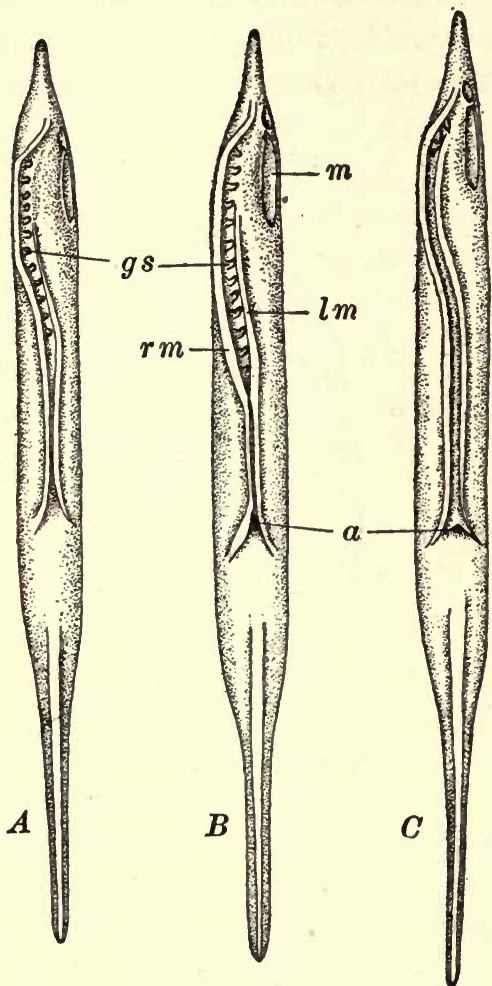

Fig. 17.-Diagrams of three larvæ of Amphioxus, viewed ventrally, showing the relations of the metapleural folds and the method of their closure. After Lankester and Willey. A. Folds still completely separate. $B$. Folds fused, and atrial cavity thereby established, from atriopore to posterior end of pharynx. $C$. Folds fused throughout, except in the region of the first two gill slits, which still open to the outside $a$, Atriopore; $g s$, primary gill sïits; $l m$, left metapleural fold; $m$, mouth; $r m$, right metapleural fold. original cœlomic space and lead to their obliteration.

From the inner face of each metapleural fold a horizontal 
ridge or shelf appears, just below the level of the body wall. These are the subatrial ridges (Fig. 18, A); they grow toward one another and fuse across the mid-line, enclosing between themselves and the ventral body wall a small space lined with ectoderm, which is the beginning of the atrial cavity (Fig. 18, B). The formation and fusion of the subatrial ridges occur first pos-
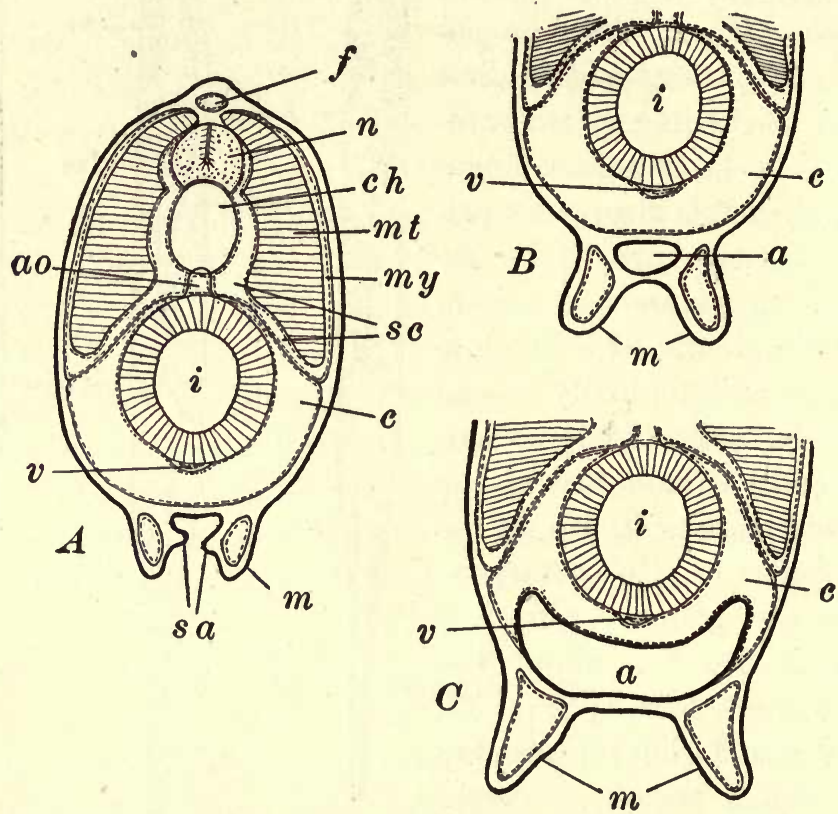

Fig. 18.-Diagrams of transverse sections through Amphioxus larvæ, showing the formation of the atrial cavity. After Lankester and Willey. A. Section through larva with eleven or twelve primary gill slits, showing subatrial ridges on metapleural folds. $B$. Section through slightly older larva (Fig. 17, B), showing first fusion of subatrial ridges to form the rudiment of the atrial cavity. $C$. Section through advanced larva showing enlargement of atrial cavity and the method of its encroachment upon the cœlom. a, Atrial cavity; ao, dorsal aorta; $c$, cœlom (splanchnocœl); $c h$, notochord; $f$, dorsal fin cavity; $i$, intestine; $m$, metapleural fold containing colomic space; $m t$, myotome; $m y$, myocœl; $n$, nerve cord; $s a$, subatrial ridges; $s c$, sclerotome; $v$, subintestinal vein.

terior to the gill-slit region, and proceed thence.forward, and by the time the secondary gill-slit series is completed the branchial region is completely covered. Anteriorly the subatrial ridges merge with the body wall closing the cavity at this end, but posteriorly the atrial cavity remains open to the outside as 
the atriopore (Fig. 17). The cavity, at first very small, gradually enlarges (Fig. 18, C) pushing upward each side of the pharynx, ultimately surrounding it on all sides except the dorsal, where alone the pharynx remains connected with the body wall. The space occupied by the atrium was previously the region of the general cœlom. The development of the atrium therefore reduces the cœlom to a number of greatly reduced and somewhat scattered spaces. The chief of these are (a) the pair of dorsal colomic canals along the dorso-lateral regions of the pharynx, from which the atrium remains separated by the suspensory folds or denticulate ligament; (b) the endostylar coelom in the ventral region below the endostyle; and $(c)$ the branchial colomic canals connecting the two preceding regions by way of very small canals in the primary branchial arches. Posterior to the atriopore the cœlom remains for a time in its normal relation, but later the atrial cavity extends into this region on the right side nearly to the anus, leaving the colom widely open only on the left side of the intestine.

The atrial cavity is formed in the branchial region just before the primary gill slits (except perhaps the more anterior) are perforated, so that from the first these open into the atrial cavity and never directly to the outside. From its mode of formation the denticulate ligament or-suspensory fold is seen to be a membrane equivalent to the body wall, and therefore covered on its atrial surface with ectoderm. Of course the remainder of the atrial cavity is lined with ectoderm, except, as we have seen, that the endodermally covered tongue bars line a small portion of it.

\section{Larval Asymmetry}

The nature and extent of the larval asymmetry of Amphioxus represent the most important difference between the development of this form and other Chordates. The asymmetry of such structures as the neuropore and anus, which have merely been displaced slightly by a later medially developing structure 
(in these cases the median fins), is not unusual or of particular importance. Nor is the alternation on opposite sides of the body of the mesodermal somites and structures primarily related to them, such as the spinal nerves and gonads. The asymmetry which is unique in Amphioxus concerns the formation of the mouth, gill slits, and other organs connected with the anterior end of the pharynx and with the oral hood. One of the remarkable facts in this connection is that the asymmetry is not indicated in early development and is wholly corrected later in larval life, so that it is wholly limited to a comparatively brief period during larval development. It is obviously a purely secondary or cœnogenetic character, and must be explained as in some way adaptive either to present or past conditions of development. We may mention but a single explanatory hypothesis-that of Willey.

The starting point of this hypothesis is the assumption of a primitively median dorsal position of the mouth, for which there is some evidence. The second consideration is the extension of the notochord forward to the tip of the snout, far in advance of the central nervous system and enteron. So far as is known, Amphioxus is the only form in which the chorda extends in this way-in all Craniates it stops in the region of the midbrain. This anterior extension of the chorda, which is obviously an adaptation to the burrowing habit assumed at the close of the larval period, occurs very early in development and necessarily prevents the mouth from appearing in the middorsal region. The net result of this is the shifting of the formative centers of the mouth, and associated structures of the oral hood, to one side, actually the left, and the correlated shifting of the formative centers of other pharyngeal organs. The whole rotation occurs in a counterclockwise direction, throwing structures of the proper left side over to the median line, or even to the right side, structures of the median line up on the right side, and structures of the right side proper, still higher up on the same side. The region of extreme torsion is around the mouth so that posteriorly the amount of displacement becomes less and less and in the posterior part of 
the pharynx the parts appear and remain in a normal position.

Such an asymmetry is in itself obviously not advantageous (adaptive) and during later development it becomes corrected by a compensatory rotary growth of the pharyngeal structures in the opposite direction, while the mouth and associated structures (Räderorgan, groove of Hatschek, etc.) move chiefly in the anterior direction.

This explanation of the origin and correction of the larval asymmetry may be accepted though its necessarily hypothetical character should be clearly recognized.

\section{The Mesodermal Somites}

At the commencement of the larval period there were fourteen or fifteen pairs of somites formed, and we have seen how each of these divides into a dorsal myotome, with its small myocœl and thickened muscular wall, and a ventral lateral plate with thin walls and large splanchnocœl, and further how the median, anterior, and posterior faces of the lateral plates break through, forming a continuous cœlom, walled externally by somatic and internally by splanchnic mesoderm (Fig. 19, A). By the time three primary gill slits have been formed the number of somites has more than doubled, and the full adult number (sixty-one in the common species of Amphioxus, Branchiostoma lanceolatum) is acquired by the time the series of primary gill slits is completed. All of these additional somites are formed from the rapidly elongating posterior region of unsegmented mesoderm, which has been carried backward past the neurenteric region by the outgrowth of the tail.

During the later development of the somites their myotomal region increases largely in vertical extent, and the narrowed myocœl sends downward a thin-walled extension below the myotome proper. This divides into two folds or outgrowths: one of these folds extends inward and upward, between the myotome or protovertebra as it is sometimes called, 
and the axial structures-notochord and nerve cord, to the region. of the dorsal fin (Fig. 19, B). This is the sclerotome; its inner or axial layer is called the skeletogenous layer and gives rise to dense fibrous connective tissue forming the notochordal and neural sheaths; its outer or myotomal layer remains thin and forms the fascia covering the muscular protovertebra:

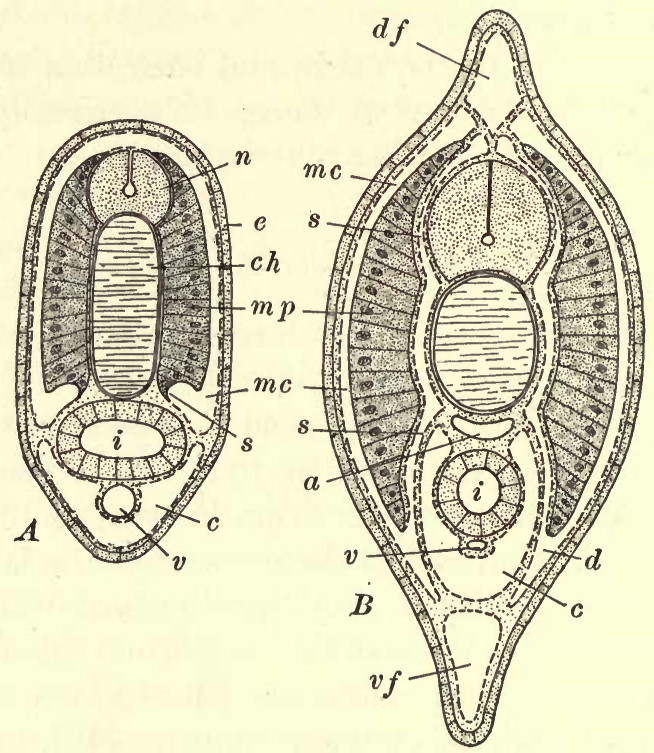

Fig. 19.-Diagrams of transverse sections through Amphioxus larvæ. $A$. Through the body region of a larva with five gill slits, showing separation of myocœl and splanchnocœl (cœlom). B. Through the region between atriopore and anus of young individual, shortly after metamorphosis, showing relations of sclerotome. After Hatschek. a, Dorsal aorta; $c$, cœlom (splanchnocœl); $c h$, notochord; $d$, dermatome; $d f$, dorsal fin cavity; $e$, epidermis; $i$, intestine; $m c$, myocœl; $m p$, muscle plate (myotome); $n$, nerve cord; $s$, sclerotome; $v$, subintestinal vein; $v f$, ventral fin cavity.

the cavity between the two layers is soon obliterated by an accumulation of connective tissue. There is no fascia on the outer surface of the protovertebra or myotome such as is found in Craniates. The second or dermal fold (dermatome) of the myocœlic wall, extends downward and outward between the somatic layer of the lateral plate and the ectoderm (Fig. 19, B), where its inner and outer layers come into apposition and form 
the cutis layer of the integument. The blood cells and many of the important blood-vessels appear to be derived from the skeletogenous layer of the sclerotome.

In the adult the lateral plate forms only the lining membrane of the cœlom, its somatic layer, the body wall, its splanchnic layer, chiefly the wall of the gut outside the endodermal epithelium. The ventral musculature develops later and appears to be derived from the parietal (somatic) layer of the lateral plate.

Among the Craniates the sclerotome is formed from a mass of mesenchyme cells, a tissue which is noticeably lacking in Amphioxus. It remains an open question whether in this organism the formation of the sclerotome by a folding of the myocœlic wall, and the correlated paucity of mesenchyme cells, is a primitive or secondary character.

\section{The Nephridia}

The development of the nephridia is very incompletely known (Legros, Goodrich). They appear in connection with each gill pouch, as these are first marked out (Fig. 14, A), as groups of mesoblast cells along the outer faces of the endodermal gill pouches, lying at the base of a narrow cœlomic groove in this region. These mesoblast cells soon form a definite blind tube extending posteriorly and ventrally from the cœlom toward the gill pouch. The mesodermal cells composing the small nephridial rudiments become differentiated, for the most part, as clear cubic cells, but a group of solenocytes is distinguished as a small collection of elongated granular cells in the neighborhood of the cœlomic opening of the tubule. Then the lower end of the nephridial tube fuses with the dorsal wall of the gill pouch, just posterior to the region of the developing tongue bar. Finally it opens into the gill pouch, about the time this becomes perforated externally, and at the same time its cœlomic opening is closed and the solenocytes elongate into the colom (Fig. 20).

The first formed nephridia apparently become functional 
when four or five slits are formed. When the anterior and posterior primary gill slits atrophy the associated nephridia seem also to atrophy and later to be re-formed in connection with the tertiary gill slits although actual details in the process are unknown.

\section{The Larva at the Critical Stage}

We may now summarize briefly the most important characters of the larva at the close of this period, in what is called

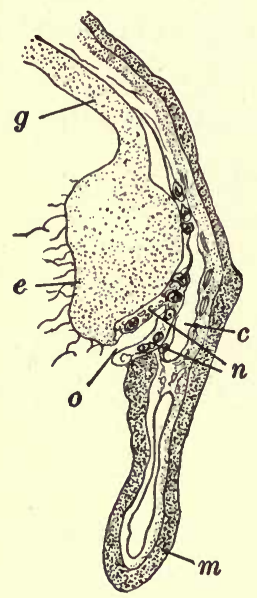

Frg. 20.-Portion of a transverse section of Amphioxus larva passing through the external (atrial) opening of a nephridium. After Goodrich. $c$, Colom; $e$, branchial epithelium; $g$, wall of gut; $m$, metapleural fold containing cœlomic space; $n$, wall of nephridium; $o$, atrial (external) opening of nephridium. the critical stage, marked by the symmetrization of the mouth and pharynx, and the reduction of the gill slits to eight symmetrical pairs. The larva (Fig. 15, B) is about $3.5 \mathrm{~mm}$. in length and tapered at each extremity. The only external opening added during this period is the atriopore, and the neuropore is about to close. The mouth has assumed a symmetrical anterior position, and in front of it a buccal cavity has been established by the outgrowth of the oral hood, from the base of which the buccal cirri have begun to grow out. The full number (61) of somites has been formed for some time, and a definite tail region has grown out posterior to the anal opening. The provisional caudal fin has been replaced by a permanent caudal fin fold continuing anteriorly as the median dorsal and ventral fins, the former continuous with the right side of the oral hood. The ventral fin has displaced the anus to the left side. The atrium is a spacious cavity receiving the external openings of the eight pairs of gill slits, of which the anterior pair remains undivided by a tongue bar, while the posterior one or two pairs are as yet in- 
completely divided. The liver or cæcum is just beginning to develop.

At this time the larva largely gives up its free swimming habit, and assumes the adult habit of burrowing in the sand or mud of the bottom, frequently remaining buried with only the anterior end protruding. The cilia of this exposed region, and of the pharynx in general, vibrate actively, carrying into the pharynx a respiratory current containing small nutritive organisms. The age of the larvæ at the critical stage varies greatly but three months may be taken as a rough approximation.

\section{THE ADOLESCENT PERIOD}

The larva now enters upon its long period of adolescence. This is characterized by the very gradual assumption of adult characteristics, chiefly through histological differentiations and increasing complexity of many regions of the body. The development of the brain and cord has been mentioned. In the pharynx pairs of gill slits (tertiary) are slowly added and the pharyngeal wall assumes the complex structure of the adult. Apparently gill slits are added slowly throughout life, and usually number upward of one hundred pairs in mature specimens. The liver pushes forward as a simple blind sac, into the atrial cavity on the right side, carrying before it a fold of fused atrial and cœlomic walls; finally it extends far forward into the pharyngeal region. The most important development of the period is the formation of the gonads.

\section{Gonads}

In reality the gonads begin to develop before the end of the larval period. They appear in the ninth or tenth segment and continue to about the thirty-fifth. They are first indicated as small groups of cells in the floor of the myocœl, in the region where the skeletogenous layer passes into the cutis layer. This is the region of the nephrostome in the Craniates, and the 
gonadial cavities which appear later have been compared with the nephrostomes, or "gono-nephrostomes," of the higher forms, and it seems likely that Amphioxus may be primitive as regards the position and origin of the germ cells. At any rate Amphioxus is primitive in that the gonads arise and remain as metameric structures, entirely separate from the excretory system.

Shortly after the completion of the larval period, in specimens about $5 \mathrm{~mm}$. long, these small groups of cells are found in the antero-ventral region of the segment, toward its inner or atrial surface, lying along the posterior face of the dissepiment separating the surrounding myocœl from that next anterior. As these cells slowly multiply they push forward into this next anterior myocœl forming, in its postero-ventral region, a small bud covered with a fold of the dissepiment. This dissepiment soon becomes sac-like and remains attached by a short stalk to the anterior face of the remainder. This solid gonadial rudiment soon develops a cavity within its mass. When the larva is about $12 \mathrm{~mm}$. long, this general region of the myocol becomes cut off by a fold the outer myotomal wall, leaving the gonad surrounded outside of its own wall by a portion of the general myocœlic space; this is the perigonadial cœlom or gonocol (Fig. $21, A)$. In the base of this fold is a blood-vessel-a branch of the posterior cardinal vein, which extends through this entire region.

Then the gonadial cells move toward the atrial side of the original gonadial sac, leaving the dissepimental wall as the visceral wall of the gonocœl while its parietal wall is formed by this latter downgrowth of the myotomal wall (Fig. 21, $B$ ). The cavity of the gonad enlarges, as the primary gonadial (ovarian or testicular) cavity, toward the outer or atrial side of which the definitive germ cells are crowded and become covered by a follicular epithelium, also formed from cells of the original mass. The later history is more completely worked out in connection with the ovary, although it is known that the development of the testis is closely similar.

The outer (atrial) region of the gonocol, lying between the 


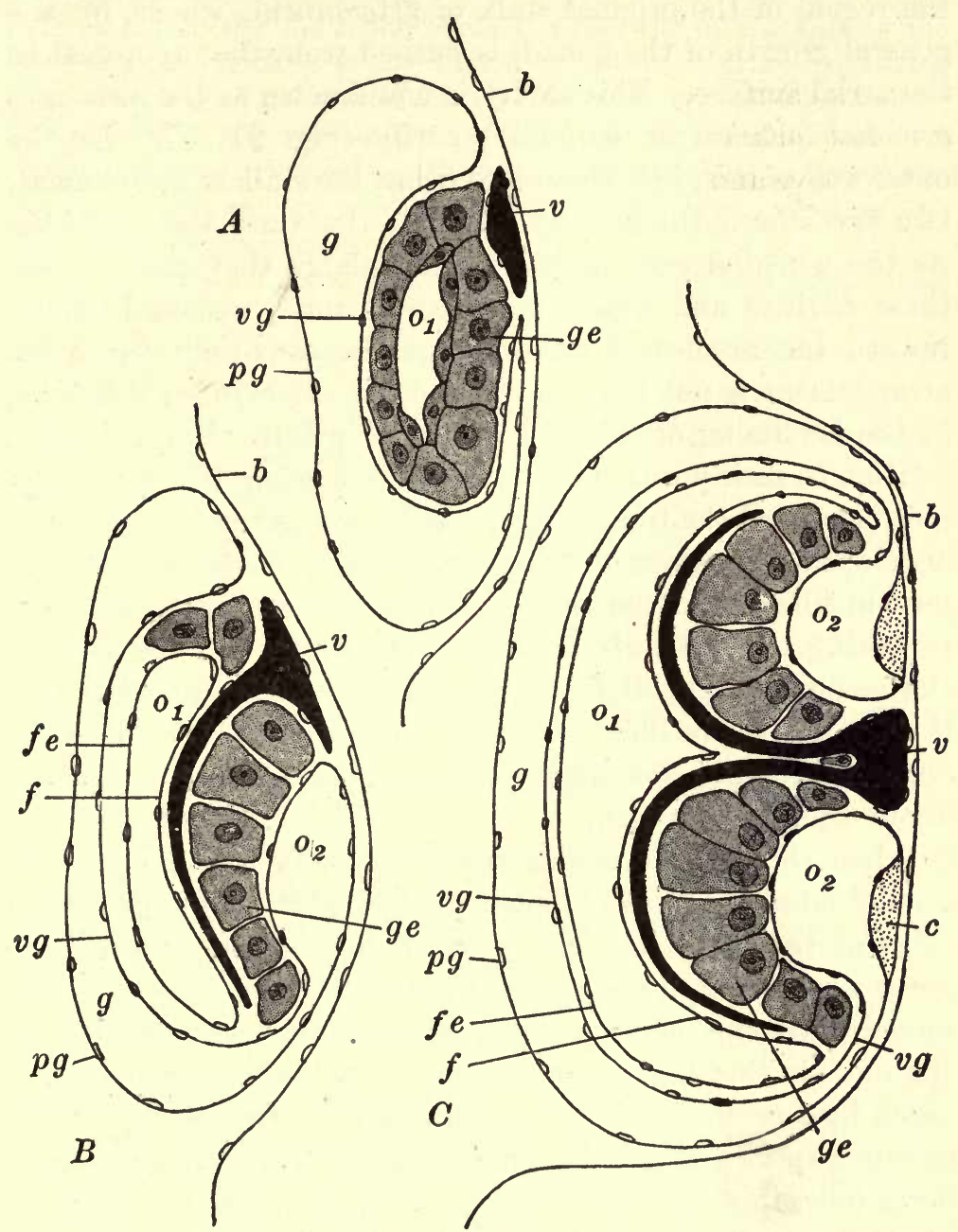

Fig. 21.-Diagrams of sections through the gonads of Amphioxus in three stages of development. After Cerfontaine. Atrial surface toward the right. $A$. Early stage. $B$. Intermediate stage. $C$, Late stage showing definitive arrangement. $b$, Peribranchial (atrial) epithelium; $c$, cicatrix; $f$, true follicular epithelium; $f e$, external layer of follicular epithelium; $g$, gonocœl; ge, germinal epithelium; $o_{1}$, primary ovarian cavity; $o_{2}$, secondary ovarian cavity; $p g$, parietal layer of gonocœl; $v$, cardinal vein; $v g$, visceral layer of gonocœl. 
genital cells and the atrial epithelium, enlarges above and below the region of the original stalk of attachment, which, by the general growth of the gonad, is pushed from the myotomal to the atrial surface. This cavity is now known as the secondary gonadial (ovarian or testicular) cavity (Fig. 21, C). In the outer wall of this, just above and below the stalk of attachment, two specialized thickenings develop; these are the cicatrices. As the gonadial cells multiply and enlarge they crowd upon these cavities and nearly obliterate them, particularly those toward the myotomal surface, but the essentially epithelial arrangement is not lost, and as the ova develop, as described at the beginning of this chapter, their polarity has a definite relation to their position in the epithelium, such that the animal pole is toward the free surface. When the germ cells are fully developed the inner and outer gonadial envelopes contain muscle fibers, the contractions of which appear to assist in rupturing the visceral layer of the gonocœl in the region of the secondary gonadial cavity, and force the germ cells into this cavity, where they remain for some time before extrusion. Sexual differentiation within the gonad appears in specimens about $18 \mathrm{~mm}$. in length.

When the fully formed germ cells, ready for laying, have accumulated in the secondary gonadial cavity, strong contractions of the body wall and ventral musculature rupture the outer membrane in the region of the cicatrices, and they are forced into the atrial cavity, whence they are carried to the outside through the atriopore by the respiratory current, aided by continued muscular contraction. Individuals of the common species of Amphioxus apparently first produce mature germ cells, that is, become adult, when they reach a length of about $2 \mathrm{~cm}$.; the age of such specimens is unknown.

\section{REFERENCES TO LITERATURE}

\section{CHAPTER I}

The literature lists here and at the end of Chapters III, V and VI, include only a few of the more important or more recent titles. References to the sources of borrowed figures are included, and a few works 
of historical importance, as well as articles containing extensive literature references.

In each reference the author's name is followed by the title of the work and the reference to the journal in which the article appeared, or to the place of publication, in case the work appeared separately. The number of the volume (Band, tome, etc.) is printed in black-face Arabic numerals, followed by the year of its publication. References to pages, parts, etc. are omitted except in a few instances where this information is necessary.

The abbreviations of the journals more frequently referred to are as follows:

Amer. Jour. Anat. American Journal of Anatomy. Baltimore and Philadelphia.

Amer. Jour. Physiol. American Journal of Physiology. Boston.

Anat. Anz. Anatomischer Anzeiger. Jena.

Anat. Hefte. Anatomische Hefte. Wiesbaden.

Anat. Record. Anatomical Record. Philadelphia.

Arch. Anat. u. Entw. Same as Arch. Anat. Physiol.

Arch. Anat. Physiol. Archiv fiir Anatomie und Physiologie. Leipzig.

Arch. Biol. Archives de Biologie. Leipzig and Paris.

Arch. d'Anat. Micr. Archives d'Anatomie Microscopique. Paris.

Arch. Entw.-Mech. Archiv fiir Entwickelungsmechanik der Organismen. Leipzig.

Arch. mikr. Anat. Archiv für mikroscopische Anatomie und Entwickelungsgeschichte. Bonn.

Arch. Naturgesch. Archiv für Naturgeschichte. Berlin.

Arch. Zellf. Archiv für Zellforschung. Leipzig.

Arch. Zool. Exp. Archives de zoologie experimentale et génêral. Paris.

Biol. Bull. Biological Bulletin. Woods Hole, Mass.

Biol. Centr. Biologisches Centralblatt. Leipzig.

Bull. Acad. Sci. Cracovie. Bulletin de l'Académie des sciences de Cracovie.

Bull. Mus. Comp. Zool. Harvard Coll. Bulletin of the Museum of Comparative Zoology at Harvard College. Cambridge, Mass.

C. R. Acad. Sci. Paris. Comptes rendus hebdomaires des séances de l'Academie de sciences. Paris.

C. R. Soc. Biol. Paris. Comptes rendus des séances et mémoires de la Société de biologie. Paris.

Ergeb. Anat. u. Entw. Ergebnisse der Anatomie und Entwickelungsgeschichte. Wiesbaden.

Intern. Monatsschr. Anat. Phys. Internationale Monatsschrift für Anatomie und Physiologie. Leipzig.

Jena. Zeit. Jenaische Zeitschrift für Naturwissenschaft. Jena.

Jour. Anat. Physiol. Journal of Anatomy and Physiology. London. 
Jour. Coll. Sci. Imp. Univ. Tokyo. Journal of the College of Science, Imperial University of Tokyo.

Jour. Exp. Zool. Journal of Experimental Zoology. Baltimore and Philadelphia.

Jour. Morph. Journal of Morphology. Boston and Philadelphia.

Mitt. Stat. Neapel. Mitteilungen aus der zoologischen Station zu

Neapel. Berlin.

Monit. Zool. Ital. Monitore Zoologico Italiano. Firenze.

Morph. Jahrb. Morphologische Jahrbuch. Leipzig.

Phil. Trans. Roy. Soc. Philosophical Transactions of the Royal Society of London.

Proc. Roy. Soc. Proceedings of the Royal Society of London.

Q. J. M. S. Quarterly Journal of Microscopical Science. London.

Sitz.-Ber. Acad. Wiss. Wien. Sitzungsberichte der Kaiserlichen Akademie

der Wissenschaften zu Wien. Mathematisch-naturwissenschaft-

liche Klasse. Wien.

Zeit. wiss. Zool. Zeitschrift für wissenschaftliche Zoologie. Leipzig.

Zool. Anz. Zoologischer Anzeiger. Leipzig.

Zool. Jahrb. Zoologische Jahrbücher. Abteilung für Anatomie und Ontogenie der Tiere. Jena.

Boeke, J., Das Infundibularorgan im Gehirne des Amphioxus. Anat. Anz. 32. 1908.

Cerfontaine, P., Recherches sur le développement de l'Amphioxus. Arch. Biol. 22. 1906.

Garbowski, T., Amphioxus als Grundlage der Mesodermtheorie. Anat. Anz. 14. 1898.

Gooprich, E. S., On the Structure of the Excretory Organs of Amphioxus. Q. J. M. S. 54. 1909.

Hammar, J. A., Zur Kenntnis der Leberentwickelung bei Amphioxus. Anat. Anz. 14. 1898.

Hatschex, B., Studien über Entwicklung des Amphioxus. Arbeiten a. d. Zool. Inst. Wien. 4. 1882. Ueber den Schichtenbau von Amphioxus. Verh. d. Anat. Gesell. 2. Anat. Anz. 3. 1888.

Kraatsch, H., Bemerkung über die Gastrula des Amphioxus. Morph. Jahrb. 25. 1897.

Kowalewsky, A., Entwickelungsgeschichte des Amphioxus lanceolatus.

Mém. de l'Acad. Impér. de St. Petersbourg. VII. 11. 1867. Weitere Studien über die Entwickelungsgeschichte des Amphioxus lanceolatus, nebst einem Beitrage zur Homologie des Nervensystems der Würmer und Wirbelthiere. Arch. mikr. Anat. 13. 1877.

Lankester, E. R. and Willey, A., The Development of the Atrial Chamber of Amphioxus. Q. J. M. S. 31. 1890.

Legros, R., Sur quelques cas d'asyntaxie blastoporale chez l'Amphioxus. Mitt. Zool. Stat. Neapel. 18. 1907. Sur le développement des 
fentes branchiales et des canalicules de Weiss-Boveri chez l'Amphioxus. Anat. Anz. 34. 1909. Published anonymously. Sur quelques points de l'anatomie et du développement de l'Amphioxus. Notes préliminaires. 1. Sur le néphridium de Hatschek. Anat. Anz. 35. 1910.

Lwoff, B., Ueber einige wichtige Punkte in der Entwicklung des Amphioxus. Biol. Centralbl. 12. 1892. Die Bildung der primären Keimblätter und die Entstehung der Chorda und des Mesoderms bei den Wirbelthieren. Bull. Soc. Impér. de Natural. de Moscou. II. 8. 1894.

MacBride, E. W., The Early Development of Amphioxus. Q. J. M. S. 40. 1898. Further Remarks on the Development of Amphioxus. Q. J. M. S. 43. 1900. The Formation of the Layers in Amphioxus and its Bearing on the Interpretation of the Early Ontogenetic Processes in Other Vertebrates. Q. J. M. S. 54. 1909.

Marḱchal, J., Sur l'ovogénèse des Sélaciens et de quelques autres Chordates. Premier mémoire: morphologie de l'élément chromosomique dans l'ovocyte I chez les Sélaciens, les Téléostéens, les Tuniciers et l'Amphioxus. Cellule. 24. 1907.

Morgan, T. H. and Hazen, A. P., The Gastrulation of Amphioxus. Jour. Morph. 16. 1900.

Samassa, P., Studien über den Einfluss des Dotters auf die Gastrulation und die Bildung der primären Keimblätter der Wirbelthiere. IV. Amphioxus. Arch. Entw.-Mech. 7. 1898.

SовоттA, J., Die Reifung und Befruchtung des Eies von Amphioxus lanceolatus. Arch. mikr. Anat. 50. 1897.

Willey, A., The Later Larval Development of Amphioxus. Q. J. M. S.

32. 1891. Amphioxus and the Ancestry of the Vertebrates. Columbia Univ. Biol. Ser. II. New York. 1894.

Wilson, E. B., Amphioxus and the Mosaic Theory of Development. Jour. Morph. 8. 1893.

ZiegLer, H. E., Die phylogenetische Entstehung des Kopfes der Wirbelthiere. Jena. Zeit. 43. 1908. 


\section{CHAPTER II}

\section{THE EARLY DEVELOPMENT OF THE FROG}

INTRODUCTION ................ . . 62

I. OUTLINE OF THE LIFE HISTORY OF THE FROG . . . . 63

II. THE GERM CELLS AND THEIR PRODUCTION . . . . . 68

A. The Egg and Sperm at the Time of Spawning • 68

B. The Formation of the Germ Cells . . . . . . . 71

1. The Reproductive Organs . . . . . . . . . . 71

2. Ö̈genesis . . . . . . . . . . . . . . 73

3. Spermatogenesis . . . . . . . . . 77

C. Spawning . . . . . . . . . . . . 77

III. THE EMBRYONIC PERIOD . . . . . . . . . . . . . . . 79

A. From Fertilization throdgh Gastrulation . . . . 79

1. Fertilization and the Development of the Symmetry of the Egg. . . . . . . . . . . . . . . . . . . . 79

2. The Symmetry of the Egg . . . . . . . . . . . . . 83

3. Cleavage . . . . . . . . . . 91

4. The Blastula . . . . . . . . . . . . . . . . 94

5. Gastrulation and Notogenesis . . . . . . . . . . . 98

6. The Mesoderm . . . . . . . . . . . . . . 107

7. The Medullary Plate . . . . . . . . . . . . . . . 110

8. Summary and Comparisons with other Forms. . . . . 111

B. The Formation of the Early Embryo . . . . . . . 116

1. The Nervous System . . . . . . . . . . . . 116

2. The Notochord . . . . . . . . . . . . . 121

3. The Enteron . . . . . . . . . . . . . 122

4. The Mesoderm . . . . . . . . . . . . 123

The frog (Rana, sp.) is important zoologically, not as a central type representative of any large group of Chordates, but as a transitional form connecting the lower and higher groups of Craniata. This relation is no less apparent embryologically than morphologically. For there are comparatively few groups-Lampreys, Ganoids, Dipnoans-whose development can be compared closely with that of the Amphibia, while the types of development seen in the Cephalochorda (Amphi- 
oxus) and among the Elasmobranchs, Teleosts, and all of the Amniota, are in various respects quite special. Many of these special conditions may be more easily understood and compared through common reference to the Amphibia so that as a form transitional between the lower and higher Craniates the frog is a type of great importance.

There are also practical and historical reasons for emphasizing the development of the frog. The size of the eggs and their abundance at a season convenient for their study, the hardiness of the embryos under laboratory conditions and during experimental manipulation, and the ease with which the eggs may be fertilized in the laboratory, all make the frog's egg a particularly valuable laboratory subject. For such reasons this egg has served as the basis for many of the great embryological classics; in this respect the egg of the frog is second only to that of the fowl. And much of the important modern experimental embryology has had this same object as its basis, so that a thorough knowledge of the development of the frog is essential to the student of biology.

\section{OUTLINE OF THE LIFE HISTORY OF THE FROG}

It will prove advantageous to recall, at this point, the most striking facts relating to the life history and development of external characters of the frog. The later development of this animal is marked by several abrupt changes in habit, accompanied by pronounced external modifications, but the earlier development is not so obviously divided into periods, marked by striking changes in habit or structure. The whole period of development may be subdivided as follows:

I. The Formation and Production of the Germ Cells.-This period terminates with spawning.

II. The Embryonic Period.-This is conveniently divided into:

A. From Spawning through Gastrulation and Notogenesis.This includes fertilization, cleavage, the formation of the germ layers, the formation of the neural tube and notochord, and the establishment of an early embryo.

B. From the Early Embryo to the Time of Hatching. 
III. The Larval Period.-From hatching to metamorphosis. The actual developmental processes of this and the latter part of the embryonic period (II, B.) are most conveniently described together.

IV. The Adolescent Period.-From the beginning of metamorphosis to sexual maturity.

The durations of these periods can be given only in the roughest way since they vary with the species and particularly with the temperature and food supply; to a lesser extent the same is true regarding size. The ages and lengths given below are to be regarded then as only approximations under favorable conditions of development.

The period of formation of the germ cells occupies the long interval between the annual spawning seasons. For the most part the germ cells are formed during the summer so that in the following spring only the final steps remain to be accomplished. Fertilization is external and maturation of the egg is not completed until the entrance of the sperm cell. Spawning occurs during the first warm days of spring in most species, although some, like the bull-frog ( $R$. catesbiana) may not spawn until summer. The cleavage of the egg terminates with the formation of a fairly typical blastula, followed by gastrulation, which is complicated during its later phases by the precocious processes of notogenesis and formation of the middle germ layer. During these early phases of development, which usually occupy about thirty-six hours, the spherical form of the egg is retained (Fig. 22, B), though a slight enlargement may result from the formation of internal cavities and the absorption of water. As notogenesis is completed the embryo begins to form, bent around the curved surface of the gastrula (Fig. 22, $C, D$ ). Soon, however, the embryo becomes slightly elongated, and shortly this elongation becomes quite marked chiefly as the result of the enlargement of the head region and the growth of the posterior part of the body (Fig. 22, E). Fig. 22, $F$, shows an embryo of about two days $(2.5 \mathrm{~mm}$.), at the stage which we may arbitrarily assume to represent the end of the first division of the embryonic period.

The neural tube is entirely closed, the blastopore roofed over, 

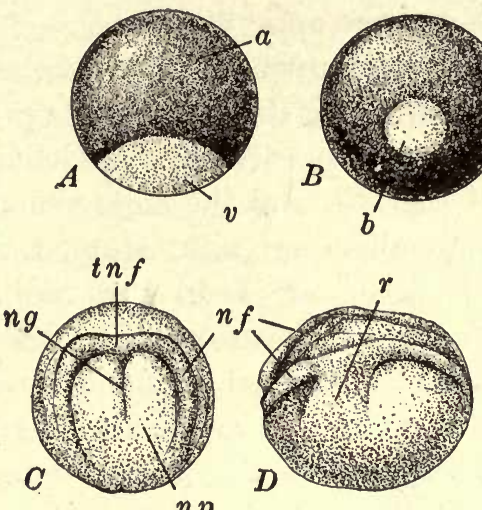

$n p$
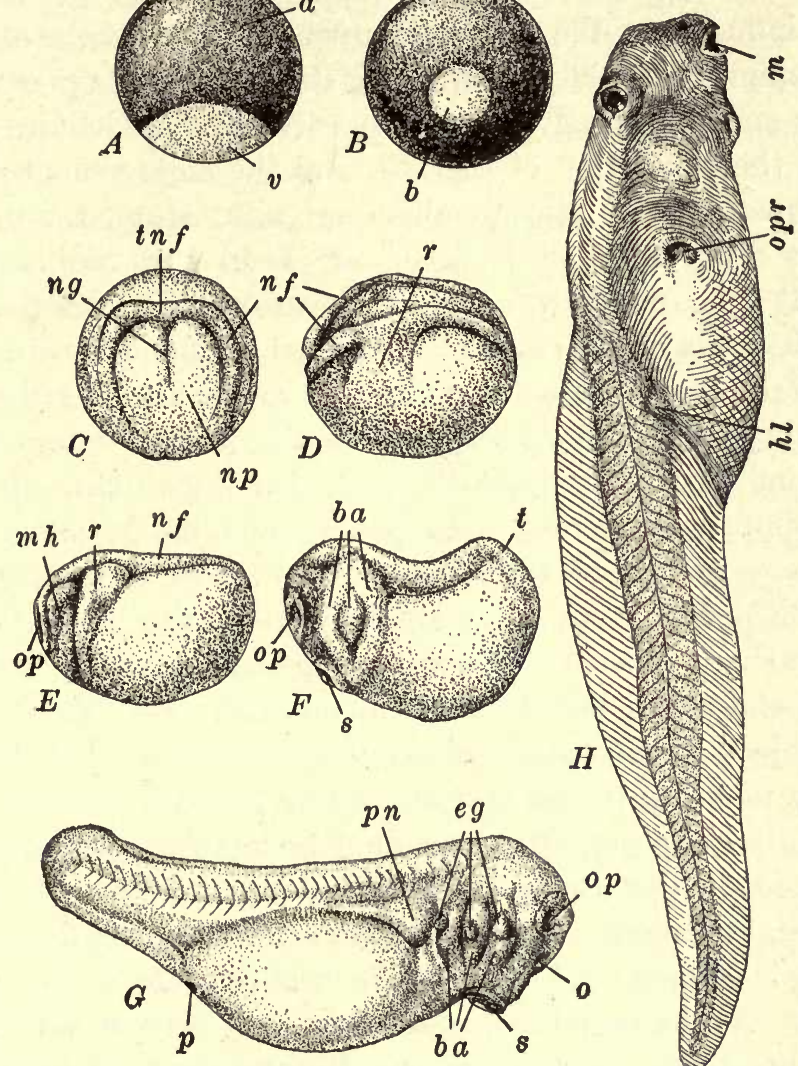

Fig. 22.- Representative stages in the development of the frog. $C-F$, after Keibel (Kopsch). $G, H$, from Ziegler's models. A. Unfertilized ovum. B. Fully formed gastrula; posterior view. $C$. Early stage in the formation of the central nervous system; dorsal view. $D$. Side view of young embryo showing the rudiments of the visceral arches. $E$. Side view of an embryo with central nervous system established, and optic vesicles indicated. F. Side view of "early embryo," showing formation of head and tail regions. $G$. Side view of embryo just before hatching. $H$. Fully formed tadpole, showing rudiments of hind-limbs. $a$, Animal pole; $b$, blastopore containing yolk plug; $b a$, branchial arches; $e g$, rudiments of external gills; $h l$, hind-limb bud; $m$, mouth; $m h$, mandibular and hyoid arches; $n f$, neural folds; $n g$, neural groove; $n p$, neural plate; $o$, olfactory and stomodæal pit; op, rudiment of optic vesicle; opr, right opening of opercular cavity, just before its closure; $p$, proctodæum; $p n$, pronephric eminence; $r$, branchial ridge (plate); $s$, oral sucker; $t$, rudiment of tail; tnf, transverse neural fold; $v$, vegetal pole. 
and in the head region are visible the rudiments of the mandibular and hyoid arches and the optic vesicle.

The influence of the yolk mass upon the form of the embryo now diminishes rapidly, and during the next few days external change consists largely in the appearance of a definite body region, the elongation of the tail, and the enlargement of the head upon which appear olfactory pits, stomodæum, and sucking disc, and just back of the head the rudiments of external gills and the pronephric elevations (Fig. 22, $G$ ).

At about six days $(5 \mathrm{~mm}$.) the embryo begins to show muscular twitchings, and about one or two weeks after fertilization the embryo wriggles its way out of the jelly and becomes a free living larva or tadpole (Fig. 22, $G$ ). This marks the end of the embryonic period. (In the higher temperatures of the laboratory the larvæ may hatch within five days after fertilization.) For some days after hatching the larvæ remain comparatively inactive, sometimes attaching themselves by their U-shaped suckers to the outside of the jelly mass, or to other objects in the water and hanging thus, singly or in groups. Or they may fall to the bottom and lie passively on one side. During the days just after hatching the larvæ are still dependent for food upon the yolk contained within the wall of the intestine, but about two to five days after hatching the mouth opening is formed, and the tadpoles begin to take in food from outside. As the tadpoles begin to feed they become active, the sucker becoming functionless and diminishing; and soon they are in almost constant motion searching for food over the bottom, or in the surface film of the water. The mouth becomes fringed with lips, covered with horny rasping papillæ and furnished with a pair of horny beaks. Their food consists of almost any kind of plant or animal débris and this is consumed in immense quantities. In captivity tadpoles thrive perfectly on a diet of any cereal, with the occasional sacrifice of one of their own number. As the alimentary tract becomes functional the digestive glands increase in size rapidly, and the long intestine, coiled like a watch spring, can easily be seen through the ventral body wall. This enlargement of the diges- 
tive tract gives the body a well-rounded form, sharply marked off from the narrow tail, upon which develop large dorsal and ventral fin-like folds of skin; this is the only locomotor organ in the tadpole. A period of rapid growth follows upon this voracious feeding; the rate of growth depending upon temperature and food supply.

Immediately after hatching external gills develop rapidly on the sides just back of the head, and for a time these are the only respiratory organs, but about the time the mouth opens four pairs of gill slits successively perforate the pharyngeal wall, and their borders become folded forming the true internal gills: thereupon the external gills gradually diminish and after a few days disappear completely. At this time the branchial region becomes covered over externally by a protecting opercular fold of integument, the opercular cavity thus formed finally remaining open on the surface only by a single excurrent pore or "spiracle" on the left side.

During the next few weeks, while the tadpole continues to feed almost incessantly, there are few external changes except the general increase in size. About four or five weeks, ordinarily, after hatching (much sooner at room temperature) the limb buds appear, first the anterior pair within the opercular cavity and therefore not visible externally, and soon after the posterior pair either side of the cloaca (Fig. 22, $H$ ). By the end of the second month these have enlarged and become jointed.

For some time previous to this the tadpoles have been coming to the surface occasionally to expel small bubbles of air from the slowly developing lungs, and to gulp down a fresh supply, and as this aërial respiration increases the internal gills retrogress and the gill slits diminish.

If developmental conditions have been favorable and food abundant, about the end of the third month the period of metamorphosis commences during which, in the space of a few days, the tadpole loses many of its characteristic structures adapted to aquatic life and rapidly, almost suddenly, assumes the characteristics of the amphibious frog. "The tadpole 
and in the head region are visible the rudiments of the mandibular and hyoid arches and the optic vesicle.

The influence of the yolk mass upon the form of the embryo now diminishes rapidly, and during the next few days external change consists largely in the appearance of a definite body region, the elongation of the tail, and the enlargement of the head upon which appear olfactory pits, stomodæum, and sucking disc, and just back of the head the rudiments of external gills and the pronephric elevations (Fig. 22, $G$ ).

At about six days (5 mm.) the embryo begins to show muscular twitchings, and about one or two weeks after fertilization the embryo wriggles its way out of the jelly and becomes a free living larva or tadpole (Fig. 22, $G$ ). This marks the end of the embryonic period. (In the higher temperatures of the laboratory the larvæ may hatch within five days after fertilization.) For some days after hatching the larvæ remain comparatively inactive, sometimes attaching themselves by their U-shaped suckers to the outside of the jelly mass, or to other objects in the water and hanging thus, singly or in groups. Or they may fall to the bottom and lie passively on one side. During the days just after hatching the larvæ are still dependent for food upon the yolk contained within the wall of the intestine, but about two to five days after hatching the mouth opening is formed, and the tadpoles begin to take in food from outside. As the tadpoles begin to feed they become active, the sucker becoming functionless and diminishing; and soon they are in almost constant motion searching for food over the bottom, or in the surface film of the water. The mouth becomes fringed with lips, covered with horny rasping papillæ and furnished with a pair of horny beaks. Their food consists of almost any kind of plant or animal débris and this is consumed in immense quantities. In captivity tadpoles thrive perfectly on a diet of any cereal, with the occasional sacrifice of one of their own number. As the alimentary tract becomes functional the digestive glands increase in size rapidly, and the long intestine, coiled like a watch spring, can easily be seen through the ventral body wall. This enlargement of the diges- 
tive tract gives the body a well-rounded form, sharply marked off from the narrow tail, upon which develop large dorsal and ventral fin-like folds of skin; this is the only locomotor organ in the tadpole. A period of rapid growth follows upon this voracious feeding; the rate of growth depending upon temperature and food supply.

Immediately after hatching external gills develop rapidly on the sides just back of the head, and for a time these are the only respiratory organs, but about the time the mouth opens four pairs of gill slits successively perforate the pharyngeal wall, and their borders become folded forming the true internal gills: thereupon the external gills gradually diminish and after a few days disappear completely. At this time the branchial region becomes covered over externally by a protecting opercular fold of integument, the opercular cavity thus formed finally remaining open on the surface only by a single excurrent pore or "spiracle" on the left side.

During the next few weeks, while the tadpole continues to feed almost incessantly, there are few external changes except the general increase in size. About four or five weeks, ordinarily, after hatching (much sooner at room temperature) the limb buds appear, first the anterior pair within the opercular cavity and therefore not visible externally, and soon after the posterior pair either side of the cloaca (Fig. 22, $H$ ). By the end of the second month these have enlarged and become jointed.

For some time previous to this the tadpoles have been coming to the surface occasionally to expel small bubbles of air from the slowly developing lungs, and to gulp down a fresh supply, and as this aërial respiration increases the internal gills retrogress and the gill slits diminish.

If developmental conditions have been favorable and food abundant, about the end of the third month the period of metamorphosis commences during which, in the space of a few days, the tadpole loses many of its characteristic structures adapted to aquatic life and rapidly, almost suddenly, assumes the characteristics of the amphibious frog. "The tadpole 
variable sizes. The egg of the frog is therefore markedly telolecithal.

In the animal pole the nucleus is contained. At the time of egg laying this is in the metaphase of the second polar division (Fig. 26, I). The first polar body has already been extruded and though very small, may be found near the light spot in the flattened area at the upper pole.
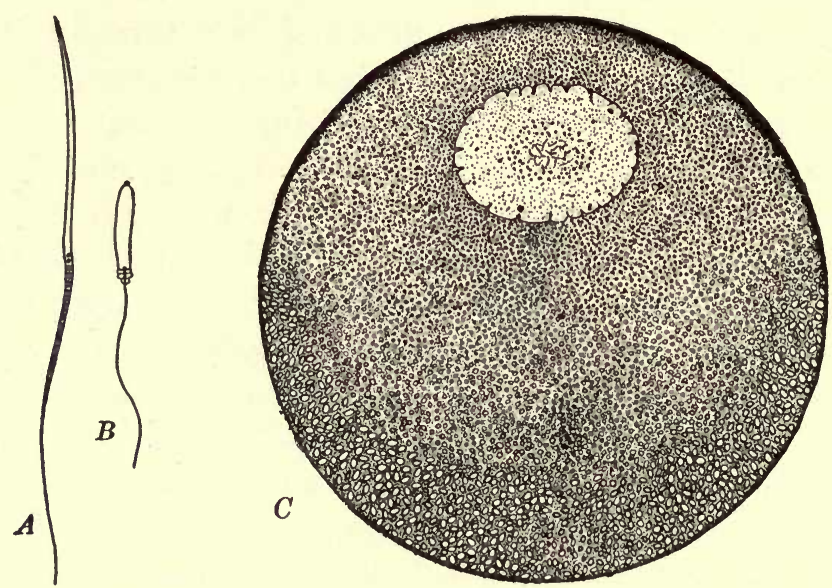

FIG. 23.-Egg and spermatozoa of frog. A. Spermatozoön of Rana fusca. After Broman. B. Spermatozoön of $R$. esculenta. After Broman. C. Section through the fully formed ovarian egg of Rana sp. From Morgan (Development of the Frog's Egg). The protoplasmic animal pole is covered with a thin layer of pigment; vegetal pole filled with yolk bodies; other deutoplasmic granules are distributed throughout the cell. The large nucleus, or germinal vesicle, surrounded by a definite nuclear membrane, lies eccentrically toward the animal pole, and contains the thread-like chromosomes and a group of nucleoli.

The specific gravity of the deutoplasm is slightly greater than that of the protoplasm, and this brings about the assumption of the definite position of the egg with the vegetal pole downward. But at this time the egg membranes are so closely adherent that its rotation to this position may be very slow.

At this time, i.e., preceding fertilization, the only symmetry of the egg is that expressed by its polarity. That is to say it has no single plane of symmetry, only an axis of symmetry (polar axis); this is the primary egg axis passing through the middles of the light and dark (vegetal and animal) poles. 
The spermatozoa of the frog are quite typical in size and form (Fig. 23, $A, B$ ). They are about $0.1 \mathrm{~mm}$. in length; the head is comparatively long, in some species tapered and curved at the apex with a laterally attached adhesive perforatorium or in others, quite blunt with a small rounded perforatorium. The anterior centrosome lies in the head, the posterior in the middle piece.

\section{B. The Formation of the Germ Cells}

\section{The Reproductive Organs}

Before describing the formation of the ova and spermatozoa it will be necessary to recall the essential arrangement of the gonads and their ducts in the mature organism. The development of the system will be described later.

The single pair of ovaries are proliferations of the cells forming the longitudinal genital ridges. These project from the body wall for some distance along either side of the dorsal attachment of the mesentery (Fig. 24). Each is surrounded by a peritoneal fold (mesovarium), which also slings the organ from the dorsal body wall and transmits its nervous and vascular supplies. Each ovary is divided transversely into a series of compartments. In each of these is a small internal cavity the thick wall of which is formed by the germinal tissue proper. After spawning the ovaries are left as small rudiments, and during the following summer eggs are formed in large numbers and their growth is practically completed before the beginning of the period of hibernation. The ova are all formed from a few primitive ova which divide repeatedly, forming small groups or nests of cells, one of which enlarges becoming the ovum proper, while the others around it become the nutritive follicle cells (membrana granulosa). As the growth of the ova is completed, the ovaries are so enlarged that they occupy a large part of the body cavity and crowd upon the other viscera.

Loosely attached to the anterior ends of the ovaries are the fat bodies-large masses of yellow streamers of lymphatic 
tissue, filled with drops of fatty substance chemically similar to the deutoplasm of the eggs. These are larger and more abundantly supplied with fat just prior to the breeding season. Their function seems, in part at least, to supply the material used in the final stages of the growth and maturing of the eggs, and also possibly, though doubtfully, for the nutrition of the

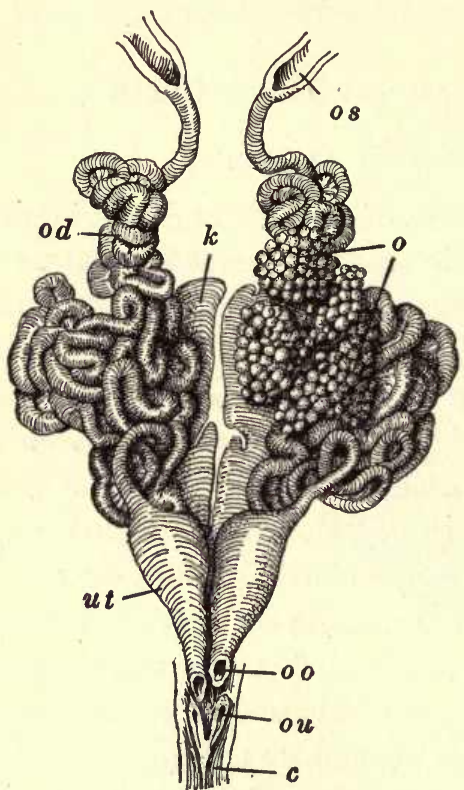

FIG. 24.

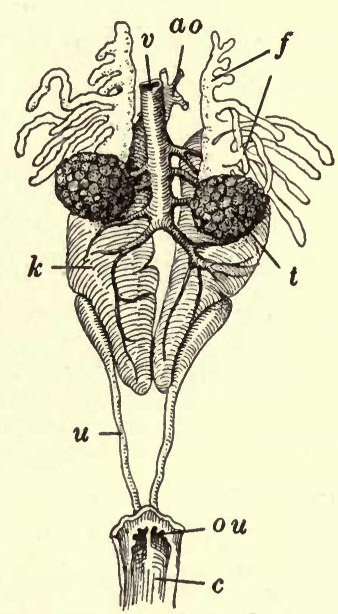

Fig. 25.

FIG. 24. - Urinogenital system of the female frog. After Wiedersheim (Ecker). $c$, Cloaca; $k$, kidney; o, ovary; od, oviduct; oo, opening of oviduct into cloaca; ou, opening of ureter into oviduct; $u$, uterus.

FIg. 25.-Urinogenital system of the male frog. After Wiedersheim (Ecker). $a o$, Dorsal aorta; $c$, cloaca; $f$, fat body; $k$, kidney; ou, opening of ureter into cloaca; $t$, testis; $u$, ureter; $v$, vena cava.

animals themselves prior to and during the spawning season, for usually no food is taken after the end of their period of hibernation till after spawning is completed.

The remaining parts of the female reproductive system are the oviducts (Müllerian ducts). These are a pair of very long, much convoluted tubes, of small diameter but with rather thick walls, suspended from the dorsal body wall by 
folds of peritoneum attached just along the outer sides of the mesovaria (Fig. 24). Each oviduct opens directly out of the body cavity at its upper end by a ciliated ostium, and at its lower extremity it opens into the cloaca. The thickness of the oviducal wall is due chiefly to the presence of glands secreting the albuminous material which forms the outer egg membrane or jelly; the lumen of the duct is lined with ciliated epithelium. Both the length of the oviducts and the thickness of their walls are subject to seasonal variation, the glands being largest and the ducts most convoluted during the time of egg laying. The lower extremities of the oviducts are thin walled and easily dilatable forming the so-called "uteri," serving as storage spaces for eggs ready to be laid.

In the male there is a single pair of ovoid testes (Fig. 25), in a position corresponding to that of the ovaries, and similarly suspended from the dorsal body wall by a peritoneal fold (mesorchium). Each testis is drained by a variabie number (ten to twelve usually) of vasa efferentia which, after penetrating the kidney, open into a longitudinal collecting duct-the vas deferens (Wolffian duct) which serves also as a ureter. Just before the vasa deferentia open into the cloaca they are dilated into the seminal vesicles, where mature sperm are stored just previous to spawning. The testes are divided into lobes like the ovaries and each lobe is further subdivided into lobules, in the walls of which the sperm develop and mature. There are fat bodies in the male similar to those in the female (Fig. 25). The testes also show much the same seasonal variation in size as the ovaries, in some species in which the formation of spermatozoa is seasonal, while in others, which form the sperm continuously, there is less variation.

\section{Oögenesis}

During the later ovarian history of the eggs the maturation processes are commenced and the deutoplasm or yolk material is accumulated (growth period). The nucleus of the early primary oöcyte passes into synizesis on that side of the nucleus 
toward the attraction sphere (Fig. 26, A). After synizesis the chromosomes scatter through the nucleus as small feathery bodies (Fig. 26, C), which stain lightly and become vacuolated, finally losing their identity. Meanwhile small yolk particles of mitochondrial nature appear in the cytoplasm, in the region of the attraction sphere and apparently under its influence. During the growth period the mitochondrial particles and yolk bodies accumulate rapidly, especially around the attraction sphere giving it the appearance of a yolk nucleus, whence they extend to other parts of the cell except in the region immediately surrounding the nucleus. Finally the "yolk nucleus" breaks down and the deutoplasm around it scatters through the cytoplasm (Fig. 26, D, E).

Toward the close of this process the nucleus moves toward one side of the cell, marking the polarity of the ovum, and from the first the yolk accumulates in the side opposite the nucleus or vegetal region, while around the nucleus in the animal region the protoplasm contains much less deutoplasm; the superficial protoplasm of the animal pole also contains many pigment granules.

As the growth period of the primary oöcyte is completed the nucleus moves up to the surface of the cell which becomes flattened or even depressed toward it; at the same time the pigment over the nucleus is partially displaced or withdrawn and the lighter fovea results. The nucleus is very large and clear; no chromatin network is visible and the only chromatic bodies in it are the nucleoli. The nucleoli are quite numerous and apparently of two kinds-true nucleoli whose real nature is doubtful, and chromatin nucleoli. The nucleus becomes elongated parallel with the surface of the egg and pigment accumulates around it, while the nucleoli become vacuolated and much enlarged. Some of the nucleoli dissolve while others fuse into large masses and the chromatin nucleoli collect in a small group near the center of the nucleus.

While these events are occurring within the nucleus a pronounced cytoplasmic modification has appeared. Along the lower or inner side of the nucleus a small cytoplasmic area has 

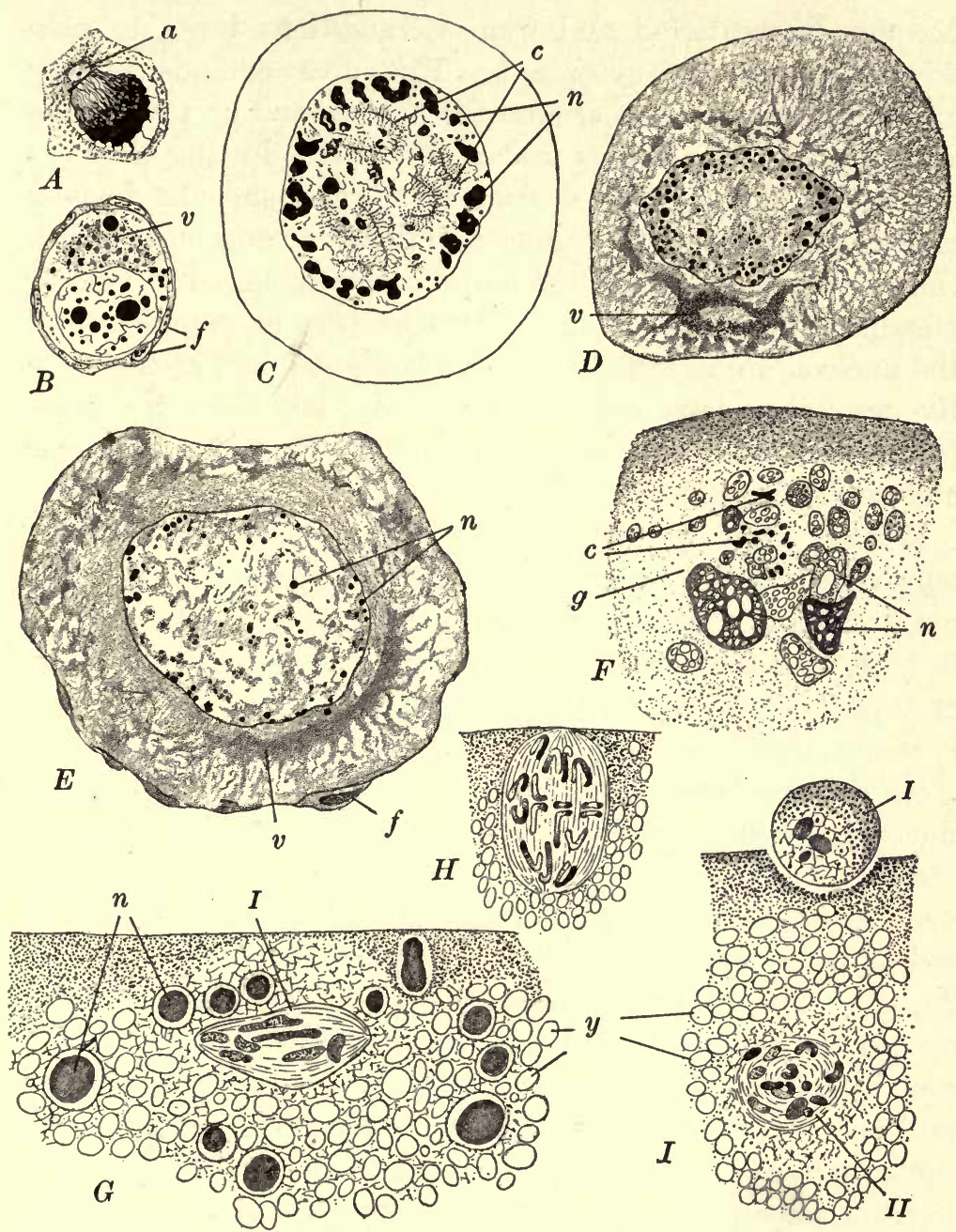

Fig. 26.-Oögenesis in the frog (R. temporaria). A-E, After Lams. $F-I$, After Lebrun. A. Primary oöcyte in synizesis. $B$. Primary oöcyte with vitelline substance of mitochondrial (chromidial) origin in the cytoplasm. $C$. Primary oöcyte showing feathery chromosomes and chromatin nucleoli. $D$. Primary oöcyte with ring-like vitelline mass. E. Primary oöcyte showing cytoplasm in two zones. $F$. Nuclear region of primary oöcyte after dissolution of the nuclear membrane, showing the small chromosomes and large chromatin nucleoli. Egg still in ovary. G. First polar spindle in primary position. From egg in body cavity. $H$. First polar spindle in metaphase. From egg in uterus. I. First polar body formed and second polar spindle forming. From egg in uterus. $a$, Attraction sphere; $c$, chromosomes; $f$, follicle cells; $g$, contents of germinal vesicle; $n$, chromatin nucleoli; $v$, vitelline substance of mitochondrial (chromidial) origin; $y$, yolk plates; $I$, first polar spindle (polar body, in $I$ ); $I I$, second polar spindle. 
become differentiated and from it radiations begin to pass downward into the cytoplasm. This area extends rapidly and the radiations pass around the nucleus and up toward the animal pole of the egg as well as centrally. Finally they extend even through the nuclear membrane and the nuclear meshwork takes on the same radiational arrangement which thus involves practically the entire animal pole. The nuclear membrane dissolves as the radiations become complete and the nucleoli are aggregated or dissolved, and just at this time the egg follicle is ruptured in some way, and the egg escapes freely into the body cavity surrounded only by its chorionic and vitelline membranes (Fig. 23, C).

Certain areas of the peritoneum are covered with cilia which beat in the direction of the oviducal ostia. These too are abundantly ciliated, and as a result of the ciliary action in both regions the eggs are soon carried to and into the upper ends of the oviducts. But by this time the first polar spindle is already formed.

As the egg leaves the ovary the small group of chromatin nucleoli becomes surrounded by a small spherical mass of fibrillar plasma; the nucleoli become more or less fused and vacuolated, and then give rise to the group of twelve small rod-like chromosomes which soon become rings or crosses (Fig. $26, F)$. The fibrillar plasma draws out into the elongated achromatic spindle, at first placed tangentially (Fig. 26, $G$ ), but soon rotating and coming to the surface of the cell in the radial position (Fig. 26, $H$ ). The spindle is quite blunt and no asters, centrospheres, or centrosomes have been seen. The chromosomes diverge as the egg is entering the oviduct, in the upper part of which the very small first polar body is cut off (Fig. 26, I).

The second polar spindle forms immediately, and by the time the egg reaches the lower end of the oviduct the second polar division has progressed as far as the mesophase or metaphase. In this condition the division is suspended, and proceeds considerably later and as a rule only after entrance of the spermatozoön. 
Entrance of the eggs into the upper part of the oviduct stimulates the jelly-secreting glands of its walls, and as the eggs are carried along singly down the oviduct by the cilia of its own walls, each is smeared over the surface of the chorion with a thin coating of viscid albuminous material arranged in two or three layers. About two hours are occupied in the passage of an ovum down the dict. At the lower ends of the oviducts the eggs collect in the uteri, where they remain stored, usually for a day or two, pending the time of spawning.

\section{Spermatogenesis}

The formation and maturation of the spermatozoa is completed within the testes. Each lobule of the testis is composed of a collection of tubules, in the walls of which the spermatogonia develop, surrounded by nutritive follicles the elements of which become in part the basal cells or Sertoli cells. So far as is known the formation of the spermatocytes and spermatids is fairly typical. The spermatid contains a large nucleus and two peripheral centrioles. During the metamorphosis of the spermatid into the spermatozoön, the inner centriole is taken into the nucleus while opposite the other the flagellum grows out. The sphere of idioplasm remains on one side of the anterior tip of the head, when this forms from the nucleus, and a part of the cytoplasm flows down around the base of the flagellum forming the middle piece; the remainder of the cytoplasm appears to be thrown off.

In some species of Rana the sperm form continuously, in others only seasonally, apparently just before hibernation begins. As the breeding season approaches they are produced more abundantly and collect in the dilated lower ends of the vasa deferentia or seminal vesicles, ready for extrusion.

\section{Spawning}

In the more common species of Rana, spawning occurs during the first warm days of early spring; some forms spawn 
later in the spring, and in a few (e.g., R. catesbiana), breeding occurs during early summer. In the first mentioned, spawning follows immediately upon emergence from the period of hibernation, when the frogs collect in small ponds or streams, or about the margins of larger bodies of water. There is no true copulation, the male merely seizing the female firmly around the body dorsally, with the forelegs just behind those of the female. This embrace or amplexus usually begins some hours, even days, before the actual extrusion of the reproductive products begins, and quite likely this affords the normal, though not essential, stimulus to their discharge from the ovaries and testes respectively. This amplexus continues throughout the entire period of spawning of a single pair, which may occupy several days or even weeks; the duration depends upon the species and upon the temperature-colder weather prolonging the period greatly.

Expulsion of the eggs usually occurs during the early morning hours and is an intermittent process. Apparently all the eggs contained in the uteri are spawned at one time, and then an interval of rest follows during which the uteri are again slowly filled. As each mass of eggs is forced out of the cloaca the male, at the same instant, expels quantities of seminal fluid containing enormous numbers of spermatozoa which mingle with the egg masses, insuring the fertilization of practically every egg. Fertilization is therefore strictly external.

In the common frogs there are no nursing habits so frequent among other Anura (e.g., Alytes, Nototrema, Rhinoderma, etc.) and the eggs are left to develop without further relation to the parent organisms, which, upon the conclusion of spawning, immediately leave the pools and scatter widely. The eggs surrounded by the jellies remain in large masses which sink to the bottow of the shallow water and there become loosely attached to sticks or débris.

The total number of eggs laid by a single individual during one season varies widely in different species, and seems to vary conversely with the size of the eggs. The European grassfrog (Rana temporaria) lays from one to two thousand large 
eggs (2-3 mm.), while the European water-frog (Rana esculenta $)$ in which the eggs are small $(1.5 \mathrm{~mm}$.) lays from five to ten thousand during each season.

\section{EMBRYONIC PERIOD}

\section{A. From Fertilization through Gastrulation}

1. Fertilization and the Development of the Symmetry of the Egg

During the first hour or two after the entrance of the sperm several changes of great importance occur within the egg. Although these are going on together and overlap to a certain extent we shall have to describe them separately.

Entrance of the Spermatozoön.-Within a few moments after ensemination, a sperm cell bores its way through the thin jelly and the chorion, and enters the egg substance; in most cases the entire spermatozoön enters. Although there is no micropyle the sperm does not enter the egg at random, but normally only in the pigmented hemisphere, more frequently about forty degrees from the animal pole, and in any meridian (Brachet). The meridian passing through both poles of the egg and the point of entrance of the sperm is known as the fertilization meridian.

Of course many sperm tend to enter the egg but the entrance of the first seems to alter the chemical structure of the egg in such a way that additional sperm are deterred from entering; frequently many such sperm may be seen in the egg jelly. Polyspermy, although not rare, is never normal in any of the frogs, and should more than one sperm succeed in entering, the development of the egg becomes abnormal.

Once within the egg substance the sperm head and middle piece move rapidly inward, following approximately a radius of the egg. The path of the sperm is marked by a distinct trail of pigment, indicating unusual metabolic activity of the region, which remains visible for some time, occasionally even to the blastula stage. The first part of the sperm path is. called the penetration path (Fig. 27, A, B). After the sperm has travelled along this path for a short distance it rotates, 
in the usual way, putting the middle piece with its centrosome in advance of the head, which begins to dissolve and to form a typical vesicular and enlarged nucleus. Then the sperm changes its course, often abruptly, and moves toward the region where the male and female nuclei will unite, unless, indeed, the penetration path may have led in that direction (Fig. 27,

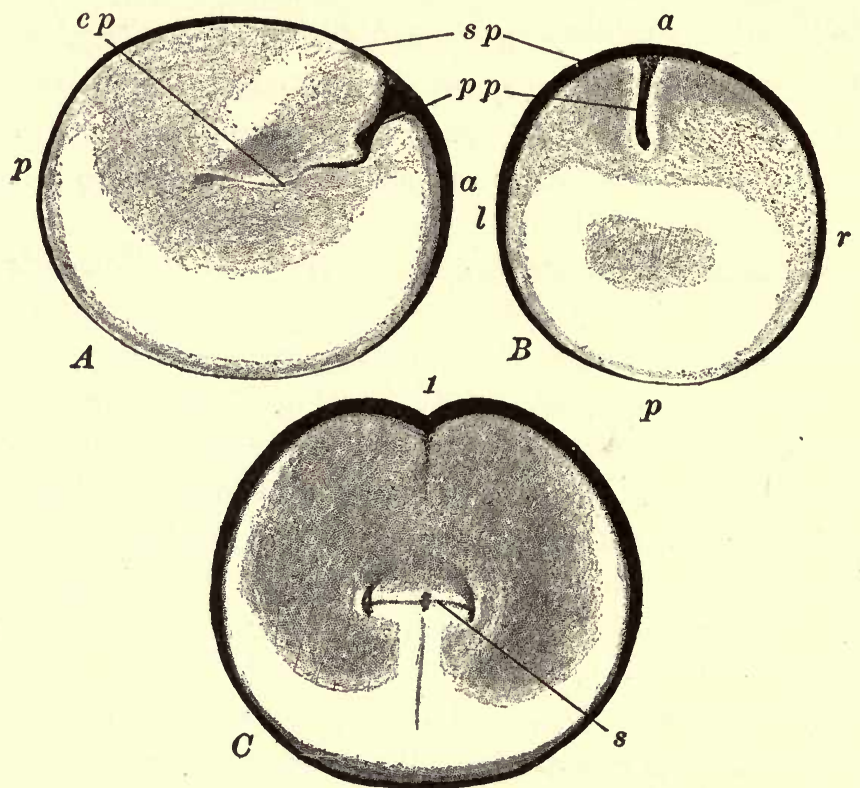

FIG. 27.-Sections through the egg of $R$. fusca, showing penetration and copulation paths, and the symmetry of the first cleavage plane. After O. Schultze. $A$. Sagittal section through the egg before the appearance of the first cleavage; $B$. Frontal section of the same stage as $A$, showing the symmetrical distribution of the egg materials. C. Frontal section through egg in two-cell stage, showing the symmetry of the egg; the penetration path is not shown. $a$, Anterior; $c p$, copulation path; $l$, left; $p$, posterior; $p p$, penetration path; $r$, right; $s$, remains of first cleavage spindle; $s p$, superficial pigment; 1 , first cleavage furrow.

A). This second part of the sperm path is known as the copulation path and like the penetration path, it is marked by a trail of pigment left in the cytoplasm.

Swelling of the Egg Membranes.-One result of the entrance of the sperm is the withdrawal of fluid from the egg substance. This fluid accumulates between the surface of the egg and the chorion forming the perivitelline space. This leaves the egg free 
to rotate within its membranes, and in a few moments after fertilization all the eggs are found with the pigmented pole uppermost. In unfertilized eggs these membranes are more adherent and while rotation occurs, it is very slow.

Probably some of the fluid in the perivitelline space is taken in from the outside, for the egg membranes, particularly the jelly, are extremely hydroscopic. The egg has been in the water only one minute when the thin jelly has visibly commenced its absorption of water. When the eggs are extruded

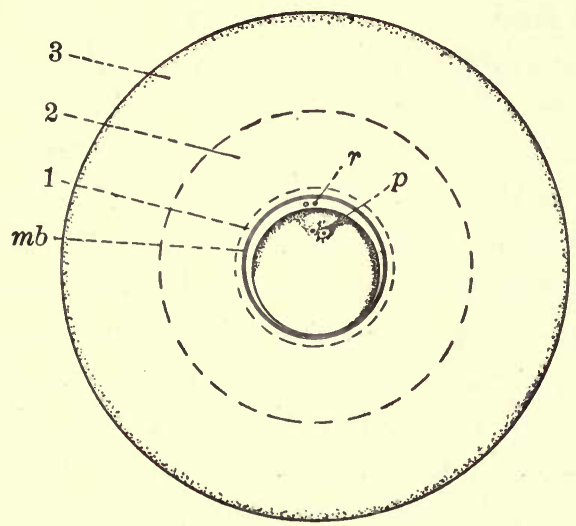

FIG. 28.-Egg of frog a short time after laying and fertilization, showing the swollen egg membranes. From Ziegler (Lehrbuch, etc.), after O. Schultze. $m b$, The so-called vitelline membrane; $p$, pigmented penetration path of the spermatozoön; $r$, polar bodies; $1,2,3$, inner, middle and outer albumenous membranes or layers of "jelly."

the thickness of the jelly is only about one-sixth the diameter of the egg; after three minutes contact with the water this is increased to one-half the diameter; and after ten to fifteen minutes its thickness equals the diameter of the egg: The swelling then becomes slower and unless fertilization has occurred it may almost cease. Usually however the absorption of water continues for several hours and the thickness of the jelly may equal twice the diameter of the egg proper.

As the jelly thickens it is seen to be arranged in definite strata-a thin denser layer closely applied to the chorion, outside this a thick layer somewhat more fluid, and on the sur- 
face a thick layer, rather firmer than the middle layer. The two thick outer layers may, in some forms, be separated by a narrow dense layer which is distinctly fibrous in structure (Fig. 28).

The functions of the jelly are various. It serves to some extent to attach the egg masses, but chiefly to protect the eggs from pressure or mechanical injury, from being eaten by other organisms, from infection of various kinds. It seems likely, too, that the jelly assists in the elevation of the temperature of the egg, for as a transparent sphere it condenses the heat rays of sunlight which it allows to enter freely and at the same time checks their radiation from the egg. The black pigment of the upper pole seems to function toward the same end by absorbing readily the heat rays, so that altogether the temperature of the egg may be considerably higher than that of the surrounding water. While the eggs, and the spermatozoa also, are very resistant to cold, they are at the same time very sensitive to warmth, so that this slight elevation of temperature has the effect of hastening development-an effect that may be quite important since the temperature of the water is often quite low at the time the eggs are laid, and the ponds in which the frogs spawn are quite likely to dry up during the summer, so that each day gained in development toward metamorphosis may mean much as regards survival.

Maturation.-Another effect of the entrance of the sperm is the completion of the maturation process in the egg nucleus. As the sperm enters, this is in the mesophase or metaphase of the second polar division (Fig. 26, I). This division is then rapidly completed and the second polar body cut off; this usually occurs about thirty minutes after entrance of the sperm. The second polar body is of the same size as the first, or smaller. The egg nucleus then reforms in the usual manner. The polar bodies are only loosely attached to the surface of the egg and frequently may be found floating in the perivitelline space.

By the time the egg nucleus has reformed the sperm nucleus has also become typical in form, and the two nuclei move 
toward the center of the egg, approach and meet in the usual manner. The path of the egg nucleus is not marked by any pigment, nor is it accompanied by any radiations such as were connected with it during its maturation. The sperm centrosome and centrosphere divide and form the poles of a small but typical cleavage figure which is not located near the center of the egg, but always toward the animal pole. The position of the first cleavage spindle is not entirely undirected, but before we can discuss this point we must consider some facts regarding the structure of the egg itself after fertilization.

\section{The Symmetry of the Egg}

Before fertilization the egg has a well-marked polarity and is radially symmetrical about its chief axis (Fig. 29, $A$ ). This form of radial symmetry (not spherical) has been termed "rotatory," i.e., radially symmetrical in any plane at right angles to the chief axis. The vegetal pole contains a large proportion of yolk, while the animal pole is relatively free from yolk and is covered externally by a thin but dense coating of brown or black pigment; moreover, the nuclear structures are in the animal pole (Fig. 23, C). The specific gravity of the lower pole is the greater, on account of the heavy yolk contained in it, and therefore the pigmented animal pole is turned upward when the egg is free to rotate. This rotation, however, is not usually completed for some minutes after the spermatozoön has entered and the egg membranes are somewhat freed from its surface.

But this radial or rotatory symmetry is not retained after the entrance of the sperm, for this affords the stimulus which leads to a rearrangement of the substance of the egg, accompanied or followed by the rapid development of a bilateral symmetry in the egg, with which that of the embryo tends strongly to coincide. The factors determining the position of this new plane of bilateral symmetry are really three-fold, one primary and two secondary. The primary factor is the polar and rotatory symmetry of the unimpregnated egg; the plane of 
bilateral symmetry always passes through the chief egg axis. The secondary factors then determine through what meridian the plane will pass. One of the secondary factors is internal and one external; the former is the point of entrance of the spermatozoön together with the direction of its penetration path, the latter is the direction of the action of gravity with respect to the egg axis. These secondary factors alone cannot direct the position of this plane, but each acts only in connec tion with the primary factor which is, in reality, the essential structure or organization of the egg as expressed by its polarity and rotatory symmetry.

We should recall that the sperm enters the upper pole in any meridian (fertilization meridian) and that its penetration path is first approximately radial, while the latter part of its path, copulation path-along which it passes after the sperm head has dissolved and become vesicular, may be at an angle with the penetration path.

Immediately upon the entrance of the spermatozoön the substance of the egg becomes more labile, and a sharper differentiation and more pronounced segregation of the various egg substances result. It is supposed that the influence of the sperm is first exerted in the cytoplasm in its own immediate neighborhood, and that the effects of its presence then spread gradually to the more remote parts of the egg; and further, that the influence of the sperm extends in a symmetrical wave like those from a vibrating body. The result of this would be that the rearrangement of the substances of the egg would be symmetrical with reference to the point of origin of the disturbance, namely, the sperm entrance point. Therefore the plane of symmetry of the egg would be that plane containing the three points: animal pole, vegetal pole, sperm entrance point (Fig. 27, B). This would be at the same time the plane containing the penetration path, and it would be marked superficially as the fertilization meridian.

Whether or not this is a true description of the effects of the sperm, the facts are that following impregnation there is a streaming of the protoplasm upward and of the deutoplasm 
downward so that the animal pole is largely freed from yolk, the vegetal pole composed more largely of it, and the polar differentiation thus more marked than heretofore. The pigment granules, whose specific gravity is really intermediate betweer, that of the yolk and of the protoplasm, show little disturbance and redistribution except in one certain region. For some reason which is not clear, the pigment granules located in a definite area at the lower margin of the pigmented pole, on the side

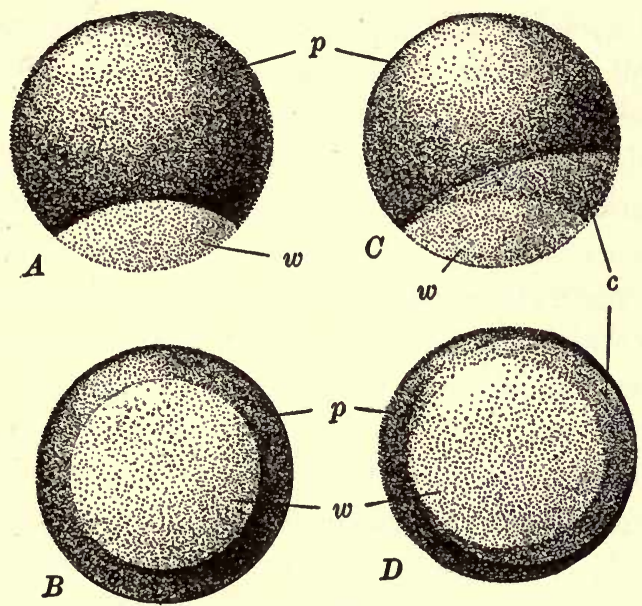

FIG. 29.-Frog's egg before and after fertilization, showing the formation of the gray crescent. A. Unfertilized egg, from side. $B$. Unfertilized egg, from vegetal pole. $C$. Fertilized egg before first cleavage, from side. $D$. Same from vegetal pole. $c$, Gray crescent; $p$, pigmented animal pole; $w$, unpigmented vegetal pole.

opposite that where the sperm has entered, and therefore in the region presumably the last to be affected by the sperm, are carried away from their original position leaving this region lighter in color. This area is crescentic in outline, the crescent extending one-half to two-thirds around the egg; it is known as the gray crescent (Fig. 29).

The rearrangement of substance which involves the formation of the gray crescent, is such that the center of the specifically lighter substance of the egg is not located precisely in the egg axis, toward the animal pole, but is displaced toward that 
side on which the gray crescent appears, i.e., on the side of the animal hemisphere farthest from the fertilization point. Normally this arrangement is sufficiently marked before the rotation of the egg is completed, so that when the egg comes to a position of rest the animal pole is not turned exactly upward. In most species of frogs the egg axis is inclined out of the vertical about thirty degrees; and of course at the same time the margin of the pigmented area is similarly tilted out of the horizontal and the gray crescent lies on that side which is the higher (Fig. 29). In the egg at rest, therefore, we may describe a definite plane which is vertical and includes both the gravitational and the polar axes; from the mode of determination of the position of the gravitational axis this plane also includes the fertilization point and meridian, and the penetration path.

While the egg is in this position the streaming and rearrangement of its materials is completed, and since the specific gravity of the different materials is concerned in the rearrangement, it takes place finally with reference to the direction of gravity in the egg at rest. The final steps in the determination of the structure of the unsegmented egg, therefore, take place with reference to this gravitational plane, which thus becomes the plane of bilateral symmetry of the egg structure. The symmetry of the egg is expressed externally at this time only by the gray crescent which is equally divided by the plane of symmetry, but this is merely an indication of the really important symmetry-that of the arrangement of the materials within the egg, which is no longer rotatory about the egg axis, but bilateral with reference to the gravitational plane.

The final development of the internal structure or organization of the egg is completed (in Rana fusca) only shortly before the first cleavage, or about an hour and a half after the entrance of the sperm (Brachet). Before the end of the first hour, the structure of the egg is gradually becoming fixed and disturbances or artificial lesions are compensated, or regulated, so that the final structure is not affected and later development is not abnormal. But after this, by the time the egg and sperm nuclei have fused, the egg structure becomes fixed 
and the egg is incapable of perfect regulation under abnormal conditions imposed upon it, so that artificial lesions or other disturbances result in abnormalities of its structure which lead to abnormalities in cleavage or embryonic development.

To summarize, the bilateral symmetry of the egg is determined primarily by the polarity of the egg which has gradually developed during its formation and maturation. How extensive this essential bipolar structure is, we do not know, but it is expressed visibly, and probably only in part, by the polar arrangement of at least three different substances having different specific densities-protoplasm, pigment, deutoplasm. Probably the arrangement of these is itself determined by some fundamental structure of the egg, but this we cannot observe directly. The bilateral symmetry is here only potential. It becomes actual only after ensemination when these substances are rearranged, first under the influence of the entering spermatozoön, which brings about the non-correspondence of the egg axis and gravitational axis, and then through the influence of gravity according to the plane fixed by these two crossing axes. In other words the bilateral symmetry is first determined by the egg structure as expressed through its polar differentiation and then through that as the result of the action of the entering spermatozoön and gravity, which latter is able to act finally only after the entrance of the sperm.

The penetration path of the sperm is usually in the direction of a radius of the egg from the entrance point, so that this portion of the sperm path tends to lie in the plane of symmetry to the same extent as, or even to a greater extent than the entrance point itself, and it may be that we should express the relation more truthfully by saying that the plane of symmetry tends to be directed first by the location of the penetration path rather than the fertilization point, since the influence of the sperm is exerted as it passes all the way along this portion of its course.

By placing the eggs under artificial conditions it has been found that the action of both these secondary factors is not essential, for normal development proceeds even when the di- 
rective effect of gravity is removed; it is not known, however, what the relation of the plane of symmetry to the fertilization point is under such conditions. And further, by placing the eggs in positions of constraint such that the sperm entrance point cannot lie in the median gravitational plane, it is found that the plane of symmetry is then that of the median gravitational plane. The secondary factors may thus be independent in their action, but in nature they usually tend to produce the same effect. However, in nature, and this also indicates their fundamental independence, there is some deviation between the plane of symmetry and either the fertilization meridian or the gravitational plane. The eggs in the interior of the mass are subject to some constraint due to pressure, and unknown factors may cause some variability in the effects of the factors named in the determination of symmetry. It is possible that the direction of the incident light (heat) rays plays some small part in the determination of the position of this plane. So that while the normal relation is that of coincidence of all these, all other relations are possible (the plane symmetry of course is always polar) and do occur with some frequency, and all that can be said is, that on the whole the median plane of the egg tends to coincide with the gravitational plane and with the fertilization meridian.

One of the reasons why the position of the plane of symmetry of the egg is of the greatest importance is that the plane of bilateral symmetry of the embryo and adult is directly related to it. Any factor which aids in the determination of the egg symmetry is at the same time influencing the symmetry of the developing embryo. By observing, in a large number of specimens, the relation between the symmetry of the egg and of the embryo it is found that the tendency for the two to correspond is very marked. And yet variations of any extent may and do occur, showing that other factors may influence and development of the embryo (Jenkinson). The symmetry of the embryo and adult can be traced directly back into the gastrula or blastula, and it seems, therefore, that whatever causes the non-correspondence between these symmetries must operate 
during cleavage and not later, although there seems to be no definite causal relation between the direction of the first cleavage plane itself and the symmetry of the embryo, although there may be certain constancies in this relation.

We may finally mention briefly the relation between the symmetry of the egg (and therefore in general of the embryo) and the symmetry of cleavage, particularly the plane of the first cleavage furrow. The position of the cleavage plane is of course the direct result of the position of the cleavage spindle; it is therefore the position of this latter which is essential. The spindle always lies at right angles to the egg axis, in agreement with the law of Hertwig. In such a plane the position of the spindle is readily influenced by at least one external factor, namely pressure, in such a way that it tends to lie at right angles to the direction of the pressure, and the resulting cleavage would therefore occur in the direction of the pressure. In a large mass of eggs this factor is probably one of considerable importance, especially in affecting the direction of the first cleavage in those eggs in the interior of the mass. The relation between the direction of pressure and the symmetry of the egg is purely accidental and consequently we find much variation in the relation of these two planes.

When the egg is not subject to pressure there is a fairly marked tendency for the spindle to lie either transversely to the plane of symmetry or in that plane (Fig. 27, $C$ ). The symmetrical structure of the egg is fairly well established by the time the spindle forms, and there are only these two positions which the spindle can occupy and yet retain symmetrical relations to the internal structure of the egg. The former relation, in which the plane of the resulting cleavage would coincide with the plane of egg symmetry, is the more frequent and in approximately 25 per cent. of eggs the first furrow deviates less than five degrees, plus or minus, from this plane. The second relation, placing the first furrow at right angles to the median plane (within five degrees, plus or minus) is found in something like 10 per cent. of eggs (Jenkinson).

But the position of the spindle seems to be influenced quite 
considerably by the direction of the copulation path of the sperm nucleus, i.e., the direction of the plane passing through the contact surface of the copulating egg and sperm nuclei, and since this is subject to much variation with respect to the median plane, and also since the postion of the spindle is easily modified by external factors, we find that the relation between the first cleavage and the median plane of the egg is not at all regular, and deviations from the two relations mentioned are very frequent and all relations occur. There is no direct relation between the plane of the first furrow and the fertilization meridian; whatever relation there is results from their common relation to the plane of egg symmetry.

We may summarize briefly the relations of the plane of symmetry of the egg, the plane of symmetry of the embryo, and the plane of the first cleavage furrow, in normally developing eggs.

The position of the plane of bilateral symmetry of the egg is determined primarily by the polarity and rotatory symmetry of the unfertilized egg, in conjunction with the point of entrance of the sperm or the direction of the penetration path, and the direction of the action of gravity, in such a way that the median plane tends to lie in the gravitation plane, which also tends to coincide with the fertilization meridian. This determination, however, is not complete and variations may and do occur.

The median plane of the bilaterally symmetrical embryo tends to a marked degree to coincide with the plane of symmetry of the fertilized egg, but all other relations in the same axis are possible and actually occur.

The plane of the first cleavage furrow tends to lie either in or at right angles to the plane of symmetry of the egg, primarily on account of the tendency of the first cleavage spindle to assume some symmetrical position with reference to the egg structure.

There is, therefore, a tendency for the gravitational plane, the point of entrance of the spermatozoön, the penetration path of the spermatozoön, the median plane of the egg, the median 
plane of the embryo, and the plane of the first cleavage furrow, all to coincide, but all relations in the same axial plane are possible among these and are actually found.

\section{Cleavage}

Cleavage of the frog's egg is total and unequal. The first cleavage spindle lies in the direction of the greatest protoplasmic extent, i.e., transversely to the egg axis, and in a position determined by several different factors as described above (Fig. $27, C)$. The first cleavage furrow becomes visible on the surface first at the animal pole, and gradually extends thence as a narrow groove around a meridian of the egg to the vegetal pole; it is completed about two and one-half hours after ensemination, or much sooner if the temperature is raised slightly. While this furrow is meridional, we have seen that it may or may not divide the gray crescent symmetrically. Throughout cleavage the blastomeres remain in close contact so that they are separated superficially by only shallow narrow grooves (Fig. 30), and do not become distinctly rounded and separate elements as in Amphioxus or in other eggs containing less yolk.

The second cleavage appears about one hour after the first; this is also meridional, at right angles to the first, dividing the egg into four adequal blastomeres. Succeeding divisions appear about an hour apart. The third cleavage is the first to divide the egg unequally; in the typical form of cleavage this appears similarly in all four quadrants, and is latitudinal or horizontal, i.e., at right angles to the first two (Fig. 30, A). Although this cleavage plane divides the protoplasmic material of the egg about equally, the accumulation of yolk in the lower pole actually displaces this middle plane above the equator of the egg, so that the cleavage furrow appears about sixty degrees from the animal pole, and the egg as a whole is divided unequally. Of the eight resulting cells, the four upper are small and richer in protoplasm, while the four lower are large and richer in yolk. This typical relation of the third cleavage is by no means invariable. A considerable proportion, in 
some lots nearly one-half, of the eggs show some departure from this arrangement, and the third cleavage may be horizontal in only one, two, or three blastomeres, and vertical in the remainder or, rarely, vertical in all four. These and also the later vertical planes frequently do not pass actually to the upper pole of the egg, and are therefore not strictly meridional,
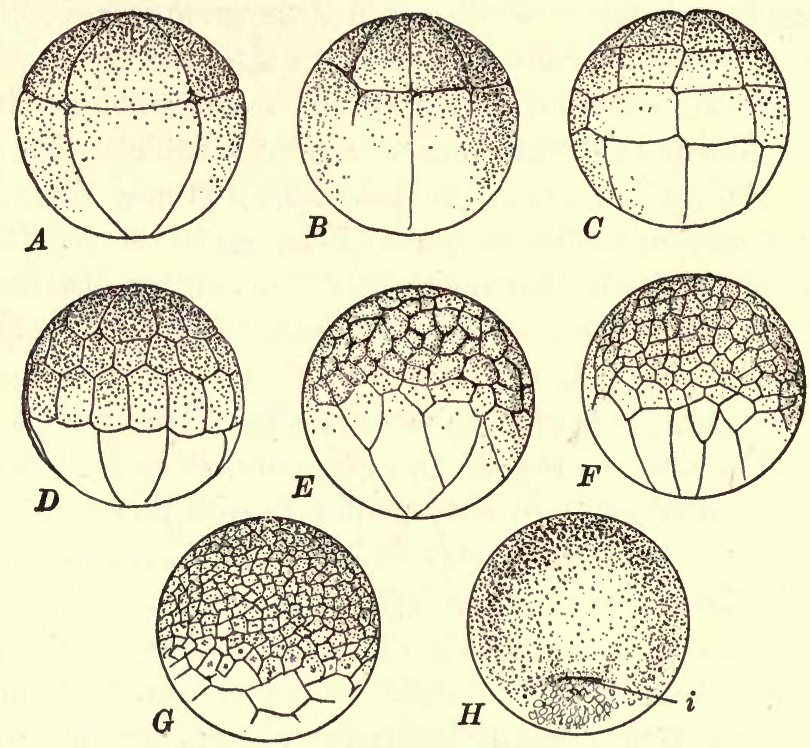

Fig. 30.-Cleavage of the frog's egg. After Morgan. Animal pole upward in all figures. (For earlier stages see Fig. 27.) A. Eight cells. $B$. Twelve cells becoming sixteen. $C$. Thirty-two cells. $D$. Forty-eight cells, more regular than usual. $E, F$. Posterior and anterior views of about 128 cell stage. $G$. Late cleavage or early blastula. $H$. Commencement of gastrulation (cell outlines indicated only in the region below the invaginating groove). $i$, Beginning of invagination.

although there is a decided tendency for them to lie in meridians. The location of the succeeding cleavages varies with that of the third. Typically the fourth cleavages (Fig. 30, B) are meridional forming eight small upper, and eight large lower cells, and the fifth again latitudinal, forming thirty-two cells arranged in four horizontal rows of eight cells each (Fig. 30,C). But the atypical appearance of some of the third cleavages may very early disturb this schema. With the appearance of the 
fifth cleavage the early, and brief, synchronism of the cleavages has become lost, the small upper cells dividing more rapidly than the large lower cells. Comparatively few eggs remain regular up to this stage, for eggs which were regular at eight cells usually become irregular at sixteen or thirty-two cells, and after that all regularity is lost (Fig. 30).

Turning back now to notice some of the internal arrangements of the cells during cleavage, we find that when four cells are cut into eight these all round off somewhat internally, as in Amphioxus, forming a small space among them, which is the beginning of the segmentation cavity or blastoccel, and which from the position of the third cleavage has from the first an eccentrie position toward the animal pole. During subsequent cleavages the blastocœl enlarges but always retains this eccentric location (Fig. 31, $A$ ).

After about thirty-two cells are formed not all of the subsequent cleavages are visible on the surface, for these early divisions, all passing through the axis of the egg, have formed cells elongated in a radial direction, and in some of these the cleavage spindles tend to take up a similar position and the resulting division occurs parallel with the surface of the mass, forming a central cell bordering the segmentation cavity and a superficial cell visible externally (Fig. 31, $B$ ). There is no period at which such a delaminating cleavage occurs throughout the cell group, but scattered cells show this arrangement, first among the cells of the upper hemisphere and then later in the lower cells, which are divided quite unequally in this way. Many of these interior cells are formed by cleavages that are not exactly tangential but considerably oblique to the surface. By the time there are sixty-four or one hundred and twenty-eight cells, approximately one-fourth of the cells are interior, and line the blastocœl, so that at this stage the wall of the blastocœl is two or more cells thick.

\section{The Blastula}

We may assume that the arrangement of the cells forming the wall of the blastocœl as a more or less definite epithelium, 
marks the end of the cleavage period and the formation of the blastula. This arrangement is really definitely established by the time thirty-two to sixty-four cells are formed, i.e., before many interior cells are present. The immediately sub-

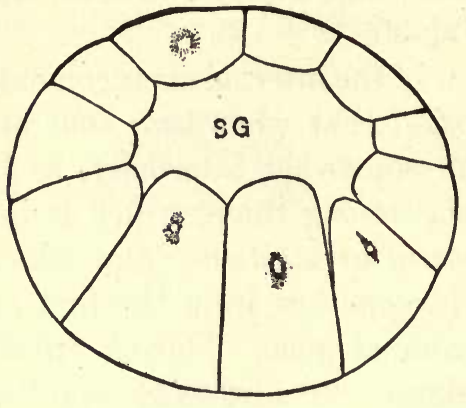

$\boldsymbol{A}$

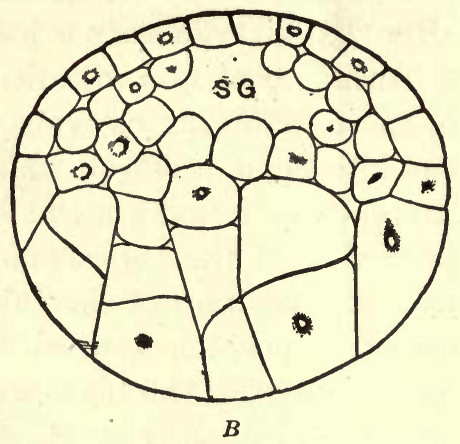

$\boldsymbol{B}$

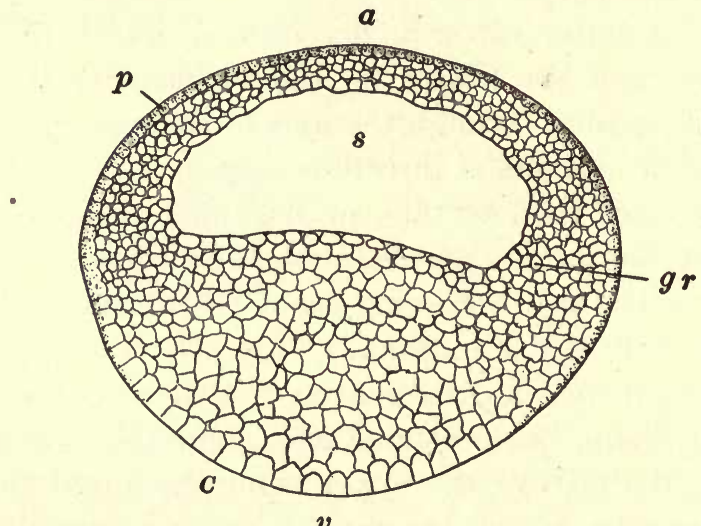

FIg. 31.-Sections through the blastula of the frog. $A, B$, from Morgan (Development of the Frog's Egg). $C$. After O. Schultze. A. Early blastula showing wall of segmentation cavity only one cell in thickness. $B$. Later stage showing multiplication of cells in wall of segmentation cavity. $C$. Late blastula showing the thinning of the roof of the segmentation cavity and the beginning of the germ ring. $a$, Animal pole; $g r$, germ ring; $p$, pigment; $s$, segmentation cavity or blastocœl; $S G$, Same as $s ; v$., vegetal pole.

sequent cleavages do not modify their essential relations; the interior cells multiply rapidly and some cells migrate inward from the surface. The early blastula is spherical and about the diameter of the egg (Fig. 31, $B, C$ ). The thinner roof and lateral walls of the eccentric segmentation cavity, are formed 
of cells derived from the animal region of the egg; these vary considerably in size and form, are irregularly and loosely arranged, and are roughly disposed in two sheets, one lining the blastocœl and one covering the surface. The thicker floor of the blastocol is formed of the larger and less numerous vegetative cells. As the number of cells continues to increase several processes go on together. The small cells of the animal region divide the more rapidly and as they multiply they move gradually from the pole toward the equator, causing a thinning of the roof and a thickening of the walls of the blastocol. This thickening toward the equator of the blastula is augmented by the rapid multiplication of cells there so that sections soon show a thicker ring, not very definitely delimited, of actively dividing cells forming what we may call the germ ring or growth zone, such as that described in the blastula and gastrula of Amphioxus (Fig. 31, C). This thinning of the animal pole increases the eccentricity of the blastocœel, which has meanwhile increased considerably in size. The later blastula increases somewhat in diameter, and accompanying this is the absorption or infiltration of water into the blastocœl, a part of the fluid content of which is, however, the secretion of its walls.

The germ ring is obviously formed of material from the animal pole of the egg, and apparently the substance contained in it can be distinguished at least as early as the eight cell stage, where it forms the upper quartet (micromeres) and the upper parts only of the lower quartet (macromeres) (Fig. 34). As the germ ring approaches the equator, one side (that of the gray crescent) commences to extend downward faster than the remainder; subsequent development proves this to be the posterior side. Soon the entire germ ring passes the equator, and by the time the blastula period is ended, it reaches, on the posterior side, a point about half way between the equator and the lower pole. These later phases in the downward movement of the animal cells can be observed externally, for these cells are easily distinguishable from the true lower pole cells on account of their dense pigmentation. 
Since the blastula retains its spherical form it is evident that the downward extension of the germ ring must displace the yolk cells in some direction, and as a matter of fact this displacement is evidenced by the elevation of the floor of the blastocœl. As the animal cells push downward the internal yolk cells rise till the floor of the blastocœl becomes first flat, and then convexly arched; at the same time the cavity widens somewhat so that in section it appears broadly crescentic (Fig. 32, $A, B$ ).

The first evidences of gastrulation now appear so that this stage must be taken to mark the completion of the blastula. We may state the characteristics of the fully developed blastula as follows. The completed blastula is spherical, in volume about one-fifth larger than the ovum, and bilaterally symmetrical; this bilaterality is accompanied by antero-posterior differentiation, and is indicated by the greater thickness of the anterior wall of the segmentation cavity, and by the more ventral extension of the pigmented cells on the posterior side, i.e., the side marked by the gray crescent in the egg. The small cells of the upper pole form the thin roof and thicker sides of the eccentric blastocœl; they are in two quite distinct sheets-an outer layer of compactly arranged cells forming a distinct epithelium known as the superficial or epidermal layer, and, lining the blastocœl, a deeper or "nervous" layer of irregularly and loosely arranged cells, gradually increasing in thickness from the pole toward the base of the blastocœlic wall, about at the level of the equator of the blastula. Just at and below the equator actively dividing cells have accumulated from the whole upper pole region. Since the fate of these cells is to form the chief axial parts of the embryo this region is called the germ ring, although it lacks the distinctness of the germ ring as it is finally found in some other forms (e.g., Teleosts). This region seems comparable with a crescentic group of actively dividing cells having a corresponding position and function in the blastula of Amphioxus (Fig. 6). The floor of the blastocœl is formed of the larger vegetative cells which form practically the lower half of the blastula; these are com- 
pacted and show no definite arrangement, except that toward the lower pole they gradually increase in size. Although some yolk is contained in all the cells, the larger lower pole cells are particularly rich in deutoplasm and are known as the yolk cells. Immediately below the germ ring the cells are intermediate in character and form what is known as the transitional zone. The position of the polar axis in the blastula, with respect to gravity, remains the same as in the egg or the cleavage period.

The pigment, while chiefly superficial and in the cells derived from the animal pole, externally extends further toward the lower pole than in the egg; it is also found to a limited extent among the animal cells below the surface, and even in the smaller vegetal cells lining the blastocœl, which resemble closely the proper cells of the animal pole. This internal pigment is not derived from that more superficially located in the earlier stages, but it is deposited in situ as a by- or endproduct of metabolism. Pigment granules are laid down wherever developmental processes, including cell division, are in rapid progress. And since the smaller cells represent regions where cell multiplication has been more active, such cells contain relatively more pigment. This relation between metabolic activity and the accumulation of pigment may explain the pigmentation of the animal pole of the egg itself, although it is customary to refer this to the adaptational relation mentioned previously, a relation which need not be negatived by this method of its formation. The dense pigmentation of the path of the sperm is also referable to an unusual degree of metabolic activity.

The blastula of the frog differs from that of Amphioxus chiefly in that the blastocœl of the former is so decidedly eccentric, relatively smaller, and its wall several cells thick, the cells differing greatly in size, and already differentiated into superficial and deeper layers, at least in the animal region. The germ ring (growth zone in Amphioxus) extends completely around the blastula of the frog. These differences for the most part seem to be the direct results of the abundance of yolk in 


\section{OUTLINES OF CHORDATE DEVELOPMEN'T}

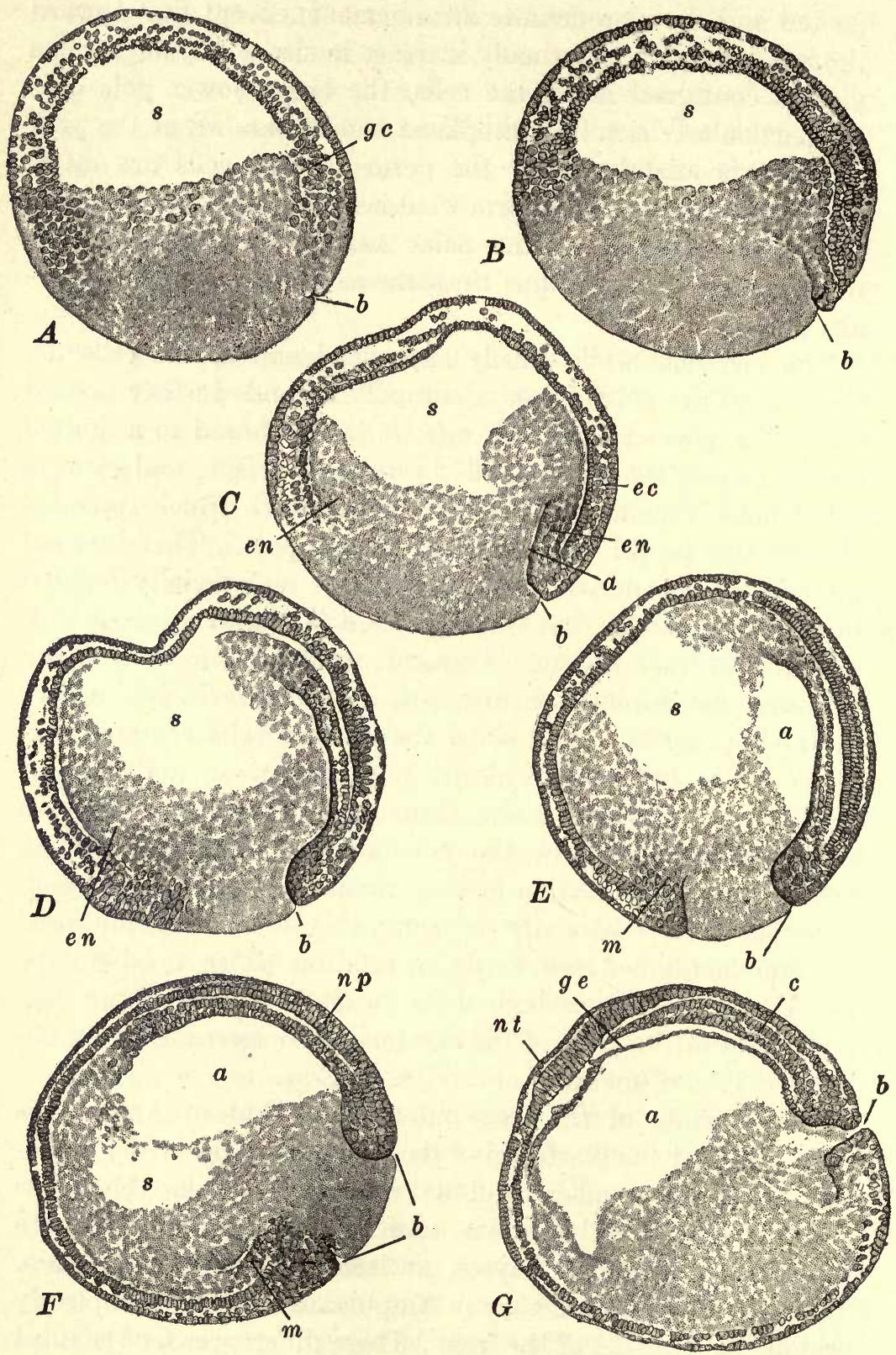

Fig. 32.- Median sagittal sections through a series of gastrulas of the frog (R. temporaria). After Brachet. The figures illustrate the change in position 
the frog egg, and its accumulation chiefly in one region. The effect of the yolk in modifying the course of development becomes more marked in the immediately succeeding phases of development, namely gastrulation and notogenesis; these processes are not at all as simple and diagrammatic as in Amphioxus.

\section{Gastrulation and Notogenesis}

The processes of gastrulation proper and of notogenesis overlap to a more considerable extent in the frog, than in Amphioxus, and are conveniently described together. Gastrulation results directly from a continuation of the downward extension of the germ ring, together with the consequent elevation of the yolk cells, which were such important features in the development of the late blastula.

The first external indication of gastrulation is the appearance of a slight irregular groove, approximately horizontal, lying across the sagittal plane on the posterior side of the egg, just at the lower margin of the germ ring, i.e., just below the equator (Figs. 32, 35, A). Thus located, the groove lies just between the animal cells and the yolk cells, and therefore comes to be lined by both kinds of cells on its opposite faces. From subsequent development we know that the formation of this groove is the beginning of invagination, the groove itself the beginning of the archenteron, its upper margin the rim of the blastopore, and the cells lining it above and below,

of the whole gastrula, as well as the phenomena of gastrulation proper. A. Commencement of gastrulation; earliest appearance of the dorsal lip of the blastopore. Internally the gastrular cleavage is indicated. $B$. Invagination more pronounced; beginning of epiboly. C. Invagination, epiboly and involution in progress. The gastrular cleavage is now indicated on the side opposite the blastopore. Rotation of the gastrula. D. Just before the ventral lip of the blastopore reaches the median line. The indentation of the wall of the segmentation cavity is an artifact. $E$. Blastopore circular and filled with yolk plug. Gastrula beginning to rotate back to its original position. Peristomial mesoderm differentiating. F. Segmentation cavity nearly obliterated. Neural plate established. G. Gastrulation completed. $a$, Archenteron; $b$, blastopore; $c$, rudiment of notochord; $e c$, ectoderm; $e n$, endoderm; $g c$, gastrular cleavage; ge, gut endoderm; $m$, peristomial mesoderm; $n p$, neural plate; $n t$, transverse neural ridge; $s$, segmentation cavity or blastocœl. 
ectoderm and endoderm respectively. Hemisection of this very early gastrula (Fig. $32, A$ ), shows that the elevation of the floor of the blastocœl is very rapid at this time, and one of the first results of the arching up of the yolk cells (endoderm) is the formation of a narrow groove all around the margin of the blastocœl, between the base of its wall and its rising floor. As the yolk continues to rise, this groove soon becomes quite marked and compressed into a narrow slit which, though originating in the manner just described, seems tobe continued ventrally all around the central cells as a definite splitting or delamination (Fig. 33, $A$ ). In effect this narrow space separates definitely the ectoderm and endoderm in the region within (above) the restricted invaginating region, which of course also gives rise to an ectodermal and an endodermal layer. This original groove is called the gastrular groove, and the delamination which extends it is the gastrular cleavage; the formation of these is not limited to the region of the dorsal lip of the blastopore, but extends entirely around the gastrula, even to the side opposite that of invagination. It remains a question whether the invagination process is the result of an active inturning of the cells forming the lower margin of the germ ring, or whether these cells are rather pulled inward by the elevation of the yolk cells, which results from the compression produced by the thickening and downgrowing germ ring; perhaps both factors are involved. However this may be, the invagination once begun continues rapidly, so that an elongating tongue of inturned cells continually pushes up under the superficial ectoderm which lies just above the invaginating region. This tongue is the invaginated endoderm derived from some of the cells of the animal pole and their descendants; at its inner limit it becomes directly continuous with the endoderm formed directly from the yolk cells, or cells of the transitional zone which have become entitled to the name endoderm, while still practically in situ, by the appearance of the gastrular groove and cleavage.

Turning for a moment to the consideration of the external modifications during gastrulation we see that the germ ring, 
once having passed the equator begins tó niarrow as a whole (i.e., diametrically), and this is chiefly accomplished by the drawing in of its lateral regions toward the mid-line posteriorly. This, together with the very active multiplication of its constituent cells, causes this portion to push down more rapidly than the remainder, carrying along the layer of invaginating endoderm, and increasing considerably the vertical extent of the archenteron. Surface views show that at the same time the archenteric groove extends laterally, becoming first crescentic, then semicircular and finally circular (Fig. 35). That is to say, the first invagination of the pigmented cells forms the dorsal lip of the blastopore; then the invagination gradually extends laterally in each direction forming the lateral lips of the blastopore; and finally the process of invagination is carried around to the side of the gastrula, almost diametrically opposite to that where it began, and forms there the ventral lip of the blastopore, and the circular blastoporal margin is thus completed. During the completion of the blastoporal rim the germ ring has continued to extend downward over the yolk on all sides, so that by the time the rim is completed by the formation of the ventral lip, this is found at a level quite below that at which invagination began on the dorsal side (Fig. 35). The invagination involves the inturning of the cells transitional between the animal and vegetal poles, so that the cells of the pigmented and white areas are brought into sharp contrast. The circle of white yolk cells left within the blastoporal rim is called the yolk plug (Figs. 22, B; 32, E, F; 33, C). As the rim draws together, i.e., as the blastopore closes, the yolk plug appears gradually to diminish in size, while it really draws within, or some would say that it is pushed within, by the overgrowing lips of the blastopore, until finally it is no longer visible upon the surface (Fig. 38, B). The blastoporal opening then remains as a narrow elongated slit leading directly into the archenteron.

The precise way in which the germ ring narrows, or as we might say, in which the blastopore closes, is a matter of some importance. It has already been stated that the dorsal lip 


\section{OUTLINES OF CHORDATE DEVELOPMENT}
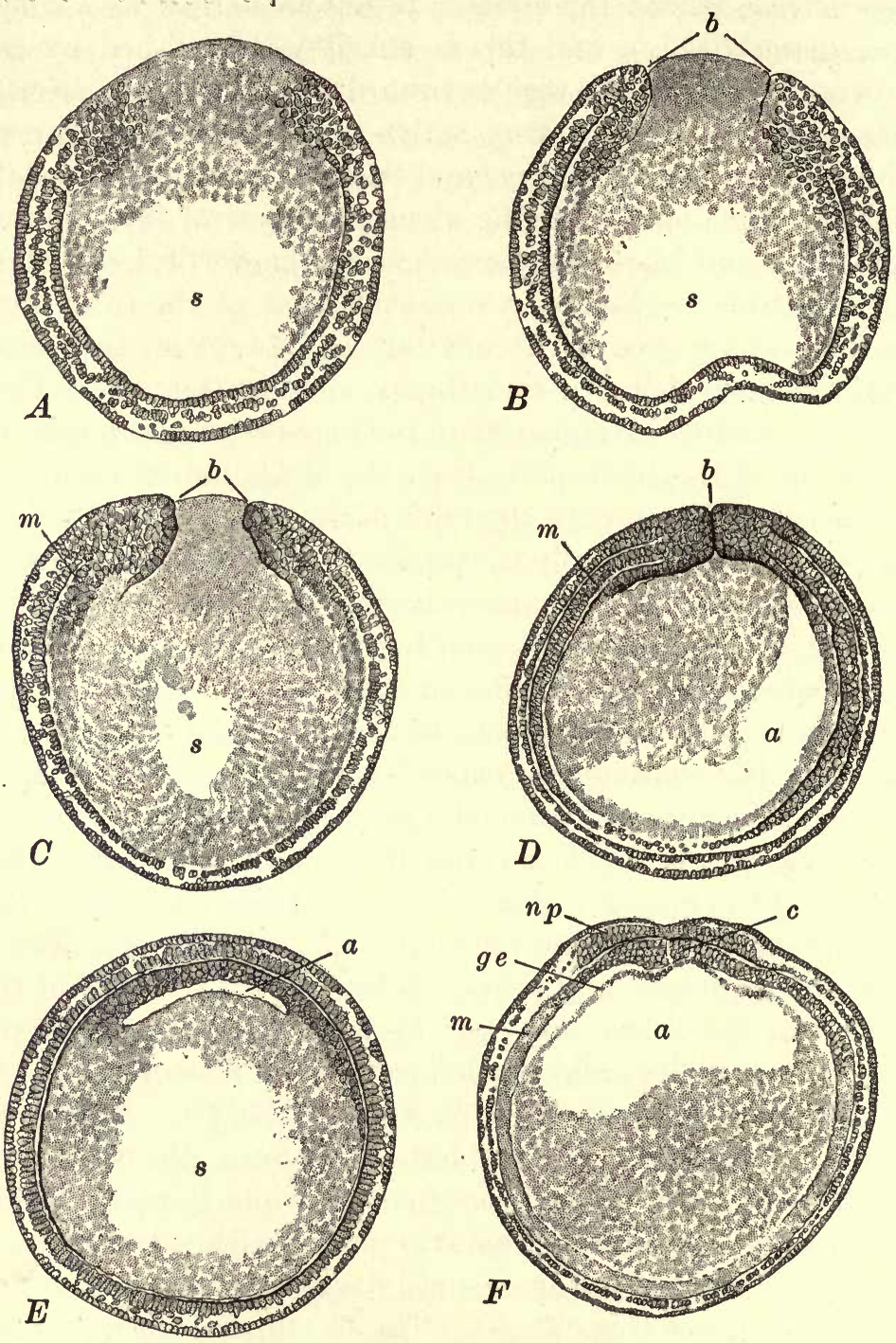

Fig. 33.-Frontal and transverse sections through gastrulas of the frog (R. temporaria) of various ages. After Brachet. A. Frontal section through gastrula of same age as Fig. 32, C. B. Frontal section through gastrula of same age as Fig. $32, D$. C. Frontal section through gastrula slightly older than Fig. 32, $F$. $D$. Frontal section through gastrula of same age as Fig. 32, G. $E$. Transverse section through gastrula slightly older than Fig. 32, D. F. Transverse section through gastrula slightly older than Fig. 32, G. a, Archenteron; $b$, blastopore; $c$, notochord; ge, gut endoderm; $m$, peristomial mesoderm; $n p$, neural plate; $s$, segmentation cavity or blastocœl. 
grows downward more rapidly than the remainder, due in part to the inflowing of the lateral portions toward the mid-line. This process is termed concrescence, or confluence, as in Amphioxus. In this way the materials found in the lateral, as well as posterior, regions of the germ ring are drawn to the median region, and as the ring there extends backward or downward, a thick median strand of tissue is left, from which are developed later, when the embryo begins to form, the rudiments of many of the important axial organs. The downward progress of the lateral and ventral margins of the blastopore is quite slow comparatively, so that the closure seems to occur mainly toward the lower pole of the gastrula. That is, as the diameter of the circular blastopore diminishes, its center, which is the center of the yolk plug, moves toward the lower pole and may finally reach this or even pass beyond it a short distance up on the opposite side. The form of the blastopore changes markedly during the later phases of its closure. The final rapid approach of the lateral margins alters its outline from a circle, so that it becomes ovoid and finally quite elongated and slitlike, in the direction of the sagittal plane of the gastrula (Figs. 35, 38, A). But before this occurs the blastopore is carried back near its point of origin by the rotation of the whole gastrula to be described shortly.

Sections through the gastrula during this period of closure of the blastopore show that many important processes are going on internally. Continuing from the stage described, where the archenteron had become a curved crevasse putting the invaginated and delaminated endoderm of its outer wall into connection, we find that the essential process of gastrulation is continued chiefly by the rising of the yolk cells from the floor of the blastocœl in advance of the archenteron, so that the inner limit of this cavity is carried upward under the animal pole and then beyond, toward the side of the gastrula opposite that of the first appearance of the archenteron (Fig. 32, $C-F$ ). The yolk cells at the same time are carried around the front of the advancing archenteron and form the outer wall of the primary gut cavity. This rearrangement of the yolk cells, involving 
their elevation in the dorsal or postero-dorsal region, draws away the yolk cells from beneath the original segmentation cavity so that as the archenteron advances the segmentation cavity recedes. Meanwhile the archenteron enlarges over the whole animal region and actually encroaches upon the blasto-
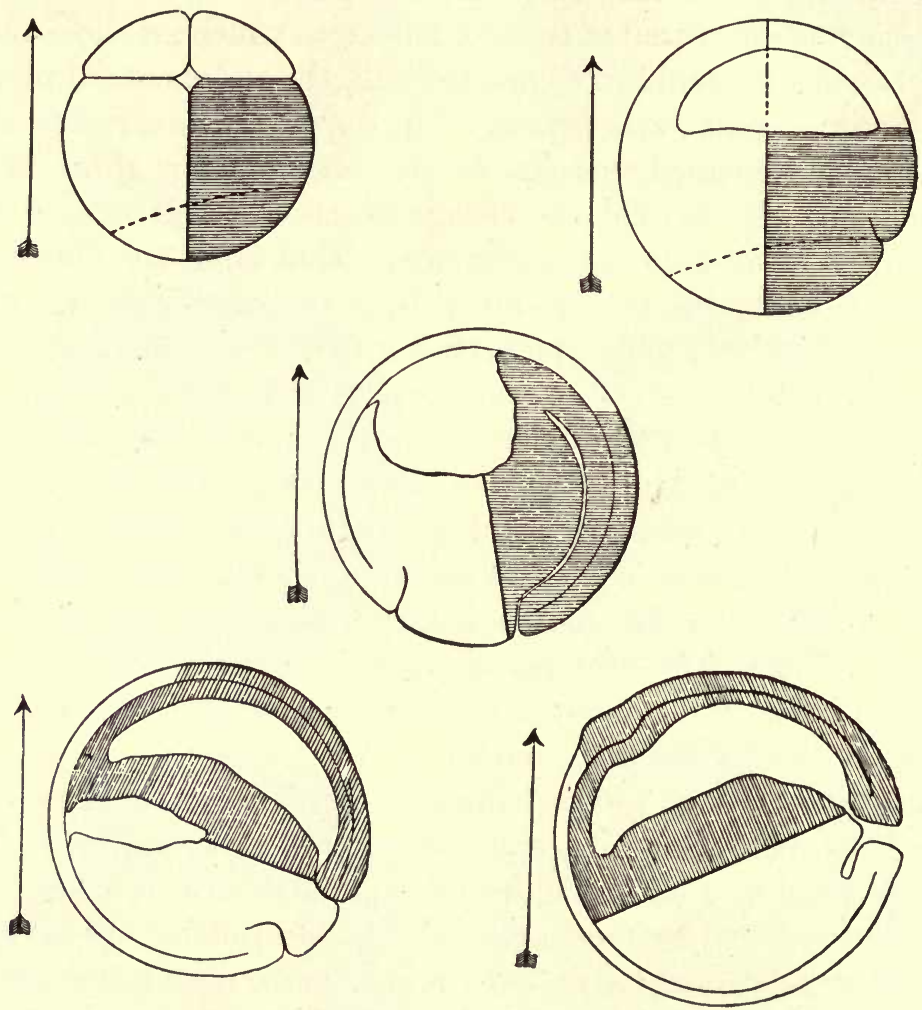

Fig. 34.-Diagrams of median sagittal sections through the frog's cleavage and gastrula stages, showing the changes in position during gastrulation. From Ziegler (Lehrbuch, etc.), after Kopsch. The arrow marks the vertical. (According to Morgan and others the figures of the first two stages should be rotated about $15^{\circ}$ in the counter-clockwise direction.)

cœl, so that as the latter moves toward the anterior side of the gastrula it diminishes in size and soon disappears (Figs. 32, 33). In some cases the wall of yolk cells separating the archenteron and blastocœl becomes thin and breaks through, before the blastocœl has completely disappeared; in this case the remnant 
of the blastocœl is added to the archenteron and the endodermal wall is completed by growth of the portion already formed.

The formation of the archenteric cavity in a region formerly occupied by yolk cells, and the gradual enlargement and shifting of this cavity as well as of the blastocœl, obviously alter the position of the center of gravity of the gastrula as a whole, and the extensive changes in the relative positions of yolk and protoplasmic cells, whose specific gravities are unlike, contribute to the same alteration. This all results in a rotation of the gastrula about a horizontal transverse axis (Fig. 34). During the early phases of gastrulation, as just described, the more rapid growth of the dorsal (posterior) lip of the blasto-
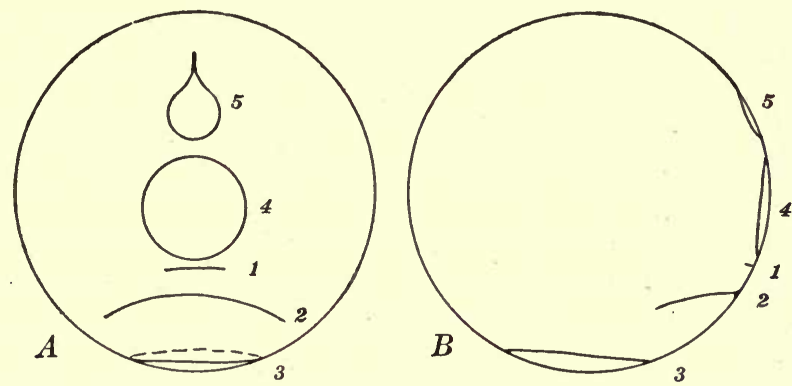

FIG. 35.-Diagrams of the frog's gastrula showing the position of the blastopore at various ages. $A$. Posterior view. $B$. Lateral view. 1-5 indicate the successive positions and forms of the blastopore. The change in position is due both to the actual growth movements of the blastopore, and to the rotation of the entire gastrula. Compare Figs. 32, 34 .

pore carries this to or even past the lower pole of the gastrula, even past the lower gravitational pole. Then as the blastopore continues to narrow, the whole gastrula rotates slowly in the opposite direction, carrying the blastopore back to the region where the dorsal lip first appeared and then on dorsally somewhat above the equator, into a postero-dorsal position, where it remains stationary for a time. Thus the shifting of the blastopore is the combined result of changes due to growth and to rotation. On account of the localization of the growth processes in the gastrula, which will be described presently, there is not a complete correspondence between the chief axis of the blas- 
tula or early gastrula and any single straight axis of the later gastrula.

The description of gastrulation from observation of median sagittal sections or hemisections does not give a complete idea of this process. We may return now to a consideration of some of the processes going on in the lateral parts of the gastrula. As the dorsal lip of the blastopore becomes crescentic, the deepening archenteron pushes laterally around through the mass of yolk cells (Fig. 33, $B, C, D$ ). But these have already become separated from the ectoderm by the gastrular groove and cleavage, except only in the region of the lower pole just anterior to the place where the anterior (ventral) lip of the blastopore will form. So that by the time the blastoporal lip becomes circular, i.e., by the time the ventral lip forms, this region has already been divided into ectoderm and endoderm, and therefore the extent of invagination at the ventral lip is greatly limited. The important result of this is that the actually invaginated endoderm is confined to a broad tongue of cells on the dorsal side (roof of the archenteron) and a ring-like strip extending around within the blastopore lip from the base of this tongue, narrowing rapidly toward the ventral side. The archenteron itself is at first a narrow slit, crescentic in cross section, but as it grows up to the animal pole and enlarges, it grows farther laterally so as to extend in a wide crescent (in transverse section) about to the level of the equator of the gastrula (Fig. 33, $E, F$ ). This leaves the yolk cells as a convex mass projecting into the archenteron from its floor.

The frog illustrates very well the way in which the process of gastrulation proper is complicated, in the Chordata, by the early formation of certain of the important axial organs which are the chief characteristics of the Chordate group; the formation of the rudiments of these structures is termed notogenesis. Gastrulation proper includes only those processes by which the singlelayered (monodermic) blastula is converted into the two-layered (didermic) organism, with definitely established ectoderm and endoderm - the gastrula. The method by which this is accomplished may vary in different groups of Chordates; in Amphi- 
oxus, we have seen, invagination is the chief process, while in the frog this is less important, and the endoderm is more largely the result of delamination or of a simple rearrangement of cells forming different parts of the wall of the blastula. Among the higher Chordates invagination may be entirely lacking and gastrulation may be entirely accomplished by other methods (involution, epiboly, delamination). As a matter of fact, even in the frog, invagination is concerned less with the formation of the inner layer than with the establishment of the notochord and the formation of the rudiment which gives rise in part to the mesoderm. It becomes necessary, therefore, to distinguish sharply between gastrulation and notogenesis. In the frog the strictly two-layered condition exists for a very brief period only, for the earliest phases of notogenesis, namely the formation of the mesoderm and chorda, occur quite precociously. The early formation of these structures may more conveniently be described together, and for the first stages we must return to the early gastrula.

\section{The Mesoderm}

In order to understand the origin of the mesoderm we must examine the early gastrula at the time the gastrular groove and cleavage extend down toward the incipient blastopore. Here the inner region of the germ ring and the yolk cells lining the blastocol are continuous, and it is here that we find those cells which are later to form the mesoderm, and although distinguishable at this time, they are not definitely delimited within this zone which is transitional between the ectoderm and endoderm. On one side these cells are continuous with ectoderm cells, on the other with endoderm or yolk cells (Figs. 32, $A ; 33, A$ ). As the lips of the blastopore extend laterally this mesoderm rudiment forms pari passu, and when the blastopore rim becomes circular the mesoderm rudiment can be distinguished in the ventral lip (Figs. 32, $F ; 33, C$ ). We may say then that the rudiment of the mesoderm appears first as a ring of cells just within the margin of the blastopore. But by the time the ring is com- 
pleted ventrally, its dorsal region, in the more rapidly developing dorsal lip, has broadened considerably so that its general form might be compared with a signet ring.

As the blastopore is completed and begins to close, the confluence of its lateral margins transports masses of the cells toward the mid-line, and leaves them as a broad median band extending upward from the dorsal lip of the blastopore. As a result of the multiplication of these cells, and of the downward extension of this lip, a considerable axial thickening is formed. And at the same time the extension of the archenteron carries the yolk cells, with which the mesoderm is closely related on one side, upward toward the animal pole, so that altogether, even in these comparatively early stages, the extent of the mesoderm is nearly as great as that of the endoderm. And soon the mesoderm is definitely delimited from the endoderm by a rearrangement of cells giving the appearance of an irregular delamination (Fig. 33, D). This delamination commences in the dorsolateral regions either side of the thickened axial mass and gradually extends thence anteriorly and laterally around the sides of the archenteron separating a thin layer of definitive endoderm walling the gut cavity, and a much thicker mass of mesoderm between this and the superficial ectoderm (Fig. 44). In the region of the lower pole of the gastrula, under the thickest mass of yolk, the delamination comes to the surface of the yolk mass forming a free circular margin of mesoderm. From this free margin cells and groups of cells break or bud off passing farther ventrally, and finally reaching the lower pole, completing thus a fairly continuous layer between ectoderm and endoderm (yolk) (Figs. 44, 61, 63). In the region of the dorsal axial mass, particularly in the region of the blastopore, the delamination is delayed and its course somewhat modified. In these regions the cells concerned in mesoderm formation have very different relations from the remainder, since throughout they are the derivatives of cells which have been invaginated from the outer layer. And here too the development of the notochord complicates the matter somewhat.

Sections cut transversely through the blastopore and the 
region just in front of it, show that the rudiments of the chorda, mesoderm, and dorsal endoderm are for a time not distinguishable (Fig. 33, E). Sections through the narrowed blastopore, while it is still filled with the yolk plug, show that the rim of the blastopore is composed of a thick undifferentiated mass of cells, representing a part of the contracted germ ring. Farther laterally the ectoderm is separated by a line or narrow space which is formed in gastrulation; the thin endoderm is separated from the middle layer by a line or space which results from delamination as described above. In front of the blastopore, in the region formed by confluence, the arrangement of the cells and germ layers is much the same, except that they are not interrupted in the mid-line. The pigmentation of the inner surface of the mass is an indication of the derivation of these cells from the outer layer through invagination.

At a later stage, when the yolk plug has withdrawn from the surface and the blastopore has become slit-like, transverse sections show several important changes in this axial mass. In front of the blastopore the separation between ectoderm and mesoderm has extended, by delamination, toward the midline, and just before reaching this, turns sharply downward toward the line of delamination between the endoderm and mesoderm, not, however, reaching quite to this, thus leaving in the mid-line a narrow vertical ridge of cells. In the regions where the endoderm and mesoderm remain continuous, a pair of slight depressions appear as shallow grooves out of the archenteron; these are continued into the cell mass a short distance as virtual grooves, indicated only by the arrangement of the pigmented cells. Further forward (Fig. 36) the lower margins of these grooves become better marked, as low lip-like structures approaching the mid-line, and the mesoderm in these regions is more extensively separated from the lining of the archenteron. Finally, still farther forward, the grooves disappear and the extension vertically of the spaces separating the mesoderm from the endoderm and ectoderm completely delimits the pair of mesoderm masses. The cells left in the mid-line between the proximal ends of the mesoderm sheets 
form a wedge-shaped elevation continuous with the endoderm; this is the rudiment of the notochord. In a still later stage the chorda begins to be cut off from the endoderm by a narrow split leaving the enteron roofed dorsally by a layer only one cell thick (Fig. 44). Passing posteriorly from the first section described above, into the region of the blastopore, we find the grooves out of the archenteron better marked and the ventral lip, as well as the dorsal, quite pronounced. These grooves are apparently indications of the enterocœlic evaginations;

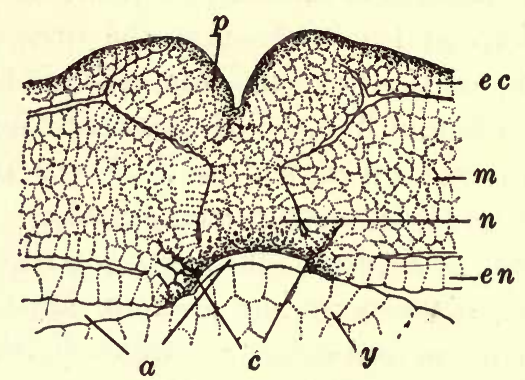

Fig. 36.-Part of a transverse section through the young embryo of $R$. fusca, showing traces of enterocœl formation. After O. Hertwig. $a$, Archenteron; $c$, enterocœls; $e c$, ectoderm; $e n$, endoderm; $m$, mesoderm; $n$, notochord; $p$, neural plate; $y$, yolk cells.

this relation will be mentioned more fully at the close of the description of notogenesis.

\section{The Medullary Plate}

The rudiment of another axial structure is developing at the same time as the chorda and mesoderm; this is the medullary plate. The medullary plate is formed in part from the median band of cells extending from the region of the dorsal lip of the blastopore nearly to the animal pole, and in part from the axial thickening due to the confluence of the lateral portions of the germ ring. In the former region the inner or nervous layer of ectoderm begins to thicken and by the time the blastopore has become circular and commenced to close, a thickened medullary plate has formed over the whole dorsal surface of the gastrula (Fig. 32, F). This is in the form of a broad plate, 
narrow just in front of the blastopore and widening gradually as it extends up toward and beyond the upper pole. By the time the yolk plug is withdrawn the margins of the medullary plate are considerably thickened so that its outline is visible externally; the median region has meanwhile become thinner and a shallow groove results (Fig. 22, C). The thickened margins are elevated slightly above the general surface of the ectoderm and form the two lateral neural ridges, extending from the sides of the blastopore along the dorso-lateral regions of the embryo, widely separated, to a level about opposite the blastopore, where they turn sharply and pass to the mid-line, meeting and forming thus the transverse neural fold, which marks the anterior limit of the medullary plate. The median groove soon becomes quite pronounced and is known as the neural groove.

Sections through the region just in front of the blastopore (Figs. 32, $G ; 33, F$ ) show that the neural plate early begins to separate from the remainder of the ectoderm. Fig. 44 shows how the medullary plate is cut away laterally and superficially from the ectoderm by a narrow split resulting from cell rearrangements; we have already seen that a similar space separates the medullary plate from the underlying notochord.

\section{Summary and Comparisons with Other Forms}

Before continuing our account of the development of the rudiments whose formation has just been described, we should summarize the events of notogenesis and their relation to gastrulation. It is evident that the distinction between gastrulation and notogenesis is real, and essential to an understanding of this period in development. Gastrulation involves only those processes which convert the monodermic embryo into a didermic embryo; notogenesis includes those processes involved in the formation of the medullary plate, notochord, and mesoderm. Gastrulation is accomplished primarily by delamination and the rearrangement of the yolk cells, and only secondarily, and to a very slight extent, by invagination. In 
the frog the process of invagination is chiefly concerned in the formation of the rudiments of the chorda and the mesoderm of the dorsal and dorso-lateral regions, although these structures are not formed wholly by invagination but also by the transport of the materials of the germ ring to an axial position, and then by delamination. In the development of the frog, therefore, invagination is a process of minor importance.

The actual materials out of which the axial structures of the embryo are formed are to be found in the animal half of the blastula, that is, in the walls and floor of the blastocœl, and these in turn are distinguishable in the eight cell stage, where they are contained in the upper quartet and the upper parts of the lower quartet; finally they can be traced back, approximately to the animal half of the egg. The later localization of the greater part of these materials in a true germ ring is an important characteristic in the development of the frog, and makes possible a close comparison of the gastrula and early embryo of this form, with the conditions in the lower as well as higher forms. It is also important that accompanying the downgrowth and closure of the germ ring there is a true confluence of its lateral margins, forming a thickened axial mass of cells, gradually elongating posteriorly through continued confluence. In this axial region the chief organs characteristic of the frog as a Chordate animal, have their origin. These organs gradually differentiate as the mass elongates posteriorly, and their rudiments, though not individually differentiated as such, are thus seen to form gradually from the cells contained laterally in the roots of the axial thickening, that is, in the germ ring itself. This germ ring is not so clearly indicated in Amphioxus, although we have seen in that form a rapidly growing ring of cells around the blastopore, wider on its dorsal side, from which are differentiated gradually the rudiments of the chorda and mesoderm, and a considerable part of the medullary plate. In Amphioxus, however, gastrulation is more nearly completed before notogenesis commences, so that while there is some overlapping it is not so extensive as to cause confusion of the two processes. In the frog not only does notogenesis commence 
so early as to obscure certain features of gastrulation, but these processes are both highly modified by the presence of a large mass of inert yolk cells. The presence of this rather immobile mass results in the formation of the embryo as on the surface of a sphere instead of as a simple elongated embryo, and such processes as the formation of enterocœlic or notochordal evaginations of the gut seem more or less abbreviated when compared with such a form as Amphioxus, where the embryonic layers are not impeded in their foldings by any such restraining influence as the yolk mass in the frog. Were we to assume that the development of Amphioxus represents truly primitive processes of development among the Chordates, we might describe the course of early development in the frog by saying that it is directed toward the accomplishment of the final result, rather than the carrying through of a definite program, so that the formation of the chief axial structures is effected more or less independently of the formal processes of development, as illustrated by Amphioxus.

However, there is considerable doubt as to whether Amphioxus does really represent, in these respects, conditions which may be considered primitive for the Chordates. Opinion remains quite divided upon the subject of the relation between developmental processes in Amphioxus and in such forms as the Amphibia. Some would point out that excepting only the Protochordates, all of the lower vertebrates have large eggs, containing a considerable quantity of yolk, definitely localized in one pole; that, indeed, the Mammals are the only Craniates which have small eggs, with little yolk, comparable with those of the Tunicates and Amphioxus. These would maintain that, while we may say that the presence of the yolk in the egg has modified the form of early development, we cannot call this modified development typical for the Chordata. It is quite likely that while the development of Amphixous is simpler and more diagrammatic than that of any Craniate, we must after all regard this as a secondary or acquired simplicity, and not the simplicity of primitiveness. From the point of view of purely comparative embryology, Amphioxus should be the first to be 
considered; from the phyletic standpoint it should be considered after the more typically Chordate frog-not that the frog is embryologically typical of all Chordates, merely that it represents that condition more truthfully than Amphioxus. On the other side, some would hold that the embryological simplicity of Amphioxus is that of true primitiveness, that Amphibian development is secondarily modified, and phyletically a modification of that of the Protochordates, interpretable only through the latter and not vice versa, and that many of the differences are the result of the accumulation of yolk in the Amphibia. We may mention from these two points of view only the development of the mesoderm as one of the central points.

Except in Amphioxus the Chordate embryo remains two layered or didermic only a very short time, on account of the very early development of the mesoderm in all other forms. In the frog the mesoderm cells are found, soon after the endoderm begins to be formed, first all around the margin of the blastopore forming an important part of the germ ring; that is, the mesoderm is first all blastoporal or peristomial. Then confluence begins and the lateral portions of the germ ring are carried to the mid-dorsal region and poured into the posteriorly elongating embryo, where they form the mesoderm bands; the mesoderm of the germ ring thus becomes axial in position and is known then as gastral mesoderm. The gastral mesoderm is here derived from the peristomial through a change in position due to confluence, and no essential distinction between the two is to be drawn. Only in the posterior region of the frog embryo immediately in front of the blastopore, are there traces of evagination in connection with the formation of the mesoderm in the form of a pair of slight grooves or slits. These may be considered as due merely to the rapid, unilateral and localized proliferation of the cells around the blastopore, such as often leads to a grooving of the surface, and as having nothing to do with the mesoderm folds and enterocolic evaginations of Amphioxus. Or, on the other hand, these grooves may be regarded as vestiges of the enterocœlic grooves of a primitive 
Amphioxus-like embryo, which has been modified by the accumulation of yolk and the precocious formation of the mesoderm before confluence. It might easily be supposed that the formation of the mesoderm in advance of the definite establishment of the gut cavity would result in the loss of function and disappearance of the enterocœls. In Amphioxus the gastral and peristomial mesoderms have unlike origins, because the mesoderm is not formed until after gastrulation, and consequently, that formed from invaginated endoderm (gastral or axial) is unlike that (peristomial or blastoporal) formed from the "germ ring" or growth zone, around the posterior end or blastoporal region of the embryo.

While decisive evidence is perhaps lacking, and much may be said on both sides, on the whole the evidence seems to favor the first view; that the method of mesoderm formation in Amphioxus is not wholly primitive, that primarily there is no distinction between gastral and peristomial mesoderm, for all is first peristomial or blastoporal, and that the mesoderm grooves of the frog are not vestiges of enterocœlic evaginations, like those of Amphioxus, but represent the primary mode of origin of the mesoderm as a proliferation from the margin of the blastopore. This would, of course, involve the conclusion that the cœlomic cavity is not phylogenetically derived from the gut cavity among the Chordata. In the frog the cœlom has no relation with the mesoderm folds, as it would have if these are vestigial enterocœlic grooves, and it will be recalled that in Amphioxus only the cavities of the more anterior somites are ever connected, even as grooves, with the gut cavity. And yet in some of the tailed Amphibia the colom is apparently sometimes directly connected with the grooves in the mesoderm folds.

Most of the differences in the arrangement of the mesoderm rudiments in Amphioxus and the frog can be traced to this difference: in the frog the mesoderm differentiates before gastrulation and confluence, in Amphioxus the mesoderm diffe: entiates after gastrulation and after confluence has begun. Doubtless these unlike relations are largely the result of the absence of the yolk mass in Amphioxus and the consequent 
mobility of the blastomeres and the epithelia which they compose.

We may assume that the stage in which the rudiments of the central nervous system, notochord, mesoderm, and gut are all definitely established, marks the end of notogenesis and the beginning of the formation of a definite embryo in a restricted sense.

\section{B. The Formation of the Early Embryo}

We may close our account of the early embryonic period by tracing briefly the further development of the rudiments formed at the close of notogenesis, up to the time the neural tube is closed. We have assumed arbitrarily to let this stage (about two days after fertilization) represent an "early embryo" (Fig. 22, $E, F$ ). At this time the embryo has elongated so that its length is about one and one-half times its depth or the diameter of the gastrula. The postero-dorsal region is narrowed and drawn out slightly into the rudiment of the tail. The dorsal surface is straight or even slightly concave and narrowed in cross section, the ventral surface remains broadly convex. The rudiments of several organs are visible externally as elevations or depressions; these will be described in connection with the internal structure. Externally the ectoderm alone still forms the covering epithelium, for as yet no part of the mesoderm has contributed to the formation of an integument. About the only change in the character of the ectoderm is the development, on some of its cells, of numerous short cilia. Just before the fusion of the neural folds the cilia begin to appear first along their margins. They extend rapidly more widely over the surface, and by the time this early embryonic stage is reached they are absent from only the ventral surface. A little later the ciliation is completed. The cilia beat in the posterior direction and give the embryo a slow rotary motion within the egg membranes.

\section{The Nervous System}

It is more convenient to describe the development of the nervous system first, partly because many of the chief external 
characteristics of this period are associated with the development of this system. The transverse neural fold, which marks the anterior limit of the central nervous system (Figs. 22, $C ; 32$, $G$ ), forms from materials located in about the middle of the roof of the blastocœl, while the posterior limit of the nervous system is just above or in front of the dorsal lip of the blastopore. The downward extension of the latter, on account of the confluence, increases the length of the rudiment of the nervous system, so that before the blastoporal margin or germ ring has fully contracted, it extends nearly from pole to pole, around the posterior side of the gastrula. The rotation of the gastrula then changes the apparent, though not the true morphological, position of the anterior margin of the medullary plate, so that when the transverse neural fold actually appears, it is on the anterior side of the gastrula, and the medullary plate itself occupies nearly the whole dorsal surface of the early embryo (Fig. 32, $G$ ). We have already described the formation of the neural or medullary. plate, the neural groove, and the lateral and transverse neural or medullary ridges.

The elevation of the neural ridges, particularly their anterior portions, soon becomes very marked, and as it continues the middle of the plate sinks downward and soon the ridges bend over toward each other and meet along the mid-line, where they fuse, transforming the neural plate into the neural tube containing the neural canal or neurocœl (Figs. 22, D, E; 38). For a long time after fusion a deep median groove marks the region where the folds have come together. The fusion of the neural ridges does not occur simultaneously throughout their extent, but first in about their middle, then extending posteriorly and anteriorly from this region (Fig. 38). This is approximately the location of the future medulla (myelencephalon); from this time, therefore, the spinal cord and brain are distinguishable (Fig. 37). The narrower cord region closes before the much wider brain. In the closure of the brain region the transverse fold plays an important part; this extends backward, roofing the expanded cavity of the brain, and meets the slowly fusing lateral folds in the region between the future fore- and mid- 
brain. This is, therefore, the last region of the neural tube to close, and may consequently be termed the neuropore; in the later embryo this is the region just posterior to the epiphysis or pineal body (see next chapter). The neuropore has a very transitory existence.

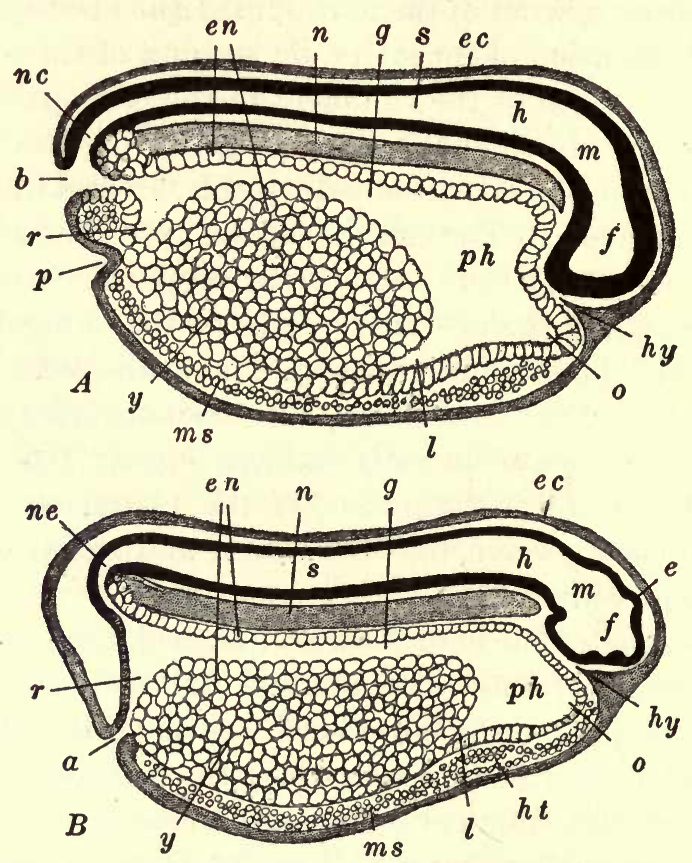

FIG. 37.-Diagrams of median sagittal sections of frog embryos. After Marshall. $A$. Just before the closure of the blastopore. $B$. Just after the closure of the blastopore. (See Fig. 38, D, E.) $a$, Anal or cloacal aperture; $b$, blastopore; $e$, epiphysis; $e c$, ectoderm; $e n$, endoderm; $f$, fore-brain; $g$, mid-gut; $h$, hind-brain; $h t$, rudiment of heart; $h y$, hypophysis; $l$, liver diverticulum; $m$, midbrain; $m s$, mesoderm; $n$, notochord; $n c$, neurenteric canal; $o$, oral evagination; $p$, proctodæum; $p h$, pharyngeal region of gut cavity; $r$, rectum; $s$, spinal cord; $y$, yolk cells.

Those cells in the nervous layer of the ectoderm forming the lateral margins of the neural plate, that is, the neural ridges proper, do not themselves form an integral part of the neural tube. When the margins of the neural plate fold together they are left dorso-laterally, between the neural tube and the definitive ectoderm. These ridges of cells become broken into cell groups, lying along the lateral regions of the neural tube, forming 
the neural crests. These are concerned later in the development of the nerves, which, together with some additional details of this period, will be described in the next chapter.

At the posterior end of the embryo, the relations of the blastopore (germ ring) and neural folds are of considerable importance from a comparative point of view. We left the blastopore in the form of an elongated slit on the postero-dorsal surface of the embryo. The lateral walls then approach, about the middle of the slit, and finally it is there constricted completely; so that the blastopore is divided into two small openings, an upper and a lower (Fig. 38, $A, B$ ). The medullary groove extends forward from the upper opening, which remains open, leading directly into the archenteron (Fig. 37). The lower opening is soon closed by the fusion of its lips. The fusion involves only the ectoderm and endoderm of the region, and occurs some distance below the surface, so that a pit-like depression is left on the surface, lined with ectoderm; this is the proctodoe $u m$. When the posterior ends of the neural folds form, they extend into, or rather out from, the middle regions which have fused, and as they become elevated and form the neural tube they cover over the upper blastoporal opening (Fig. 38, C) which thus becomes the neurenteric canal, like that of Amphioxus or other Chordates, and similarly formed, putting the neurocœl and gut into communication. The lateral margins of the blastopore are formed of the remains of the germ ring, and when they meet, dividing the blastopore, they form a median cell mass in which ectoderm, endoderm, and mesoderm are fused in a more or less undifferentiated mass. This mass can no longer be called the germ ring, although it is really equivalent to the lateral parts of this; it is known as the primitive streak, and the groove that remains for a time on its surface, indicating its origin from originally separate lateral portions, is the primitive groove. The primitive streak and groove of the frog are homologous with the similarly named structures in Amphioxus and in the Amniota. The cells of the primitive streak continue to multiply rapidly, and bud forth strands of ectoderm into the neural folds and upon the surface of the body, mesoderm into the lateral bands, and en- 
doderm into the wall of the gut. This leads to the formation of a postero-dorsal protuberance which is the rudiment of the

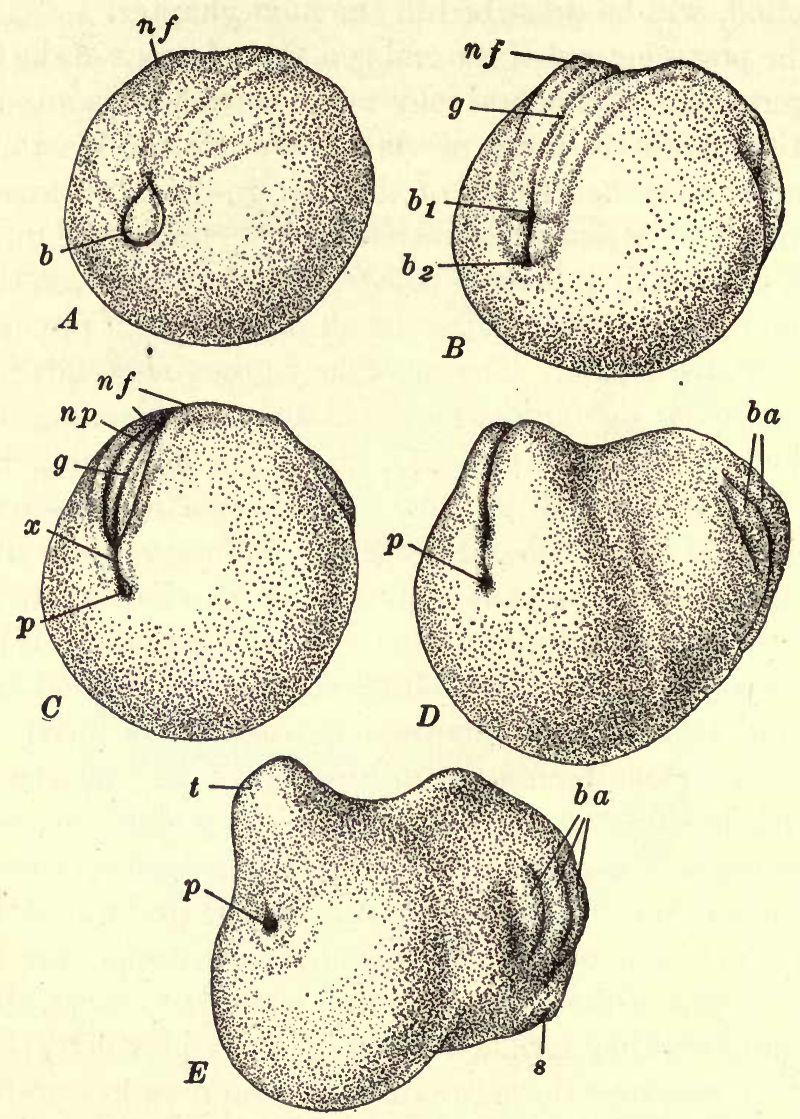

FIG. 38.-Posterior ends of a series of young frog embryos, showing the later history of the blastopore, and the relation of the neural folds to it. The embryos are viewed obliquely from the postero-lateral aspect. After F. Ziegler. A. Blastopore nearly closed; neural folds just indicated. $B$. Blastopore becoming divided into neurenteric and proctodæal portions; neural folds becoming elevated. $C$. Neurenteric canal forming; neural folds closing together. $D$. Neural folds in contact throughout. $E$. Neural folds completely fused; tail commencing to grow out. $b$, Blastopore, containing yolk plug; $b_{1}$, rudiment of neurenteric canal (dorsal part of blastopore); $b_{2}$, rudiment of proctodæal pit (ventral part of blastopore); $b a$, branchial arches; $g$, neural groove; $n f$, neural folds; $n p$, neural plate; $p$, proctodæal pit; $s$, rudiment of oral sucker; $t$, rudiment of tail; $x$, neural folds roofing the blastopore and establishing the neurenteric canal.

tail, but in the stage we are describing this is only just indicated (Fig. 38, D, E). 
At the opposite end of the body the enlarged brain protrudes, forming a head region faintly indicated externally. A sagittal section (Fig. 37, A) shows that the future regions of the brain are but faintly marked out. The chief characteristic of the brain is its abrupt bending or flexure around the anterior tip of the notochord; the region immediately in front of the chorda is that of the future mid-brain, while the large fore-brain lies entirely below the level of the chorda and the remainder of the neural tube. In the mid-line, just beneath the end of the forebrain, a tongue-like proliferation of ectoderm cells extends inward a short distance. This is the rudiment of the hypophysis.

The simple rudiments of the chief sense organs are also indicated at this early stage. The eyes are distinguishable, even before the brain closes, as small patches of deeply pigmented ectodermal epithelium in the antero-lateral regions of the medullary plate. When the neural tube is completed, they form a pair of hollow ventro-lateral outgrowths from the fore-brain to the superficial ectoderm. Frequently they can be seen externally, marked by a pair of slight darkened elevations, either side of the fore-brain region (Fig. 22, $E, F$ ). The ears are indicated as a pair of thickened patches of the inner or nervous layer of the ectoderm opposite the hind-brain region. They are scarcely visible externally at this time. The olfactory organs develop as thickened circular patches of ectoderm below and in front of the optic rudiments. At this stage a pair of slight depressions may sometimes be detected on the surface, marking the future olfactory pits.

\section{The Notochord}

By the time the neural tube is completed the chorda has become completely delaminated from the outer surface of the endoderm, except only in the region of the primitive streak where its formation is still progressing posteriorly. The separation of the chorda from the endoderm, or rather the enteroderm (see below), occurs in the posterior direction, beginning near but not quite at the anterior tip. 


\section{The Enteron}

At the close of gastrulation and notogenesis the archenteron is a nearly hemispherical cavity on the dorsal side of the embryo, open posteriorly through the blastoporal opening. Its roof and sides are left as a thin layer of endoderm-the enterodermafter the chorda and mesoderm have been split off; its floor is formed of the thick mass of yolk cells. By the time the neural tube is completed and the embryo has elongated slightly, the enteric cavity or mesenteron has enlarged considerably, chiefly in front of the yolk mass, which retains a postero-ventral position in the wall of the gut (Fig. 37). This anterior enlarged region of the mesenteron is known as the fore-gut, the entire wall of which is but one cell thick; this is the region of the embryonic pharynx and later of the œesophagus and stomach as well. At the posterior end of the mesenteron there is a slight enlargement, just in front of the neurenteric canal, which is the hind-gut or rectal portion of the intestine. Connecting these two regions the narrow mid-gut or intestinal region proper, is that containing the yolk cells, which are also to be regarded as enteroderm.

In the fore-gut of this stage there is an antero-ventral outpocketing toward the ectoderm just below the fore-brain, indicating the region where the mouth will form later. A posteroventral outgrowth beneath the anterior end of the yolk mass is the rudiment of the liver. Sections passing through the sides of the fore-gut show that even in this early stage the rudiments of the first two or three visceral pouches are present in the form of vertical outgrowths from the side walls of the pharynx to the ectoderm, with which they fuse. Along the region of the fusion the ectoderm is depressed so that these are externally visible as vertical depressions just back of the head (Fig. 22, E, $F)$. Externally two of these are marked at this time as the external branchial grooves, and the ridges left between and in front of them are the rudiments of the second or hyoid, and first or mandibular arches respectively. The hyoid arch is sometimes known here as the "gill plate"; it extends dorsally nearly to 
the margin of the nervous system. The mandibular arches are less marked; these appeared even before the medullary plate became folded together, as a pair of low ridges diverging from the antero-lateral regions of the plate and sometimes called the "sense plates." . They lie between the olfactory and optic rudiments and form the antero-lateral regions of the embryonic head.

\section{The Mesoderm}

The delamination of the mesoderm from the surface of the endoderm commenced in the dorso-lateral regions anteriorly, and spread thence posteriorly and ventrally. We have seen that posteriorly the mesoderm finally passes into the region of the germ ring, or what now represents a portion of that, the primitive streak, where it continues to be formed and budded off anteriorly as the primitive streak extends posteriorly. And ventrally the delamination ceased in the ventro-lateral regions of the endoderm, and the mesoderm then extended gradually toward the ventral side through the multiplication of its own cells and their downward extension, and through the splitting off of scattered groups of cells from the endoderm toward the ventral side. In this early embryo the mesoderm forms a distinct layer separating ectoderm and endoderm throughout (Fig. 44), except in the primitive streak and in the head region, where the mesoderm is never in the form of a definite sheet, but is represented by scattered cells filling the spaces between the wall of the mesenteron and the nervous system and ectoderm (Fig. 45). In the pharyngeal region the mesoderm becomes interrupted by the extension of the gill pouches out to the ectoderm; between successive pouches groups of mesoderm cells are enclosed which become the rudiments of the visceral arches - mandibular, hyoid, and branchial.

Through the trunk region the typical mesodermal structures develop rapidly. The axial region toward the chorda thickens and becomes solid; this is the segmental plate or myotomal region (Fig. 39). Farther laterally the thinner layer is the lateral plate; 
this splits into two more or less distinct sheets, the somatic and splanchnic layers, in contact with ectoderm and endoderm respectively. The space between these is the splanchnocœl, the rudiment of the body cavity or colom. In the stage we are describing the cœlom does not extend completely through the lateral plate as it does finally. The cœlom does however extend into the segmental plate, as the rudiment of the myocols,

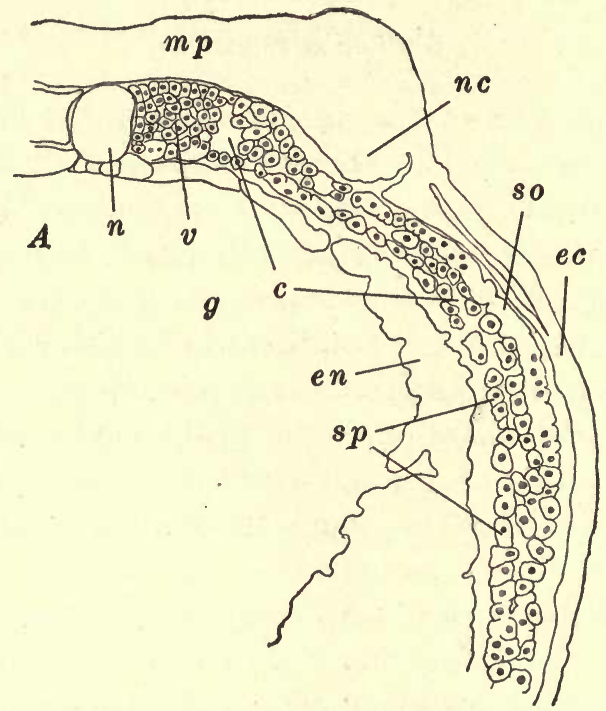

Fig. 39.-Part of a section through the anterior body region of an embryo of R. sylvatica, just beginning to elongate, illustrating the differentiation of the mesoderm. After Field. $c$, Cœlom; $e c$, ectoderm; $e n$, endoderm; $g$, gut cavity; $m p$, medullary plate; $m y$, myotome; $n$, notochord; $n c$, rudiment of neural crest; $s o$, somatic layer of mesoderm; $s p$, splanchnic layer of mesoderm.

appearing toward the surface of this thicker mass. As the neural folds approach, the cells of this axial mesoderm are rearranged in such a way that the longitudinal bands are cut transversely into segments or somites. This process begins just back of the pharynx and extends rapidly toward the posterior ends of the bands (Figs. 44, E; 53). It is important that the mesoblast of the head region is not clearly divided into segments. By the time the neural tube is completed three or four pairs of somites have been formed. At first these are 
continuous with the lateral plate but shortly after they are marked out, the two regions become separate and the lateral plate itself is never segmented but remains uninterrupted. The cœlomic spaces of the somites, the myocœls, then disappear without leaving any trace. These processes are progressive posteriorly, the formation of the cœlom, somites, etc., continuing as the mesoderm forms from the primitive streak.

In the embryo of this period indications of two important structures are present in connection with the mesoderm but not as readily recognizable and definite rudiments; these are the pronephros and the heart. The connections between the second, third and fourth somites and the lateral plate remain as small masses of cells, in close relation with the somatic layer of the lateral plate. These are the cells forming the rudiments of the pronephric tubules. The definite formation of these rudiments and that of the pronephric duct must be left until a later stage is described. Just below the pharynx and in front of the liver, the mesoderm cells are arranged in somatic and splanchnic layers, and between these the colomic space is well marked on either side, while elsewhere in the ventral region it has not appeared (Fig. 37, B). Between the mesoderm and the ventral side of the pharynx a few loose cells are scattered along. This is the region where the heart is soon to appear but this, too, must be described later.

(References to the literature will be found at the end of Chapter III.) 
THE LATER DEVELOPMENT OF THE FROG:

\section{ORGANOGENY}

PAGE

INTRODUCTION . . . . . . . . . . . . . . . . . . . . . 127

I. THE NERVOUS SYSTEM . . . . . . . . . . . . . . . . 128

1. The Central Nervous System . . . . . . . . . . . . . 128

2. The Peripheral Nervous System . . . . . . . . . . . . 135

A. The Cranial Nerves . . . . . . . . . . . . . . . 136

B. The Spinal Nerves . . . . . . . . . . . . . . . 142

C. The Sympathetic System . . . . . . . . . . . 143

II. THE SPECIAL SENSE ORGANS . . . . . . . . . . . . . 144

1. The Eye . . . . . . . . . . . . . . . . . . . . 144

2. The Ear . . . . . . . . . . . . . . . . . . . . . . 149

3. The Olfactory Organ . . . . . . . . . . . . . . . . . . 153

4. The Sense Organs of the Lateral Line . . . . . . . . . . 156

III. THE ALIMENTARY TRACT AND ITS APPENDAGES . . 158

1. The Derivatives of the Fore-gut. . . . . . . . . . . . . 159

2. The Derivatives of the Mid-gut . . . . . . . . . . . . 168

3. The Derivatives of the Hind-gut . . . . . . . . . . . . . . 169

IV. THE MESODERMAL SOMITES . . . . . . . . . . . . . . 170

V. THE VASCULAR SYSTEM . . . . . . . . . . . . 174

1. The Heart . . . . . . . . . . . . . . . . . . . . . 174

2. The Origin of the Blood and Vessels . . . . . . . . . . . 178

3. The Arterial System . . . . . . . . . . . . . . . . . 179

4. The Venous System . . . . . . . . . . . . . . . . . 185

5. The Lymphatic System and the Spleen . . . . . . . . . 189

6. The Formation of the Septum Transversum . . . . . . . . . 191

VI. THE URINOGENITAL SYSTEM . . . . . . . . . . . . . . 192

1. The Excretory System . . . . . . . . . . . . . . . . 192

A. The Pronephros and the Pronephric Duct . . . . . 193

B. The Mesonephros (Wolffian Body) . . . . . . . . 198

2. The Reproductive System . . . . . . . . . . . . . . . 201

A. The Gonoducts . . . . . . . . . . . . . . . . . 202

B. The Gonads . . . . . . . . . . . . . . 203

3. The Adrenal Bodies . . . . . . . . . . . . . . . . . 206 
VII. THE SKELETON AND TEETH . . . . . . . . 207

1. The Vertebral Column . . . . . . . . . . . . . . 208

2. The Skull . . . . . . . . . . . . 210

A. The Cranium and Sense Capsules . . . . . . . 210

B. The Visceral Arches . . . . . . . . . 216

C. The Dermal Elements . . . . . . . . . 219

3. The Teeth . . . . . . . . . . . 220

4. The Appendicular Skeleton . . . . . . . . . 221

In this chapter we shall trace the chief events in the development of the frog tadpole, from the stage described at the close of the preceding chapter, i.e., just after elongation is inaugurated by the enlargement of the head and the outgrowth of the tail. The more important changes in external form and in habit have been described in the introduction to the preceding chapter, and we may turn at once to the description of the internal processes of development.

The developmental history of no single species of frog is known with even fair completeness, and we should note that this chapter presents a composite account of the development of the genus Rana. No attempt has been made, save in occasional instances, to distinguish the species serving as the basis for different sections; these vary somewhat in details, but since details are largely omitted, little confusion is likely to follow such treatment. The species of Rana chiefly serving as the material for this account are the American species, sylvatica, palustris, and virescens, and the European temporaria, esculenta, fusca, and muta.

While the early history of the frog embryo is fairly well known, there are still many gaps in our knowledge of the later development of the tadpole, gaps which sometimes seem of remarkable proportions in view of the extent to which the frog is used as an object of embryological study.

No accurate and convenient description of the age of the frog larva has been determined, since the rate of development varies so markedly with temperature changes before the opening of the mouth, and afterward with the abundance of food. At the time of hatching, which is usually between one and two weeks after fertilization, the larvæ of most species are approxi- 
mately 6-7 mm. in total length. Another frequent reference point is the time of the opening of the mouth, which usually occurs in tadpoles of 9-10 mm., only a few days after hatching. The limbs appear as small buds in tadpoles of 11-12 mm.; the fore-limbs are of course concealed underneath the operculum, but they develop at about the same time and rate as the hindlimbs.

\section{THE NERVOUS SYSTEM}

\section{The Central Nervous System}

In the preceding chapter we described the formation of the neural tube and noted the differentiation between the narrow spinal cord and the dilated brain region. Posteriorly the cord is bent downward toward the blastopore, and the cavity of the cord is continuous with the archenteron by way of the neurenteric canal. Anteriorly the brain is strongly flexed around the tip of the notochord. The neuropore, which is located anteriorly from the tip of the chorda, has just closed and remains connected with the surface ectoderm by a broad cone of pigmented cells (Fig. 37, A).

The development of the brain from the stage described is comparatively simple. In its early history it differs from most other forms in two important respects; no neuromeres or brain segments are indicated, and the division of the primitive brain into its primary fore-, mid-, and hind-brain regions is incompletely indicated and appears relatively late. The chief morphological characteristics of the brain result largely from two groups of processes, (a) thickenings and thinnings, (b) outgrowths and ingrowths of the wall. In describing these processes it is convenient to distinguish the roof, floor, and sides of the brain tube.

One of the chief features of the brain is its well-marked ventral flexure, the large anterior part of the brain lying below the level of the notochord (Figs. 37, 40); this flexure remains a permanent characteristic of the brain, although as we shall see, it soon becomes masked by the unequal growth of the neigh- 
boring regions. Just opposite the tip of the chorda the floor of the brain becomes slightly thickened as the tuberculum posterius, and in the roof, obliquely upward and forward from this, appears a rather extensive dorsal thickening (Fig. 41). With the aid of these landmarks we may map out the location of the future brain regions. A plane passing from the tuberculum posterius in front of the dorsal thickening, marks approximately the limit between the primary fore-brain or prosencephalon, and the mid-brain or mesencephalon; while a plane passing from the tuberculum posterius behind the dorsal thickening, marks the limit between mesencephalon and the primary hind-brain or rhombencephalon. The beginning of the rhombencephalon is also indicated by a considerable transverse extension of the brain tube; posteriorly the rhombencephalon passes insensibly into the spinal cord.

We may now proceed to describe the more important events in the development of each of these primary divisions of the brain. We should note in advance that the prosencephalon forms the olfactory lobes and cerebral hemispheres (telencephalon) and the between-brain (diencephalon): the mesencephalon forms the region of the optic lobes and chiasma: the rhombencephalon forms the cerebellum (metencephalon) and the medulla oblongata or spinal bulb (myelencephalon).

The Prosencephalon.-The cells of the ectodermal cone opposite the neuropore (Fig. 40), soon scatter, as the tissues of the head push out in advance of the brain, and no trace is left of the original location of this structure, save a slight bay or olfactory recess, which soon disappears. Below this level the anterior wall of the fore-brain remains somewhat thickened for a time, as the lamina terminalis. This extends to the ventral side of the brain where the optic stalks extend out from the fore-brain. These are hollow and their cavities are continuous with the cavity of the prosencephalon. The regions of the anterior and posterior borders of their attachment early become considerably thickened as the torus transversus and the rudiments of the optic chiasma and thalami, respectively (Figs. 41, 42); the former becomes the seat of the anterior, and 
certain other, commissures of the brain. The narrow depression between these two thickenings is the recessus opticus, i.e., the passage to the cavities of the optic stalks. The posterior side of the prosencephalon extends backward beneath the tip of the notochord forming a well-marked outgrowth, the infundibulum.

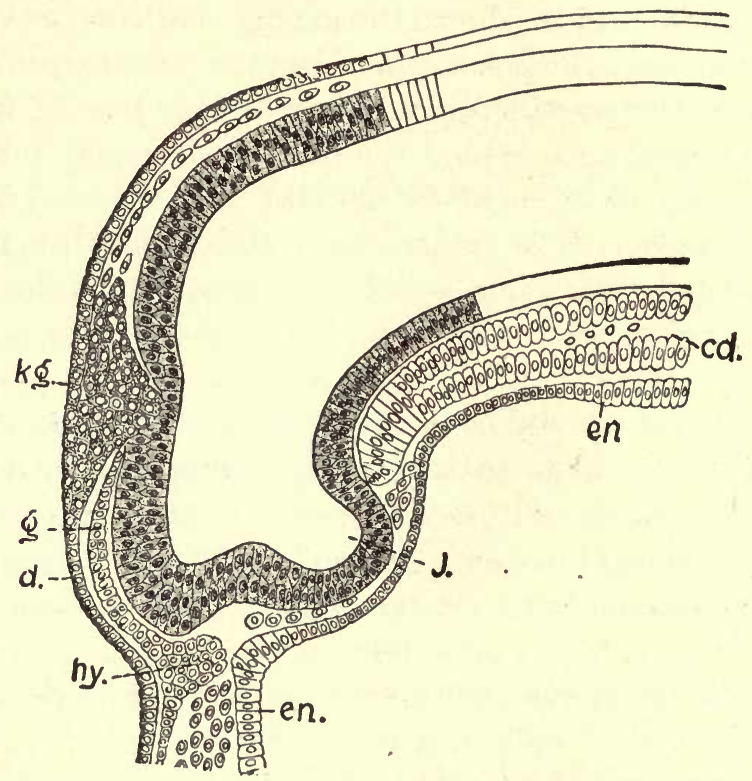

Fig. 40.-Median sagittal section through the brain of an embryo $R$. fusca, of $2.3 \mathrm{~mm}$. From Von Kupffer (Hertwig's Handbuch, etc.). cd, Notochord; $d$, superficial layer of ectoderm ("deckschicht"); $e n$, endodermal lining of pharynx; $g$, inner or nervous layer of ectoderm; $h y$, hypophysis; $J$, infundibulum; $\mathrm{kg}$, conical proliferation of ectoderm cells at the point of closure of the neural folds.

Somewhat later the entire dorsal wall of the prosencephalon becomes thinner, and toward its posterior limit an evagination appears which is the beginning of the epiphysis or pineal body (Fig. 42). In front of this the roof ultimately becomes nonnervous and forms a series of highly vascular folds projecting down into the cavity of the brain; this is the choroid plexus of the third ventricle. Later there develop, between this choroid plexus and the epiphysis, the habenular ganglia and commissure, and much later there develops, in front of this, a dorsal out- 
growth of the wall, the paraphysis. That part of the prosencephalon extending from the choroid plexus and epiphysis on the dorsal side, to the infundibulum on the ventral side, is known as the diencephalon or between-brain, while the remaining anterior portion is the telencephalon or secondary fore-brain.

Considerably later (about $7 \mathrm{~mm}$. or time of hatching) the rudiments of the cerebral hemispheres appear, growing outward

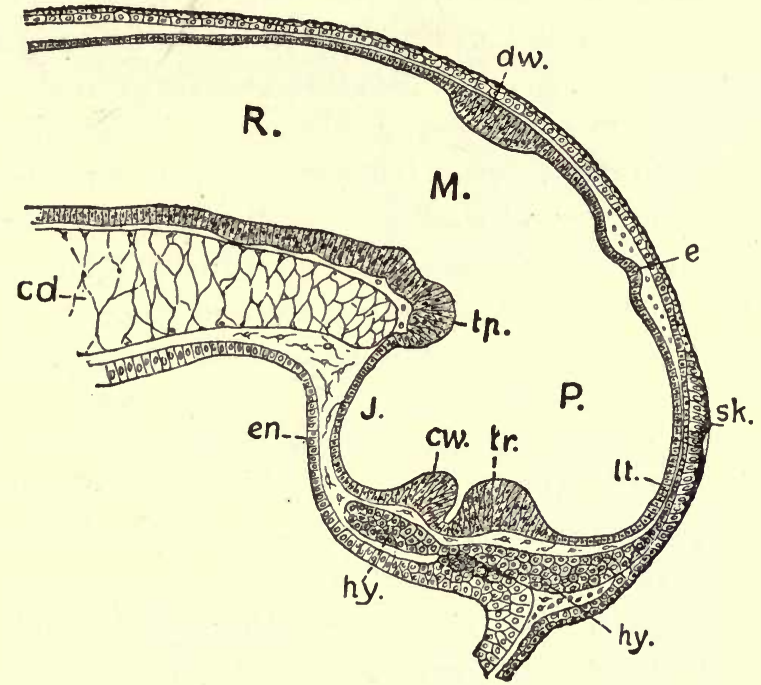

Fig. 41.-Median sagittal section through the brain of an embryo $R$. fusca, of $2.3 \mathrm{~mm}$. length, but in a more advanced stage than that of Fig. 40. From Von Kupffer (Hertwig's Handbuch, etc.). cd, Notochord; $c w$, rudiment of optic. chiasma; $d w$, dorsal thickening; $e$, rudiment of epiphysis; $e n$, endodermal lining of pharynx; $h y$, hypophysis; $J$, infundibulum; $l t$, lamina terminalis; $M$, mesencephalon; $P$, prosencephalon; $R$, rhombencephalon; $s k$, rudiment of olfactory placode; $t p$, tuberculum posterius; $t r$, torus transversus.

and forward from the sides of the telencephalon (Fig. 42, A). These ultimately become very large and extend far in front of the median region. They have thick inner and outer walls, and contain extensions of the cavity of the telencephalon known as the lateral or first and second ventricles, while the remaining median cavity of the telencephalon and diencephalon is known as the third ventricle. The ventricles of the cerebral hemispheres are laterally compressed, and open out of the third ventricle by a pair of openings known as the foramina of 
Monro. The thickenings of these anterior extensions of the telencephalon and of the regions of the optic thalami and anterior commissure produce an apparent straightening of the original ventral flexure of the brain; this does not really disappear, however, and the infundibulum continues to extend below and in front of the tip of the notochord.

The hypophysis, while originally not a part of the brain, becomes intimately related with it. This forms very early as a strand of cells extending inward from the inner layer of the surface ectoderm (Figs. 37, 40), below the telencephalon and just above the future mouth region (oral plate). These cells multiply and form a definite mass lying between the endoderm and the infundibulum, just at the tip of the chorda. This rudiment later cuts off from the ectoderm, and after fusing with the dorsal wall of the pharynx, comes into intimate relation with the lower surface of the infundibulum, forming the essential part of the pituitary body.

The mesencephalon undergoes only slight modification during the larval period. While its roof and floor remain thin, its ventro-lateral walls thicken as the crura cerebri, connecting with the prosencephalic wall: its dorso-lateral walls form the large rounded optic lobes. In front of these the anterior limit of the mid-brain is marked later by the posterior commissure. The cavity of the mesencephalon is called the aqueduct of Sylvius; it becomes narrow and connects the third ventricle with the cavity of the rhombencephalon.

The rhombencephalon is an elongated part of the brain lying wholly above the notochord. The usual division of this part of the brain into an anterior metencephalon and a posterior myelencephalon, is scarcely indicated in the frog. The wide cavity of the rhombencephalon is known as the fourth ventricle; it is continuous anteriorly with the aqueduct of Sylvius and posteriorly with the cavity of the spinal cord. The metencephalon is the region of the cerebellum; this is very small in the frog, and appears late in larval life, as a dorsal and dorso-lateral thickening. Ventrally the walls of the two regions are uniformly continuous. 

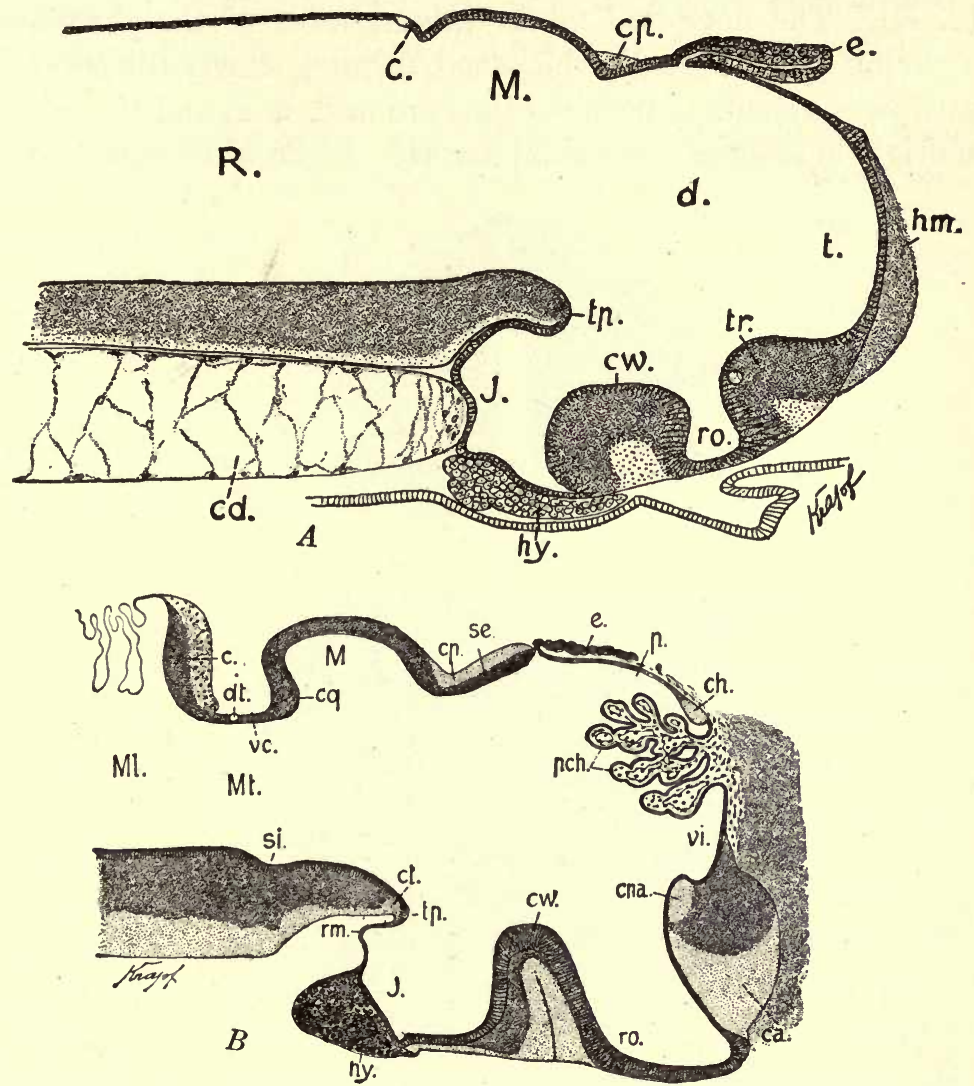

Fig. 42.- Median sagittal sections through the brain of the frog. From Von Kupffer (Hertwig's Handbuch, etc.). A. Of a larva of $R$. fusca of $7 \mathrm{~mm}$. in which the mouth was open. $B$. R. esculenta at the end of metamorphosis. $c$, Cerebellum; $c a$, anterior commissure; $c d$, notochord; $c h$, habenular commissure; $c p$, posterior commissure; $c p a$, anterior pallial commissure; $c q$, posterior corpus quadrigeminum; $c t$, tubercular commissure; $c w$, optic chiasma; $d$, diencephalon; $d t$, tract of IV cranial nerve; $e$, epiphysis; $h m$, cerebral hemisphere; $h y$, hypophysis; $J$, infundibulum; $M$, mesencephalon; $M l$, myelencephalon; $M t$, metencephalon; $p$, antero-dorsal extension of diencephalon; pch, choroid plexus of third ventricle; $R$, rhombencephalon; $r m$, recessus mammillaris; ro, optic recess; $s e$, roof of diencephalon; $t$, telencephalon; $t p$, tuberculum posterius; $t r$, torus transversus (telencephali); $v c$, valvula cerebelli; $v i$, ventriculus impar (telencephali) (third ventricle). 
The broad roof of the myelencephalon is wholly non-nervous and forms the folded choroid plexus of the fourth ventricle (Fig. $42, B)$. The floor and ventro-lateral walls of the rhombencephalon become greatly thickened, forming chiefly the nervous pathways extending between the cord and brain and the nuclei of origin of most of the cranial nerves. Posteriorly the myelen-
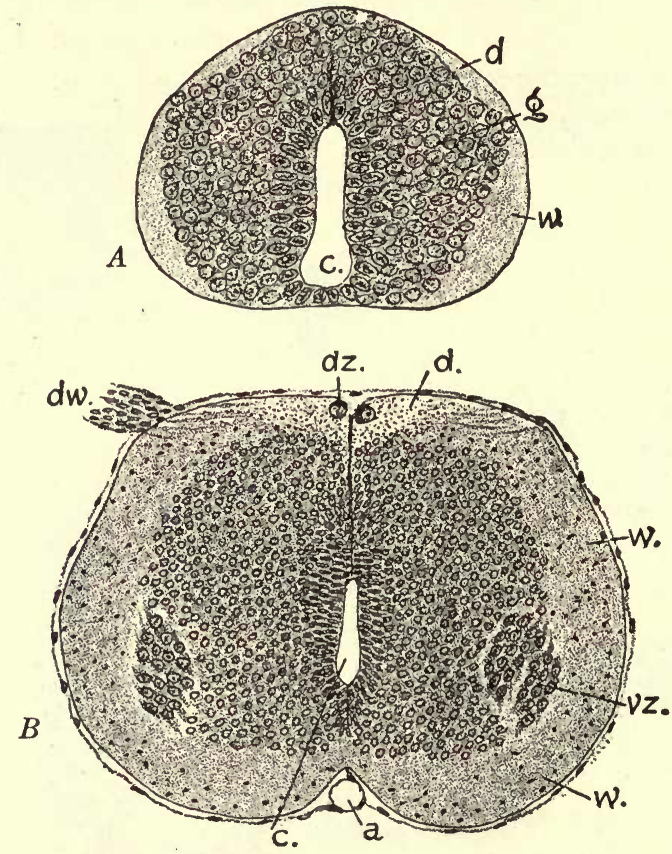

Fig. 43.-Transverse sections through the spinal cord of R. fusca. From Von Kupffer (Hertwig's Handbuch, etc.). A. Through the anal region of a larva of $7 \mathrm{~mm}$. B. Through the anterior body region of a larva during metamorphosis. $a$, Spinal artery; $c$, central canal; $d$, dorsal column (white substance); $d w$, dorsal root of spinal nerve; $d z$, atrophied dorsal cells; $g$, gray substance; $v z$, ventral cells; $w$, dorso-lateral column (white substance).

cephalon narrows gradually and passes insensibly into the spinal cord.

The spinal cord is at first flexed posteriorly toward the blastopore (Fig. 37), but when the tail begins to grow out this flexure disappears. The cavity of the cord is the central canal; it is lined with a layer of non-nervous cells known as ependymal cells, while the true nerve cells composing the greater part of its wall 
are termed the germinal cells (Fig. 43). Part of the germinal cells become the supporting or glia cells, while the remainder become the functional nerve cells or neuroblasts. The closure of the neural folds is complete and no dorsal fissure is left in the cord.

The thickening of the walls of the cord begins dorsally and dorso-laterally, so that the central canal is given a ventral location, its floor formed only by a layer of ependymal cells. Later the ventral wall thickens slightly and the ventro-lateral walls extend below its level forming a shallow groove, the ventral fissure. The central canal becomes compressed laterally, and is later completely surrounded by neuroblasts (gray matter), the outgrowths of which form a superficial layer known as the white matter of the cord (Fig. 43).

\section{The Peripheral Nervous System}

We should recall in a few words the morphological arrangement of the spinal and cranial nerves. The spinal nerves, of which there are but ten pairs in the adult, although in the tadpole upward of forty pairs, arise from the cord by a dorsal or afferent root, on which is located the spinal ganglion, and a ventral or efferent root. These roots join to form the trunk of the spinal nerve which is then divided into dorsal and ventral rami and a ramus communicans which passes to the sympathetic system (Fig. 46). Of the cranial nerves, connected with the brain, there are commonly described ten pairs, considerably varied in morphological, as well as in functional, characteristics. Those regarded as the most typical are primarily related with the gill clefts and are therefore known as the branchiomeric nerves; these are the V, VII, IX, and X. Each of these arises by a single, though in some cases compound, root of mixed character, i.e., afferent and efferent, passes into a large ganglion, beyond which it gives off a horizontal branch, and then divides into two branches which pass anteriorly and posteriorly to the gill cleft with which the nerve is associated. The III, IV, and VI cranial nerves are simple, purely efferent, 
supplying the muscles of the eye-ball. The development of the nerves commonly described as I and II will be considered in connection with the development of the olfactory and optic sense organs.

Embryologically the rudiments of the cranial nerves are composite structures, three elements entering into their formation. These are, (a) cell masses derived from the neural crests, (b) cells from ectodermal patches on the surface of the head, $(c)$ cell processes extending out from neuroblasts in the ventrolateral walls of the spinal cord. In the spinal nerves the elements from the surface ectoderm are lacking.

\section{A. THE CRANIAL NERVES}

The rudiments of certain of the cranial nerves appear very early, and we may therefore describe them first although they are more complicated than the later appearing spinal nerves. While the central nervous system is still in the form of a flat plate we have already seen that its margin is considerably thickened, on account of the proliferation of the cells of the inner or nervous layer there. These thickened margins are visible on the surface of the embryo as the medullary ridges. Transverse sections of this stage (Fig. 44) show that these thickened masses of inner ectoderm become delaminated, both from the outer stratum of ectoderm and from the medial portion of the medullary plate which then goes to form the neural tube proper. These lateral cell masses are the beginnings of the neural crests. In the head region these masses become very large, and as the neural plate begins to close each becomes transversely divided into three masses. Posteriorly to the brain region the neural crests are much smaller, but are typically formed. As the neural tube closes these cell masses are left in situ along the sides of the brain and cord (Corning, Brachet).

The three divisions of the head portion of each crest soon become quite distinct. The anterior section, which begins in the mid-brain region, is to be recognized as the rudiment of the trigeminal ganglion ( $\mathrm{V}$ nerve), the middle section as the rudi- 
ment of the acustico-facialis ganglion (VIII and VII nerves), and the posterior as the rudiment of the ganglion of the glosso-
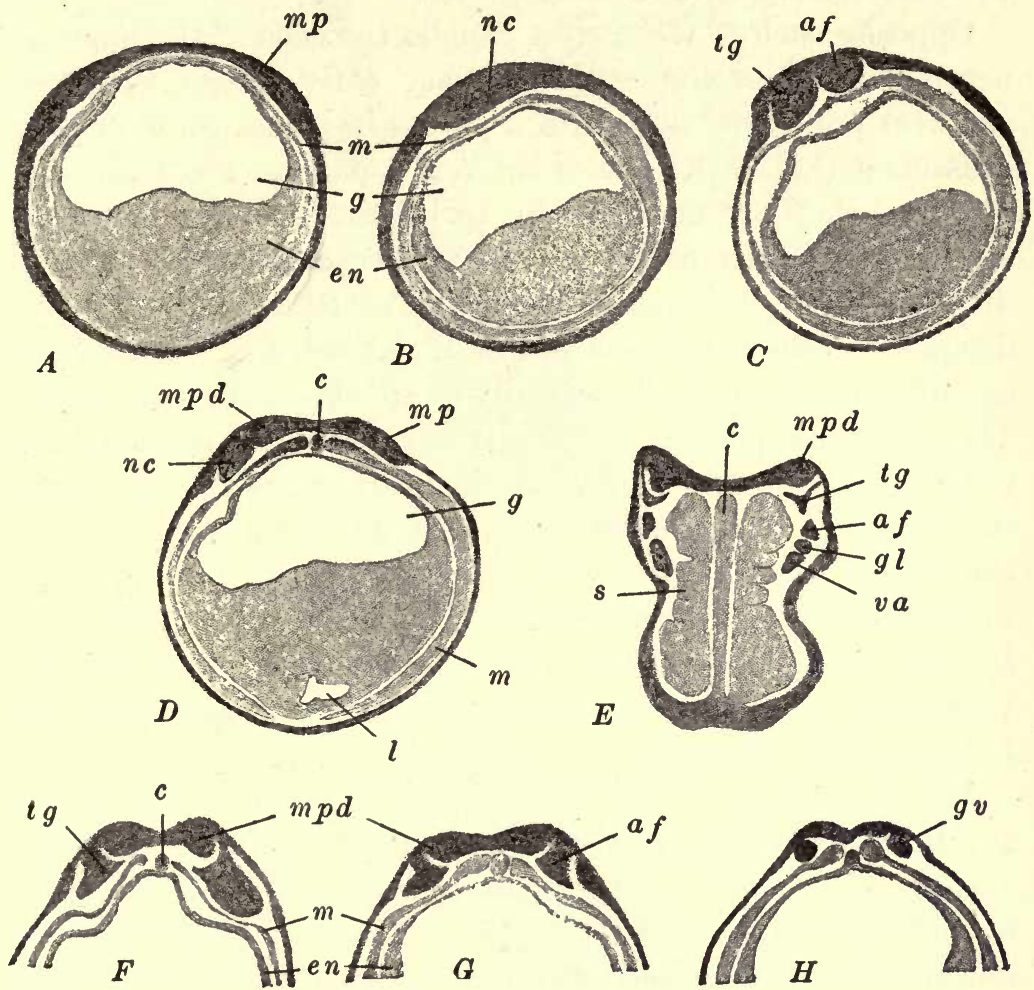

FIG. 44.--Sections through young frog embryos ( $R$. fusca), illustrating the development of the crest ganglia and placodes. After Brachet. $A$. Transverse section through the neural plate of an embryo before elongation begins. $B$. Sagittal section, to one side of the mid-line, through an embryo of the same age as $A$. (This is also the stage of Fig. 32, G.) $C$. Sagittal section, to one side of the mid-line, through an embryo just beginning to elongate. $D$. Transverse section through an embryo slightly older than that of $A$ and $B$. E. Frontal section through an embryo with three or four pairs of mesodermal somites. $F, G, H$. Three transverse sections through an embryo just beginning to elongate (same age as $C$ ), showing the trigeminal, acustico-facial and glossopharyngealvagus crest ganglia. af, Acustico-facialis ganglion; $c$, notochord; en, endoderm; $g$, gut cavity; $g l$, glossophary ngeal ganglion; $g v$, glossopharyngeal-vagus ganglion; $l$, liver diverticulum; $m$, mesoderm; $m p$, primitive medullary plate; $m p d$, definitive medullary plate; $n c$, neural crest; $s$, mesodermal somites; $t g$, trigeminal ganglion; $v a$, vagus (pneumogastric) ganglion.

pharyngeal (IX) and vagus (X) nerves. The delamination of these rudiments from the medullary plate is not quite complete, 
and when the neural tube is fully formed, each may be seen to be connected with the dorsal surface of the myelencephalon by a slender chain of cells (Fig. 45).

Opposite each of these crest ganglia the cells of the inner or nervous layer of the ectoderm very early (stage with 3-4 somites) proliferate and form a patch, in places three or four cells deep (Fig. 45). These thickened patches are known as placodes: they are undoubtedly to be interpreted as vestigial sense organs. In each placode two separate elements are distinguishable, a superficial sensory element, which usually disappears (the exceptions will be noted below), and a deeper ganglionic portion which is usually retained to a varying extent. The ganglionic portion of the placode typically fuses with the cells of the associated crest ganglion, and together they form the rudiment of the chief sensory or afferent components of the cranial nerve. From this point onward we may describe separately the history of the chief cranial nerves. (Reference is unavoidably made to the visceral arches and clefts whose development must be described later, in connection with the history of the pharynx).

The Trigeminal Nerve $(V)$.- This is the chief nerve of the mouth and mandibular arch. The trigeminal portion of the neural crest is very large, extending from the eye to the hyomandibular cleft (Fig. 44, $C, E, F$ ). The crest ganglion grows downward and comes ventrally into contact with the mesoderm of the mandibular arch. These ectodermal and mesodermal cell groups then fuse, cells of the apposed surfaces intermingling, and finally a cell mass is formed in which the two elements are indistinguishable; this becomes the mesenchyme of the mandibular arch (Fig. 45, $A, B$ ).

The dorsal and superficial cells of the crest ganglion retain their nervous character and come into relation with the large placode of this region. The superficial or sensory portion of this placode disappears, but its deeper or ganglionic portion enlarges and divides into two parts. An anterior part separates from the surface ectoderm as the ophthalmic ganglion of the ophthalmic branch of the V nerve, whose fibers grow out ante- 


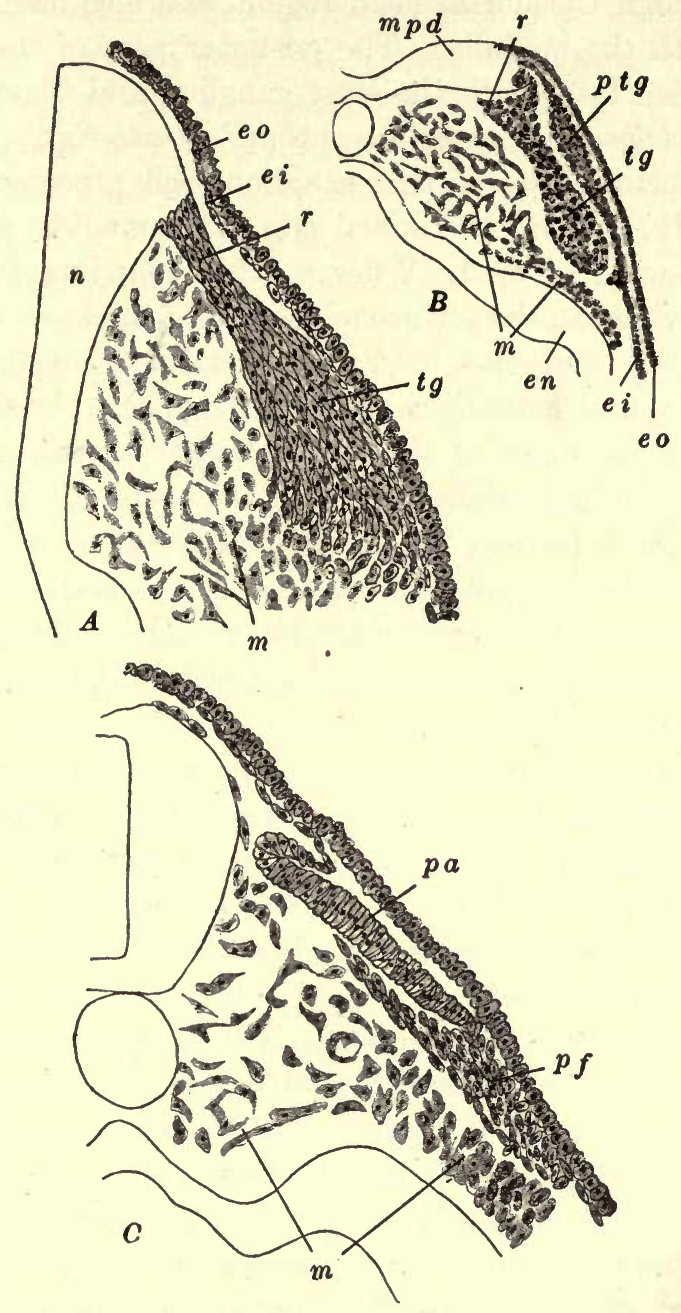

Fig. 45.-Portions of sections through the head of the frog ( $R$. fusca), illustrating the formation of the placodes and the history of the crest ganglia. After Brachet. A. Transverse section through the trigeminal ganglion of an embryo of $3 \mathrm{~mm}$. B. Transverse section through the acustico-facialis ganglion of an embryo with three or four pairs of mesodermal somites. C. Transverse section through the facial ganglion and auditory placode of an embryo of $2.8 \mathrm{~mm}$. $e i$, inner or nervous layer of ectoderm; $e n$, endoderm; $e o$, outer layer of ectoderm; $m$, mesoderm; $m p d$, definitive medullary plate; $n$, nerve cord; $p a$, auditory placode; $p f$, facial placode; $p t g$, trigeminal placode; $r$, spinal prolongation of ganglion; to, trigeminal ganglion. 
riorly, through the dorsal head region, and also medially connecting with the medulla. The posterior part of the placode ganglion then fuses with the crest ganglion and together they form the trigeminal ganglion proper (Gasserian ganglion). From the neuroblasts of this ganglion, cell processes extend centripetally, entering the dorsal side of the medulla and forming the sensory root of the $\mathrm{V}$ nerve, while centrifugal processes rapidly grow out to the surface of the head (cutaneous branch of the $\mathrm{V}$ nerve), and also in front of and behind the mouth (mandibular and maxillary branches). These branches, as indeed those of most of the other branchiomeric nerves as well, are established before the time of the opening of the mouth. The cells which formed the original attachment of the crest ganglion with the medulla, appear to form the medullary sheath cells of the root of this nerve, while the sheaths of the peripheral branches are apparently derived from other migratory cells of the crest ganglion itself.

The Facial and Auditory Nerves (VII and VIII).--It is convenient to describe these together, since both are derived from the acustico-facialis crest ganglion and its associated placode. The VII nerve is the nerve of the hyo-mandibular cleft, while the VIII nerve is not one of the branchiomeric series, but is purely sensory (auditory).

The early history of these nerves is similar to that of $\cdot V$; the major part of the crest ganglion contributes to the mesenchyme of the hyoid arch. The nervous part of the crest ganglion is somewhat more extensive than that of the $\mathrm{V}$ nerve, i.e., a greater part of the original ganglion remains of nervous function. The first important distinctive character here, consists in the fact that the sensory or superficial part of the placode does not disappear, but, continuing to enlarge, gradually sinks below the surface of the head, invaginates, and forms the rudiment of the ear, the auditory sac (Fig. 45, C). The deeper placode ganglion cells in connection with this sensory epithelium remain in close contact with the sac forming the rudiment of the VIII nerve. From the neuroblasts of this rudiment processes grow into the medulla forming the root of the VIII nerve. 
The remainder of the placode ganglion joins with the nervous portion of the crest ganglion, forming the ganglion of the VII nerve, from which centripetal processes grow out connecting the ganglion with the medulla, while centrifugal processes extend into the hyoid arch and neighboring regions (hyomandibular and palatine nerves).

The Glossopharyngeal and Vagus (Pneumogastric) Nerves ( $I X$ and $X$ ).--These are the nerves of the remaining visceral clefts, the first to fourth branchial clefts (third to sixth visceral arches). The IX nerve is limited to the region of the first branchial cleft, while the $\mathrm{X}$ nerve is distributed around the remaining gill clefts, and is to be regarded as a compound nerve, equivalent to several branchiomeric nerves. The crest ganglion of these nerves forms the large posterior section of the crest in the head region (Fig. 44, $E, H$ ). Essentially it resembles that of $\mathrm{V}$; it rapidly grows very large, extends farther ventrally and posteriorly, and contributes to the formation of mesenchyme to a considerably less extent than the ganglion of the $\mathrm{V}$ nerve. The placode of the IX nerve develops typically; its superficial sensory portion disappears, while its ganglionic portion comes only slightly into relation with the crest ganglion. Posterior to this, the large placode of the X nerve appears simultaneously and has a similar early history, save that it fuses more extensively with the nervous portion of the crest ganglion. When the fibers grow out from the IX and $\mathrm{X}$ ganglia they pass together into the medulla as a single root. The IX ganglion is only incompletely separated from that of $\mathrm{X}$ by the passage of the anterior cardinal vein (see below). From the IX portion of the ganglion, which is thus almost wholly placodal in origin, fibers grow peripherally into the usual relation with the first branchial cleft, while from the mixed ganglion of the $\mathrm{X}$ nerve, branches grow out with typical relations to all the remaining clefts.

From the vagus ganglion certain other important processes grow out posteriorly. From the placode a considerable tongue of cells grows posteriorly, forming the rudiment of the sense organs of the lateral line (see below), and accompanying this, 
fibers grow out from the ganglion as the rudiment of the lateral line nerves (Fig. 53), this branch is present throughout the tadpole stage but disappears at metamorphosis. The vagus ganglion also sends out processes which grow posteriorly, to the thoracic and abdominal viscera; these form the visceral branch of the $\mathrm{X}$ nerve.

The branchiomeric cranial nerves include, beside the afferent or sensory components, whose development has just been outlined, efferent or motor fibers in varying numbers. These do not arise by a morphologically separate root, but neuroblasts in the wall of the medulla send out processes (axons), which leave the cord in association with the afferent roots described above. They are distributed with the branches which pass posterior to the gill clefts.

The development of the III, IV, and VI cranial nerves is incompletely known. They form, comparatively late, as outgrowths of neuroblasts located in the ventral parts of the medulla. These processes extend through the mesenchyme of the head into the orbit and innervate the muscles attached to the eye-ball. The III nerve is the first to appear (Held) at a time when 8-9 somites are present $(5-6 \mathrm{~mm}$.).

\section{B. THE SPINAL NERVES}

The spinal nerves differ from the cranial nerves in two important respects: (a) they are related primarily with the mesodermal somites instead of the visceral clefts, and (b) there are no placodal elements connected with them.

The neural crests continue from the head region as much smaller strands of cells; these become broken into segments which are the rudiments of the spinal ganglia. From the neuroblasts of each ganglion, cell processes grow out, some centripetally into the cord, forming the dorsal root of the spinal nerve, others centrifugally, forming the peripheral afferent fibers which are distributed chiefly to the skin and other sensory surfaces (Fig. 46). The ventral root of the spinal nerve is formed by outfrowths (axons) from neuroblasts in the ventral side of the cord. 
They first appear in the anterior part of the body in the larva of about $4 \mathrm{~mm}$. As they grow outward they meet the dorsal root, just beyond the ganglion, and pass thence in part to the mesodermal myotomes, and in part are distributed with the sympathetic system. The two most anterior myotomes are without spinal nerves; these are occipital in position and later disappear.

\section{THE SYMPATHETIC SYSTEM}

The development of the sympathetic system is very imperfectly known in the frog. The first definite indication of it appears in the embryo of about $6 \mathrm{~mm}$., as slight collections of cells on the spinal nerves about the level of the dorsal aorta. From what is known here and to be inferred from the conditions in other lower vertebrates, it may be said that these cell groups are composed of elements from the spinal ganglia and certain of the posterior cranial ganglia. These cells, coming from the ganglia, migrate ventro-medially and form a pair of longitudinal sympathetic cords along the sides of the dorsal aorta (Fig. 46). From the cells of these cords processes appear to grow back to the spinal ganglia forming the nerve trunk; $v$, ventral (motor, rami communicantes, and also peripherally to the visceral organs and surfaces. Along the paths thus marked out fibers grow out from other spinal ganglion cells, and quite likely other cells migrate from the cord 
forming additional sympathetic elements. From the sympathetic cords, cells also migrate peripherally, forming the peripheral sympathetic ganglia in connection with the great blood vessels and the thoracic and abdominal viscera. The ganglion of the III cranial nerve is also sympathetic in character, but its origin is uncertain in the frog, as is also the origin of the sympathetic fibers of the head region in general.

\section{THE SPECIAL SENSE ORGANS}

\section{The Eye}

In the preceding chapter we described the earliest traces of the eyes. These consist of a pair of patches in the superficial ectoderm of the medullary plate, before this has begun to fold

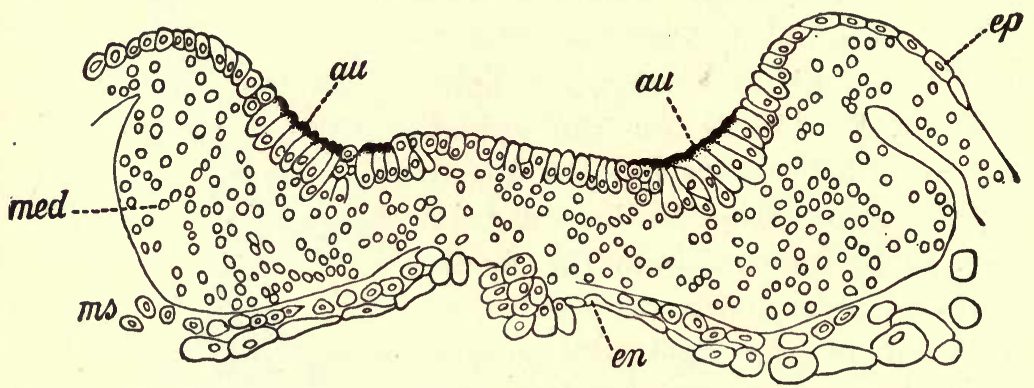

FIg. 47.-Transverse section through the anterior part of the medullary plate of an embryo of $R$. palustris, in which the medullary ridges are just forming. From Froriep (Hertwig's Handbuch, etc.), after Eycleshymer. au, Optic grooves; $e n$, endoderm; $e p$, ectoderm (epidermis); med, medullary plate; $m s$, mesoderm.

together. The cells of these patches are distinguished by their comparatively large size and by the presence of pigment in their outer ends (Fig. 47). When the medullary plate folds into a tube these patches are carried inward and are left toward the antero-ventral border of the fore-brain (telencephalon). Their pigment then gradually disappears, but it is clear that their originally free ends now border the cavity of the brain. Before the brain folds have entirely closed together, the regions surrounding and including these patches have already evaginated and formed the pair of optic stalks and vesicles extending 
quite to the surface of the head. The cavities of these structures are continuous with the brain cavity (third ventricle), so that the relation of the optic cells to the optic vesicle is the same as to the original cavity of the brain, and the free surfaces of these cells are now turned away from the surface of the head; the true optic cells form the most distal part of the optic vesicle.

The sensory, or recipient, and the nervous elements of the eye (retina and optic nerve fibers), originally on the surface of the head, are the essential parts of the eye; they now form the optic vesicles, and are to be distinguished from the various accessory parts (the choroid and sclerotic coats, the aqueous

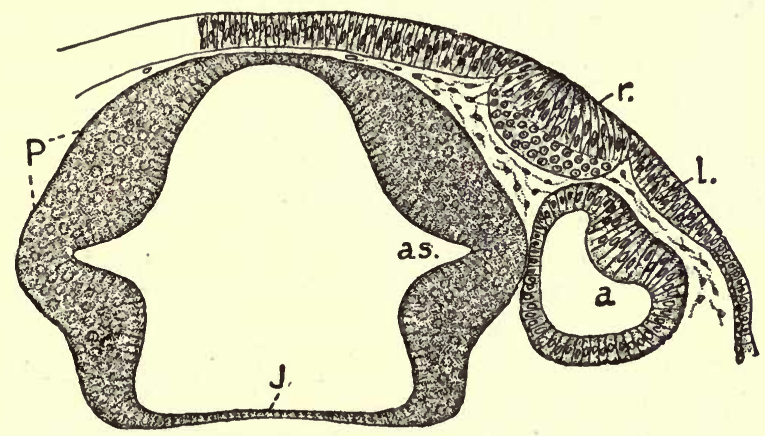

Fig. 48.-Frontal section through the fore-brain and optic vesicle of an embryo of $R$. fusca, in which the tail is just growing out. From Von Kupffer (Hertwig's Handbuch, etc.). a, Optic vesicle; as, opening of optic stalk out of fore-brain; $J$, posterior wall of infundibulum; $l$, rudiment of lens (placode); $P$, wall of prosencephalon; $r$, rudiment of olfactory organ.

and vitreous humors, and the cornea), which are derived chiefly from the mesoderm (mesenchyme) of the region, and from the ectoderm outside of the optic vesicle (lens and cornea, in part).

Upon reaching the surface of the head the optic vesicle is converted into the optic cup. The peripheral part of the vesicle becomes flat and then folds into the proximal part, forming a roughly hemispherical, two-layered cup, obliterating the original cavity of the optic vesicle and establishing a new cavity (Figs. 49, 50). The extent of the optic cup is increased 
immediately by the outgrowth of its free margin, which gradually draws partially together, leaving a small opening toward the surface of the head; this is the rudiment of the pupil. In the optic cup then we distinguish an inner and an outer layer, and a central cavity; these are respectively, the rudiments of the true retinal layer, the pigment layer, and the posterior chamber of the eye.

During the growth and invagination of the optic cup the optic stalk remains attached to its ventral side, so that the retinal

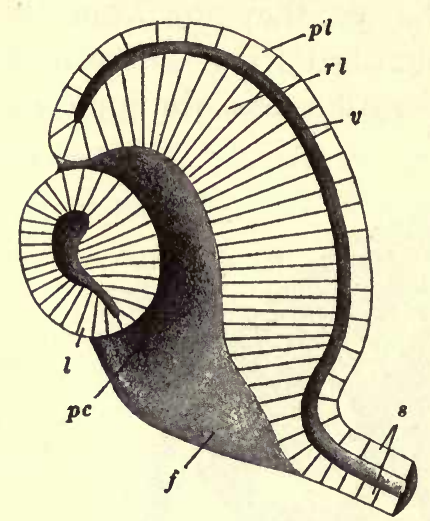

Frg. 49.-Plastic figure of hemisected optic vesicle, lens and optic stalk of the frog. $f$, Choroid fissure; $l$, lens; $p c$, posterior chamber of eye; $p l$, outer or pigmented layer of optic cup; $r l$, inner or retinal layer of optic cup; $s$, optic stalk; $v$, original cavity of optic vesicle.

layer of the cup is continuous with the lower side of the optic stalk (Fig. 49). The infolding of the retinal layer is not a simple invagination, but, owing to this ventral attachment of the optic stalk, the infold is continued as a groove from the middle of the vesicle to the ventral border of the cup where it joins the stalk. This groove remains narrowly open for a time and is known as the choroid fissure, the pupil appearing as a dilatation of its upper end in the middle of the cup (Figs. 49, 128). That part of the cup opposite the pupil is referred to as the fundus region.

While the vesicle is invaginating the rudiment of the lens is formed as a thickening of the ectoderm opposite the pupillary region (Figs. 48, 50). This thickening involves only the deeper or nervous layer of the ectoderm and is, in all essential respects, similar to the ganglionic portion of the ectodermal placodes described in connection with the cranial nerves. This lens placode is immediately anterior to the placode of the $\mathrm{V}$ cranial nerve. By the time of hatching this forms a prominent rounded thickening, which is cut off from the ectoderm as a solid spheroidal cell mass (Fig. 50). After hollowing out internally 


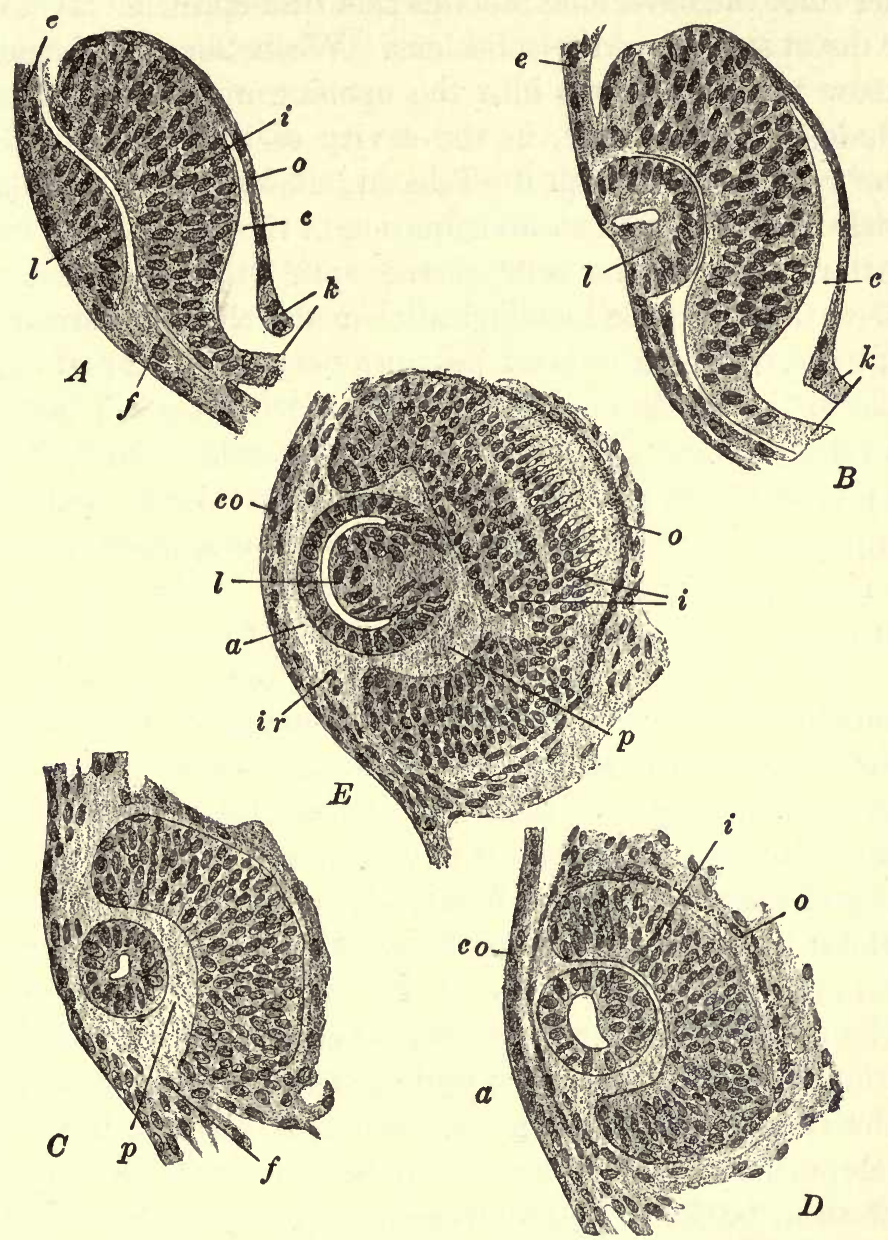

FrG. 50.-The development of the eye in the Urodele, Siredon pisciformis. After Rabl. $A$. Of embryo with about twenty-five pairs of somites, showing the thickening of the lens rudiment. $B$. Invagination of the lens and formation of the optic cup. C. Lens separating from the superficial ectoderm in an embryo of about thirty-five pairs of somites. $D$. Thickening of the inner wall of the lens. $E$. Shortly before hatching; differentiation of the rods and cones in the retinal layer. $a$, Anterior chamber of eye; $c$, cavity of primary optic vesicle; co, cornea; $e$, ectoderm of head; $f$, choroid fissure; $i$, inner or retinal layer of optic cup; $i r$, rudiment of iris; $k$, optic stalk; $l$, lens; $o$, outer or pigmented layer of optic cup; $p$, posterior chamber of eye. 
it again becomes solid through the elongation of the cells of its inner side: the outer side remains as a thin epithelial layer over the distal surface of the solid lens. When the pupil begins to narrow the lens moves into the opening and finally remains included just within it, in the cavity of the optic cup. other vertebrates, except the Teleosts, the lens forms as a hollow vesicle resulting from an invagination of the surface ectoderm.)

After hatching the cells of the optic cup layers begin to differentiate into the histological elements characteristic of the adult eye. The outer layer becomes very thin and pigmented, while in the thick inner layer the characteristic cell layers of the retina appear (Fig. 50, D, E). The cells in contact with the pigment layer gradually differentiate as the rods and cones; this process begins in the fundus region and spreads thence to the more peripheral regions of the retina. Other cells of the retina are neuroblasts and send out nerve processes; those from the cells bordering the cavity of the optic cup (posterior chamber) grow down, by way of the margins of the choroid fissure, to the optic stalk. Entering the ventral and posterior sides of this, they pass thence to the ventral wall of the forebrain, forming there the optic chiasma and optic thalami. The cavity of the optic stalk is finally obliterated by the collection of these fibers; in the brain the optic recess marks the region of its original opening (Figs. 41, 42,43). The component cells of the stalk become largely non-nervous, forming the supporting neuroglia cells. The optic stalks are commonly known as the II pair of cranial nerves, but it is evident from their development that they are tracts of the brain, without any similarities to the true cranial nerves.

The eye develops very slowly during the larval period after hatching, but at the time of metamorphosis it is rapidly perfected. The details in the later development are not very well known. The rudiment of the vitreous body, occupying the posterior chamber of the eye, is of mixed origin, although purely ectodermal. It arises from outgrowths of cells from the inner surface of the lens placode and also from the retinal layer of the cup. These elements intermingle and form the 
stroma of the vitreous humor or body. In its essential composition it thus resembles a typical cranial nerve ganglion which is derived from placode cells and neural crest; of course, in this region, the neural crest is not typically present, but the retinal cells have somewhat similar relations to the neural tube proper.

A day or two before hatching the choroid fissure of the optic cup begins to close, first in the fundus region. Blood vessels have already entered the posterior chamber and the margins of the fissure embrace these. The region last to fuse, at the margin of the cup, becomes enlarged as the ventral choroid knot, and from this the iris develops, gradually extending dorsally around the outer side of the pupil. The cornea and outer coats of the eye-ball, as well as muscles are formed from the mesenchyme around the optic cup.

\section{The Ear}

Like the eye this organ is a complex, derived from diverse sources; the essential parts, composing the membranous labyrinth, are derived from the ectoderm of the surface of the head, while the accessory parts, tubo-tympanic cavity and columella, are of endodermal and mesodermal origin. We shall describe first the formation of the membranous labyrinth or internal ear.

We have already mentioned the first formation of the auditory organ as the auditory placode, which appears just as the neural folds close together (Fig. 45, C). As the embryo begins to elongate each of these placodes becomes depressed below the surface of the head and invaginates, forming the ovoid auditory sac or otocyst, about $0.2 \mathrm{~mm}$. in diameter (Fig. 51, A). For a time this remains connected with the surface of the head by a narrow tube of cells, but just before hatching it becomes completely closed and separates entirely from the surface ectoderm, sinking in toward the lateral surface of the myelencephalon (Fig. 51, B). The superficial layer of the ectoderm does not share in the formation of the auditory sac but remains continued across the surface of the head outside the invaginating 


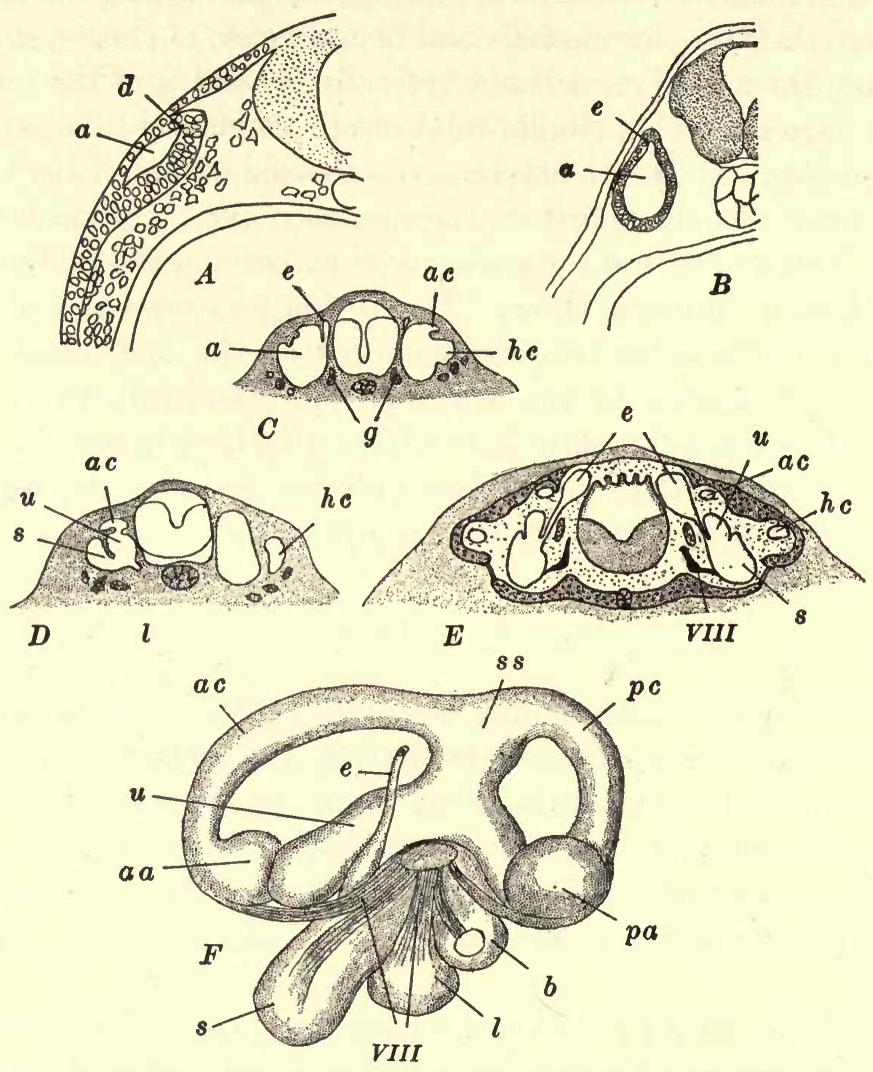

Fig. 51.-The development of the auditory organ in the frog and toad. $A$, $B, F$, after Krause; $C, D, E$, after Villy. $A$. Section through the auditory vesicle of an embryo just beginning to elongate. $B$. Section through the auditory vesicle that has very nearly separated from the superficial ectoderm. $C$. Transverse section, somewhat oblique, through the auditory organs of a $12 \mathrm{~mm}$. $R$. temporaria. $D$. Slightly more advanced stage than $C$. $E$. Section through the auditory organs of a $25 \mathrm{~mm}$. R. temporaria. $F$. Membranous labyrinth of the toad (Bufo vulgaris). a, Auditory sac; $a a$, anterior ampulla; $a c$, anterior vertical semicircular canal; $b$, pars basilaris; $d$, dorsal outgrowth of primitive auditory vesicle (rudiment of endolymphatic duct); $e$, endolymphatic duct; $g$, ganglion of auditory (VIII) nerve; $h c$, horizontal semicircular canal; $l$, lagena or cochlea; $p a$, posterior ampulla; $p c$, posterior vertical semicircular canal: $s$, saccule; $s s$, sinus superior; $u$, utricle; VIII, auditory nerve. 
auditory epithelium. The wall of the auditory sac is only one cell in thickness, except in its medio-ventral region where the ganglionic portion of the placode is located. A small fingerlike outgrowth of the sac extends a short distance dorsally, from its medio-dorsal region: this is the rudiment of the endolymphatic duct. From this condition the auditory sac changes very little until after the opening of the mouth (10-12 mm.) when it continues its development.

The next differentiations of the auditory sac result from the formation of various ridges and septa extending into its cavity (Fig. 51, $C, D, E$ ). The first of these appears obliquely along the outer and posterior walls of the sac, and finally divides the otocyst into two regions, an inner and upper part, known as the utricle, and a lower and outer part, the saccule; these two divisions remain connected by a small perforation in the septum. The endolymphatic duct is connected with a dorsal extension of the saccule, while from the utricle the three semicircular canals grow out. These canals are formed first by the growth of couples of ridges into the cavity of the utricle, approximately in the relative positions of the future canals; the couples meet and fuse save at their ends, so that the cavities enclosed by them open directly into the utricle. Each rudiment then begins to enlarge and pushes above the surface of the utricle, first as a ridge, which becomes plate-like and then tunneled between the canal and the wall of the utricle. The posterior canal is formed somewhat later than the anterior and the horizontal canals. The ampullæ are added to the canals by additional constrictions of the wall of the utricle.

Shortly after the appearance of the semicircular canals, the saccuie commences to differentiate. First there appears a postero-ventral outpocketing, the rudiment of the lagena or cochlea, which forms a simple sac of considerable size. Then posteriorly to this appears a second ventral extension, the basilar chamber (pars basilaris).

Upon the division of the auditory sac into utricle and saccule, the endolymphatic duct remained in connection with the latter. The duct slowly elongates dorsally, along the surface of the 
hind-brain, on the dorsal side of which its tip forms an expansion known as the endolymphatic sac. These sacs enlarge very considerably, fuse together, and finally, after metamorphosis, form a vascular structure, covering a large portion of the roof and sides of the myelencephalon: they remain throughout life, connecting with the saccule by the narrow endolymphatic ducts which pass through openings in the walls of the auditory capsules (Fig. 51, $F$ ).

The epithelial lining of the membranous labyrinth becomes truly sensory only in certain patches with which fibers of the VIII cranial nerve are related. These patches are located in the cochlea (3), utricle (1), saccule (1), and the ampullæ (3). The perilymph and the cartilaginous and bony labyrinths are laid down around the membranous labyrinth by the surrounding mesenchyme.

The parts of the middle ear develop relatively late and are not well differentiated until after metamorphosis. As in other vertebrates the tubo-tympanic cavity is a derivative of the pharynx, more precisely of the region of the first gill pouch (the spiracular pouch, between the hyoid and mandibular arches; see below). In the frog this gill pouch is vestigial: it is never perforated, indeed does not contain a cavity, and is represented only by a fold of the endodermal wall of the pharynx which does not quite reach to the surface ectoderm. From the dorsal end of this vestige a rod of cells grows out and terminates as a solid knob, beneath the eye (Fig. 57). During metamorphosis spaces appear in this rod and in the terminal knob, and it moves back into the region of the ear, losing its connection with the pharynx. After metamorphosis it acquires a secondary connection with the pharynx, and its distal portion enlarges very considerably, occupying the space between the ear and the integument of the side of the head: the integument here later becomes the tympanic membrane, the expanded cavity is the tympanic cavity, and the narrow connection with the pharynx is the Eustachian tube.

In the adult the auditory capsule and the tympanic membrane are connected by a rod, the columella, extending across 
the tympanic cavity. This is the last part of the auditory apparatus to be formed. Its inner end is formed as a cartilage (the operculum) appearing in the tissue plugging the large foramen ovale in the outer side of the cartilaginous auditory capsule. From this region another cartilage, the plectrum, differentiates, toward the close of metamorphosis, along the dorsal wall of the tympanic cavity, finally extending to the tympanic membrane where it connects with a ring-like cartilage developed from the palato-quadrate cartilage (see below). The elements of the columella are thus not genetically related with the visceral skeleton (palatosquadrate cartilage). While the opercular part of the columella is becoming chondrified, the tympanic cavity extends dorsally and finally surrounds it completely, leaving it in its definitive position crossing the tympanic cavity.

\section{The Olfactory Organ}

The olfactory organs appear very early, before the brain closes, as a pair of ectodermal thickenings either side of the head, above and anterior to the future mouth region. Only the deeper nervous layer of the ectoderm is involved in the thickening, and the superficial layer disappears here, leaving only its pigment scattered among the remaining cells. These thickenings are immediately anterior to the lens placodes and may be termed the olfactory placodes (Fig. 52, A): these appear to have no relation to the median anterior ectodermal thickening which is present at this time. These placodes soon fold inward forming simple olfactory pits, the rudiments of the true olfactory cavities, their walls becoming the olfactory epithelium (Fig. 52, B). A few cells detach from the inner surface of the olfactory placode and mingle with cells derived from the surface of the telencephalon: these appear to be equivalent to the crest ganglion, and from them are formed, apparently, the sheath cells of the true olfactory nerve fibers, which are outgrowths of the sensory cells of the olfactory placode. 

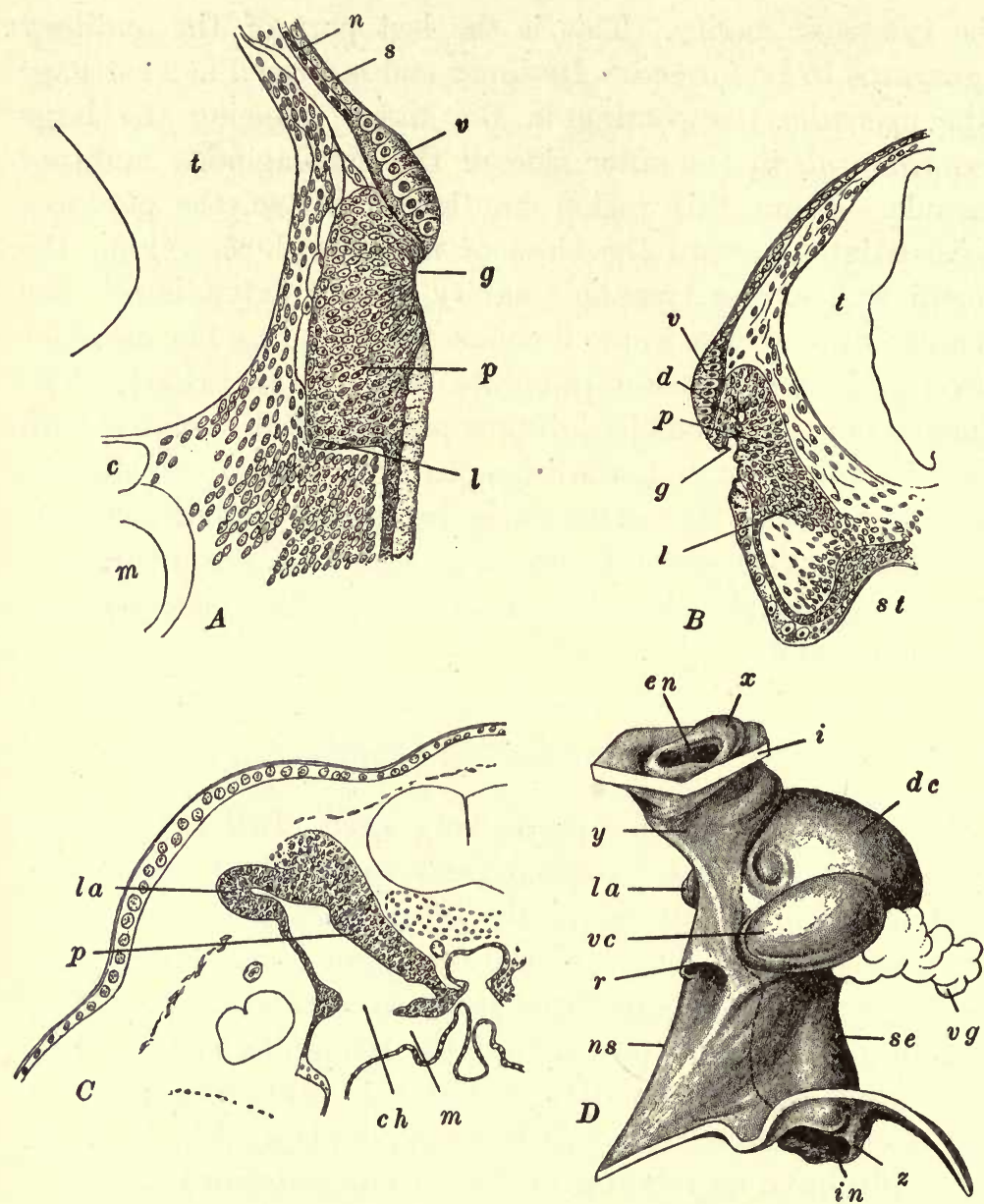

Frg. 52.-The development of the olfactory organ in $R$. fusca. After Hinsberg. $A, B, C$. Sections through the olfactory groove and organ of $5 \mathrm{~mm} ., 6 \mathrm{~mm}$., and $11 \mathrm{~mm}$. larvæ, respectively. $D$. Lateral view of a model of the olfactory organ of a $31 \mathrm{~mm}$. larva. The dotted line marks the limit between the sensory and non-sensory portions of the epithelial lining of the olfactory cavities. c, Notochord; $c h$, internal nares (choanæ); $d$, dorsal lumen; $d c$, dorsal sac; en, external nares; $g$, olfactory groove; $i$, cut edge of integument; in, internal nares (choanæ); $l$, elongation toward the mouth; $l a$, lateral appendix; $m$, mouth cavity; $n$, inner or nervous layer of ectoderm; $n s$, part of chamber lined with non-sensory epithelium; $p$, olfactory placode; $r$, ridge marking the limit between middle and ventral chambers; $s$, superficial layer of ectoderm; $s e$, part of chamber lined with sensory epithelium; st, stomodæum; $t$, telencephalon; $v$, thickened bands of superficial ectoderm cells (possibly the vestige of a primitive sense organ); $v c$, ventral sac; $v g$, ventral nasal gland attached to Jacobson's organ; $x$, elevation around external nares; $y$, canal leading to olfactory cavity; $z$, fold around internal narial opening. 
About the time of hatching $(6 \mathrm{~mm}$.) a thick strand of cells extends from each olfactory pit to the roof of the pharynx just within the limit of the stomodæum (Fig. 52, B). Later (9-12 mm.) each strand acquires a lumen which opens into the olfactory sac, and also into the pharynx as the internal nares or choanoe (Fig. 52, C).

The extent of the olfactory cavity is increased by a cavity formed in a dorsal cell proliferation of the olfactory epithelium. This region soon forms a separate dorso-lateral lobe which disappears entirely during metamorphosis.

The surface of the head pushes out above the olfactory sac so that the duct leading to it is considerably elongated, and meanwhile, on account of the enlargement of the fore-brain and the formation of the cartilage of the skull in this region, the openings of the olfactory tubes or external nares are carried to the dorsal side of the head, so that the entire olfactory organ extends along a straight perpendicular axis (Fig. 52, D).

During metamorphosis the olfactory organ becomes considerably complicated by the appearance of various foldings and out-pocketings of its walls, and by a sharp flexure of its axis. We may mention only the more important of these outgrowths. The first is an extension from the ventral side of the olfactory chamber; here a solid mass of cells proliferates, acquires a cavity, and, enlarging rapidly, turns transversely toward the medial side. This is the rudiment of Jacobson's organ; a large glandular mass develops upon its medial end. Other outgrowths are formed from parts of the olfactory sac that are non-nervous, i.e., lined with indifferent cells. One of these appears opposite Jacobson's organ and soon becomes a large sac whose cavity is added to the olfactory chamber. Another appears anteriorly at the base of the olfactory duct; this receives the ducts of the lachrymal glands. Later a dorsal sac grows out from the medial and posterior walls of the tube. During late metamorphosis the axis of the olfactory organ is sharply bent on account of the posterior shifting of the internal nares. In addition to the glands of Jacobson's organ, other glands appear as outgrowths of the 
olfactory chamber and in the posterior wall of the internal nares.

\section{The Sense Organs of the Lateral Line}

As mentioned above, the sense organs of the lateral line are derived from the placode of the $\mathrm{X}$ cranial nerve, and are innervated by the ramus lateralis of this nerve. When the embryo has elongated to about $4 \mathrm{~mm}$. a small dorso-lateral section of the vagus ganglion separates from the remainder (Fig. 53, $A, B$ ) remaining closely in relation with the ectodermal placode (Harrison). The placode now begins to elongate posteriorly; the deeper cells multiply rapidly and form a long narrow tongue which pushes along through the epidermis just outside the basement membrane (Fig. 53, $C$ ). Finally, just before hatching, it reaches to the tip of the tail. Differentiation of this cord progresses posteriorly commencing in the older anterior part. The cells become grouped at intervals, each group representing the rudiment of a sense organ of the lateral line. In each rudiment a few central cells become sensory and are surrounded by a layer of enveloping cells (Fig. 53, D). These groups push up through the epidermal layer to the surface of the body, and the sensory cells develop hair-processes.

As the placodal rudiment grows posteriorly it is accompanied by outgrowths (axons) from neuroblasts of the dorso-lateral portion of the vagus crest ganglion. These processes lie within the basement membrane of the epidermis, but when the definite sense organs develop the nerve fibers pass up among the sensory cells. The cells forming the medullary sheaths of these fibers appear to wander along the fibers from the vagus ganglion.

Similar series of integumentary sense organs are formed in definite rows on the head, and dorsally from the lateral line; these are innervated by branches of the VII, IX, and X nerves. The VIII nerve and the auditory organ also belong in this group of sense organs. At metamorphosis they all disappear save the auditory organ and nerve. 

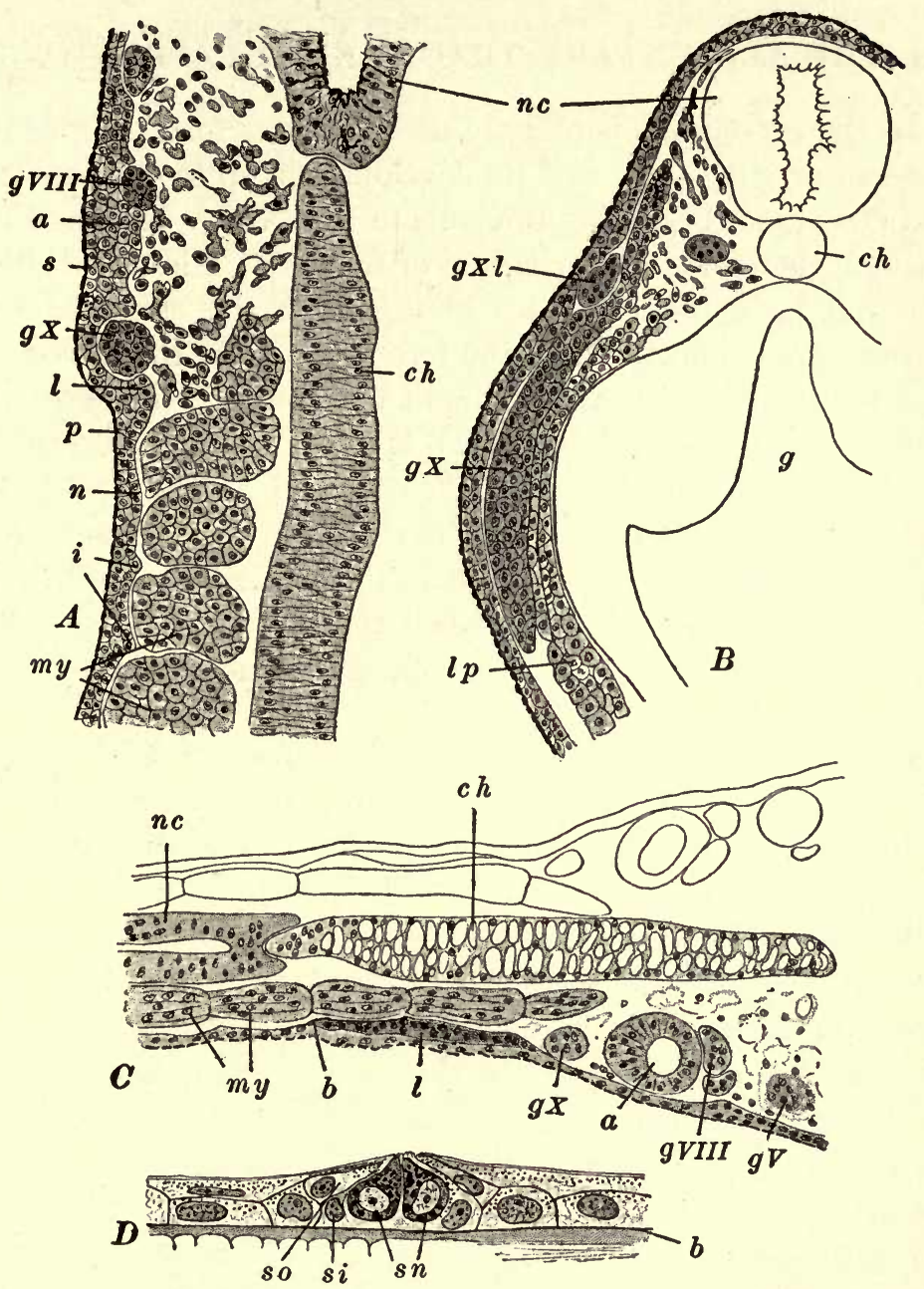

Fig. 53.-The development of the lateral line organs in $R$. sylvatica. After Harrison. A. Part of a frontal section through the level of the notochord of a $3.3 \mathrm{~mm}$. embryo. B. Part of a transverse section through the vagus region of a $4 \mathrm{~mm}$. embryo. $C$. Part of a frontal section through a $4 \mathrm{~mm}$. embryo of $R$. virescens. D. Section through the lateral line organ of a $15.5 \mathrm{~mm}$. larva of $R$. sylvatica. $a$, Auditory vesicle (in $A$, its rudiment); $b$, basement membrane of epidermis; $c h$, notochord; $g$, gut; $g V$, trigeminal ganglion, of $\mathrm{V}$ cranial nerve; $g V I I I$, acoustic ganglion of VIII cranial nerve; $g X$, vagus ganglion; $g X l$, ganglion of the lateral nerve (branch of the vagus); $i$, intersegmental thickenings of the epidermis (ectoderm); $l$, rudiment of lateral line nerve; $l p$, lateral plate of mesoderm; $m y$, myotomes; $n$, inner or nervous layer of epidermis (ectoderm); $n c$, nerve cord; $p$, pigment in epidermis; $s$, superficial layer of epidermis (ectoderm); $s i$, inner sheath cells of lateral line organ; $s n$, sensory cells of lateral line organ; so, outer sheath cells of lateral line organ. 


\section{THE ALIMENTARY TRACT AND ITS APPENDAGES}

In the preceding chapter we described the formation of the enteron or gut cavity and its development up to the time the embryo is just beginning to elongate (Fig. 37). We have described, therefore, the formation of the fore-, mid-, and hindgut and have seen that up to this time the chief differentiations are connected with the fore-gut. After the mesoderm and the notochord have been split off from the endoderm, the wall of the enteron is but one cell layer in thickness, excepting the floor of the mid-gut which is occupied by the large yolkmass. The hind- and mid-gut cavities are narrow, while the fore-gut expands widely in front of the yolk-mass, and in connection with it we have described the first indications of the mouth region, of two or three visceral pouches, and of the liver.

We may proceed now to outline the further development of each section of the alimentary tract. Before taking up the history of the fore-gut we must notice the development of certain structures associated with the mouth.

The stomodreum has already been mentioned as a shallow median depression of the head, just below the olfactory and fore-brain region. At hatching this is still rather shallow, but its floor has come into contact with the wall of the enteron, establishing a fusion known as the oral plate (Figs. 57, 58, $A$ ), for this is the region where the mouth forms a few days after hatching (9-10 mm.). In the older larvæ the inner boundary of the stomodæal region is marked by the internal nares or choanæ, which lie just within the boundary between ectodermal and endodermal territory. The formation of the oral sucker, just below the stomodæal invagination (Fig. 57), was described in the previous chapter.

The margins of the stomodæum are at first formed chiefly by the mandibular ridges, but soon the integument above these becomes drawn out in the form of lips. The mouth itself remains small in the tadpole, but the lips, of which there are an upper and a lower, soon project considerably in front of 
the mouth, enlarging the stomodæal cavity; the lower lip is the larger and is freely movable (Fig. 58, B). The lips form the important feeding organs of the tadpole, and as such they become furnished with various horny structures, described as teeth and jaws (Fig. 58, B), but not at all to be compared with the true teeth and jaws which develop later. These horny structures are purely larval organs and are entirely lost at the time of metamorphosis. The "teeth" develop from strands or piles of cells of the deeper layer of the epidermis. Each cell, as it nears the surface, undergoing cornification, becomes conical in form, and pushes through the skin, soon to be replaced by the next cell ("tooth") underlying it. The upper lip bears three medially interrupted rows of these "teeth," the lower lip four complete rows. Toward the base of each lip, near the mouth, a closely set row of teeth, together with intermediate horny cells, form a continuous ridge; these ridges, of which that of the lower lip is the larger, form the "jaws" or beak of the tadpole. During metamorphosis the horny teeth and jaws are lost, the lips are retracted, and as the mouth rapidly enlarges the true teeth and definitive jaws are formed.

\section{The Derivatives of the Fore-gut}

The organs derived from the fore-gut region that we shall describe, are the pharynx, œsophagus, stomach, liver and pancreas; in connection with the pharynx we shall describe the development of the visceral pouches and arches, the internal and external gills, the thymus, the ultimobranchial bodies, epithelioid bodies, the thyroid body, tongue, and the lungs.

In front of the yolk-mass the fore-gut is widely expanded transversely as the pharyngeal cavity (Fig. 37); antero-ventrally its wall is fused with the ectoderm as the oral plate, while postero-ventrally the liver region is indicated as a small cavity extending backward beneath the yolk. Dorsal to the yolk the fore-gut is narrowed as the rudiment of the osophagus. Along the sides of the pharynx a series of vertically elongated ridges, or solid outfoldings, appear and extend to the surface ectoderm 
with which they fuse (Figs. 54, 57). These are the visceral pouches. They appear very early and the formation of the first two or three pairs was mentioned in the preceding chapter. Altogether six pairs are formed, decreasing in size and impor-

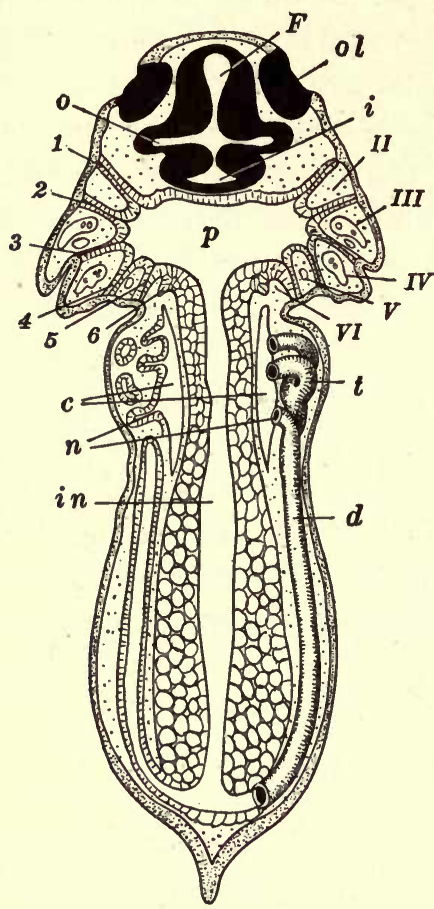

Fig. 54.-Diagram of a frontal section of a frog larva at the time of hatching. After Marshall (modified). $c$, Cœlom; $d$, pronephric duct; $F$, fore-brain; $i$, infundibulum; in, intestine; $n$, nephrostome; $o$, base of optic stalk; ol, olfactory pit (placode); $p$, pharynx; $t$, pronephric tubules; $I I$, hyoid arch; $I I I-V I$, first to fourth branchial arches; 1 , hyomandibular pouch; 2-6, first to fifth branchial pouches. tance posteriorly. As these pouches extend out to the ectoderm they divide the mesodermal tissue lying between ectoderm and endoderm, into a series of vertical rods known as the visceral arches. The most anterior visceral pouch is the hyomandibular; in front of it, between this and the mouth, lies the mesodermal mandibular arch. The remaining pouches are the first to fifth branchial pouches. Between the hyomandibular and first branchial pouches is the hyoid arch, while between successive branchial pouches are the branchial arches. Like the pouches these diminish in size posteriorly until the last is quite small and incompletely marked. Some additional facts regarding the last branchial pouch will be mentioned later in connection with the ultimobranchial bodies.

About the time the mouth is opened spaces appear within each branchial pouch; these cavities become continuous with the pharyngeal cavity, and soon perforate the area of ectodermal and endodermal fusion, forming the branchial clefts or gill clefts. The second and third clefts are perforated earliest, and soon after, the first and fourth. The hyomandibular 
pouch is never perforated; shortly before the opening of the second and third clefts it loses its connection with the ectoderm and gradually disappears. The formation, from the dorsal wall of the hyomandibular pouch, of the rudiment of the tubotympanic cavity of the ear, has already been described (Fig. 57).

The chief structures associated internally with the visceral arches are the aortic arches and the visceral skeleton, the development of which will be described in later sections. From the external surface of certain visceral arches are developed the gills, both external and internal. Reference has previously been made to the external gills: these appear just before hatching, as small outgrowths from the outer surfaces of the dorsal ends of the first and second branchial (second and third visceral) arches (Fig. 22, G.) Later a small external gill appears on the third branchial arch. The two anterior pairs grow very rapidly and about the time the mouth opens they form large branched or lobed processes, extending out from the sides of the pharynx (Figs. 64, 65). They become extremely vascular and are the earliest respiratory organs of the tadpole. The posterior pair remains small and comparatively simple.

After a few days the external gills become covered by the operculum, and then they are gradually absorbed and finally disappear completely. The operculum makes its first appearance before the perforation of the mouth, as a pair of outgrowths from the posterior borders of the hyomandibular arches. These folds grow backward rapidly, and just as the external gills reach their maximum development, the operculum extends to and outside of them, enclosing them in an opercular cavity. This cavity finally becomes entirely enclosed on the right side by the fusion of the posterior border of the right opercular fold with the surface of the body; this fusion extends across the ventral side also, and thus puts the right cavity in connection wlth the left (Fig. 58, B). The left fold remains partly free and the margins of the opening are drawn out forming a short opercular tube or "spiracle."

The internal gills appear just after the gill clefts are perforated, about the time the mouth opens, as a series of small 
elevations on the postero-external faces of the branchial arches (Figs. 64, 65). Like the external gills, these are covered with a thin layer of ectoderm cells which move in, covering their original endodermal coat. These rudiments soon become doubled on the first three branchial arches, but remain in a single row on the fourth branchial arch. They enlarge rapidly and form long branched processes or gill filaments, along the whole border of the arch below the external gills. The filaments become very vascular and project freely into the opercular cavity, where they are bathed in the respiratory current entering the mouth and passing out through the gill clefts and opercular tube.

The inner borders of the branchial arches become serrated by the formation of a series of papillæ, forming a kind of filtering organ (Fig. 64, D, E). The continued widening of the dorsal part of the pharynx throws the branchial portion into a ventral position, and then this whole region becomes partly separated from the dorsal region by the anterior and posterior folds of the pharyngeal floor; these are the velar plates (Fig. 64, D, E).

As the period of metamorphosis approaches, the opercular cavity and the gill clefts become occluded by the rapid proliferation of the cells lining these spaces, and of the gills themselves. The most of this solid mass of cells becomes completely resorbed. Various structures of the young frog are derived from vestiges of the gill clefts.

The thymus body appears just before hatching, as a solid internal proliferation from the epithelium of the upper side of the first branchial pouch (second visceral, or hyobranchial pouch) (Fig. 56). A similar smaller proliferation from the hyomandibular pouch has only a transitory existence. The thymus rudiment enlarges slowly, separating from the wall of the pouch at about $12 \mathrm{~mm}$. After metamorphosis the thymus bodies are seen toward the outer surface of the head, just back of the auditory capsule and jaw articulation.

From the dorsal ends of the other branchial clefts somewhat similar bodies are formed; these become lymphoid and appear to have no correspondence with the thymus rudiments. From 
the ventral ends of the anterior gill pouches additional epithelial proliferations are formed about the time the internal gills

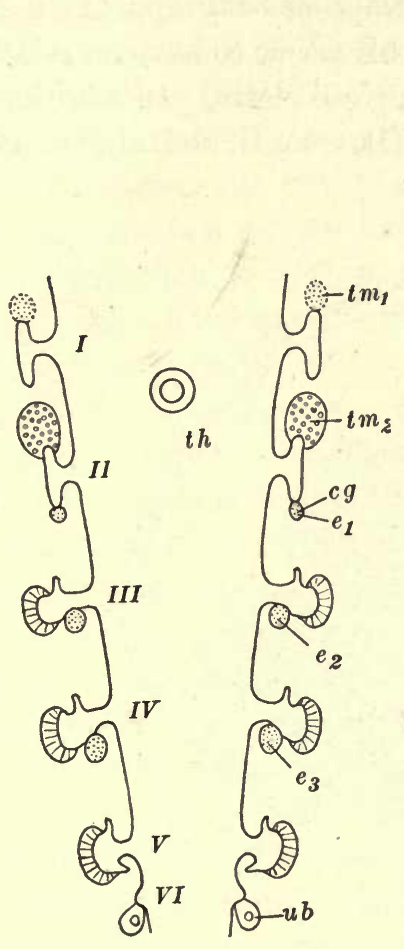

Fig. 55.

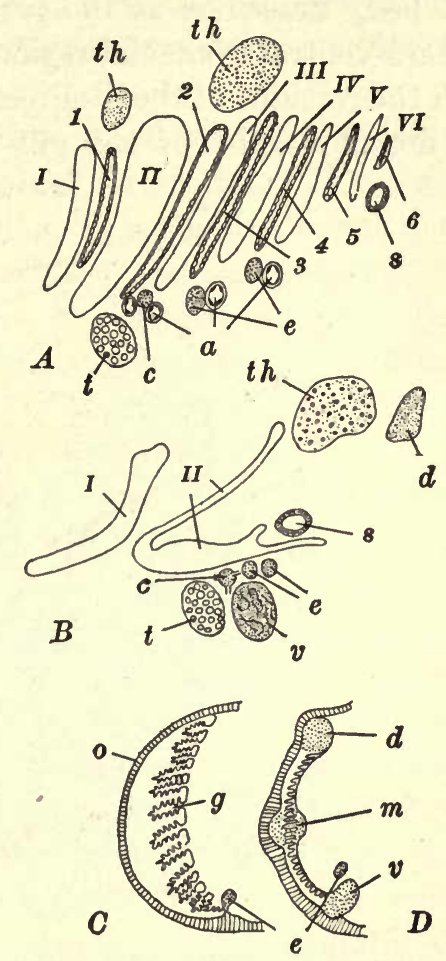

FIG. 56.

Frg. 55.-Diagram of the branchial pouch derivatives in the frog. After Maurer, with Greil's modification. $c g$, Carotid gland; $e_{1}, e_{2}, e_{3}$, epithelioid bodies; th, thyroid body; $t m_{1}, t m_{2}$, thymus bodies; $u b$, ultimobranchial body; $I-V I$, first to sixth visceral pouches. (I, hyomandibular; $I I-V I$, first to fifth branchial pouches.)

FIG. 56.-Diagrams of the derivatives of the visceral pouches and arches in the frog. After Maurer, with Greil's modification. A. Lateral view, frog larva. $B$. Lateral view, after metamorphosis. $C$. Transverse section through gill of frog larva. D. Transverse section through gill region, just after metamorphosis; the gills have not quite disappeared. $a$, Afferent branchial arteries; $c$, carotid gland; $d$, dorsal gill remainder; $e$, epithelioid bodies; $g$, internal gills; $m$, middle gill remainder; $o$, operculum; $s$, suprapericardial or postbranchial body; $t$, thyroid body; th, thymus bodies; $v$, ventral gill remainder; $I-V I$, visceral arches; $I$, mandibular arch; $I I$, hyoid arch; $I I I-V I$, first to fourth branchial arches; 1-6, visceral pouches; 1 , hyomandibular pouch; 2 , hyobranchial pouch; 3-6, first to fourth branchial pouches.

appear. Those derived from the first branchial pouch form the carotid glands, while the others form the so-called epithelioid 
bodies. These remain present throughout life, lying just below the aortic arches (Fig. 56).

A body described as the pseudothyroid body appears in the postero-ventral branchial region; this seems to have no relation with the remains of the disappearing gill clefts. In addition to the dorsal remains of the gill clefts, mentioned above, mid-

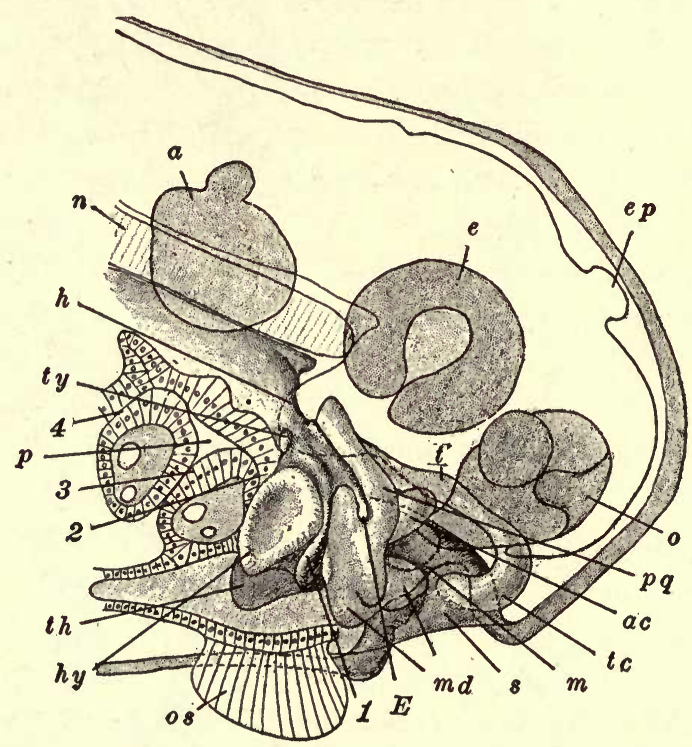

FIg. 57.-Semi-diagrammatic optical section of the head of a $7.5 \mathrm{~mm}$. larva of $R$. temporaria, illustrating the relations of the visceral pouches and chondrocranium. After Spemann. The wall of the pharynx toward the observer has been removed, so that the visceral arches are shown in section. a, Auditory organ; $a c$, anterior ascending process of the palato-quadrate cartilage; $e$, eye; $E$, rudiment of Eustachian tube; $e p$, epiphysis; $h$, hypophysis; $h y$, hyoid cartilage; $m$, oral membrane; $m d$, mandibular cartilage; $n$, notochord; $o$, olfactory organ; $o s$, oral sucker (in section); $p$, pharyngeal cavity; $p q$, palato-quadrate cartilage; $s$, stomodæum; $t$, trabecular cartilage; $t c$, trabecular cornu; $t$, rudiment of thyroid body; ty, rudiment of thymus; $1-4$, first to fourth visceral pouches.

dle and ventral traces may be seen for some time after metamorphosis.

A pair of ultimobranchial bodies, known also as post-branchial or suprapericardial bodies, are found in the frog, lying posterior to the fifth visceral pouch. These are formed as solid proliferations from the pharyngeal wall just back of the fifth visceral pouch (fourth branchial pouch). They clearly repre- 
sent the vestiges of a sixth pair of visceral pouches, although they never extend out as far as the surface ectoderm. They soon separate from the pharyngeal wall and acquire internal cavities, remaining in the floor of the pharynx, in a suprapericardial position (Figs. 54, 55, 56).

The remaining structures of the pharyngeal region have no genetic relationship with branchial structures. The thyroid body appears just before hatching, as a median evagination, narrow and elongated, from the floor of the pharynx (Fig. 57). This slowly pinches off from the pharyngeal epithelium, forming a solid rod of cells, and a few days after the opening of the mouth it divides into a pair of bodies, which then enlarge rapidly and become very vascular.

The lungs appear just before hatching as a pair of solid proliferations from the ventral wall of the posterior part of the fore-gut, between the yolk-mass and the heart. These rudiments slowly extend posteriorly along the sides of the fore-gut, and early acquire cavities proximally. Some time after the opening of the mouth, the wall of the fore-gut between and around the openings of these diverticula, becomes depressed as a transverse groove, the laryngeal chamber (Fig. 58, B), which is then partly constricted off from the alimentary tract; the opening that remains is the glottis. The lungs now rapidly elongate, pushing out into the body cavity and becoming very vascular (Fig. 72); the mesodermal constituents surrounding the endodermal lining of the lungs, are derived from the splanchnic mesoderm.

The tongue appears very late, just before metamorphosis. It is first indicated as an elevation in the floor of the anterior part of the pharynx, just back of the region from which the thyroid body was derived. In front of this elevation, between it and the lower jaw, the floor of the pharynx is depressed and glandular. During metamorphosis the rapid anterior extension of the tongue carries this glandular area upward so that it lies on the free anterior tip of the tongue.

The liver appears very early, even before the embryo begins to elongate, as a postero-ventral extension of the cavity of the 

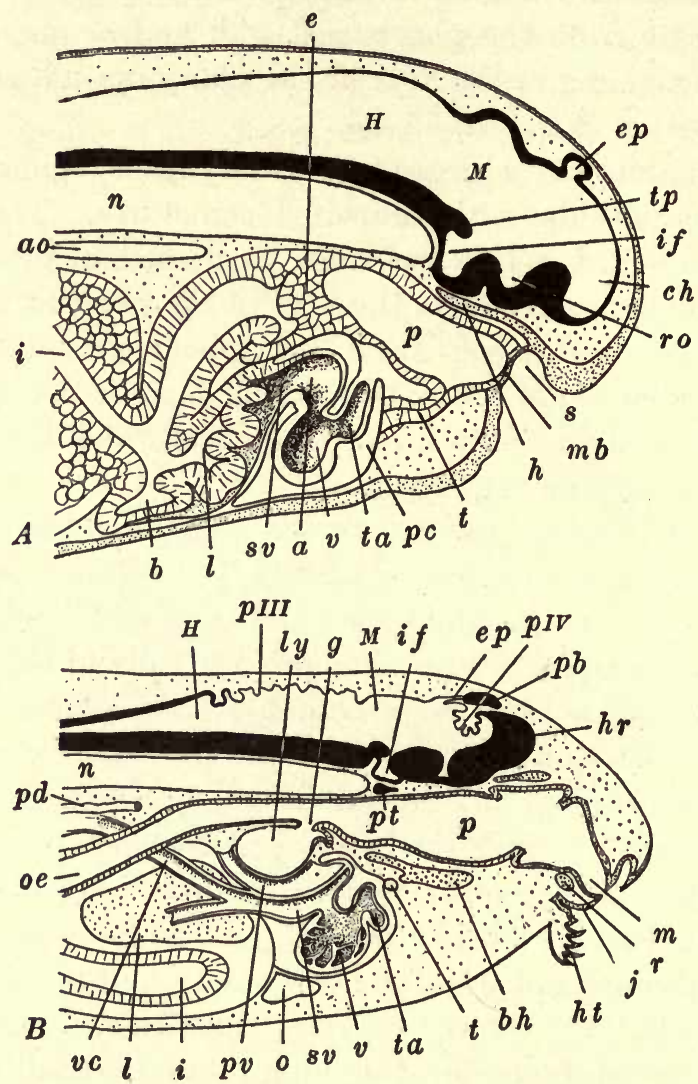

FIG. 58.-Diagrams of median sagittal sections of the anterior ends of frog larvæ. After Marshall. $A$. Of a larva just before the opening of the mouth. $B$. Of a $12 \mathrm{~mm}$. larva (at the appearance of the hind-limb buds). $a$, Auricle; $a o$, dorsal aorta; $b$, gall bladder; $b h$, basihyal cartilage; $c h$, cavity of cerebral hemisphere (lateral ventricle); $e$, epithelial plug closing the osophagus; $e p$, epiphysis; $g$, glottis; $h$, hypophysis; $H$, hind-brain; $h r$, cerebral hemisphere; $h t$, horny "teeth"; $h v$, hepatic vein; $i$, intestine; if, infundibulum; $j$, lower jaw; $l$, liver; $l y$, lary ngeal chamber; $m$, mouth; $M$, mid-brain; $m b$, oral membrane (oral septum); $n$, notochord; $o$, median portion of opercular cavity; oe, œesophagus; $p$, phary nx; $p b$, pineal body; $p c$, pericardial cavity; $p d$, pronephric (mesonephric) duct; $p t$, pituitary body; $p$, pulmonary vein; $p I I I$, choroid plexus of third ventricle; $p I V$, choroid plexus of fourth ventricle; $r$, rostral cartilage; ro, optic recess; $s$, stomodæum; $s v$, sinus venosus; $t$, thyroid body; $t a$, truncus arteriosus; $t p$, tuberculum posterius; $v$, ventricle; $v c$, inferior (posterior) vena cava. 
fore-gut, beneath the yolk-mass (Fig. 37). This rudiment enlarges very slowly at first, the solidity of the yolk preventing its penetration. The liver lies just posterior to the heart and separated from it only by a mass of scattered mesoderm cells, which come to be added to the anterior wall of the liver diverticulum, forming its mesodermal components. Some of the yolk cells adjoining the liver appear to be added to it, forming true hepatic cells. After hatching, the wall of the anterior part of the diverticulum becomes folded, and later forms the chief part of the definitive liver (Fig. $58, A)$. The posteroventral extension of the diverticulum is the rudiment of the gall-bladder, which becomes somewhat separated from the anterior hepatic portion; the original opening of the diverticulum out of the fore-gut remains as the bile-duct (Figs. 58, 59). In later stages the liver enlarges very considerably and shifts its position pos-

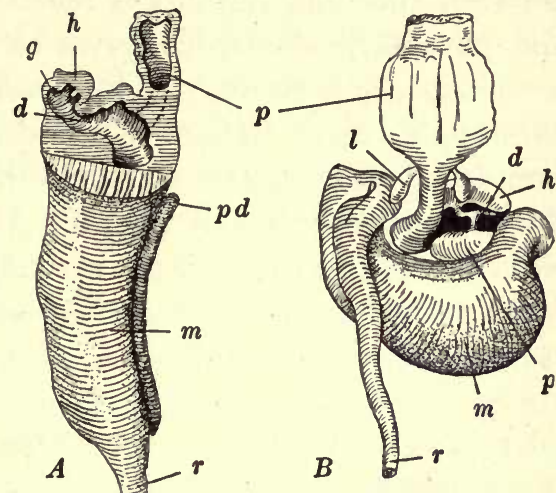

FIG. 59.-Models of the digestive tract of frog embryos. After Hammar (Maurer.) $A$. Lateral view of the tract of a $7 \mathrm{~mm}$. larva. The anterior portion has been opened by a median sagittal section. $B$. Dorsal view of the tract of an $8.5 \mathrm{~mm}$. larva. $d$, Ductus choledochus; $g$, gall bladder; $h$, liver; $l$, lung; $m$, mid-gut; $p$, pancreas; $p d$, dorsal rudiment of pancreas; $r$, rectum. teriorly; the gall-bladder also becomes very large in the tadpole.

The pancreas develops in the region where the liver diverticulum originally opens out of the fore-gut (Fig. 59). It arises from three rudiments. A dorsal rudiment appears as a solid outgrowth of the dorsal wall of the fore-gut, from which it soon separates entirely. Right and left ventral rudiments grow out from the fore-gut, just in the posterior margin of the opening of the bile-duct. These ventral rudiments, retaining a common connection with the gut, then enlarge and, passing around the sides of the bile-duct, fuse together in front of it. 
Later the dorsal rudiment unites with the fused ventral parts, and the entire pancreas is then connected by the pancreatic duct with the ventral wall of the gut. The opening of the pancreatic duct marks the boundary between the fore- and mid-gut regions during these early stages; later the opening of the duct shifts just within the margin of the bile-duct.

The chief steps in the differentiation of the csophagus, stomach, and intestines occur just after hatching. The region between the lung rudiments and the openings of the hepatic and pancreatic ducts, elongates as the region from which the œsophagus and stomach are formed (Fig. 59). Shortly after hatching $(8 \mathrm{~mm}$.$) the anterior end of the œsophagus becomes$ completely occluded by a proliferation of its wall just anterior to the laryngeal opening (Fig. 58, $A$ ). The œsophagus remains closed until shortly after the opening of the mouth (10-11 mm.) when it reacquires cummunication with the pharynx. The stomach appears as a dilation of the posterior portion of the fore-gut. Its axis is at first longitudinal, but soon it becomes bent so as to lie transversely. Throughout the larval period the stomach remains comparatively small and not clearly marked off from œsophagus and intestine.

\section{The Derivatives of the Mid-gut}

Up to the time of hatching the mid-gut remains as a narrow opening, dorsal to the yolk-mass which forms the floor of this, the intestinal region; its roof and sides are but one cell thick (Fig. 37). After hatching, the yolk is rapidly absorbed and the intestine begins to elongate. The process of yolk absorption is most rapid during the first week after hatching; in part the yolk cells degenerate, and in part they become modified into the glandular epithelium of the intestinal wall. Some of the cells of the endodermal lining of the intestine seem to wander outside the wall of the gut, into the mesentery (see below) and contribute to the formation of lymphatic tissue.

As a result of the elongation of the intestine it becomes thrown into a transverse or duodenal loop, extending across 
the body cavity from the posterior end of the stomach (Fig. 59). The elongation of the intestine continues rapidly, and soon it becomes thrown into a double spiral which occupies the entire ventral part of the body cavity. At its maximum length it is about nine times the length of the body. The œsophagus and stomach also elongate somewhat during this period, so that the pancreas and liver are pushed back into the body cavity. The relations of the mesentery are described below. During the period of metamorphosis the entire digestive tract shortens to about one-third its maximum larval length; this shortening affects chiefly the intestine and stomach.

One structure developing in connection with the enteron has not been mentioned as yet; this is the hypochordal rod. This has no direct relation with the notochord. It appears in tadpoles of about eight somites (3-4 mm.) as a median ridge along the outer surface of the endodermal wall of the midgut (Fig. 70, C). Later this ridge extends both anteriorly and posteriorly, as the part first formed separates from the enteric wall; it becomes entirely free at about $4.6 \mathrm{~mm}$. Finally it extends the entire length of the gut posterior to the dorsal pancreas; through the tail it lies above the postanal gut. It is a narrow rod, only two or three cells in diameter, lying between the dorsal aorta and the notochord. Shortly after the opening of the mouth $(13 \mathrm{~mm}$.) it breaks into short pieces and its cells either disappear or scatter; in the older larva no traces remain.

\section{The Derivatives of the Hind-gut}

This is the smallest section of the enteron. We have described, in the preceding chapter the formation of the neurenteric canal and proctodæum, and the terminal dilation of the enteron which becomes the rectal region (Fig. 37). Just after the tail has begun to elongate $(4 \mathrm{~mm}$.) the fusion between the rectal and proctodæal walls becomes perforated by the anal opening, so that the gut opens directly to the outside. The proctodæal region becomes the cloaca of the tadpole and frog, 
and receives not only the opening of the rectum, but the openings of the excretory and reproductive ducts as well. The urinary bladder is formed just before metamorphosis as a ventral outgrowth from the cloaca.

As the tail grows out, the nerve cord and notochord extend into it, while the true enteron remains limited to the body region, and the neurenteric canal consequently is drawn out posteriorly. It soon cuts off from the nerve cord, but for a time its antero-ventral limb remains open into the rectum and is known as the postanal gut. This gradually closes, and by the time of hatching it is represented only by a strand of cells extending posteriorly from the rectum, nearly to the tip of the tail; finally it disappears entirely. Throughout the larval stage the rectum remains short and only slightly dilated; during metamorphosis it enlarges and elongates, forming a considerable terminal portion of the alimentary canal.

\section{THE MESODERMAL SOMITES}

All of the remaining systems are primarily associated with the mesoderm. In the preceding chapter we described the early history of the mesoderm and in a few words we may recall its arrangement at the time the larva is about to commence its elongation.

In the body region the mesoderm is already differentiated into the thickened proximal portion along the chorda, known as the segmental or vertebral plate, and the thinner peripheral lateral plate, which passes around the sides of the yolk-mass to the ventral surface. Dorso-laterally the lateral plate is split into two sheets, the outer or somatic layer, and the inner or splanchnic layer, separated by a narrow splanchnocel or rudimentary body cavity (Fig. 70, A). Through most of the body region the vertebral and lateral plates are continuous and the cavity of the lateral plate is continued into the vertebral plate as the myoccl. At this stage, however, in the anterior body region, the vertebral plate is already transversely divided into three or four pairs of somites, which have separated, distally, from the lateral plate. 
In the region of the head and pharynx the mesoderm is in the form of scattered groups of cells, mesenchymal in character, filling the irregular spaces among the organs of these regions, brain, sense organs, ganglia, gillpouches, etc. Some of the details of the later history of this mesoderm have already been mentioned in connection with the visceral pouches, and others will be considered with the history of the vascular and skeletal systems. We may mention here, however, the essential facts regarding the development of the somites and lateral plate.

The formation and differentiation of the somites and lateral plate occur progressively in the posterior direction, so that in a young larva all of the process may be read in a series of transverse sections. The cavity of the somite, the myocœl, lies toward its surface; the

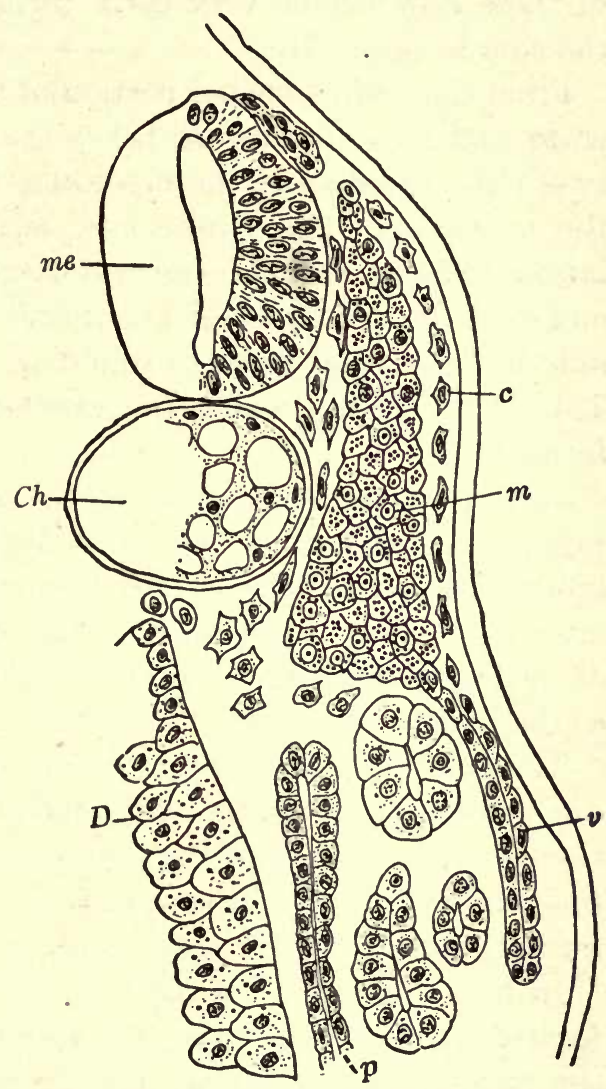

Frg. 60.-Transverse section through the sixth mesodermal somite of a $5 \mathrm{~mm}$. larva of $R$. temporaria, illustrating the arrangement of the mesoderm. From Maurer (Hertwig's Handbuch, etc.). $c$, Cutis plate; $C h$, notochord; $D$, gut wall; $m$, myotome (muscle plate); $m e$, nerve cord; $p$, lateral plate; $v$, ventral process of myotome and cutis plate.

outer wall, only one cell thick, forms the cutis plate or dermatome, lying just beneath the surface ectoderm (Fig. 60). The inner wall of the somite is much thickened as the myotome or muscle plate; through the continued thickening 
of the myotome the myocol is early obliterated. The myotomal cells or muscle cells, elongate antero-posteriorly through the entire segment; the formation of muscle fibrillæ in these cells begins very early $(5 \mathrm{~mm}$.) on the side toward the chorda (Fig. 53).

From the ventro-medial portion of the myotome, cells proliferate and move downward below the chorda, and upward between the chorda and the myotome, forming the rudiment of the sclerotome. The somite now separates entirely from the lateral plate, and soon the sclerotome separates from the somite, and extends dorsally around the nerve cord, forming a considerable mesenchymal mass surrounding this and the notochord. This is the region where the cartilaginous vertebral column forms later.

Just after the separation of the sclerotome $(5 \mathrm{~mm}$.) the myotome and dermatome send down a ventro-lateral outgrowth, which soon separates from the myotome and forms later the ventral musculature (Fig. 60); from the myotomes of the limb regions these outgrowths extend into the rudiments of the limbs, later giving rise to their voluntary musculature.

The cutis plate breaks into groups of branched mesenchyme cells, some of which become applied to the inner surface of the ectoderm and form the dermal layer of the dorsal half of the embryo, while others pass in between the mytomes, forming the connective-tissue septa or myocommata.

In the trunk region of the frog, thirteen pairs of somites are formed altogether, but the two anterior pairs disappear about the time the limbs appear, leaving eleven in the adult. The region of these two transitory somites later becomes incorporated into the head, as the occipital region. The accompanying table, based upon the observations of Elliot, summarizes the history of the somites and spinal nerves of the body region of the embryo. In the tadpole there is, of course, a large, and varying, number of somites in the tail region; Harrison has counted about forty-five pairs in a $5.5 \mathrm{~mm}$. larva of Rana virescens. All posterior to the thirteenth (eleventh of the adult series) disappear during metamorphosis. 
TABLE OF SOMITES, VERTEBRA, AND RELATED NERVES OF THE TADPOLE (ELLIOT)

\begin{tabular}{|c|c|c|c|}
\hline \multirow{2}{*}{$\begin{array}{l}\text { Cartilaginous } \\
\text { elements in } \\
\text { sclerotome }\end{array}$} & \multicolumn{2}{|r|}{ Somites } & Nerves \\
\hline & Embryo & Adult & Adult \\
\hline \multirow{3}{*}{$\begin{array}{l}\text { Occipital } \\
\text { region of } \\
\text { skull }\end{array}$} & 1 & $\begin{array}{l}\text { Absent (Disappears at forma- } \\
\text { tion of limbs) }\end{array}$ & Root of vagus nerve \\
\hline & 2 & $\begin{array}{l}\text { Absent (Disappears at forma- } \\
\text { tion of vertebræ) }\end{array}$ & $\begin{array}{l}\text { No nerve. Ganglion in embryo } \\
\text { only }\end{array}$ \\
\hline & 3 & 1 & $\begin{array}{l}\text { Ganglion and nerve in embryo. } \\
\text { Absent in adult }\end{array}$ \\
\hline 1 verteora & \multirow{2}{*}{4} & \multirow{2}{*}{2} & \multirow{2}{*}{1 spinal nerve ("hypoglossal") } \\
\hline 2 vertebra & & & \\
\hline 3 vertebra & 5 & 3 & \multirow{2}{*}{ brachial plexus } \\
\hline 4 vertebra & 6 & 4 & \\
\hline 5 vertebra & \multirow[b]{2}{*}{8} & 5 & \multirow{3}{*}{$\left.\frac{4}{5}-\frac{5}{6}\right\}$ to body wall } \\
\hline 6 vertebra & & 6 & \\
\hline 7 vertebra & 9 & 7 & \\
\hline 8 vertebra & 10 & 8 & \multirow{3}{*}{ sciatic plexus } \\
\hline 9 vertebra & 11 & 9 & \\
\hline & 12 & 10 & \\
\hline $\begin{array}{l}\text { Part of uro- } \\
\text { style }\end{array}$ & $\overline{13}$ & 11 & 10 to pelvic region \\
\hline
\end{tabular}

Before the separation of the somites and lateral plate, the latter shows traces of segmentation in the region adjoining the somites, from which later the pronephros is formed (see below). This region may therefore be termed nephrotome or intermediate cell mass. These traces disappear very quickly, and the lateral plate itself is never segmented. The cavity of the lateral plate, the general body cavity, gradually extends ventrally, and finally divides the entire lateral plate into somatic and splanchnic layers. Except in the extreme anterior and posterior ends of the trunk, the cavities of the two sides meet and fuse across the mid-ventral line, establishing a continuous body cavity and 
completely separating the somatopleure, or body wall, consisting of the somatic mesoderm and integument, from the splanchnopleure, or gut wall, consisting of splanchnic mesoderm and enteric wall.

In the pharyngeal region, the layers of mesoderm remain fused together medially, below the gut; consequently the splanchnocol is paired in this region, where the heart develops later. The median fusion is a vestige of a ventral mesentery. Along the dorsal side of the enteron, the splanchnic layers of mesoderm push in between the chorda and the enteron, and form the dorsal mesentery by which the gut remains connected with the dorsal wall of the body cavity, and through which later the vessels, nerves, etc., pass to and from the gut. When the yolk is absorbed and the narrowed gut passes to the ventral side of the body cavity, the mesentery forms a thin double fold of membrane. Then as the intestine elongates the mesentery is thrown into folds corresponding with those of the gut.

Through the absence of a ventral mesentery, save in the heart region, the body cavity is continuous from side to side beneath the gut; dorsally the mesentery interrupts such a communication. Later on, the body cavity becomes incompletely divided transversely into anterior and posterior parts, but this and the formation of the pericardial portion of the body cavity, are more conveniently described in connection with the vascular system.

\section{THE VASCULAR SYSTEM}

\section{The Heart}

The first parts of the vascular system to appear are the heart and the large veins connected with its posterior end. We have already said that the cardiac region lies beneath the hinder part of the pharynx, immediately anterior to the liver and posterior to the thyroid body. In this region the somatic and splanchnic layers of the lateral plate are separated by a wide cavity which is the beginning of the pericardial cavity (Fig. 61). This is at first directly continuous posteriorly with 
the general body cavity, though we shall see that later it becomes completely closed off. Dorsally there is no definite cœlomic space in this, the pharyngeal region. The pericardial

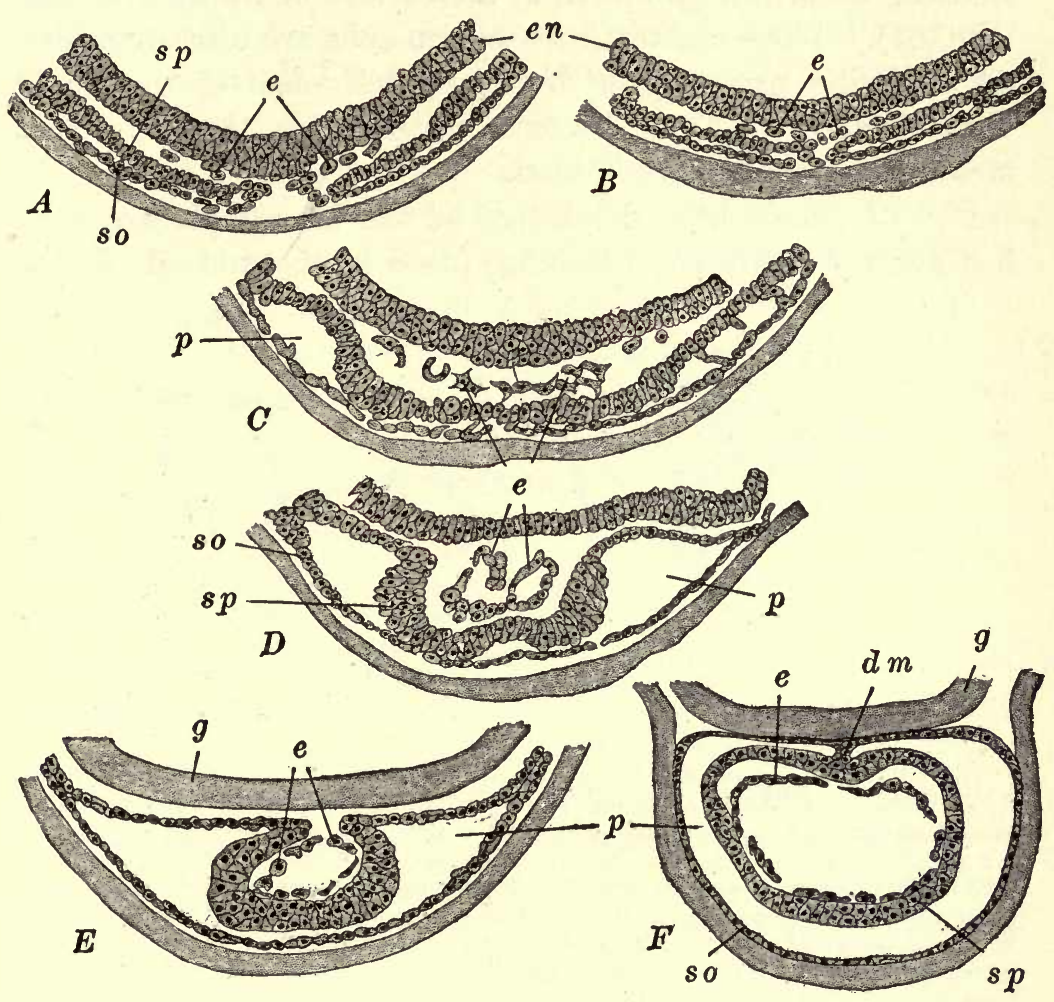

Frg. 61.-Sections showing the formation of the heart in the frog. $A-D$. Series of transverse sections through corresponding regions of a series of embryos of $R$. temporaria. After Brachet. $E$. F. Sections through the same region in older embryos of $R$. sylvatica. A. $2.6 \mathrm{~mm}$. embryo. Mesoderm approaching the mid-line; endothelium appearing. $B$. Older embryo of same length as $A$. C. $3 \mathrm{~mm}$. embryo showing enlargement of pericardial cavity and the beginning of the folding of the somatic mesoderm. D. $3.2 \mathrm{~mm}$. embryo. Endothelial cells becoming arranged in the form of a tube. $E$. Embryo of about $3 \mathrm{~mm}$. $F$. Embryo of 5-6 $\mathrm{mm}$. Heart tube established; dorsal mesocardium still present. $d m$, Dorsal mesocardium; $e$, cardiac endothelial cells; $e n$, endoderm; $g$, wall of gut (pharynx); $p$, pericardial cavity; so, somatic layer of mesoderm; $s p$, splanchnic layer of mesoderm.

wall and the muscular wall of the heart are derived from the lateral plate mesoderm, while the inner lining of the heart, the endothelium, is derived from scattered mesoderm cells.lying 
between the splanchnic mesoderm and the enteron, cells that have been formed from the endoderm in the same way that much of the lateral plate mesoderm has been, i.e., through a splitting off of cell groups from the surface of the enteric wall (Fig. 61). These scattered mesoderm cells are often regarded as belonging primarily with the ventral ends of the hyoid visceral arches. They become distinct by the time two mesodermal somites are formed.

Fig. 61 shows how the layers of the lateral plate extend beneath the pharynx, remaining fused in the mid-line as the
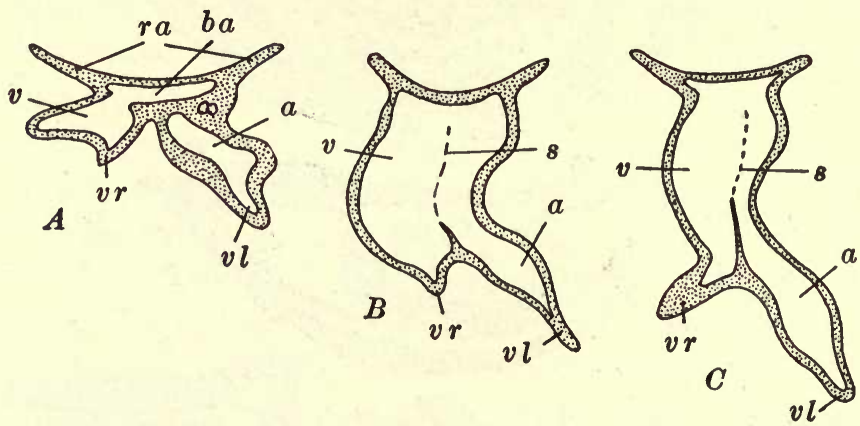

FIG. 62.-Diagrams of frontal projections of the hearts of early frog embryos. After Weber. A. Heart of an embryo of $2.7 \mathrm{~mm}$. showing the median bulbus arteriosus and the separate auricular and ventricular cavities. $B$. Heart of a $3.2 \mathrm{~mm}$. embryo showing the fusion of the auricular and ventricular cavities. The broken line marks the incomplete separation between the endothelial auricular and ventricular regions. $C$. Heart of a $3.5 \mathrm{~mm}$. embryo. At this stage the ventricle is strongly looped ventrally. $a$, Auricle; $b a$, bulbus arteriosus; $r a$, roots of aortic arches; $s$, incomplete septum between endothelial tubes of auricle and ventricle; $v$, ventricle; $v l$, root of left vitelline vein; $v r$, root of right vitelline vein.

ventral mesocardium. The inner or splanchnic wall of the pericardial cavity now folds together dorsally, enclosing the endothelial cells, which have become arranged in the form of a short tube. Finally the splanchnic folds meet and fuse dorsally, forming a tube outside of the endothelial tube and connected with the dorsal wall of the pericardial cavity; this tube forms the muscular wall of the heart and the connection is the dorsal mesocardium.

The endothelial tube, which is to be regarded as the primary rudiment of the heart, really consists of a pair of short tubes 
(Weber); these very early fuse together anteriorly forming a median region, the future bulbus aortoe (Fig. 62). From the antero-lateral margins of the bulbus, two short strands of cells extend forward in the floor of the pharynx as the rudiments of the bifurcated truncus arteriosus or ventral aortoe. Posteriorly the two endothelial tubes are only incompletely fused and are asymmetrically developed. That of the right side forms a dilated flexed tube which is the rudiment of the ventricle and the right vitelline vein, while that of the left side is more elongated and is dilated posteriorly as the rudiment of the auricle, continuing posteriorly as the left vitelline vein (Fig. 62). Both vitelline veins pass directly into the liver and yolk-mass. These two cardiac tubes gradually fuse more extensively and their cavities become somewhat confluent, so that the ventricular region is in a small degree formed of the left tube also; the more posterior auricle similarly receives a small addition from the end of the right tube with which the right vitelline vein is continuous.

The heart rudiment begins to elongate at once and is thrown, by horizontal folds, into an S-form, whereupon the dorsal mesocardium disappears, leaving the heart tube attached to the pericardial wall only at its ends. The posterior limb of the heart lies toward the left side and abuts against the liver; this forms the region of the sinus venosus and auricles. The anterior section, and the right or middle section which is the region of the ventricle, soon swing downward, becoming relatively ventral in position, while the auricle then extends through nearly the entire dorsal part of the pericardial cavity (Fig. 66).

These limbs of the heart tube are very early separated from one another by constrictions. Shortly after the opening of the mouth the auricle becomes divided into right and left auricles by the downgrowth of the interauricular septum from its dorsal wall. The sinus venosus remains connected with the right auricle. The left auricle receives the pulmonary veins, but these are only slightly represented during the tadpole stage. The wall of the ventricle becomes much thickened by the ingrowth of a muscular network from its inner surface. A few days after 
the mouth opens, the bulbus aortæ becomes divided into the anterior and posterior parts characteristic of the adult frog, and in the former, now known as the truncus arteriosus, a longitudinal fold appears separating its cavity into right and left channels.

\section{The Origin of the Blood and Vessels}

Details regarding the exact method of origin of the blood vessels of the frog are scanty. For the most part they seem to

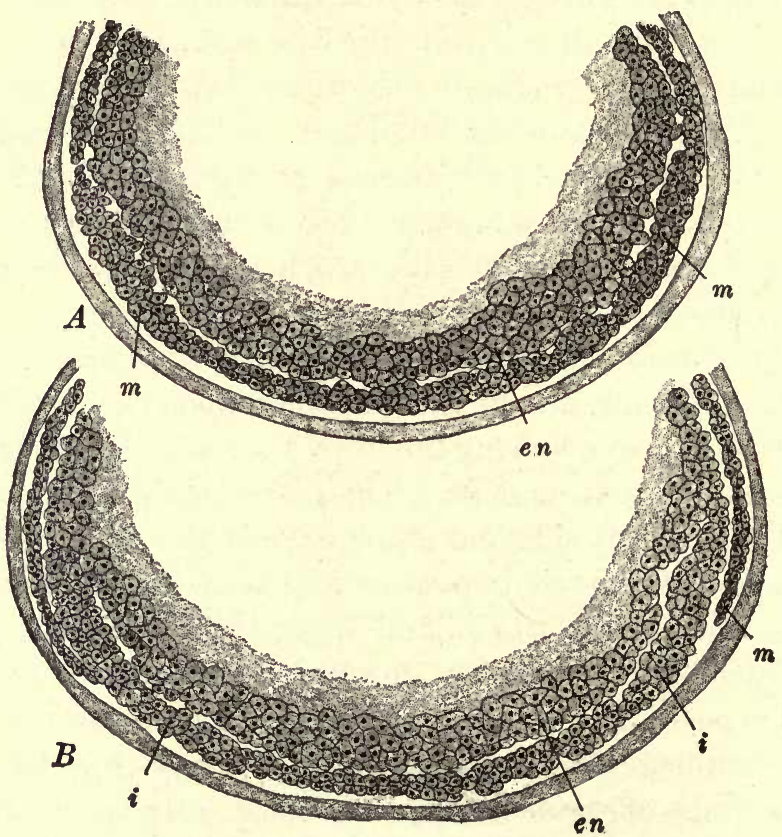

FrG. 63.- Sections showing the formation of the blood islands in the frog. After Brachet. A. Part of a transverse section through the middle of the yolk region of a $2.8 \mathrm{~mm}$. embryo of $R$. temporaria. B. Same of a $3.2 \mathrm{~mm}$. embryo. $e n$, Endothelium; $i$, blood island; $m$, mesoderm.

arise (4-4.5 mm.) as irregular and often isolated lacunar spaces in the mesenchyme and splanchnic mesoderm. The cells bordering these spaces gradually form a definite boundary and the sinuses thus formed are linked into continuous vessels. In 
some cases the smaller vessels seem to be preformed as short solid strands of cells, which become rearranged to form the walls of hollow tubes.

At first these vessels, like the heart itself, are devoid of cellular (corpuscular) elements. Some have described the origin of blood corpuscles directly from the walls of the vessels, but it seems doubtful whether such a process is at all common. For the most part the blood corpuscles are formed from a large group of blood islands, groups of cells occupying the ventral side of the yolk-mass, between the liver diverticulum and the ventral margin of the original blastoporal region (Fig. 63).

The ventro-lateral surfaces of the endodermal yolk-mass, as we have seen, give off the mesoderm by delamination, but in this ventral region the superficial cells of the yolk-mass split off irregularly in groups. These cell groups are the blood islands (Brachet). While some of these cells are converted into the walls of the veins of the yolk, they are mostly transformed into red blood corpuscles, which thus enter the circulation by way of these veins. The corpuscles enter the circulation in larvæ of about $5 \mathrm{~mm}$., and for some time their origin from the yolk region is indicated by their abundant yolk content; not until after hatching do they assume the histological characteristics of the definitive corpuscles.

\section{The Arterial System}

The earliest arteries to appear (about $4 \mathrm{~mm}$.) are.the paired lateral dorsal aortoe, dorsal to the pharyngeal region. At first a series of separate spaces or lacunæ in the mesenchyme of the head, these soon connect forming definite vessels extending forward into the cranial region. Posterior to the pharynx these vessels unite forming the median dorsal aorta, which then extends to the posterior extremity of the embryo.

The blood vessels of the visceral arches develop very early. Those of all the branchial arches are essentially similar, while the arteries of the hyoid and mandibular arches are considerably modified from the branchial type and are largely vestigial 
in character. There is some variation here among different species of Rana; we shall outline the history of these vessels in $R$. esculenta, as described by Maurer. Here, in each branchial
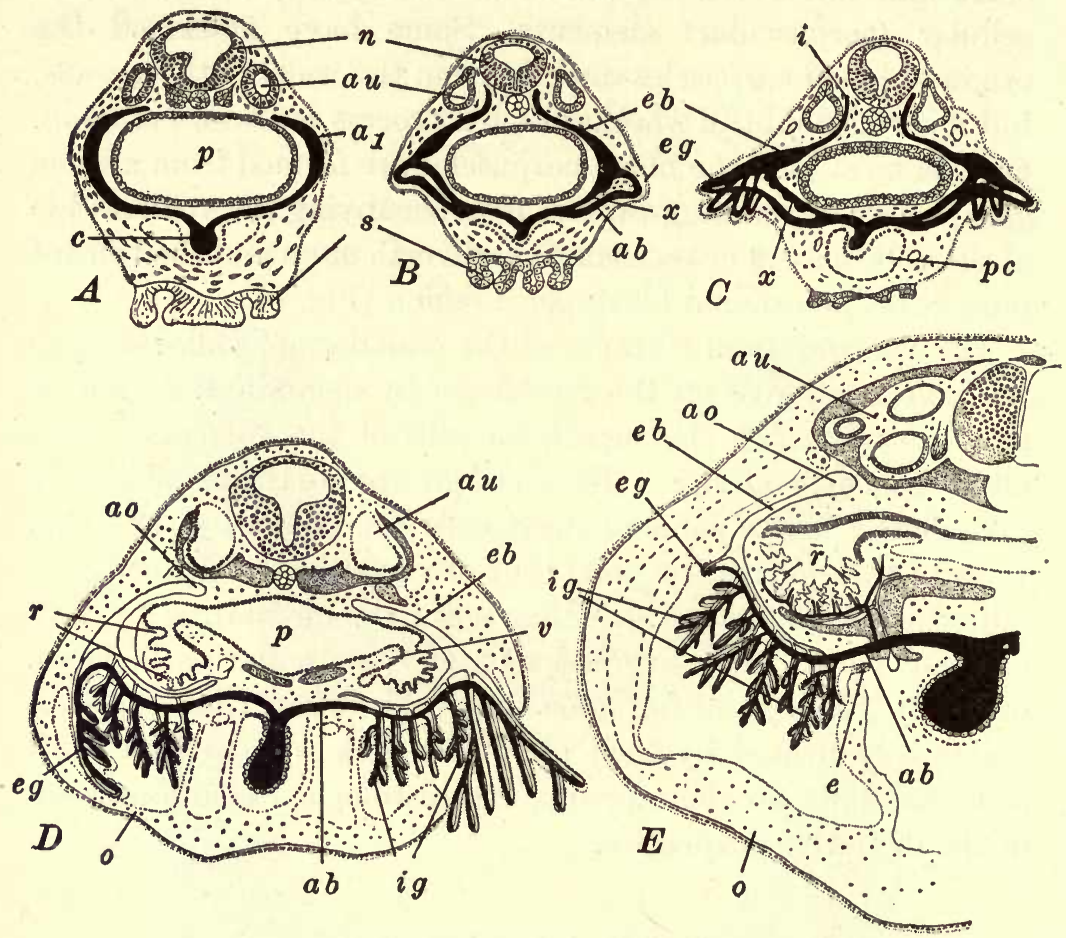

Fig. 64.-Sections through the branchial region of tadpoles of $R$. esculenta, showing the development of the gills and the history of the aortic arches. After Maurer. A. $4 \mathrm{~mm}$. larva showing the continuous first branchial aortic arch. B. $5 \mathrm{~mm}$. larva showing the anastomosis between the afferent and efferent portions of the aortic arch. C. $6 \mathrm{~mm}$. larva with vascular loops in the external gills. D. $13 \mathrm{~mm}$. larva. On the left the opercular cavity is closed and the external gill is beginning to atrophy, while on the right this cavity is still open and the external gill well developed and projecting through the opercular opening. $E .17 \mathrm{~mm}$. larva. Vessels of the second branchial arch. External gill represented only by a minute pigmented vestige. $a_{1}$, First branchial aortic arch; $a b$, afferent branchial artery; $a o$, root of lateral dorsal aorta; au, auditory organ; $c$, conus arteriosus; $e$, epithelioid body; $e b$, efferent branchial artery; $e g$, external gill; $i$, internal (anterior) carotid artery; $i g$, internal gills; $n$, nerve cord; $o$, operculum; $p$, pharynx; $p c$, pericardial cavity; $r$, gill rakers; $s$, oral sucker; $v$, velar plate; $x$, anastomosis between afferent and efferent branchial arteries.

arch a lacunar vascular space appears (about $4.5 \mathrm{~mm}$.) which early connects ventrally with the truncus arteriosus, and dor- 
sally with the lateral dorsal aorta, forming thus, before the gills appear, a continuous aortic arch in each branchial arch (Fig. 64, A). There are, therefore, in the branchial arches, four pairs of aortic arches; these are really the third to sixth pairs of aortic arches, the first and second being formed in the mandibular and hyoid visceral arches.

When the external gills appear an additional vessel develops dorso-laterally to the aortic arch, along the base of the gill, forming its supply. This vessel opens out of the ventral end
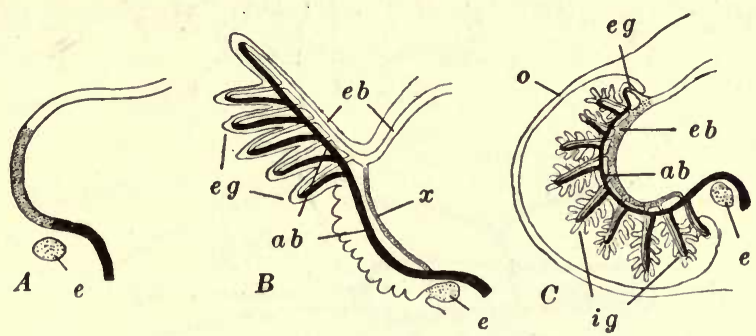

Fig. 65.-Diagrams of the aortic arch of the adult frog and tadpole. After Maurer. A. The continuous aortic arch of the adult; showing the parts corresponding with the larval vessels. B. First external gill and associated vessels in young tadpole. $C$. Internal gill and associated vessels in the tadpole after the disappearance of the external gills. $a b$, Afferent branchial artery; $e$, epithelioid body; $e b$, efferent branchial artery; $e g$, external gill; $i g$, internal gill; $o$, operculum; ' $x$, direct anastomosis between afferent and efferent branchial arteries.

of the aortic arch and joins it again toward its upper end (Fig. $64, B$ ); the lower end of the aortic arch may then be termed the afferent branchial artery, its dorsal end the efferent branchial artery. The small vessels of the external gills form loops connecting the dorsal and ventral parts of this second vessel. Then as the external gills disappear and the internal gills develop on the branchial arches, the direct ventral connection between the original aortic arch and the second vessel, becomes interrupted by the disappearance of a part of the aortic arch, and the vascular networks of the internal gills connect the two vessels. In this way the original aortic arch becomes almost entirely the efferent branchial artery, while the second vessel serves as the afferent branchial artery (Figs. 64, 65). 
When the internal gills disappear, during metamorphosis, the lower end of the efferent branchial artery (original aortic arch) reacquires a direct connection with the afferent branchial artery

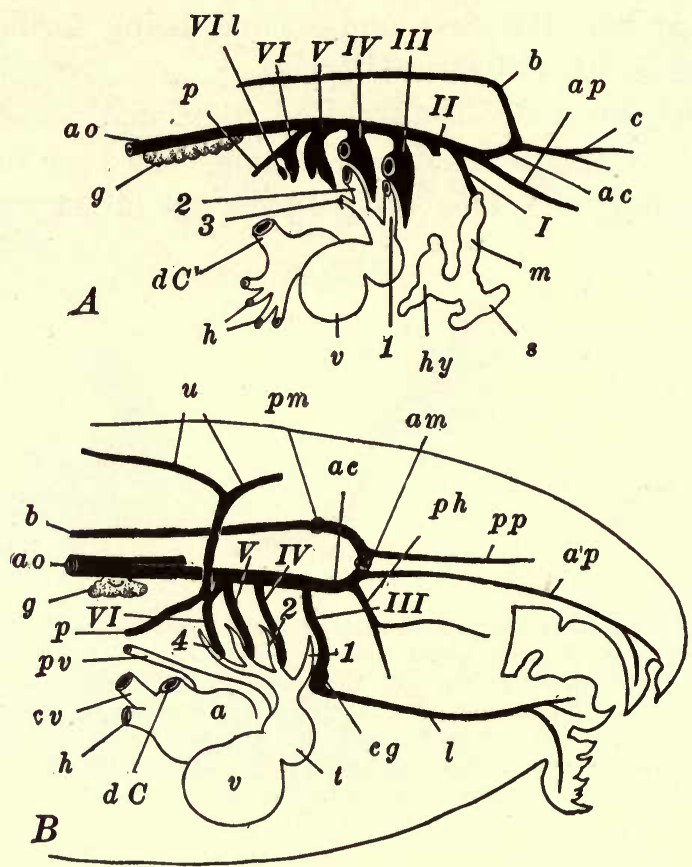

Fig. 66.-Diagrams of the branchial blood vessels in frog larvæ. After Marshall. A. Of a $7 \mathrm{~mm}$. larva (shortly after hatching). The vessels supplying the external gills are removed, only their roots being indicated. $B$. Of a $12 \mathrm{~mm}$. tadpole. The vascular loops in the gills are omitted. $a$, Auricle; ac, anterior (internal) carotid artery; $a m$, anterior commissural artery; $a o$, dorsal aorta; $a p$, anterior palatine artery; $b$, basilar artery; $c$, anterior cerebral artery; $c g$, carotid gland; $c v$, posterior (inferior) vena cava; $d C$, ductus Cuvieri; $g$, pronephric glomerulus; $h$, hepatic veins; $h y$, hyoidean vein; $l$, lingual artery; $m$, mandibular vein; $p$, pulmonary artery; $p h$, pharyngeal artery; $p m$, origin of posterior oommissural artery; $p p$, posterior palatine artery; $p v$, pulmonary vein; $s$, vein of oral sucker; $t$, truncus arteriosus; $u$, cutaneous artery; $v$, ventricle; $1-4$, first to fourth afferent branchial arteries; $I, I I$, efferent arteries of the mandibular and hyoid arches; $I I I-V I$, first to fourth efferent branchial arteries; $V I l$, lacunar vessel of the fourth branchial arch.

and the blood again passes directly from the truncus to the dorsal aorta (Fig. 65, $A$ ). This connection enlarges as the gill capillaries diminish, and finally these direct paths remain as the only vessels of the branchial arches. 
In the fourth branchial arch, which lacks external gills, the history is essentially modified only to the extent of the omission of the vessels related to these structures. The vessels of this arch appear considerably later than in the anterior arches.

The development of the vessels of the mandibular and hyoid arches seems to be quite variable among the different species of Rana. The most consistent account is that of Marshall and Bles, of $R$. temporaria. Here, in tadpoles of about $5 \mathrm{~mm}$. a lacunar vessel representing the aortic arch (the second of the whole series) appears in the hyoid arch, and a small outgrowth of the lateral dorsal aorta extends toward it, but never actually joins it, disappearing about the time the mouth opens (Fig. 66, $A$ ). At hatching a small outgrowth of the truncus arteriosus may be seen extending into the lower end of the hyoid arch; this has a very brief duration. At the same time the vestige of the aortic arch has divided into dorsal and ventral portions; of these, the former soon disappears while the latter, now known as the hyoidean vein, connects with a large vascular sinus in the region of the oral sucker.

The vessels of the mandibular arch appear shortly before hatching; these are, a lacunar vessel in the lower part of the arch, representing the original aortic arch, and a small outgrowth of the lateral dorsal aorta into the dorsal part. Soon these unite and also join the hyoidean vein. After the mouth opens, the outgrowth from the lateral dorsal aorta separates from the other vessels and grows forward as the pharyngeal artery, while the hyoidean vein disappears with the oral sucker (Fig. 66, B).

The continuations of the lateral dorsal aortæ into the head form the roots of the anterior or internal carotid arteries, whose numerous branches supply the organs of the whole dorsal part of the head; the internal carotids become connected by two transverse commissural arteries passing anterior and posterior to the infundibulum (Fig. 66). The ventral part of the head is supplied by the lingual or external carotid arteries; these vessels appear, some time before the opening of the mouth, as a pair of sinuses in the floor of the buccal cavity and 
pharynx. About the time the mouth opens, they extend backward and connect with the ventral ends of the efferent branchial arteries of the first branchial arch, in the region where the carotid gland (see above) develops later.

About the time of hatching, outgrowths of the dorsal aorta, just back of the pharyngeal region, extend laterally into the region of the pronephros or head kidney (see below). These

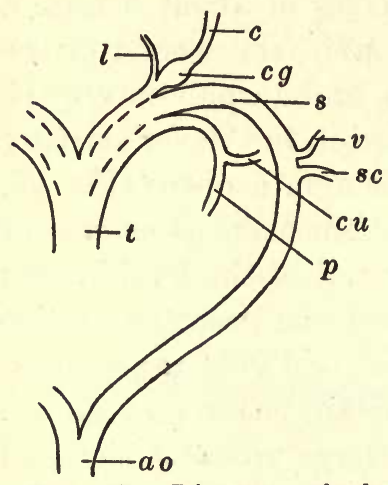

FIG. 67.-Diagram of the aortic arches and their chief branches in an adult frog. Ventral view. $a o$, Dorsal aorta; $c$, carotid artery; $c g$, carotid gland; $c u$, cutaneous artery; $l$, lingual artery; $p$, pulmonary artery; $s$, systemic arch; $s c$, subclavian artery; $t$, truncus arteriosus; $v$, vertebral artery.

later become very large and form the vascular glomi of this kidney (Figs. 66, 72); traces of these remain, long after the pronephros itself has disappeared.

During metamorphosis, as the gills disappear, the branchial blood vessels are considerably modified. We have seen that a continuous aortic arch is reëstablished in each of the four branchial arches by the fusion of the afferent and efferent arteries. The first branchial aortic arch (third of the whole series) remains as the root of the anterior carotid artery, and is known as the carotid arch (Fig. 67). The lateral dorsal aorta between the first and second (third and fourth) aortic arches, becomes reduced to a solid strand of connective tissue, and the second (fourth) pair of aortic arches consequently become the roots of the dorsal aorta, and are known as the systemic arches. The third (fifth) aortic arch, after becoming a solid strand of tissue, disappears entirely. The fourth (sixth) arch remains as the root of the pulmonary and cutaneous arteries of the adult, and is known as the pulmocutaneous arch.

The pulmonary arteries appear just after hatching as small outgrowths from the upper ends of the efferent branchial arteries of the fourth branchial arch. (Figs. 66, 67). They extend backward to the lung rudiments, which they reach 
before the vessels of this arch have acquired a connection with the truncus arteriosus. Later the cutaneous arteries leave the pulmonary, and extend dorsally, spreading over the skin of the back and sides. Some time after metamorphosis that part of the aortic arch between the origin of these vessels and the lateral dorsal aorta, known as the ductus Botalli, slowly atrophies and becomes a solid strand.

Longitudinal septa appear in the truncus arteriosus, dividing it into three channels. One of these leads to the carotid arches, and in the heart receives blood from the left side, i.e., fully aërated blood which has been received through the left auricle from the lungs and skin. Another channel leads from the right side of the heart and carries the venous blood to the pulmo-cutaneous arches. The remaining channel connects with the systemic arches; in the heart its closer connection is with the left side.

\section{The Venous System}

The large veins of the yolk-mass are in reality the first parts of the vascular system differentiated. These are the paired, but asymmetrical, omphalomesenteric veins (known also as the vitello-intestinal or the vitelline veins) arising on the ventral surface of the yolk, in the region of the blood islands described above, and passing along the lateral surfaces of the yolk and liver diverticulum, to enter the sinus venosus. This posterior chamber of the heart appears to be formed chiefly by the fusion of these large veins, although it receives later a pair of large veins, the ductus Cuvieri or Cuvierian sinuses, coming from the body wall opposite the sinus venosus. As the liver develops, both of the vitello-intestinal veins break up into capillary nets within its substance, and the parts of the two veins between the liver and the heart fuse into a single hepatic vein. Posteriorly from the liver the right vein seems to disappear as a definite channel, while the left partly remains as the root of the definitive hepatic portal vein, ultimately receiving branches from the digestive tract and its appendages. 
The ductâs Cuvieri pass from the sinus venosus obliquely upward in a nearly vertical plane, to the body wall where they divide, passing thence anteriorly and posteriorly. The anterior branches are the anterior cardinal veins. These continue forward as the superior jugular veins, receiving blood from the brain and dorsal parts of the head, and from the region of the eye and ear (facial branch). The inferior jugular veins, coming from the region of the mouth, sucker, and ventral surface of the head, open into the roots of the ductûs Cuvieri, just before these open into the sinus venosus.

The posterior branches of the ductus Cuvieri are the posterior cardinal veins. These are primarily the veins of the body wall and the excretory systems. They pass posteriorly through the pronephric region, and thence along the medial side of the pronephric ducts (see below) (Figs. 68, 72, 76) receiving blood from the veins of the body wall (segmental veins). A median caudal vein, passing forward through the entire length of the tail, just ventral to the dorsal aorta or caudal artery, upon reaching the body cavity divides above the cloacal regior, and its branches connect directly with the extremities of the posterior cardinal veins, so that these receive blood from the tail also. In the region of the head kidney or pronephros (see below) each of these veins forms a large sinusoidal system among the tubules of this excretory organ. Shortly after the opening of the mouth, as the definitive kidney or mesonephros (see below) commences to develop, the arrangement of the posterior cardinal veins is profoundly modified. The hinder parts of the two veins begin to fuse together at about $15 \mathrm{~mm}$., and ultimately form a median vessel, which may be termed the median cardinal vein (Fig. 68). Anteriorly this vessel effects a new and direct connection with the sinus venosus.

This connection is brought about by the development of the posterior or inferior vena cava or postcaval vein. This important vessel is first indicated by the marking out of a definite pathway in the vessels of the dorsal side of the liver, vessels which are branches of the left vitello-intestinal vein (Shore). This vessel then leaves the surface of the liver and passes through 
the suspensory fold of the liver (mesohepaticum) to the right posterior cardinal vein, with which it connects, just in front of the beginning of the median cardinal vein (i.e., immediately

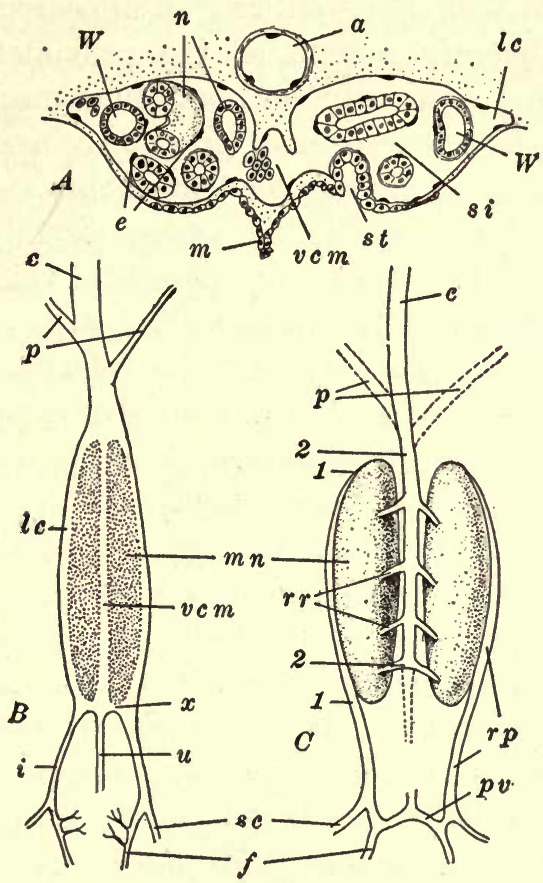

FIg. 68.-The development of the posterior part of the venous system in the frog. After Shore. A. Portion of a transverse section through the posterior mesonephric region of an $18 \mathrm{~mm}$. tadpole. $B$. Diagram of the veins of a 25-30 $\mathrm{mm}$. tadpole. $C$. Diagram of the veins of the adult frog. $a$, Dorsal aorta; $c$, vena cava; $e$, nuclei of the endothelial lining of the mesopheric sinus, continuous with the vascular endothelium; $f$, femoral vein; $i$, iliac vein; $l c$, lateral mesonephric channel of the posterior cardinal vein; $m$, mesentery; $m n$, mesonephros; $n$, mesonephric tubules; $p$, posterior cardinal veins (in $C$ showing their original location); $p v$, pelvic vein; $r p$, renal-portal vein; $r r$, revehent renal veins; $s c$, sciatic vein; st, nephrostome; $u$, caudal vein; $v c m$, median mesonephric channel of the posterior cardinal vein; $W$, Wolffian duct; $x$, connection between caudal vein and the lateral mesonephric channels; $1-1$, part of the renal-portal vein formed from the lateral channel of the posterior cardinal; 2-2, part of the renalportal vein formed from the median channel of the posterior cardinal vein.

posterior to the pronephric region). This new channel enlarges rapidly and ultimately becomes the largest blood vessel of the body. Through the liver it passes directly to the sinus 
venosus, and the hepatic vein comes to open directly into it instead of into the sinus venosus.

As the pronephroi degenerate the pronephric sections of the posterior cardinal veins diminish also, and by the time of metamorphosis they have entirely disappeared. The ductûs Cuvieri consqeuently remain as the proximal parts of the anterior cardinal veins only, and are sometimes known as the anterior; or superior venœ cava or precaval veins. As the result of these changes, all of the blood from the posterior parts of the body wall, and from the tail, passes directly to the heart through the median cardinal and postcaval veins (Fig. 68).

The development of the mesonephroi, which begins as the pronephroi diminish, entirely alters the relations of the median cardinal vein. On each side the tubular components of the mesonephros, whose development will be described below, push into this vein, dividing it roughly into three parallel channels, one median and two lateral (Fig 68). The caudal vein remains for a time, opening directly into the posterior end of the median channel, while iliac veins, coming from the hindlegs, open into the lateral channels. The caudal vein disappears later, of course, while the iliac veins remain as the chief vessels leading to the mesonephric region.

The arrangement of the vessels in the adult may now be understood easily. The iliac veins and lateral channels of the median cardinal vein, with which they are continuous, become the afferent or advehent mesonephric veins or the renal portal veins (Fig. 68, C). The small veins from the posterior body wall (posterior vertebral veins) open into the renal portal veins. The vascular spaces of the mesonephros remain connected, by a series of short pathways-the revehent mesonephric or renal veins, with the median channel of the median cardinal vein, which therefore remains alone as the posterior continuation of the postcaval vein.

Summarizing we may say that the inferior vena cava or postcaval vein is composed of four different elements. An hepatic section derived from the left vitelline vein, is followed by a short section which represents a new structure; next comes a 
very short region derived from the original right posterior cardinal vein, and finally, the entire posterior section is formed from the median channel, derived from the fused right and left posterior cardinal veins. The renal portal veins consist of two sections: a posterior part is derived from the iliac vein, and an anterior part is formed from the lateral channel of the median cardinal vein, which represents the hinder part of the original posterior cardinal veins.

A pair of lateral veins develops late, in the ventral abdominal wall, for a time opening directly into the sinus venosus. Posteriorly these connect with the iliac veins, and then continue, fusing together medially. The anterior portions of these vessels then lose their connection with the sinus venosus and the anterior part of the right vessel disappears entirely, the left vein forming a new connection with the hepatic portal vein, when it is known as the anterior abdominal vein.

The rudiments of the pulmonary veins are indicated very early (about $6 \mathrm{~mm}$.) as proliferations of the endothelium on the dorsal side of the sinus venosus (Federow). These cells later form a definite tube opening proximally into the left side of the auricle, and distally leaving the wall of the sinus venosus and passing dorsally to the rudiments of the lungs. At the base of the lung it bifurcates, each branch passing along the medio-ventral side of each lung rudiment. Later, when the lungs become functional the pulmonary veins discharge into the left auricle.

\section{The Lymphatic System and Spleen}

The first indications of this system appear shortly before hatching. In the larva of $6.5 \mathrm{~mm}$. (Knower) a single pair of anterior lymph hearts is present, as small sac-like outgrowths of a pair (usually the fourth) of intersegmental veins (i.e., veins running between the fourth and fifth myotomes, and opening into the posterior cardinal veins at the posterior limit of the pronephros). These hearts lie between the peritoneum and the integument, below the level of the myotomes. The endothelial wall of the lymph hearts is continuous with that 
of the veins. Outside the endothelium is a syncytial layer or network of striated muscle fibers, which commence rhythmic contraction about the time the mouth opens.

Shortly after hatching (7.5-8 mm.) two lymphatic vessels may be seen passing anteriorly and posteriorly from each heart, along the lateral nerve, in the connective tissue beneath the integument. The anterior vessel extends forward into the head region, while the posterior vessel extends along the sides of the trunk for a considerable distance. The openings of these
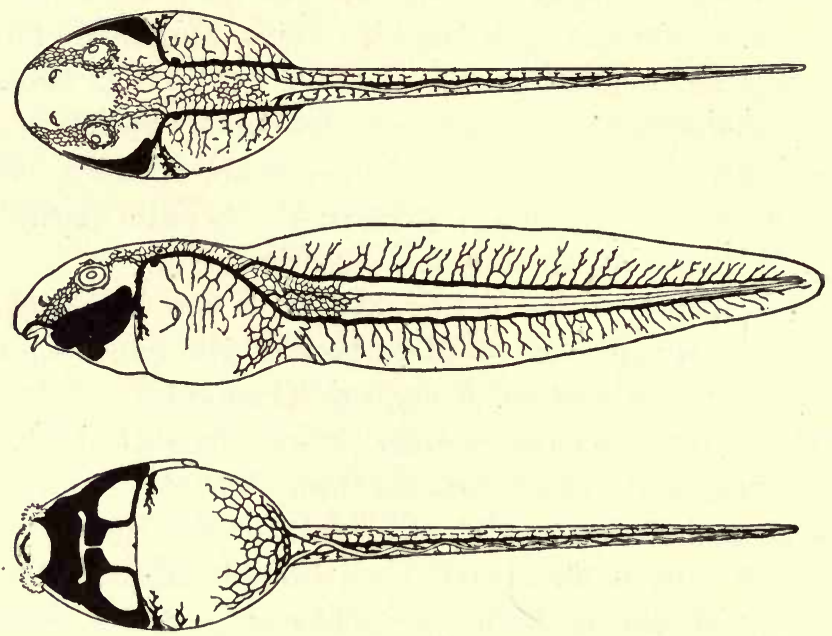

Fig. 69.-Dorsal, lateral and ventral views of the lymphatics in a $26 \mathrm{~mm}$. tadpole of $R$. temporaria. From Hoyer. For description see text.

vessels into the lymph hearts, and of the hearts into the veins, are guarded by long valves. These vessels are formed as blind tubular outgrowths from the endothelium of the lymph heart; they grow rapidly and give off a rich network of fine lymphatic capillaries and vessels which spread generally among the other tissues and especially just beneath the skin.

In the older tadpole of about $26 \mathrm{~mm}$. (Hoyer) the lymphatic system is quite extensively developed. At this time the anterior vessel runs forward and downward, connecting with a large lymph sinus around the mouth and heart and branchial region (Fig. 69), while the posterior trunk passes to the base 
of the tail, where it divides into dorsal and ventral branches. The dorsal and ventral branches of each side then unite forming two large vessels which extend through the tail, lying above and below the myotomes (Fig. 69).

The large subcutaneous lymph sacs, so characteristic of both the tadpole and the adult frog, are formed very early from the network growing out from these vessels. The small lymphatics in the subcutaneous connective tissue branch abundantly and anastomose freely, forming a rich network; their walls then disappear and the wide lymph sacs are left, still connected with the lymph hearts by way of the lateral trunks described.

The thoracic ducts also appear to arise from the anterior lymph hearts, as a pair of outgrowths which extend posteriorly, between the dorsal aorta and the posterior cardinal veins. When the hind-legs appear, from one to three pairs of posterior lymph hearts develop in connection with the intersegmental veins of the region, in much the same way that the anterior hearts developed. They open for a time into the posterior cardinal veins, and later, therefore, into the renal-portal veins, whether by the intersegmental veins or by the ischiadic branch is not clear.

The spleen is first indicated in larvæ of about $10 \mathrm{~mm}$. by a collection of mesenchymal lymphoid cells in the mesentery, around the mesenteric artery, just dorsal and posterior to the stomach (Radford). These cells multiply and in a $15 \mathrm{~mm}$. larva form a definite projection from the mesentery, covered therefore, by a cœlomic or peritoneal epithelium. During this period of enlargement, the spleen appears to receive some cells which wander out from the intestinal epithelium. Later this organ becomes very vascular and in the 25-27 $\mathrm{mm}$. larva it forms a definite ovoid body, in the position where it is found in the adult.

\section{The Formation of the Septum Transversum}

We have seen that the pericardial cavity is formed as a median ventral section of the cœlom. This remains completely 
closed anteriorly and laterally, but posteriorly it is at first directly, though incompletely, open into the general body cavity or peritoneal cavity. During the early stages the liver forms the hinder wall of the pericardial cavity, medially, but it still remains open postero-laterally, either side of the liver, and medio-ventrally, below it. When the ductûs Cuvieri are formed, passing from body wall to sinus venosus, they traverse this region of the cœlom and aid in establishing the hinder wall of the pericardial cavity. Incomplete peritoneal folds from the body wall accompany the ductûs Cuvieri from the body wall to the heart; these are known as the lateral mesocardia. Dorsally the lateral mesocardia remain incomplete for a long time, but ventrally they gradually extend to the body wall entirely across the colom and form a complete ventral partition, between pericardial and peritoneal cavities. The transverse peritoneal fold thus formed is the pericardio-peritoneal septum, or septum transversum. Its median ventral portion appears to be formed by the peritoneum originally covering the anterior face of the liver; this separates from the liver and becomes added to the septum transversum. On the right side it becomes continuous with the posteriorly directed suspensory fold of the liver (mesohepaticum). Not until after metamorphosis is the septum transversum fully united dorsally with the dorsal mesentery, and the separation of the pericardial and peritoneal cavities entirely completed.

\section{THE URINOGENITAL SYSTEM}

The excretory and reproductive systems develop independently and at widely different times, but in their definitive state they form a complex, in which structures orignally excretory, have assumed morphological and functional relations with the reproductive system, while other parts function, at different times, both as excretory and reproductive organs.

\section{The Excretory System}

A functional excretory system is already established, before the rudiments of the reproductive system have more than made 
their appearance. This is the embryonic or larval pronephric system or larval kidney, known also as the head kidney. This kidney is limited to early larval life and is replaced during the tadpole stage by an excretory organ which remains the definitive kidney of the adult; this is the mesonephros, which, it should be noted, retains as its efferent duct, the duct of the original pronephros. We have then to describe the development of the pronephros and the pronephric duct, the development of the mesonephros, and the disappearance of the pronephros. We shall see how, during the later stages, the arrangement of these parts is complicated by the relation between the excretory and the reproductive systems. Since these organs are symmetrically paired we may describe only the organs of one side.

\section{A. THE PRONEPHROS AND THE PRONEPHRIC DUCT}

In a preceding section we described the position and relations of that part of the mesodermal somite known as the intermediate cell mass or nephrotome, and said that this formed a rudiment of the pronephric system. The first indication of the pronephros is seen before the nephrotomal region has separated from either the myotome or the lateral plate, as a solid thickening of the somatic mesoderm anteriorly (Fig. 70, B). This thickening, which begins before the cavity (cœlom) of the lateral plate and nephrotome appears, gradually extends posteriorly along the nephrotomal level, and finally reaches to the region opposite the cloaca, although this is not until the anterior part of the rudiment has become quite markedly differentiated. Anteriorly, in the region of somites 2-4, the pronephros itself is formed, while the posterior remainder forms the pronephric or segmental duct. As the thickening of the anterior pronephric region becomes marked, the rudiment here begins to extend ventro-laterally, like an epaulet, over the outer surface of the dorsal margin of the lateral plate (Fig. 70, $C$ ). Spaces appear in this cell mass, about the same time that the colom appears in the lateral plate; in the lateral or distal part 
of the thickening a continuous space is formed, from which there extend medially or proximally, toward the lateral plate, three small irregular canals which open into the upper margin

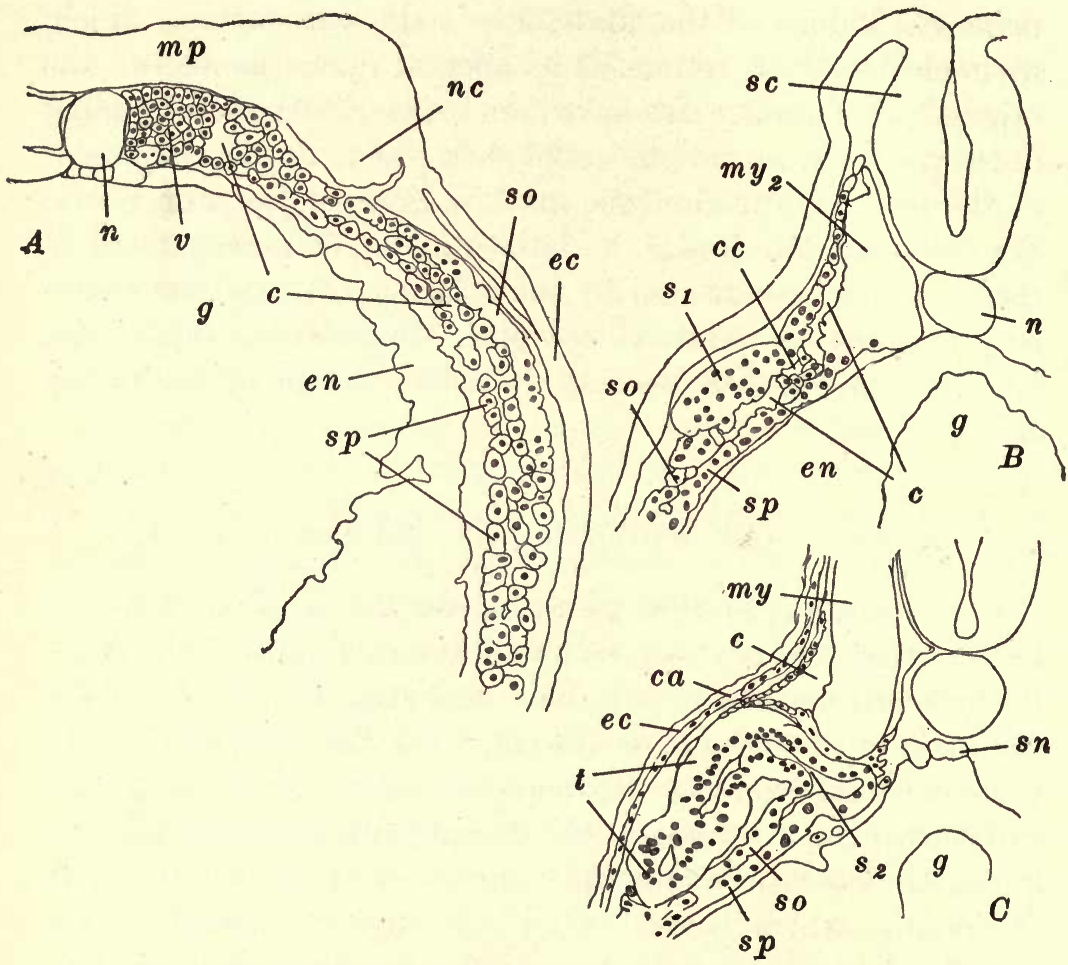

FIG. 70.- Sections through frog embryos (R. sylvatica) illustrating the formation of the pronephros. After Field. A. Through the anterior body region of an embryo at the commencement of its elongation. $B$. Through the anterior end of the pronephric rudiment of an embryo in which the neural folds are just closed together. $C$. Through the second nephrostome of an embryo of about $3.5 \mathrm{~mm}$. c, Cœlom; $c a$, rudiment of pronephric capusle; $c c$, communicating canal; $e c$, ectoderm; $e n$, endoderm; $g$, gut cavity; $m p$, medullary plate; $m y$, myotome; $m y_{2}$, second myotome; $n$, notochord; $n c$, rudiment of neural crest; $s_{1}, s_{2}$, first and second pronephric nephrostomes; $s c$, spinal cord; $s n$, subnotochordal rod (hypochorda); so, somatic layer of mesoderm (in $A$ the reference line points to the rudiment of the pronephros); $s p$, splanchnic layer of mesoderm; $t$, pronephric tubule; $v$, vertebral plate of mesoderm.

of the cœlom of the lateral plate opposite the middle of each of the three pronephric somites (Field). From the posterior end of this peripheral space or common trunk, the cavity leads 
directly into the cavity of the pronephric duct, which is continuous posteriorly with this portion of the pronephric rudiment.

There are now established the primary elements of the pronephros. The three short canals with their cellular walls are the rudiments of the three pronephric tubules, and their openings into the cœlom are the nephrostomes. The tubules and also the proximal part of the pronephric duct, now elongate rapidly, and as a consequence become thrown into complicated loops and folds (Figs. 71, 72) forming a conspicuous mass whose position is marked externally by a slight elevation. The pronephric duct gradually acquires a lumen throughout its extent; at about 4.5 $\mathrm{mm}$. the duct effects a connection with the wall of the cloaca, and its cavity then opens into the cloacal chamber (Fig. 54). The nephrostomes become lined with large cilia which produce a current out of the colom, passing by way of the pronephric duct to
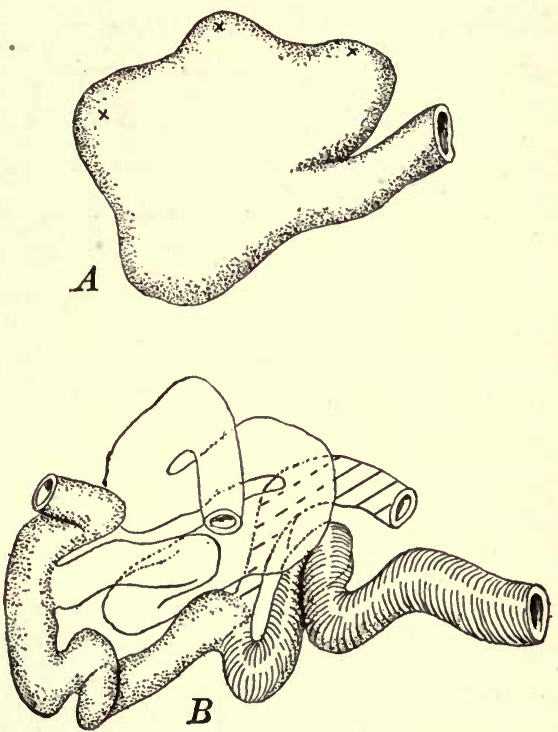

Fig. 71.- Total views of the pronephros of the frog (R. sylvatica). After Field. $A$. Right pronephros of an embryo of about 3.5 $\mathrm{mm}$. The crosses mark the location of the nephrostomes. $B$. Right pronephros of a larva of about $6 \mathrm{~mm}$. First tubule dotted; second white; third obliquely ruled; pronephric (segmental) duct shaded with lines. the cloaca.

Meanwhile the pronephros acquires a rich vascular supply, both arterial and venous. It will be remembered that the posterior cardinal veins are passing along the pronephric ducts, and in the region of the pronephros itself these veins become greatly enlarged. As the pronephric tubules elongate, they push up into the posterior cardinal sinus, which is ultimately 
nearly filled by them. Each tubule carries around it a reflected layer of the thin vascular wall and so is completely bathed in the venous stream (Fig. 72).
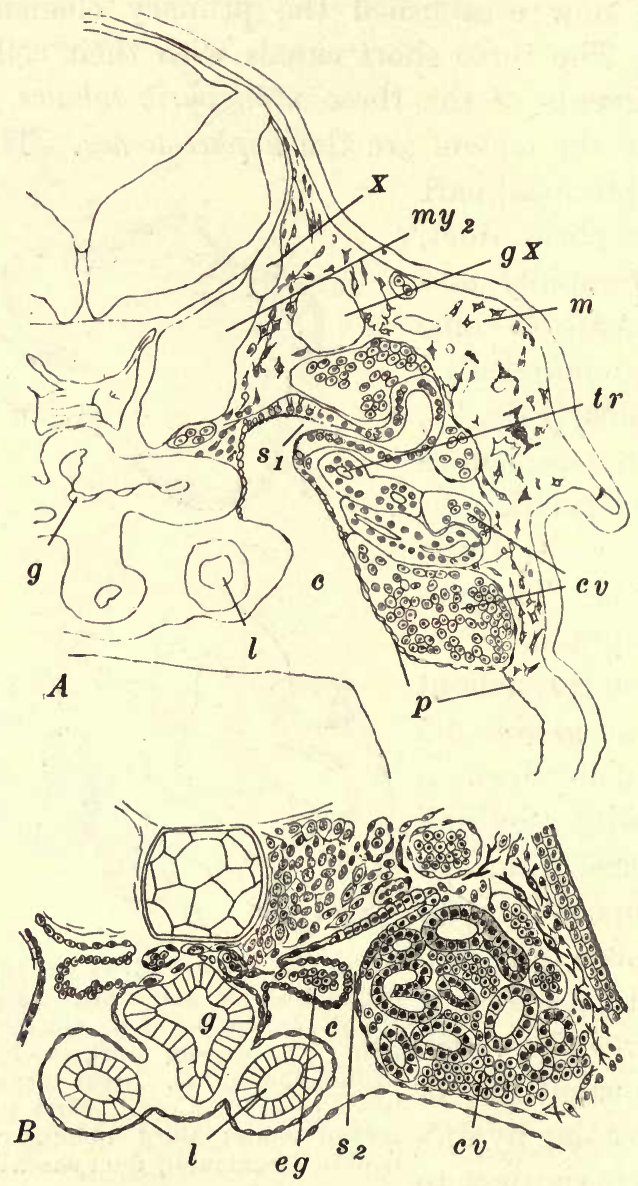

Fig. 72.- Sections through frog larvæ illustrating the later development of the pronephros. A. Through the first nephrostome of a larva of $R$. sylvatica of about $8 \mathrm{~mm}$., with prominent external gills. After Field. $B$. Through the region of the second nephrostome of a $12 \mathrm{~mm}$. larva of $R$. temporaria. After Fürbringer. $c$, Cœlom; $c v$, sinuses of posterior cardinal vein; $e g$, external glomerulus; $g$, gut cavity; $g X$, ganglion nodosum (part of the ganglion of the vagus nerve); $l$, lung; $m$, mesenchyme; $m y_{2}$, second myotome; $p$, peritoneum; $s_{1}, s_{2}$, first and second pronephric nephrostomes; $t r$, common trunk; $X$, root of vagus nerve.

At the same time an arterial supply is derived from the dorsal aorta. This is the pronephric glomus already mentioned. 
The first indication of this is a horizontal fold of the splanchnic or medial wall of the dorsal cœlom just opposite the second nephrostome. This fold appears at about $4.5 \mathrm{~mm}$. and its development is in general parallel with that of the pronephros. It soon extends the entire length of the pronephric region and becomes considerably elevated, projecting freely into the cœlom opposite the nephrostomes (Fig. 72). In it vascular spaces soon appear, some of which form the long convoluted vessels of the glomus proper, while others go to form a vessel, connecting with a branch of the dorsal aorta, which is apparently one of the segmental arteries passing ventrally from the aorta. Later this section of the body cavity is cut off, as the pronephric chamber, by the lateral projection of the lung (Fig. 72, B) which carries a fold of peritoneum across to the peritoneum covering the pronephros, with which it fuses for a short distance. This pronephric chamber remains open into the body cavity both anteriorly and posteriorly to the lung region.

A definite pronephric capsule is formed early from two sources. The ventro-lateral walls of the myotomes, which we have seen give rise elsewhere to mesenchyme, here, in the pronephric region, evaginate over the dorsal and lateral surfaces of the pronephros, and meet folds coming up from the somatic layer of the lateral plate (about $6 \mathrm{~mm}$.). These folds form a definite connective tissue layer enclosing the pronephros and the pronephric sinus of the posterior cardinal vein.

The pronephros reaches its full development in the larva of about $12 \mathrm{~mm}$., when it consists of a large mass composed of the coiled proximal end of the pronephric duct and the three tubules, each of which has acquired blind tubular outgrowths, the whole mass interpenetrated by the vascular sinus of the posterior cardinal vein. In the larva of about $20 \mathrm{~mm}$. the pronephros commences to degenerate. At this time the pronephric duct becomes closed just back of the pronephros; the tubules become variously dilated and constricted, breaking into irregular sections and gradually disappearing (Fig. 73, C). The nephrostomes approach one another and finally meet, opening into a common cavity known as the common nephro- 
stome, which is then closed, and the nephrostomes thus cut off from the body cavity (Fig. 73, C). The glomus shrinks, and by the time of metamorphosis only a few scattered traces of the pronephros remain, although the glomus remains indicated for some months after metamorphosis. The pronephric ducts do not take part in this process of degeneration, posterior to the pronephric region; they remain, closed anteriorly. The disappearance of the pronephros is correlated with the development of the second excretory system, the mesonephros, to the formation of which we shall now turn.

\section{B. THE MESONEPHROS OR WOLFFIAN BODY}

This begins to develop in tadpoles of 8-10 $\mathrm{mm}$. Its rudiment is formed by the nephrotomes of the seventh to twelfth somites, and it is consequently both somatic and splanchnic in origin. The nephrotomes of these segments fuse into a continuous longitudinal strip of irregularly arranged cells, lying between the pronephric duct and the dorsal aorta, along the posterior cardinal vein. In this mass, cell groupings appear, forming definite swellings of the cord. These are the rudiments of the mesonephric vesicles; they are not strictly metameric, but are somewhat more numerous than the mesodermal segments.

All of these rudiments have essentially the same history (Hall). First each becomes divided into a large ventral chamber and a small dorsal one; the larger chamber is a primary mesonephric unit, the smaller a secondary mesonephric unit (Fig. $73, B)$. The secondary units divide similarly, though much later, into secondary units proper and tertiary mesonephric units. All three series of units develop similarly though successively, and we shall therefore describe only the history of the primary series.

From the vesicular primary unit two outgrowths are formed (Fig. 74). One, known as the inner tubule, extends dorsolaterally to the pronephric duct and opens into it. The other, the outer tubule, grows ventro-medially to the peritoneum with which it fuses and opens into the body cavity. The 
connections of the inner tubules with the pronephric duct convert this into the mesonephric or Wolffian duct, which remains as the ureter of the mesonephros or definitive kidney. The inner tubules elongate and become coiled, forming the tubular portion of the later kidney.

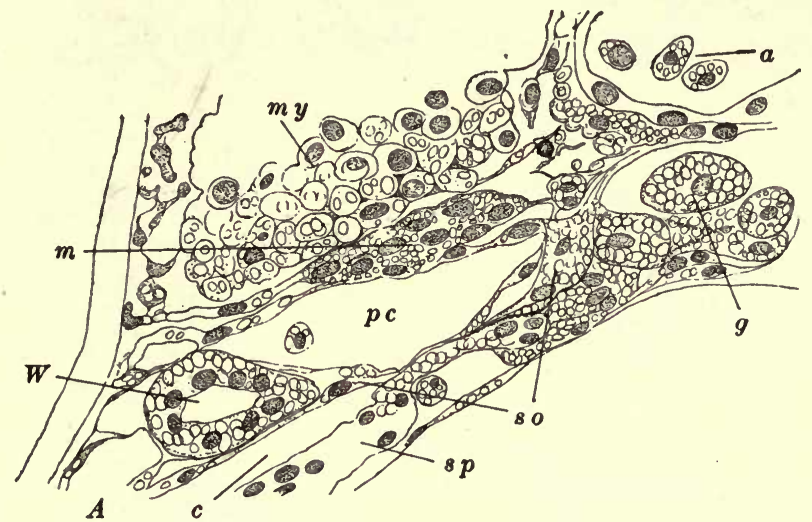

A
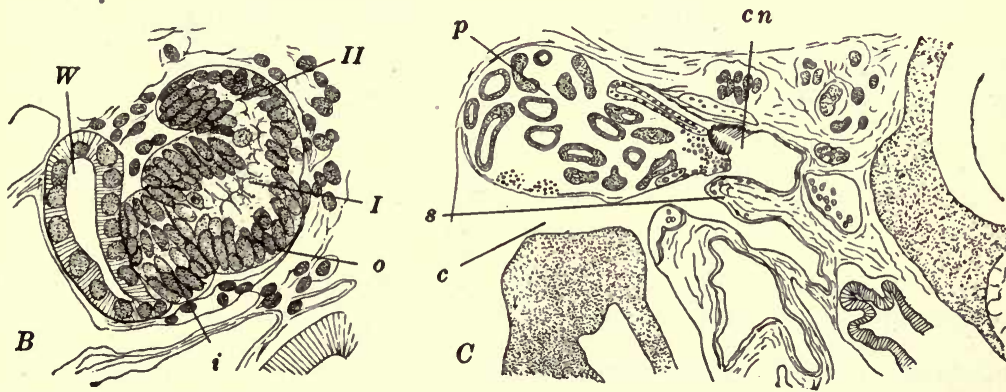

Fra. 73.- Sections through the developing mesonephros and the common nephrostome of $R$. sylvatica. After Hall. A. Section through the eighth somite of an $8.5 \mathrm{~mm}$. larva. B. Section through the mesonephric rudiment of a $25 \mathrm{~mm}$. larva. $C$. Section through the common nephrostome of a $25 \mathrm{~mm}$. larva. $a$, Dorsal aorta; $c$, colom; $c n$, common nephrostome; $g$, germ cell; $i$, inner tubule; $m$, mesonephric rudiment; $m y$, myotome; $o$, outer tubule; $p$, remains of pronephros; $p c$, posterior cardinal vein; $s$, shelf cutting off the common nephrostome from the remainder of the cœlom; so, somatic mesoderm; $s p$, splanchnic mesoderm; $W$. Wolffian duct; $I$, primary mesonephric tubule; $I I$, secondary mesonephric tubule.

Subsequently the outer tubules have a rather unusual history in the frog. From the proximal portion an outgrowth appears, which forms the capsule (Bowman's capsule) around the gloo 
merulus associated with each tubule, the two forming the Malpighian body. Each glomerulus is connected with a small

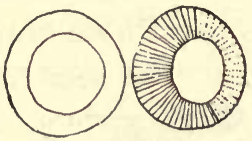

$A$

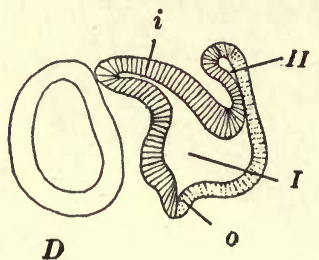

$\boldsymbol{D}$

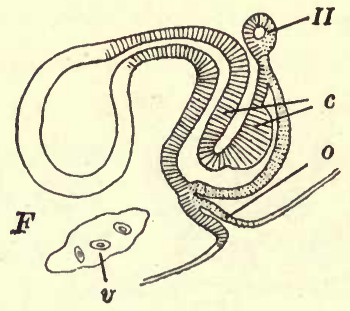

$B$
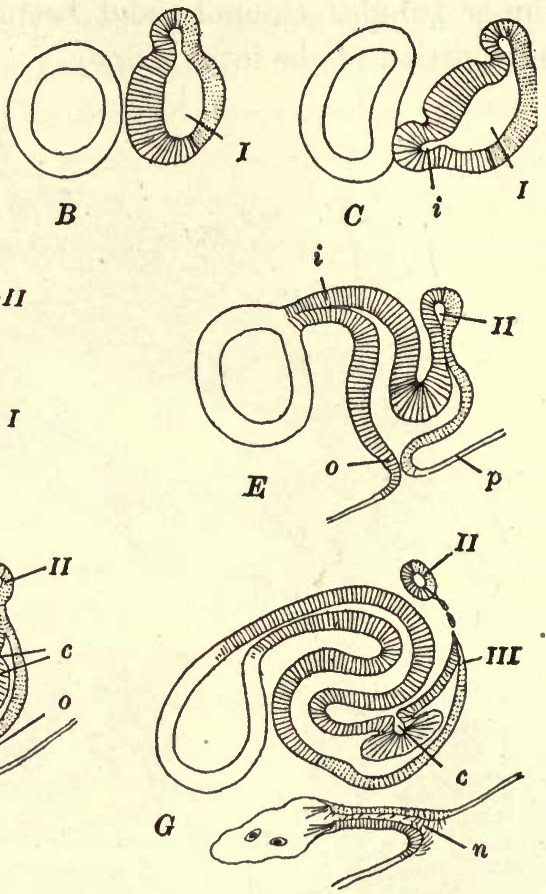

Fra. 74.- Series of diagrams illustrating the development of the primary mesonephric tubules in $R$. sylvatica. After Hall. The Wolffian duct is drawn in outline simply. The mesonephric vesicles are shaded; the somatic part of the tubule is shaded by continuous lines, the splanchnic part by dotted lines. $A$. Wolffian duct and simple mesonephric vesicle. B. Mesonephric vesicle dividing into the large primary mesonephric unit and the small dorsal chamber. The latter elongates antero-posteriorly and represents the rudiment of the secondary and later mesonephric units. C. Formation of the rudiment of the inner tubule. $D$. Inner tubule extending upward and toward the mesonephric duct; formation of rudiment of outer tubule. $E$. Outer tubule fused with peritoneum and rudiment of nephrostome thus established. Bowman's capsule forming. Commencement of differentiation of secondary mesonephric tubules. $F$. Separation of nephrostomal rudiment from remainder of tubule. $G$. Connection cf nephrostome with branch of posterior cardinal vein. Separation of secondary tubule, and beginning of tertiary tubule indicated. $c$, Bowman's capsule; $i$, inner tubule; $n$, nephrostome; $o$, outer tubule; $p$, peritoneum; $v$, branch of posterior cardinal vein; $I$, primary mesonephric tubule; $I I$, secondary mesonephric tubule; $I I I$, tertiary mesonephric tubule.

twig derived from the dorsal aorta, and structurally resembles essentially a miniature glomus like that of the pronephros. 
This part of the outer tubule now separates from the remainder, retaining the connection with the inner tubule, while the distal part retains its connection with the body cavity; this connection now becomes ciliated and forms a typical nephrostome. This nephrostomal region is short and effects a new connection at its inner end, with the sinus of the posterior cardinal vein. It will be remembered that, as the tubules of the mesonephros enlarge $(15 \mathrm{~mm}$.) this body seems to extend freely into the posterior cardinal vein, which has a sinusoidal character here and interpenetrates the substance of the mesonephros, its walls being closely reflected around the surfaces of the tubules.

During the later development of the mesonephros, the secondary and tertiary units acquire, similarly, connections with the mesonephric duct, Malpighian bodies, and nephrostomal connections with the body cavity and cardinal vein. Additionai outer tubules and nephrostomes are formed later, but it is not clear whether they form as independent evaginations of the peritoneum or by splitting off from those previously formed; perhaps both processes occur. The number finally formed is very large (about two hundred according to Marshall and Bles.)

Upon the development of the reproductive organs certain of the mesonephric tubules become modified and take on new functions; these processes will be described below.

Just before metamorphosis the urinary bladder appears as a median ventral evagination of the wall of the cloaca, nearly opposite the openings of the mesonephric ducts or ureters. The rudiment forms just at the border between the ectoderm and endoderm lining the cloaca. It is at first a long narrow sac, directed anteriorly; later its diameter increases and as it enlarges it becomes bifid at its extremity.

\section{The Reproductive System}

In order to make the devlopment of this system easier to understand we may first repeat, very briefly, the arrangement and composition of the adult system as described in the pre- 
ceding chapter (Figs. 24, 25). In the male each of the paired testes, suspended from the dorsal body wall by a double fold of peritoneum, the mesorchium, is connected by a series of small tubules, the vasa efferentia, with the upper end of the Wolffian duct or mesonephric duct. The vasa efferentia are modified mesonephric tubules, and the mesonephric duct therefore functions here both as excretory (ureter) and reproductive (vas deferens) duct, i.e., as a urinogenital duct. The homolog of the oviduct or Müllerian duct of the female, is represented in the male by a vestigial cord.

In the female the ovaries are similarly suspended by mesovaria. They are not directly connected with the reproductive ducts or oviducts, and consequently, in this sex the Wolffian or mesonephric duct remains purely excretory in function (ureter). The oviduct is a long convoluted tube which develops from the peritoneal epithelium, quite independently of the excretory ducts.

\section{A. THE GONODUCTS}

It will be convenient to describe the formation of the gonoducts first. The vas deferens of the male is the original mesonephric duct or ureter, and nothing need be added to the account of its development given above, save to point out that in connection with each duct a glandular seminal vesicle develops, just in front of the opening of the duct into the cloaca. In the female the mesonephric duct remains unmodified, and the seminal vesicles are barely represented.

The Müllerian ducts for a long time develop similarly in both sexes, although they become fully developed and functional, as the oviducts, only in the female. The Müllerian duct appears during the early stages of the degeneration of the pronephros, just beneath the common nephrostome, the formation of which has been mentioned. Here a peritoneal proliferation forms an elongated thickening along the dorso-lateral wall of the body cavity. From the dorsal border of this thickening a thin shelf of cells forms and projects downward (Fig. 75), parallel with the peritoneal thickening. The free lower margin of this shelf 
or flap then fuses with the peritoneal wall forming a short compressed tube open at both ends. This tube then extends anteriorly and posteriorly: anteriorly it reaches to the anterior end of the body cavity and then turns ventrally, stopping near the base of the lung, where its open end forms the rudiment of the ostium or infundibulum of the oviduct. Posteriorly the formation of the shelf and its closure continute parallel with and just to the outer side of the mesonephric duct, and entirely
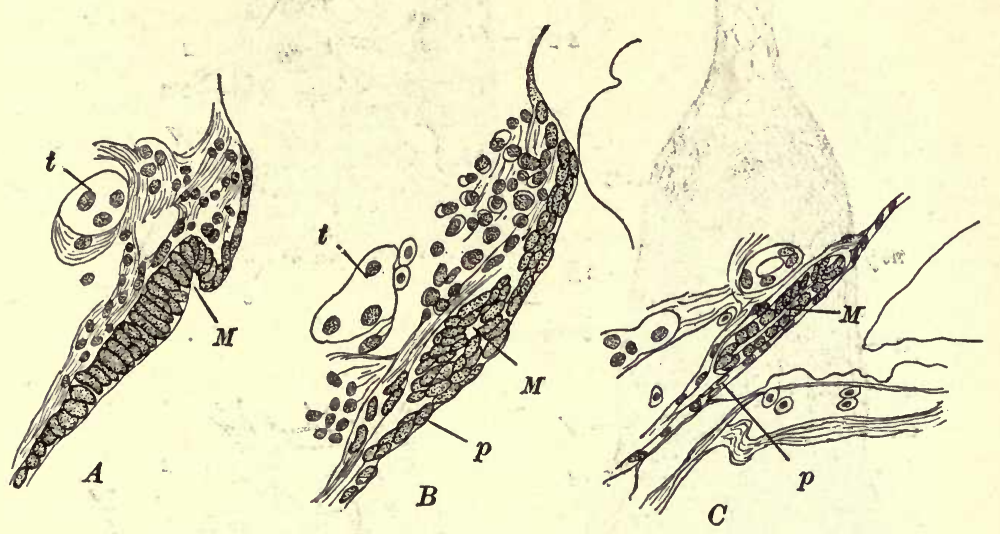

FIG. 75. - Sections through the developing Müllerian duct of a $34 \mathrm{~mm}$. tadpole of $R$. sylvatica. After Hall. A. Section passing through the beginning of the Müllerian evagination. $B$. Section posterior to $A$. Duct established but still connected with peritoneum. $C$. Section still farther posterior, showing the separation of the duct from the peritoneum. $M$. Müllerian duct; $p$, peritoneum; $t$, third pronephric tubule.

independent of it, until it reaches the cloaca, with which it connects some time after metamorphosis.

In the male the development of the Müllerian duct eeases at this stage, but in the female it continues to thicken and to elongate, so that it becomes entirely free from the body wall, though remaining suspended from it by a double fold of peritoneum; finally it acquires the characteristics described in the beginning of the preceding chapter.

\section{B. THE GONADS}

The primitive germ cells are distinguishable quite early (about $6 \mathrm{~mm}$.) as a definite though slight ridge along the 
median dorsal side of the endodermal wall of the intestine. The cells composing this ridge resemble closely the other cells of this part of the enteric wall, and are to be distinguished chiefly by their behavior (Fig. 76, $A$ ). The mesentery is formed shortly after this stage, and when the mesodermal folds push

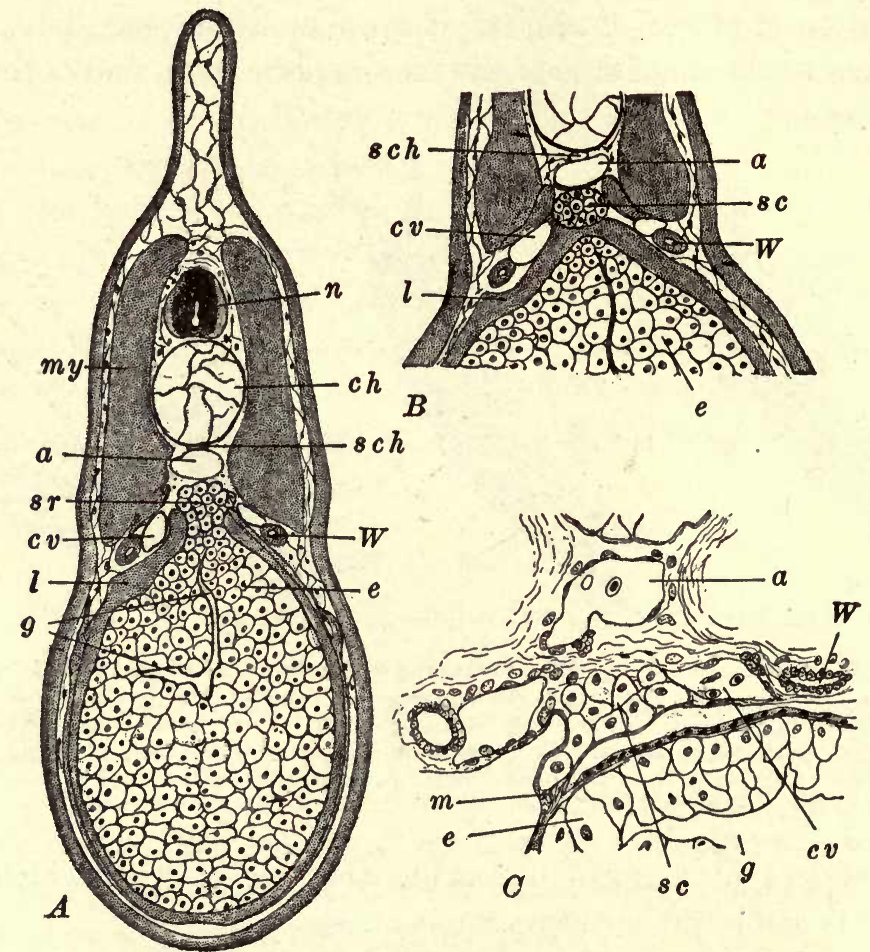

FIg. 76. -Sections showing the origin of the sex-cells (germ cells) in $R$. sylvatica. After Allen. $A, B$, Sections of a $7.5 \mathrm{~mm}$. larva showing $(A)$ sex-cell ridge of endoderm and $(B)$ its separation as the sex-cell cord. $C$, Part of a section of an $8.3 \mathrm{~mm}$. larva showing the beginning of the migration of the sex-cells. $a$, Dorsal aorta; $c h$, notochord; $c v$, posterior cardinal vein; $e$, endoderm cells; $g$, gut cavity; $l$, lateral plate of mesoderm; $m$, mesentery; $m y$, myotome; $n$, nerve cord; $s c$, sex-cell cord; $s c h$, subchordal rod (hypochorda); $s r$, sex-cell ridge; $W$, Wolffian duct.

in toward the mid-line, above the gut, this ridge of primitive germ cells seems to separate from the gut and to move dorsally, so that when the mesentery is formed they are found in its base, near the body wall (Fig. 76, B, C). Here they form a 
definite median strand of cells between the posterior cardinal veins (8-9 mm.) (Allen).

This germinal strand now divides longitudinally and the halves move more laterally, projecting slightly into the body cavity as the genital ridges, near the attachment of the mesentery and just beneath the cardinal veins. The genital ridges now become more conspicuous through the proliferation of the primitive germ cells and the peritoneal cells covering them, to which are added mesenchyme cells from the body wall. In this cell mass the mesenchyme elements are concerned in the formation of the stroma of the ridge. The peritoneum continues to form a thin superficial covering and later forms the suspensory folds (mesorchia, mesovaria) of the gonads, as the ridges may be called when they project freely into the body cavity. As the primitive germ cells begin to multiply, they form the nests of cells described in the preceding chapter, the further development of which need not be repeated here.

Before metamorphosis begins the anterior third or half of each genital ridge commences to degenerate and becomes converted into the fat body (see above). The posterior portion has previously acquired secondary

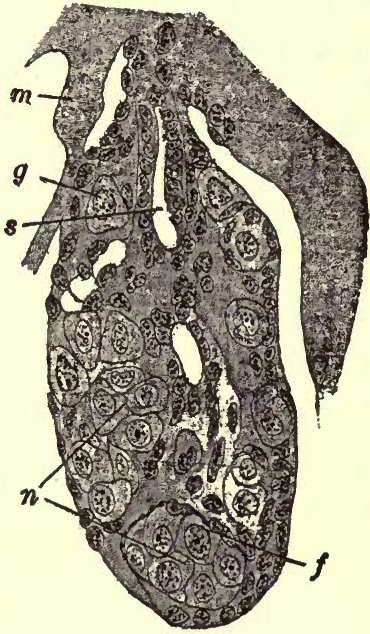

Fig. 77.-Section through the gonad of a $35 \mathrm{~mm}$. tadpole of $R$. temporaria. After Bouin. $f$, Follicle cells; $g$, primitive germ cell; $m$, mesentery; $n$, "nests" formed by multiplication of the primitive germ cells; $s$, genital strand (sex cord). connections with the mesonephric duct in the following manner. From the stalks of a few (7-8, Nussbaum) of the Malpighian bodies of the posterior part of the mesonephros, there grow out solid strands of cells known as the sexual cords. These become tubular and gradually extend downward into the substance of the gonad, either forming or connecting with spaces within this organ (Fig. 77). From this point onward their history is different in the two sexes. In the 
male, the sexual cords, after metamorphosis, establish intimate connections with the cavities of the testis and form the efferent ducts (vasa efferentia) by which the spermatozoa are conducted from the gonad to the gonoduct (vas deferens). In the female, while the intragonadial portions of the sexual cords may give rise to cavities there, the parts connecting the gonad and the mesonephros undergo degeneration and remain vestigial in the adult, forming what is known as Bidder's organ.

The sexes are morphologically indistinguishable during the early stages of development, and in $R$. temporaria it is not until the tadpole reaches a length of about $30 \mathrm{~mm}$. (Bouin) that the sex can be distinguished. About this time the ovary acquires a central lumen; the sex cords appear larger in the male, and the form of the nests of germ cells can be distinguished, in that in the testis groups of similar cells are formed while in the ovary the cells become arranged as a follicle surrounding a large central primitive ovum.

\section{The Adrenal Bodies}

The adrenal bodies of the adult frog consist of a thin layer of irregularly distributed tissue on the ventral surface of the pelvic portion of the mesonephros and intimately connected with it. Histological examination shows that the tissue consists of a coarse network of cell strands with occasional groups of darkly staining "phæochrome" tissue. The spaces within this meshwork are occupied by sinusoids of the efferent renal (median posterior cardinal) vein. These two kinds of tissue are known respectively as the cortical and the medullary tissues, not because they have such a relation here, but because the corresponding elements of the adrenals in higher forms have such a disposition.

The cortical substance of the adrenal appears first, in the larva of about $12 \mathrm{~mm}$. in the form of small cell groups along either side of the wall of the median posterior cardinal vein (Fig. 78, A). They lie below the level of the mesonephroi, and 
beneath the peritoneal epithelium from which they appear to have arisen. Just after metamorphosis these cell groups separate from the peritoneum, and begin to send out branching and anastomosing processes which soon form a network, like that of the cortical substance of the adult.

The medullary or phæochrome substance is derived originally from ganglia of the sympathetic nervous system. After the central network is established there appear within the sympathetic ganglia of the mesonephric region groups of cells, the precise origin of which is not clear, having the properties of
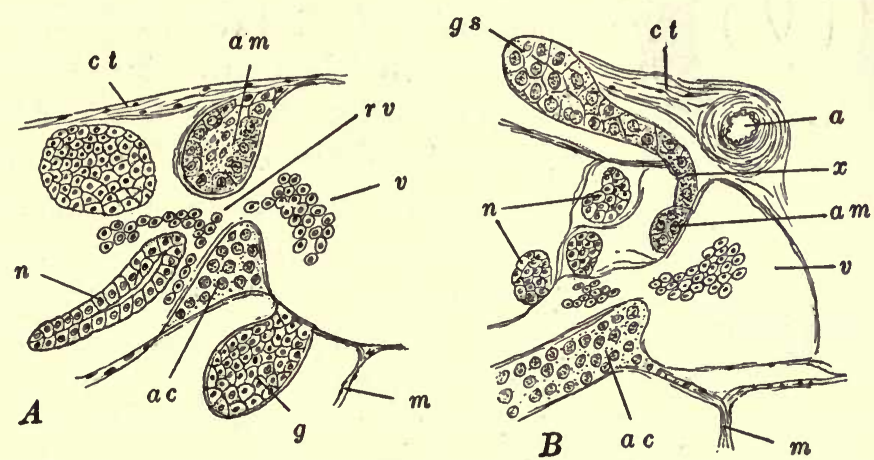

Fig. 78.-Parts of sections through young $R$. temporaria, showing the origin of the adrenal bodies. After Srdínko. $A$. Through $30 \mathrm{~mm}$. tadpole. $B$. Through $11 \mathrm{~mm}$. frog after metamorphosis. $a$, Dorsal aorta; $a c$, cortical cells of adrenal body; $a m$, medullary cells of adrenal body; $c t$, connective tissue; $g$, gonad; $g s$, sympathetic ganglion; $m$, mesentery; $n$, mesonephros; $r v$, revehent renal vein; $v$, vena cava; $x$, point where ganglion cells enter mesonephros and adrenal body.

these phæochrome cells. Some of these cell groups remain in the sympathetic ganglia, while others appear to migrate into the rudiment of the adrenal body, where they become scattered through the cortical tissue (Fig. 78, $B$ ). The method by which they extend into the adrenal is not clearly known in the frog.

\section{THE SKELETON AND TEETH}

In describing the development of the skeletal system we shall limit our account to the establishment of the essential 
structures of the tadpole, merely indicating the trend of later development. The later history of the skeleton falls largely without our province.

\section{The Vertebral Column}

The formation of the notochord has been described previously, but its differentiation deserves a further word. The

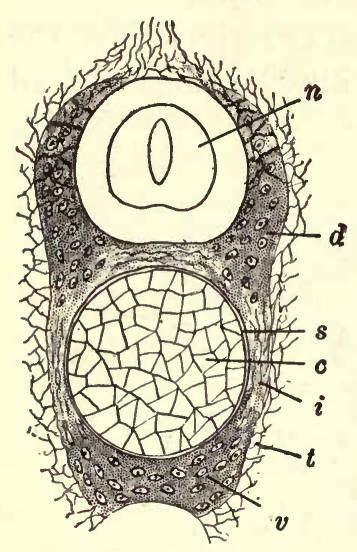

Fig. 79.- Transverse section through the vertebral column in the body region of a larva of Xenopus capensis. After Schauinsland. $c$, Notochord; $d$, dorsal vertebral cartilaginous arch; $i$, inner chorda sheath of sclerotome; $n$, nerve cord; $s$, chorda sheath; $t$, perichondrial connective tissue; $v$, ventral (hypochordal) vertebral cartilage. chorda cells early become flattened antero-posteriorly, and about the time the embryo begins to elongate, vacuoles appear within the protoplasm of the cells and also between adjacent cells. The chorda becomes surrounded by three sheaths. The primary or elastic sheath is formed on the surface of the chorda by the action of the superficial chorda cells. The secondary or fibrous sheath is formed within the primary sheath by the chorda epithelium, which is composed of a layer of cells just within the primary sheath. Considerably later a skeletogenous sheath is laid down outside the primary sheath; this is formed by the sclerotomal outgrowths of the somites, whose formation was described above (Fig. 79). The skeletogenous layer is continued dorsally around the nerve cord, and it also extends a short distance laterally from the chorda, between successive myotomes.

The vertebral column is formed within this skeletogenous layer. First there appears (about $15 \mathrm{~mm}$.) a metameric series of cartilages, along the dorso-lateral surfaces of the chorda in the base of the neural arch. A metameric series of cartilages appears also in the skeletogenous layer along the median ventral surface of the chorda (Fig. 79). The cartilages of the dorsal series become united on each side, so that a pair of con- 
tinuous strips extends along the entire chorda, while the ventral elements similarly fuse forming a median ventral strip.

Separate vertebræ now become marked out by the appearance, in these continuous cartilages, of metameric rings of fibrous tissue; these are the beginnings of the intervertebral ligaments. They appear opposite the middle of each mesodermal segment, consequently the segments of the vertebral column (vertebræ) alternate with the muscle segments. Cartilage now begins to form across the notochord, between successive vertebræ, so that the notochord becomes completely segmented, remaining only intravertebrally. In each of these transverse partitions appears a curved split, concave anteriorly. The intervertebral cartilages then fuse with the adjoining vertebræ and thus determine the procœlous character of the vertebral centra.

The ventral cartilages now grow up around the sides of the chorda, meeting and fusing with the dorsal series. From the former there extend outward short cartilaginous processes which become the transverse processes of the vertebra. Laterally from these, bits of cartilage are formed later which represent ribs. These fuse with the tips of the transverse processes so that no separate ribs are subsequently distinguishable. In the meantime outgrowths from the dorsal elements have extended inward beneath the nerve cord, as well as laterally and dorsally to it (neural arch).

Bony tissue appears first, before the beginning of metamorphosis, in the region between the dorsal and ventral series of cartilages. It soon invades the entire cartilaginous structure, forming a complete shell around the intravertebral notochordal remains, and dorsally around the nerve cord. In addition to the articulation of the procœlous vertebral centra, intervertebral articulations develop on short processies of the neural arches.

The foregoing description applies only to the nine vertebræ of the body. In the greater part of the tal cartilage is not formed. But in the region of the future urostyle three longitudinal strips of cartilage are formed as in the trunk, but these fuse completely enclosing the chorda in a cylinder of cartilage which is never segmented into vertebræ. 


\section{The Skull}

The fully formed skull is a complex organ formed by the association, and more or less extensive fusion, of several diverse elements; these are (a) the cranium, (b) the sense capsules, (c) the visceral arches (in part), (d) the notochord (in part), (e) vertebral elements, $(f)$ membrane or derm bones. Before proceeding to describe the formation and association of these elements in the frog, we should note that here no embryologically distinct vertebral elements are included in the skull; their inclusion in the above list is based upon phyletic and comparative grounds, and upon the behavior of the anterior somites.

\section{A. THE CRANIUM AND SENSE CAPSULES}

In tadpoles of about $7 \mathrm{~mm}$. the first rudiments of the cranium are formed as a pair of curved strands of dense tissue, soon becoming cartilaginous, along the ventro-lateral surfaces of the fore-brain. These are the rudiments of the trabeculoe or trabecular cartilages (Fig. 80, A). They rapidly extend forward and fuse across the mid-line between the olfactory organs, forming there the rudiment of the internasal plate; each rod then continues forward as the trabecular cornu, which expands slightly, partly enclosing the olfactory organ and forming the olfactory capsule. In front of the olfactory capsule the trabeculæ unite with the rudiments of a pair of labial or suprarostral cartilages, lying in the extended upper lip. Posteriorly the trabeculæ extend beneath the mid-brain, embracing between their ends the anterior extremity of the notochord, which it will be recalled extends forward to the mid-brain region. Soon similar tissue thickenings extend posteriorly each side of the notochord in the hind-brain region; these are the indications of the parachorda or parachordal cartilages. The rudiments of the parachordals now fuse with the posterior ends of the trabeculæ, enclosing the tip of the chorda and forming a continuous plate beneath the hind-brain, known as the parachordal plate (Fig. 80, A). 
In the tadpole at this stage (shortly after hatching) there are present also rudiments of parts of the visceral arches; the general development of these will be described below, but it is necessary to mention here one of these elements, the palato-quadrate, since from the beginning it takes part in the formation of the cranium. The paired palato-quadrate rudiments are formed as short,
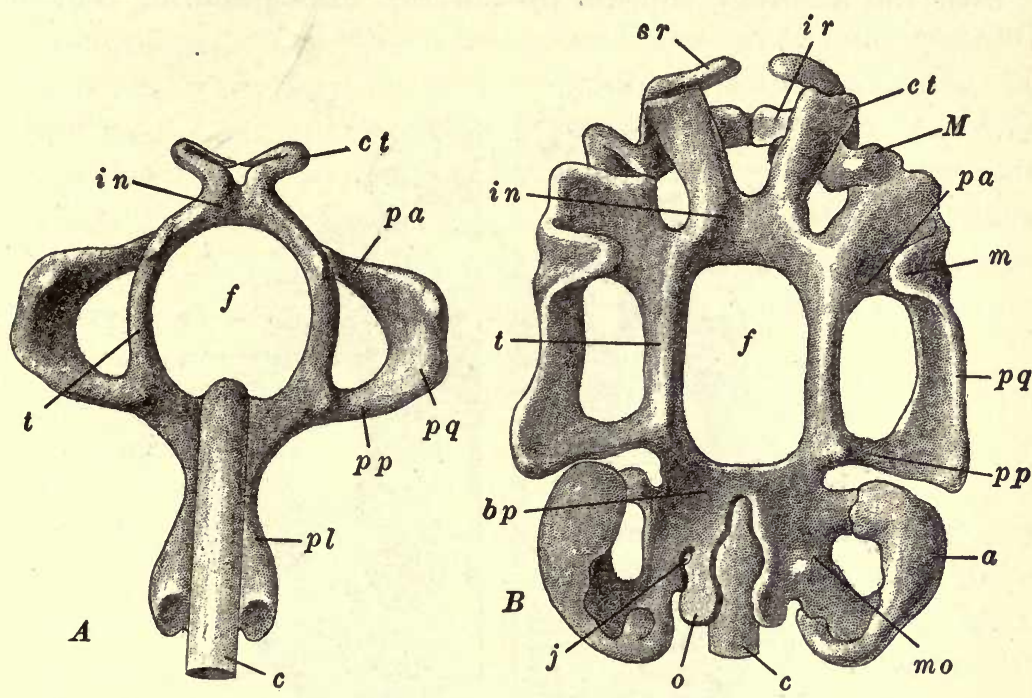

FIG. 80.-Dorsal views of the chondrocranium of the frog larva. A. Chondrocranium of a $7.5 \mathrm{~mm}$. larva of $R$. temporaria. After Gaupp, from Stöhr-Ziegler model. B. Chondrocranium of a $14 \mathrm{~mm}$. larva of $R$. fusca. After Gaupp, from Ziegler model. $a$, Auditory capsule; $b p$, basal plate; $c$, notochord; $c t$, trabecular cornu; $f$, basicranial fontanelle; in, internasal plate; $i r$, infrarostral cartilage; $j$, jugular foramen (for IX and $\mathrm{X}$ cranial nerves); $m$, muscular process; $\boldsymbol{M}$, Meckel's cartilage; $m o$, mesotic cartilage; $o$, occipital process; $p a$, anterior ascending process of palato-quadrate cartilage; $p l$, parachordal plate; $p p$, posterior ascending process of palato-quadrate cartilage; $p q$, palato-quadrate cartilage; $s r$, suprarostral cartilage; $t$, trabecular cartilage.

flattened, crescentic rods, lateral to the trabeculæ. They soon connect with the trabeculæ by anterior ascending processes a short distance back of the olfactory region, and by posterior ascending processes opposite the extremity of the chorda. The rudiments of the cranium thus marked out are now converted into a continuous cartilaginous structure having the appearance illustrated in Fig. 80, A. The large basi-cranial fontanelle in front of the chorda is the seat of the infundibulum and pituitary body. 


\section{2}

Immediately subsequent events concern chiefly the posterior part of the cranium. The auditory organ becomes partially enclosed by a connective tissue capsule which is early chondrified, forming a cap open toward the mid-line (Fig. 80, B). A cartilage (mesotic cartilage) then extends posteriorly and laterally from the parachordal plate and becomes united with the auditory capsule by anterior and posterior ventral

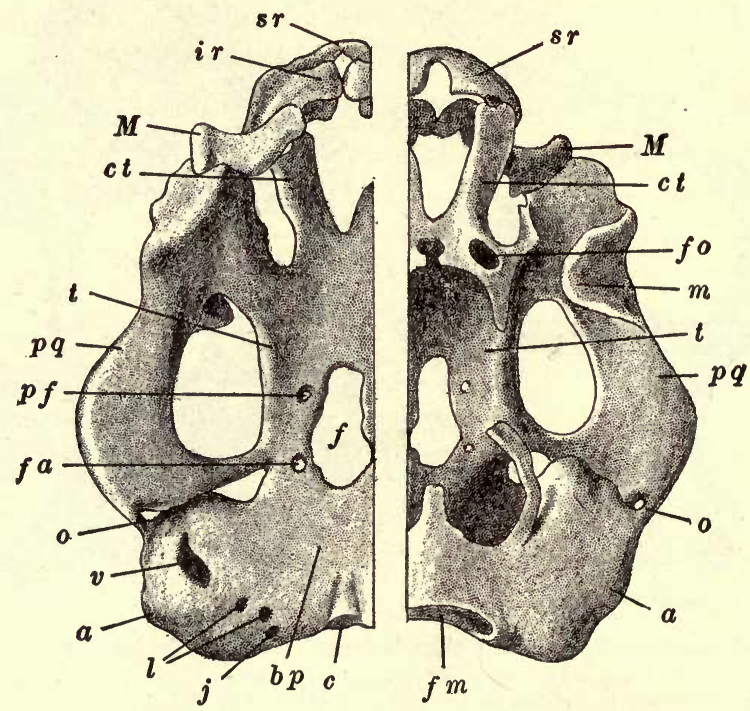

Fig. 81.-Chondrocranium of $29 \mathrm{~mm}$. larva of $R$. fusca. After Gaupp, from Ziegler. To the left, the ventral surface; to the right, the dorsal surface. $a$, Auditory capsule; $b p$, basal plate; $c$, notochord; $c t$, trabecular cornu; $f$, basicranial fontanelle; $f a$, foramen for carotid artery; $f m$, foramen magnum; $f o$, foramen for olfactory nerve; $i r$, infrarostral cartilage; $j$, jugular foramen for IX and X cranial nerves; $l$, perilymphatic foramina; $m$, muscular process; $M$, Meckel's cartilage; $o$, otic process of palato-quadrate; $p f$, palatine foramen; $p q$, palato-quadrate cartilage; $s r$, suprarostral cartilage; $t$, trabecular cartilage: $v$, secondary fenestra vestibuli.

connections, leaving between them a wide space. Posteriorly to this mesotic cartilage the floor of the cranium is continued as the occipital cartilage. This also fuses with the floor of the auditory capsule leaving, however, a small space which represents the jugular foramen transmitting the IX and $\mathrm{X}$ cranial nerves. The floor of the posterior part of the cranium, 
composed of the occipital and mesotic cartilages and the parachordal plate, is known as the basal plate.

By the time the tadpole has reached a length of about 14 $\mathrm{mm}$. the chondrocranium has acquired the form shown in Fig. 80, B. A still later stage is illustrated in Fig. 81. Comparison of these two figures brings out most of the facts of later development. We need therefore mention specifically only a few details. No traces of the notochord finally remain; it is partly replaced by, and partly converted into cartilage of the basal plate. The occipital region slowly extends vertically forming the hinder wall of the cranial cavity, and fuses extensively with the auditory capsule. Finally the occipital cartilage extends dorsally around the nerve cord, enclosing the foramen magnum. In the frog the occipital cartilage shows no definite indications of its vertebral origin.

The auditory capsule becomes more complete externally, remaining open into the cranial cavity by a large foramen. From the inner surface of the capsule cartilage grows in, surrounding the elements of the membranous labyrinth previously described. On the outer side of the capsule an opening is formed, the secondary fenestra vestibuli, which becomes plugged by the movable operculum. In connection with the ear we described above the development of the plectrum or columella, and its connection later with the annular cartilage in the superficial tympanic membrane. We should repeat that the columella is not related with the elements of the visceral skeleton in the frog.

In the orbital region the trabeculæ gradually grow across the basicranial fontanelle, closing it and forming the floor of this part of the cranial cavity. They also extend vertically forming the lateral walls of the cranial cavity, separating it from the orbits; these walls are perforated only for the passage of nerves and blood vessels. Anteriorly, cartilages from the trabeculæ also extend dorsally across the mid-line forming a narrow dorsal bridge. The large supracranial fontanelle between this bridge and the supraoccipital region is not closed by cartilage. 

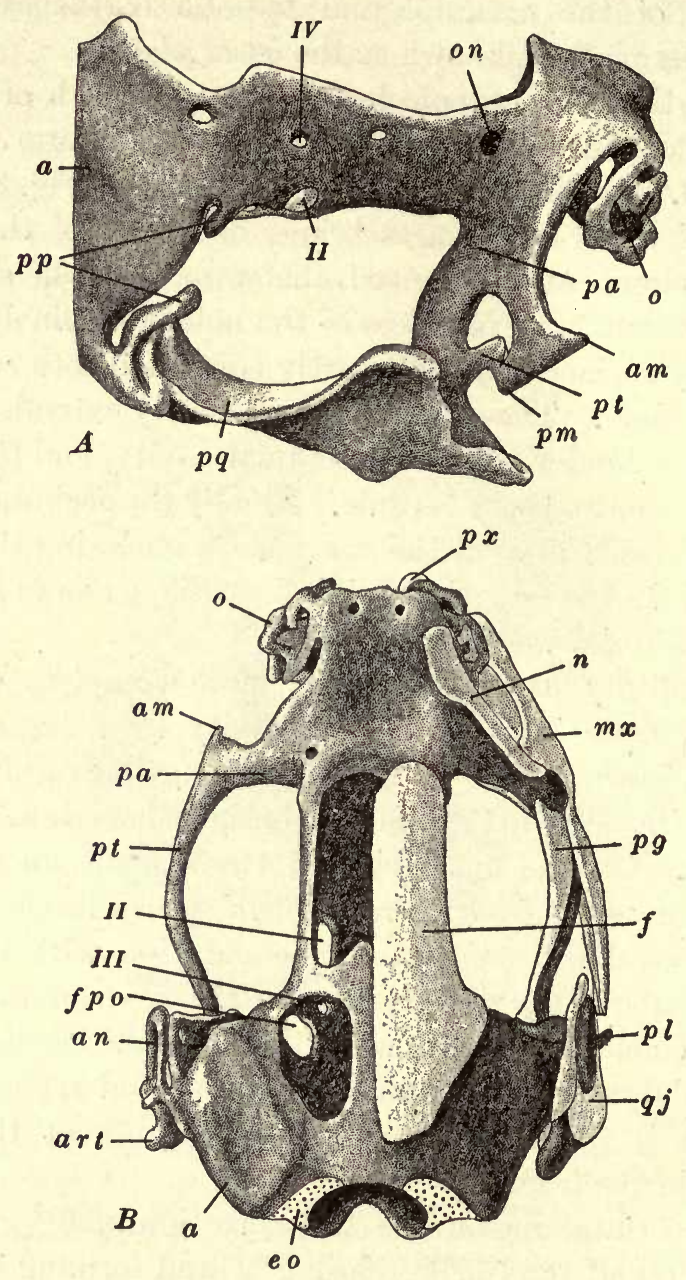

FIG. 82.-A. Anterior portion of chondrocranium of $R$. fusca during metamorphosis. Lateral view. After Gaupp, from Ziegler. B. Skull of $2 \mathrm{~cm}$. $R$. fusca, after metamorphosis. Dorsal view. Membrane bones removed from left side. After Gaupp, from Ziegler. a, Auditory capsule; am, anterior maxillary process; an, annulus tympanicus; art, articular process of palato-quadrate cartilage; $e o$, exoccipital bone; $f$, fronto-parietal bone; $f p o$, proötic foramen; $m x$, maxillary bone; $n$, nasal bone; $o$, olfactory cartilages; on, orbito-nasal foramen; $p a$, anterior ascending process of palato-quadrate; $p g$, pterygoid bone; $p l$, plectrum; $p m$, posterior maxillary process; $p p$, posterior ascending process of palato-quadrate; $p q$, palato-quadrate cartilage; $p t$, pterygoid process of palatoquadrate; $p x$, premaxillary bone; $q j$, quadrato-jugal bone; $I I$, foramen for optic nerve; $I I I$, foramen for III cranial nerve; $I V$, foramen for IV cranial nerve. 
The ethmoid region remains comparatively simple throughout the tadpole stage; its extreme complication comes later. During the larval period the internasal septum extends dorsally, forming the anterior wall of the cranial cavity, perforated by the olfactory nerves. The trabecular cornua remain separate from the olfactory capsules and connect anteriorly with the suprarostral or labial cartilages. During metamorphosis the labial cartilages and the anterior ends of the cornua disappear in front of the olfactory capsules (Fig. 82).

The formation of the bony elements of the skull occurs relatively late in the frog. As a matter of fact, the derm or membrane bones appear before those which are formed in the cartilage cranium, but they will be described later. There are, in the frog's skull the following elements formed as cartilage bones in the original cranium.

(a) The exoccipitals (lateral occipitals) which form from the posterior parts of the occipital cartilage and auditory capsule; the occipital condyles and the median dorsal and ventral parts of the occipital region remain cartilaginous.

(b) The proitics, which form from the anterior parts of the auditory capsules and the parts of the basal plate and orbital region adjacent to the auditory capsules.

(c) The columella, whose development has been described in another place.

(d) The ethmoids which form as vertical elements in the anterior part of the inner wall of the orbit; later the two ethmoids unite above and below, forming a band-like element around the cranium. This is often known as the sphenethmoid or orbito-sphenoid.

In the palato-quadrate cartilage, bone appears only in the region of the articulation with the lower jaw (see below). This region does not form a distinct element of the skull, however, but unites with a membrane bone, the two together forming the quadrato-jugal.

With the exception of the ethmoids, these elements are all present by the end of metamorphosis: the ethmoids form some weeks later. 


\section{B. THE VISCERAL ARCHES}

The elements of the visceral skeleton are formed in the pharyngeal visceral arches, which are established by the fusion of the serial gill pouches with the ectoderm. We have described the formation of the mandibular, hyoid and four branchial visceral arches. In each of these save the last branchial, skeletal elements appear. The mandibular and hyoid skeletal arches appear about the time the full number of visceral pouches is established, as condensations in the mesenchyme of the visceral arch regions, soon becoming cartilaginous. The mandibular arch appears first as a short rod, lying transversely to the axis of the embryo, in the floor and sides of the mouth cavity (Fig. 57). It is very early divided into two parts, the separation between them marking the jaw articulation. The dorsal section, or upper jaw rudiment, known as the palatoquadrate, has already been described; the ventral section, or lower jaw rudiment, becomes subdivided into Meckel's cartilage, or lower jaw proper, and the infrarostral cartilage. The last two elements remain ventral to the olfactory region as small relatively undifferentiated elements during early development, but the palato-quadrate rapidly enlarges and grows backward, becoming roughly parallel with the trabeculæ and fusing with them at two points as described above. Later (about $21 \mathrm{~mm}$.) the posterior or quadrate portion of this cartilage forms a connection with the auditory capsule. During metamorphosis, as the mouth enlarges and extends far posteriorly, the arrangement of the jaw elements is very considerably modified. The upper end of Meckel's cartilage, which has been in the olfactory region, rapidly extends posteriorly and reaches to the quadrate cartilage, below and in front of the auditory capsule. The quadrate, meanwhile, elongates ventro-laterally. That part of the palato-quadrate lying in the orbital region, softens and largely disappears, and the anterior connection with the trabecula is drawn back in its place and remains as the seat of the future pterygoid and palatine regions. The jaw articulation is thus carried rapidly from the anterior to the posterior region 
of the cranium, and through the elongation of the quadrate, also some distance laterally from the cranial wall (Fig. 82).

The infrarostral cartilages, which very early fuse across the mid-line forming the apex of the lower jaw, also elongate at this time, and now fuse with the Meckelian cartilages as the mento-Meckelian cartilages. Later on each becomes bony and fuses with the dentary, the chief membrane bone of the lower jaw (see below). A small median element between the infrarostrals fuses with them.

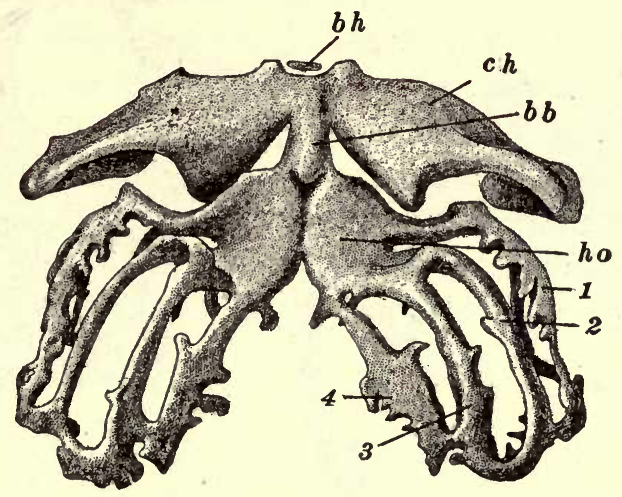

FIG. 83.-Hyoid and branchial arches of a $29 \mathrm{~mm}$. larva of $R$. fusca. Ventral view. After Gaupp, from Ziegler. $b b$, Basibranchial (first), or copula; $b h$, basihyal; ch, ceratohyal; ho, hypobranchial plate; $1-4$, first to fourth ceratobranchials.

The annulus tympanicus surrounding the tympanic membrane of the frog, forms as an outgrowth of the quadrate cartilage; it becomes separate and gradually extends to the surface of the head, forming first a crescentic, then a circular cartilage, and later bone, long after the completion of metamorphosis (Fig. 82, $B$ ).

The development of the hyoid and branchial arches may conveniently be described together. These all appear first as paired rods of dense tissue, lying in the corresponding visceral arches. The hyoid arch forms about the same time as the mandibular, the first branchial just after hatching, the second branchial at 9-10 mm., and the third and fourth branchials shortly after. 
On each side, the hyoid cartilage or ceratohyal, extends dorsally, connecting with the palato-quadrate just behind the jaw articulation; ventrally it unites with its fellow (Fig. 83). The first branchials also unite ventrally. The other branchial cartilages do not reach the mid-ventral line, but the lower end of each unites with that anterior to it; later they similarly connect dorsally. In the ventral region of the pharynx a median element, the copula (basibranchial) appears, between

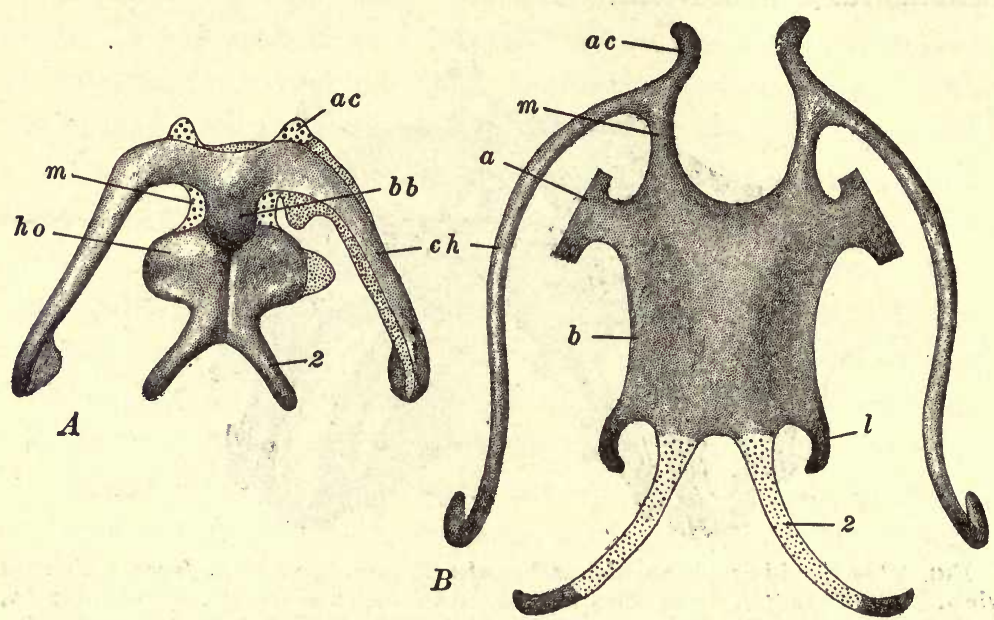

FIG. 84.-A. Hyobranchial apparatus of $R$. fusca, toward the end of metamorphosis. The left side is shown in a more advanced stage than the right, in that less cartilage is present. The original cartilage is indicated by fine stipples. The coarse stipples indicate the cartilage added during the early part of metamorphosis: After Gaupp, from Ziegler. B. Hyobranchial apparatus of a $2 \mathrm{~cm}$. R. fusca, after metamorphosis. After Gaupp, from Ziegler. $a$, Alar process; $a c$, anterior process of hyoid cornu; $b$, body of hyobranchial cartilage; $b b$, basibranchial (first), or copula; $c h$, ceratohyal (hyoid cornu in $B$ ); ho, hypobranchial plate; $l$, postero-lateral process of hyobranchial cartilage; $m$, manubrium; 2 , remains of second ceratobranchial (postero-medial process of hyobranchial cartilage).

the hyoid and the first branchial, connecting with the ventral ends of both these arches. The basihyoid cartilage is represented only by a small median copula in front of the hyoid cartilage. The lower. ends of the first branchials become flattened and expanded as the hypobranchial plate, with which the ventral ends of the other three branchials then fuse. Only the lateral or middle sections of the branchial cartilages, between the 
visceral pouches, then remain separate from one another as the first to fourth ceratobranchials.

The arrangement of these arches is profoundly modified during metamorphosis, when the gill slits close and the jaw articulation moves backward. The hyoid bar, or ceratohyal, loses its connection with the palato-quadrate, and becomes considerably reduced in diameter. The copula becomes reduced and a pair of new cartilages develops each side of it, connecting with the hypobranchial plate and hyoid elements; these are the manubrial cartilages (Fig. 84, $A$ ).

The hyobranchial apparatus of the fully metamorphosed frog consists of a broad median plate of cartilage formed of the fused manubria, copula, and hyobranchial plate (Fig. 84, $B$ ). The ceratohyals remain as slender processes of this, known as the hyoid cornua. Other processes of the median cartilage develop anew, and only the posterior processes formed from the second branchials, represent primary elements of the branchial series; the other elements disappear entirely.

\section{THE DERMAL ELEMENTS}

The derm bones of the skull begin to appear in the dermal layer of the integument covering the head and lining the mouth before any other bony elements of the skeleton are indicated.

The first derm bone to appear is the median parasphenoid, in the roof of the mouth of the tadpole of about $20 \mathrm{~mm}$. (shortly after the hind-legs appear). This bone finally becomes daggershaped and covers the large basicranial fontanelle of the chondrocranium. The paired frontals and parietals appear somewhat later, roofing the cranium and covering the supracranial fontanelle; these elements later unite forming the paired frontoparietals (Fig. 82, B). A pair of nasals roof the olfactory capsules, while within the capsules appear the septo-nasals (intranasals).

During metamorphosis the dermal elements of the mandibular arch and the other bones of the mouth appear. Premaxilloe and maxillo form the margin of the upper jaw, connecting later 
with the teeth (see below), while in the lower jaw Meckel's cartilage becomes surrounded by the dentary and angular; the dentary then connects with the infrarostrals of Meckel's cartilage. Paired vomers beneath the olfactory capsules, and palatines across the anterior margins of the orbits develop next. The pterygoids develop along the inner faces of the palatoquadrate cartilages, and the squamosals along their outer surfaces, finally extending back over the auditory capsules. The quadrato-jugal represents a dermal element developed in connection with the posterior angle of the palato-quadrate cartilage, and fused with the cartilage quadrate bone of the palatoquadrate itself. With the exception of this element the derm bones remain easily separable from the cartilage bones and the remains of the chondrocranium, even in the skull of the fully grown frog.

\section{The Teeth}

Teeth are present in the adult on the premaxillæ, maxillæ, and vomers. During the larval period the teeth are functionally replaced by the horny "jaws" and "teeth," so that the true teeth develop relatively late. They form independently of the bones with which they are later associated. During metamorphosis a series of dermal papillæ forms around the margin of the upper jaw and covering the vomers. These are the tooth germs; they project into an associated thickening of the epidermis. The Malpighian cells of the epidermis covering each tooth germ become the enamel organ and secrete a layer of enamel over the surface of the hollow cone of the dentine which is formed by the surface of the dermal papilla. The cellular core of the papilla remains as the pulp cavity of the tooth. The teeth are elongated by the formation of bony tissue at their bases, and they gradually push through the epidermis. This basal mass of bony tissue serves also to attach the teeth to the inner sides of the jaw, and to the surface of the posterior part of the vomer; their attachment does not occur until some time after metamorphosis. 
The teeth are continually wearing away and dropping out of the jaw; they are replaced by new teeth which develop similarly deeper in the dermis. There are therefore always present in the jaws, teeth in various stages of development.

\section{The Appendicular Skeleton}

The elements of the pectoral and pelvic arches and limbs do not appear until just before metamorphosis. We shall make only the briefest reference to these structures.

The pectoral arch appears as a pair of crescentic cartilages around the lateral and ventral parts of the body, opposite the anterior end of the vertebral column. Just below its middle each rod forms an articulation (glenoid cavity) with the head of the humerus. Above this are formed the bony scapula and the cartilaginous terminal suprascapula. Below the glenoid cavity the arch is divided into the posterior coracoid and the anterior procoracoid elements. The coracoid becomes bony, while in connection with the procoracoid a dermal element, the clavicle, develops later. The lower ends of the coracoids and procoracoids become united on each side by a cartilaginous epicoracoid. Later the two epicoracoids fuse together in the mid-line.

Posterior to the epicoracoids a median cartilage develops which is the rudiment of the sternum. Later this fuses with the epicoraciids, and its proximal section becomes bony while posteriorly it forms the cartilaginous xiphisternum. Anterior to the epicoracoids a similar omosternum is formed.

The pelvic arch also appears first as a pair of cartilaginous rods, but these are early in contact medio-ventrally, and soon fuse together. These articulate with the femora, and the parts dorsal to the articulations (acetabula) form the ilia, which connect with the transverse processes of the last or ninth vertebra (sacral vertebra). The postero-ventral region of each arch becomes the bony ischium, while the antero-ventral part remains cartilaginous as the pubis.

While the pelvic arch is, like the pectoral, originally at right angles to the vertebral column, after metamorphosis it rotates 
so as to lie nearly parallel with the vertebral column and at the same time the ilia elongate enormously, throwing the attachment of the hind limbs far backward.

The details regarding the development of the elements of the limb skeleton are outside our province. For information the student may be referred to the papers of Zwick (1898), Tschernoff (1907), and Schmalhausen (1907, 1908), and to the literature there cited.

\section{REFERENCES TO LITERATURE}

\section{CHAPTERS II and III}

Allen, B. M., An Important Period in the History of the Sex-cells of Rana pipiens. Anat. Anz. 31. 1907.

Assheton, R., On the Development of the Optic Nerve of Vertebrates, and the Choroidal Fissure of Embryonic Life. Q. J. M. S. 34. 1892. On the Growth in Length of the Frog Embryo. Q. J. M. S. 37. 1894. On Growth Centers in Vertebrate Embryos. Anat. Anz. 27. 1905.

Bataillon, E., L'embryogenèse complète provoquée chez les Amphibiens par piqûre de l'œuf vierge, larves parthénogénésiques de Rana fusca. C. R. Acad. Sci. Paris. 150.1910.

Bialaszewicz, K., Beiträge zur Kenntnis der Wachstumsvorgänge bei Amphibienembryonen. Bull. Acad. Sci. Cracovie. 1908. Review in Arch. Entw.-Mech. 28. 1909.

Bourn, $\mathrm{P}$., Histogenèse de la glande génitale femelle chez Rana temporaria. Arch. Biol. 17. 1901.

Brachet, A., Recherches sur l'ontogénèse des Amphibiens urodèles et anoures. (Siredon pisciformis-Rana temporaria). Arch. Biol. 19. 1902. Recherches sur l'origine de l'appareil vasculaire sanguin chez les Amphibiens. Arch. Biol. 19. 1903. Gastrulation et formation de l'embryon chez les Chordés. Anat. Anz. 27. 1905. Recherches expérimentales sur l'œuf de Rana fusca. Arch. Biol. 21. 1905 (1904). Recherches expérimentales sur l'œuf non segmenté de Rana fusca. Arch. Entw.-Mech. 22. 1906. Recherches sur l'ontogénèse de la tête chez les Amphibiens. Arch. Biol. 23. 1908. Recherches sur l'influence de la polyspermie expérimentale dans le développement de l'œuf de Rana fusca. Arch. zool. exp. V. 6. 1910. Etudes sur les localisations germinales et leur potentialité reélle dans l'œuf parthénogénétique de Rana fusca. Arch. Biol. 26. 1911. 
Braem, F., Epiphysis und Hypophysis von Rana. Zeit. wiss. Zool.

63. 1898.

Braus, H., See Hertwig, Handbuch, etc.

Broman, I., Ueber Bau und Entwicklung der Spermien von Rana fusca.

Arch. mikr. Anat. 70. 1907.

Carnoy, J. B. et Lebrun, H., La vésicule germinative et les globules polaires chez les Batraciens. Cellule. 17. 1900.

Conning, H. K., Ueber einige Entwicklungsvorgänge am Kopf der Anuren. Morph. Jahrb. 27. 1899.

Dustin, A. P., Recherches sur l'origine des gonocytes chez les Amphibiens. Arch. Biol. 23.1908.

Elliot, A. I. M., Some Facts in the Later Development of the Frog, Rana temporaria. I. The Segments of the Occipital Region of the Skull. Q. J. M. S. 51. 1907.

Erlanger, R. von, Ueber den Blastoporus der anuren Amphibien, sein Schicksal und seine Beziehungen zum bleidenden After. Zool. Jahrb. 4. 1890.

Eycleshymer, A. C., The Development of the Optic Vesicles in Amphibia. Jour. Morph. 8. 1893. The early Development of Amblystoma, with Observations on some other Vertebrates. Jour. Morph. 10. 1895.

Federow, V., Ueber die Entwickelung der Lungenvene. Anat. Hefte. 40. 1910.

Felix, W., See Hertwig, Handbuch, etc.

Field, H. H., The Development of the Pronephros and Segmental Duct in Amphibia. Bull. Mus. Comp. Zool. Harvard College. 21. 1891. Die Vornierenkapsel, ventrale Musculatur und Extremitätenanlagen bei den Amphibien. Anat. Anz. 9. 1894. Bemerkungen über die Entwickelung der Wirbelsäule bei den Amphibien. Morph. Jahrb. 22.1895.

Filatow, D. P., Zur Entwickelungsgeschichte des Exkretionssystems der Amphibien. Anat. Anz. 25. 1904.

Froriep, A., See Hertwig, Handbuch, etc.

Fürbringer, M., Zur Entwickelung der Amphibienniere. Heidelberg. 1877.

GaupP, E., Ecker und Wiedersheim's Anatomie des Frosches. Braunschweig. 1896, 1904. Ontogenese und Phylogenese des schalleitenden Apparates bei den Wirbeltieren. Ergeb. Anat. u. Entw. 8. 1899 (1898). See also Hertwig, Handbuch, etc.

Giannelli, L., Sulle prime fasi di sviluppo del pancreas negli Anfibii anuri (Rana esculenta). Monit. Zool. Ital. 14. 1903.

G๘тTе, A., Die Entwickelungsgeschichte der Unke (Bombinator igneus). Leipzig. 1875.

Göppert, E., Die Entwicklung und das spätere Verhalten des Pankreas 
der Amphibien. Morph. Jahrb. 17. 1891. See also Hertwig, Handbuch, etc.

Greil, A., Ueber die sechsten Schlundtaschen der Amphibien und deren Beziehungen $\mathrm{zu}$ den suprapericardial (postbranchialen) Körpern. Verh. Anat. Ges. 18. Anat. Anz. 25. 1904. Ueber die Anlage der Lungen, sowie der ultimobranchialen (postbranchialen, supraperikardialen) Körper bei anuren Amphibien. Anat. Hefte. 29. 1905. Ueber die Homologie der Anamnierkiemen. Anat. Anz. 28. 1906.

Guieysse, A., Étude de la régression de la queue chez les tétards des Amphibiens anoures. Arch. d'Anat. Micr. 7. 1905.

HaLl, R. W., The Development of the Mesonephros and the Müllerian Ducts in Amphibia. Bull. Mus. Comp. Zool. Harvard College. 45. 1904.

Hamecher, H. Jr., Ueber die Lage des kopfbildenden Teils und der Wachsthumszone für Rumpf und Schwanz (Fr. Kopsch) zum Blastoporusrande bei Rana fusca. Intern. Monatschr. Anat. Phys. 21. 1904.

Harrison, R. G., Experimentelle Untersuchungen über die Entwicklung der Sinnesorgane der Seitenlinie bei den Amphibien. Arch. mikr. Anat. 63. 1904.

Hewd, H., Entwickelung des Nervengewebe bei den Wirbeltiere. Leipzig. 1909.

Hempstead, M., Development of the Lungs in the Frogs, Rana catesbiana, R. sylvatica and $R$. virescens. Science. 12. 1901.

Hertwig, O., Die Entwicklung des mittleren Keimblattes der. Wirbelthiere. Jena. Zeit. $16(9) .1883$.

Hertwig, O., Editor, Handbuch der vergleichenden und experimentellen Entwickelungslehre der Wirbeltiere. Jena. 1906. (19011906).

Hinsberg, V., Die Entwickelung der Nasenhöhle bei Amphibien. Arch. mikr. Anat. 58. 1901.

Hochstetter, F., Beiträge zur vergleichenden Anatomie und Entwicklungsgeschichte des Venensystems der Amphibien und Fische. Morph. Jahrb. 13. 1887. See also Hertwig, Handbuch, etc.

Hoyer, H., Ueber das Lymphgefässsystem der Froschlarven. Verh. Anat. Ges. 19. Anat. Anz. 27. 1905. Untersuchungen über das Lymphgefässsystem der Froschlarven. II. Bull. Acad. Sci. Cracovie. 1908.

IKedA, S., Contributions to the Embryology of Amphibia: The Mode of Blastopore Closure and the Position of the Embryonic Body. Jour. Coll. Sci. Imp. Univ. Tokyo. 17. 1902.

Jenkinson, J. W., On the Relation between the Symmetry of the Egg 
and the Symmetry of Segmentation and the Symmetry of the Embryo in the Frog. Biometrika. 7. 1909.

Johnston, J. B., The Nervous System of Vertebrates. Philadelphia. 1906.

Keibel, F., See Hertwig, Handbuch, etc.

Knower, H. McE., The Origin and Development of the Anterior Lymph Hearts and the Subcutaneous Lymph Sacs in the Frog. Anat. Record. 2. 1908.

Konopacka, M., Die Gestaltungsvorgänge der in verschiedenen Entwicklungsstadien centrifugirten Froschkeime. Bull. Acad. Sci. Cracovie. 1908.

Kopsch, F., Beiträge zur Gastrulation beim Axolotl- und Froschei. Verh. Anat. Ges. 9. Anat. Anz. 10. 1895. Ueber das Verhältnis der embryonalen Axen zu den drei ersten Furchungsebenen beim Frosch. Intern. Monatschr. Anat. Phys. 17. 1900.

Котне, K., Entwicklungsgeschichtlichle Untersuchungen über das Zungenbein und die Ohrknöchelchen der Anuren. Arch. Naturgesch. 76. 1910.

Krause, W., See Hertwig, Handbuch, etc.

Kupffer, K. von, See Hertwig, Handbuch, etc.

Kuschakewitsch, S., Die Entwicklungsgeschichte der Keimdrüsen von Rana esculenta. Ein Beitrag zum Sexualitätsproblem. Festschr. f. R. Hertwig. Jena. 1910.

Lams, H., Contribution a l'étude de la genèse du vitellus dans l'ovule des Amphibiens (Rana temporaria). Arch. d'Anat. Micr. 9. 1907.

LEBRUN, H., La vésicule germinative et les globules polaires chez les Anoures. Cellule. 19. 1902.

McClendon, J. F., Cytological and chemical Studies of Centrifuged Frog Eggs. Arch. Entw.-Mech. 27. 1909. On the Effect of Centrifugal Force on the Frog's Egg. Arch. Zellf. 5. 1910.

Marcelin, R. H., Histogénèse de l'épithélium intestinal chez la Grenouille (Rana esculenta). Revue Suisse Zool. 11. 1903.

Marshall, A. M., Vertebrate Embryology. London and New York. 1893.

Marshall, A. M. and Bles, E. J., The Development of the Kidneys and Fat-bodies in the Frog. Studies Biol. Lab. Owens College. 2. 1890. The Development of the Blood-vessels in the Frog. Studies Biol. Lab. Owens College. 2. 1890.

Maurer, F., Schilddrüse, Thymus und Kiemenreste der Amphibien. Morph. Jahrb. 13. 1887. Die Kiemen und ihre Gefässse bei anuren und urodelen Amphibien, und die Umbildungen der beiden ersten Arterienbogen bei Teleostiern. Morph. Jahrb. 14. 1888. Die erste Anlage der Milz und das erste Auftreten von lymph- 
atischen Zellen bei Amphibien. Morph. Jahrb. 16. 1890. See also, Hertwig, Handbuch, etc.

Maximow, A., Ueber embryonale Entwickelung der Blutzellen bei

Selachiern und Amphibien. Verh. Anat. Ges. 24. Anat. Anz.

37. 1910

Mollier, S., See Hertwig, Handbuch, etc.

Morgan, T. H., The Formation of the Embryo of the Frog. Anat.

Anz. 9. 1894. Half-embryos and Whole-embryos from One of the First Two Blastomeres of the Frog's Egg. Anat. Anz. 10. 1895. The Development of the Frog's Egg. An Introduction to Experimental Embryology. New York. 1897. The Relation between Normal and Abnormal Development of the Embryo of the Frog. X. A Re-examination of the Early Stages of Normal Development from the Point of View of the Results of Abnormal Development. Arch. Entw.-Mech. 19. 1905. Experiments with Frog's Eggs. Biol. Bull. 11. 1906. The Origin of the Organforming Materials in the Frog's Embryo. Biol. Bull. 11. 1906. Morgan, T. H. and Tsuda, U., The Orientation of the Frog's Egg. Q. J. M. S. 35.1894.

Moser, F., Beiträge zur vergleichenden Entwicklungsgeschichte der Wirbeltierelunge (Amphibien, Reptilien, Vögel, Sänger). Arch. mikr. Anat. 60. 1902.

Neumayer, L., See Hertwig, Handbuch, etc.

Norris, H. N., The Origin of the so-called "ventraler Kiemenrest" and of the Corpus propericardiale of the Frog. Anat. Anz. 21. 1902. Nussbaum, M., Zur Mechanik der Eiablage bei Rana fusca. Arch. mikr. Anat. 46. 1895.

Oeder, R., Die Entstehung der Munddrüsen und der Zahnleiste der Anuren. Jena. Zeit. 41. (34). 1906.

Peter, K., See Hertwig, Handbuch, etc.

Poll, H., See Hertwig, Handbuch, etc.

RabL, C., Theorie des Mesoderms. Morph. Jahrb. 15. 1889. Ueber den Bau und die Entwickelung der Linse. I. Selachier und Amphibien. Zeit. wiss. Zool. 63. 1898. III. Säugethiere, Rückblick und Schluss. Zeit. wiss. Zool. 67. 1899.

Radford, M., Development of the Spleen. Jour. Anat. Physiol. 42. 1908.

Radwańska, M., Die vorderen Lymphherzen des Frosches. Bull. Acad. Sci. Cracovie. 1906.

Robinson, A. and Assheton, R., The Formation and Fate of the

Primitive Streak, with Observations on the Archenteron and

Germinal Layers of Rana temporaria. Q. J. M. S. 32. 1891.

Roux, W., Beiträge zur Entwickelungsmechanik des Embryo. Nr. 4.

Die Richtungsbestimmung der Medianebene des Froschembryo 
durch die Copulationsrichtung des Eikernes und des Spermakernes. Arch. mikr. Anat. 29. 1887. Ueber die Lagerung des Materials des Medullarrohres im gefurchten Froschei. Verh. Anat. Ges. 2. Anat. Anz. 3. 1888. Ueber die ersten Teilungen des Froscheies und ihre Beziehungen zu der Organbildung des Embryo. Anat. Anz. 8. 1893.

Rufrini, A., L'ameboidismo e la secrezione in rapporto con la formazione degli organi e con lo sviluppo delle forme esterne del corpo. Anat. Anz. 33. 1908.

Schanz, F., Das Schicksal des Blastoporus bei den Amphibien. Jena. Zeit. 21 (14). 1887.

Schauinsland, H., See Hertwig, Handbuch, etc.

Schmalhausen, J. J., Die Entwickelung des Skelettes der vorderen Extremität der anuren Amphibien. Anat. Anz. 31. 1907. Dio Entwickelung des Skelettes der hinteren Extremität der anuren Amphibien. Anat. Anz. 33. 1908.

Schmitt-Marcel, W., Ueber Pseudo-Hermaphroditismus bei Rana temporaria. Arch. mikr. Anat. 72. 1908.

Schultze, O., Untersuchungen über die Reifung und Befruchtung des Amphibieneies. I Zeit. wiss. Zool. 45. 1887. Die Entwicklung der Keimblätter und der Chorda dorsalis von Rana fusca. Zeit. wiss. Zool. 47. 1888. Ueber das erste Auftreten der bilateralen Symmetrie im Verlauf der Entwicklung. Arch. mikr. Anat. 55. 1900. Ueber die Nothwendigkeit der freien Entwicklung des Embryo. Arch. mikr. Anat. 55. 1900.

Schwink, F., Ueber die Gastrula bei Amphibieneiern. Biol. Centr. 8. 1888. Ueber die Entwickelung des mittleren Keimblattes und der Chorda dorsalis der Amphibien. München. 1889. Untersuchungen über die Entwicklung des Endothels und der Blutkörperchen der Amphibien. Morph. Jährb. 17. 1891.

Shore, T. W., On the Development of the Renal-portals and Fate of the Posterior Cardinal Veins in the Frog. Jour. Anat. Physiol. 36. 1901.

Spemann, H., Ueber die erste Entwickelung der Tuba Eustachii und des

Kopfskelets von Rana temporaria. Zool. Jahrb. 11.1898.

SRdínko, O. V., Bau und Entwickelung der Nebenniere bei Anuren. Anat. Anz. 18. 1900.

StöHr, P., Ueber die Entwicklung der Hypochorda und des dorsalen

Pancreas bei Rana temporaria. Morph. Jahrb. 23. 1895.

Tretjakoff, D., Die vordere Augenhälfte des Frosches. Zeit. wiss. Zool. 80. 1906.

TschernofF, N. D., Zur Embryonalentwickelung der hinteren Extremitäten des Frosches. Anat. Anz. 30. 1907.

Ussow, S. A., Vergleichend-embryologische Studien des axialen Skel- 


\section{OUTLINES OF CHORDATE DEVELOPMENT}

ettes. Stomodaeum-Ektochorda (das vordere Ende der Chorda). Anat. Anz. 35. 1909.

Villy, F., The Development of the Ear and Accessory Organs in the Common Frog. Q. J. M. S. 30. 1890.

Weber, A., Étude de la torsion de l'ébauche cardiaque chez Rana esculenta. Bibliographie Anatomique. 18. 1908 (1909).

Wetzel, G., Der Wassergehalt des fertigen Froscheies und der Mechanismus der Bildung seiner Hülle im Eileiter. Arch. Entw.-Mech. 26. 1908.

Weysse, A. W., Ueber die ersten Anlagen der Hauptanhangsorgane des Darmkanals beim Frosch. Arch. mikr. Anat. 46. 1895.

Wilson, H. V., Formation of the Blastopore in the Frog Egg. Anat. Anz. 18. 1900. Closure of Blastopore in the normally placed Frog Egg. Anat. Anz. 20. 1902.

Wintrebert, P., Sur le déterminisme de la métamorphose chez les Batraciens anoures. Series of articles in C. R. Soc. Biol. Paris. 62, 63, 65. 1907-1908.

ZiegLeR, F., Zur Kenntnis der Oberflächenbilder der Rana-Embryonen. Anat. Anz. 7. 1892.

ZiegLeR, H. E., Lehrbuch der vergleichenden Entwickelungsgeschichte der niederen Wirbeltiere. Jena. 1902.

Zwick, W., Beiträge zur Kenntnis des Baues und der Entwicklung der Amphibiengliedmassen, besonders von Carpus und Tarsus. Zeit. wiss. Zool. 63. 1897. 
CHAPTER IV

\section{THE EARLY DEVELOPMENT OF THE CHICK}

THE EMBRYONIC MEMBRANES AND APPENDAGES

INTRODUCTION .

I. THE EGG AND ITS PRODUCTION . . . . . . . . . . 232

1. The Egg . . . . . . . . . . . . . . . . . . . . . . 232

2. The Reproductive Organs of the Fowl . . . . . . . . . . 234

3. The Formation of the Egg and its Early Development . . . 236

A. The History of the Ovarian Ovum to Ovulation . . 236

B. The Period from Ovulation through Fertilization . . 240

C. From the Beginning of Cleavage to the Time of Laying 241

D. Gastrulation . . . . . . . . . . . 248

II. THE FORMATION OF THE EMBRYO . . . . . . . . . . 251

III. THE EARLY DEVELOPMENT OF THE EMBRYO . . . . 260

1. The Head-fold . . . . . . . . . . . . . . . . . 260

2. Mesoderm . . . . . . . . . . . . . . 262

3. Endoderm . . . . . . . . . . . . . . . . 267

4. The Nervous System . . . . . . . . . . . . . . . 269

5. The Vascular System . . . . . . . . . . 271

IV. THE EMBRYO OF ABOUT THIRTY HOURS (10-12 PAIRS OF SOMITES) . . . . . . . . . . . . . . . 275

V. THE SEPARATION OF THE EMBRYO FROM THE EXTRAEMBRYONIC STRUCTURES . . . . . . . . . . . . . 279

VI. THE ESTABLISHMENT OF THE EXTERंNAL FORM OF THE EMBRYO . . . . . . . . . . . . . . . . . . 282

VII. THE EMBRYONIC MEMBRANES AND APPENDAGES . . 286

1. The Yolk-sac . . . . . . . . . . . . . . . . . . 286

2. The Amnion . . . . . . . . . . . . . . . . . . . . 292

3. The Allantois . . . . . . . . . . . . . . . 295

MANY of the great embryological classics are based upon the development of the chick. The pioneer works of Harvey, of Malpighi, of Wolff, Pander, and Von Baer, bear witness to the fact that answers to many of the fundamental problems of the science of embryology were sought in the development of this 
form; and from its study came many of the long dominant conceptions of the process, as well as the morphology, of development.

To-day, when the development of the chick is better known, as a whole, than that of.perhaps any other vertebrate, it remains an extremely important subject, not so much because of its historical importance, nor because of its very great practical convenience on account of its abundance, ease of manipulation, and freedom from seasonal limitations in its use, as for other more significant reasons.

The development of the chick is fairly typical of the development of the members of the largest and most important Chordate division, the Sauropsida. And besides, it suggests interpretations of many of the very special features of mammalian development. The egg of the fowl represents the climax of the process of yolk accumulation, which begins in Amphioxus and steadily increases through the Ganoids, Amphibia, and Elasmobranchs. Consequently we find here, in pronounced form, many interesting phases of the influence of deutoplasm upon development. Indeed so great is the accumulation of yolk here, that it remains no longer contained within the limits of the embryo proper, and instead of exercising a retardative influence upon the rate of development, it becomes so related to the embryo that development is greatly hastened. In this form the presence of the great mass of yolk results chiefly in an extensive modification of the external form of the embryo, particularly during its early phases. The embryo develops for some time as a flat disc, upon the surface of the yolk mass, so that it gives a sort of map-like spherical projection of a Chordate embryo. And the morphological separation of embryo and yolk, freeing the former from the influence of the inert deutoplasm, enables the young chick to proceed to a remarkably advanced stage of development during the three weeks of its brief embryonic life.

Moreover, the chick embryo possesses, in comparatively simple form, certain embryonic membranes and appendages, which, in the Mammal, are highly specialized and come to play 
an important rôle in the modification of embryonic form, and an essential rôle in relating the embryo to the walls of the maternal cavity in which it develops, a relation that is singly the most important distinction of Mammalian development.

As an introduction to the subject we may outline, in a few words, the more striking points in the life of the young chick. Fertilization is internal, following a process of copulation, and after laying, the eggs are brooded by the mother during their three weeks incubation. This ensures, besides protection, the temperature necessary for development. Normally this is about $38^{\circ} \mathrm{C}$.; development ceases entirely at temperatures above $41^{\circ}$, and becomes very slow when it falls to $25^{\circ}$ or even to $28^{\circ}$. Newly laid eggs may remain alive, however, without undergoing any advance, for a considerable period at much lower temperatures than these; development then proceeds when the temperature is raised.

The processes of maturation, fertilization, cleavage, and blastula formation are completed before the ovum leaves the body of the parent, while the egg is passing down the oviduct. Thus the hen's egg, as laid, may be roughly compared to the sced of a plant, in which a simple embryo is already formed and surrounded with nutritive material for its later development.

The chief steps in the formation of the definitive embryo, occur during the first day of incubation, and during the second day a complicated series of folds appear, which largely effect a separation of the embryo from the yolk-mass, with which it then remains in connection by a narrow stalk. At the same time a very extensive circulatory system develops, putting the embryo into relation with its outlying food supply (Fig. 118). Development now becomes very rapid since, through the morphological separation of embryo and yolk, the usual retardative effect of the latter is obviated, while an efficient physiological connection is established through the precocious appearance of the circulation.

During the second and third days of incubation, appear the embryonic membranes and appendages, which provide for respiration, extend the nutritive surfaces, and afford the spaces 
within which the embryo may fully extend. In general the development of the embryo occurs progressively from the anterior end, posteriorly. Thus the brain and other structures of the head are very large and in a fairly advanced stage of development, while the posterior part of the trunk and the tail are still in process of formation. The heart, which appears very far forward, is also a very prominent feature of the early embryo. From about the fifth day the development and enlargement of the body region reduce the relative prominence of the head region, and the typically bird-like form of the embryo is acquired about the eighth day.

On the twentieth day, usually, the chick makes a small perforation through the shell and begins to breathe with its lungs, and on the following day the young chick breaks entirely from the shell. The chick is representative of those birds whose young are "precocious," for it is able to run about actively, to pick up food, and to lead a generally active life, within a few hours after hatching.

\section{THE EGG AND ITS PRODUCTION}

\section{The Egg}

We shall find it convenient to describe first the general structure of the hen's egg in its newly laid condition, although this is not the true ovum; for the "egg" is not laid for some time, usually twenty-one to twenty-three hours, after fertilization, and during these hours the process of cleavage is completed, gastrulation and germ layer formation are well advanced, so that the "egg" already contains a multicellular embryo.

The true ovum, or egg cell proper, is the large yolk, or vitellus, surrounded by a thin but rather tough vitelline membrane (Fig. 85). The extreme animal pole is nearly free from yolk and appears at the time of laying as a circular whitish area, the blastoderm, 3-4 mm. in diameter: this pole is the less dense and is therefore turned upward when the vitellus is free to rotate. The blastoderm of this stage will be fully described later, but we may now distinguish in it two regions, a central translucent 
area pellucida, and a whitish peripheral area opaca. The great mass of the ovum or vitellus is composed of the deutoplasm or "yolk," of which two forms are present, known as white and yellow yolk. The white yolk occupies the region just beneath the blastoderm, and extends thence as a flask-shaped mass, to

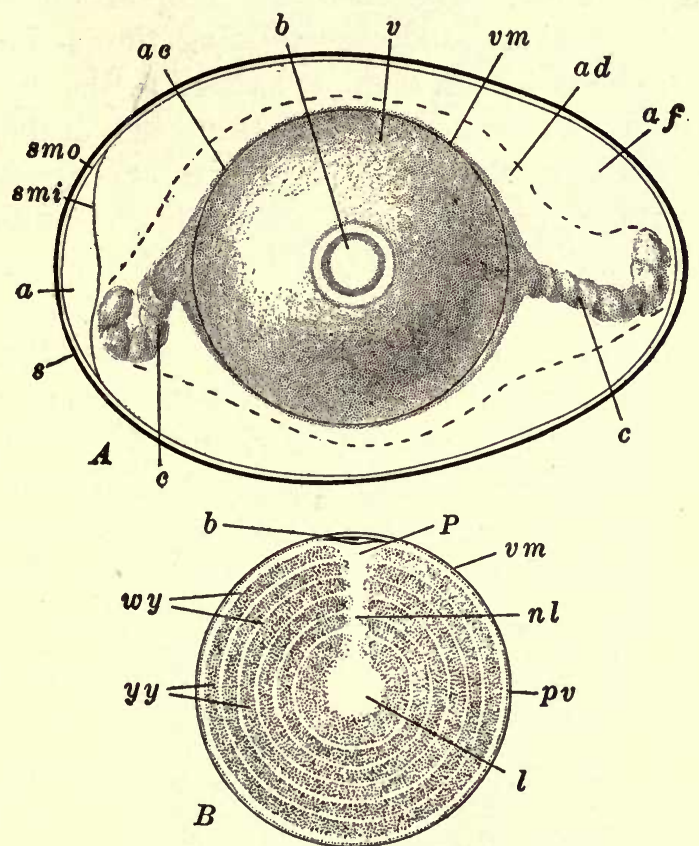

Frg. 85.-Semidiagrammatic illustration of the hen's egg at the time of laying A. Entire "egg." Modified from Marshall. B. Diagram of a vertical section through the vitellus or ovum proper, showing the concentric layers of white and yellow yolk. $a$, Air chamber; $a c$, chalaziferous layer of albumen; ad, dense layer of albumen; af, fluid layer of albumen; $b$, blastoderm; $c$, chalaza; $l$, latebra; $n l$, neck of latebra; $P$, nucleus of Pander; $p v$, perivitelline space; $s m i$, inner layer of shell membrane; smo, outer layer of shell membrane; $v$, vitellus or "yolk"; $v m$, vitelline membrane; $w y$, layers of white yolk; $y y$, layers of yellow yolk.

the center of the whole vitellus; surrounding this the deutoplasm is arranged in several concentric layers, thick layers of the yellow yolk alternating with thinner strata of white yolk (Fig. $85, B)$. These two forms of yolk differ in physical characteristics other than color, and also in chemical composition; both are made up of yolk spheres or plates, which in the white yolk are smaller and quite variable in size and form. 
Surrounding the vitellus or egg cell, are several nutritive and protective egg membranes, all of the tertiary class, i.e., formed by the accessory reproductive organs, the vitellus alone having been formed within the ovary; there are no secondary or chorionic membranes. Immediately surrounding the vitellus is the "white" or albumen. The chemical composition of this is quite complex, various albumens forming the predominating constituents. Two denser, opaque, twisted cords, the chalazoe, extend through the albumen from opposite sides of the vitellus, toward the apices of the shell. These are continuous with a very thin, dense layer of albumen surrounding the vitellus, the chalaziferous layer. Outside of this the albumen forms a rather thick dense layer, and superficially there is a still thicker layer of more fluid albumen. In the hard-boiled egg the albumen can often be seen to be laid around the vitellus in spiral sheets.

The entire ovum is enclosed in a definite ovoid shell, very resistant to pressure applied gradually, though easily broken by a sharp blow. The shell is covered superficially by a thin structureless cuticle perforated by numerous pores. The chief substance of the shell is composed of loosely arranged particles of the carbonates and phosphates of calcium and magnesium, in an organic matrix. The inner surface of the shell is formed by a thinner but denser layer of inorganic salts. The dried shell is very porous and affords an easy pathway for the passage of gases and water vapor.

Lining the shell is the tough shell membrane. This is a double sheet of fibrous connective tissue; at the blunter end of the shell its two layers are separated by an air space, which is often of considerable size in eggs that have been laid for some time.

\section{The Reproductive Organs of the Fowl}

A better understanding of the structure of the egg can be had from the study of its formation, but first we must review the main facts regarding the reproductive system of the fowl. These organs are asymmetrically developed, those of the right side 


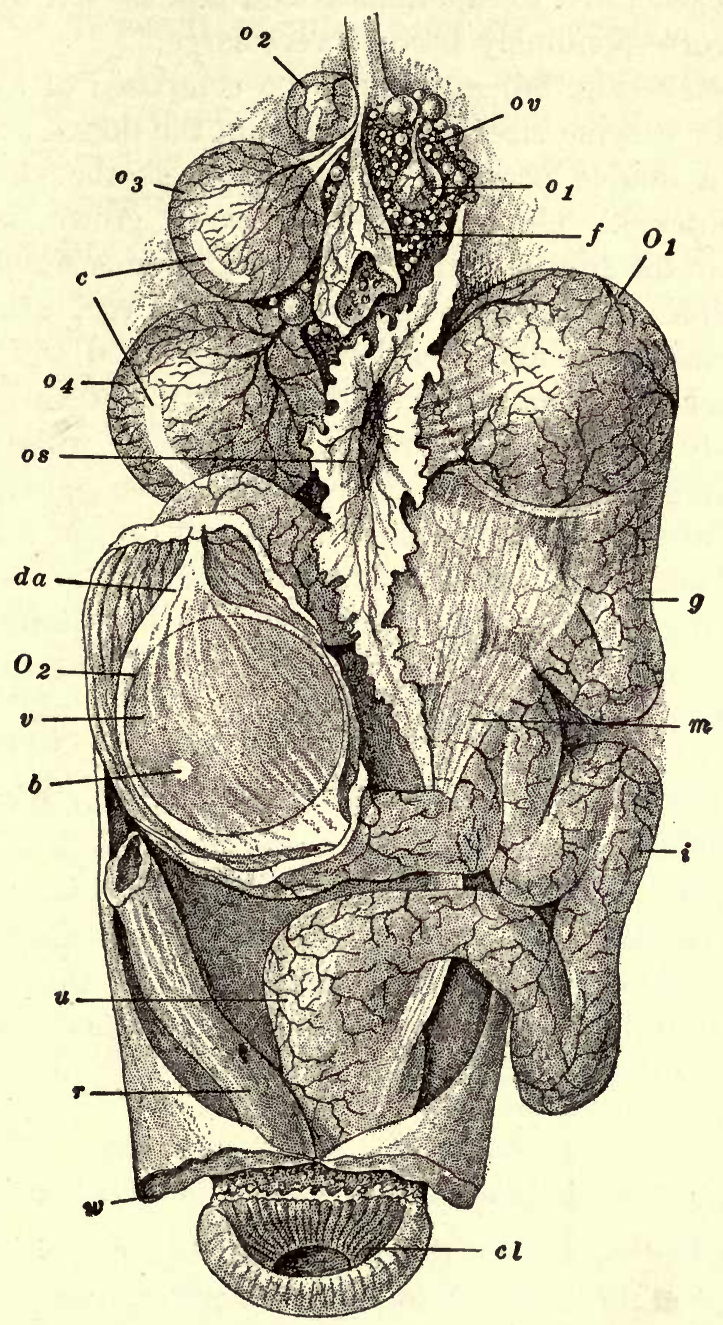

FIG. 86. - The reproductive system of the fowl. After Duval (Coste). The figure shows two eggs in the oviduct, whereas normally only one egg is in the oviduct at a time. $b$, Blastoderm; $c$ cicatrix; $c l$, cloaca; $d a$, dense layer of albumen; $f$, empty egg follicle from which the ovum has escaped; $g$, glandular portion of oviduct; $i$, isthmus; $m$, mesovarium; $o_{1}-o_{4}$, ovarian ova in various stages of growth; $O_{1}$, ovum in upper end of oviduct; $O_{2}$, ovum in middle portion of oviduct (the oviduct has been cut open to show the structure of this ovum); os, ostium or infundibulum; $o v$, ovary containing ova in various stages of growth; $r$, rectum; $u$, uterus; $v$, vitellus; $w$, ventral body wall, opened and reflected. 
having degenerated to functionless vestiges; the left ovary and oviduct correspondingly become very large.

The ovary (Fig. 86) appears to be composed of a mass of globules of varying size, suspended from the dorsal abdominal wall by a double peritoneal fold known as the mesovarium (cf. mesentery). These globules are partly grown, immature ova, and in the adult hen they vary in size from a simple cell up to the full-sized vitellus. The oviduct is a large, rather thickwalled, muscular tube, considerably convoluted and showing a well-marked regional differentiation. Its upper end opens out of the body cavity from the region of the ovary, while its lower end discharges directly into the cloaca. Three general regions are to be distinguished: the first, or oviduct proper, is the most extensive and is itself divisible into three sections. The abdominal opening of the oviduct is a wide, flaring, funnel-shaped opening called the ostium, or infundibulum, or sometimes the fimbriated opening, from its fringe-like margin; its walls are thin and muscular, and it is lined internally with cilia. The ostium leads directly into the long convoluted glandular portion, and this is followed by the short third section of the true oviduct, the $i s t h m u s$. The second general region is the so-called uterus, a dilated portion, also with glandular walls. The short terminal region is a thin-walled vagina, which opens into the cloaca just dorsally to the opening of the rectum. The functional characteristics of these regions will be described in the following paragraphs.

\section{The Formation of the Egg and its Early Development}

The early developmental history of the egg may conveniently be described in three periods: $(A)$ ovarian development to the time of ovulation; $(B)$ from ovulation through fertilization; $(C)$ from the beginning of cleavage to the time of laying.

\section{A. THE HISTORY OF THE OVARIAN OVUM TO OVULATION}

The rhythm of egg production in the domestic fowl is unusual in that, as a rule, a long period of egg formation and laying, extending over several months, is followed by a relatively 
brief period of nearly or quite complete cessation. This is quite in contrast to the great majority of animals, in which a large number of ova are produced within a very brief period, a condition probably correlated with the very large size of the ova, for the formation of even a single egg requires a considerable expenditure of energy and substance. Moreover, there is space in the organs of reproduction for but a very few ova of such large dimensions. Such a succession in the formation of the ova makes it possible to observe, in a single ovary, most of the steps in the formation of the fully grown ovum.

The first phase of oögenesis, the multiplication of the oögonia, occurs during the embryonic life of the chick, and is practically completed by the time of hatching. All of the ova produced later, during the period of adult life, are thus in the form of primary oöcytes at the "birth" of the chick. Surrounded by the non-germinal cells of the "germinal" epithelium, the oögonia or primitive ova multiply rapidly. Some of them leave the epithelium and migrate into the stroma of the ovary where they degenerate. The remaining oögonia, which commence to enlarge while still continuing their multiplication, together with the rapidly proliferating epithelial cells, then form elongated strands or cords, extending from the epithelium into the stroma. Soon these definitive oögonia cease multiplication and are then to be termed primary oöcytes (Fig. $87, A$ ). The strands then break up into cell groups or "nests," each consisting of a single primary oöcyte surrounded by a number of the original epithelial cells; these latter take up a definite epithelial arrangement around the oöcyte, and thus form the primitive egg follicle (granulosa cells). This arrangement of the cells occurs a few days after hatching. The structure of the oöcyte follicle at this age is shown in Fig. 87, $B$.

The egg cell, both nucleus and cytoplasm, now begins to enlarge and deutoplasmic granules are laid down all around the centrally located nucleus, and throughout the cytoplasm, except. in its peripheral region which remains comparatively free from yolk. This peripheral protoplasmic layer is definitely thickened at one point, namely, toward the attached surface of the ovum 
or follicle, forming there the germinal disc or spot. When the ovum reaches a diameter of something more than $0.5 \mathrm{~mm}$. the nucleus migrates into the germinal disc, where it remains
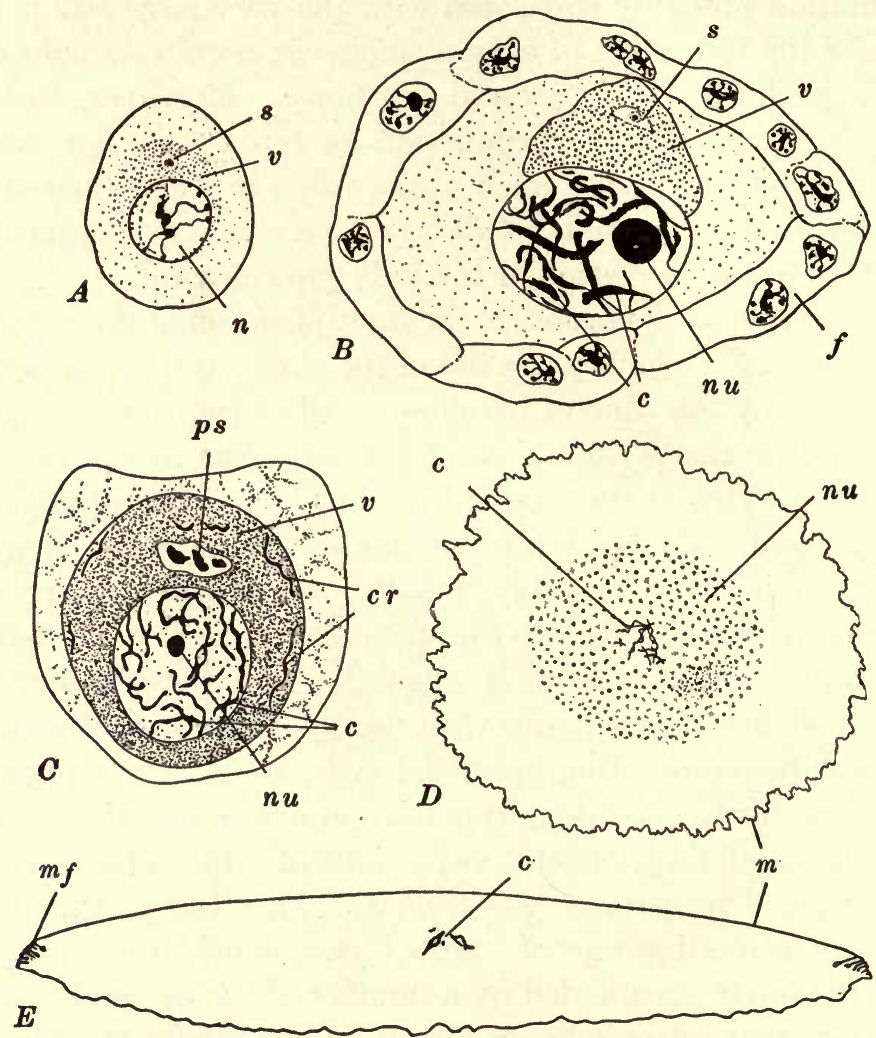

Fra. 87.-Growth stages in the oögenesis of the hen's egg. After Sonnenbrodt. A. Oöcyte measuring $0.012 \times 0.016 \mathrm{~mm}$., the nucleus of which is 0.006 $\mathrm{mm}$. in diameter. $B$. Oöcyte measuring $0.018 \times 0.028 \mathrm{~mm}$., the nucleus of which is $0.0105 \times 0.014 \mathrm{~mm}$. Enclosed in follicle. $C$. Oöcyte measuring $0.040 \times 0.045$ $\mathrm{mm}$., the nucleus of which is $0.020 \times 0.022 \mathrm{~mm}$. $D$. The nucleus only, of an oöcyte measuring $5.84 \times 6.16 \mathrm{~mm}$., the nucleus itself measuring $0.214 \times 0.238 \mathrm{~mm}$. Total view showing the small chromosomes in the midst of a collection of chromatin nucleoli. $E$. Vertical section through the nucleus only, of an oöcyte, the follicle of which measured $37 \mathrm{~mm}$. in diameter. The nucleus itself is $0.455 \mathrm{~mm}$. in diameter and $0.072 \mathrm{~mm}$. in greatest thickness. $c$, Chromosomes; $c r$, extranuclear chromsome-like bodies; $f$, follicle; $m$, nuclear membrane; $m f$, folds in nuclear membrane; $n$, nucleus; $n u$, chromatin nucleolus; $p s$, pseudochromosomes; $s$, centrosphere; $v$, yolk nucleus or vitellogenous body.

throughout the remainder of its ovarian history (Fig. 88). It should be noted that the eccentricity of the nucleus, which 
marks the animal pole of the ovum, is toward the attached surface.

The formation and accumulation of yolk now become more rapid. The surface of the ovum is in the form of a zona radiata, through the pores of which nutritive substances diffuse from

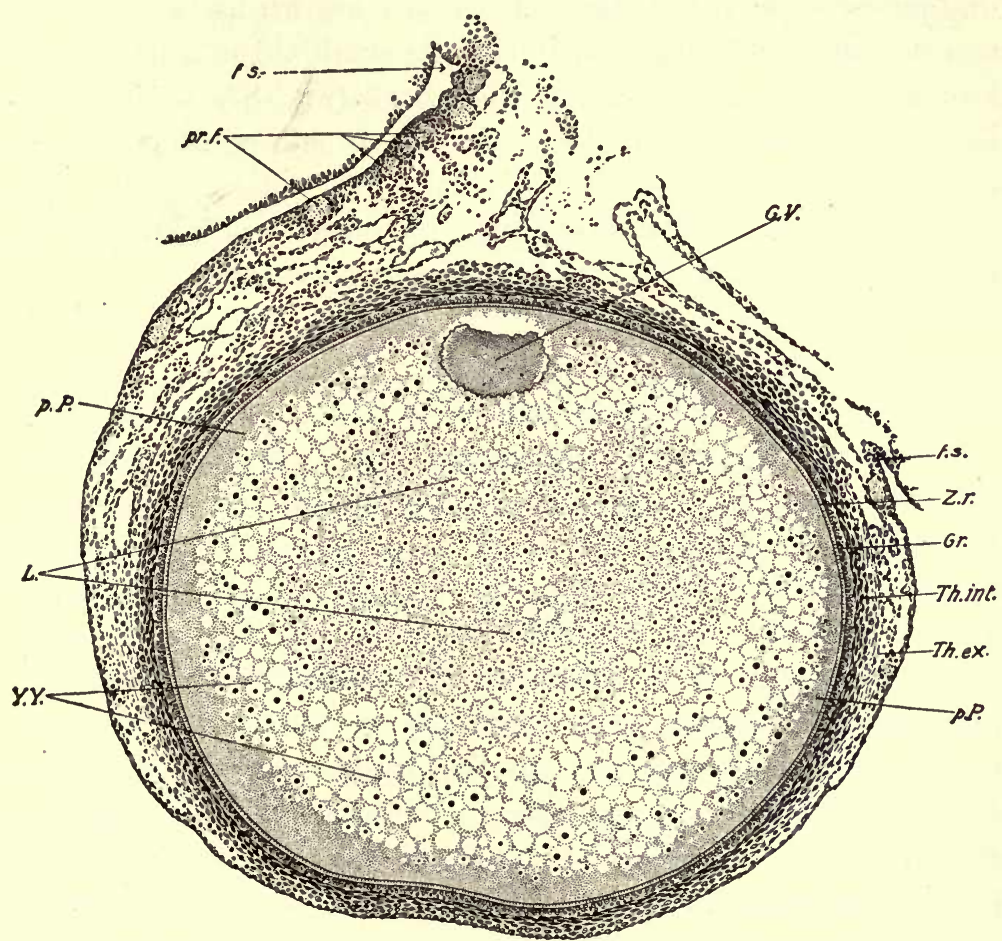

FIG. 88.- Section through the pigeon's ovarian ovum, $1.44 \times 1.25 \mathrm{~mm}$. From Lillie (Development of the Chick). f.s., Stalk of follicle; G.V., germinal vesicle or nucleus; Gr., granulosa cells; L., latebra; p.P., peripheral protoplasm; pr.f., primordial follicles; Th.ex., theca externa; Th. int., theca interna; Y.Y., yellow yolk; Z.r., zona radiata.

the follicle cells, which thus function as nurse cells (Fig. 88). The rate of growth of the egg during its final stages may be determined by the arrangement of the concentric layers of white and yellow yolk (Fig. 85, B), which mark daily periodicities in the formation of deutoplasm (Riddle). Toward the close of the growth period the follicle becomes more clearly membranous, and along the side opposite its attachment, which is 
comparatively non-vascular, a modified band appears; this is the cicatrix, where the follicle ruptures when the ovum escapes from the ovary.

Just before the egg leaves the ovary its nucleus, lying flattened against the vitelline membrane, reaches the enormous diameter of about $0.3 \mathrm{~mm}$. It is now known as the germinal vesicle, since the condensation of its small chromatin content leaves the nucleus as a large clear spot (Fig. 87, D, E). The last events before ovulation are the breaking down of the nuclear wall and the formation of the first polar spindle. This rotates into position and the primary oöcyte, prepared for its first maturation division, pauses to await ovulation.

\section{B. THE PERIOD FROM OVULATION THROUGH FERTILIZATION}

At the time a completed egg is laid, or very shortly thereafter, the region of the ostium or infundibulum of the oviduct, becomes very active and seems to grasp the ovarian follicle containing the primary oöcyte, through muscular or ciliary action, or both. The follicle then becomes ruptured, apparently by the pressure exerted by the contraction of the infundibulum, or by its pulling away from the region of the ovary, and the oöcyte, contained within the infundibulum, is withdrawn from the follicle, and ovulation is accomplished. In some cases it may be that the follicle is ruptured before it can be grasped by the infundibulum and the freed oöcyte is subsequently received by the fimbriated opening. The oöcyte always enters the infundibulum with its chief axis transverse to the long axis of the oviduct, and this relation is retained during the entire passage of the ovum down the oviduct.

The spermatozoa, after their receipt by the female, make their way to the extreme upper end of the oviduct, where they collect, remaining alive and capable of functioning for two weeks or more. Upon its entrance into the oviduct, therefore, the primary oöcyte becomes bathed in a fluid containing sperm cells, and fertilization immediately ensues. The details regarding the penetration of the spermatozoa are not fully described, 
but it is known that this occurs immediately after ovulation, while the ovum is in the infundibulum. Polyspermy is normal, five to twenty-four spermatozoa having been counted within a single ovum (Patterson). Entrance of the spermatozoa affords the stimulus to the completion of maturation, which offers no unusual features. After the second maturation division the egg nucleus unites with one of the sperm nuclei and the first cleavage spindle is typically established.

\section{FROM THE BEgINNING OF CLEAVAGE TO THE TIME OF LAYING}

Before continuing our account of the development of the ovum we must outline the series of events occurring during the passage of the egg down the oviduct; we shall follow the accounts given by Patterson, and Pearl and Curtis.

The first cleavage furrow appears about three hours after ovulation. During this interval the egg has traversed the entire glandular portion of the oviduct, the walls of which have secreted the denser layers of albumen and the chalazæ (40 to 50 per cent. of the entire weight of albumen). The egg is carried along chiefly by peristaltic contraction of the oviducal wall, and as it passes it is rotated about the long axis of the oviduct, so that the germ dise describes a spiral path; this accounts for the spiral arrangement of the albumen around the yolk. During the next hour or so (Pearl) the egg traverses the isthmus, the walls of which secrete the shell membrane over the surface of the dense albumen as the egg enters this region. The fluid layer of albumen is added while the egg is traversing the isthmus and the upper part of the uterus. The formation of this fluid albumen, which passes through the shell membrane already laid down, is completed five to seven hours after the egg enters the uterus (nine to eleven hours after entering the oviduct). Before this the calcareous shell substance has begun to be laid down on the shell membrane. The egg usually occupies twelve to sixtcen hours longer in completing its passage through the uterus and vagina. At the end of this 
time, twenty-one to twenty-seven hours after ovulation, gastrulation has begun and the egg is ready to be laid. If the completely formed egg reaches the vagina during the middle of the day ( 8 A. M. to 4 P. M.) it may be laid promptly; otherwise it is retained within the vagina until the following day. In the latter case, since development continues during the entire period of its retention, which may be quite prolonged, the embryo may be in a fairly advanced stage when the egg is finally deposited. Thus, variation in the period of retention accounts for the variation in developmental stages observed in different eggs as laid.

TABLE SHOWING THE CHIEF EVENTS IN THE EARLY HISTORY OF THE HEN'S EGG

\begin{tabular}{|c|c|c|c|}
\hline $\begin{array}{l}\text { Hours after } \\
\text { ovulation }\end{array}$ & $\begin{array}{l}\text { Location in } \\
\text { oviduct }\end{array}$ & Action of oviduct & $\begin{array}{c}\text { Action of germ } \\
\text { disc }\end{array}$ \\
\hline 0 & Infundibulum & Reception of ovum & $\begin{array}{l}\text { Maturation and fer- } \\
\text { tilization }\end{array}$ \\
\hline 0 to 3 & $\begin{array}{l}\text { Glandular por- } \\
\text { tion }\end{array}$ & $\begin{array}{l}\text { Secretion of chala- } \\
\text { zæ, chalaziferous, } \\
\text { and dense. layers } \\
\text { of albumen }\end{array}$ & $\begin{array}{l}\text { First cleavage fur- } \\
\text { row }\end{array}$ \\
\hline 3 to 4 & Isthmus & $\begin{array}{l}\text { Secretion of shell } \\
\text { membrane and } \\
\text { fluid albumen }\end{array}$ & $\begin{array}{l}\text { Formation of eight } \\
\text { cells }\end{array}$ \\
\hline 4 to $21(27)$ & $\begin{array}{l}\text { Uterus and } \\
\text { vagina }\end{array}$ & $\begin{array}{l}\text { Secretion of shell } \\
\text { and fluid albumen. } \\
\text { Retention prior to } \\
\text { laying }\end{array}$ & $\begin{array}{l}\text { Gastrulation begun, } \\
\text { or completed if egg } \\
\text { is long retained }\end{array}$ \\
\hline
\end{tabular}

We may now return to describe the processes of development occurring during the period prior to laying. The unicellular germ disc consists of a quite definitely circumscribed area at the animal pole of the vitellus. The disc is about $3 \mathrm{~mm}$. in diameter and less than $0.5 \mathrm{~mm}$. in thickness. Beneath, the 
protoplasm merges with a well-marked region of the white yolk called the nucleus of Pander, which connects with the central white yolk by a narrow stalk called the latebra (Fig. $85, A)$. In the disc itself two regions may be distinguished, a large central area, which is to form the blastoderm proper, and a narrow marginal area of denser appearance, known as the periblast. Peripherally the periblast continues for some distance, perhaps completely, over the surface of the vitellus as an extremely thin protoplasmic layer.

The first cleavage furrow appears as a short shallow groove, near the middle of the germ disc, in length approximately onehalf the diameter of the disc (Fig. 89, A). Sections show that this cleavage also fails to extend completely through the disc vertically (Fig. 90, $A$ ). It is not known that the first cleavage plane coincides with the median sagittal plane of the embryo: indeed it seems probable that, as in other eggs of this extremely meroblastic type, there is no correspondence between these two planes. The position of the main embryonic axis is, however, fairly uniform, though not completely fixed. It lies approximately at right angles to the long axis of the whole egg, the anterior end of the embryo directed to the left, when the sharper end of the egg is held pointing away from the observer. In the few cases actually observed, the first cleavage plane seems to have no definite relation to these axes.

The second cleavage is also vertical, and approximately at right angles to the first, giving four adequal cells, all still incomplete (Fig. 89, B). About an hour after the first cleavage, the third appears. Typically, though not in a majority of instances, this is a fairly regular cleavage, parallel with the first, and dividing the disc into two rows of four cells each. Frequently this cleavage is more or less irregular in form, and the synchronism of division is lost by this time (Fig. 89, $C$ ). The subsequent stage, consequently, may be said to consist only approximately of sixteen cells. These are very irregular, but the general tendency of the fourth plane is to separate each of the eight cells into a central and a distal cell.

During the appearance of the third and fourth cleavages, 
the central portion of the germ disc has become divided horizontally by a cleavage plane, connecting the lower margins of

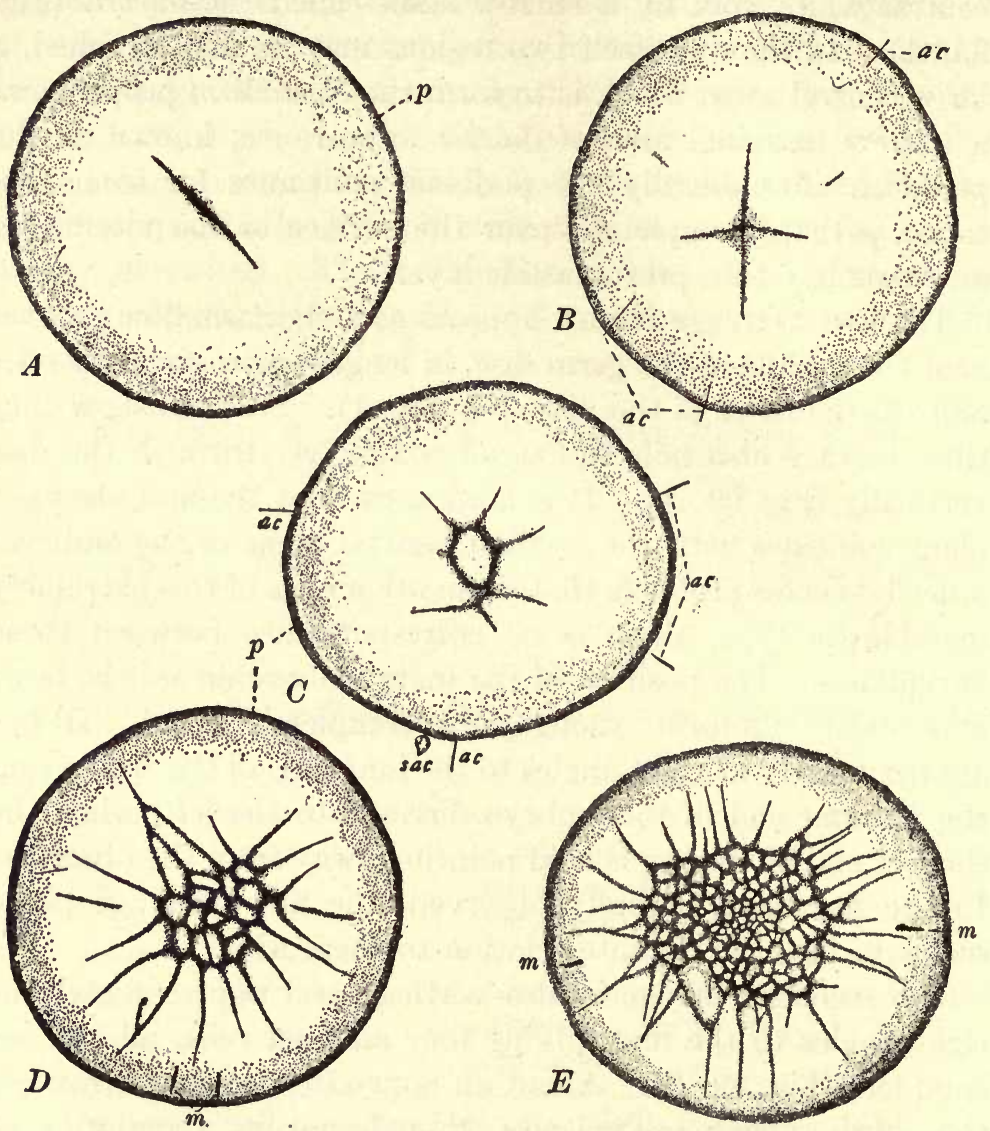

Fig. 89.-Cleavage in the hen's egg. Surface views of the blastoderm and the inner part of the marginal periblast only. From Patterson. The anterior margin of the blastodise is toward the top of the page. A. Two-cell stage. About three hours after fertilization. $B$. Four cells. About three and one-fcurth hours after fertilization. $C$. Eight cells. About four hours after fertilization. $D$. Thirty-four cells. About four and three-fourths hours after fertilization. $E$. One hundred and fifty-four cells upon the surface; the blastoderm averages about three cells in thickness at this stage. About seven hours after fertilization. $a c$, Accessory cleavage furrows; $m$, radial furrow; $p$. inner part of marginal periblast; sac, small cell formed by the accessory cleavage furrows.

the first and second planes. In this way a small group of central cells become completely circumscribed, and are to be 
distinguished from the marginal cells, which remain incomplete both below and distally, retaining their connection with the periblast (Fig. 90, B). A definite though narrow space appears, accompanying the horizontal cleavage, which separates the superficial cellular elements from the underlying undivided
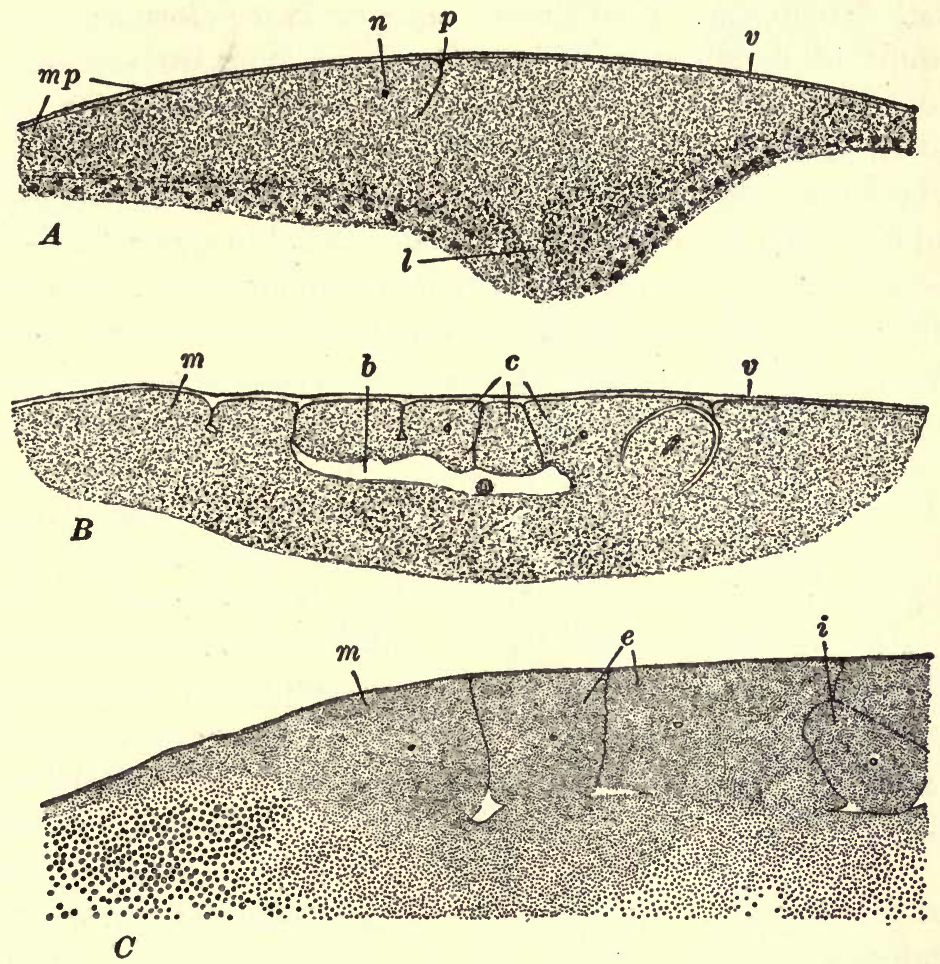

Fig. 90.- Vertical sections through the chick blastoderm during the process of cleavage. After Patterson. A. Section through the two-cell stage. $B$. Median section through the thirty-two cell stage. $C$. Part of a longitudinal section through the sixty-four cell stage. $b$, Blastocœl or segmentation cavity; $c$, central cells; $i$, inner cell cut off by horizontal cleavage plane; $l$, neck of latebra; $m$, marginal cell; $m p$, marginal periblast; $n$, nucleus; $p$, first cleavage; $v$, vitelline membrane.

protoplasm. This space is the beginning of the segmentation cavity or blastocœl; the protoplasm beneath it is termed the central periblast. The original periblast region is now distinguished as marginal periblast. The two periblastic regions 
retain their connection with one another peripherally, in the deeper region of the marginal cells.

We should note here a few details ragarding the history of the accessory or supernumerary spermatozoa. During the period intervening between fertilization and the early cleavages, these form nuclei which migrate to the outlying portion of the blastodisc. There they may divide once or twice, forming small groups of daughter nuclei. These divisions are sometimes accompanied by slight indications of cytoplasmic division, and the short superficial grooves thus formed are known as the accessory cleavages (Fig. 89). These are visible during the fourand eight-cell stages; they are usually radial in direction, lying just across; or without, the margin of the blastodisc. No true cells are formed by such cleavages. The accessory sperm nuclei degenerate rapidly, the accessory cleavages fade out, and by the time thirty-two cells are formed no traces of these structures are left.

During subsequent cleavages the number of central cells increases rapidly, by additions from the dividing marginal cells, and through their own multiplication, which becomes very rapid as the cells diminish in size. Additional horizontal cleavages appear in the central cells, converting the roof of the blastocœl into a layer several cells in thickness (Fig. 90, $C$ ). No cells are added to the germ disc from the floor of the segmentation cavity. The marginal cells become greatly shortened through the continued cutting off of central cells, and finally they are limited to the extreme margin of the blastodisc.

About the time two or three hundred cells are formed, intercellular furrows extend out into the marginal periblast (Fig. 89, $E)$. Up to this time both central and marginal periblast have been entirely free from nuclei, but soon these areas, which are still directly continuous, become converted into a nucleated syncytium. The details of this process have not been described for the chick, but are well known in the pigeon (Blount). In the latter form, when the marginal cells have become reduced to an approximately spherical form by the cutting off of central 
cells, their nuclei continue to divide, while accompanying cytoplasmic divisions are either incomplete or entirely lacking. A large number of free nuclei is thus formed in the margin of the blastodisc. These nuclei, continuing to multiply, wander out into the marginal periblast, converting this into a nucleated though non-cellular tissue. From this region some of the nuclei migrate inward below the blastodisc, converting the central periblast also into a nucleated structure, except in its middle area, above the nucleus of Pander, which appears to remain free from nuclei. The nucleated rim of the periblast forms a part of what is known later as the germ wall.

The circular blastoderm now begins to extend radially, partly on account of the growth of its own cells, and partly (continuing Blount's account) by the addition of cells from the marginal periblast. The region of the original blastodisc now becomes thin-
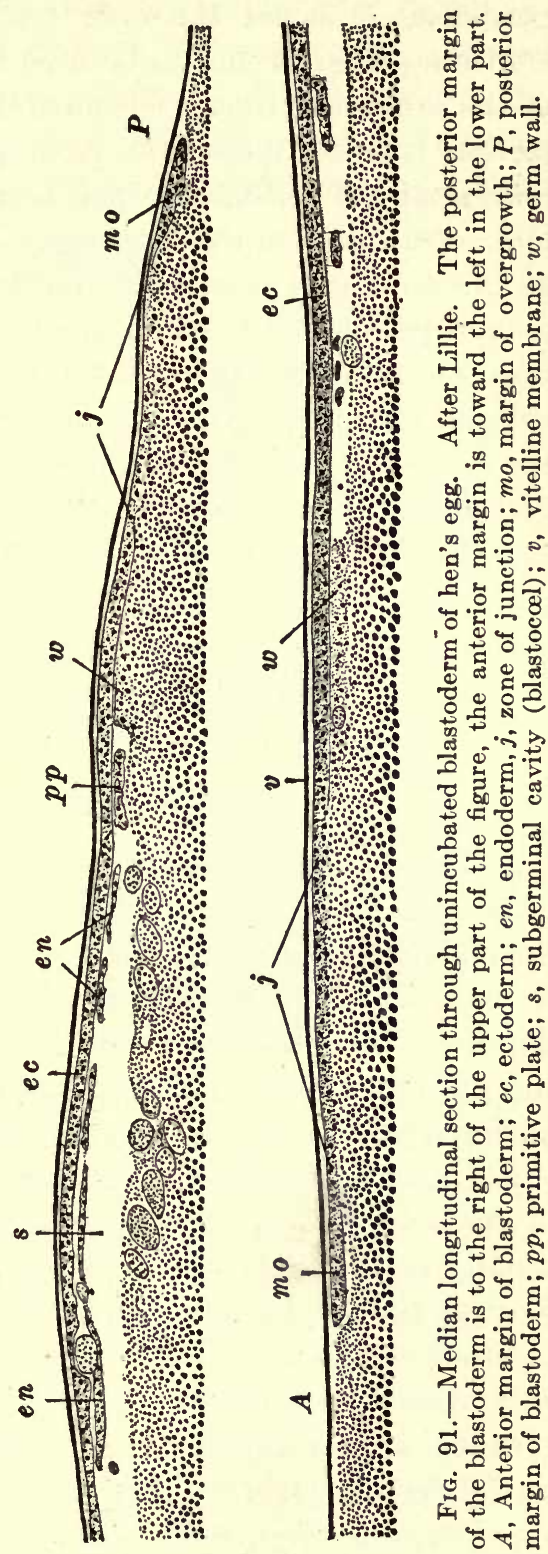
ner and more transparent, and is known as the area pellucida, 
while its circular margin, apparently derived largely from the periblast, is called the area opaca. The ring-like periblast continues to grow and to become nucleated more peripherally, at the same time that it is contributing cells to the blastoderm, so that it steadily increases in diameter. The inner nucleated margin of the periblast, which becomes of cellular composition and contributes to the later extra-embryonic tissues, is known as the germ wall (Fig. 91). Later the cells of the blastoderm extend peripherally, overlapping the inner margin of the germ wall, so that there is a narrow region transitional between pellucid and opaque areas. We should not overlook the fact that the lower surface of the periblast is directly continuous with the yolk-mass, and is peripherally continuous with a very thin superficial layer of protoplasm; this latter is sometimes referred to as a part of the germ wall. The thinning of the blastoderm, mentioned above, may be regarded as indicating the completion of the blastula stage and the beginning of gastrulation, to which we may next turn our attention.

\section{GASTRULATION}

In the chick the formation of the primary germ layers, ectoderm and endoderm, i.e., gastrulation proper, is quite easily distinguished from the processes of notogenesis and mesoderm formation. In the following account of gastrulation many details are supplied from Patterson's account of this process in the pigeon, for this period in the development of the chick is incompletely known, although it is clear that the pigeon and chick are in agreement as to essentials. The thinning of the blastoderm through the rearrangement of its cells, continues rapidly as the diameter of the area pellucida increases. It is most marked in a wedge-shaped area in the posterior part of the blastoderm, the most posterior portion of which may come to be only one cell in thickness (Fig. 92, $A$ ). In this region the subgerminal or segmentation cavity increases in depth, and around the hinder margin of the thinner area the germ wall (area opaca) appears to be interrupted more or less completely, so that the blastoderm cells border directly upon the yolk. 
The first step in the real process of gastrulation is the turning under of a few of the marginal cells in this posterior region of the blastoderm, where it is free from the germ wall. As the involution of superficial cells continues, this margin soon becomes thickened, and since the involuted cells are the rudiment of the endoderm, the thickening represents the lip of the
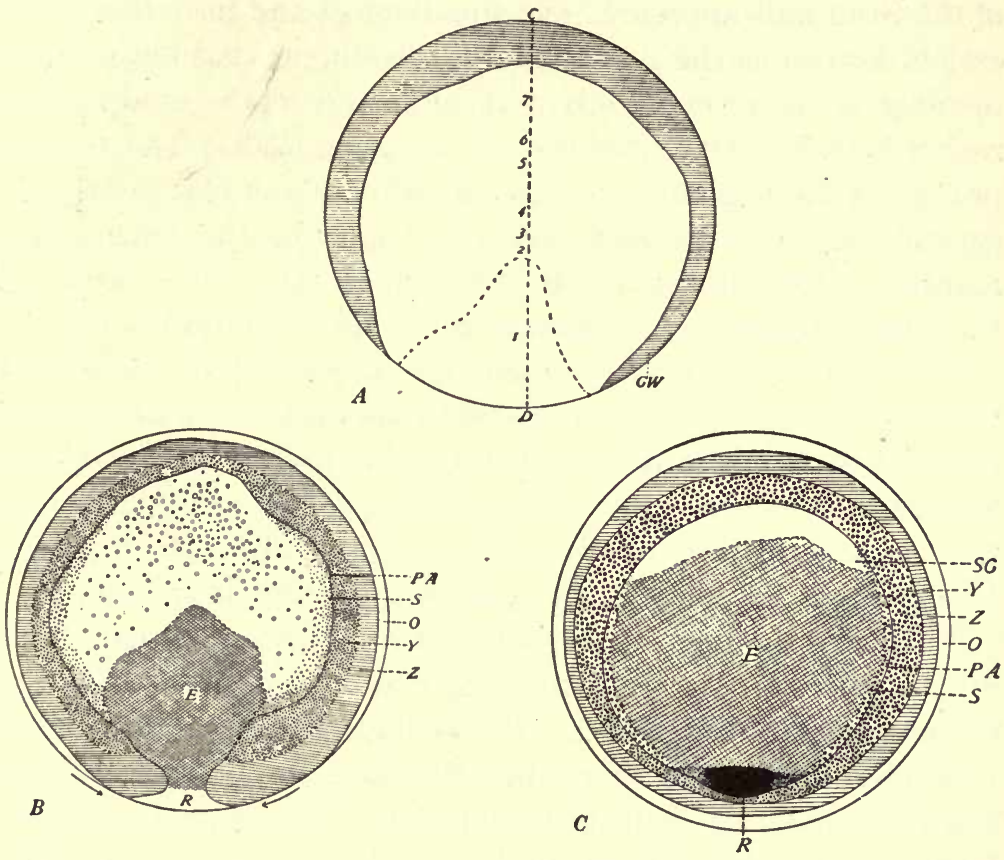

FIG. 92.-Diagrams of reconstructions of the pigeon's blastoderm. From Lillie (Development of the Chick) after Patterson. A. Thirty-one hours after fertilization. $B$. Thirty-six hours after fertilization. $C$. Thirty-eight hours after fertilization. $E$, Gut endoderm; $G W$, germ wall; $O$, margin of overgrowth; $P A$, outer margin of area pellucida; $R$, in $B$, margin of involution; in $C$, mass of cells left after closure of the blastopore; $S$, beginning of yolk-sac endoderm; $S G$, anterior part of subgerminal cavity (blastocœl), as yet free from endoderm; $Y$, yolk zone; $Z$, zone of junction. The numbers $1-7$ along the line $C-D$, in $A$, indicate the number of cells in the thickness of the blastoderm in these regions.

blastopore, which is here reduced to a short crescent, between the two free extremities of the germ wall (Fig. 92, B). Once established, the endoderm rapidly grows forward between the yolk and the ectoderm, as the upper cells of the blastoderm may now be called, extending through the segmentation cavity 
laterally as well as anteriorly (Fig. 91). During these early stages the endoderm cells are considerably scattered, and are arranged as a solid mass or definite layer only in the region of the blastoporal margin. As the endoderm cells gradually extend across the segmentation cavity, two other processes become apparent (Fig. 92, C). First, the free posterior extremities of the germ wall approach, and finally meet and fuse; this process, is known as the closure of the blastopore, the blastoporal opening being present only virtually, and represented by the region between the extremities of the germ wall. The second process is the beginning of the extension of the blastoderm, or gastrula, from every side save the region of the blastoporal margin. The endoderm cells soon extend out to the margin of the segmentation cavity in every direction except anteriorly.

The closure of the blastopore produces a small thickened region, representing the contracted blastoporal margin, which is left just within (anterior to) the germ wall, where this now becomes continuous posteriorly. The process of extension of the blastoderm now involves this region and leaves the contracted blastoporal margin well within the area pellucida.

Normally the egg is laid twenty-two to twenty-three hours after fertilization, in approximately this condition, with gastrulation not quite completed. The structure of the unincubated blastoderm may therefore be described as follows. Three general regions are to be distinguished. The original area pellucida is surrounded by a complete area opaca, and beyond this is a narrow margin where the blastoderm cells are extending over the surface of the yolk. The pellucid area is formed by a layer of ectoderm cells, slightly thickened toward the middle of the area; posteriorly it includes also a sheet of endoderm cells. The endoderm is in the form of a definite layer only toward the posterior margin, elsewhere the endoderm cells are scattered through the segmentation cavity, in the process of migrating toward its anterior margin. A narrow space between the endoderm and yolk, really the remains of the segmentation cavity there, is the rudiment of the archenteron. The area opaca, save in its posterior region, is formed by the thickened 
margin of the blastoderm resting upon the germ wall and fusing peripherally with it. Posteriorly, where the germ wall is narrower, there is a thickened region of the cellular germ disc which represents the contracted blastoporal margin. In this region the ectoderm and endoderm are continuous.

During the first few hours of incubation, or even before laying in cases where the eggs have been retained for some time longer than usual in the vagina, the endoderm extends completely across the segmentation cavity and becomes organized into a fairly definite layer.

From the preceding description it will be seen that the process of gastrulation in the chick is essentially a process of involution. There is no true invagination, and the process of epiboly is not immediately concerned in the establishment of the primary inner layer. Moreover, the process of epiboly is here greatly limited, being restricted to a narrow posterior section of the blastoderm. In other words, the entire blastoporal region is greatly reduced, doubtless in correlation with the excessive amount of yolk in the ovum.

The formation of the middle germ layer and the chief axial structures of the embryo, is not intimately bound up with the gastrulation process, as in the forms previously described. We may now consider these processes in connection with the general development of the whole embryonic region.

\section{THE FORMATION OF THE EMBRYO}

The earliest indication of the true embryo becomes visible during the early hours of incubation, immediately after the entire area pellucida has become two layered, through the complete extension of the endoderm. It appears first as a slightly thickened band, not very well marked, extending directly from a point approximately in the middle of the area pellucida, nearly to its posterior margin. This is called the primitive streak; it is constituted at first solely by a thickening in the ectodermal layer (Fig. 93). Once established, the primitive streak grows very rapidly, chiefly through posterior elongation, 
the anterior end remaining relatively fixed. The blastoderm, too, shares in this elongation and becomes irregularly oval, and then pear-shaped, the blunter end being anterior.
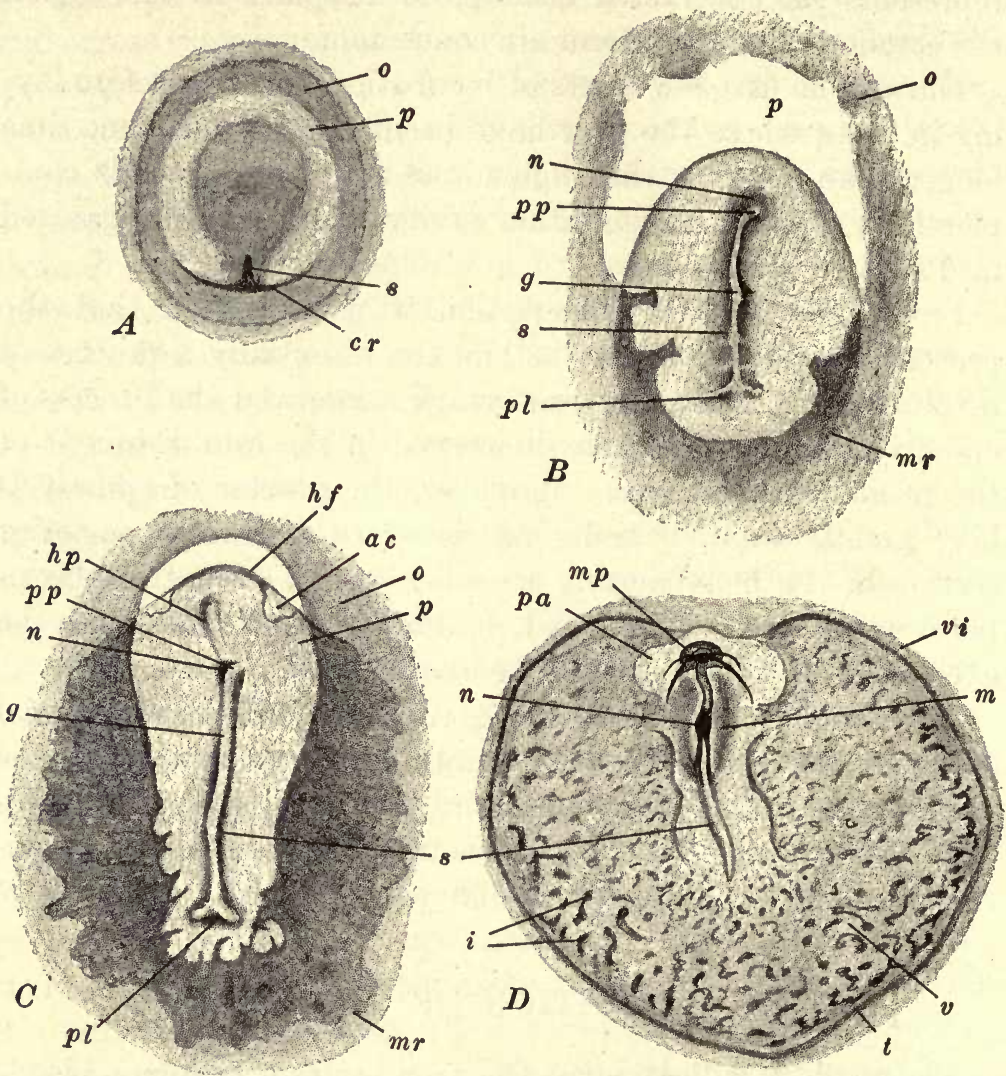

Fig. 93.-Total views of chick blastoderms. $A$. After Duval (modified); $B-D$, after Lillie. $A$. Unincubated blastoderm, with primitive streak just form ing. $B$. Primitive streak formed, head process not yet indicated. $C$. Head process formed; head-fold just commencing. $D$. Just before the establishment of the first mesodermal somites. ac, Amnio-cardiac vesicle; $c r$, crescent-shaped thickening at the posterior side of the blastoderm, in the region of endoderm and mesoderm formation; $g$, primitive groove; $h f$, head-fold; $h p$, head process; $i$, blood islands; $m$, axial thickening of mesoderm; $m p$, medullary plate; $m r$, margin of mesoderm; $n$, Hensen's node; $o$, area opaca; $p$, area pellucida; $p a$, proamnion; $p l$, primitive plate; $p p$, primitive pit; $s$, primitive streak; $t$, sinus terminalis (marginal sinus); $v$, area vasculosa; $v i$, area vitellina interna.

As the primitive streak elongates a narrow transparent line appears along its middle; this is the primitive groove. Anteriorly 
the primitive groove terminates in a small depression, the primitive pit, near the anterior end of the primitive streak, where a slight thickening, known as the primitive knot or Hensen's node, is visible (Fig. 97). In front of this another larger though less definite thickening, known as the head process, soon may be made out. Posteriorly the primitive streak is somewhat transversely extended, just within the border of the area pellucida, forming a more or less well marked crescentic area, through which the primitive groove may be continued, giving this a bifurcated appearance here. Between this broadened hinder end of the primitive streak and the margin of the pellucid area, a wide thickened region can sometimes be discerned; this is known as the primitive plate.

Many important structural details of the primitive streak region may be determined through the study of sections. Figures 94, 101 illustrate sections through representative regions of an early primitive streak. The ectoderm is broadly thickened as the rudiment of the medullary plate, and along the mid-line is the primitive streak, soon marked by the rapid proliferation of ectoderm cells, which are thrown off in the space between ectoderm and endoderm. These cells are the rudiment of the mesoderm. The endoderm forms a uniformly thin sheet of cells across the entire blastoderm.

A little later (Fig. 94, B) the primitive groove is indicated, bordered by a pair of primitive folds. Proliferation of mesoderm cells from the inner surface of the ectoderm is now very rapid, and these cells soon migrate distally throughout a large part of the space between the two primary germ layers. Near the midline they become intimately related with the endoderm, often giving the misleading appearance of having been derived directly from that layer (Fig. 101). Soon the cells along the middle of the primitive streak have multiplied so extensively that they form a dense mass, in which clearly marked limits of the germ layers cannot be made out. More laterally, however, the layers are completely distinct. The ectoderm extends out over the germ wall and yolk; the endoderm, now more than one cell thick, reaches only to the germ wall, with which it fuses, 
the mesoderm forms a loosely arranged sheet of cells, thinning peripherally and extending for a short distance between the ectoderm and germ wall.

The conditions found near the extremities of the primitive streak deserve special mention. Posteriorly, the primitive plate shows a transversely extended region of mesoderm pro-
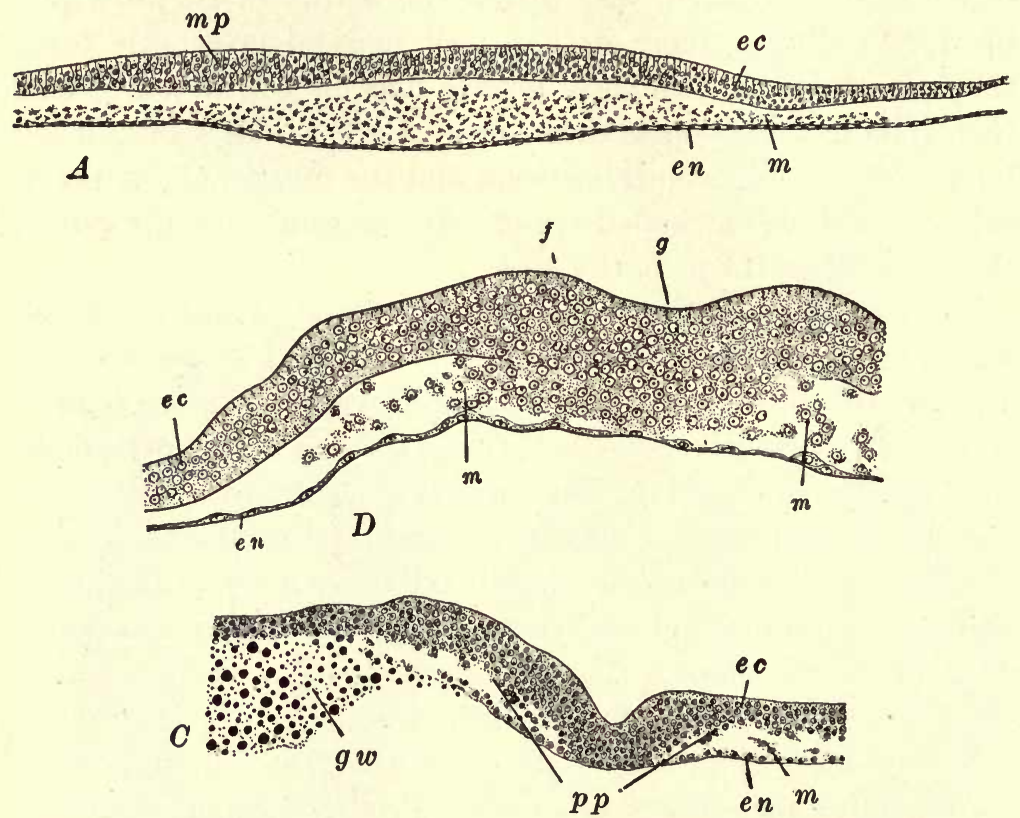

FIG. 94.- Transverse sections through various levels of the blastoderm at different ages. $A$. Through the head process of an embryo of about sixteen hours. After Duval. B. Through the primitive streak of an embryo of seventeen and one-half hours. After Lillie. $C$. Through the primitive plate of an embryo of about the same age as $A$. After Lillie. ec, Ectoderm; en, endoderm; $f$, primitive folds; $g$, primitive groove; $g w$, germ wall; $m$, mesoderm; $m p$, medullary plate; $p p$, primitive plate.

liferation, which continues across the posterior side of the primitive plate, so that the pellucid area behind the primitive streak is composed of all three germ layers (Fig. 94, $C$ ). In the region of the head process, i.e., in front of the primitive pit and streak, conditions are not essentially unlike those found farther back. The primitive groove is absent, and the cells of the three layers are more intimately fused. Relatively 
little mesoderm is proliferated from the anterior part of the head process, so that in front of this, the blastoderm is for a considerable time composed of only the two primary layers.

Surface views of entire blastoderms now show considerable modifications in the structure and relations of the primary pellucid and opaque areas. The outline of the elongated pellucid area becomes irregular; as a whole it is divisible into a posterior darker region where all three layers are present, and an anterior lighter area, crescentic in.form, composed of ectoderm and endoderm only (Fig. 93, B). Later the irregular anterior border of the mesoderm can be seen to advance along the sides of the area pellucida, in front of the level of the head process (Fig. 93, C). Finally the mesoderm extends completely around the margin of the pellucid area, leaving only a small area directly in front of the head process in the two layered condition; this area is known as the proamnion (Fig. 93, D).

Meanwhile the area opaca has been undergoing very marked modifications. This has broadened rapidly, and in its lateral and posterior regions, where the mesoderm has extended more widely over the germ wall, it has assumed an irregularly mottled appearance. Sections show this to be due to the formation of differentiated cell groups known as blood islands, the beginning of the vascular system. The presence of these blood islands marks that portion of the opaque area known as the area vasculosa (Fig. 93, $C, D$ ). This appears first immediately behind the embryo, but rapidly spreads laterally and anteriorly. Peripherally it becomes bounded by a definite blood vessel known as the sinus terminalis. Beyond this the opaque area is formed only of ectoderm and endoderm, extending widely over the yolk and known as the area vitellina. The blood islands are formed of compact cell masses scattered through the deeper portion of the germ wall. The cells composing them have apparently, though not certainly, differentiated in situ; they become covered superficially by a layer of scattered germ wall cells, which comes to be known later as celomic "mesoderm" (Fig. 95). While this layer, and the blood islands, early become directly continuous with the mesoderm of the pellucid 
area derived from the primitive streak, it is commonly supposed

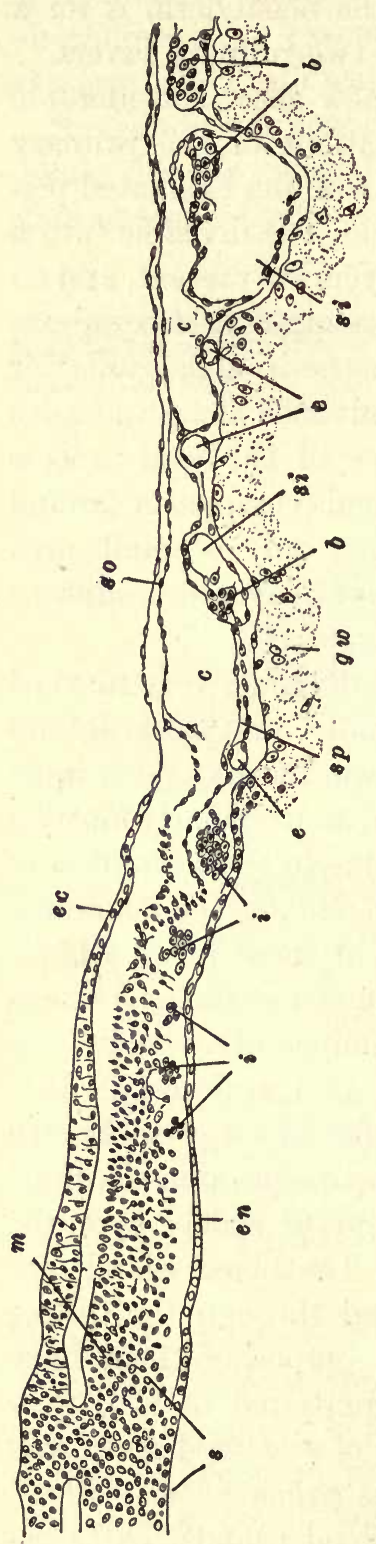

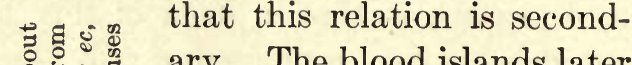
ary. The blood islands later become hollowed out, their constituent cells forming both the walls and the corpuscular contents of the irregular vascular lacunœ. These soon anastomose with one another forming a complex network throughout the area vasculosa; still later this net connects with the vascular structures of the pellucid area and of the embryo. The cellular portion of the germ wall remaining after the colomic "mesoderm" and the blood islands are differentiated, forms the

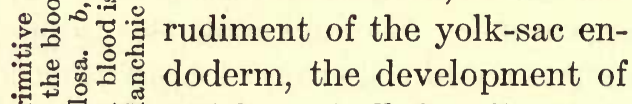
语语 †.

on 证 政 和 in on of this 政 is the s.

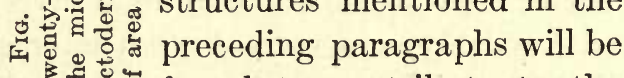
政 found to contribute to the formation of the definitive embryo. As a matter of fact, of 
all the structures so far described, only those in the vicinity of the head process, represent actual embryonic rudiments; these are, that thickening of the ectoderm described as the medullary (neural) plate, and the axial endoderm and mesoderm lying directly beneath it. The substance of the primitive streak, therefore, lies posterior to the embryo proper, and the boundary between the two is indicated by the position of the primitive pit.

As the embryo becomes more definitely established, it elongates posteriorly, while the primitive streak correspondingly shortens. The relation between the two structures is such that the embryo draws its substance from the anterior end of the primitive streak, or, we may say, the materials of the anterior end of the primitive streak are slowly redifferentiated and payed into the hinder end of the embryo. The region where this process of redifferentiation is proceeding is indicated as Hensen's node (primitive knot). Finally the primitive streak is wholly transformed into embryonic structures, but this is not fully accomplished until a relatively late stage in development (second day of incubation). To the question as to how much of the definitive embryo is formed of structures primarily anterior to the primitive streak, no precise answer can be given. In a general way, however, it may be said that the region of the primary location of the primitive pit corresponds roughly with the boundary between hind- and midbrain, and that structures developing anteriorly to this level were related originally to the region of the head process, rather than to the primitive streak.

We are now prepared to understand the essential homologies of the primitive streak, having learned of its relation to the embryo, on one hand, and to the structures of the gastrula, on the other. Theoretically the primitive streak is to be interpreted as the result of a modified process of concrescence (confluence). The chief alterations of the typical process of concrescence (confluence) are apparently due to the relatively enormous mass of yolk, and the consequent modification of the gastrula into a flat sheet, more or less intimately related to 
the yolk through the germ wall. Thus the endoderm is involuted from only a limited extent of the margin of the blastodisc. The region where ectoderm and endoderm become continuous is the rim of the blastopore; therefore we may say that

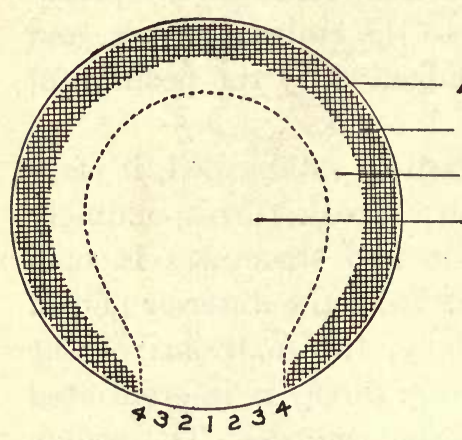

A

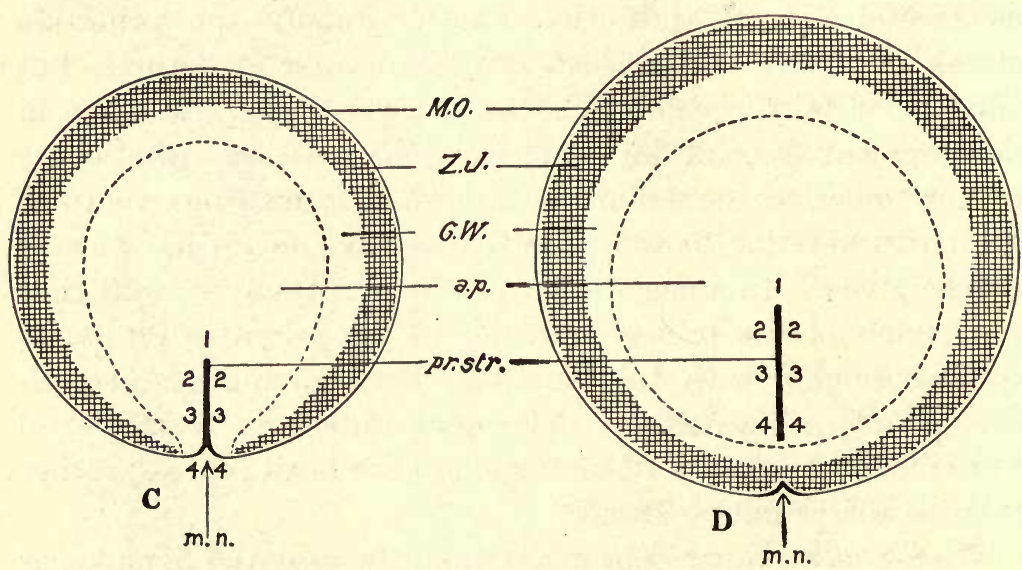

FIG. 96.-Diagrams illustrating the idea of confluence (concrescence) as applied to the chick. From Lillie (Development of the Chick). The central area bounded by the broken line represents the area pellucida; external to this is the area opaca, showing the germ wall $(G . W$.$) , zone of junction (Z . J$.$) , and$ margin of overgrowth $(M . O)$. m.n., Marginal notch.

the rim of the blastopore here is a short crescent at the posterior side of the blastula. The blastoporal opening is consequently represented by a space, really only virtual, between this and the contiguous periblast and yolk. Closure of this 
vestigial blastopore occurs after the growth of the blastoderm begins, so that when the lips of the blastopore approach and fuse, in the typical manner of confluence, the region of their fusion is limited to the posterior region of the blastopore (Fig. 96). We recognize the primitive streak, therefore, as the fused halves of the blastoporal margin; the primitive groove is consequently to be interpreted as a vestige of the blastopore itself. Later in development we know that the anus develops at the posterior end of the remains of the primitive streak, while from the beginning the primitive pit, at its anterior end, represents the vestige of the neurenteric canal, as described for Amphioxus and the frog.

The so-called head process thus represents, theoretically, the earliest portion of the true embryo to be differentiated out of the primitive streak. As a matter of fact, however, it is difficult to observe a true genetic relation between the primitive streak and the earliest portion of the head process, which seems to form precociously. This theoretical distinction is also the basis for the further distinction between gastral and peristomial mesoderm in the chick. Really such a distinction is not evident here, but it is sometimes useful to regard as gastral, that mesoderm originating in the primary head process, and as peristomial, that arising more posteriorly from the primitive streak proper. Ultimately, of course, the "peristomial" mesoderm becomes "gastral" in its relations to other structures.

The fact that the whole process of gastrulation is itself vestigial, to a certain extent, offers an interpretation of the independent formation of the medullary plate, which occurs unusually early, and of the mesoderm, which does not appear until the primitive streak is largely established. This latter fact, together with the development of the mesoderm from cells of ectodermal character, are probably both related to the marked restriction of the endoderm; this is reduced both in extent and in importance, for most of the early functions of the endoderm are here, in correlation with the extreme amount of yolk, taken over by the periblast and the cells of the germ wall. 

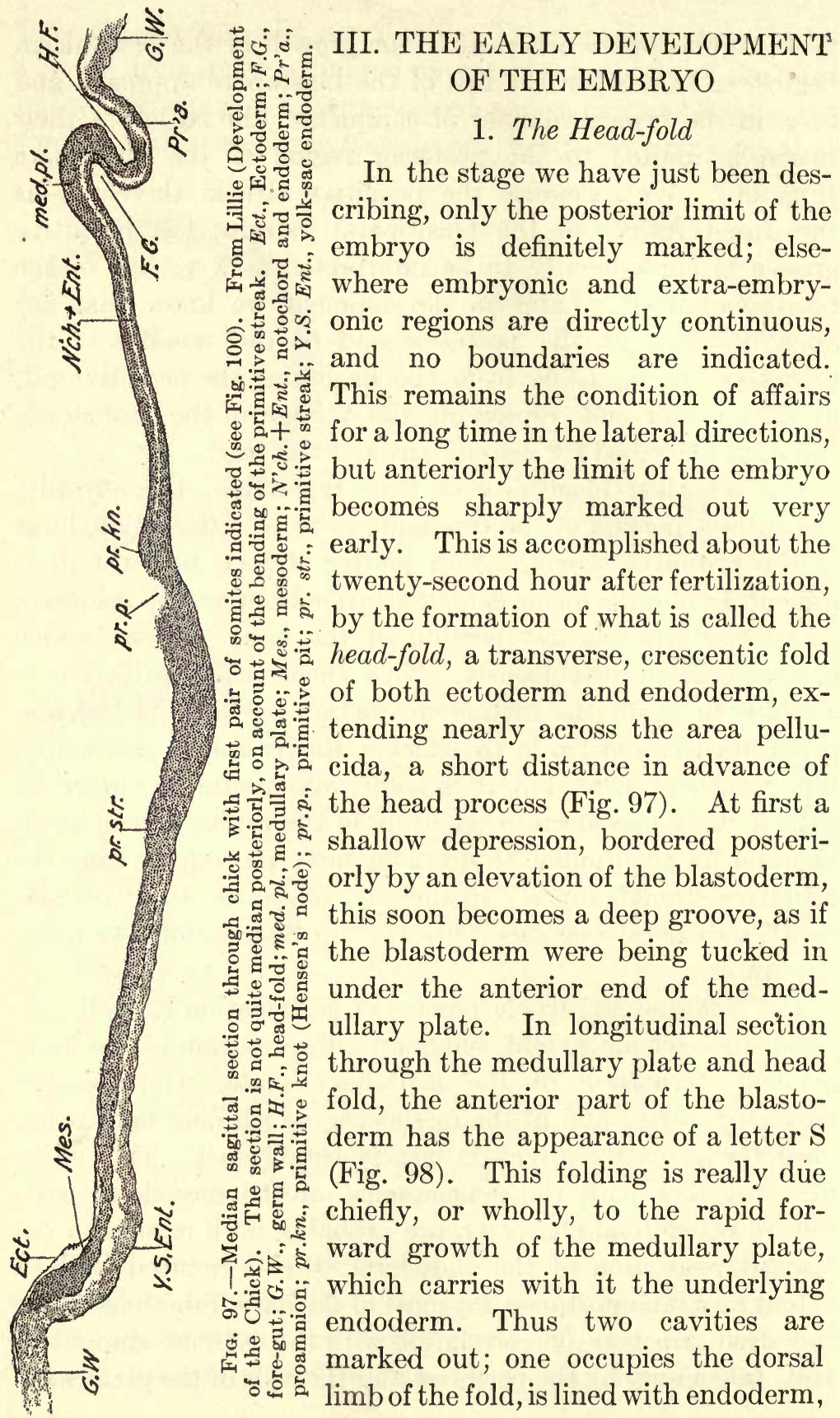

\section{The Head-fold}

In the stage we have just been describing, only the posterior limit of the embryo is definitely marked; elsewhere embryonic and extra-embryonic regions are directly continuous, and no boundaries are indicated. This remains the condition of affairs for a long time in the lateral directions, but anteriorly the limit of the embryo becomes sharply marked out very early. This is accomplished about the twenty-second hour after fertilization, by the formation of what is called the head-fold, a transverse, crescentic fold of both ectoderm and endoderm, extending nearly across the area pellucida, a short distance in advance of the head process (Fig. 97). At first a shallow depression, bordered posteriorly by an elevation of the blastoderm, this soon becomes a deep groove, as if the blastoderm were being tucked in under the anterior end of the medullary plate. In longitudinal section through the medullary plate and head fold, the anterior part of the blastoderm has the appearance of a letter $\mathrm{S}$ (Fig. 98). This folding is really due chiefly, or wholly, to the rapid forward growth of the medullary plate, which carries with it the underlying endoderm. Thus two cavities are marked out; one occupies the dorsal limb of the fold, is lined with endoderm, 
and is an extension of the archenteric space between endoderm and yolk; the other occupies the ventral limb, is lined with ectoderm, and is continuous with the space entirely outside the embryo. The dorsal, endodermally lined cavity is the rudiment of the fore-gut. The lower space is sometimes called the cavity of the head-fold; it is merely an external space lying under the head of the embryo. The boundary between embryonic and

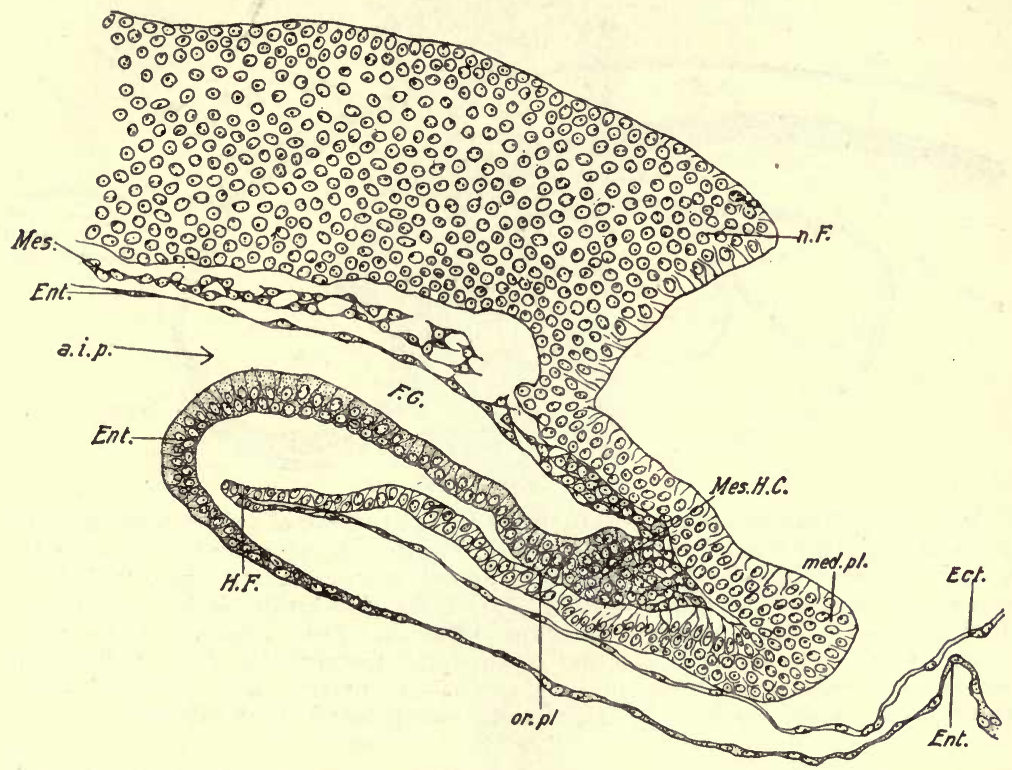

FIG. 98.--Median sagittal section through the head end of an embryo with four pairs of somites (twenty-three to twenty-four hours). From Lillie (Development of the Chick). a.i.p., Anterior intestinal portal; Ect. ectoderm; Ent., endoderm; F.G., fore-gut; H.F., head-fold; med. pl., anterior limit of medullary plate; Mes., mesoderm; Mes. H.C., mesodermal head cavity; n. F., neural fold: or. $p l$., oral plate (oral membrane).

extra-embryonic structures is just along the middle, i.e., most posterior limit, of this latter fold or cavity; at this level the morphologically anterior limit of the medullary plate passes abruptly into the extra-embryonic ectoderm of the blastoderm, in the region of the proamnion.

Laterally the head-fold and fore-gut narrow and finally disappear into the flat blastoderm. The posterior curvatures of the lateral extremities of the head-fold mark the lateral sur- 
faces of the head, but otherwise the lateral limits of the embryo proper are not definitely marked until twenty-two to twentyeight hours later, i.e., forty-two to fifty hours after fertilization. We may proceed, therefore, to describe the development of the essential structures of the embryo, during the period from the formation of the head-fold up to the time when the embryo begins to be completely marked off from the extra-embryonic

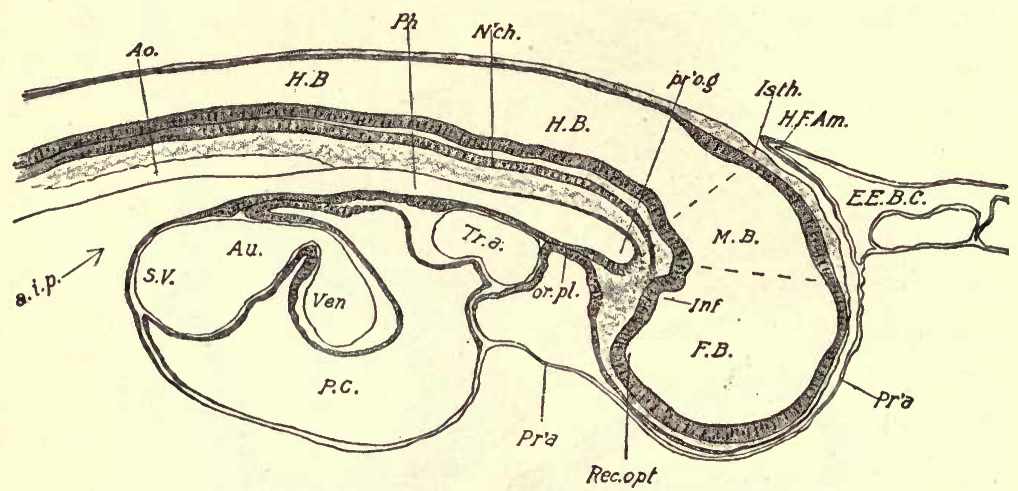

Fig. 99.- Median sagittal section through the head end of a chick with eighteen pairs of somites (about forty hours). From Lillie (Development of the Chick). a.i.p., Anterior intestinal portal; Ao., dorsal aorta; Au., auricle; E.E.B.C., exocœlom (extra-embryonic body cavity); F.B., fore-brain; H.B., hind-brain; H.F.Am., head-fold of amnion; Inf., infundibulum; Isth., isthmus; M.B., midbrain; $N^{\prime} c h$. , notochord; or.pl., oral plate (oral membrane); P.C., pericardial cavity; Ph., pharynx; Pr'a., proamnion; pr'o.g., preoral gut; Rec.opt., optic recess; $S . V$. , sinus venosus; Tr.A., truncus arteriosus; Ven., ventricle.

region of the blastoderm. We shall describe the history of the mesoderm first, not only because this seems the easiest method of approach, but because the differentiations within the mesoderm afford valuable landmarks in describing other structures. Thus the age of the chick embryo is usually indicated by reference to the number of pairs of mesodermal somites formed.

\section{Mesoderm}

While the head-fold is becoming well established (Fig. 100), the mesoderm is in the form of a pair of sheets extending from the sides of the primitive streak (peristomial) and head process (gastral), across the whole extent of the area pellucida to the 
germ wall, where it is continuous with the vascular area. The inner or axial border of each sheet is considerably thickened, and often distinguished as the vertebral plate, the remaining distal portion being known as the lateral plate. The mesoderm also

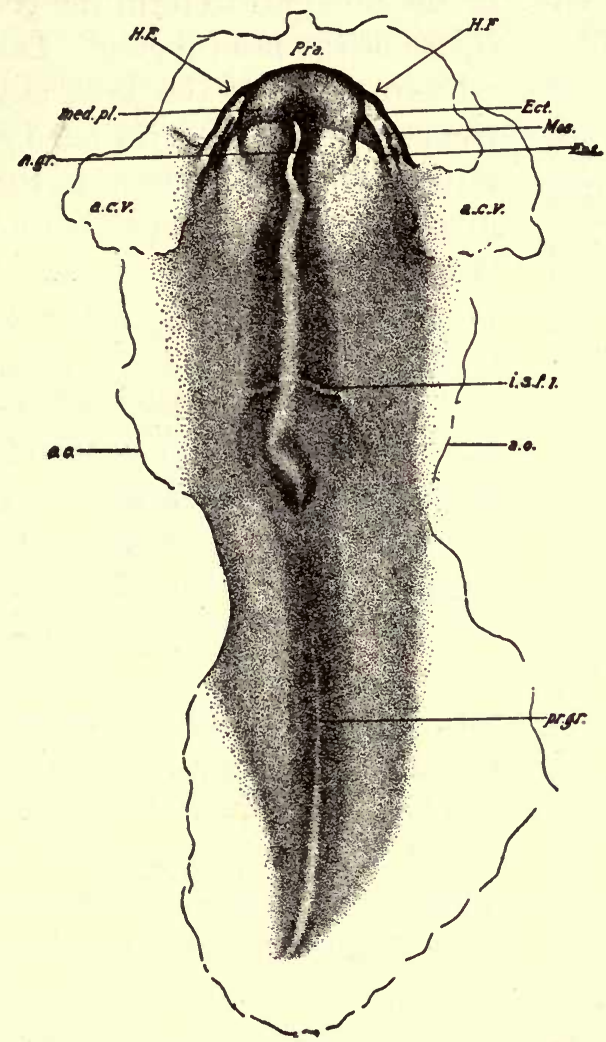

Frg. 100.-Chick embryo of about twenty hours, showing first intersomitic furrow. Dorsal view. From Lillie (Development of the Chick). a.c.v., Amniocardiac vesicle; a.o., inner margin of area opaca; Ect., ectoderm; Ent. endoderm, H.F., head-fold; i.s.f.1., first intersomitic furrow; med.pl., medullary plate; Mes., mesoderm; n.gr., neural groove; $P r^{\prime} a .$, proamnion; $p r . g r$. , primitive groove.

extends posteriorly from the primitive streak, but at this time there is none in the anterior part of the blastoderm in front of the head process and head-fold (proamnion), although around the margin of the pellucid area it is carried forward as a pair of wing-like extensions, so that in surface view its anterior limit is markedly concave (Fig. 93, D). 
In the vicinity of the head process (Fig. 101), the vertebral plates at this stage rapidly lose their definite character and the cells scatter throughout the region, combining with the cells

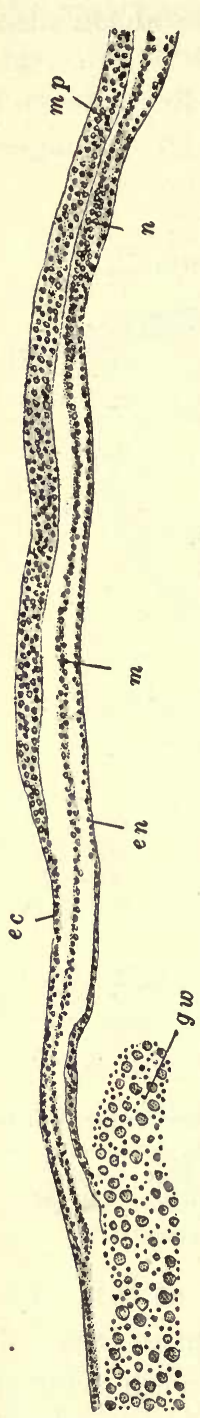

g: continually being budded off from the walls of the fore-gut, to form the general mesenchyme of the head region. Later scattered cells are added to the mesenchyme directly from the ectoderm of the head region. The history of the mesoderm farther back, just in front of the end of the primitive streak, is much the same as in the body region proper, although all of the embryo thus far developed out of the primitive streak shares in the formation of the head only. Here the vertebral plate thickens still more and its cells become rearranged so as to form a short transverse break in the continuity of the plate (Fig. 100). On each side the cells immediately in front of this become grouped in a definite mass and form the first pair of mesodermal somites, continuous anteriorly with the forward extensions of the vertebral plates (Figs. 100, 104). This rearrangement of cells continues posteriorly, and soon a second split appears on each side, a short distance behind the first, cutting out a definite block of the cells of the vertebral plate. This is the second pair of somites. Additional pairs of somites are blocked out in regular fashion, as the embryonic region grows at the expense of the primitive streak. The formation of somites is not actually completed until about the fifth day of development, by which time about fifty-two pairs have been formed. The first somites formed remain the most anterior in position. The first four pairs are ultimately in- 
cluded in the hinder part of the head region of the embryo, and although no other definite boundary of the head appears for some time, its future limit may be marked by the position of the fourth somites.

This division of the vertebral plate into somites expresses the primary segmentation or metamerism of the body, and is fundamental, the metameric arrangement of other organs being secondary and dependent upon this. Sections through the somites (Fig. 102) show that their superficial cells are arranged

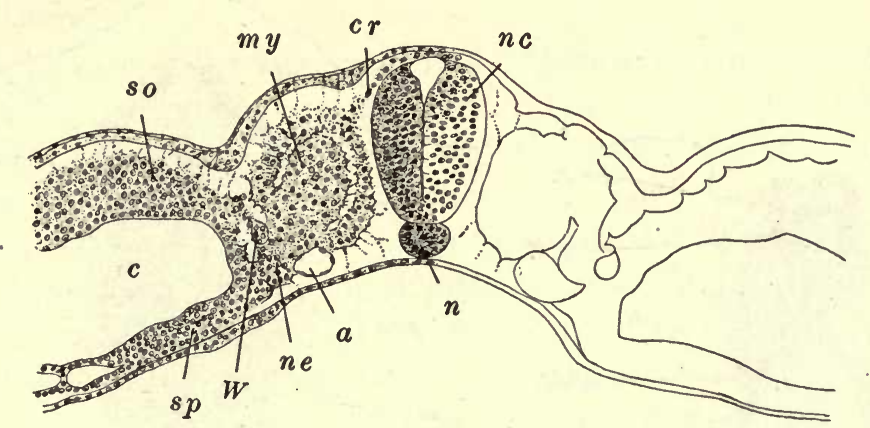

Fig. 102.-Transverse section through the last somite of a chick of about forty-eight hours (twenty-nine pairs of somites). After Lillie. a, Lateral dorsal aorta; $c$, cœlom; $c r$, neural crest; $m y$, myotome; $n$, notochord; $n c$, nerve cord; $n e$, nephrotome; so, somatic mesoderm; $s p$, splanchnic mesoderm; $W$, Wolffian duct.

in the form of an epithelium, while the cells of the central parts are loosely and irregularly arranged. This central portion, although only virtually a cavity, is termed the myocal; it corresponds, theoretically, with the region of the enterocol of other forms.

The more lateral portions of the mesoderm, the lateral plates, remain unsegmented. They are connected with the somites by an intermediate, transitional band, also unsegmented in the chick, known as the nephrotome or intermediate cell mass: The dorsal and ventral surfaces of the nephrotome are continuous with the corresponding surfaces of the somites, the separation between the two bodies being indicated by a depression resulting chiefly from the thickening of the somites. 
The intermediate cell mass in part forms the rudiment of the excretory system, and in part contributes to the formation of mesenchyme: its history will be described in connection with the later stages of development.

The lateral plate very early becomes separated into two distinct sheets by the development of a wide cavity within it. This cavity is the cœlom, which is here, as in the frog, to be described as a schizocœl. The outer or upper sheet of lateral

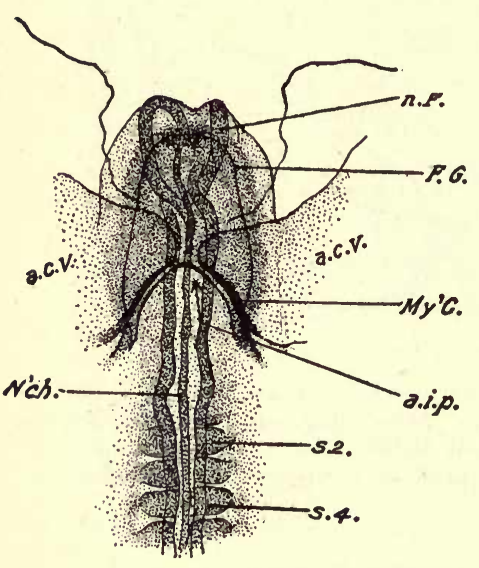

$A$

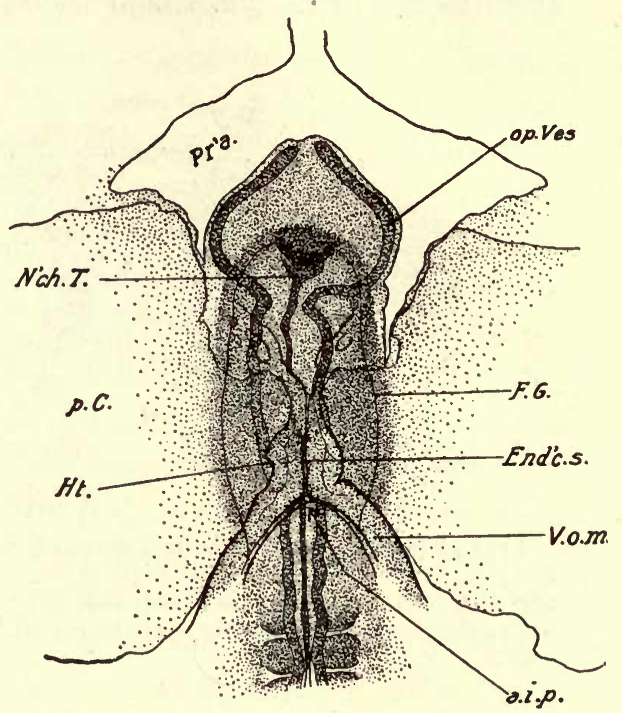

$B$

Fig. 103.-Ventral views of the head ends of chick embryos. From Lillie (Development of the Chick). A. Embryo with five pairs of somites (about twenty-three hours). B. Embryo with seven pairs of somites (about twentyfive hours). a.c.v., Amnio-cardiac vesicle; a.i.p., anterior intestinal portal; End'c.s., endocardial septum; F.G., fore-gut; Ht., heart; $M y^{\prime} C$. , myocardium; $N^{\prime} c h$., notochord; $N^{\prime} c h . T$., anterior tip of notochord; n.F., neural fold; op.Ves., optic vesicle; p.C., parietal cavity (cœlomic); Pr'a., proamnion; s.2, s.4, second and fourth mesodermal somites; V.o.m., omphalo-mesenteric vein.

plate cells is the somatic mesoderm; this unites with the overlying ectoderm to form the somatopleure (Figs. 102, 105, 108). The inner or lower sheet is the splanchnic mesoderm; this unites with the underlying endoderm to form the splanchnopleure. The somatic and splanchnic layers of mesoderm remain united proximally, in the region of the intermediate 
cell mass. The somatopleure, splanchnopleure and colom, later become separated into embryonic and extra-embryonic regions, but during these early stages they form continuous structures extending laterally out to the germ wall, and anteriorly into the head region. From the embryonic portions of these structures develop, respectively, the body wall, the gut wall and vascular organs, and the pericardial, pleural, and peritoneal cavities: their extra-embryonic portions give rise to the embryonic membranes and appendages, and to the extra-embryonic portions of the vascular system and colom (exocœlom).

The history of the mesoderm and colom in the region of the head-fold deserves a special word. The cœlom very early enlarges, either side of the head region, forming a pair of large spaces called the amnio-cardiac vesicles. These grow inward toward the head-fold, and by the time this is well established (4-6 pairs of somites) they push into the lower limb of the headfold, between its ectodermal and endodermal layers (Fig. 105). Here they finally meet and fuse, forming a median cœlomic space bounded, of course, by a mesodermal wall. This is the rudiment of the pericardial cavity, and its formation and subsequent enlargement, bring about a wide separation of the ectoderm and endoderm, or as we may now say, of the somatopleure and splanchnopleure, of the head-fold, the latter being carried much the farther posteriorly (Figs. 98, 99). The later development of this region and of the vascular system in general, may more conveniently be postponed, until after an account of the history of the ectodermal and endodermal layers during these early stages.

\section{Endoderm}

We left the embryonic endoderm as a thin sheet of cells extending forward from the primitive streak, and we had described the important events connected with the formation of the fore-gut from its anterior margin (Figs. 97, 98). In the region between the fore-gut and the primitive streak, i.e., in the 
remainder of the embryonic region thus far formed, the endoderm and mesoderm are united in a secondary median fusion continuous with the cells of the primitive knot. Very soon the mesoderm and endoderm separate again and the median

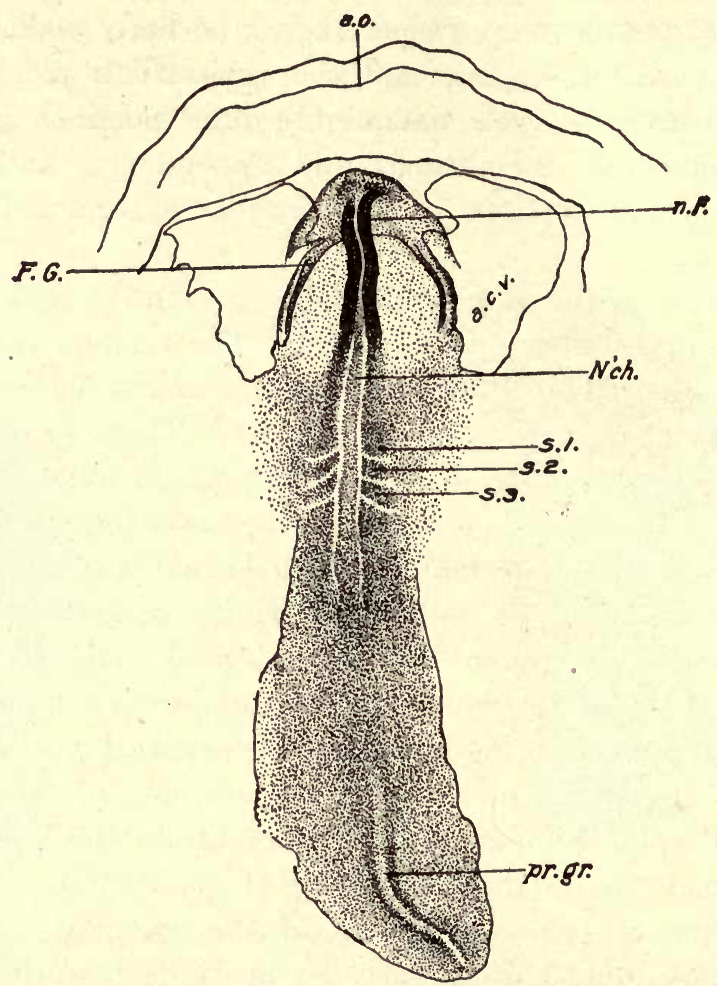

Fig. 104.-Chick embryo with three pairs of somites (about twenty-three hours). Dorsal view. From Lillie (Development of the Chick). a.c.v., Amniocardiac vesicle; a.o., inner margin of area opaca; $F$. G., fore-gut; $N^{\prime} c h .$, notochord; $n . F$. ., neural fold; pr.gr., primitive groove; s.1, s.2, s.3, first, second and third somites.

cells, then associated with the mesoderm, become arranged as a small longitudinal rod, the notochord (Fig. 102). Since the germ layers are fused in the primitive knot, and redifferentiate anteriorly out of it, it is of little consequence whether the notochord is regarded as of mesodermal or endodermal origin; it is more closely associated with cells which later are 
clearly mesodermal. As the primitive streak shortens and the primitive knot moves posteriorly, the notochord continues to differentiate, and so to elongate posteriorly.

The cavity of the fore-gut is the rudiment of the pharynx. It remains a wide but shallow cavity throughout these early stages, open posteriorly, by way of the anterior intestinal portal, upon the surface of the yolk, where the endoderm remains spread out as a thin flat sheet (Figs. 97, 98, 103). Near the anterior extremity of the head-fold, the endoderm lining the pharynx comes ventrally into contact with the ectoderm, and later fuses with it. This forms the oral plate, which becomes the inner wall of the stomodoum; it is perforated early the third day of incubation. There is a pair of lateral extensions of the fore-gut, where its walls come into contact with the ectoderm, marking the positions of the future first gill pouches.

\section{Nervous System}

The rudiments of the central nervous system are the most conspicuous structures of the chick embryo during its early development. We have already noted the formation of the medullary plate, a broad ectodermal thickening developing precociously, before any other part of the embryo is definitely established (Figs. 94, 101). The medullary plate is converted into the medullary tube in the usual manner. A medullary groove appears soon after the head-fold becomes marked; the marginal medullary folds then become elevated and grow rapidly so that they form a pair of high conspicuous ridges on the surface of the anterior part of the blastoderm. They extend posteriorly, gradually diminishing in height, until finally they sink into the general level of the blastoderm in the region of the primitive knot. The formation of the medullary folds and central nervous system in general, as of the somites, notochord, and other parts of the embryo, is progressive posteriorly, so that steps in the formation of all these parts can be read in any series of transverse sections extending from the primitive knot anteriorly. 
The elevated medullary folds, or neural folds, soon bend over toward one another and first meet a short distance back from the anterior limit of the head; this is known to be the region of the future mid-brain (Fig. 105). Here the folds soon fuse together forming a superficially continuous sheet of ectuderm, and an underlying medullary or neural tube. From this point the fusion extends very rapidly posteriorly, very slowly anteriorly. The point where the final closure occurs anteriorly, and consequently the final separation from the superficial ectoderm, is known as the neuropore; it is the region later distinguished as the lamina terminalis, and is regarded as the morphologically anterior limit of the brain. Topographically, however, this is not the most anterior part of the central nervous system, for during these early stages the rudiment of the brain grows forward and downward, in front of the fore-gut, so that its anterior end becomes bent like a crook. Its floor remains closely applied to the roof of the fore-gut, its extension being due to the growth of the anterior and dorsal regions chiefly (Fig. 99). Thus the morphologically anterior end of the brain really comes to lie on its antero-ventral aspect.

Certain details in connection with the neural folds and their closure deserve special mention. The actual crests of the neural ridges are flattened, and when the ridges fold together these flattened surfaces form a broad vertical contact. The neural tube is formed by the fusion of the lower or inner margins of these surfaces; the upper margins fuse across the mid-line, restoring a continuous superficial ectoderm. The cells left between the upper and lower margins, derived approximately from the very apices of the neural ridges, are thus left between the neural tube and the surface ectoderm; these are. the neural crests (Fig. 102). They do not fuse across the midline, but remain as a pair of longitudinal bands along the dorsolateral surfaces of the neural tube. The neural crests are the rudiments of the ganglia of the cranial and spinal nerves; they are not uniformly developed throughout their extent and may be better marked in some sections than in others.

The anterior portion of the neural tube expands very con- 
siderably. Its most anterior region expands transversely, the lateral extensions thus formed representing the rudiments of the optic vesicles (Figs. 106, 107). This is obviously, therefore, the region of the primary fore-brain or prosencephalon, which now includes the entire anterior portion of the nerve tube. Its posterior limit is marked by a slight constriction just back of the optic vesicle rudiments. A little later (9-10 pairs of somites) another constriction appears marking the posterior limit of the mid-brain or mesencephalon. The third section of the brain, the hind-brain or rhombencephalon is much the longest section of the brain (Fig. 107). It is marked by a series of irregular constrictions, ultimately five in number forming six segments, or neuromeres, in this region. No posterior limit of the hind-brain can be made out in these early stages, but from later development it is known that all in front of the fourth mesodermal somites really belongs to the head, and consequently this level may be taken to mark approximately the posterior limit of the brain. The hind-brain narrows posteriorly and the remainder of the neural tube is the rudiment of the spinal cord, which remains narrowed and approximately uniform in diameter.

\section{Vascular System}

We may now return to a description of the formation and development of the embryonic vascular system, postponed from an earlier page. The formation of the vascular rudiments in the area opaca continues, in the manner already described (Fig. 95), and when the extra-embryonic cœlom forms, dividing the mesoderm into somatic and splanchnic layers, the bloodvessels remain associated with the latter, i.e., with the splanchnopleure (Fig. 105, A). The vessels of the pellucid area develop first from its margin, near the area opaca, and gradually extend toward the embryo. They first appear shortly after the head-fold, just as the somites begin to be cut out. The formation of the conspicuous blood islands does not occur in the pellucid portion of the blastoderm; only the tubular vessels 


\section{OUTLINES OF CHORDATE DEVELOPMENT}

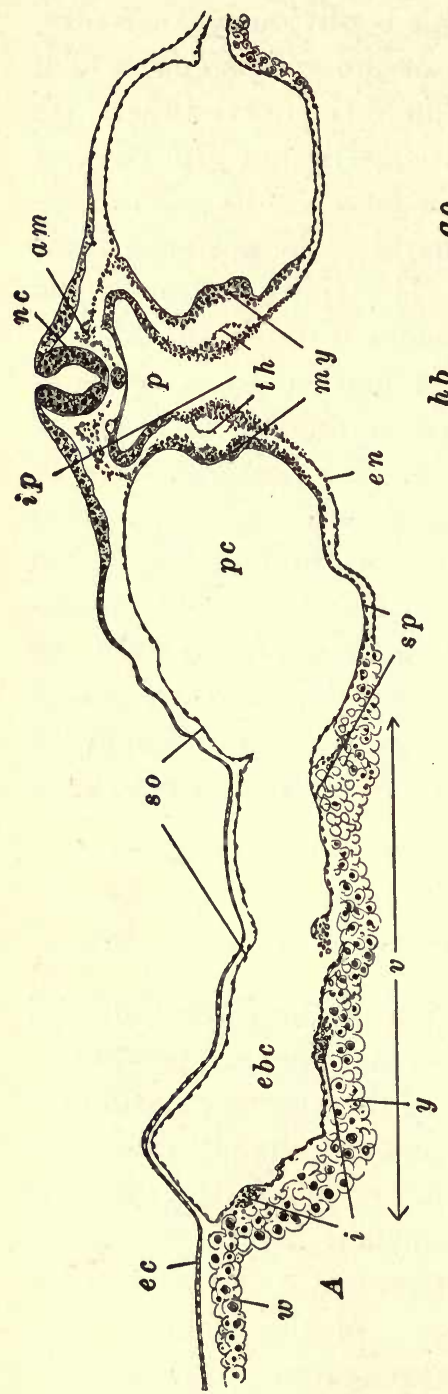

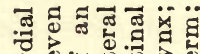

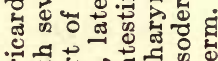

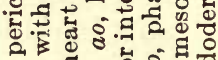
느 의 0.0

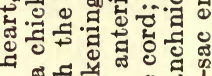
¿ 40 廿 융

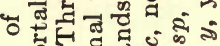

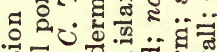

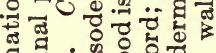

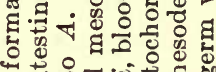

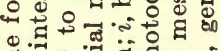
๘

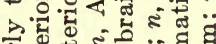

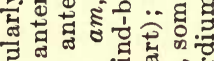
.

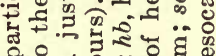
ณึ

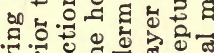

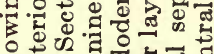

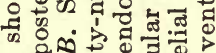
on 0 卷

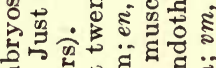
द्व ㄱ. Ð ०

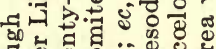
डै

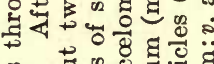

$\sim$ 党 . क जै 050.

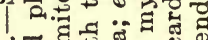
10 뭉 ๘ี

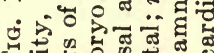
质.5

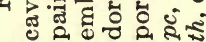


develop here. Thus the cellular or corpuscular elements of the early vascular system arise only in the area opaca, and most extensively in its posterior region. Following Rückert, we may say that the vessels of the pellucid area are formed by the rearrangement of small groups of cells in the splanchnic mesoderm of the area. These first form short sections of tubular vessels which very soon connect with the more peripheral vessels of the opaque area. In this way a continuous vascular network develops centripetally, finally reaching the embryo about the time six pairs of somites are formed.

Soon after this, vessels appear in the embryo itself. The first of these are the paired dorsal aortae of the body region (Fig. 105). These are to be regarded as enlarged and straightened inner (axial) margins of the vascular network of the pellucid area. Posteriorly they diverge widely, passing as the vitelline arteries, into the general vascular net (Fig. 107). Anteriorly they become prolonged forward toward the head region, where they connect with a pair of vessels differentiated within the mesenchyme of the head.

The heart is clearly a specialized portion of the system of vessels. Like the dorsal aortæ, the rudiment of the heart is paired. When the vessels grow into the head region, which is now elevated above the level of the general splanchopleure, they are related to the ventral, rather than to the dorsal region (Fig. 105). The amnio-cardiac vesicles (see above) become vascularized in the same manner as the rest of the pellucid area, and a pair of ventral aorte is formed beneath the fore-gut (Fig. 103). Posteriorly to the opening of the fore-gut (anterior intestinal portal) these vessels diverge and pass into the vascular net as the rudiments of the vitelline veins. Reference to the transverse section illustrated in Fig. 105, A, shows that beneath the fore-gut the mesoderm has a considerable vertical extent. This is commonly regarded as splanchnic mesoderm, although it is a region where somatic and splanchnic layers become continuous. The paired rudiments of the heart pass along the inner or axial surface of this vertically extended mesoderm, and thus come into close relation, shortly fusing 


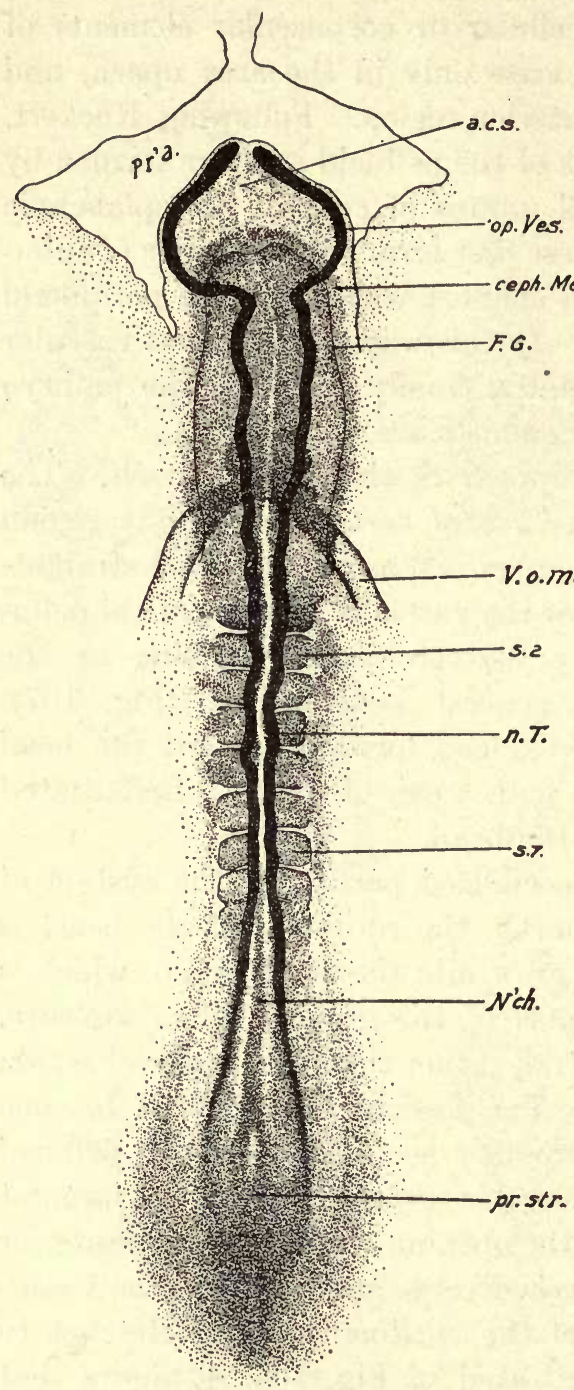

Fig. 106.-Chick embryo with seven pairs of somites (about twenty-five hours). Dorsal view. From Lillie (Development of the Chick). a.c.s., Anterior cerebral suture; $c e p h$. Mes., cephalic mesoderm; F.G., fore-gut; $N^{\prime} c h$. notochord; $n$. T., neural tube; op.Ves., optic vesicle; $P r^{\prime} a .$, proamnion; $p r$. $s t r .$, primitive streak; s. 2, s. 7 , second and sevent b somites; V.o.m., omphalomesenteric vein. together to form a inedian, thin-walled tube. This becomes the endothelial lining of the heart; the muscular wall of the heart is formed by the addition of an external layer of mesoderm; for the splanchnic mesoderm of each side forms a fold around the endothelial rudiment (Fig. 105, B). These folds then approach and fuse across the mid-line, both above and below the endothelial tube. For a brief period after their fusion they remain in the midline forming a dorsal mesocardium, connecting the heart with the tissues just beneath the fore-gut, and a ventral mesocardium, extending from the heart to the splanchnopleure. The ventral mesocardium breaks away almost immediately after its formation, while the dorsal mesocardium remains for a time, but then disappears, except at the anterior and posterior extremities of the heart. The heart is then left as a short median tube con- 
sisting of endothelial and rudimentary muscular layers, freely suspended in a cavity which is the beginning of the pericardial cavity. Anteriorly it is continuous with a short pair of vessels extending into the head-fold; these are the ventral aortæ mentioned above. Posteriorly the heart is directly continuous with the vitelline veins.

The dorsal aortæ have meanwhile extended forward into the head, connecting with their cephalis sections which have developed directly within the mesenchyme of the region. These now unite with the ventral aortæ (Fig. 107, B) around the sides of the anterior end of the fore-gut; these connections are the rudiments of the first, or mandibular aortic arches. Thus the embryonic circulatory system is established and connected with the extra-embryonic vessels.

\section{THE EMBRYO OF ABOUT THIRTY HOURS (10-12 PAIRS OF SOMITES)}

We may summarize, now, the events of this early period of development by mentioning the structures of the chick embryo at about its thirtieth hour of incubation, when it includes ten to twelve pairs of mesodermal somites (Figs. 107, 108).

In the freshly opened egg of this age, the circular blastoderm is seen to have extended approximately one-fourth of the way around the vitellus (i.e., its diameter is roughly $25 \mathrm{~mm}$.). The embryo appears as a definite whitish streak, approximately 4 $\mathrm{mm}$. in length, enlarged anteriorly, and posteriorly fading gradually into the surrounding area. Thus by far the greater part of the blastoderm is extra-embryonic. Three definite areas may be distinguished in the extra-embryonic blastoderm (Fig. 108). (1) Immediately surrounding the embryo is the clear area pellucida, now greatly restricted and not very distinct. save in the head region which includes the proamnion. The area opaca is now clearly divided into (2) the area vasculosa and (3) the area vitellina. The vascular area, save for a slight interruption anteriorly, has grown entirely around the embryo. It has extended both peripherally, over the yolk, andee ntrally 

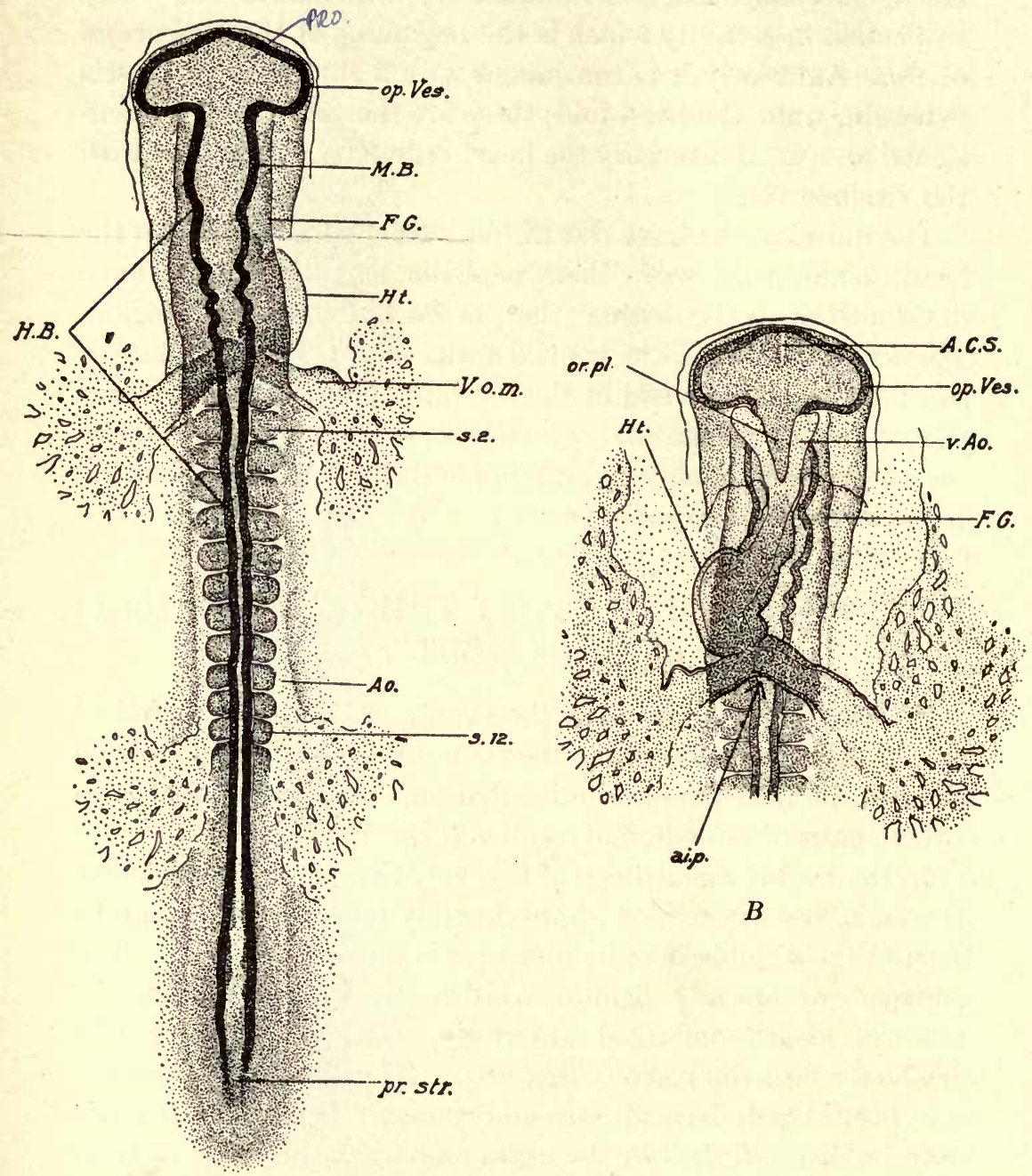

$B$

\section{A}

FIG. 107.-Chick embryo with twelve pairs of somites (about thirty-three hours). From Lillie (Development of the Chick). A. Dorsal view of entire embryo. B. Ventral view of anterior end. A.C.S., Anterior cerebral suture; a.i.p., anterior intestinal portal; Ao., dorsal aorta; F.G., fore-gut; H.B., hindbrain; $H t$., heart; M.B., mid-brain; op. Ves., optic vesicle; or.pl., oral plate; $p r . s t r .$, primitive streak; $s .2, s .12$, second and twelf th somites; v.ao., ventral aorta; V.o.m., omphalo-mesenteric vein. 
encroaching upon the pellucid area. is further separable into two regions, an inner band, the vitellina interna, and a narrow area vitellina externa.

The examination of sections through the blastoderm shows that the areas pellucida and vasculosa are composed of the three germ layers, the area vitellina of ectoderm and endoderm only. Except in the region about the head of the embryo, the area pellucida is extensively vascularized, but the vessels are still comparatively free from corpuscles. The pellucid and opaque areas are further characterized by the presence of extraembryonic cœlom between the two sheets of mesoderm (Fig. 108). The vascular area is definitely limited peripherally by the sinus terminalis. The area vitellina externa is the region where the extension of the blastoderm is actually being carried forward. Here are found conditions similar to those described in the early part of this chapter. The extreme margin ("margin of overgrowth"-Lillie) is formed by a single layer of superficial cells called "ectoderm," while between this margin and the area vitellina interna is a band ("zone of junction"-Lillie) which is the equivalent of the marginal periblast, where the processes of true blastoderm formation are chiefly localized,

The broad area vitellina

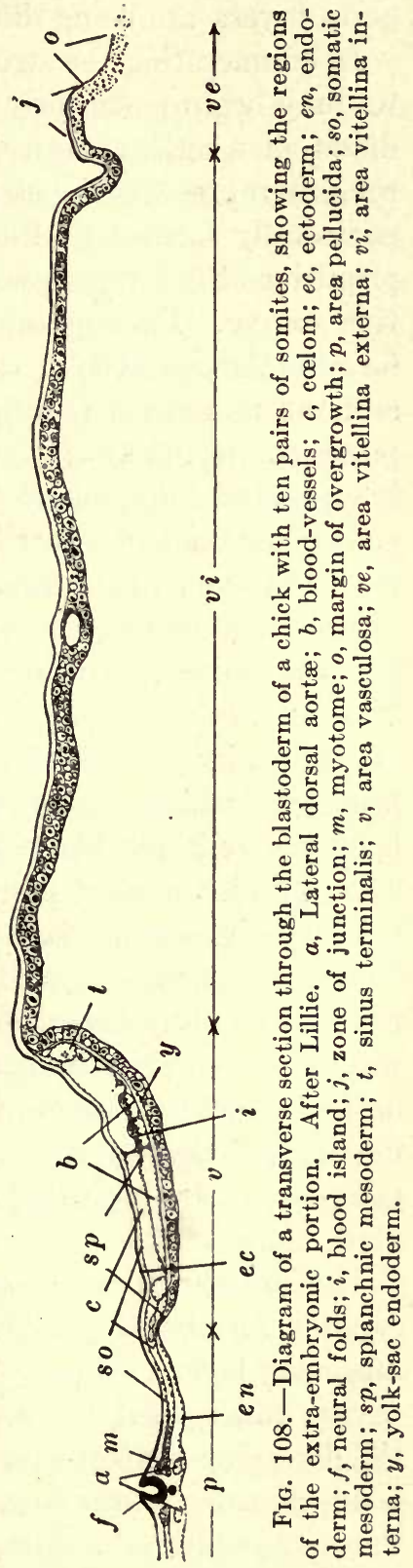


and where the extra-embryonic portions of the two primary germ layers are being differentiated.

In enumerating the structures of the embryo itself (Fig. 107) we may begin posteriorly and pass forward, since, read in this direction, a series of transverse sections through a single embryo roughly represents the steps in the development of most of the parts fully formed at this time. Posteriorly there is a short primitive streak region with thickened neural plate and primitive groove. Passing anteriorly the neural folds rise and meet forming the neural tube; the notochord becomes distinguishable, and the mesoderm is differentiated into vertebral and lateral plates connected by the intermediate cell mass; the lateral plate is separated into somatic and splanchnic layers by the extraembryonic colom. In the trunk and hinder part of the head ten to twelve pairs of mesodermal somites are formed.

The long head region extends forward from about the fourth pair of somites (counting from the anterior end of the series). The head is now almost completely separated from the yolk ventrally, by the endodermal head-fold, while the ectodermal head-fold, which also separates the lateral surfaces of the head from the yolk and blastoderm, has more than half this extent. The brain is the most prominent feature of the embryo, and its primary divisions, fore-, mid-, and hind-brain, are well marked. Neural crests are distinct in sections from the mid-brain posteriorly. The fore-gut, widely open posteriorly upon the yolk by way of the anterior intestinal portal, is the only strictly endodermal structure thus far differentiated. This is the pharyngeal region, and the rudiments of the first or hyomandibular pair of gill pouches are indicated laterally, while in its floor is the oral plate.

The large vitelline veins unite in front of the level of the first somites, forming the heart, which now has both endothelial and muscular layers. The elongated heart lies in the pericardial cavity and is bent to the right. Anteriorly it continues into the diverging ventral aortæ, which pass around the sides of the head, dorsally, as the first or mandibular pair of aortic arches; these are continuous with the wide lateral dorsal aortæ which 
pass backward and become continuous posteriorly with the extra-embryonic vascular network.

From this point onward we shall not attempt to continue a description of the development of the embryo as a whole. Next we shall outline the processes leading to the more complete formation of the embryo, and to its well-marked separation from the yolk and blastoderm on every side. Then, after a consideration of the formation of the embryonic membranes and appendages, we shall give, in the next chapter, a brief resume of the more essential steps in the organogeny of the later embryo. (Throughout this chapter, and more especially this part of it, the student is referred for additional details to such an account as Lillie's "Development of the Chick," and to the literature there cited).

\section{THE SEPARATION OF THE EMBRYO FROM THE EXTRA-EMBRYONIC STRUCTURES}

The remaining primitive streak region loses its typical structure soon after the fortieth hour of incubation (about 18 pairs of somites) (Fig. 112), and thereafter it remains, as a locus of continued cell proliferation known as the tail bud (Fig. $109, A)$. Shortly after this, about the forty-sixth hour (about 26 pairs of somites) a definite infolding of the blastoderm occurs posteriorly, marking the hinder limit of the embryo. This fold is the tail-fold, in many respects the counterpart of the headfold although appearing so much later. This fold, in which all three of the germ layers are involved (Fig. 109, B), slowly grows forward forming a hind-gut, comparable with, though much less extensive than, the fore-gut. The tail bud, thus separated from the yolk beneath, continues to elongate slowly for a considerable time. Mesodermal somites continue to be added posteriorly, through the trunk region, which extends as far as the thirty-fifth somite, formed about the seventieth hour, and finally through the incurved tail region (Fig. 109, B). A total of fifty-two pairs of somites have been counted, although some 


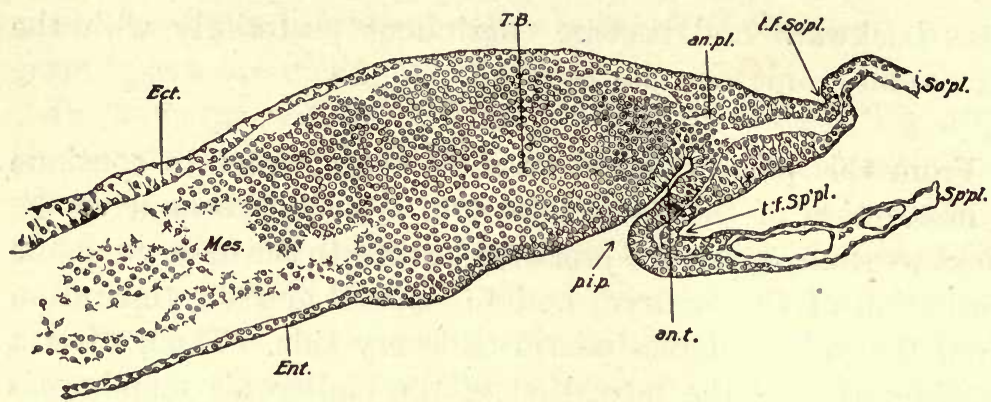

$A$

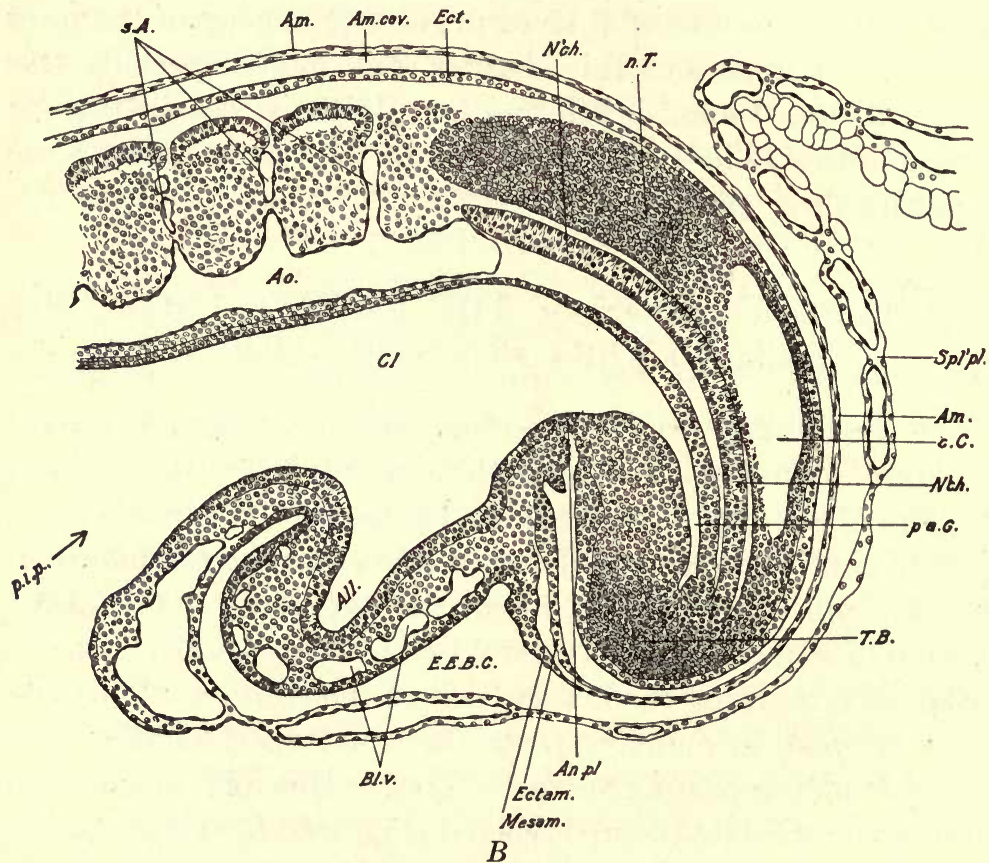

Fig. 109.- Sagittal sections through the posterior ends of chick embryos. From Lillie (Development of the Chick). A. Median section through an embryo with about twenty-one pairs of somites (about forty-four hours). B. Lateral section through embryo with about thirty-five pairs of somites (about seventytwo hours). All., allantois; $A m$., amnion; $A m$. cav., amnionic cavity; $A n$. pl., anal plate; An. $t$., anal tube; $A o$. , dorsal aorta; $B l$. v., blood-vessels in wall of allantois; $c . C$., central canal of spinal cord; $C l$., cloaca; Ect., ectoderm; Ectam., ectoderm of amnion; E.E.B.C., exocœlom; Ent. endoderm; Mes., mesoderm; Mesam., mesoderm of amnion; $N^{\prime} c h$., notochord; $n . T$., nerve cord; $p^{\prime} a . G$. ., postanal gut; p.i.p., posterior intestinal portal; s.A., segmental arteries, between the somites; $S o^{\prime} p l$., somatopleure; $S p l$ ' $p l$., $S p$ ' $p l$., splanchnopleure; T.B., tail bud; t.f.So' $p l$., tail-fold in somatopleure; $t . f . S p^{\prime} p l$., tail-fold in splanchnopleure. 
ten of these disappear again during the eighth day of embryonic life, leaving a total of forty-two pairs.

The formation of the tail-fold is but one phase in the general process of the separation of the embryo from the remainder of the blastoderm, and at the same time from the yolk. The formation of the head-fold we have already described. We may recall that this extended backward along each side of the head. Posteriorly this now becomes continued either side of the trunk, by a somewhat similar lateral fold, which finally reaches to the

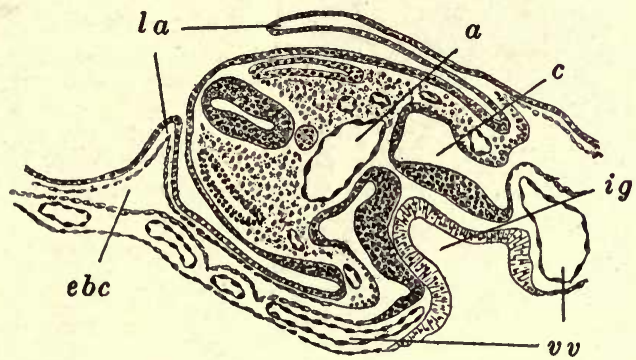

Frg. 110.-Diagrammatic transverse section through the region where the gut is open out over the yolk (yolk-stalk umbilicus), in a chick of about fortyeight hours (about twenty-eight pairs of somites). After Duval. a, Dorsal aorta; $c$, cœlom; $e b c$, exocœlom; $i g$, intestinal groove; la, lateral folds of amnion; $v v$, vitelline vein.

tail-fold. These folds involve both somatopleure and splanchnopleure (Fig. 110). The lateral folds gradually extend inward toward the mid-line beneath the embryo, converting the flat sheet of endoderm into a median fold above the yolk; this is the intestinal groove, the rudiment of the true intestine (Fig. 110). The head-and tail-folds continue to extend posteriorly and anteriorly, respectively, increasing the extent of the foreand hind-gut. These connect with the intestinal groove by the anterior and posterior intestinal portals.

Gradually these folds all approach beneath the embryo, cutting it off from the yolk, and marking a clear distinction between embryonic and extra-embryonic regions. Finally, about the fifth day, the lateral folds of the splanchnopleure fuse together extensively, and the gut is entirely closed off except in a restricted region in the hinder part of the trunk (Fig. 115). 
This remains open to the yolk nearly throughout embryonic life. The walls of this open portion of the gut elongate into a tubular stalk known as the yolk stalk. In reality this is double, composed of an outer wall of somatopleure, and an inner tube of splanchnopleure; the former is sometimes known as the somatic stalk, the latter as the splanchnic stalk or yolk stalk proper. The splanchnic stalk is the pathway by which

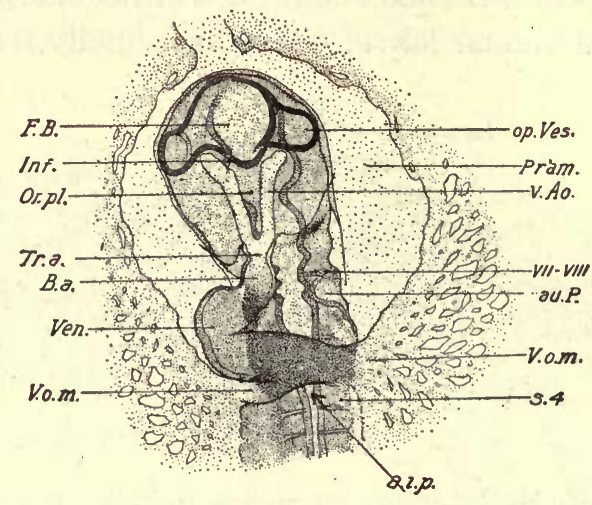

Fig. 111.-Ventral view of the anterior end of a chick embryo with sixteen pairs of somites (about thirty-eight hours). From Lillie (Development of the Chick). a.i.p., Anterior intestinal portal; au.P., auditory pit; B.a., bulbus arteriosus; F.B., fore-brain; Inf., infundibulum; op.Ves., optic vesicle; Or.pl., oral plate; Pr'am., proamnion; s.4, fourth somite; Tr.a., truncus arteriosus; v.Ao., ventral aorta; Ven., ventricle; V.o.m., omphalo-mesenteric (vitelline) vein; $V I I-V I I I$, acustico-facialis ganglion.

the blood-vessels pass between embryonic and extra-embryonic structures. The narrow space between the two stalks is, of course, cœlomic, connecting embryonic or true cœlom, and extra-embryonic cœlom or exocœlom.

The inner limbs of the lateral folds of the somatopleure form the body wall of the embryo, of which we may now describe lateral and ventral surfaces, as well as the dorsal, which has hitherto been the only free surface of the embryo, save in the head and tail.

\section{THE ESTABLISHMENT OF THE EXTERNAL FORM OF THE EMBRYO}

Rapid growth and extensive changes in the external form of the embryo have been proceeding, as the embryo has been 

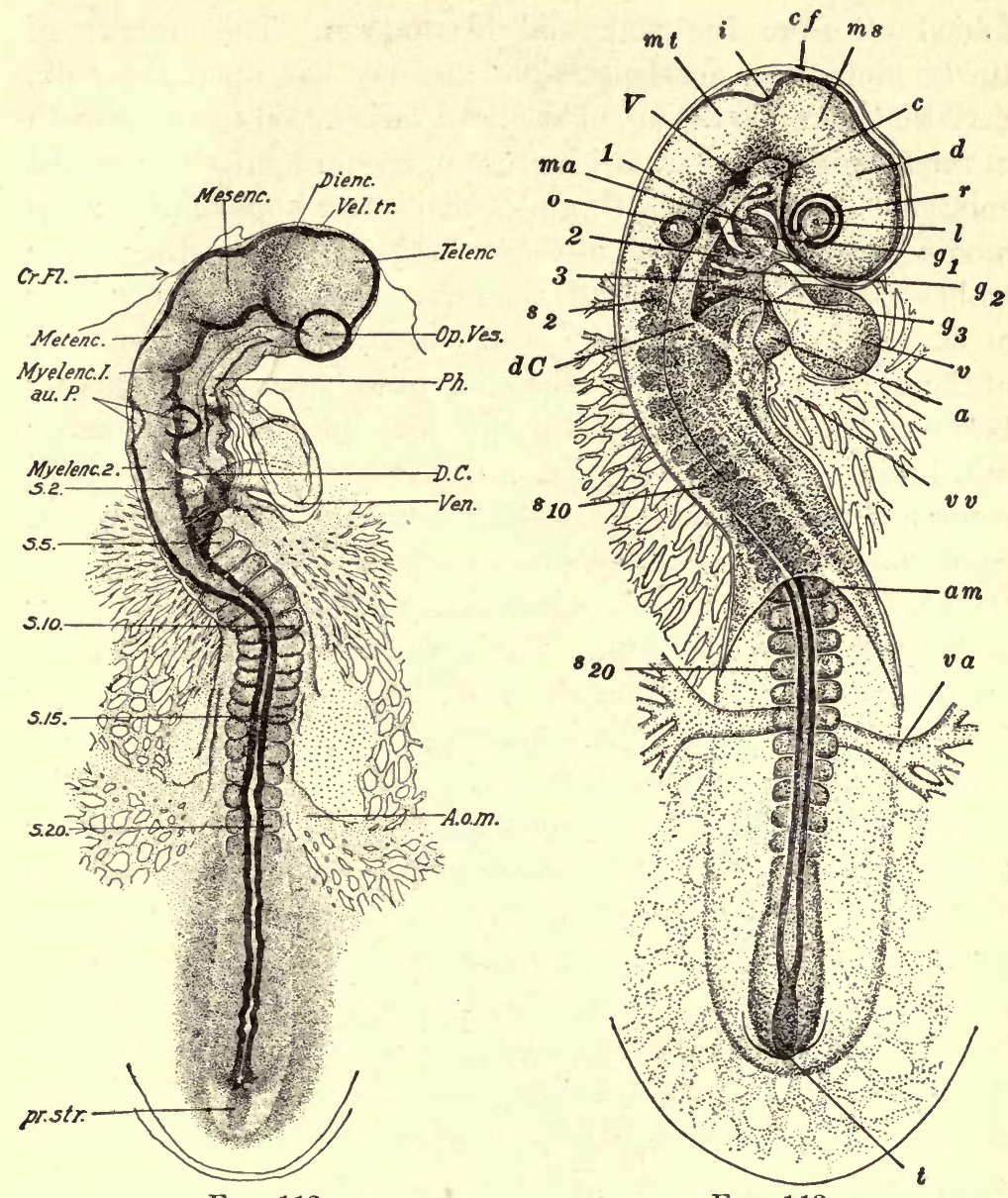

FIG. 112.

FIg. 113.

Fig. 112.-Chick embryo with twenty pairs of somites (about forty-three hours). Dorsal view. From Lillie (Development of the Chick). A.o.m., Vitelline artery; au.P., auditory pit; $C r . F l$., cranial flexure; D.C., ductus Cuvieri; Dienc., diencephalon; Mesenc., mesencephalon; Metenc., metencephalon; Myelenc. 1 , and 2, anterior and poster ior divisions of the myelencephalon; Op.Ves., optic vesicle. Ph., pharynx; $p r . s t r .$, primitive streak; s.2, s.5, etc., second, fifth, etc., somites; Telenc., telencephalon; Vel.tr., velum transversum; Ven., ventricle.

FIG. 113. - Chick embryo with twenty-seven pairs of somites (about fortyeight hours). Viewed from above. After Lillie. $a$, Auricle; $a m$, posterior margin of amnionic folds; $c$, carotid loop; $c f$, cranial flexure; $d$, diencephalon; $d C$, ductus Cuvieri; $g_{1}, g_{2}, g_{3}$, first, second and third gill elefts; $i$, isthmus; $l$, lens; $m a$, mandibular arch; $m s$, mesencephalon; $m t$, metencephalon; $o$, otocyst (auditory sac); just to the right of the otocyst is a thickening representing the ganglion of the VII and VIII cranial nerves; $r$, retinal layer; $s_{2}, s_{10}, s_{20}$, second, tenth, and twentieth somites; $t$, tail-bud; $v$, ventricle; $v a$, vitelline artery; $v v$, vitelline vein; $1,2,3$, first, second and third aortic arches; $V$, ganglion of $V$ cranial nerve. 
. folded off from the yolk and blastoderm. The embryo of thirty hours was nearly straight and lay flat upon the yolk, save that a simple flexure of the head was caused by the growth of the fore-brain downward in front of the fore-gut. Continued enlargement of the head (brain) leads to two important general modifications of the form of the embryo, first, a twisting of the embryonic body, and second, the formation of certain bendings or flexures in the axis of the head and trunk. As the cavities of the brain enlarge, a sharp bending of the neural axis appears between the fore- and mid-brain, soon involving the entire mid-brain. The development of this cranial flexure (Fig. 112), combined with the extensive enlargement of the fore-brain, would force this region down into the yolk but for the fact that the head now falls over upon one side, its left, which now comes to lie over upon the yolk. The trunk retains for a time its original position flat upon the yolk, and thus a twist appears just back of the head. This change in the position of the head begins about the thirty-sixth hour (15 pairs of somites), the cranial flexure having appeared somewhat earlier, about the thirtieth hour (10-12 pairs of somites). Gradually the region of the twist moves posteriorly, leaving the more anterior parts thrown over (Figs. 113, 114), and finally, about the close of the fourth day, the entire embryo comes to lie upon its left side.

Long before this, about the end of the second day, another flexure of the longitudinal axis appears; this is the cervical flexure. A broad ventral sweep through the entire hind-brain region, extending a short distance into the neck, results in the more complete flexure of the fore-brain, which thus comes to be directed posteriorly rather than ventrally, its true ventral surface approximating the ventral surface of the pharyngeal region. Later the entire trunk becomes arched dorsally, bringing the head and tail fairly close together on the ventral side (the morphologically ventral side now lies turned toward what was the right side, previous to the torsion of the embryo). During the later phases of embryonic development these last flexures disappear to a considerable extent, leaving permanently well-marked only the original cranial flexure. 


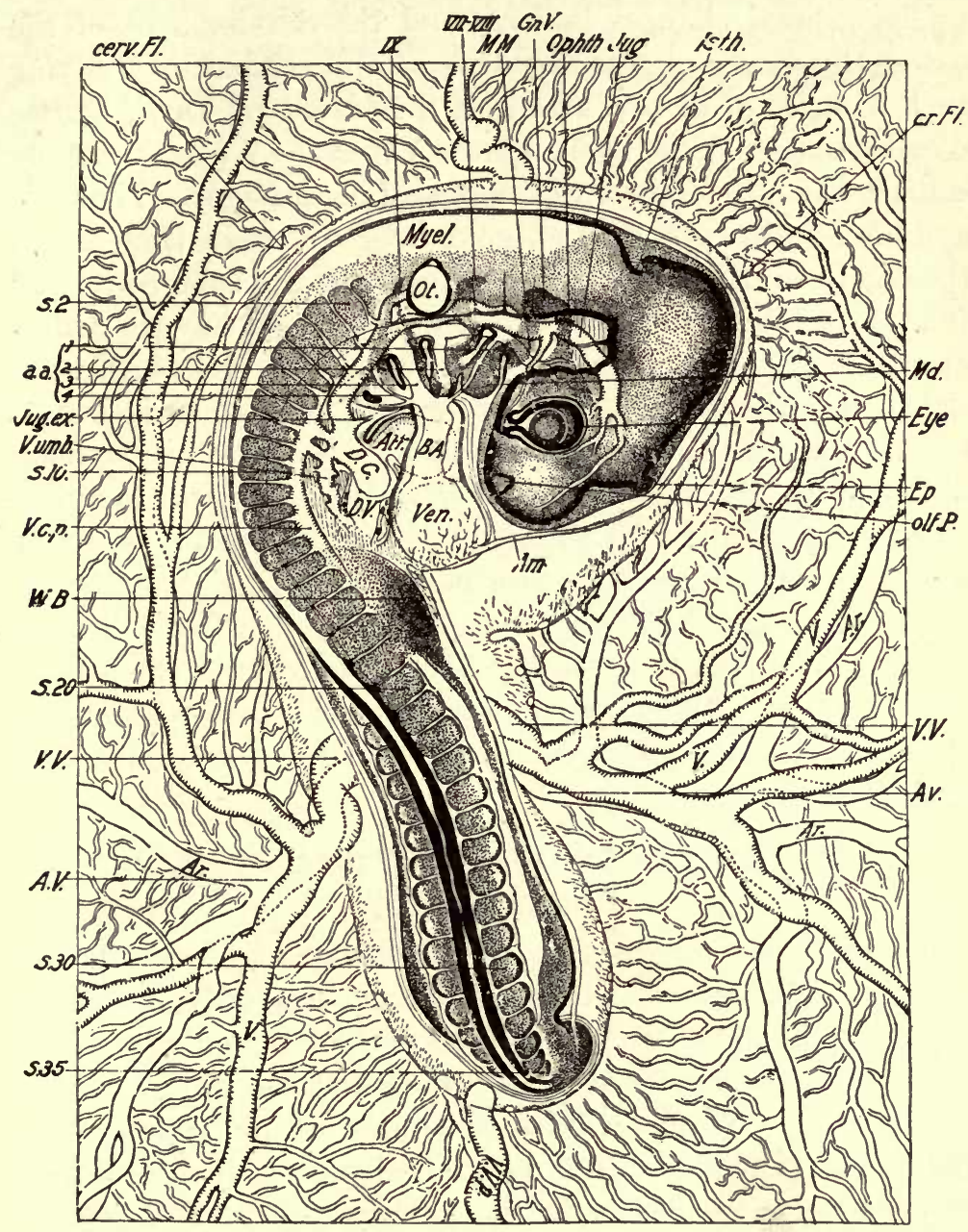

FIG. 114.-Chick embryo with adjacent portion of area vasculosa, with thirtyfive pairs of somites (about seventy-two hours). Dorsal view. From Lillie (Development of the Chick). a.a., 1, 2, 3, 4, First to fourth aortic arches; $A m$., amnion; $A r .$, branches of vitelline arteries; Atr., auricle; $A . V .$, vitelline artery; B.A., bulbus arteriosus; cerv. $F l$., cervical flexure; $c r . F l$., cranial flexure; D.C., ductus Cuvieri; D.V., ductus venosus; $E$ p., epiphysis; $G n$. V., ganglion of V cranial nerve; Isth., isthmus; $J u g$., external jugular vein; $M d$., mandibular arch; M.M., maxillo-mandibular branch of $\mathrm{V}$ cranial nerve; Myel., myelencephalon; olf.P., olfactory pit; Ophth. ophthalmic branch of V cranial nerve; Ot., otocyst; s.2, s.10, s.20, etc., second, tenth, twentieth, etc., somites; $V$., branches of the vitelline veins; V.c.p., posterior cardinal vein; V.umb., umbilical vein; V.V., vitelline vein; V.V.p., posterior vitelline vein; W.B., wing-bud. 
The general changes in form and the development of the externally visible organs may best be appreciated by studying such a series of embryos as that illustrated by Duval (Atlas d'embryologie, Paris, 1889; Plates VII-X). We may merely call attention to a few of the more striking features. The foreand hind-limbs appear, at approximately the same time, about the eightieth hour, as bud-like outgrowths from the body wall (Fig. 114). These enlarge rapidly; their segments are distinguishable at the beginning of the seventh day, and during the eighth day they assume the outlines of the wings and legs. During the eighth day too, the heart is drawn within the body wall, the visceral arches and gill clefts disappear, save as they remain represented in the adult by the jaws (mandibular arch) and the external auditory meatus (hyomandibular gill cleft), and the abdominal viscera are much enlarged. The following day the facial portion of the head begins to enlarge, the feather papillæ become well marked, and the general musculature begins to develop to such an extent as to affect the general contours of the body.

\section{THE EMBRYONIC MEMBRANES AND APPENDAGES}

We have now to describe the development of certain extremely important structures which are primarily extra-embryonic, and have to do with the protection, nutrition, and respiration of the embryo, rather than with the actual formation of proper organs. These structures are the yolk-sac, the amnion, and the allantois; strictly speaking the allantois arises as an embryonic structure, but it soon becomes extra-embryonic in position, and its functional value is derived from its later relation. These three structures are all characteristic of the embryos of the higher Craniates-Reptiles, Birds, and Mammals, and consequently these classes are often grouped together under the term Amniota.

\section{The Yolk-sac}

We have seen that at the thirtieth hour of incubation the blastoderm has extended over approximately one-fourth the 
surface of the yolk. Throughout its pellucid and vascular areas it is divided by the exocolom, into somatopleure and splanchnopleure (Fig. 108). Its outlying area vitellina for the most part

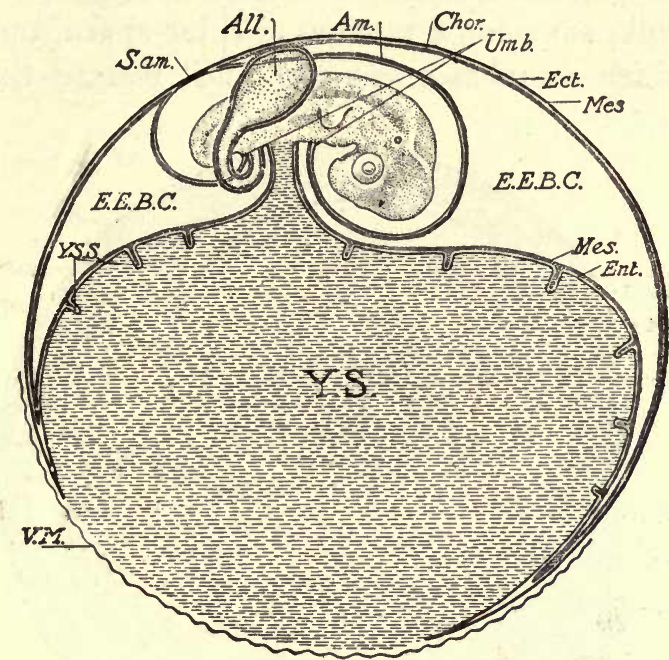

Figs. 115, 116, 117.-Diagrams of the relations of the embryonic membranes in the chick. Figures and description from Lillie (Development of the Chick). The ectoderm and endoderm are represented by plain lines; the mesoderm by a cross-hatched line or band. The yolk-sac is represented by broken parallel lines. In Fig. 115 the allantois is represented as a sac. In Figs. 116 and 117, where it is supposed to be seen in section, its cavity is represented by unbroken parallel lines. The stalk of the allantois is exaggerated in all the diagrams to bring out its connection with the embryo.

Fig. 115.-Fourth day of incubation. The embryo is surrounded by the amnion which arises from the somatic umbilicus in front and behind; the sero-amniotic connection is represented above the tail of the embryo; it consists at this time of a fusion of the ectoderm of the amnion and chorion. The allantois is represented as a sac, the stalk of which enters the umbilicus behind the yolk-stalk; the allantois lies in the extra-embryonic body-cavity (exocœlom), and its mesodermal layer is fused with the corresponding layer of the chorion above the embryo. The septa of the yolk-sac are represented at an early stage. The splitting of the mesoderm has progressed beyond the equator of the yolk-sac, and the undivided portion is slightly thickened to form the beginning of the connectivetissue ring that surrounds the yolk-sac umbilicus. The ectoderm and endoderm meet in the zone of junction, beyond which the ectoderm is continued a short distance. The vitelline membrane is ruptured, but still covers the yolk in the neighborhood of the yolk-sac umbilicus. The albumen is not represented in this figure. (For explanation of lettering see Fig. 117.)

consists of ectoderm and endoderm only, and is bounded peripherally by a region where the further extension of the blastoderm is being effected. The extension of the blastoderm con- 
tinues in the manner previously described, the differentiation of the mesoderm and the subsequent formation of exocœlom, somatopleure, and splanchnopleure, slowly proceeding centrifugally. During the fifth day the blastoderm extends completely over the yolk, save for a narrow circular space, opposite the embryo, which remains uncovered until toward the close of

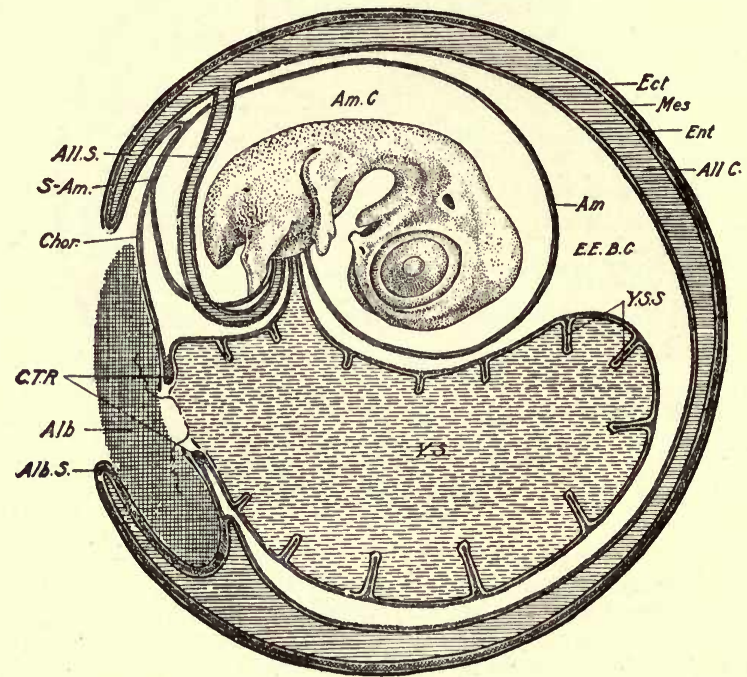

FIG. 116.-Ninth day of incubation. The yolk-sac umbilicus has become much narrowed, it is surrounded by the mesodermal connective-tissue ring, and by the free edges of the ectoderm and endoderm. The vitelline membrane still covers the yolk-sac umbilicus and is folded into the albumen. The allantois has expanded around the amnion and yolk-sac and its outer wall is fused with the chorion. It has pushed a fold of the chorion over the sero-amniotic connection, into which the mesoderm has penetrated, and thus forms the upper fold of the albumen-sac. The lower fold of the albumen-sac is likewise formed by a duplication of the chorion and allantois; it must be understood that lateral folds are forming also, so that the albumen is being surrounded from all sides. The stalk of the allantois is exaggerated so as to show the connection of the allantois with the embryo; it is supposed to pass over the amnion, and not through the cavity of the latter, of course. (For explanation of lettering see Fig. 117.)

embryonic life (Fig. 115). The mesoderm and associated structures of the blastoderm push around more slowly, but finally arrive at the blastodermal margin, which becomes a narrow ring called the yolk-sac umbilicus. The yolk-sac itself is the splanchnopleural portion of the extra-embryonic blastoderm, one surface applied directly to the surface of the yolk, the 
other forming the inner lining of the exocœlom (Fig. 115). The lower pole of the yolk-sac is open at the yolk-sac umbilicus, and the upper pole is of course directly continuous with the yolk stalk or splanchnic stalk, and therefore with the embryonic midgut (Fig. 115). While the yolk-mass is now practically enclosed in a layer of endoderm (splanchnopleure) it cannot be said to be

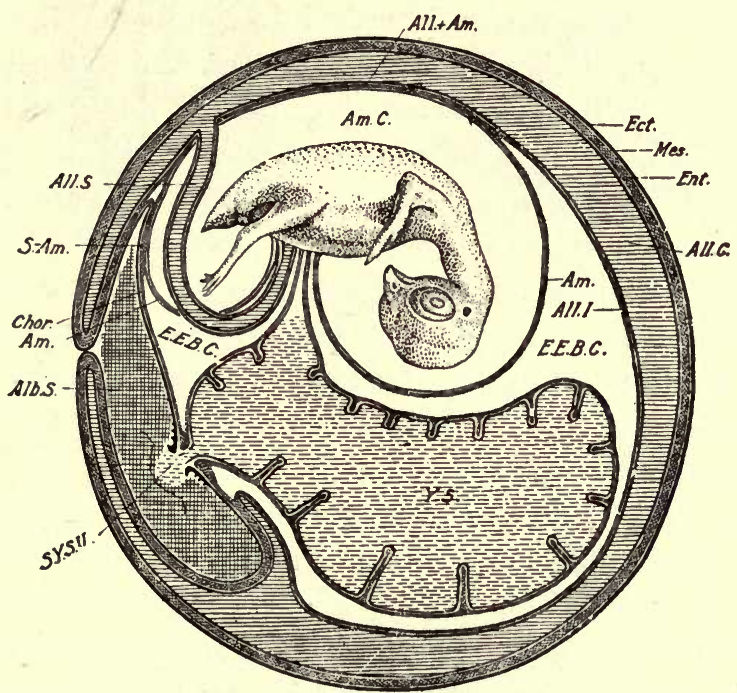

Fig. 117.-Twelfth day of incubation. The conditions represented in Fig 116 are more advanced. The albumen-sac is closing; its connection with the cavity of the amnion by way of the sero-amniotic connection will be obvious. The inner wall of the allantois has fused extensively with the amnion. The umbilicus of the yolk-sac is much reduced, and some yolk protrudes into the albumen (sac of the yolk-sac umbilicus). Alb., albumen; Alb.S., albumen-sac; All., allantois; All.I., inner wall of allantois; All.C., allantoic cavity; All.S., allantoic stalk; $A l l .+A m$. , fusion of allantois and amnion; $A m$. , amnion; Am.C., amnionic cavity; Chor., chorion; C.T.R., connective-tissue ring; Ect., ectoderm; E.E.B.C., exocœlom (extra-embryonic body-cavity); Ent., endoderm; Mes., mesoderm; S.-Am., sero-amnionic connection; S.Y.S.U., sac of the yolksac umbilicus; Umb., umbilicus; V.M., vitelline membrane; Y.S., yolk-sac, Y.S.S., septa of yolk-sac.

included within the enteron, for it remains extra-embryonic in position until the very close of embryonic life. The yolk is never taken directly into the embryonic digestive cavity and there absorbed. The endodermal lining of the yolk-sac early becomes differentiated as a yolk-digesting and absorbing organ, 
and the nutritive materials taken up by it are received into the yolk-sac circulation and carried to the embryo.

The yolk-sac is characterized by a wealth of blood vessels. The vessels of the vascular area, already mentioned, are the beginnings of the yolk-sac circulation. In the rich network of small vessels, larger pathways soon develop; the first of these are the paired anterior vitelline veins, which pass from the anterior margin of the blastoderm directly into the posterior end of the heart (Fig. 118, A). Soon after these become well established, the pair of vitelline arteries develop (about thirty-eight hours) as lateral branches of the dorsal aorta in the middle trunk region (Fig. 118, $A, B$ ). These arteries become distributed by large trunks throughout the yolk-sac, and supply its abundant capillaries. The blood re-collects into the marginal sinus or sinus terminalis, and passes thence into the anterior vitelline veins. By the end of the third day the two anterior vitelline veins have fused, just within the sinus terminalis, and between this point and the heart the right vein begins to disappear (Fig. 118, B), Soon it disappears entirely, and for a time the left anterior vitelline vein alone returns the blood to the heart, but before long, venous trunks appear, parallel with the main branches of the vitelline arteries, and by the end of the fourth day (Fig. 118, $C$ ) the greater part of the blood returns to the heart through these, the lateral vitelline or omphalo-mesenteric veins, which unite into the ductus venosus immediately before entering the heart. Thus both the supply and the return of the yolk-sac circulation pass along the wall of the yolk stalk or splanchnic stalk.

The digestive and absorptive surface of the yolk-sac becomes enormously increased by a folding process, which begins at the time the larger vessels begin to be marked out. The folding (Fig. 116) finally comes to be very complicated, and accompanying the folds is a continuation of the network of large capillaries which thus come to have an intimate and extensive relation with the nutritive supply. The inner surface of the yolk-sac finally acquires a structure not unlike that of the lung, its spaces or meshes being filled with the yolk material. 

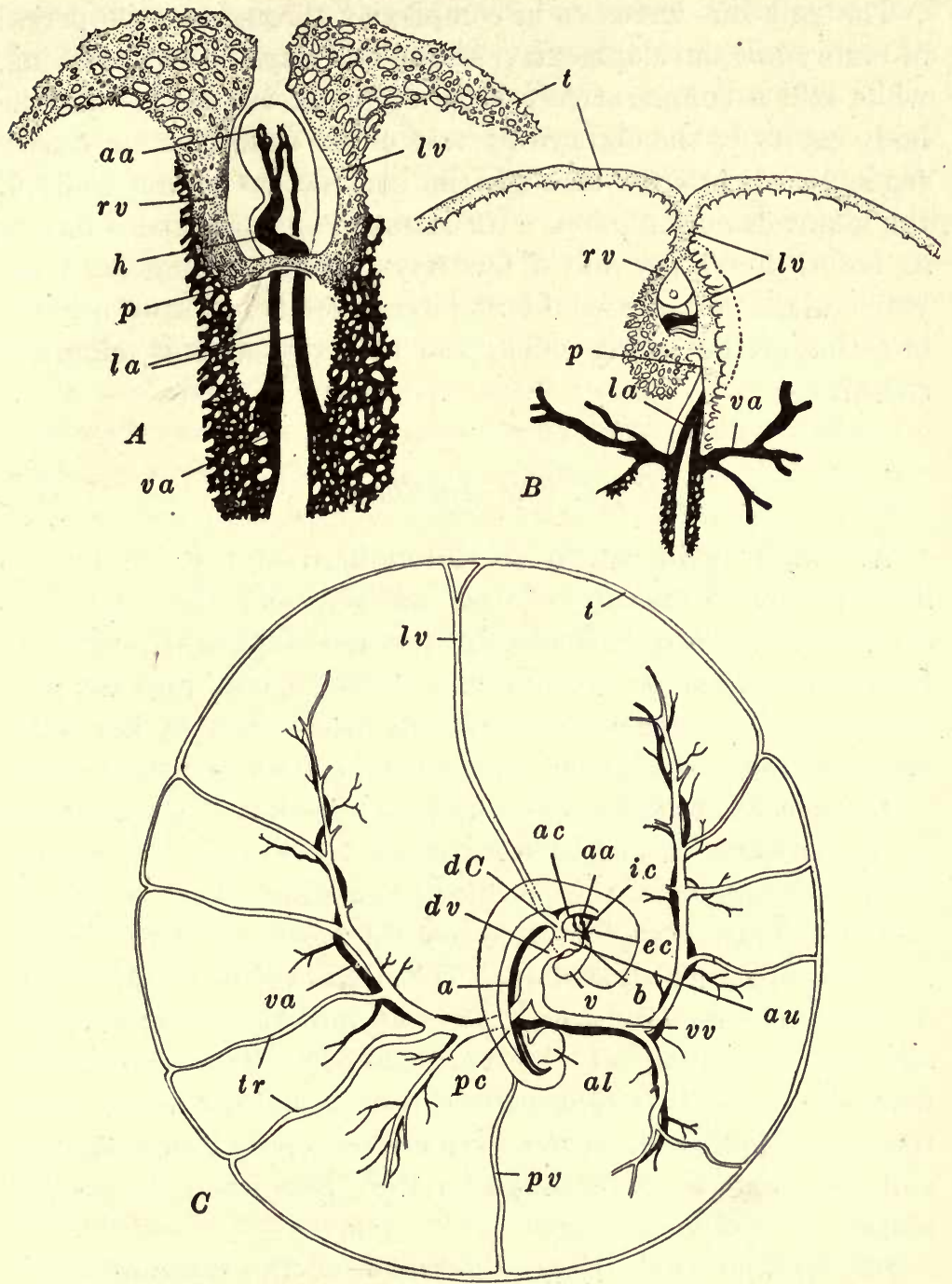

FIG. 118.-Diagrams of the circulation in the chick embryo and area vasculosa. The vascular network of the area vasculosa is omitted for the most part. $A$. Anterior and central parts of the embryo and vascular area at about thirtyeight hours (sixteen pairs of somites). Viewed from beneath. After Popoff. $B$. Median and anterior parts of vascular area and embryo at about seventy-two hours (twenty-seven pairs of somites). Viewed from beneath. After Popoff. $C$. The main vascular trunks of the fourth day. After Lillie (modified). $a$, Dorsal aorta; $a a$, aortic arches (first and second in $A$, second, third and fourth in $C$ ); $a c$, anterior cardinal vein; $a l$, allantois; $a u$, auricle; $b$, bulbus arteriosus; $d C$, ductus Cuvieri; $d v$, ductus venosus; $e c$, external carotid artery; $h$, heart; 
The yolk-sac increases in complexity throughout the period of embryonic development. About two days before hatching, while still a comparatively large organ, it is pushed into the body cavity of the embryo by way of the somatic stalk cavity (cœlom), apparently through the contraction of the walls of the allantois and amnion. Its entrance is completed a day or so before hatching, and a few days after hatching both the yolk and the yolk-sac itself become completely absorbed into the intestine of the young chick, and the yolk stalk is definitely closed.

\section{The Amnion}

We must again return to the embryo of thirty hours to describe the formation of the amnion, and the associated chorion, which are derived from the somatopleural portion of the extra-embryonic blastoderm. In a general way we may say that these structures arise from the crests of the outer limbs of those folds which cut the embryo off from the extraembryonic blastoderm. The first indication of the amnion is in the blastoderm, just in front of the head of the embryo, in the anterior region of the so-called proamnion, where, about the thirtieth hour, a slight transverse thickening of the ectoderm becomes apparent in sections. This thickening is the beginning of the ectamnion, which plays an important rôle throughout the formation of the true amnion. The proamnion, it will be recalled, is that part of the blastoderm which for a time remains mesoderm-free. Hence no cœlom, somatopleure, and splanchnopleure are present in the region where the amnion makes its first appearance.

Just in front of the head, the region of the ectamnion, endoderm as well as ectoderm, becomes folded upward as the head-fold of the amnion (Fig. 99). This begins to extend back-

$i c$, internal carotid artery; $l a$, lateral dorsal aorta; $l v$, left anterior vitelline vein; $p$, anterior intestinal portal; $p c$, posterior cardinal vein; $p v$, posterior vitelline vein; $r v$, right anterior vitelline vein; $s$, sinus venosus; $t$, sinus terminalis; $t r$, venous trunks of the area vasculosa; $v$, ventricle; $v a$. vitelline artery; $v v$, vitelline or omphalo-mesenteric vein. 
ward over the head of the embryo, but before it has gone very far the mesoderm, with the accompanying exocœlom, extends into the fold, and the endodermal layer is withdrawn. The lateral extremities of the head-fold of the amnion turn posteriorly along the sides of the head, as the lateral folds of the amnion. These are somatopleural from their beginning, and may be described as elevations of the blastoderm in the region where the outer limbs of the lateral embryonic folds pass into the general surface of the blastoderm (Fig. 110).

As these folds of the amnion extend posteriorly, the ectamnion serves actively to draw the more lateral regions toward the mid-line where they are fused together, and in this way the lateral folds are gradually drawn up over the embryo (Lillie). This process occurs progressively in a posterior direction. About the time the embryo has thus become covered over as far back as the level of the vitelline arteries (48 hours, 26-28 pairs of somites), a posterior tail-fold of the amnion appears, (Fig. 109, A), similar to its head-fold save that it is somatopleural, i.e., contains cœlom and mesoderm, from its commencement. This tail-fold extends laterally and then anteriorly, and soon its extremities meet the lateral folds. About the seventieth hour (35 pairs of somites) the amnionic folds are completely closed together above the embryo; this closure occurs about at the level of the hind-limb buds.

As the somatopleural amnion folds come together above the embryo, the ectodermal and mesodermal layers of each side fuse together and the original contact surfaces break through, so that the colomic cavities of the amnionic folds become continuous across the mid-line. This results in the formation of two complete membranes, each composed of two cell layers, around the embryo. The inner of these, close to the embryo, is the true amnion, and the space between this and the embryo is the amnionic cavity (Figs. 119, 125). From the method of its formation it will be seen that the amnion is continuous with the somatic stalk, while the amnionic cavity is a space entirely outside the embryo, and has no connection with any other cavity. The outer membrane is now called the chorion. It is, 
of course, the somatic portion of the blastoderm, and at this time has no direct connection with any other structure. The space between amnion and chorion is exocœlom. This cavity and the amnionic cavity are filled with a watery fluid. Later on, as we shall see, this space is largely occupied by the allantois, which comes into intimate connection with the inner surface of the chorion.

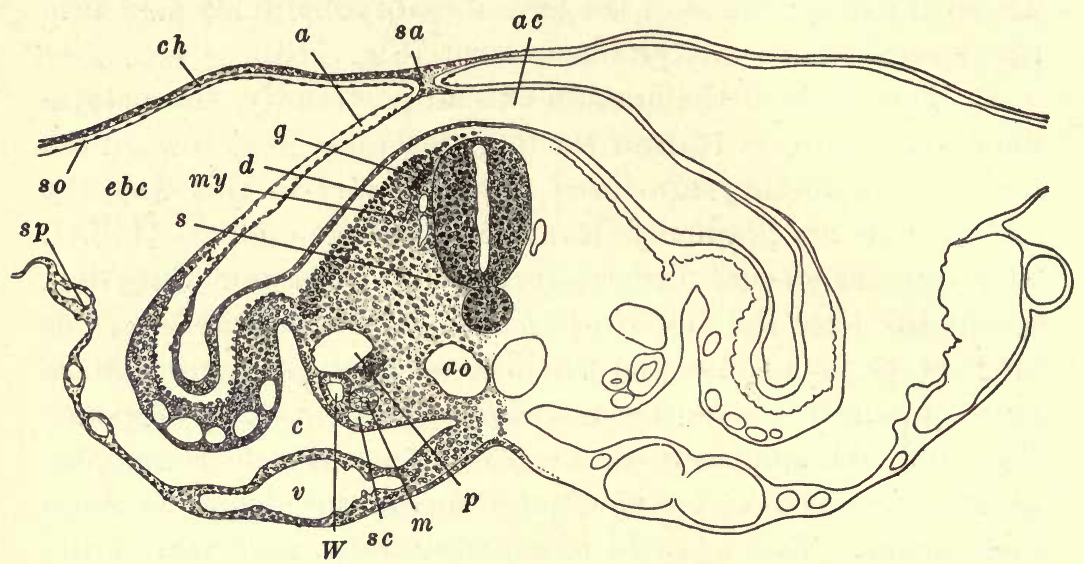

Fig. 119.-Transverse section of chick embryo with thirty-five pairs of somites (about seventy-two hours), passing through the region of the twentythird somite. After Lillie. $a$, Amnion; $a c$, amnionic cavity; $a o$, dorsal aortæ; $c$, embryonic cœlom; $c h$, chorion; $d$, dermatome; $e b c$, exocœlom; $g$, rudiment of spinal ganglion; $m$, mesonephric tubules; $m y$, myotome; $p$, posterior cardinal vein; $s$, sclerotome; $s a$, sero-amnionic connection; $s c$, subcardinal vein; $s o$, somatic mesoderm; $s p$, splanchnic mesoderm; $v$, vitelline artery; $W$, Wolffian duct.

One important fact has not been mentioned thus far. In the region where the amnionic folds finally close together over the embryo, the amnion and chorion remain firmly united (Fig. 119). This forms the sero-amnionic fusion, which affects in an important way the later arrangement of these membranes, as well as the disposition of the allantois, when it extends into this region (Figs. 115, 116).

The regular arrangement of the lateral folds of the amnion is disturbed somewhat by the twisting of the embryo, which is going on as these folds are becoming elevated and fused. As the embryo turns upon its left side the amnionic folds do not similarly alter their morphological relation to what was 
originally the median plane. That is, they continue to close together along what was originally marked out as the mid-line, and they finally close immediately above the right hind-limb bud. It follows from this that the left lateral fold becomes much the more extensive, passing from the lower (left) side of the embryo, around to the upper (right) side and thus covering the whole morphologically dorsal surface of the embryo, while the right lateral fold covers only a portion of the upper (morphologically right) surface of the embryo. This is correlated with the fact that the right fold is thicker than the left and is usually thrown into irregular transitory folds or wrinkles.

Soon after the amnion is fully formed, muscle fibers are differentiated in its mesodermal layer. Waves of contraction then slowly pass along the amnion and keep up a movement of the amnionic and cœlomic fluids. It is also supposed that they keep the embryo in slight motion, preventing adhesions between the embryo and its appendages. The amnion also serves, probably, as a water cushion, protecting the embryo against deforming pressures. The later relations of the amnion to the allantois and the albumen of the egg will be mentioned below.

\section{The Allantois}

The allantois is an embryonic appendage of the greatest importance. Unlike the yolk-sac, amnion, and chorion, it is developed from the embryo itself, and not from the extraembryonic structures. When fully developed it is the most extensive of all these accessory organs, the arrangement of which becomes considerably modified by its growth. Functionally, too, it is of the utmost importance, it is primarily the embryonic respiratory organ, but it serves also as a reservoir for excretory products, and through the development of muscle fibers in its wall it effects certain movements of the embryo. and assists in the final inclusion of the yolk-sac within the embryonic body.

The morphological relations of the allantois to the embryo 
will be better understood if we recall certain facts regarding the development of the tail. The tail-fold of the blastoderm differs essentially from the head-fold in that the blastoderm where it appears includes mesoderm, and is already differentiated into somatopleure and splanchnopleure (Fig. 109, A). Preceding the formation of the tail-fold of the somatopleure, the splanchnopleure is folded forward beneath the posterior end of the embryo, establishing a short hind-gut. Soon after, the true, or

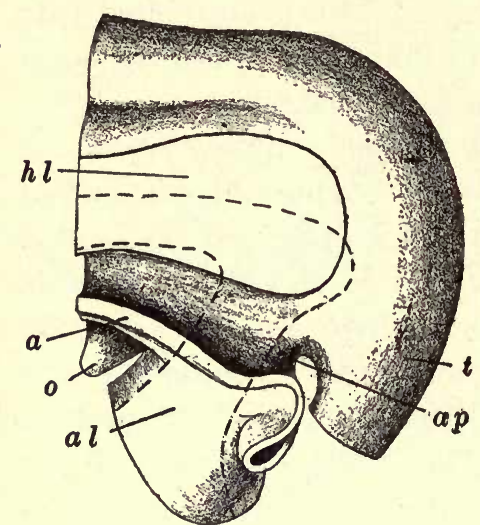

Fig. 120.- Model of part of the caudal end of a four-day chick, showing the relation of the amnion to the allantoic stalk. After Ravn( modified). The broken line marks the cavity of the hind-gut and allantoic stalk. $a$, Cut edge of amnion; al, allantoic stalk; $a p$, anal plate; $h l$, cut surface of hind-limb bud; $o$, vitelline artery; $t$, base of tail (the tip has been removed). somatopleural, tail-fold pushes inward, a short distance behind the splanchnic fold. The embryonic region immediately anterior (morphologically) to the somatopleural tail-fold is the rudiment of the tail, the tail bud (Fig. 109, B). As this elongates, a posterior extension of the hind-gut continues into it, as the postanal gut, for the anus is formed on the ventral side of the base of the tail. The anterior extent of the hindgut is increased by the forward extension of the splanchnopleural tail-fold. The region beneath the hind-gut, between the splanchnopleural and somatopleural tail-folds, is mesodermal and is in reality a deep ventral mesentery. The cavity of the hind-gut now pushes downward into this ventral mesentery, forming an elongated depression, just in front of the anal region (Fig. 109, $B$ ). This groove-like outgrowth is the rudiment of the allantois, which thus makes its appearance about the commencement of the third day of incubation (about 28 pairs of somites). In front of this the hind-gut narrows again; the dorsal portion of this expansion, and the region just posterior to it, form the rudiment of the cloaca (Fig. 120). 
The allantois is clearly an outgrowth or appendage of the hind-gut, and consequently its cavity is directly continuous with the enteron; it is lined with endoderm, and its outer wall is mesodermal. Before the close of the fourth day (Fig. 121) the allantois has extended for some distance out into the exocœlom, lying to the right of the tail. Its terminal portion has enlarged into a dilated vesicle connected with the gut by a narrow tubular allantoic stalk. It has an abundant blood supply, derived from a pair of branches of the dorsal aorta,

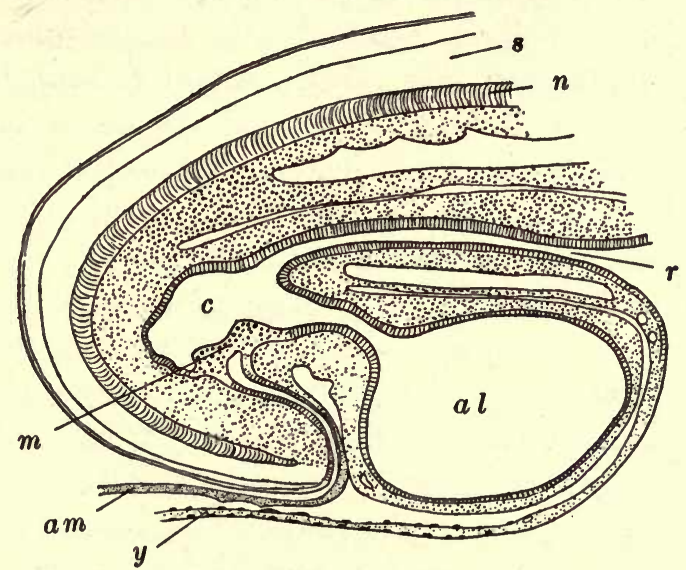

Fig. 121.-Median sagittal section through posterior end of four-day chick. After Gasser (Maurer). al, Allantois; $a m$, amnion (tail-fold); $c$, cloaca; $m$, cloacal membrane; $n$, notochord; $r$, rectum; $s$, spinal cord; $y$, wall of yolk-sac (endoderm and splanchnic mesoderm).

the allantoic arteries; its blood collects into a single allantoic vein and is returned to the heart through the large left umbilical vein (Fig. 138).

Once established, the allantois grows very rapidly through the exocœlom, between the chorion and the yolk-sac and amnion (Figs. 115, 116). Its vesicle enlarges rapidly and by the end of the sixth day has covered the entire embryo; two days later it has passed nearly around the yolk-sac. As the allantois enlarges it effects certain important fusions with other structures. Superficially it fuses with the chorion, the mesodermal layers of the two membranes uniting so intimately 
that in effect the chorion forms a part of the outer membrane of the allantois. Along the dorsal surface of the embryo the inner wall of the allantois meets the amnion, and the apposed mesodermal layers of these two membranes fuse together, forming a layer of muscle fibers which function for a time similarly to those of the amnion, and then disappear.

A modified region of the allantois should be mentioned here. During the early days of incubation the albumen of the egg condenses toward what may be described as the posteroventral side of the yolk, from which it is separated only by the remains of the vitelline membrane, in the region where the yolk-sac remains open (Fig. 116). About the ninth or tenth day, the albumen, through continued loss of water, forms a relatively small, dense mass, incompletely separated from the yolk by the intervention of the yolk sac (Fig. 117). The allantois reaches the albumen first from the lower side. It is now fused with the chorion throughout its extent, and as it pushes onward, it sends a short fold in between the albumen and the yolk-sac, and a longer limb around the outer surface of the albumen, almost covering it over. These folds of fused chorion and allantois are the beginnings of the albumen sac. The albumen sac is completed by the downgrowth of a similar fold of fused chorion and allantois from the upper side. These folds finally enclose the yolk completely (Fig. 117). The upper fold is not quite like the lower on account of the obstruction offered to the progress of the allantois, by the sero-amnionic fusion. When the rapidly extending allantois reaches this area of fusion of the amnion and chorion, it pushes the chorion out above the fused area, carrying a fold of the chorion before it in its onward extension (Fig. 116). This fold of the chorion, with the allantois within, is similar to the outer limb of the ventral part of the albumen sac, but the inner limb of the latter is consequently not represented on the dorsal side. Dorsally the inner wall of the albumen sac is formed only by the original chorion of the region, the seroamnionic connection being included (Fig. 117). About the time the albumen sac is completed, by the fusion of the mem- 
branes forming its outer wall, the sero-amnionic fusion becomes perforated, and the albumenous material partly passes into solution in the amnionic fluid. For the most part, however, the albumen is absorbed through the walls of the albumen sac. This is fully accomplished toward the close of the embryonic period, and the albumen sac then remains as an appendage of the yolk-sac, with which it passes into the body of the embryo at the end of incubation.

The most characteristic feature of the allantois is the extreme vascularity of its outer wall. The withdrawal of the albumen from the greater part of its surface leaves the fused chorion and outer allantoic wall in direct apposition to the shell membrane. The chorionic layers become extremely thin and the shell membrane becomes very porous, so that there is an easy exchange of gases between the outside air and the capillaries of the outer allantoic wall. These capillaries are very. wide and extremely abundant, so altogether the allantois is a very efficient respiratory organ.

The inner wall of the allantois, as already mentioned, fuses with the amnion in the region dorsal to the embryo, during the second week of incubation. Here as elsewhere in the inner wall, muscle fibers may develop. Later on, as the period of incubation draws toward its close, it fuses also with the yolksac. The cavity of the allantois remains connected with the cloaca by the tubular allantoic stalk. It is filled with a fluid containing the excretory products, received from the cloaca where the excretory ducts discharge.

Von Baer's account of the principal events associated with the hatching of the young chick, is summarized in the two following paragraphs quoted from Lillie ("Development of the Chick," p. 232).

"About the fourteenth day the growing embryo accommodates itself to the form of the egg so as to lie parallel to the long axis with its head usually toward the broad end near to the airchamber. Sometimes, however, the embryo is turned in the reverse position (Von Baer). The head is bent toward the breast, and is usually tucked under the right wing. Important 
changes preparatory to hatching take place on the seventeenth to nineteenth days. The fluid decreases in the amnion. The neck acquires a double bend so that the head is turned forward, and, in consequence, the beak is toward that part of the membranes next to the air-chamber. The intestine is retracted completely into the body-cavity, and on the nineteenth day the yolk-sac begins to enter the body-cavity. On the twentieth day the yolk-sac is completely included, and practically all the amniotic fluid has disappeared. The chick now occupies practically all the space within the egg, outside of the air-chamber. The umbilicus is closing over. The ductus arteriosi begin to contract, so that more blood flows through the lungs. The external wall of the allantois fused with the chorion still remains very vascular.

"Now, if the chick raises its head, the beak readily pierces the membranes and enters the air-chamber. It then begins to breathe slowly the contained air; the chick may be heard, in some cases, to peep within the shell two days before hatching, a sure sign that breathing has begun. But the circulation in the allantois is still maintained and it still preserves its respiratory function. When the chick makes the first small opening in the shell, which usually takes place on the twentieth day, it begins to breathe normally, and then the allantois begins to dry up and the circulation in it rapidly ceases. It then becomes separated from the umbilicus, and the remainder of the act of hatching is completed, usually on the twenty-first day."

References to the literature are given at the end of Chapter V. 
CHAPTER V

THE LATER DEVELOPMENT OF THE CHICK: ORGANOGENY

I. THE NERVOUS SYSTEM . . . . . . . . . . . 302

1. The Central Nervous System . . . . . . . . . . . 302

2. The Cranial and Spinal Ganglia . . . . . . . . . . . . 309

3. The Peripheral Nervous System . . . . . . . . . . . . 311

A. The Spinal Nerves and the Sympathetic System . . 311

B. The Cranial Nerves . . . . . . . . . . . . . . . 313

II. THE SPECIAL SENSE ORGANS . . . . . . . . . . 316

1. The Eye . . . . . . . . . . . . . . . . . . . . . 316

2. The Ear . . . . . . . . . . . . . . . . . . . . . . 321

3. The Olfactory Organ . . . . . . . . . . . . . . . . . . . . . 325

III. THE ALIMENTARY TRACT AND ITS APPENDAGES . . 326

1. The Organs of the Fore-gut . . . . . . . . . . . . . . . . 326

2. The Organs of the Hind-gut . . . . . . . . . . . . . 334

3. The Mid-gut . . . . . . . . . . . . . . . . . . . . 334

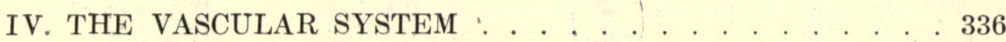

1. The Heart . . . . . . . . . . . . . . . . . . . 337

2. The Aortic Arches and the Arterial System . . . . . . . . 340

3. The Venous System. . . . . . . . . . . . . . . . . . 343

4. The Lymphatic System and Spleen . . . . . . . . . . . 349

V. THE CAVITIES OF THE BODY . . . . . . . . . . . 350

VI. THE LATER HISTORY OF THE MESODERMAL SOMITES . 352

VII. THE URINOGENITAL SYSTEM . . . . . . . . . . . . . 354

1. The Excretory System . . . . . . . . . . . . . . . . 355

A. The Pronephros and the Pronephric Duct (Wolffian Duct) . . . . . . . . . . 355

B. The Mesonephros . . . . . . . . . . . . . . 356

C. The Metanephros . . . . . . . . . . . . . . 358

2. The Reproductive System . . . . . . . . . . . . . . . . . . 359

A. The Reproductive Ducts . . . . . . . . . . . 360

B. The Gonads . . . . . . . . . . . . . . . . 361

3. The Adrenal Bodies . . . . . . . . . . . . . . . . 363

Iт remains now for us to describe the chief events in the development of the systems and organs of the embryo, from the 
condition at which we left them, for the most part about the thirtieth hour. We shall limit this account to little more than the bare mention of the more important morphological alterations of structure, considering the systems separately.

\section{THE NERVOUS SYSTEM}

\section{The Central Nervous System}

We have already traced the development of the central nervous system up to the formation of the spinal cord and
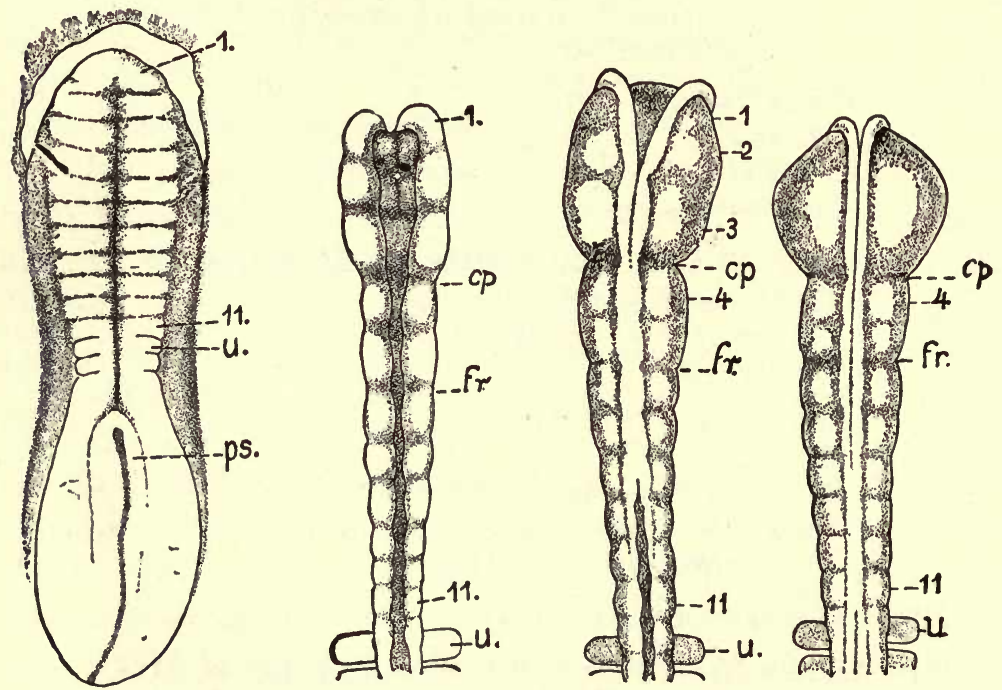

Fig. 122.- Neuromeres in the brain of the chick embryo. Dorsal views. From Von Kupffer (Hertwig's Handbuch, etc.) after Hill. A. Embryo of twenty-four hours; two pairs of somites. B. Embryo of twenty-four hours; five pairs of somites. C. Embryo of twenty-five and one-half hours; six pairs of somites. D. Embryo of twenty-six hours; seven pairs of somites. $c p$, Posterior limit of prosencephalon; $f r$, posterior limit of mesencephalon; $p s$, primitive streak; $u$, first somite; $1-11$, neuromeres.

brain, and the establishment, in the latter, of the primary hind-, fore-, and mid-brains. In connection with the primary fore-brain we noted the rudiments of the optic vesicles, and in the primary hind-brain the series of six neuromeres, or brain segments, which are clearly distinguishable at the thirty-hour stage described. 
In earlier stages neuromeres have made a brief, transitory appearance in the fore- and mid-brains as well. Thus in the embryo of about twenty-three hours (4 pairs of somites), Hill has distinguished eleven neuromeres, all told. Of these three are in the region of the future fore-brain: these disappear about the twenty-sixth hour (7 pairs of somites). The midbrain includes only two neuromeres, which remain distinguishable until about the thirty-fifth hour (14 pairs of somites). The six neuromeres of the hind-brain are the best marked, and are visible for several days (Fig. 122).

The next important advance consists in the transverse constriction of the primary fore- and hind-brains each into two sections, making in all five regions in the brain. The primary fore-brain, or prosencephalon, is divided into the anterior telencephalon, or secondary fore-brain, and the posterior diencephalon, or between-brain. Then following the undivided mid-brain, or mesencephalon, come the two divisions of the primary hind-brain, or rhombencephalon; the anterior of these is known as the metencephalon, the posterior as the myelencephalon. The myelencephalon continues directly into the spinal cord.

We may now proceed to describe the principal structures arising in connection with each of the secondary divisions of the brain.

The telencephalon includes only the first neuromere. This portion of the brain, it will be recalled, is directed backward beneath the fore-gut, its morphologically anterior end actually being directed postero-dorsally (Fig. 123). The telencephalon expands vertically and the optic vesicles remain connected with its ventral side. As the optic vesicles push out toward the surface of the head, their connections with the brain become narrowed as the optic stalks. The cavities of the vesicles remain continuous, through the stalks, with the cavity of the telencephalon (Fig. 127), and a median depression in the floor of the telencephalon, between the openings of the optic stalks, becomes well marked as the recessus opticus (Fig. 123). This optic recess, and a short stretch of the brain-wall 

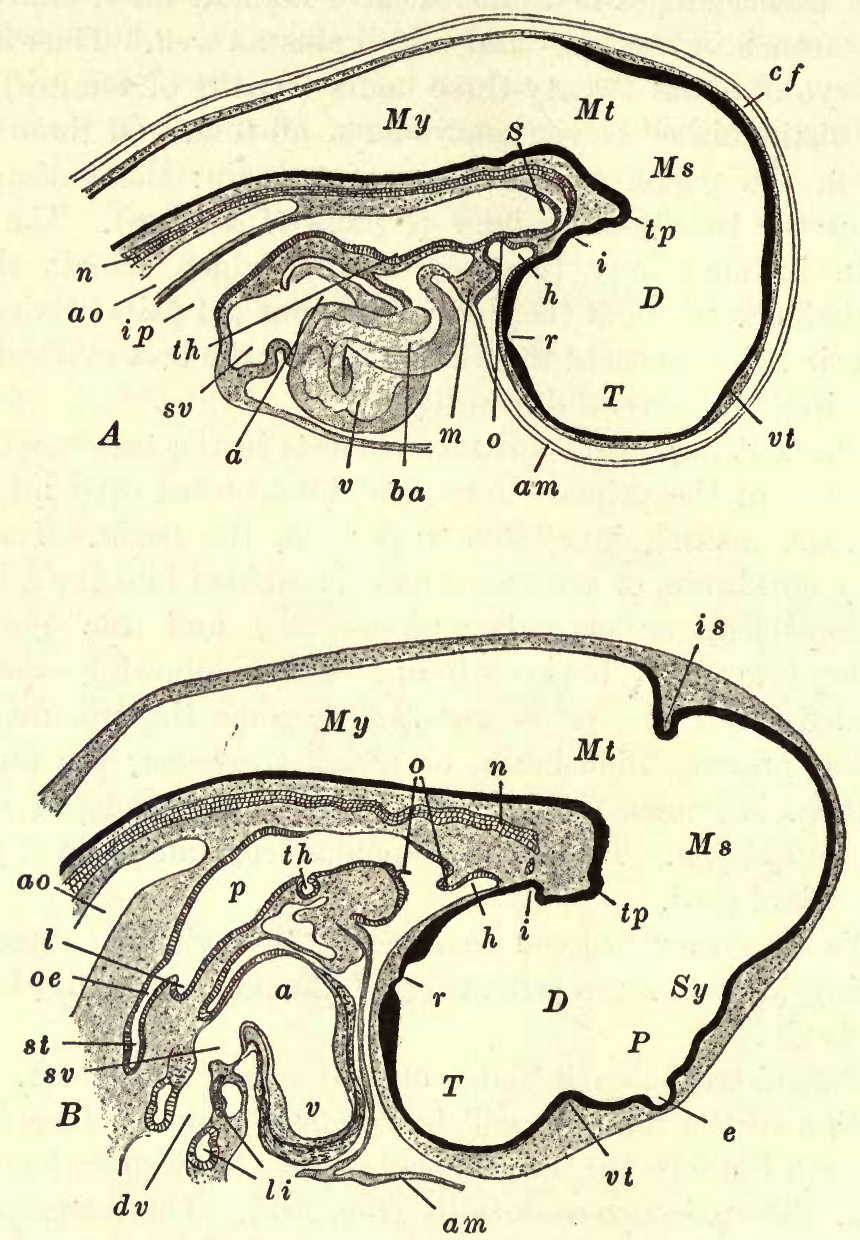

Fig. 123. - Sagittal sections through the head of the chick. In $A$ the beart is shown in optical section. After Lillie. $A$. Of an embryo with twenty-two or -three pairs of somites (about forty-four hours). B. Of an embryo with thirtynine pairs of somites (end of the fourth day). a, Auricle; $a m$, amnion; $a o$, dorsal aorta; $b a$, bulbus arteriosus; $c f$, cranial flexure; $D$, diencephalon; $d v$, ductus venosus; $e$, epiphysis; $h$, hypophysis; $i$, infundibulum; $i p$, anterior intestinal portal; $i s$, isthmus; $l$, rudiment of lung; $l i$, liver; $m$, mandibular $\operatorname{arch} ; M s$, mesencephalon; $M t$, metencephalon; $M y$, myelencephalon; $n$, notochord (degenerating); $o$, oral membrane (oral plate); oe, œesophagus; $p$,pharynx; $P$, parencephalon; $r$, optic recess; $S$, Seessel's pocket (preoral gut); st, stomach; $s v$, sinus venosus; $S y$, synencephalon; $T$, telencephalon; $t h$, rudiment of thyroid body; $t p$, tuber culum posterius; $v$, ventricle; $v t$, velum transversum. 
immediately in front of it, not now distinct but differentiating later, together mark, morphologically, the true anterior end of the central nervous system.

The anterior and lateral portions of the telencephalon now begin to expand, giving the effect of a constriction at its posterior limit. This apparent constriction of the dorsal wall of the fore-brain is the velum transversum, which marks the separation between telencephalon and diencephalon. In these

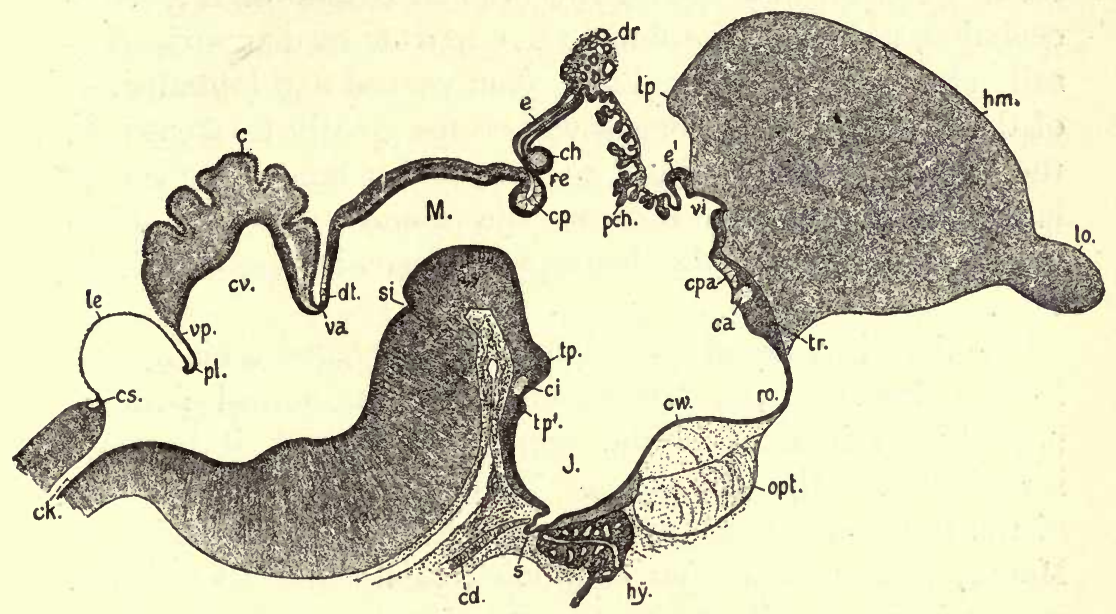

FIg. 124.-Median sagittal section through the brain of the chick of twelve to thirteen days. From Von Kupffer (Hertwig's Handbuch, etc.); $c$, Cerebellum; $c a$, anterior commissure; $c d$, notochord; $c h$, habenular commissure; $c i$, infundibular commissure; $c k$, central canal of spinal cord; $c p$, posterior commissure; $c p a$, anterior pallial commissure; $c s$, spinal commissure; $c v$, cavum cerebelli; $c w$, optic chiasma; $d r$, epiphysial gland; $d t$, decussation of the trochlear (IV) nerve; $e$, epiphysis; $e^{1}$, paraphysis; $h m$, cerebral hemisphere; $h y$, hypophysis; $J$, infundibulum; le, ependymal lamina of the roof of the fourth ventricle; $l o$, olfactory lobe; $l p$, posterior lobe of cerebral hemisphere; $M$, mesencephalon; opt, optic chiasma; $p c h$, choroid plexus third ventricle; $p l$, choroid plexus of fourth ventricle; $r e$, epiphysial recess; $r o$, optic recess; $s$, saccus of infundibulum; $s i$, posterior intracephalic furrow; $t p$, tuberculum posterius; $t p$, tuberculum mammillare; $t r$, torus transversus; $v a$, velum medullare anterius; $v i$, median ventricle of telencephalon; $v p$, velum medullare posterius.

early stages no other sharp distinction between these two regions can be determined.

Toward the close of the second day a pair of lateral extensions of the telencephalon push out rapidly and expand dorsally, anteriorly, and posteriorly; these are the rudiments of 
the cerebral hemispheres. The cavity of the telencephalon is continued into the cerebral hemispheres as the lateral ventricles, known also as the first and second ventricles of the brain. The reduced median cavity of the telencephalon remains, forming the anterior portion of the third ventricle; the openings of the lateral ventricles out of the third ventricle are known as the foramina of Monro. The cerebral hemispheres enlarge rapidly and soon grow out, either side of the mid-line, far in front of, and above, the original extent of the telencephalon, which remains limited to a narrow median strip dorsally and anteriorly (Fig. 124). The ventral and lateral walls of the cerebral hemispheres soon become greatly thickened, as the corpora striata, or basal ganglia, which finally become so large that they nearly obstruct the lateral ventricles. Elsewhere the walls of the hemispheres remain comparatively thin.

The dorsal region of the original telencephalon remains embedded between the hemispheres. On its dorsal surface, immediately in front of the velum transversum, it becomes invaginated as the paraphysis. That part of its walls in front of the recessus opticus, as far as the level of the foramina of Monro, forms the lamina terminalis; this remains very thin except in its middle where the anterior commissure develops later.

The diencephalon includes the second and third neuromeres. Its antero-dorsal and antero-ventral limits are marked, respectively, by the velum transversum and the recessus opticus. Posteriorly it is marked off from the mesencephalon ventrally by an elevation in the floor, the tuberculum posterius, and dorsally by a broad depression of the wall (Fig. 123).

Like the telencephalon, this also is vertically extended. Its cavity forms, together with the median cavity of the telencephalon, the third ventricle. Just behind the recessus opticus the ventral and ventro-lateral walls are thickened as the optic chiasma, and just back of this is a well-marked evagination, the rudiment of the infundibulum. The lateral walls of the diencephalon remain thin for a time, but later become greatly 
thickened as the optic thalami. The roof remains thin and considerably expanded; in the region of the velum transversum the roof of the diencephalon and telencephalon becomes modified into the folded choroid plexus of the third ventricle (Fig. 124). Back of this the diencephalic roof is evaginated as the tubular epiphysis or pineal body. Finally a thickening in the dorsal wall marks the posterior dorsal limit of the diencephalon; this is the rudiment of the posterior commissure.

The mesencephalon, or mid-brain, forms the topographically anterior part of the brain, projecting considerably in advance of all the other structures of the embyro (Figs. 113, 114, 123). It includes the fourth and fifth neuromeres. It remains relatively undifferentiated for a considerable period, but later its dorsal and dorso-lateral walls evaginate and thicken, forming the optic lobes, which include a part of the original cavity of the mesencephalon. The central portion of its cavity, now greatly restricted, remains as the aqueduct of Sylvius, or iter, continuing posteriorly from the third ventricle. The ventral and ventro-lateral walls of the mesencephalon thicken to form the great nervous pathways from the optic lobes to other centers, known as the crura cerebri. Back of the optic lobes the mesencephalon remains undilated as the isthmus, leading directly to the metencephalon (Fig. 124).

The metencephalon, lying dorsal to the anterior tip of the notochord, consists of only the sixth neuromere, and is thus very short. The cavity of the metencephalon is not separable from that of the succeeding section, the myelencephalon; together they are known as the fourth ventricle. The walls of the metencephalon thicken slowly, though steadily, during the early days of incubation. Later the dorsal and dorso-lateral regions become very greatly thickened, forming the cerebellar hemispheres, or cerebellum. The ventral and ventro-lateral walls also thicken very considerably to form the pons Varolii.

The myelencephalon, the last division of the brain, includes the seventh to eleventh neuromeres, indications of which remain visible, ventrally, until about the fourth day. Its cavity, as said above, is the fourth ventricle. The roof of this section 
remains thin and non-nervous, and ultimately forms the choroid plexus of the fourth ventricle. The ventral and ventrolateral walls become greatly thickened as the medulla oblongata or spinal bulb. This region becomes flexed ventrally forming the pontine flexure (Fig. 124). The cervical flexure, previously. mentioned, is gradually disappearing, but the original cranial flexure of the mesencephalic region remains as a permanent feature of the brain.

Posteriorly the elongated myelencephalon passes into the spinal cord. This is of lesser diameter than the medulla, and at first is vertically elongated with somewhat thickened lateral walls. Its cavity, the central canal, continuous with the fourth ventricle, is narrow but deep. The lateral walls continue to thicken and finally the cord becomes approximately circular in section, and its cavity is reduced to a very small tube by the fusion of its dorsal walls. The median dorsal and ventral walls of the cord remain thin. As the lateral walls begin to thicken their constituent cells become highly differentiated. The original epithelial elements of the neural tube become differentiated as the ependymal cells; these are non-nervous, supporting cells. The free ends of the ependymal cells, bordering the central canal, become ciliated. The embryonic nerve cells, or germinal cells, originally scattered through the epithelial cells, multiply rapidly and form both neuroblasts, which give rise to the gray matter of the cord, and non-nervous supporting cells or glia cells. From the neuroblasts various processes grow out, for the most part remaining within the central nervous system and forming in part the white matter. Some of the neuroblasts of the ventro-lateral regions (ventral cornua) send out processes which leave the central system as the components of the ventral or motor (efferent) roots of the spinal nerves.

From the functional standpoint the most important facts concerning the development of the central nervous system are the histogenetic processes going on in the different regions of the walls of the brain and cord, especially the establishment of the various centers (nuclei) and tracts. Although of the greatest importance and interest, all such details lie without 
the scope of this work. (Further information on this general subject is found, together with references to the literature, in Johnston's "Nervous System of the Vertebrates," Philadelphia, 1906.)

\section{The Cranial and Spinal Ganglia}

These structures are derived from the neural crests, whose formation has already been described. The neural crests finally extend the entire length of the nervous system. In the trunk region they begin to extend laterally between the ectoderm and the mesodermal somites. In the intervals between successive somites the neural crest becomes interrupted, the cells apparently, though not certainly, being converted into mesenchyme. By this process each crest is broken into a metameric series of cell-groups. These groups are the rudiments of the spinal ganglia, and therefore also of the dorsal (afferent) roots of the spinal nerves. Each group contains neuroblasts as well as indifferent cells, and very early comes into close relation with the cells of the adjoining somite.

In the region of the somites of the head the neural crests are poorly developed, in correlation with the absence of ganglia in these segments. But throughout the remainder of the head they are well developed and form the rudiments of the ganglia and certain of the branches of the cranial nerves. As in the trunk region, the continuous crests are broken up by the conversion of certain regions into mesenchyme. The regions remaining form the rudiments of the ganglia of the X, IX, VIII, VII, and V cranial nerves. The ganglia of the IX and X nerves arise together and are divided, toward the close of the second day into separate cell-groups by the formation of the gill-pouches of the region (Fig. 130). Thus the ganglion of the IX nerve, ganglion petrosum, is left just above the third visceral arch, and that of the $\mathrm{X}$ nerve, ganglion jugulare-nodosum, above the fourth and fifth visceral arches. Each of these acquires a connection with an ectodermal $k$ ranchial sense organ, or 
placode, just above the second and third visceral pouches. These placodes are vestigial organs in terrestrial vertebrates, and this connection, as well as the existence of the placodes themselves, is of very brief duration.

The ganglia of the VII and VIII nerves arise in common, from an enlarged part of the crest lying immediately in front of the rudiment of the ear. This cell-group, the acusticofacialis ganglion (Fig. 114), connects with the brain in the region of the third neuromere of the myelencephalon. It also connects (about the forty-eighth hour) with a well-marked pla-

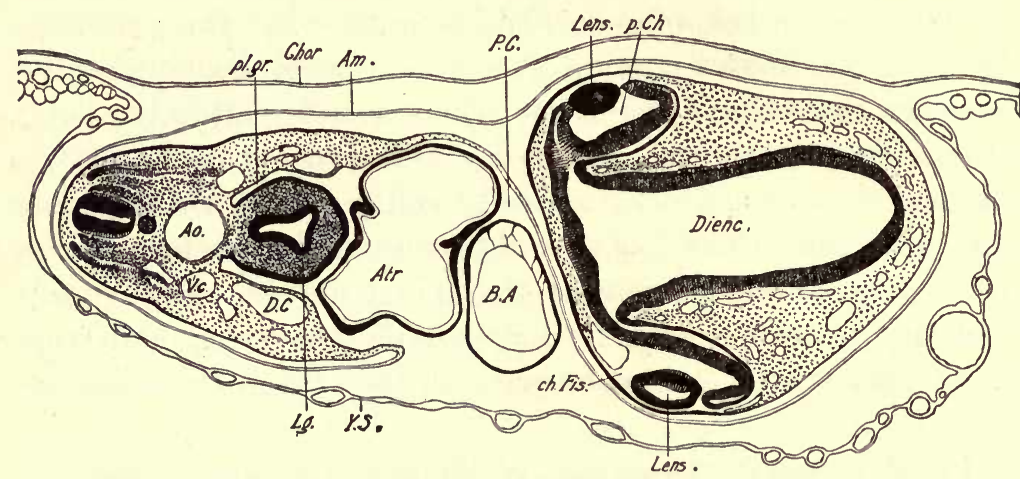

FIG. 125.-Transverse section, passing through the eyes and heart, of an embryo with about thirty-five pairs of somites (about seventy-two hours). From Lillie (Development of the Chick). Am., Amnion; Ao., dorsal aorta; Atr., auricle; B.A., bulbus arteriosus; Ch.Fis., choroid fissure; Chor., chorion; D.C., ductus Cuvieri; Dienc., diencephalon; Lg., rudiment of lung; P.C., pericardial cavity; p.Ch., posterior chamber; pl.gr., pleural groove; V.c., posterior cardinal vein; Y.S., yolk-sac.

code on the first visceral pouch. The ganglion of the $\mathrm{V}$ nerve, trigeminal ganglion, is the most anterior of the series (Fig. 130). It connects with the first neuromere of the myelencephalon and sends outgrowths into the regions of both upper and lower jaws, as the rudiments of certain branches of the $\mathrm{V}$ nerve. A part of the mesenchyme of the mandibular and hyoid arches is also formed from the cells of these rudiments, and especially from the neural crest anterior to the $\mathrm{V}$ ganglion. During an early stage in their formation from the crests, the rudiments of the V and VII ganglia connect with the surface ectoderm 
around the first visceral pouch, and appear to receive cells proliferated from this layer. This connection is not to be confused with the much later connections established by the VII, IX, and $\mathrm{X}$ ganglia with the epibranchial placodes.

\section{The Peripheral Nervous System}

We must distinguish here between the spinal and cranial nerves. The spinal nerves, of which there are some thirtyeight pairs distinguishable at certain stages, all develop essentially alike, while the twelve pairs of cranial nerves may differ widely form one another and from the spinal nerves, in their development, as they do in morphology and in function.

\section{A. THE SPINAL NERVES AND THE SYMPATHETIC SYSTEM}

Each spinal nerve is a complex, including parts of unlike function and relation to other structures. For our present purposes it will be necessary to distinguish only the dorsal or afferent and the ventral or efferent portions, and in each of these the somatic (somatopleural) and splanchnic (splanchnopleural) pathways. Brief reference to the sympathetic system may be included here because it is obviously derived, to a certain extent, from the same rudiments as are the spinal nerves.

The rudiments of the somatic afferent portions of the spinal nerves are the cell-groups derived from the neural crests described above, i.e., the spinal ganglia. During the third day the neuroblasts of the spinal ganglia send out processes (axons, axis cylinder processes) in two directions, centripetally into the spinal cord, and centrifugally, meeting and joining the ventral root; the cell bodies thus remain as the spinal ganglion cells. The ventral root of each spinal nerve is efferent and is composed of outgrowths (axons) from both somatic and splanchnic neuroblasts located in certain regions of the ventral cornua of the spinal cord. The dorsal and ventral (afferent and efferent) roots unite a short distance from the cord forming the spinal nerve trunk. This trunk then immediately divides into three 
main branches (Fig. 126); first is given off ventrally a large branch to the sympathetic system, the ramus communicans (see below). These fibers are finally distributed to splanchnopleural derivatives, and are derived from both dorsal and ventral roots. The trunk then separates into dorsal and ventral branches, each containing fibers from both roots, which are distributed chiefly, though not wholly, to somatopleural struc-

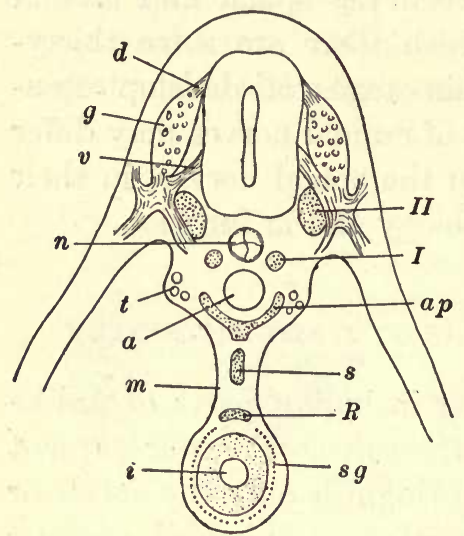

FIG. 126.-Diagram of the chief elements of the sympathetic nervous system of the chick, in transverse section. After His, Jr. $a$, Dorsal aorta; $a p$, aortic plexus; $d$, dorsal (afferent) root of spinal nerve; $g$, spinal ganglion; $i$, intestine; $m$, mesentery; $n$, notochord; $R$, Remak's ganglion; $s$, splanchnic plexus; $s g$, sympathetic elements in intestinal wall; $t$, mesonephric tubules; $v$, ventral (efferent) root of spinal nerve; $I$, primary sympathetic cord; $I I$, secondary sympathetic cord.

pathetic cords (Fig. 126). Other migratory neuroblasts from the spinal ganglia then form somewhat similarly, a pair of secondary sympathetic cords just above the primary cords. The secondary cords then become connected segmentally with. the spinal ganglia, by processes from the secondary cord cells which extend back into the ganglia and through the dorsal spinal root into the spinal cord. The primary sympathetic cords give rise to the prevertebral plexuses, but otherwise disappear en- 
tirely during the sixth and seventh days. The secondary sympathetic cords are the rudiments of the major portion of the definitive sympathetic cords of the adult, and the groups of fibers connecting these cords with the spinal ganglia become the rami communicantes. The secondary sympathetic cords become ganglionated, and additional processes grow out, connecting with the ganglia derived from the primary cords, whose cell processes are finally distributed in a complex manner to the visceral surfaces. The cardiac plexus, and the plexuses of the viscera, arise chiefly from neuroblasts migrating along the $\mathrm{X}$ nerves from the hind-brain and $\mathrm{X}$ ganglion.

Two other important groups of fibers pass by way of the rami communicantes; these are (1) the visceral afferent fibers belonging with the dorsal spinal root and ganglion, which pass by way of the sympathetic trunks to their distribution in the visceral sensory surfaces, and (2) the visceral efferent (motor) fibers from the spinal cord and ventral root, to their distribution in the visceral musculature. These latter fibers, although arising in the cord and forming an important part of the spinal nerve, are really to be regarded as components of the sympathetic system.

\section{B. THE CRANIAL NERVES}

The cranial nerves exhibit in their development a variety that parallels their diversity in morphology and in function. It is possible, however, to relate many of them to parts of the typical spinal nerves, and like these they have two sources, ganglia from the neural crests, and neuroblasts within the spinal cord. The details of their development are complicated and we may include here only a few of the more important facts in connection with each nerve. The more posterior cranial nerves show greater similarity to the spinal nerves, and as we pass forward they diverge more and more widely from this type.

The XII Cranial Nerve (Hypoglossus).--This is the nerve associated with that part of the cord which has most recently become included within the medulla, i.e., the region of the first 


\section{4}

OUTLINES OF CHORDATE DEVELOPMENT

four mesodermal segments. It arises as two pairs of roots, similar to the ventral spinal nerve roots, in the region of the last two segments of the head. The two roots join and the nerve then passes around, posteriorly to the last gill-pouch, to the floor of the pharynx, where it is distributed to the muscles of the tongue; it is thus visceral efferent.

The XI Cranial Nerve (Spinal Accessory).-The development of this nerve in the chick is unknown. The XI nerve is to be regarded as a separated caudal portion of the $\mathrm{X}$ nerve and since it is visceral efferent (although supplying voluntary muscles in the higher vertebrates), its development is probably not unlike that of the visceral efferent elements of the $\mathrm{X}$ nerve.

The $X$ Cranial Nerve (Vagus or Pneumogastric).-This large nerve is really a complex, consisting of the nerves associated with the third and fourth visceral pouches. It is chiefly visceral, both afferent and efferent, and in addition to its visceral-pouch branches, it sends branches back to certain of the viscera.

The formation of the primary vagus ganglion has been described. This divides into two parts, one remaining in its original position (ganglion nodosum), the other moving down between the fourth and fifth visceral arches (ganglion jugulare). Its ventral root is multiple, a large number of outgrow ths from the cells of the medulla converging to the ganglion nodosum, from which connections are made with the sympathetic system. From the ganglion jugulare branches grow out to the gill-pouch region, and a large branch passes posteriorly to supply the thoracic and abdominal organs. Neuroblasts accompany these branches and later form the sympathetic ganglia of the organs innervated.

The IX Cranial Nerve (Glossopharyngeal).-This is to be regarded as a separate anterior portion of the vagus group. It is the nerve of the second visceral pouch (first branchial pouch). Its development is essentially similar to that of the branchial portions of the $\mathrm{X}$ nerve, with which it forms a connection.

The VIII Cranial Nerve (Auditory).-As already noted the VIII nerve arises in common with the VII. A posterior part 
of the common acustico-facialis ganglion becomes intimately related with the rudiment of the ear (auditory sac) and later differentiates in situ. It has no efferent fibers equivalent to the ventral spinal roots or to the efferent components of the cranial nerves so far described.

The VII Cranial Nerve (Facial).-That portion of the acustico-facialis ganglion remaining after the differentiation of the VIII ganglion and nerve, remains as the geniculate ganglion of the VII nerve. This connects with the medulla in the usual fashion, and distally fibers grow out into the vicinity of the first or hyomandibular visceral pouch (spiracle) with which this nerve is primarily related. In the chick it is chiefly visceral efferent, but the development of these motor components is not known, although the cells composing its nuclei of origin in the medulla, have the usual position relative to other centers.

The V Cranial Nerve (Trigeminal).-The trigeminal ganglion derived from the neural crest connects with the medulla in the usual way and early becomes partially divided. From the smaller anterior portion (profundus ganglion) fibers grow forward as the deep ophthalmic branch, while from the posterior part (trigeminal ganglion proper) arise the branches distributed to the upper and lower jaws. This nerve is chiefly somatic afferent, its small efferent component developing typically during the fourth day.

The IV and VI Cranial Nerves (Trochlear and Abducent).These two nerves have many characteristics in common. They are purely motor, distributed to muscles of the eye-ball (superior oblique and external rectus, respectively), and consequently have no ganglia outside the medulla. They appear relatively late (fourth day), the IV from the dorsal surface of the isthmus, the VI from the ventral side of the myelencephalon.

The III Cranial Nerve (Oculomotor).- This too is chiefly motor, supplying the remaining muscles of the eye-ball. Its fibers grow out from the ventral side of the mid-brain and extend into the mesenchyme around the developing eye. Associated with its motor elements are afferent components 
growing out from the ciliary ganglion. The neuroblasts forming the ciliary ganglion appear to migrate from the neural tube and from the profundus ganglion of the $\mathrm{V}$ nerve. These afferent fibers seem to arise in the muscles of the eye-ball.

The so-called I (Olfactory) and II (Optic) cranial nerves will be considered in connection with the development of the sense organs with which they are associated.

\section{THE SPECIAL SENSE ORGANS}

\section{The Eye}

We have described in the preceding chapter the formation of the optic vesicles from the primary fore-brain, and the differentiation of the optic stalks, which remain related with the ventral side of the diencephalon either side of and posterior to the recessus opticus (Fig. 125). The optic stalks and vesicles are the rudiments of only the esseniial, or sensory (recipient) and nervous elements, of the eye. All of the other accessory parts of this complex organ are differentiated from other tissues.

While the tubular optic stalk remains comparatively short, the optic vesicle enlarges rapidly and soon (thirty hours) reaches the surface ectoderm of the head. The continued dilation of the vesicle occurs mostly above the level of the stalk, - which therefore remains related with the ventral side of the vesicle, as of the brain (Fig. 128). Now there appears a thickening of the ectoderm opposite the optic vesicle; this is the rudiment of the lens. Both the optic vesicle and the rudiment of the lens then proceed to invaginate, independently, but in the same direction (Figs. 127, 128).

The invagination of the optic vesicle, which converts it into the two-layered optic cup, is not a simple hemispherical invagination. The invaginating region begins about in the middle of the outer wall of the vesicle and extends thence downward, to the attachment of the optic stalk, as a vertical groove. The distal wall of the vesicle rapidly folds in toward the proximal wall and nearly obliterates the original cavity of the vesicle, just as the invaginating endoderm of the Amphioxus gastrula 
obliterates the blastocoel. The result is the formation of a twolayered optic cup; its inner layer, which may now be distinguished as the retinal layer is already quite thickened, while its
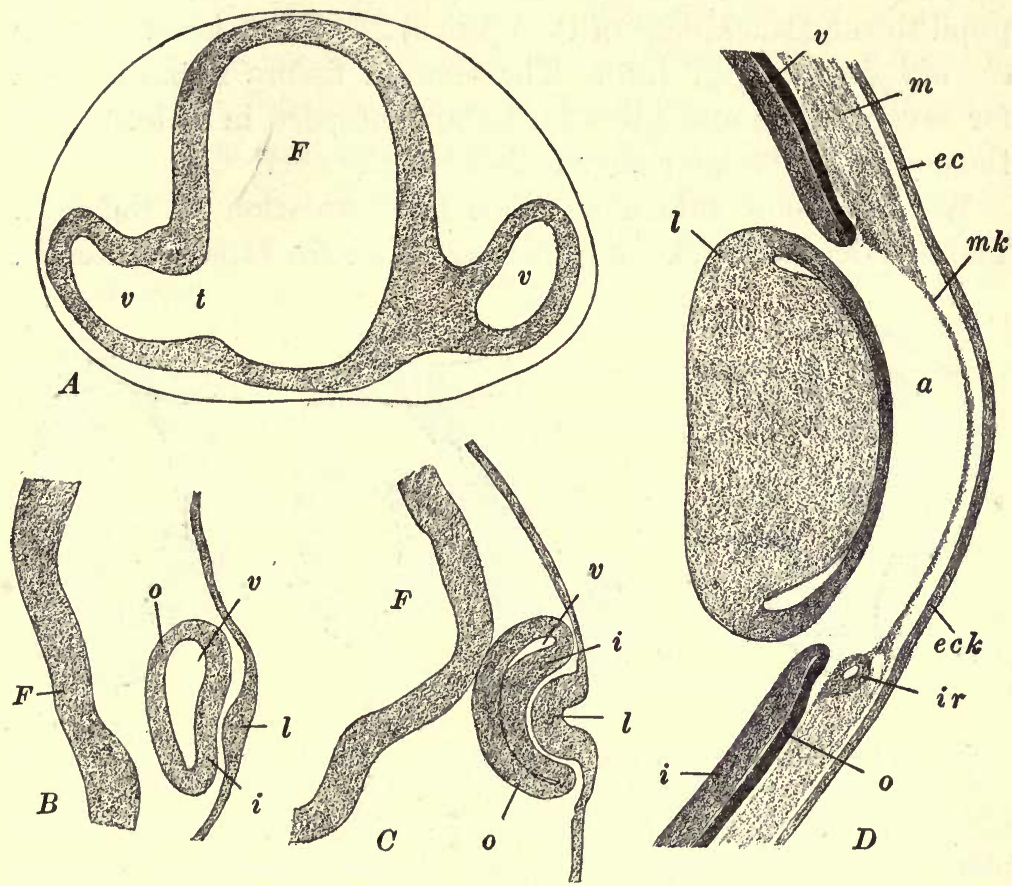

Fig. 127.-Diagrams illustrating the development of the eye in the chick. After Froriep (slightly modified). $A$. Transverse section through part of the fore-brain and optic vesicles of a chick, late the second day of incubation. On the left the section passes through the optic stalk, on the right to one side of it. $B$. Transverse section through the optic vesicle and associated structures at the end of the second day. $C$. Same, slightly later. $D$. Section through the pupillary region of the eye, the latter part of the fifth day. $a$, Anterior chamber of eye; $e c$, ectoderm of head (epidermis); eck, ectoderm of cornea; $F$, cavity (or wall, in $B$ ) of fore-brain; $i$, inner or retinal layer of optic cup; ir, mesodermal rudiment of iris; $l$, lens; $m$, mesoderm; $m k$, mesoderm of cornea; $o$, outer or pigment layer of optic cup; $t$, optic stalk; $v$, cavity of primary optic vesicle (in $C$ and $D$ nearly obliterated by the invagination of the optic vesicle to form the optic cup).

outer layer has become quite thin (Figs. 127, 128). The new cavity of the optic cup is the rudiment of the large posterior chamber of the eye. The cavity of the cup is at first widely open toward the lens and ectoderm, but its margin rapidly 
draws together somewhat. In the middle of the cup, opposite the lens, it remains open as the circular rudiment of the pupil. Ventrally from this the rim draws together more closely, leaving only a narrow slit-like opening extending from the pupil to the attachment of the optic stalk; this is known as the choroid fissure (Fig. 128). The choroid fissure remains open for several days and takes an important part in determining the course of the later development of the eye.

We must now return to trace the formation of the lens. The ectodermal thickening mentioned above rapidly extends,
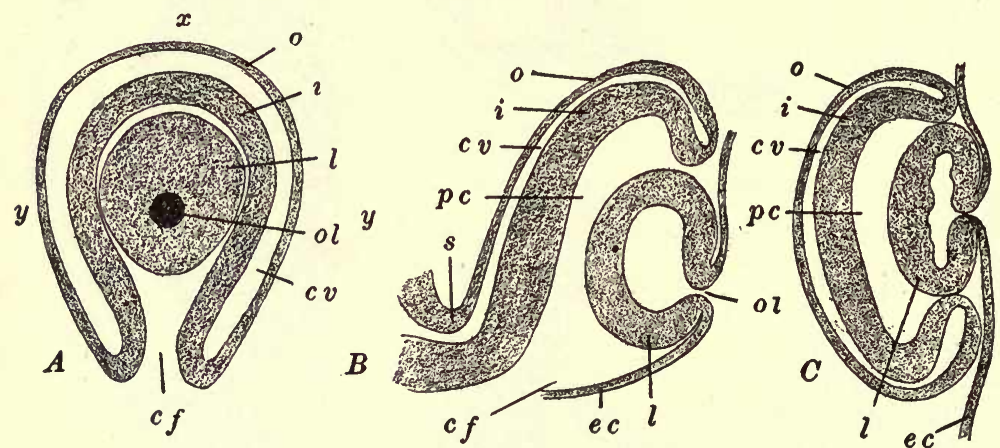

Fig. 128.-Diagrams of sections through the eye of the chick embryo at the end of the second day. After Lillie. The dorsal margin is toward the top of the page in $A$ and $B$. $A$. Eye as viewed directly. $B$. Vertical section through the line $x-c f$, in $A$. $\quad C$. Horizontal section through the line $y-y$, in $A$. cf. Choroid fissure; $c v$, cavity of primary optic vesicle; $e c$, superficial ectoderm of head; $i$, inner or retinal layer of optic cup; $l$, lens; $o$, outer or pigmented layer of optic cup; ol, opening of lens sac from surface of head; $p c$, posterior chamber of eye; $s$, optic stalk, continuous with the floor and lateral wall of the diencephalon.

and proceeds to invaginate toward the invaginating retinal layer. Its wall thickens as it invaginates simply, and soon after the invagination of the retinal layer is completed, the lens separates from the ectoderm, forming a flattened vesicle lying in the cavity of the optic cup.

These events are completed during the third day, and now the optic cup enlarges very rapidly for a time, forming a large cavity (posterior chamber) still open through the pupil and the choroid fissure. From the rudiments now established and from the surrounding mesenchyme, the parts of the fully formed eye are all derived. We may mention only a few of the 
more important events in the further history of the structures.

The optic cup extends ventrally as well as in other directions, so that the attachment of the optic stalk appears no longer connected with the ventral side, but rather with the middle of the proximal hemisphere, nearly opposite the pupil and lens; this is known as the fundus region. Associated with this change is a decided alteration in the character and relations of the choroid fissure, which will be described below.

About the sixth or seventh day the thick inner layer of the optic cup becomes clearly differentiated into a proximal or retinal zone, and a distal or lenticular zone. The former includes rather more than the proximal hemisphere, while the latter forms a broad band bordering the pupil. The retinal portion becomes transformed later into the sensory and nervous parts of the eye; the thin outer layer in the retinal zone of the cup is not sensory, but is transformed into the pigmented layer of the retina. Both the inner and outer layers of the cup in the lenticular zone share in forming the rudiments of the iris and ciliary process. The separation between the retinal and lenticular zones is marked in the developed eye by the ora serrata.

In the lenticular zone the thin outer layer fuses with the inner layer, becomes pigmented, and together with mesenchyme cells of the region they form the iris (Fig. 127, D). The proper muscles of the iris are not derived from the mesenchyme, but from bud-like outgrowths of the lenticular zone (ectoderm). The ciliary process is at first a series of folds of the lenticular zone around the base of the iris, but soon these folds are invaded by mesenchyme cells which form the muscles of the ciliary process.

During the expansion of the optic cup the margins of the choroid fissure have come into close apposition, nearly closing the fissure. Owing to the originally ventral attachment of the optic stalk the retinal layer is continuous, either side of the fissure, with the lower side of the stalk, and the connection extends through about one quadrant of the optic cup, i.e., from the fundus nearly around to the margin of the retinal 
zone. Some of the retinal layer cells form neuroblasts, and from these nerve processes (axons) grow out, along the inner surface of the retina toward the attachment of the optic stalk. Finally they extend, by way of the margin of the choroid fissure, into the stalk, from all parts of the retina, and thence into the wall of the diencephalon (optic thalami) to the optic lobes. The consequent thickening of the ventral walls of the optic stalks forming the optic tracts, leads to the disappearance of the original cavity of the stalk. The solid stalks or optic tracts, are commonly known as the II cranial or optic nerves (Fig. 128).

In the meantime the free inner margins of the choroid fissure have extended up into the cavity of the optic cup (posterior chamber), and finally they fuse forming a low ridge, the whole length of the original fissure, enclosing between them a bit of mesenchyme which had extended into the fissure from without. Finally the lips of the fissure fuse together externally also, and the internal ridge is recognized as the rudiment of the pecten of the eye. This enlarges rapidly and ultimately forms a folded, fan-like organ, projecting some distance upward into the posterior chamber.

The general content of the posterior chamber, known as the vitreous humor, is formed from the innermost cells of the retinal and lenticular zones. These cells send out branching processes through the chamber and later add a secretion, the two materials together making up the humor.

Just after the rudiment of the lens separates from the surface ectoderm, its inner wall becomes thickened by the elongation of its single layer of cells. Finally their elongation becomes so marked that the original cavity of the lens vesicle is obliterated and the lens become solid. The epithelium on its outer face remains simple. This primary lens vesicle forms only the nucleus of the final structure, for around this are laid down, beginning about the eighth day, a large number of concentric layers of cells, derived from the periphery of the lens region, where the thin outer lens epithelium becomes continuous with the thick inner layer. These cells are formed 
irregularly, but finally they assume a definite and regular arrangement. Layers are formed during the entire growth period of the organism and the number finally laid down is very large. At first the lens is in contact with the surface ectoderm, but it soon moves within the pupil, leaving a space between itself and the ectoderm. This is the beginning of the anterior chamber of the eye (Fig. 127, D); it extends beyond the margin of the iris, and soon becomes invaded by mesenchyme cells, part of which are added to the iris, while others form the larger part of the cornea.

The cornea is only to a slight degree formed by the surface ectoderm without the iris and pupil; only its superficial epithelium is thus derived. The major portion of the cornea is derived from mesenchyme cells which first form a single layer within the ectodermal epithelium (Fig. 127, D), and then later enter in large numbers between these two epithelia, forming layers of corneal cells. From the mesenchyme surrounding the entire optic cup are derived the choroid and sclerotic coats of the eye-ball.

\section{The Ear}

Like the eye, the fully formed ear is a complex, formed of elements of diverse origin, which become morphologically and functionally associated during development. The ear develops somewhat later than the eye, in the region of the myelencephalon. Making the customary distinction between the primary or sensory, and the secondary or accessory portions, we see that the true sensory portion is formed from the surface of the head, rather than the wall of the nervous system. The accessory parts are derived from the hyomandibular visceral pouch and the mesenchyme of the region.

The first indication of the ear appears about the thirtieth hour as a circular thickening in the ectoderm on the side of the head, just in front of the level of the first mesodermal somite. This patch enlarges, becomes depressed and invaginated, and about the beginning of the third day it has formed a consider. 
able sac, the auditory sac or otocyst, connected with the surface by a narrow canal. The otocyst very early becomes vertically elongated and soon shows internally an oblique ridge on its inner or medial surface, indicating its division into an upper and inner part, and a lower and outer part, known respectively as the superior and inferior chambers. The superior chamber is extended vertically as a short tube with which the canal leading to the surface is connected. This tube is the rudiment of the endolymphatic duct (Fig. 129, A). On account of the dorsal expansion of the superior chamber along the outer side
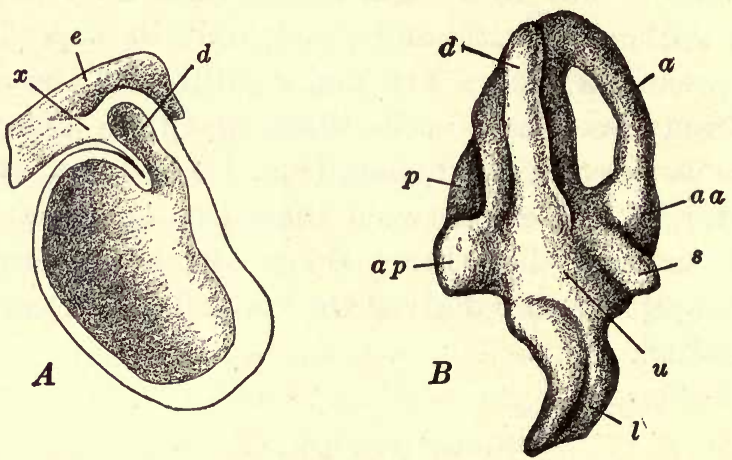

Fig. 129.-Two stages in the development of the auditory organ of the chick. $A$. Hemisected model of the auditory sac (otocyst) just before its separation from the superficial ectoderm of the head. After Krause. B. Median view of a model of the left membranous labyrinth of an embryo of seven days and seventeen hours. After Röthig and Brugsch. a. Anterior vertical semicircular canal; $a a$, ampulla of anterior vertical semicircular canal; $a p$, ampulla of posterior vertical semicircular canal; $d$, ductus endolymphaticus; $e$, superficial ectoderm of head; $l$, lagena (cochlea); $p$, rudiment of posterior vertical semicircular canal; $s$, rudiment of saccule; $u$, utricle; $x$, connection between auditory sac and superficial ectoderm.

of the endolymphatic duct, the latter appears to open into the inner side rather than into the apex of the superior chamber. The narrow canal of the duct becomes closed during the fifth day, and the otocyst soon thereafter loses all connection with the surface ectoderm.

We may now mention the more important steps in the development of each of these three primary regions of the otocyst. The endolymphatic duct grows dorsally during the seventh and eighth days, and its extremity dilates forming the 
endolymphatic sac. This finally extends above the central nervous system, and lies embedded in the mesenchyme along the dorso-lateral surface of the myelencephalon.

The superior chamber of the otocyst gives rise to the semicircular canals and the utricle. The semicircular canals are indicated the fifth day, as three slight grooves in the wall of the superior chamber, approximately in the relative positions of the fully developed canals, save that the posterior canal is at first oblique to the other two. These grooves deepen and their margins meet converting them into canals, which remain open into the chamber at their extremities. The canals push out from the surface of the otocyst carrying a thin sheet of its wall which becomes perforated between the body of the superior chamber and the canals, leaving them free, except at their attached ends. The cavity of the superior chamber remaining, after the formation of the semicircular canals, is the utricle; this receives also the opening of the endolymphatic duct, and ventrally it opens into the inferior chamber of the otocyst. The ampullo of the canals appear as dilations very early (seventh day).

The inferior chamber of the otocyst is the seat of origin of the saccule and the lagena or cochlea. The saccule appears (seventh day) as a swelling on the inner or medial wall of the extreme upper (dorsal) end of the inferior chamber. The ventral end of this chamber pushes downward as the rudiment of the lagena or cochlea, while the intermediate region remains as the cochlear duct. The lagena grows out for some distance, turning inward (medially) at its tip, forming as a whole a hook-shaped structure (Fig. 129, B).

The epithelial lining of the otocyst has meanwhile become thinner, except in certain patches which mark the location of the macula, crista, and papilla of the fully formed ear. In these regions the epithelium assumes the typical characteristics of the acustic epithelium, and into each patch there extend nerve fibers (axons) from the cells of the VIII nerve ganglion, which is intimately fused with the antero-ventral face of the otocyst. 
The mesenchyme surrounding the otocyst differentiates into various structures during the development of the otocyst. First is formed a membranous layer which fuses with the external surface of the complex otocyst and its derivatives, forming the membranous labyrinth. Surrounding this the mesenchyme forms a loose tissue which becomes the perilymph, and around all of this comes finally a dense mesenchyme where the cartilaginous and later the bony labyrinths are laid down.

We have thus far described only the structures of the internal ear. It remains now to mention the chief facts regarding the development of the middle and outer ears. These develop partly from the pharyngeal cavity, and partly from the region of the hyomandibular visceral pouch and the surrounding mesenchyme. The hyomandibular pouch develops in two parts, a large ventral portion corresponding in general with the typical gill-pouch, and a smaller dorsal portion (Fig. 130, $B$ ). The former is transitory and disappears without becoming perforated, while the latter, which is perforated for a short time only, enlarges and becomes differentiated as a part of the middle ear or tympanic cavity. The major portion of this cavity is, however, derived from the cavity of the pharynx adjoining the dorsal portion of the hyomandibular pouch. This part of the pharynx becomes incompletely cut off from the remainder of the pharyngeal cavity by a horizontal shelf or partition, a narrow slit remaining open in the mid-line. The narrow pharyngeal space thus formed, and the dorsal portion of the hyomandibular pouch, enlarge distally, between the otocyst and the surface of the head, as the rudiment of the tympanic cavity; the narrow medial portion of the pharyngeal space becomes the Eustachian tubes, opening into the pharynx by the slit mentioned.

The mesenchyme of the dorsal wall of the tympanic cavity becomes differentiated into the two inner auditory ossicles. The cavity then extends dorsally around either border of this mesenchymal region, which is thus formed into a stalk containing the two ossicles and connecting the surface of the head with the wall of the otocyst (twelve days). 
Meanwhile the external auditory meatus forms, as a depression on the surface of the head between the dorsal and ventral portions of the original hyomandibular pouch. This depression deepens and finally the tympanic cavity meets it; the membrane separating the two cavities does not perforate, but remains as the tympanic membrane or ear-drum. In the interior of this membrane, the mesenchyme which remains between its outer ectodermal and inner endodermal layers, differentiates into the stapes, which comes into relation with the malleus and incus, already marked out in the mesenchymatous rod crossing the tympanic cavity.

\section{The Olfactory Organ}

This develops much later than the eye and ear, a fact which is possibly correlated with the secondary importance of the olfactory sense in the birds. Its appearance is made at the close of the second day, as a pair of circular thickenings in the superficial ectoderm just anterior to the eyes. These thickenings are due to the elongation of the epithelial cells and mark the primary differentiation of the olfactory epithelium. Each patch soon becomes invaginated, forming an olfactory pit, which remains open to the surface of the head. Outgrowth of the fore-brain produces an apparent shifting of the external openings of the olfactory pits to the ventro-lateral surfaces of the head in the margin of the stomodæum. The two pits are separated by a broad ridge, produced by the enlargement of the fore-brain; this is the fronto-nasal process. The outer border of the opening of the olfactory pit also becomes elevated, and about the fifth day becomes joined with the fronto-nasal process by a bridge of tissue, extending across the olfactory opening and dividing it into upper and lower portions. This bridge enlarges as the rudiment of a part of the upper jaw, and the two openings thus have quite different fates. The upper is carried outward and upward and forms the external nares; the lower is carried downward and inward (relatively) as the internal nares (choano). 
The olfactory pit has already begun to deepen before the division of its external aperture. The true olfactory epithelium, which is distinguished from the adjacent non-sensory epithelium by the fact that the former remains only one cell thick, is limited to the deeper part of the pit, so that the olfactory and respiratory portions of the olfactory chamber are sharply distinguished even in these early stages. During the fourth to eighth days, the internal nares are carried farther back by the development of the palate, and the three pairs of turbinates make their appearance, growing in from the outer or lateral wall of the chamber. The lower turbinate extends into the respiratory portion, the middle and upper turbinates into the olfactory portion; later, however, the epithelium covering the middle turbinate loses its olfactory character and becomes like that of the respiratory part.

The true olfactory epithelium contains neuroblasts as well as ordinary epithelial cells. Superficially the former send out to, or above, the surface of the epithelium, short processes which are sensory or receptive in character. These same cells send out also long processes (axons) which grow into the wall of the adjacent telencephalon and form the true olfactory nerves. The sensory epithelium of the olfactory organ is therefore a neuro-epithelium. The I cranial nerve called the olfactory, is composed of these fibers.

\section{THE ALIMENTARY TRACT AND ITS APPENDAGES}

We have already described the formation of the main divisions of the embryonic gut; these are the fore-gut, with which we described the pharynx and oral plate, the hind-gut with the postanal gut, and the mid-gut connecting with the splanchnic stalk. We shall now review the early development of the various regions of the tract and of the appendages or derivatives of each portion.

\section{Organs of the Fore-gut}

We left the fore-gut, at the thirtieth hour, as the short but wide cavity of the head-fold, extending from the oral plate 
to the anterior intestinal portal (Fig. 99). Later we have seen that, through the process of fo'ding the embryo off the yolk, the splanchnopleural gut is rapidly extended posteriorly from the fore-gut and anteriorly from the hind-gut, and is closed in entirely, save where it communicates with the yolk-sac by way of the splanchnic stalk. That part of the gut which is thus formed primarily by the approach of the lateral embryonic folds is distinguished as the mid-gut, the definitive fore- and hindgut being formed primarily by the head- and tail-folds. Embryologically the fore- and hind-gut are more important than the mid-gut, for in connection with these regions develop all of the important appendages of the alimentary tract.

In connection with the fore-gut we have to describe the formation of the pharynx and visceral arches and pouches, the thyroid, the thymus and post-branchial bodies, the whole respiratory tract, the œsophagus and stomach, the liver, and the pancreas; in addition we must include the stomodæum and the structures derived from it, the hypophysis and the organs of the buccal cavity.

The stomodoum is an ectodermally lined depression on the lower side of the head; the oral plate is at its bottom (Figs. $99,123, A)$. The depth of the stomodæum is increased by the formation and growth of the jaws. As previously noted, the oral plate becomes perforated during the third day and then gradually disappears, the stomodæum itself, however, is the seat of several important organs. The hypophysis appears about the forty-fourth hour, as an elongated evagination from its mid-dorsal wall, just in front of the oral plate (Fig. 123). It grows directly toward the ventral surface of the diencephalon, in the region of the infundibulum, which it reaches at about the beginning of the third day. Later it becomes glandular, loses its connection with the epithelium of the stomodæum and joins with the infundibulum to form the glandular portion of the pituitary body.

The cavity of the stomodæum and the future buccal cavity are practically coincident, whether precisely or not can hardly be said, on account of the disappearance of the oral plate 
before the formation of any other landmark. The maxillary and mandibular arches, whose formation is described below, extend forward, around the sides of the stomodxum forming the rudiments of the jaws; the buccal cavity is considerably enlarged by their formation and their enlargement as the beak. An incomplete palate is formed later, above which the pharyngeal cavity extends. On the upper surface of the beak is formed a superficial horny egg-tooth, which is used in perforating the shell and shell membrane at the close of incubation; and on the margins of the jaws slight, transitory ridges appear, representing the vestiges of the enamel organ-all that there is left of the true teeth of other vertebrates. Although the tongue extends forward into the buccal cavity, it is really a pharyngeal derivative.

The pharynx is the most complicated region of the embryonic gut. On account of the obliquity of the oral plate, the antero-dorsal portion of the pharynx may be described as pre-oral; this region is known as Seessell's pouch, but when the oral plate disappears this is indistinguishable (Figs. 123, A; 130). We have already described, in connection with the ear, the separation of this antero-dorsal portion of the pharynx as the rudiment of a part of the tympanic cavity and the Eustachian tubes, which open into the pharynx through a median fissure in the palate, the tubal fissure.

Beginning the second day there grow out from the walls of the wide pharynx toward the surface of the head, a series of paired, vertically elongated pouches, the visceral pouches (Fig. 130). Of these there are four pairs, diminishing in size and importance posteriorly. The first and largest is the hyomandibular pouch, the other three are the branchial or gill-pouches, the last of which is very feebly developed. These visceral pouches, containing extensions of the pharyngeal cavity, push out to the surface ectoderm with which they fuse intimately, dividing the body wall of the region into a series of vertical sections between them; these are the visceral arches. The visceral arches are composed chiefly of mesenchyme, in which develop later the aortic arches and the cartilaginous visceral 

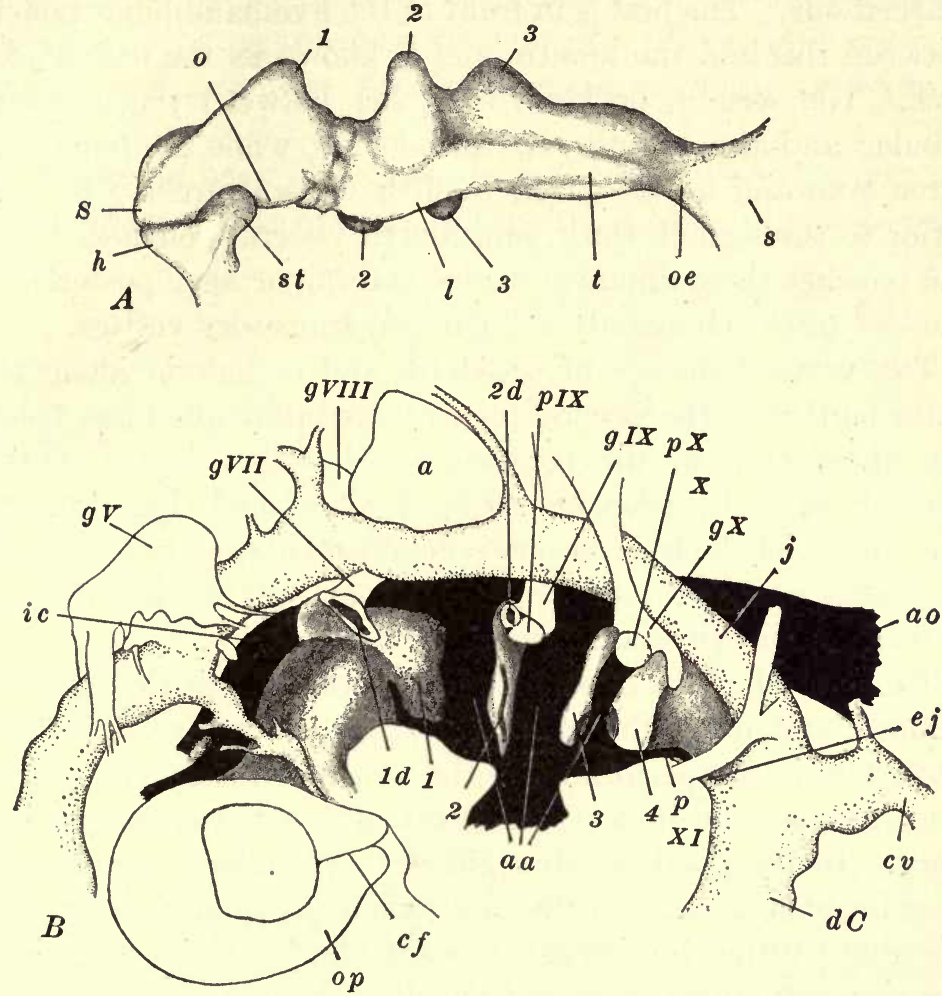

FIG. 130.-Models of the pharynx and associated structures in the chick. After Kastchenko. $A$. Ventro-lateral view of pharynx at the beginning of the third day. B. Lateral view of the pharynx and associated nervous and vascular structures, at the end of the third day. Nervous structures are left unshaded; arteries in solid black; veins lightly stippled; pharyngeal structures darkly stippled. a. Auditory sac; $a a$, aortic arches; $a o$, dorsal aorta; $c f$, choroid fissure; $c v$, posterior cardinal vein; $d C$, ductus Cuvieri; $e j$, external jugular vein; $g V$, Gasserian ganglion of $V$ cranial nerve; $g V I I$, geniculate ganglion of VII cranial nerve; $g V I I I$, acustic ganglion of VIII cranial nerve; $g I X$, ganglion petrosum of IX cranial nerve; $\mathrm{g} X$, ganglion nodosum of $\mathrm{X}$ cranial nerve; $h$, hypophysis; $i c$, internal carotid artery; $j$, internal jugular vein; $l$, rudiment of larynx; $o$, oral evagination of fore-gut; oe, œsophagus; $o p$, optic vesicle enclosing lens; $p$, pulmonary artery; $p I X$, placode of IX cranial nerve; $p X$, placode of $X$ cranial nerve; $s$, stomach; $S$, Seessel's pocket (preoral gut); st, stomodæum; $t$, rudiment of trachea; $1-4$, first to fourth visceral pouches (or their ventral portions, in $B$ ); $1 d, 2 d$, dorsal portions of first and second visceral pouches; $I X$, glossopharyngeal nerve; $X$, vagus nerve; $X I$, hypoglossal nerve. 
arches; each is covered externally by ectoderm, and internally and laterally by endoderm. Five visceral arches are thus marked out. The first is in front of the hyomandibular pouch, between this and the mouth, and is known as the mandibular arch. The second, or hyoid arch, lies between the hyomandibular and the second visceral pouches, while the remaining three branchial arches (third to fifth visceral arches) lie posterior to the second, third, and fourth visceral pouches. Like the pouches these diminish in size and importance posteriorly, the last (fifth) being only a slight and transitory vestige.

The vertical fusions of ectoderm and endoderm along the outer borders of the visceral pouches are interrupted just below the upper ends of the pouches, so that dorsal and ventral portions of each fusion are to be distinguished (Fig. 130, B). The surface of the head becomes depressed in the lines of fusion, so that a series of vertical grooves marks externally the disposition of the visceral arches and pouches.

The second visceral pouch (first branchial) is the best developed, and about the end of the second day both upper and lower fusions are perforated as the vestiges of a gill cleft; these perforations close during the fourth day without leaving any trace. In the third pouch a gill cleft is similarly formed and closed a little later. In the first pouch (hyomandibular) only the dorsal fusion is perforated (spiracular cleft) shortly before the perforation of the second pouch. No cleft appears in the fourth pouch, only the dorsal portion of which fuses with the ectoderm.

After the fourth day the visceral pouches become reduced. The first undergoes a change in function and takes an essential part in the formation of the tympanic cavity, as described above. For the most part the other visceral pouches finally disappear, but from parts of their epithelial walls the thymus and the post-branchial bodies are derived. The thymus is chiefly derived from part of the dorsal epithelium of the third visceral pouch, but the fourth contributes to a small extent. A transitory anterior portion of the thymus is derived from the epithelium of the second pouch. The thymus be- 
comes a very considerable embryonic structure, extending finally throughout the neck region. The post-branchial bodies are epithelial buds connected with the fourth visceral pouches, They are to be regarded as vestiges of a fifth pair of visceral pouches and are thus serially homologous with the thymus elements (Fig. 131).

The thyroid body also arises in connection with this part of the pharynx. This appears during the middle of the second

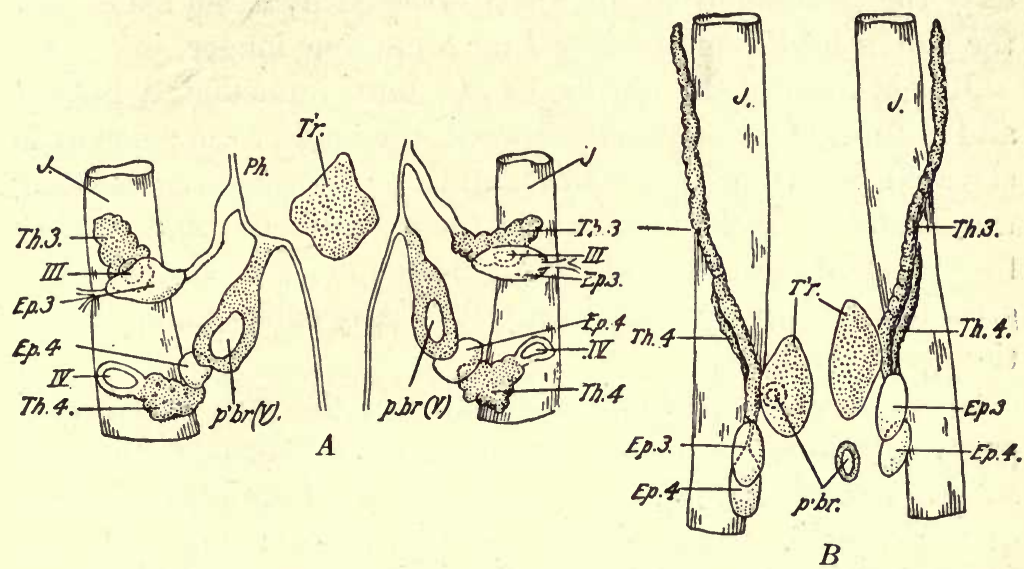

Fig. 131.-Derivatives of the visceral pouches and associated organs, in the chick. From Lillie (Development of the Chick). After Verdun (Maurer). Combined from frontal sections. A. In embryo of seven days. $B$. In embryo of eight days. Ep.3, Ep.4. Epithelioid bodies derived from third and fourth visceral pouches; $J$, jugular vein; $p^{\prime} b r ., p^{\prime} b r .(V)$., postbranchial bodies derived from fifth visceral pouch; Ph., pharynx; Th.3., Th.4., thymus bodies derived from third and fourth visceral pouches; $T ' r$., thyroid body; $I I I, I V$, third and fourth visceral clefts.

day as a thickened patch of cells in the floor of the pharynx, between the lower ends of the second visceral pouches. This plate of cells evaginates toward the close of the second day (Fig. 123), and soon appears as an entirely closed sac below the pharynx. Later (seventh day) it divides into a pair of vesicles which enlarge and migrate a short distance posteriorly.

Finally we must describe, as an appendage of the pharynx, the pulmonary tract. For a short distance behind the branchial region the pharynx becomes narrower and is deepened by the formation, late the second day, of a ventral groove (Fig. 130, 
A). This groove is the beginning of the whole pulmonary system. The depression becomes well marked early the third day as the laryngo-tracheal groove, and its posterior end expands transversely forming the rudiments of the lungs. The groove then becomes cut off from the pharyngeal cavity as the rudiment of the trachea, remaining open out of the pharynx only at its anterior end; this opening is the glottis, behind which the larynx develops later. From the eighth to the eleventh days the glottis and larynx are obstructed by a cell mass, and the glottis itself remains closed for some time longer.

Just in front of the glottis, in fact both immediately behind and in front of the thyroid rudiment, the floor of the pharynx is elevated (fourth day), the two papillæ thus formed representing the beginning of the tongue. As these rudiments enlarge they fuse together, the thyroid having been cut off meanwhile, and grow forward into the buccal cavity, finally extending nearly to the tip of the jaws.

The bifurcated posterior extremity of the laryngo-tracheal groove or lung rudiment, grows backward through the surrounding mesenchyme; the tubes thus formed are the rudiments of the bronchi. By a sort of budding process they form the bronchioles and terminal alveoli of the lung proper. The mesodermal parts of the entire pulmonary tract are derived from the splanchnic mesoderm of the primary gut-wall. The air-sacs are also formed from terminal dilations of branches of the bronchial tubes.

Passing backward from the pharynx the gut is considerably narrowed for a short distance as the osophagus, but it soon expands again as the rudiment of the stomach (third day). Before these organs are differentiated, however, the liver appears. This makes its appearance toward the close of the second day, in the region where the fore-gut is at that time open by the anterior intestinal portal, i.e., just back of the future posterior limit of the stomach.' Here two evaginations of the ventral wall of the gut, or portal, push out, one above the other in the mid-line; they extend forward as pouch-like outgrowths, through the mesoderm of the ventral mesentery, below 
the stomach region. This is the region where the great veins are converging toward the heart (ductus venosus, ductûs Cuvieri, see below). The anterior liver diverticulum appears some hours before the posterior, and as it grows forward it lies to the left, the later posterior diverticulum then extends rather toward the right side, and although it becomes the larger, it does not grow as far forward as the anterior diverticulum. During the third and fourth days both diverticula branch and anastomose, forming a network of liver tissue (Fig. 123, B) around the large vein (ductus venosus). The liver soon enlarges enormously and early becomes very vascular, through the development of vessels connecting with the ductus venosus, directly among the meshes of the liver substance.

The bile duct and gall-bladder arise from the short ventral region of the gut between the two liver diverticula; this region grows out carrying with it the openings of the diverticula, which thus come to open into a common chamber, the ductus choledochus or common bile duct. The gall-bladder develops in connection with the duct of the posterior liver diverticulum. The common bile duct is a transitory structure, and when it disappears the two liver (bile) ducts again open separately into the gut.

About the same time and in the same general region as the liver, the pancreas develops, from three separate diverticula. A dorsal median diverticulum grows out, directly opposite the posterior liver diverticulum, about the end of the third day. Right and left ventral pancreatic diverticula appear later, pushing out from the walls of the ductus choledochus close to the gutwall. When the ductus choledochus disappears these pancreatic rudiments open separately into the gut. As these diverticula enlarge they branch distally and by a budding process form the glandular units of the organ. The bodies of the three rudiments finally join, forming a common gland, although their ducts remain opening separately near the two bile ducts. 


\section{Organs of the Hind-gut}

Embryologically the most important appendage of the hindgut is the allantois. In describing the formation of this organ we mentioned the essential steps in the establishment of the hind-gut itself, and described the formation of the postanal gut and the beginning of the cloaca (Figs. 120, 121). On the ventral side of the hind-gut, between the outgrowing allantois and the base of the tail the ectoderm becomes pitted in toward the cloaca forming the proctodøum. The ectodermal epithelium of the proctodæum fuses with the endodermal lining of the cloaca forming the anal plate. This was originally toward the dorsal surface of the embryo, but was pushed into a ventral position by the formation and growth of the tail-bud (Fig. 121).

The cloaca becomes a deep but narrow cavity; it receives, antero-dorsally, the opening of the terminal portion of the intestine, and the allantoic stalk connects antero-ventrally. Laterally, either side of the rectal opening, the urinogenital ducts discharge into the cloaca. During the fifth and sixth days the postero-ventral portion of the cloaca is temporarily closed by the fusion of its walls. The cavity which re-forms here is the rudiment of the bursa Fabricii, which acquires an opening directly into the proctodæum just outside the anal plate. Thus the embryonic cloaca gives rise chiefly to that part of the adult cloaca into which the urinary and genital ducts open. The anal plate finally becomes perforated early in the third week of incubation.

\section{The Mid-gut}

The embryonic mid-gut gives rise to the intestinal tract extending from the hepatic and pancreatic diverticula to the cloaca. Its establishment through the approach and fusion of the lateral splanchnopleural folds has been described in the preceding chapter. 'It remains open by the splanchnic stalk to the yolk-sac until toward the close of the embryonic period (see above). 
Although really derived from the embryonic fore-gut, we may include here certain details in the later development of of the œsophagus and stomach, the formation of which was mentioned above. The stomach and intestine extend through the body cavity, from the dorsal wall of which they are suspended by a double mesodermal fold, the dorsal mesentery and mesogastrium, which represents the original dorsal fusion of the lateral splanchnopleural folds involved in the establishment of the intestinal groove and tube. The similarly formed ventral
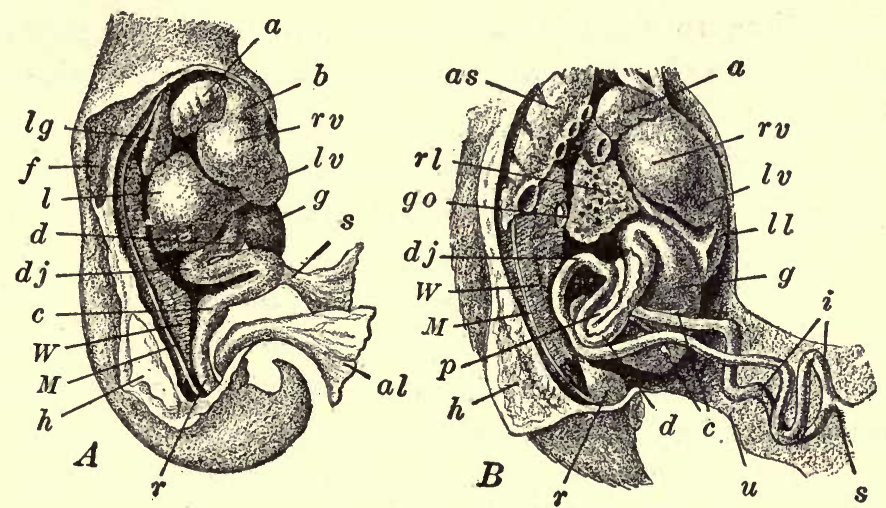

Fig. 132.- Partially dissected viscera of the chick, from the right side. After Duval. A. Of a six-day chick, enlarged slightly less than six times. $B$. Of a thirteen day chick, enlarged two and one-half times, showing the elongated intestine and its extension into the umbilical stalk. $a$, Right auricle; $a l$, allantois; $a s$, abdominal air sac; $b$, bulbus arteriosus; $c$, cæcal processes; $d$, loop of duodenum; $d j$, duodenal-jejunal flexure (a relatively fixed point during the elongation of the intestine); $f$, fore-limb bud (cut through); $g$, gizzard; go, gonad; $h$, hind-limb bud (cut through); $i$, loops of small intestine; $l$, liver; $l g$, lung; $l l$, left lobe of liver; $l v$, left ventricle; $M$, rudiment of Müllerian duct (tubal ridge); $p$, pancreas; $r$, rectum; $r l$, right lobe of liver; $r v$, right ventricle; $s$, yolk stalk; $u$, umbilical stalk; $W$, Wolffian body or mesonephros.

mesentery disappears immediately after its formation, save in the region of the stomach and liver, where it forms the gastrohepatic ligament.

The œesophagus elongates with the neck, and during the seventh to eleventh days is closed just back of the glottis by cells proliferated from its wall. The crop appears as a posterior dilation of the osophagus just in front of the stomach. During the fifth to seventh days the enlarging stomach becomes differ- 
entiated into the anterior proventriculus, with thick glandular walls, and the posterior gizzard, with its thick muscular coats. The horny lining of the gizzard is derived from the secretion of specific glands in its own wall.

Beginning the third or fourth day of incubation the mid-gut, including the stomach, commences to elongate, and as a result the tract becomes first simply looped, and then complexly folded (Fig. 132). First the stomach bends to the right, returning to the median region near its opening into the intestine. The first section of the intestine is the duodenum; this is a very short section, receiving the ducts of the liver and pancreas. The duodenum elongates very little and remains as a relatively fixed region, closely attached to the dorsal body wall. Similarly the terminal portion of the intestine, the rectum and large intestine, elongates only slightly. Between the duodenum and the large intestine the jejunum or vitelline portion of the small intestine elongates considerably, and is consequently thrown first into an S-shape. The yolk-sac connects with the apex of the lower loop (Fig. 132). Later this whole section shows secondary loops or twistings along each primary loop. At the connection of the small and large intestines the two intestinal caeca grow out during the second week.

\section{THE VASCULAR SYSTEM}

The formation of the vascular system and its development up to the thirtieth hour, we have already described; and we have also mentioned the important steps in the development of the extra-embryonic circulation (yolk-sac and allantois). We must now trace the important steps in the development of the true embryonic circulation, from the stage where we left it. Let us recall that we left the heart as a bent tube, suspended in the pericardial cavity; anteriorly it leads, by way of the first or mandibular pair of aortic arches, into the dorsal aorta, the chief branches of which (vitelline arteries) supply the extraembryonic organs. The embryonic venous system at that time consisted only of the roots of the vitelline veins, returning 
the blood from the yolk-sac and opening directly into the posterior end of the heart. The main vessels of the yolk-sac and allantois are described in the preceding chapter, and we shall need to add little to those accounts of the extra-embryonic circulation.

\section{The Heart}

The sharp bending of the heart to the right divides it roughly into anterior and posterior limbs (Fig. 133, A), and as it continues to elongate an additional loop appears, directed posteriorly from near the apex of the original loop. The entire structure then swings underneath the pharynx and the loops become less widely spread out (Fig. 133, B). The extent of the heart is increased posteriorly by the addition of a region formed by the fusion of the roots of the paired lateral vitelline or omphalomesenteric veins; the chamber thus formed is the sinus venosus.

During the third day certain constrictions appear, dividing the tube into its primary chambers, and each of these shows characteristic modifications in form, so that by the end of the third day the following regions are distinctly marked. The sinus venosus, formed by the confluence of the omphalomesenteric veins, is a wide triangular cavity with thin walls; it receives also the embryonic veins, the ductus venosus and the paired ductûs Cuvieri (see below). Its apex is anterior, where it opens into the atrium or auricle by the sinu-auricular aperture; this opens into the postero-dorsal region of the auricle. The auricle is formed from the originally posterior loop of the heart tube, now dorsal in position. The auricle already shows signs of its future division in that it is laterally expanded; the sinus venosus is more directly connected with its right side and its left side extends forward nearly to the limit of the pericardial cavity. The wall of the auricle remains thin like that of the sinus venosus; its cavity opens downward into the dorsal region of the ventricle.

The ventricle is formed essentially from the secondary pos- 
terior loop of the heart tube, and is now separated from the auricle by the narrowed auriculo-ventricular aperture (Fig. 133). The ventricle occupies the ventral region of the pericardial

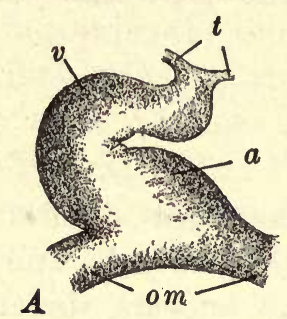

$$
r a
$$

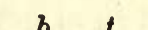

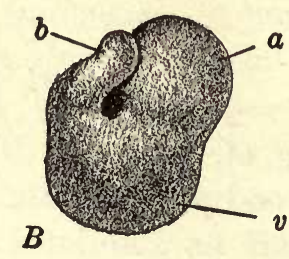

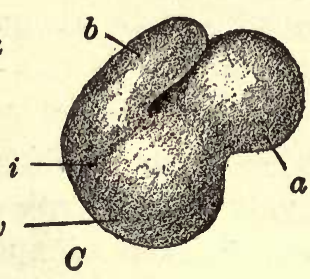

Fia. 133.-The development in the heart of the chick. $A, F$, after Hochstetter; $B-E$, after Greil. $A-E$, ventral views of the heart; $A$, of a forty-hour embryo; $B$, of a $2.1 \mathrm{~mm}$. embryo; $C$, of a $3.0 \mathrm{~mm}$. embryo; $D$, of a $5.0 \mathrm{~mm}$. embryo; $E$, of a $6.5 \mathrm{~mm}$. embryo. $F$. Frontal section through the heart of a 9 $\mathrm{mm}$. embryo. $a$, Auricle; $b$, bulbus; $d$, roots of dorsal aorta; $e$, median endothelial cushion; $i$, interventricular groove; $l a$, left auricle; $l e$, lateral endothelial cushion; $l v$, left ventricle; om, vitelline artery; $p$, left pulmonary artery; $r a$, right auricle; $r v$, right ventricle; $s$, interventricular septum, $s a$, interauricular septum; $t$, roots of aortic arches; $v$, ventricle.

cavity beneath the auricle. The walls are already thickened and spongy. Anteriorly a slight constriction separates the 
ventricle from the bulbus arteriosus, the most anterior chamber of the heart, formed from the anterior loop of the original heart tube and now passing obliquely upward to the antero-dorsal wall of the pericardial cavity, where it connects with the truncus arteriosus in the floor of the pharynx. The wall of the bulbus is also much thickened at this time.

Long before the end of the third day the heart is beating regularly and rapidly. Its first irregular twitching begins toward the middle of the second day and before the end of this day its contraction becomes quite regular.

The later development of the heart may be sketched only roughly. There are further changes in the position of the heart, as the net result of which the ventricle assumes a posterior, and the auricles an antero-dorsal, location; the auricles also grow ventrally around the bulbus, which finally occupies a median ventral position.

The bulbus arteriosus finally loses its identity as a separate chamber. Three semilunar valves develop, about in its middle, and its anterior section then becomes transformed into the proximal parts of the truncus arteriosus (finally the root of the dorsal aorta) and the pulmonary artery, while its posterior section is absorbed into the ventricles. Similarly the sinus venosus becomes incorporated into the right auricle, and the sinu-auricular valves, which had developed from the wall of the sinuauricular aperture, entirely disappear. Thus of the original four chambers of the heart only two remain separate. The cavities of these two chambers, the auricle and ventricle, then become secondarily divided longitudinally, so that the heart may again be described as four-parted, though in a very different sense.

From the postero-dorsal wall of the auricle, between the openings of the sinus venosus and the pulmonary vein (see below) a thin partition grows downward and forward, during the fourth day, and soon reaches a thickened cushion of cells lying on the upper and lower sides of the auriculo-ventricular aperture, thus completely dividing the primary auricular cavity into right and left cavities (Fig. 133, F). This interauricular septum early 
becomes fenestrated and is not completely re-formed until after hatching. At the same time the ventricle is becoming divided into right and left portions by an interventricular septum. This commences as an extension of the spongy wall of the ventricle near its posterior apex; it becomes a thick partition, rapidly extends anteriorly, meeting and fusing with the cushion of the auriculo-ventricular aperture, with which the interauricular septum has already connected. Finally the division of the ventricle is completed save for a small antero-ventral aperture, the interventricular foramen, by which the root of the dorsal aorta later connects with the left ventricle.

The bulbus arteriosus too becomes divided before its fusion with the ventricle, by a partition extending from the anterior margin of the pulmonary aortic arch (see below) back to the ventricles. The effect of this is to connect the pulmonary aortic arches with the right ventricle and the systemic aortic arch with the left ventricle. When this bulbus region is absorbed, as described above, the pulmonary arteries and the dorsal aorta arise directly from the right and left ventricles, respectively, and the separate blood streams are established.

\section{The Aortic Arches and the Arterial System}

At the thirtieth hour the heart is connected with the dorsal aorta by a single pair of aortic arches running through the first or mandibular visceral arch. Later an aortic arch forms in each visceral arch, connecting the truncus arteriosus or ventral aorta with the lateral dorsal aortæ. The second or hyoid aortic arch forms during the latter part of the second day and at the end of that day the third aortic arch is completed. The fourth is formed by the end of the third day and during the fourth and fifth days fifth and sixth aortic arches are formed in the tissues posterior to the last (fourth) visceral pouch (Fig. 134, $A$ ). Of these arches the fourth and sixth are the best developed, while the fifth is present only as a transitory and incompletely developed vestige.

This embryonic aortic arch system is converted into the adult 
condition chiefly by a series of degenerations. During the third and fourth days the first (mandibular) and second (hyoid) arches disappear, leaving only their continuous ventral roots as the root of the external carotid artery. The lateral dorsal aortæ have meanwhile continued forward, as the internal carotid arteries, supplying the brain and other organs of the head. These parts of the lateral dorsal aortæ become separated, during the sixth and seventh days from the remainder of the lateral dorsal aortæ by an interruption in these vessels just back of the dorsal attachment of the third aortic arch; thus the internal carotid arteries arise only from the third aortic arch, which is consequently known as the carotid arch (Fig. 134, B). The
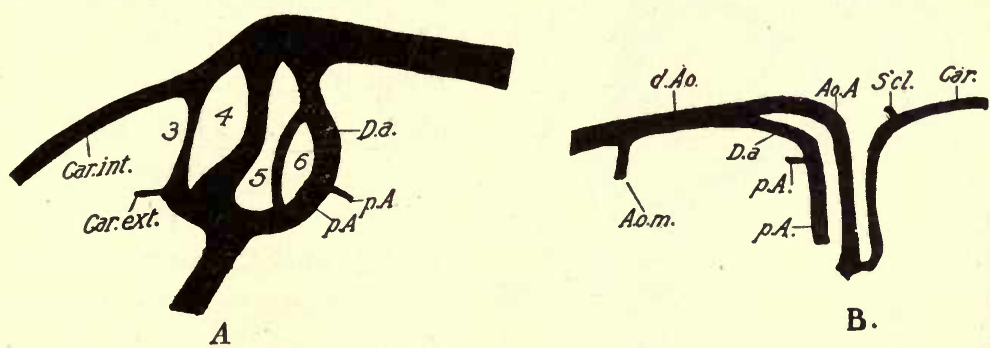

Fig. 134.-Aortic arches of the chick. From Lillie (Development of the Chick), $A$, after Locy. A. Of the left side of a chick of four and one-half days; from an injection. $B$. Reconstruction from sagittal sections of an eight-day embryo. Ao. A. Arch of the aorta (systemic arch); A.o.m., vitelline artery; Car., carotid artery; Car. ext., external carotid artery; Car. int., internal carotid artery; D.a., ductus arteriosus; d.Ao., dorsal aorta; p.A., pulmonary artery; $S^{\prime} c l$., subclavian artery; $3-6$, third to sixth aortic arches (first to fourth branchial aortic arches).

ventral roots of the first and second arches (external carotids) remain as branches from the bases of the carotid arch (roots of the third arches), supplying the mandibular region.

The fourth, or systemic arch, is at first symmetrically developed like the others, but during the fifth and sixth days it becomes reduced on the left side and correspondingly enlarged on the right. Finally the left systemic arch wholly disappears, and the anterior part of the left division of the truncus remains only as the stem of the left carotid artery. Meanwhile, it will be recalled, the right side of the truncus has connected with the 
left ventricle alone, so that the blood from this side of the heart is now carried by the third arches to the carotids, and by the right systemic or fourth arch to the general dorsal aorta.

The fifth aortic arches having already disappeared, only the sixth is left connecting now with the right ventricle. The sixth arches ultimately form the roots of the pulmonary arteries,

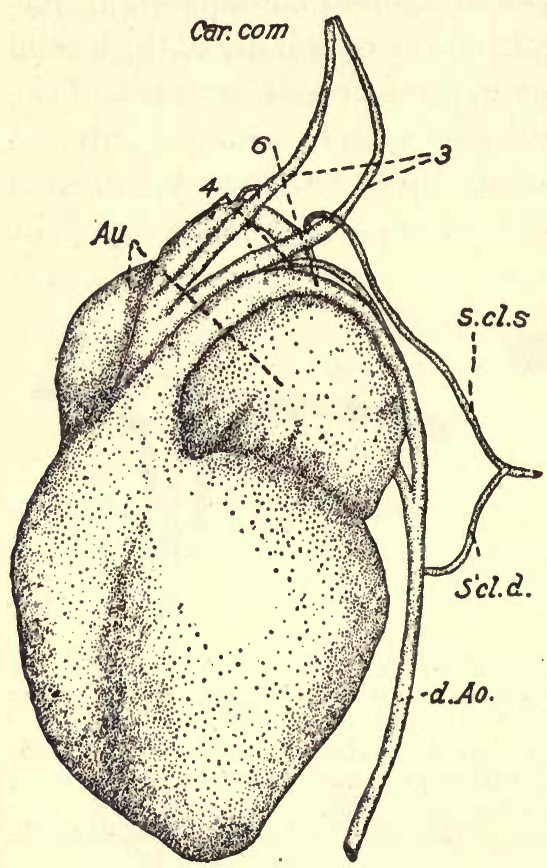

Fig. 135.-The heart and aortic arches of a chick embryo the latter part of the sixth day. From a dissection. From Lillie (Development of the Chick) after Sabin. Au. Auricles; Car. com., common carotid artery; $S^{\prime} c l . d .$, S.cl.s., primary and secondary subclavian arteries; $3,4,6$, third (carotid), fourth (systemic), and sixth (pulmonary) aortic arches.

but throughout embryonic life they remain, on each side connected with the roots of the definitive dorsal aorta. The true pulmonary arteries are small vessels passing backward from the upper ends of the sixth or pulmonary arches (Fig. 134). That part of each arch between the origin of the pulmonary artery and the dorsal aorta is known as the ductus Botalli, and shortly after hatching the two ductûs Botalli become closed as strands of connective tissue, turning the whole of the blood stream of these arches, coming from the right side of the heart, into the pulmonary arteries. Thereupon the dorsal remainder of the left lateral dorsal aorta disappears and leaves the dorsal aorta connected with the heart only by the right systemic arch (Fig. 135).

Certain branches of the aortic arches and dorsal aorta deserve a special word. The dorsal aorta gives off segmental branches between the somites, known as the segmental arteries (Fig. 109, 
$B)$. Some of these become enlarged as the roots of the arteries supplying the limbs, the subclavian and sciatic arteries. A branch from the carotid artery grows out and connects with the subclavian, finally forming its true root, its original root from the aorta disappearing about the ninth day. The sciatic artery gives off branches, the umbilical arteries, supplying the allantois; the right umbilical artery is the smaller and finally disappears.

During embryonic life the chief branches of the dorsal aorta, and really those first formed, are the pair of large omphalomesenteric or vitelline arteries, distributed to the yolk-sac by way of the splanchnic stalk. The proximal parts of these become fused as a single vessel from the base of which is derived the anterior mesenteric artery. The posterior mesenteric and celiac arteries are derived directly from the dorsal aorta. The dorsal aorta also gives off a series of small twigs supplying the excretory organs, certain of which enlarge forming the renal and genital arteries (Fig. 138).

\section{The Venous System}

We have thus far described only the veins of the extraembryonic circulation, for at the thirtieth hour the embryonic veins are not developed. It will not be necessary to add anything regarding the strictly extra-embryonic portions of these vessels, but their intra-embryonic terminations take an important part in the formation of the definitive embryonic circulation.

The first embryonic veins to appear, about the middle and latter part of the second day, are the anterior cardinal veins. Coming from the brain they extend along its ventro-lateral walls, beneath the auditory sacs, receiving as they pass, branches from the general head region, including the three anterior somites. Just back of the head they also receive later, branches from the floor of the pharynx (external jugular veins); the anterior cardinals themselves become known as the internal jugular veins. The proximal parts of the anterior cardinal 
veins are considerably enlarged as the ductûs Cuvieri, which turn inward and downward and pass into the sinus venosus (Fig. 137).

From the upper end of each ductus Cuvieri an outgrowth extends posteriorly as the rudiment of the posterior cardinal vein, which passes along the Wolffian duct (see below), finally reaching nearly to the base of the tail. The posterior cardinal veins receive the intersomitic or intersegmental veins, except the first three, and the vessels of the nephros (mesonephros, see below). The veins of the fore-limbs also discharge into the posterior cardinals near the ductûs Cuvieri. The anterior and posterior cardinal veins are consequently the chief somatic veins of the early embryo, and it should be noted that all of the somatic veins connect with the heart by way of the ductûs Cuvieri.

The splanchnic veins of the digestiv - tract and its appendages are primarily related with the intra-embryonic portions of the great veins of the yolk-sac, the omphalomesenteric veins. We have already seen how the proximal ends of these veins unite to form the sinus venosus; they continue to fuse posterior to the sinus venosus, and form thus the ductus venosus, around which, as we have seen, the liver develops. The ductus venosus and the ductûs Cuvieri are the only vessels emptying directly into the heart, until the time when the pulmonary veins appear. The omphalomesenteric veins, entering the embryo, pass across the body cavity to the mid-line, beneath the gut and between the two liver diverticula.

It should be noted here that between each omphalomesenteric vein and the dorsal body wall an extensive fusion occurs, forming an incomplete oblique partition through that part of the body cavity immediately posterior to the heart. These fusions are known as the lateral mesocardia, and they are of considerable importance in the later history of the cavities of the body (Fig. 139). The ductûs Cuvieri pass from the dorsal body wall to the sinus venosus through the anterior parts of the lateral mesocardia.

Posterior to the ductus venosus the two omphalomesenteric 
or lateral vitelline veins anastomose, at about the age of three days, on the dorsal side of the intestine, posterior to the pan.creatic rudiment (Fig. 136). Thereupon the base of the left

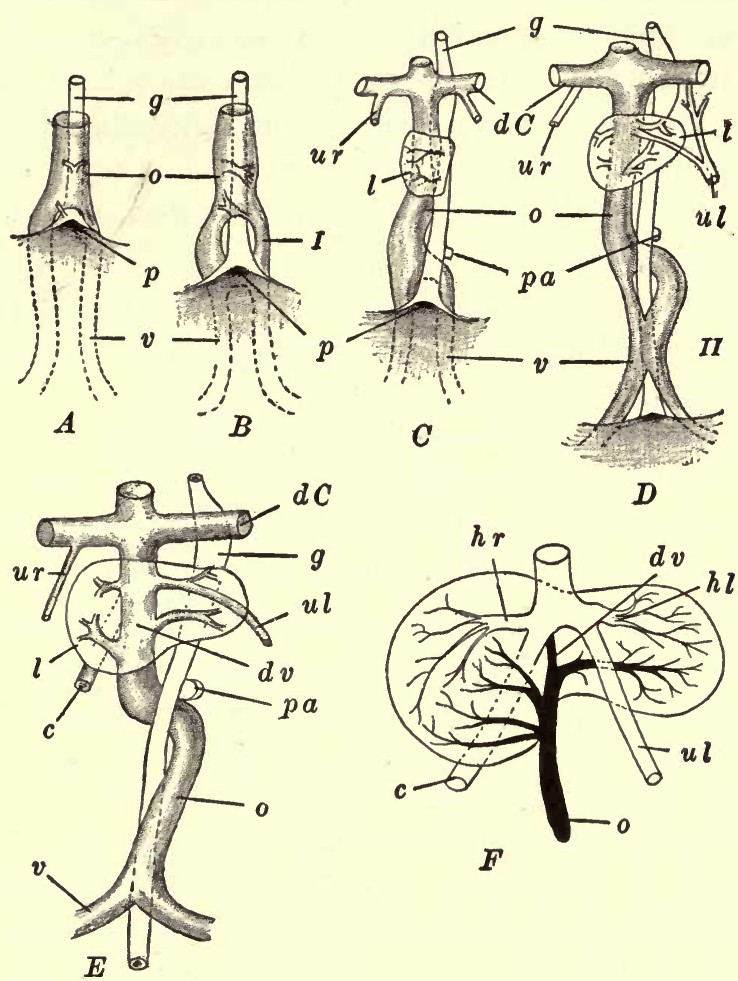

FIG. 136.-Diagrams illustrating the formation of the omphalomesenteric and umbilical veins, in the chick. After Hochstetter. A. At about fifty-eight hours. B. At about sixty-five hours. Veins joined dorsal to the gut. $C$. At about seventy-five hours. Veins again separate. D. At about eighty hours. Secondary union of veins around the gut. $E$. At about one hundred hours. Definitive arrangement of the vessels. c. Vena cava posterior (inferior); $d C$, ductus Cuvieri; $d v$, ductus venosus; $g$, gut; $h l$, left hepatic vein; $h r$, right hepatic vein; $l$, liver; $o$, omphalo-mesenteric vein; $p$, anterior intestinal portal; $p a$, rudiment of pancreas; $u l$, left umbilical vein; $u r$, right umbilical vein; $v$, vitelline vein; $I, I I$, primary and secondary venous rings around the gut.

vein rapidly disappears, so that during the fourth day all of the blood from the yolk-sac is returned to the heart by the right vein, for it will be recalled that the original or anterior vitelline veins have previously fused and connected with the right 
omphalomesenteric vein; and now a large vein coming directly from the posterior part of the yolk-sac similarly opens into the same trunk.

By the end of the fourth day the two omphalomesenteric veins again anastomose still farther back, and now the intermediate portion of the right vein disappears. The embryonic course of the omphalomesenteric veins may therefore be described as follows (Fig. 136); they enter the body symmetrically, passing directly to the ventral side of the intestine just in front of the anterior intestinal portal; here they fuse into a single vessel which passes anteriorly around the left side of the intestine to its dorsal surface and thence across to the right side, where it enters the liver.

This portion of the omphalomesenteric vein becomes the trunk of the hepatic portal vein in the following manner. As the vein passes through the liver to the ductus venosus, which is now embedded in it, it branches abundantly supplying the vascular spaces of the liver tissue, and soon the strands of liver cells push into the large vessel so that it becomes entirely broken up into small vessels and capillaries in the liver. The ductus venosus then remains as the efferent vessel, or hepatic vein, while the base of the omphalomesenteric vein itself be comes the afferent hepatic vessel, the hepatic portal vein. This arrangement is practically completed during the sixth day. Before this time the veins of the digestive tract appear; these collect into the mesenteric vein, which becomes the chief branch of the hepatic portal. A typical subintestinal vein is indicated the fourth day, coming from the tail and connecting with the left omphalomesenteric vein; it soon disappears without taking an essential part in the formation of any permanent venous structure.

Veins of considerable phyletic importance are the umbilical veins, which represent the lateral veins of the Elasmobranchs and the abdominal vein of the Amphibia. These appear early in the body wall, primarily as the veins of the limb-buds, opening into the ductûs Cuvieri (Fig. 137). During the fourth day they connect with the veins of the allantois, and shortly there- 
after the right vein disappears, while proximally the connection of the left vein with the ductus Cuvieri is lost, and this vessel acquires a new connection with the intra-hepatic vessels and ductus venosus. Through this pathway the veins of the allantois then connect with the embryonic circulation.

The largest venous trunk of the fowl is the inferior vena cava or postcaval vein. As in other forms, this vein is in part an

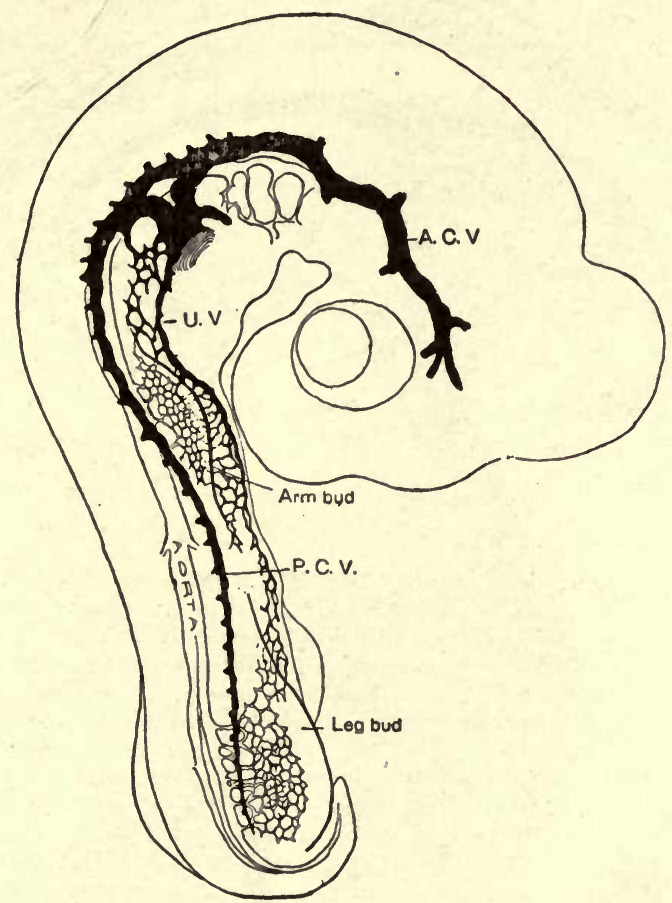

Fig. 137.- Injected chick embryo of the third day, showing the arrangement of the cardinal veins and the formation of the umbilical vein from capillary networks. From Evans. A.C.V., Anterior cardinal vein; P.C.V., posterior cardinal vein; U.V., umbilical vein.

independent formation, and in part formed from the posterior cardinal system. The first part of the vena cava appears during the fourth day as a posterior outgrowth of the ductus venosus, which connects with a series of venous spaces in the dorsal wall of the liver, on the right side (Fig. 136). Soon this 
vein connects with the right posterior cardinal vein. The posterior cardinal veins pass along the dorsal side of the kidneys (mesonephroi, see below) receiving their vessels. But during the fourth day a system of venous spaces appears on the ventral side of the kidney; these vessels are known as the $s u b$ cardinal veins (Fig. 138). During the sixth day the inferior

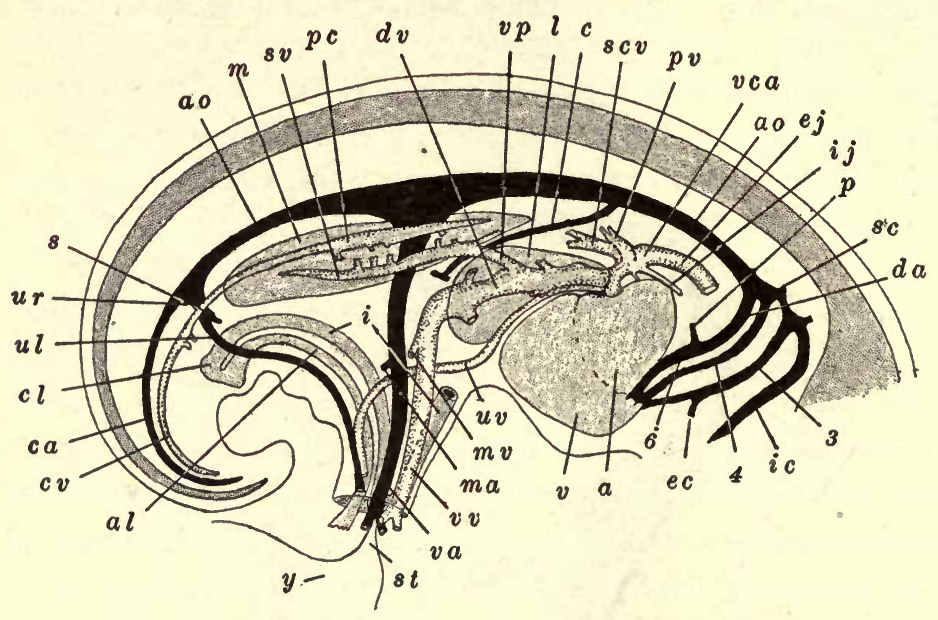

Fig. 138.-Diagrammatic lateral view of the chief embryonic blood-vessels of the chick, during the sixth day. After Lillie. a. Auricle; al, allantoic stalk; $a o$, dorsal aorta; $c$, cœliac artery; $c a$, caudal artery; $c l$, cloaca; $c v$, caudal vein; $d a$, ductus arteriosus; $d v$, ductus venosus; $e c$, external carotid artery; $e j$, external jugular vein; $i$, intestine; $i c$, internal carotid artery; $i j$, internal jugular vein; $l$, liver; $m$, mesonephros; $m a$, mesenteric artery; $m v$, mesenteric vein; $p$, pulmonary artery; $p c$, posterior cardinal vein; $p v$, pulmonary vein; $s$, sciatic artery; $s c$, subclavian artery; scv, subclavian vein; st, yolk-stalk; sv, subcardinal vein; $u l$, left umbilical artery; $u r$, right umbilical artery; $u v$, left umbilical vein; $v$, ventricle; $v a$, vitelline artery; $v c a$, anterior vena cava (anterior cardinal vein); $v p$, posterior vena cava; $v v$, vitelline vein; $y$, yolk-sac; $3,4,6$, third, fourth, and sixth aortic arches.

vena cava connects with the right subcardinal vein, the two subcardinal veins anastomose posterior to this connection, and the anterior parts of both posterior cardinal veins disappear. The net result of these changes is that the hinder parts of the posterior cardinals form the afferent renal trunks or renal portal veins, the subcardinals form the efferent renal vessels leading into the inferior vena cava, through which all of the blood from the embryonic kidneys (mesonephroi) is returned directly to the 
heart. This arrangement continues until this embryonic kidney is replaced by the definitive kidney of the adult (metanephros), when the posterior cardinal veins, then the renal veins, connect directly with the subcardinal veins and the vena cava, and the renal portal system disappears. The subclavian veins which originally opened into the proximal parts of the posterior cardinals, acquire openings into the ductûs Cuvieri near the vertebral and external jugular veins.

\section{The Lymphatic System and Spleen}

The development of the lymphatic system is imperfectly known in the chick. A pair of anterior or cervical lymph hearts appears during the fifth day, and from these lymphatic networks grow out extending posteriorly, parallel with the lateral veins of the body; by the eighth day these nets are developed into definite lymphatic vessels. A pair of posterior or caudal lymph hearts appears early the seventh day as a series of lateral outgrowths from the first five coccygeal veins (tributaries of the posterior cardinal veins). These branches anastomose with one another, forming an irregular sac or lymph heart, on each side, which remains connected with the second, third, and fourth coccygeal veins. The walls of the hearts become muscular and rhythmically contractile during the eighth and ninth days.

During the ninth day the posterior lymph hearts connect with a system of lymphatic spaces around the posterior section of the dorsal aorta, and this space connects in turn with the thoracic ducts. These lymphatic trunks are visible on the eighth day, when they are described as two solid strands of mesenchyme, extending from the thyroid body to the roots of the cœliac artery. They become hollowed out and connect with the ductûs Botalli, the dorsal aorta and the ductûs Cuvieri. These canals then approach and fuse, and about the twelfth day connect with the posterior lymph hearts by way of the lymphatic vessel around the posterior dorsal aorta. (It is entirely probable that the connections between the thoracic 
ducts and the blood-vessels represent the primarily formative outgrowths of the ducts from the vascular endothelium, from which the cords have extended and fused secondarily, but direct observations to this effect are wanting.)

The anterior lymphatic hearts apparently disappear early. The posterior lymph hearts attain their maximum development during the fourteenth and fifteenth days, when they begin to retrogress and disappear entirely ten to fourteen days after hatching.

The spleen arises, during the fourth day, from a proliferation of peritoneal cells in the base of the mesentery just above the pancreatic region. It enlarges rapidly through continued cell proliferation and the accumulation of a mesenchymatous stroma. Its spaces, without definite endothelial walls, are directly continuous with the sinusoidal origins of its efferent vein, and from these spaces splenic cells enter the blood stream and become converted into blood corpuscles.

\section{THE CAVITIES OF THE BODY}

The folding-off of the embryo from the yolk completes the roughing-in of the body cavity. From the very beginning the general embryonic body cavity shows signs of the differentiation of the region surrounding the heart as the pericardial cavity. We have already traced the origin of this part of the cœlom, in describing the origin and formation of the heart. We have also seen how the body cavity proper is formed and closed, and how it is partially divided longitudinally by the dorsal mesentery. It remains now for us to consider the essential steps in the complete separation of the pericardial cavity and the further subdivision of the primary body cavity.

Throughout the early stages of development the pericardial cavity is only incompletely closed off from the body cavity, since it is only partly closed posteriorly by the mesoderm in the wall of the anterior intestinal portal, and the vessels which are entering the heart from the yolk-sac. The formation of the lateral mesocardia (see above) extends the separation of the 
two cavities, but they still remain connected above and below this partition. The pericardial cavity soon becomes restricted to the median region of the body and the general body cavity then pushes forward along the sides of the pericardial cavity. These antero-lateral extensions of the body cavity are the rudiments of the pleural cavity; they soon extend inward toward the median line, dorsally to the pericardial cavity with which, however, they still connect above the lateral mesocardia. The pericardial cavity still connects with the general body cavity beneath the lateral mesocardia (Fig. 139).

The complete closure of the pericardial cavity is begun during the fourth day, by the formation of the septum. transversum. This partition is established by the formation of tissue connecting the lateral mesocardia dorsally and ventrally, or ventrolaterally, with the body wall. While the septum transversum soon becomes complete between pericardial and body cavities,

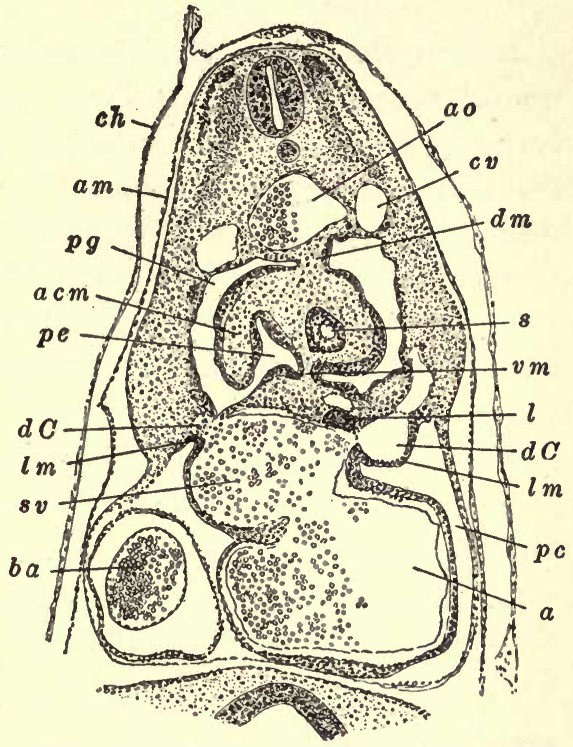

Fig. 139.-Part of a transverse section through the lateral mesocardia of a chick with thirty-five pairs of somites (about seventy-two hours). After Lillie. $a$, Auricle; $a c m$, accessory mesentery; $a m$, amnion; $a o$, dorsal aorta; $b a$, bulbus arteriosus; $c h$, chorion; $c v$, posterior cardinal vein; $d C$, ductus Cuvieri; $d m$, dorsal mesentery; $l$, liver; $l m$, lateral mesocardium; $p c$, pericardial cavity; $p e$, pulmo-enteric recess; $p g$, pleural groove; $s$, stomach; $s v$, sinus venosus; $v m$, ventral mesentery.

it remains for a time incomplete between pleural and body cavities. As the lungs begin to expand they push out into the pleural cavities, the walls of which supply the greater part of their mesodermal tissue. Finally the lungs extend posteriorly as well as laterally and as they reach the region of the septum transversum this gradually becomes completed 
(about the tenth day) as the pleuro-peritoneal membrane, closing off the pleural cavity from the body cavity proper or peritoneal cavity.

\section{THE LATER HISTORY OF THE MESODERMAL SOMITES}

In the chick of thirty hours we saw how the embryonic mesoderm is divided into three general regions, (a) the axial somites, (b) the intermediate cell mass or nephrotome, (c) the distal lateral plate, continuous with the extra-embryonic mesoderm (Fig. 102). We have already described the chief structures derived from the lateral plate-the vascular system and the cœlom and its derivatives, and it remains now to describe the structures derived from the somites and intermediate cell mass.

The following table, quoted from Lillie (Development of the Chick, pp. 184-185) gives a resumé of the general disposition of the somites.

"In an embryo of 42 somites (about ninety-six hours), the value of the somites as determined by their relations and subsequent history is as follows:

1 to 4 . Cephalic; entering into the composition of the occipital region of the skull.

5 to 16 . Prebrachial; i.e., entering into the region between the wing and the skull.

17 to 19 . Brachial.

20 to 25 . Between wing and leg.

26 to 32 . Leg somites.

33 to 35 . Region of cloaca.

36 to 42 . Caudal.

"More somites are formed later, the maximum number recorded being 52 (see Keibel and Abraham, Normaltafeln). In an eight-day chick the number of somites is again about 42 , including the four fused with the skull. Thus the ten somites formed last are again lost."

The somites, excepting those at each end of the series, have essentially a similar history, differing only in later details of development (Fig. 119). Each gives rise to three structures: (a) the muscle plate or myotome, (b) the cutis plate or dermatome, 
(c) the sclerotome. The somites form as solid segmental cell masses; their superficial cells are arranged as a rather dense wall, epithelial in character, which encloses a loosely arranged central mass of mesenchymal nature. The densely arranged cells soon become limited to the dorsal and dorso-lateral regions of the somites. The dorsal portion, in particular the region toward the nerve cord, forms the rudiment of the myotome or muscle plate, while the dorso-lateral region gives rise to the cutis plate. In the more loosely arranged core, the formation of intercellular substance begins very early, producing a truly mesenchymatous structure. This part of the somite then extends over toward the notochord and nerve cord, as the rudiment of the sclerotome.

The myotome becomes thin and turns under the thicker cutis plate, finally extending downward and outward entirely beneath it, occupying a position between the spinal ganglion and the cutis plate. The cells of the myotome elongate anteroposteriorly, through the whole extent of the segment, and each becomes converted into a striated muscle fiber. Later the myotomes enlarge, as their component cells multiply and grow, and each extends down into the body wall. Opposite the limbbuds, outgrowths of the myotomes extend into these, forming their musculature. The entire voluntary musculature of the chick develops from the myotomes; the involuntary musculature is mesenchymal in origin, chiefly splanchnic. The cutis plate extends laterally, as the embryo grows, and after thinning considerably, breaks up into a mesenchyme which spreads underneath the ectoderm, forming the foundation of the thin dermis layer of the integument.

The sclerotomal cells, multiplying and continuing the formation of intercellular substance, extend dorsally, between the nerve cord and the myotome, and ventrally, around the notochord and dorsal aorta, and finally fill all the spaces around these axial structures. Later the sclerotomes acquire a secondary segmentation, in that each becomes transversely divided opposite the middle of the somite; the posterior half of one sclerotome then unites with the anterior half of the succeeding, 
forming a sclerotomal segment. The sclerotome forms the axial skeleton of the embryo (except the major portion of the skull) and the segments are the rudiments of the vertebrox, which thus alternate with the muscle segments, the arrangement of which marks the primary segmentation of the embryo.

All details of the formation of the skeletal system lie without the scope of the present chapter, and we shall merely call attention to the fact that the skeleton arises in part from the sclerotomes and in part from the general mesenchyme. The vertebral column is the part derived from the sclerotomes. These cells condense and the cartilaginous rudiments of the vertebræ begin to appear during the fifth day around the notochord (centra) and nerve cord (neural arches). The sclerotomes of the head somites form the occipital region of the skull; the remainder of the skull is formed from the mesenchyme around the brain and sense capsules. Cartilage begins to form in the skull during the sixth day. The visceral skeleton forms from the mesenchyme of the visceral arches, cartilage appearing here during the sixth day also. The skeleton of the pectoral and pelvic arches and limbs is formed from the mesenchyme of these regions, cartilage appearing during the sixth and seventh days. The clavicles, like the derm bones of the skull and anterior visceral arches, ossify directly from mesenchyme, without being preformed in cartilage. (For simple accounts of the development of the skeleton the student is referred to Marshall, "Vertebrate Embryology," and Lillie, "Development of the Chick," where full references to the literature will be found.)

\section{THE URINOGENITAL SYSTEM}

In the chick, as in other vertebrates, the excretory and reproductive sytems arise separately and come into relation only secondarily. We may therefore begin with an account of the origin of the excretory system, and through this lead to the development of the reproductive system, and to an account of their association. 


\section{The Excretory System}

The intermediate cell masses, or nephrotomes, form the rudiments of the excretory system which, as in all Amniota, is complicated by the succession of three nephric systems, pronephros, mesonephros, and metanephros, of which the first two are purely embryonic, only the last giving rise to the excretory system of the adult. The nephroi develop only through the neck and trunk regions, for in the head and tail no lateral plate, nephrotome, and somite are differentiated in the mesodermal segment.

\section{A. THE PRONEPHROS AND THE PRONEPHRIC DUCT} (WOLFFIAN DUCT)

The pronephros is wholly of vestigial character in the chick, functionless even in the embryo. The pronephric duct, however, is retained as the duct of the embryonic kidney, and is hence known as the mesonephric or Wolffian duct. On account of its vestigial character the pronephros develops variably, even in different regions in a single individual. It is limited to the fifth to fifteenth or sixteenth somites, but becomes typically developed only in the tenth to fifteenth.

In the latter region a small bud of cells grows upward from the middle of the postero-dorsal surface of each nephrotome. These buds, appearing about the middle of the second day, are the rudiments of the pronephric tubules, so-called although they remain solid here. The buds or tubules elongate gradually, and their terminal portions bend over posteriorly, each uniting with the next posterior tubule, forming thus a continuous longitudinal strand, which is the rudiment of the pronephric or Wolffian duct. Toward the close of the second day the duct becomes hollow anteriorly. It pushes backward rapidly, above the nephrotomes, growing independently, until about the sixtieth hour it reaches the cloaca, with which it fuses; its lumen is completed throughout at the end of the third day. In front of the tenth somite no duct is formed and the tubules are re- 
duced to small transitory buds entirely disappearing during the latter part of the second day.

Soon after the tubule appears in each segment, the nephrotome is separated from the somite by the conversion of its proximal part into mesenchyme, and the distal part then appears added to the tubule. The only cavity of the pronephric tubule is one sometimes appearing in this added portion of the nephrotome, and is to be regarded as a continuation of the cœlom of the lateral plate into the nephrotome region; when present its opening to the cœlom would therefore represent a nephrostome. No Malpighian bodies are developed in connection with these tubules, and the whole pronephros disappears by the end of the third day.

\section{B. THE MESONEPHROS}

The mesonephros is the functional embryonic kidney; its duct is the original pronephric or Wolffian duct. The mesonephros begins to develop toward the end of the second day in the region immediately posterior to the pronephros. Mesonephric tubules finally develop in all segments from the thirteenth or fourteenth to the thirtieth; the most anterior tubules are thus present in segments developing pronephric tubules also. In front of the twentieth segment, however, the mesonephros remains rather vestigial and develops typically only from the twentieth to the thirtieth segments.

In this latter region the narrow nephrotomal band widens, separates entirely from the lateral plate and the somites, and the original arrangement of its cells in dorsal and ventral layers is lost. We should note that the Wolffian duct passes along, between the nephrotomes and the somatic layer of the lateral plate, while along the opposite sides of the nephrotomes is the dorsal aorta; the posterior cardinal veins soon appear just above the Wolffian ducts. On the ventral side of the nephrotome, about opposite the middle of the segment, its cells become condensed into a spherical mass, in which a definite space appears; this is the rudiment of the primary mesonephric tubule 
(Fig. 140). This rudiment then extends upward to the Wolffian duct with which it communicates. On the side opposite this extension another outgrowth appears which forms the Malpighian body.
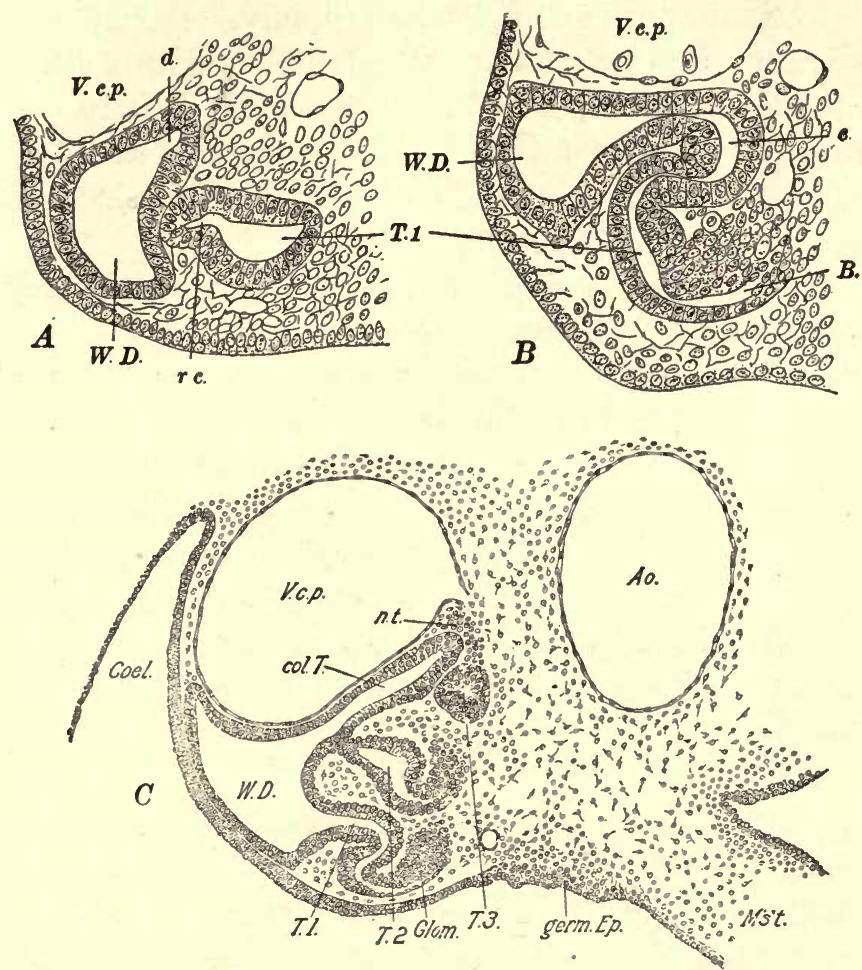

FIG. 140.-The development of the mesonephros. $A, B$. Transverse sections through the mesonephric tubules of the duck embryo with forty-five pairs of somites. After Schreiner. $C$. Transverse section through the middle of the mesonephros of a chick of ninety-six hours. From Lillie (Development of the Chick). Ao., Dorsal aorta; B., rudiment of Bowman's capsule; c., collecting duct; $C \propto l$., cœlom; Col. T., collecting tubule; $d$., dorsal outgrowth of the Wolffian duct; Glom., glomerulus; germ. Ep., germinal epithelium; M's't., mesentery; n.t., nephrogenous tissue; $r$., rudiment of conducting portion of primary tubule; $T .1,2,3$, primary, secondary, and tertiary mesonephric tubules; V.c.p., posterior cardinal vein; W.D., Wolffian duct.

From the nephrotome, just above the primary tubule, several additional secondary mesonephric tubules are formed, and finally, other dorsal tertiary tubules are added, so that six 
or seven tubules are formed in each segment; all these tubules develop similarly. No nephrostomes, or cœlomic connections, are formed save in the four or five most anterior tubules, which are themselves transitory structures. The formation of the mesonephric tubules is completed during the fourth and fifth days, when they begin to elongate rapidly. During the next three or four days they become convoluted and form altogether a large mass, sometimes known as the Wolffian body, projecting from the dorsal body wall. The tubules of each segment open into a common dilation of the Wolffian duct, distinguished as the collecting tubule.

The mesonephros becomes very vascular through the formation of abundant sinuses from the accompanying posterior cardinal veins, which are its afferent vessels. The walls of these sinuses are in direct contact with the tubules. The blood collects along the ventral side of the mesonephros in the socalled subcardinal veins, which connect, as we have seen, with the inferior vena cava. The mesonephros begins to degenerate the tenth or eleventh day, and by the time of hatching it has completely disappeared, save in so far as parts of it remain connected or associated with the reproductive system.

\section{THE METANEPHROS}

The metanephros is the permanent kidney of the adult, and it also functions probably, together with the mesonephros, during the latter part of embryonic life. Metanephric structures appear toward the end of the fourth day as outgrowths from each mesonephric or Wolffian duct, just as this turns to enter the cloaca. Each outgrowth becomes a sac and then a tube, turning anteriorly and rapidly growing forward along the inner side of the posterior cardinal vein, and finally extending anteriorly, above the mesonephros, as far as the twenty-fifth somite. This tube is the rudiment of the ureter and collecting tubules of the metanephros; the latter are formed as the result of a complicated system of branches of the original duct as it grows forward (Fig. 141).

The secreting tubules or true metanephric tubules, are formed 
from the cells of the nephrotomes of the last two or three segments of the body (31-33). In this region the nephrotomal structure is not clearly differentiated, and it is referred to simply as the metanephrogenous tissue. As the metanephric diverticulum grows out it is accompanied on its inner face, throughout all its branching, by cells of this tissue. During the seventh or eighth day typical nephric vesicles appear, like those of the mesonephros, and acquire openings into each branch of the collecting duct; Malpighian bodies develop in the usual manner. During the next three or four days the entire metanephros is established, and the mesodermal cells surrounding the tubules and ducts form the stroma and capsule of the kidney. During the fifth and sixth days the terminal portions of the Wolffian ducts and the metanephric diverticula become absorbed into the wall of the cloaca so that the ureters acquire openings separate from those of the Woffian ducts; the latter are hence, after the degeneration of the mesonephros, not at all excretory in function. No nephrostomes appear in connection with the metanephros.

From the preceding description it will be seen that the inner or medullary part of the definitive kidney is derived from the branched outgrowth from the Wolffian duct, while the outer cortical layer of secretory tubules and Malpighian bodies is derived from the metanephrogenous tissue (nephrotomes) of the last two or three body somites, and is therefore homologous with the glandular part of the mesonephros.

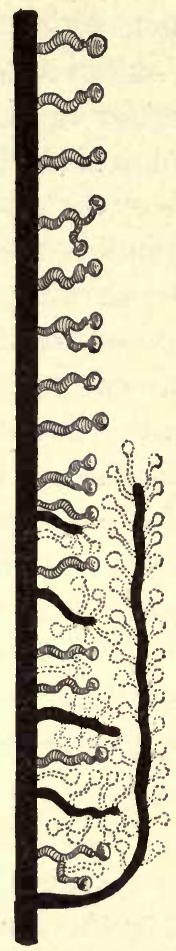

FI G. 141 .Diagram of the arrangement of the nephric ele$m$ en $t \mathrm{~s}$ in the chick. Af ter Felix. Pronephric duct, and metanephric ducts (ureters) in black; mesonephric tubules cross hatched; metanephric tubules in dotted outlines.

\section{The Reproductive System}

Before describing the development of this system it seems necessary to recall, in a few words, the composition of the adult 
reproductive system in the Amniota. In these forms the gonoducts are derived from the original mesonephric or Wolffian duct, which is now represented by two longitudinal ducts, the Wolffian duct, stricto sensu, and the Müllerian duct. As a matter of fact, we shall see that the Müllerian duct develops independently of the mesomephric duct, but phyletically it is clear that both ducts are to be regarded as derivatives of a common mesonephric duct. The mesonephros itself largely degenerates, of course, but some part of it remains functionally connected with the reproductive system in the male, and as a purely vestigial structure in the female. Consequently in the male Amniote the Wolffian duct proper is freed from excretory function, and serves only as the gonoduct or vas deferens, effecting a connection with the gonad through the remains of certain mesonephric tubules; the Müllerian duct is either vestigial or entirely wanting. In the female, on the contrary, the Müllerian duct is the functional gonoduct, or oviduct, while the Wolffian duct and mesonephros either disappear entirely or remain as functionless vestiges.

\section{A. THE REPRODUCTIVE DUCTS}

Nothing need be added to the account already given of the development of the Wolffian duct. We shall see below how, in the male, this connects with the testis; in the female the Wolffian duct disappears along with the mesonephros.

The Müllerian ducts develop similarly in the male and female; they appear during the fourth day. Each is formed as a thickened longitudinal band in the peritoneum, along the outer surface of the mesonephros, near its attachment to the body wall, i.e., just along the outer side of the Wolffian duct. This band invaginates, forming first a groove and then a tube, lying just beneath the surface of the anterior end of the mesonephros. The extreme anterior end of this canal remains open into the body cavity, as the rudiment of the ostium or infundibulum. The greater part of the Müllerian duct is formed by the backward extension of the tube thus formed. It grows posteriorly as a 
solid rod, gradually becoming tubular, and reaches the cloaca during the seventh day, although it does not acquire an opening into the cloaca during embryonic life, indeed not until the fowl is about six months old. The duct becomes surrounded by a thick coat of mesenchyme cells and appears as a ridge on the surface of the mesonephros.

After the eighth day, in the male both Müllerian ducts and in the female the right duct, cease to develop and immediately begin a series of degenerative changes. In the female the left duct continues to enlarge, and as the mesonephros disappears, it remains as a conspicuous organ, attached to the dorsal body wall by a double fold of peritoneum, the mesovarium. Further differentiation into the regions of the adult oviduct already lescribed, begins before the end of the second week of incubation.

\section{B. THE GONADS}

The early development of the gonads is alike in both sexes, and it is not until the end of the first week that the sexes can be distinguished. This early period is known as the indifferent period. The gonads appear on the fourth day, as longitudinal bands of thickened peritoneal epithelium, along the dorsal wall of the body cavity, between the mesonephros and the attachment of the mesentery. This band of "germinal epithelium" later appears on the inner surface of the mesonephros, on account of the enlargement of this organ. The germinal epithelia develop symmetrically and extend through the posterior half or third of the mesonephric region.

The peritoneal cells of the so-called "germinal epithelium" are apparently not to be regarded as the true germ cells. As in other groups, the primordial germ cells are differentiated very early in development, and migrate into this peritoneal or germinal epithelium, where they begin to multiply (Fig. 142). The mesenchyme cells of the mesonephros, beneath the peritoneum, become added to the developing gonad and later form its stroma or connective tissues. 
During the fifth day strands of cells appear, extending between the substance of the gonad and the mesonephric tubules of the region. These are the rudiments of the sexual cords (Fig. 142). While not definitely demonstrated as yet, it seems probable that the sexual cords are outgrowths of the mesonephric tubules (Malpighian bodies) which extend into the gonad. About the end of the first week of development the sexes are distinguishable

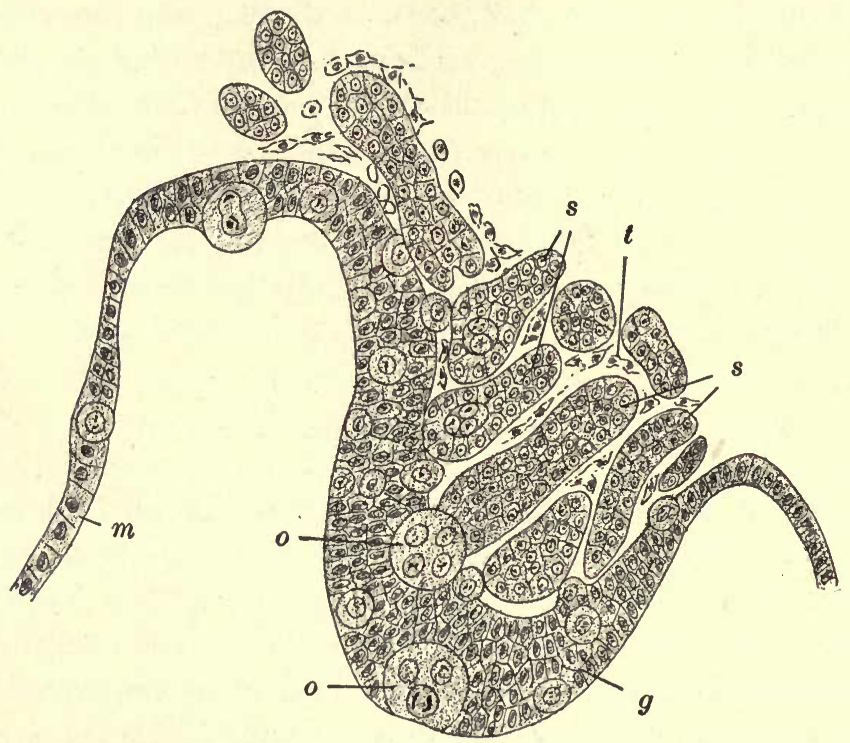

Fig. 142.- Section through the gonad of a chick, the middle of the fifth day, showing the sex cords reaching the germinal epithelium. After Semon. $g$, Germinal epithelium; $m$, epithelium of the mesentery (peritoneum); $o$, primitive ova; $s$, sex cords; $t$, connective-tissue stroma.

through the enlargement of the sexual cords in the male, and the greater thickness of the germinal epithelium in the female.

Testis.-During the second week the primordial germ cells appear all through the stroma, and even migrate into the sexual cords, which continue to increase in size and number until they form altogether a considerable bulk. While the greater part of the mesonephros degenerates, a vestige remains as the paradidymis, that part with which the sexual cords connect. The tubules of this region become the vasa efferentia (epididymis) through the formation, about the end of the third week, 
of a lumen in each sexual cord, which thus puts the cavities of the testis into communication with the mesonephric tubules. The sexual cords thus become the rete efferentia. The primordial germ cells, or spermatogonia as they may now be called, lie in the walls of the dilated inner ends of the rete efferentia (sexual cords), into the cavities of which their cell products may be discharged and pass thence, by the vasa efferentia and the vas deferens, to the cloaca. The original "germinal epithelium" becomes converted into a flat covering layer continuous with the peritoneal folds (mesorchia) slinging the testis from the dorsal body wall.

Ovary.-The early development of the ovary parallels that of the testis. Like the right oviduct, the right ovary, after developing for a time, degenerates and disappears. In the left or definitive ovary the primordial germ cells behave as in the testis, at first, but after a brief period their migration ceases and those which have left the primitive germinal epithelium degenerate, together with the sexual cords. The epithelial cells, and the primordial germ cells contained in the epithelium, continue to multiply rapidly, and the mesenchymal stroma becomes abundant. The inner surface of the germinal epithelium forms strands of cells projecting into the stroma of the ovary, and containing primordial germ cells or oögonia. These strands segment into separate cell masses or nests, each including an oögonial cell; the further growth and development of the oögonia have been described in the beginning of the preceding chapter.

The mesonephros thus has no share in the formation of the reproductive system of the female; its posterior section may be recognized in the vestigial paroöphoron, while the homolog of the epididymis of the male is to be seen in the parovarium.

\section{THE ADRENAL BODIES}

Brief reference to the development of the adrenal bodies may be made here, although they are not a part of the renal system. These bodies have a double origin, arising in part from periton- 
eal proliferation and in part from sympathetic ganglion cells; the part derived from the peritoneal cells effects secondary connections with certain mesonephric components.

During the fourth day the peritoneal cells in front of the germinal epithelia proliferate and extend through the mesenchyme anterior to the mesonephroi and along the dorsal aorta. As these cells multiply they become arranged in definite strands or solid cords; these cords then connect with the renal vesicles of the adjacent portion of the mesonephros. By the eighth day a definite and highly vascular rudiment is established on each side. About this time cells of a sympathetic ganglion located on the antero-dorsal side of the adrenal rudiment, begin to extend into it, penetrating among the primary cords. During the later stages these peritoneal and sympathetic components assume the relations found in the adult adrenal, forming then the cortical and medullary cords respectively.

\section{REFERENCES TO IITERATURE}

\section{CHAPTERS IV AND V}

Abel, W., Further Observations on the Development of the Sympathetic Nervous System in the Chick. Jour. Anat. Physiol. 47. 1912.

Assheton, R., An Experimental Examination into the Growth of the Blastoderm of the Chick. Proc. Roy. Soc. 60. 1896. Gastrulation in Birds. Q. J. M. S. 58. 1912.

Bartelmez, G. W., The Bilaterality of the Pigeon's Egg. A Study in Egg Organization from the First Growth Period of the Oöcyte to the Beginning of Cleavage. Pt. I. Jour. Morph. 23. 1912.

Beard, J., The Development of the Peripheral Nervous System of Vertebrates. Pt. I. Elasmobranchii and Aves. Q. J. M. S. 29. 1888.

Belogolowy, G., Zur Entwickelung der Kopfnerven der Vögel. Ein Beitrag zur Morphologie des Nervensystems der Wirbelthiere. Bull. Soc. Natural. Moscou. 22. 1910.

BLount, M., The Early Development of the Pigeon's Egg with especial Reference to the Supernumerary Sperm Nuclei, the Periblast and the Germ Wall. Biol. Bull. 13. 1907.

Brachet, A., Die Entwickelung der grossen Körperhöhlen und ihre Trennung von einander (Perikardial-, Pleural-, and Peritoneal- 
höhle). Die Entwickelung der Pleuro-Perikardialmembran unc! des Zwerchfells. Ergeb. Anat. u. Entw. 7. 1897 (1898).

Choronschitzky, B., Die Entstehung der Milz, Leber, Gallenblase, Bauchspeicheldrüse und des Pfortadersystems bei den verschiedenen Abtheilungen der Wirbelthiere. Anat. Hefte. 13. 1900.

CoHn, F., Zur Entwicklungsgeschichte des Geruchsorgans des Hünchens. Arch. mikr. Anat. 61. 1903.

Dantschakoff, W., Untersuchungen über die Entwickelung des Blutes und Bindegewebes bei den Vögeln. I. Die erste Entstehung der Blutzellen beim Hühnerembryo und der Dottersack als blutbildendes Organ. Anat. Hefte. 37. 1908.

Disse, J., Die erste Entwickelung des Riechnerven. Anat. Hefte. 9. 1897.

Duval, M., Atlas d'embryologie. Paris. 1889.

Edwards, C. L., The Physiological Zero and the Index of Development for the Egg of the Domestic Fowl, Gallus domesticus. Amer. Jour. Physiol. 6. 1912.

Evans, H. M., On the Development of the Aortæ, Cardinal and Umbilical Veins, and other Blood Vessels of Vertebrate Embryos from Capillaries. Anat. Rec. 3. 1909.

Felix, W., See Hertwig's, Handbuch, etc.

Felix, W. and Bühler, A., See Hertwig's Handbuch, etc.

Foster, M. and Balfour, F. M., The Elements of Embryology. 2nd. ed. London. 1883.

Froriep, A., See Hertwig's Handbuch, etc.

Fülleborn, F., Beiträge zur Entwickelung der Allantois der Vögel. Berlin. 1894 (1895). (Diss.)

Gasser, E., Die Entstehung der Cloakenöffnung bei Hühnerembryonen. Arch. Anat. u. Entw. 1880.

Gaupp, E., See Hertwig's Handbuch, etc.

Göppert, E., See Hertwig's Handbuch, etc.

Greil, A., Beiträge zur vergleichenden Anatomie und Entwicklungsgeschichte des Herzens und des Truncus arteriosus der Wirbelthiere. Morph. Jăhrb. 31. 1903.

Guyer, M., The Spermatogenesis of the Domestic Chicken (Gallus gallus domesticus). Anat. Anz. 34. 1909.

Hertwig, O., (Editor), Handbuch der vergleichenden und experimentellen Entwickelungslehre der Wirbeltiere. Jena. 1906.

Hill, C., Developmental History of Primary Segments of the Vertebrate Head. Zool. Jahrb. 13.1900.

Hirota, S., On the Sero-Amniotic Connection and the Fœtal Membranes in the Chick. Jour. Coll. Sci. Imp. Univ. Tokyo. 6. 1894.

Hıs, W., Untersuchungen über die erste Anlage des Wirbeltierleibes. Die erste Entwickelung des Hühnchens im Ei. Leipzig. 1868. 
Unsere Körperform und das physiologische Problem ihrer Entstehung. Leipzig. 1874.

His, W., JR., Ueber die Entwickelung des Bauchsympathicus beim Hühnchen und Menschen. Arch. Anat. u. Entw. 1897. Suppl. Hochstetter, F., Beiträge zur Entwicklungsgeschichte des Venensystems der Amnioten. 1. Hühnchen. Morph. Jahrb. 13. 1888. See also Hertwig's Handbuch, etc.

D'Hollander, F.-G., Recherches sur l'oögenèse et sur la structure et la signification du noyau vitellin de Balbiani chez les Oiseaux. Arch. d'anat. micr. 7. 1904.

Kastschenko, N., Das Schlundspaltengebiet des Hühnchens. Arch. Anat. u. Entw. 1887.

Keibel, F. and Abraham, K., Normaltafeln zur Entwickelungsgeschichte des Huhnes (Gallus domesticus). Jena. 1900.

Kopsch, F., Ueber die Bedeutung des Primitivstreifens beim Hühnerembryo und über die ihm homologen Teile bei den Embryonen der niederen Wirbeltiere. Intern. Monatschr. Anat. Physiol. 19. 1902.

Krause, R., See Hertwig's Handbuch, etc.

Krause, W., See Hertwig's Handbuch, etc.

KupfFer, K. v., See Hertwig's Handbuch, etc.

Lillie, F. R., The Development of the Chick. New York. 1908.

Locy, W. A., The Fifth and Sixth Aortic Arches in Chick Embryos with Comments on the Condition of the same Vessels in other Vertebrates. Anat. Anz. 29. 1906.

MaLl, F. P., Entwickelung der Branchialbogen und -Spalten des Hühnchens. Arch. Anat. u. Entw. 1887.

Maurer, E., See Hertwig's Handbuch, etc.

Mienzejewski, L., Beitrage zur Entwicklung des Lymphgefasssystems der Vögel. Bull. Acad. Sci. Cracovie. 1909.

Miller, A. M., The Development of the Posteaval Vein in Birds. Amer. Jour. Anat. 2. 1903.

Mitrophanow, P. J., Beobachtungen über die erste Entwickelung der

Vögel. Anat. Hefte. 12.1899.

Neumayer, L., See Hertwig's Handbuch, etc.

Pander, C., Beiträge zur Entwickelungsgeschichte des Hühnchens im

Ei. Würzburg. 1817. (Diss.)

Patterson, J. T., The Order of Appearance of the Anterior Somites in the Chick. Biol. Bull. 13. 1907. On Gastrulation and the Origin of the Primitive Streak in the Pigeon's Egg. Preliminary Notice. Biol. Bull. 13. 1907. Gastrulation in the Pigeon's Egg-A Morphological and Experimental Study. Jour. Morph. 20. 1909. Studies on the Early Development of the Hen's Egg. 
I. History of the Early Cleavage and of the Accessory Cleavage. Jour. Morph. 21.1910.

Pearl, R., Studies on the Physiology of Reproduction in the Domestic Fowl. I. Regulation in the Morphogenetic Activity of the Oviduct. Jour. Exp. Zool. 6. 1909. II. (with Curtis, M. R.) Data regarding the Physiology of the Oviduct. Jour. Exp. Zool. 12. 1912 .

Peebles, F., The Location of the Chick Embryo upon the Blastoderm. Jour. Exp. Zool. 1. 1904.

Peter, K., See Hertwig's Handbuch, etc.

Poll, H., See Hertwig's Handbuch, etc.

PopofF, D., Die Dottersack-Gefässe des Huhnes. Wiesbaden. 1894.

Ravv, E., Ueber den Allantoisstiel des Hühnerembryos. Verh. Anat.

Ges. 12. Anat. Anz. 14. 1898.

Rемак, R., Untersuchungen über die Entwickelung der Wirbelthiere. Berlin. 1855.

RIdDle, O., On the Formation, Significance and Chemistry of the

White and Yellow Yolk of Ova. Jour. Morph. 22. 1911.

Rösler, H., Ueber die erste Anlage der Lungen und der Nebengekröse eininger Vogelarten. Anat. Hefte. 44. 1911.

Röthig, P., and Brugsch, T., Die Entwickelung des Labyrinths beim Huhn. Arch. mikr. Anat. 59. 1902.

RǗckert, J., See Hertwig's Handbuch, etc.

SALA, L., Sullo sviluppo dei cuori linfatici e dei dotti toracici nell'embrione do pollo. Ricerche Lab. Anat. Norm. R. Univ. di Roma. 1900.

Schauinsland, H., See Hertwig's Handbuch, etc.

Schreiner, K. E., Ueber die Entwicklung der Amniotenniere. Zeit. wiss. Zool. 71. 1902.

Semon, R., Die indifferente Anlage der Keimdrüsen beim Hühnchen und ihre Differenzierung zum Hoden. Jena. Zeit. 21 (14). 1887.

Sonnenbrodt, Die Wachstumsperiode der Oöcyte des Huhnes. Arch. mikr. Anat. 72. 1908.

SouliE, A. H., Recherches sur le développement des capsules surrénales chez les vertébrés supérieurs. Jour. anat. et physiol. Paris.

39.1903.

Twining, G. H., The Embryonic History of Carotid Arteries in the Chick. Anat. Anz. 29. 1906.

Verdun, M. P., Sur les dérivés branchiaux du Poulet. C. R. Soc. Biol. Paris. X, 5. 1898.

Virchow, H., Dottersyncytium, Keimhautrand und Beziehungen zur Konkrescenzlehre. Ergeb. Anat. u. Entw. 6. 1896 (1897).

Williams, L. W., The Somites of the Chick. Amer. Jour. Anat. 11. 1910. 
CHAPTER VI

THE EARLY DEVELOPMENT OF THE MAMMAL. THE MAMMALIAN EMBRYONIC MEMBRANES AND APPENDAGES

INTRODUCTION $\cdot 0.0368$

I. THE EGG AND ITS FORMATION . . . . . . 371

1. The Reproductive Organs of the Female . . . . . . . . . 371

2. The Ovum and its Ovarian History . . . . . . . . . . . 372

3. Maturation . . . . . . . . . . . . . . . . . . 376

4. Ovulation . . . . . . . . . . . . . 377

II. FERTILIZATION AND THE EARLY PHASES OF DEVEL-

OPMENT . . . . . . . . . . . . . . . . . . . 379

1. Fertilization ............ . . . . 379

2. Cleavage . . . . . . . . . . . . . . . . . . 380

3. The Blastodermic Vesicle . . . . . . . . . . . . . . . 383

A. The Growth of the Blastodermic Vesicle . . . . . . 384

B. The Formation of the Embryo and the Embryonic Layers . . . . . . . . . . . . . . 384

III. THE DEVELOPMENT OF THE EXTERNAL FORM OF THE HUMAN EMBRYO . . . . . . . . . . . . . . 399

IV. THE EMBRYONIC MEMBRANES AND APPENDAGES OF THE EUTHERIAN MAMMALS . . . . . . . 417

1. Implantation . . . . . . . . . . . . . . 421

2. The Amnion and Chorion . . . . . . . . . . . . . . 424

3. The Yolk-sac . . . . . . . . . . . . 431

4. The Allantois . . . . . . . . . . . . . . . . . 434

5. The Placenta . . . . . . . . . . . . . . . . 437

WE shall not undertake, in the present chapter, to give an account, however brief, covering the whole embryonic history of a Mammal. We shall rather attempt to describe certain phases or aspects of mammalian development, selected on account of their interest or importance for the general student. We shall give first a description of the mammalian ovum, its formation, the early processes of cleavage and the formation 
of the embryonic layers, and the formation of the embryo and its chief rudiments. This will be followed by a brief account of the development of external form of the human embryo. Then in conclusion we shall outline the more salient facts regarding the embryonic membranes and appendages, and the establishment of those relations between the embryo and the maternal organism which are such fundamental characteristics of the true (Eutherian) Mammals. For the whole subject of mammalian organogeny the student may be referred to the excellent and recent accounts given in such texts as those of O. Hertwig, Keibel-Mall, Minot, McMurrich, etc.

The whole life-history of the Mammal may be roughly divided into four periods, each marked by one or two striking characteristics, but often not otherwise clearly separated. First is the true embryonic period or period of gestation, during the greater part of which the organism is retained within the uterine cavity of the mother, drawing its nourishment from the uterine walls. This period extends from the time of fertiliza. tion to the time of birth, and its duration is widely variable ir different species, though usually quite constant in any single form, due, perhaps, in part, to the fact that conditions of temperature, nutrition, etc., are subject to only slight variation. To mention a few examples, the period of gestation is, in the mouse twenty to thirty days (Daniel), rat about twenty-one days, in the rabbit thirty to thirty-two days, guinea-pig sixty-four to seventy days, cat about nine weeks, dog fifty-nine to sixtythree days, sheep about twenty-one weeks, pig about four months, cow about nine months, man about nine months (270-280 days), deer ten months, horse about eleven months, elephant about twenty months.

The time of birth, or parturition, marks the most abrupt physiological and morphological transition in the entire lifehistory. There is wide variation, among different forms, of the comparative stage to which development has proceeded when this event occurs. Some organisms, like the calf or the colt, may be termed precocious, since they are able, within a few hours after birth, to run about actively and to live with a 
minimum of parental protection and care. Others, such as the kitten or young rabbit, are born in a much less advanced stage and, with unopened eyes and uncoördinated movements, remain almost helpless for several days. Some of the marsupial Mammals (Metatheria) are quite remarkable in that the young are born after a very brief period of gestation (about eight days in the opossum (Didelphys) and in Dasyurus), at a relatively very early stage of development, not able even to perform the simple act of sucking. In these forms there are special adaptations to this condition, and the young are at once transferred to a special external cavity of the mother, the marsupium or pouch, where development proceeds.

In no case is the young Mammal entirely independent of the maternal organism for some time after parturition, for there follows the second general period in the life-history, that of lactation, during which the young organism is wholly or partly dependent for its nourishment upon the mammary secretion of the mother. During this period development continues, of course, but at a slower rate, and toward its close there is a gradual transition to conditions of complete independence, save that some degree of parental care may be exercised for a time longer. The duration of the period of lactation is variable, even in a given species.

Two other periods in the life-cycle of the Mammal need only to be mentioned; these are the period of adolescence, during which growth and development continue at a still slower rate, and the period of adult life or sexual maturity. The transition between these periods is often marked by a series of structural and physiological alterations in characteristics other than those of the reproductive system.

Embryologically as well as morphologically, the Mammalia present many similarities to the Sauropsida. The mammalian ovum is nearly yolkless, and yet in its development it exhibits many of the phenomena of yolk-influence-characteristics of eggs of the extreme telolecithal (meroblastic) type-such as the formation of a modified germ disc, of a (yolkless) yolk-sac, and other less striking characteristics. While the mammalian 
egg resembles that of Amphioxus in size and deutoplasmic relations, it exhibits almost none of the regularities of cleavage. blastula formation, and gastrulation that we should expect to be associated with a small, homolecithal ovum.

The origin of the most fundamental modifications of the sauropsid type of development lies in the replacement of the intra-oval yolk-mass by a source of food and energy lying outside of the ovum and embryo, i.e., the maternal uterine walls, and in the early and extensive relation between the embryo and this new source of nutrition.

In the following account of certain phases of mammalian development, we shall not be limited to any single form throughout, but shall describe in general, elementary terms, the mammalian type of development, using various forms as illustrations of the topics considered.

\section{THE EGG AND ITS FORMATION}

\section{The Reproductive Organs of the Female}

In the Mammals there is always a single pair of ovaries, suspended in the postero-dorsal region of the body cavity by peritoneal mesovaria (Fig. 143). They are whitish, rounded or ovoid bodies, of rather small dimensions (human, 3-4 cm. long, by $2-3 \mathrm{~cm}$. wide, by $0.7-1.2 \mathrm{~cm}$. thick; rabbit, about $2 \times 0.8 \mathrm{~cm}$. The ovaries are not directly connected with the gonoducts, although the openings of the oviducts are suspended in the same peritoneal mesovaria, and are located very near to the ovaries, so that the ova, when discharged from the ovary pass only a short distance through the body cavity before entering the oviduct (Fig. 143).

The Müllerian ducts, or oviducts in the broad sense, are muscular tubes, highly differentiated into three regions. The upper or anterior portion forms the Fallopian tube or oviduct, stricto sensu (Fig. 143). The inner end of this, where it opens out of the body cavity near the ovary, is expanded and its margin is drawn out into finger-like or fringe-like processes; this is the infundibulum, ostium or fimbriated opening. The 
second section is the uterus, thicker walled than the Fallopian tube, and of greatly varying extent in different Mammals, correlated with the number of young produced at one time, for this is the part of the oviduct occupied by the developing embryos. Lastly is the terminal vagina, which opens directly to the outside in all placental Mammals.

The vaginal region is practically always a single, median structure, formed by the fusion of the lower ends of the two

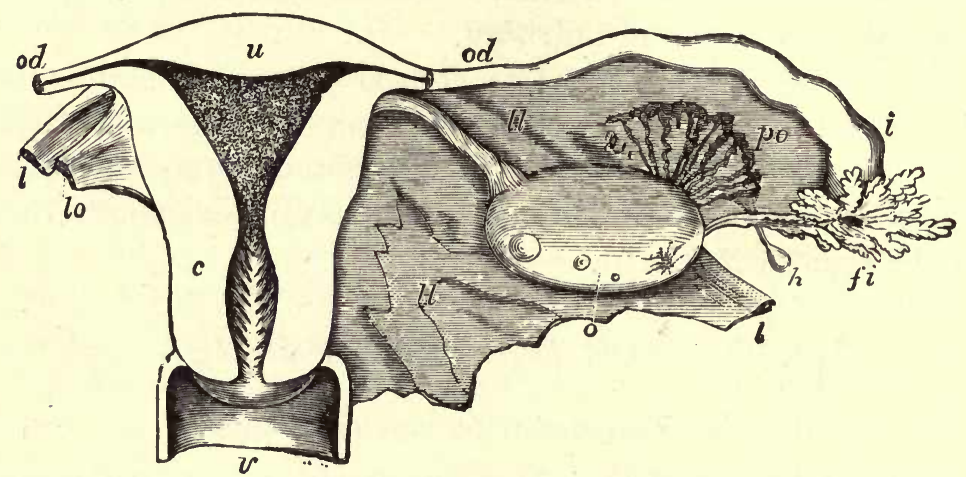

FIg. 143.-Diagrammatic representation of the human female reproductive organs. Dorsal (posterior) view. From Quain's Anatomy. The posterior walls of the uterus and vagina have been removed to show their cavities. $c$, Cervix of uterus; $f$, fimbriated opening or ostium of oviduct; $h$, hydatid; $i$, wider distal part of oviduct; $l$, round ligament; $l l$, the broad ligament; $l o$, ligament of ovary; $o$, ovary (naturally the ovary has an oblique or nearly vertical position); od, oviduct or Fallopian tube; po, parovarium; $u$, fundus of uterus; $v$, upper part of vagina.

oviducts. Different groups of Mammals exhibit various degrees in the extent of the fusion of the uterine sections also. Thus in the Rodents the vaginæ alone are fused, the uteri remaining entirely distinct (uterus duplex), in the Carnivors and most Ungulates the uteri are partly fused, partly free (uterus bicornis), and in the Primates the uteri are completely fused and only the Fallopian tubes remain paired (uterus simplex).

\section{The Ovum and its Ovarian History}

The ova of the placental Mammals are among the smallest known. When fully formed they are usually $0.1-0.3 \mathrm{~mm}$. in 
diameter, although these limits are occasionally exceeded; for example, the ovum of the mouse measures about $0.06 \mathrm{~mm}$., deer 0.07-0.10 mm., guinea-pig $0.09 \mathrm{~mm}$., dog about $0.18 \mathrm{~mm}$., human $0.22-0.32 \mathrm{~mm}$., cat $0.135-0.15 \mathrm{~mm}$., rabbit $0.11-0.12 \mathrm{~mm}$.

The cytoplasm of the ovum ordinarily exhibits two general regions, a clear exoplasm or cortical layer surrounding an opaque

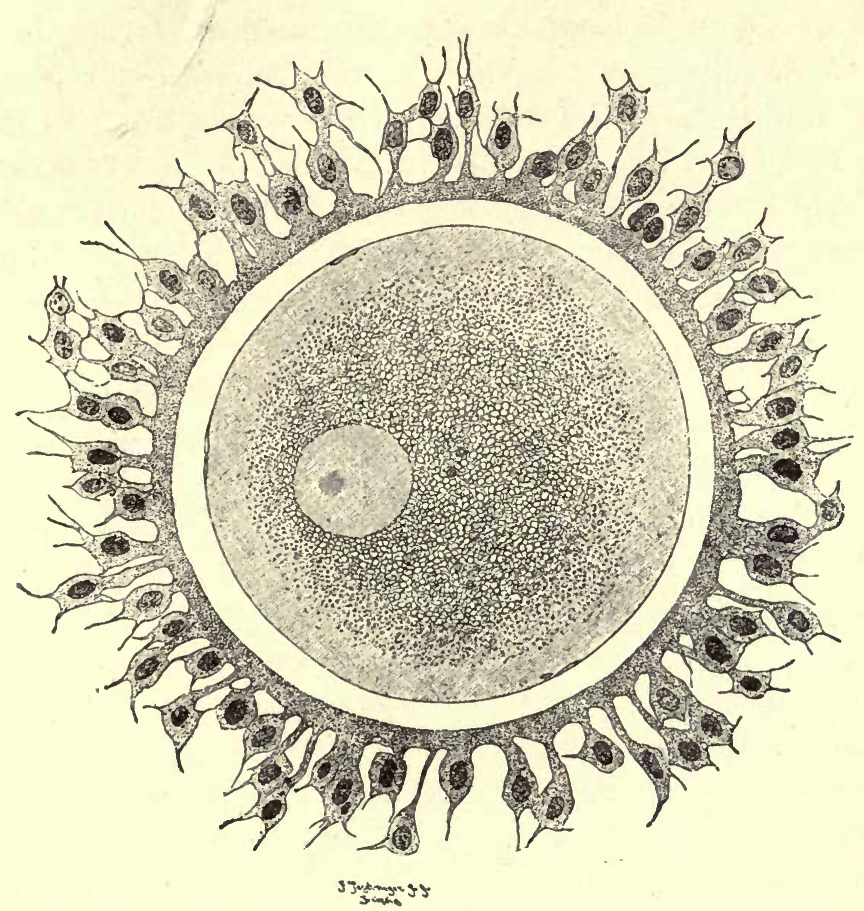

FIG. 144.-Fully grown human oöcyte just removed from the ovary. Outside the oöcyte are the clear zona pellucida and the follicular epithelium (corona radiata). The central part of the oöcyte contains deutoplasmic bodies and the eccentric nucleus (germinal vesicle). Superficially there is a well-marked exoplasm, or cortical layer. From Waldeyer (Hertwig's Handbuch, etc.).

endoplasm containing small granules of deutoplasmic material (Figs. 144, 146). The nucleus or germinal vesicle of the fully formed oöcyte is relatively large, usually $0.025-0.040 \mathrm{~mm}$. in diameter; it is spherical, possesses a definite nuclear membrane, a large nucleolus (karyosome), and has a slightly eccentric position. 
The presence of a vitelline membrane is not definitely known; surrounding the ovum, however, is a thick transparent membrane apparently of chorionic nature (i.e., of follicular origin -secondary egg membrane), known as the zona pellucida. This often has, either throughout or at least peripherally, the appearance of being perforated by minute pores or canals, and hence is often called the zona radiata. A micropyle is not known. The zona pellucida is usually separated from the surface of the fully grown egg by a narrow perivitelline space. In many Mammals, at the time the ovum escapes from the ovary it is, and for a time remains surrounded by a few layers of regularly arranged cells forming the corona radiata (Fig. 144). This is a part of the ovarian egg follicle, and in order to understand its relations we must outline the earlier ovarian history of the ovum.

The formation of the ova begins in the ovary before the time of birth, and in the case of the Mammals all of the ova which are to be produced during the period of fertility, are at that time definitely established, although only partly differentiated. In other words the period of the multiplication of the oögonia is completed during embryonic life. Subsequently there occur the phases of oögonial growth and the maturation processes. In the embryonic ovary the primordial germ cells divide repeatedly and soon form large numbers of cells arranged in small groups or "nests" (Fig. 145). The cells composing these have all had a similar history, but are destined to have very different fates. In each group one cell enlarges and becomes a definitive ovum, while its sister cells and their descendants are to form the egg follicle. All of the stages in the history of the formation of the ovum and follicle can be found in the ovary of a fertile individual.

The follicle appears first as a small group of flattened cells, forming a single layer around the slightly enlarged central oögonium (Fig. 145). As the follicle cells multiply they become cubical and then columnar, forming a definite epithelium. As the ovum increases steadily in size the follicle more than keeps pace with it, so that as the follicular epithelium becomes three 
to four cells deep, spaces begin to appear within the follicle, usually toward one side. On the side opposite these spaces the follicular cells multiply more rapidly and form a definite accu-

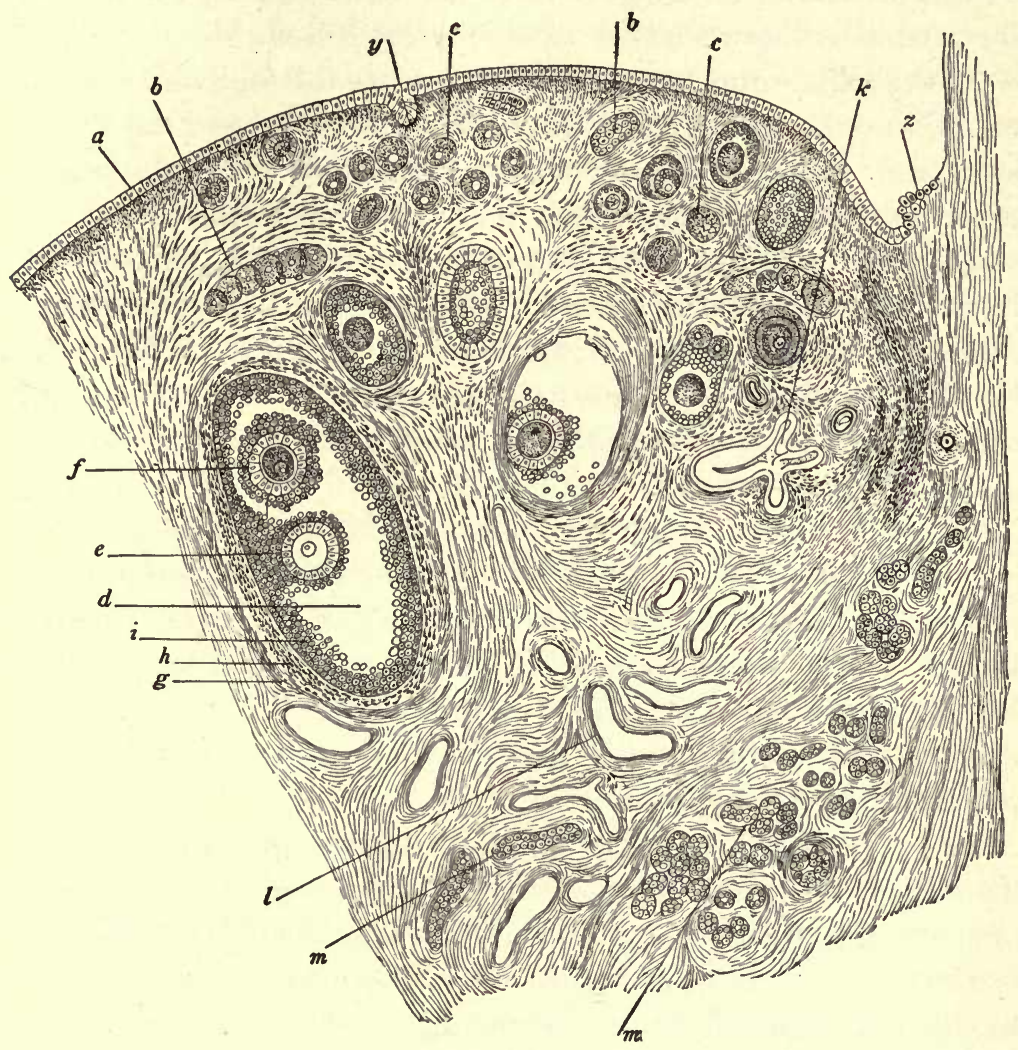

Fig. 145.- Section through part of the ovary of a dog. After Waldeyer. $a$. "Germinal epithelium"; $b$, egg tubes; $c$, small ovarian follicles; $d$, older ovarian follicles; $e$, ovum surrounded by discus proligerus; $f$, second ovum in follicle with $e$. (Only rarely are two ova thus found in a single follicle.) $g$. Outer capsule of the follicle; $h$, inner capsule of the follicle; $i$, membrana granulosa; $k$, collapsed, degenerating follicle; $l$, blood-vesesls; $m$, sections through tubes of the parovarium; $y$, involuted portion of superficial epithelium; $z$, transition to peritoneal epithelium.

mulation known as the discus proligerus, in the midst of which the ovum is buried (Fig. 145). The follicle cells immediately surrounding the ovum become markedly elongated forming the 
corona radiata, mentioned above: These cells appear to be directly connected with the ovum by fine pseudopodial processes affording the pathways by which substances enter the ovum, providing for its growth. The other cells of the follicle form what is known as the stratum granulosum.

As the follicle and egg approach maturity the follicular cavity, containing the liquor folliculi, becomes very large, and the whole structure becomes enclosed in a definite capsule consisting externally of connective-tissue fibers and cells formed from the stroma of the ovary, and internally of a thick layer of cells, blood-vessels, and nerves. Within this lies the basement membrane of the follicular epithelium (granulosa cells). At the close of the oögonial growth period, the cells of the corona radiata form a thick membrane (zona pellucida) around the egg, and the protoplasmic processes remain only partially and indistinctly (zona radiata). The full-grown follicle is very large (9-14 mm. in man) forming a well-marked projection from the surface of the ovary. The mammalian follicle is usually known as the Graafian follicle. It was first described in 1677 by Regnerus de Graaf and was regarded as what we should now call the ovum, until Von Baer's description in 1827 of the true mammalian ovum.

As to the history of the egg itself during the growth stage, little need be said here. A differentiated region around the nucleus appears very early, before the follicle has definitely formed. This becomes a sort of "yolk nucleus" giving rise to the deutoplasmic content of the egg; it disappears while the follicle is still single layered. During the growth period, which is also a period of "organization" of the ovum, the nucleus appears to give off chromatic substance into the cytoplasm. A pair of small centrioles with surrounding centrosphere may be seen during the early stages of growth.

\section{Maturation}

At the close of the growth period the nucleus forms alarge clear vesicle, with very little chromatin other than that of the 
large chromatin reservoir or karyosome. It is now ready to enter upon the period of maturation. As a rule, in the Mammals, the first polar body is given off while the ovum is still within the ovary (in the mouse about one hour before ovulation), and the second polar spindle is established; the second polar division is then completed only after fertilization, which occurs, of course, in the oviduct. It is not necessary to describe the details of the maturation process here, for in most respects it offers nothing unusual. With respect to the size of the polar spindles and polar bodies, however, the Mammals are rather remarkable, for these, especially the first polar body, are very large, often one-fourth the diameter of the ovum itself, and in some cases even larger. Occasionally an abnormally large first polar body may be formed so that the egg divides almost equally; the later history of such cases is not known. The first polar body usually divides soon after its formation; amcboid movements have been observed in the first polar body of the mouse. Centrioles are distinctly present, but the asters are only slightly indicated or absent.

A large second polar spindle is formed at once and moves toward the surface of the egg (secondary oöcyte,) when the process of maturation is inhibited while the processes of ovulation and fertilization occur. The second polar body is then formed while the ovum is in the upper part of the oviduct; it is smaller than the first. The polar bodies do not remain attached to the surface of the ovum (Fig. 147) and are easily lost sight of entirely. The number of chromosomes can be most easily determined during these phases; some of the somatic numbers determined are the following: man, twentytwo in the male, twenty-four in the female (Guyer), mouse twenty-four, cat between twenty-eight and thirty-two.

\section{Ovulation}

The escape of the ovum (secondary oöcyte) from the Graafian follicle and the ovary, is termed ovulation. The capsule of the maturing follicle becomes very vascular, and at one place very 
thin and easily ruptured; this is the cicatrix or stigma. It has been suggested that the rupture of the follicle may be due to the continued accumulation of the liquor folliculi. However this may be, when the follicle bursts the liquor flows out into the periovarial cavity, carrying along the ovum, still surrounded by the corona radiata. The fimbriæ of the oviduct are also enclosed in this periovarial cavity, and through the ciliary action of the epithelium covering these and lining the upper part of the oviduct, and probably also through peristaltic contractions of the oviducal walls, the ovum is carried through the ostium and into the oviduct. During this passage the first polar body frequently breaks through the chorion (zona pellucida or radiata) and through the corona radiata as well, so that it is entirely lost from the region of the ovum (mouse, Kirkham).

Returning to the history of the follicle itself, we find it undergoing very important changes, as the result of which it is converted into the corpus luteum. The emptied follicle soon becomes a nearly solid mass of cells, known as lutein cells, large rounded cells containing quantities of pigmented granules or lutein. The origin of these cells is somewhat uncertain; they appear to be derived from the stratum granulosum cells of the follicle, although they may come from the inner capsule of the follicle (stroma cells). Their pigment is yellowish in man, hence the name corpus luteum; in other Mammals it may be pinkish (pig, rabbit), red (mouse), brown (sheep), etc.

In cases of non-pregnancy following ovulation, thecorpus luteum is rapidly converted into fibrous connective tissue and is absorbed, but when pregnancy follows, the corpus luteum retrogresses very slowly and disappears only after parturition. There is considerable evidence (Marshall, L. Loeb) that the corpus luteum produces an internal secretion (hormone) of great physiological importance in effecting the fixation of the ovum to the walls of the uterus (implantation, see below).

The general conditions determining the occurrence of ovulation are unknown in most instances. In the lower Mammals it is associated with a general physiological condition known as aestrus or heat, which may possibly itself be determined by 
internal ovarian secretions (hormones). Usually, too, the period of cestrus is preceded by a proœstrus, or menstruation. In man, however, these two conditions are more or less independent, and no very constant relation is apparent. In the rabbit, cat, and ferret, ovulation occurs only after coitus (nine to ten hours after, in the rabbit, and usually within fifty hours in the cat), while in the dog, rat, and mouse, and in many Ungulates and Primates, it occurs independently of coitus. In many cases ovulation follows upon parturition, in the mouse about fourteen hours after the birth of a litter, and in the rat within about eighteen hours.

\section{FERTILIZATION AND THE EARLIER PHASES OF DEVELOPMENT}

\section{Fertilization}

In most of the Mammals fertilization occurs in the upper part of the oviduct, almost immediately upon the entrance of the ovum. After the introduction of the spermatozoa into the vagina, they make their way to the upper ends of the oviducts, where they may remain alive and capable of functioning for several days or even weeks. Apparently the ovum too may remain in the oviduct, alive and capable of development, for several days or even a fortnight, in case fertilization does not occur at once.

The details of the sperm entrance, the formation of the second polar body, the establishment and fusion of the egg and sperm pronuclei, and the formation of the first cleavage figure, are not unusual and need not be described here (Fig. 146). Monospermy is typical of the Mammalia, and since there is no micropyle, the spermatozoön has to penetrate the zona pellucida (radiata), as well as the corona radiata, which may remain surrounding the egg during fertilization and even until a late cleavage stage. Long before it finally breaks down it becomes soft and easily penetrated (Fig. 146). In the rat the vigorous movements of the spermatozoa within and among the cells of the corona tear this to pieces and leave the egg naked after 
twenty to twenty-five minutes. In the mouse, at least, the entire spermatozoön enters the ovum, but the tail soon disappears. Some of the details of the process of fertilization in the cat, are shown in Fig. 146.

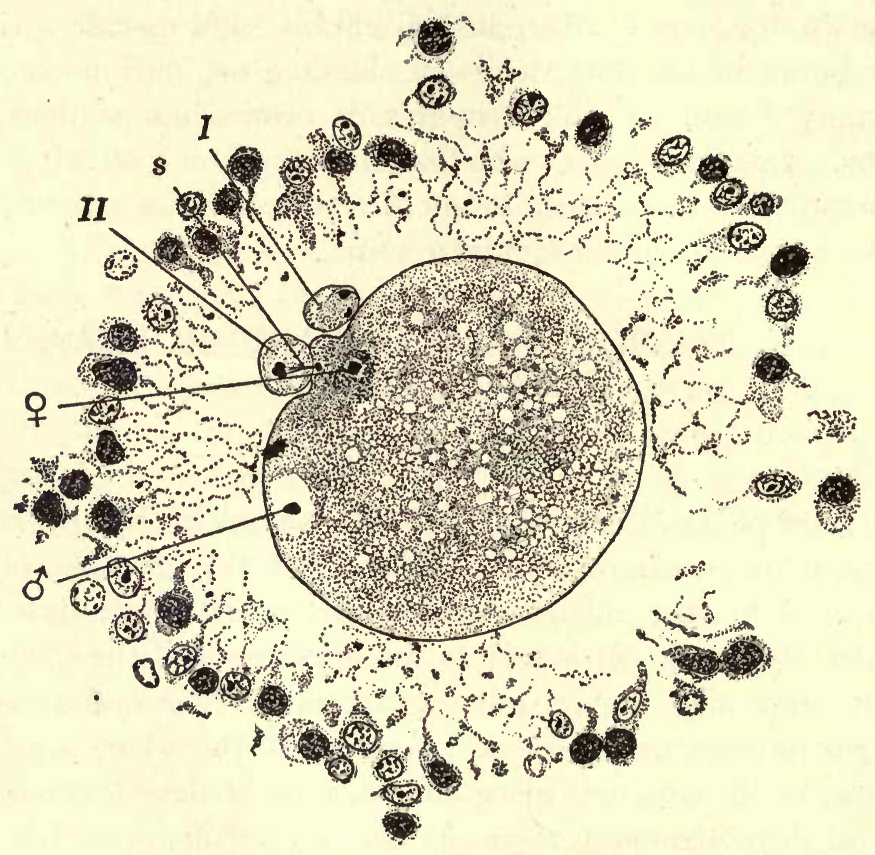

FIG. 146.-Reconstruction of four sections through the fertilized ovum of the cat. From Longley (combined from two figures). No zona pellucida is visible in these sections. The corona radiata is disintegrating. $s$, Remains of second polar spindle; $I$, first polar body; $I I$, second polar body; $\sigma^{7}$, sperm pronucleus; ๑, egg pronucleus.

\section{Cleavage}

Before taking up the details of cleavage and early development, we should say, in preface, that in the placental Mammals these processes of cleavage, formation of the blastoderm and early cell layers, are in many respects unique and often very difficult of comparison with the corresponding processes in other forms. As mentioned above, many of these peculiarities result from the fact that the mammalian ovum was originally markedly 
telolecithal, probably like that of the Sauropsida, and we are already familiar with the fact that the presence of a large yolkmass profoundly modifies the simple processes of cleavage, gastrulation, etc. Accompanying the return of the mammalian egg to the nearly alecithal condition, however, we do not find a corresponding return to the simpler early developmental processes characteristic of the primarily alecithal egg. On the contrary these processes are modified in new directions, not known elsewhere.

Added to these modifications is another series of alterations resulting from the very early development of a mechanism by which the segmenting ovum becomes intimately related with the uterine walls. Altogether, then, we find conditions here that are very special and not closely paralleled in other animals. So profoundly have the early stages of development become modified that there is sometimes difficulty in clearly idertifying and homologizing with other types, certain structures of the early mammalian embryo, such as the germ layers, primitive streak, etc. In the following description we shall adopt the convenient and more customary descriptive terms, but it should be clearly recognized that there are possible contradictions, and some students of mammalian embryology would say, positive errors, in applying the customary terminology to the early history of the mammalian embryo.

In most of the Mammals the early development is very slow. The cleavage stages usually occur as the egg is passing slowly down the oviduct. In the mouse the first and second cleavages occur about twenty-four hours and forty-eight hours, respectively, after coitus, and about eighty hours elapse before the ovum reaches the uterus. In the rabbit the first cleavage occurs fourteen to fifteen hours after ovulation, and nearly four days are occupied in the passage to the uterus. In the dog eight to ten days elapse before the ovum reaches the uterus. A few instances are known, on the other hand, where the egg arrives in the uterus just as cleavage begins (bat, hedgehog, and other Insectivors). In the European roe-deer, where fertilization occurs in the autumn, or perhaps in mid-summer, the ovum 
develops only as far as a cleavage stage that season; its development then continues the following spring.

There is considerable variation in the details of cleavage in the Mammalia, and since we are not describing any single form,
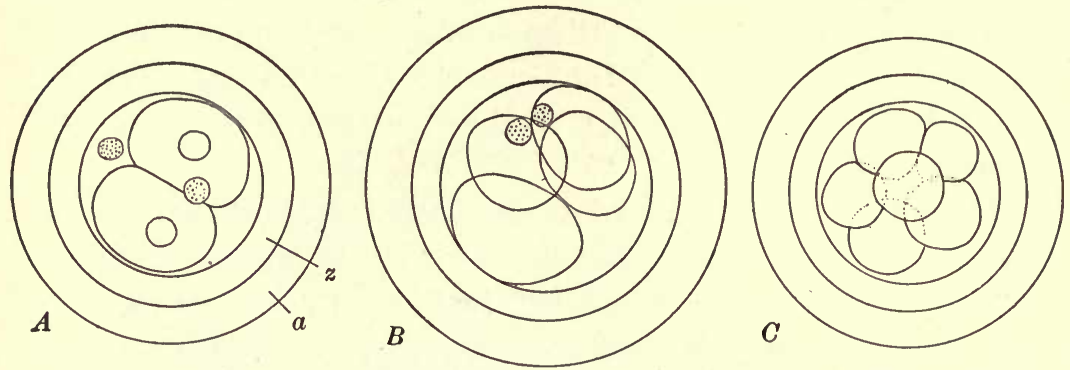

Fig. 147.-Cleavage of the ovum of the rabbit. After Assheton. A. Twocell stage, twenty-four hours after coitus, showing the two polar bodies separated. $B$. Four-cell stage, twenty-five and one-half hours after coitus. C. Eight-cell stage. $a$, Albumenous layer derived from the wall of the oviduct; $z$, zona radiata.

we must be limited to very general terms; many of the details may be learned from the accompanying figures (Figs. 147, 148). Cleavage is total and at first adequal, very early becoming quite
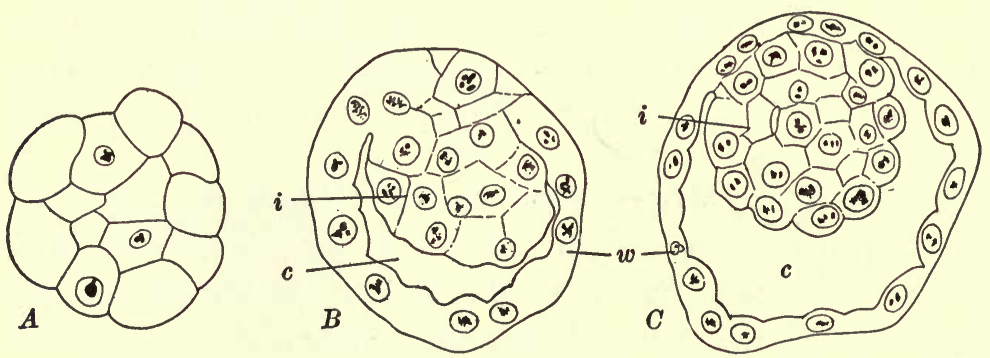

Fig. 148.- Morula and early blastodermic vesicles of the rabbit. After Assheton. The zona radiata and albumenous layer are not shown. $A$. Section through morula stage, forty-seven hours after coitus. $B$. Section through very young vesicle, eighty hours after coitus. Taken from uterus; ordinarily the ova have not reached the uterus at this age. $C$. Section through more advanced vesicle, eighty-three hours after coitus. Taken from uterus. c, Cavity of blastodermic vesicle; $i$, inner cell mass; $w$, wall of blastodermic vesicle (subzonal layer, trophoblast).

unequal, and in many cases very irregular, so that stages of three-, four-, five-cells, etc., may be found. The first cleavage plane, and usually the second, pass nearly through the chief 
egg axis, but the promorphological relations of these cleavages are not known.

In a comparatively early stage the cells take on a fairly definite arrangement. Thus on the surface of the cleavage group we may see a regular and usually continuous, epitheliumlike layer, surrounding, or nearly surrounding, a central mass of large, irregularly arranged cells (Fig. 148). The egg usually remains closely surrounded by the zona pellucida during these stages and the superficial cell layer is known as the subzonal layer, the central mass as the inner cell mass. This arrangement of cells forms what is known as the morula stage, equivalent to the blastula stage of other forms. (The identification of this stage as a gastrula ("metagastrula," Van Beneden) leads to some difficulties in the interpretation of the homologies of the layers of later stages.) The fully formed morula consists of thirty-six to seventy-two cells, of which twenty-four to thirty compose the inner cell mass.

\section{The Blastodermic Vesicle}

As cleavage continues in the cells of the morula, vacuoles appear among the cells of the inner cell mass, toward one side of the morula only. These vacuoles rapidly enlarge and flow together toward one pole, while the cells of the inner mass remain grouped together at the opposite pole (Fig. 148). The structure thus formed is termed the blastodermic vesicle; the fluid of the cavity is supposed to represent the yolk-mass of the sauropsid blastula or gastrula.

In all cases the uterus is reached by the time the blastodermic vesicle is formed, and the immediately subsequent events in development may be described under two heads, (a) the growth or enlargement of the blastodermic vesicle; (b) the formation of the embryonic rudiment and its layers (germ layers). The implantation or embedding of the vesicle in the uterine wall will be described in connection with the history of the fotal membranes. Although these processes overlap to a considerable extent we may describe them separately, as a matter of convenience. 


\section{A. THE GROWTH OF THE BLASTODERMIC VESICLE}

The enlargement of the vesicle results from the flattening of the cells of the subzonal layer, as well as from their multiplication, and the wall of the vesicle thus becomes very thin (Fig. 149). Growth of the vesicle is always rapid, and often it becomes very large. In the rabbit the ovoid vesicle reaches dimensions of about $4.5 \times 3.5 \mathrm{~mm}$. by the seventh day of de-

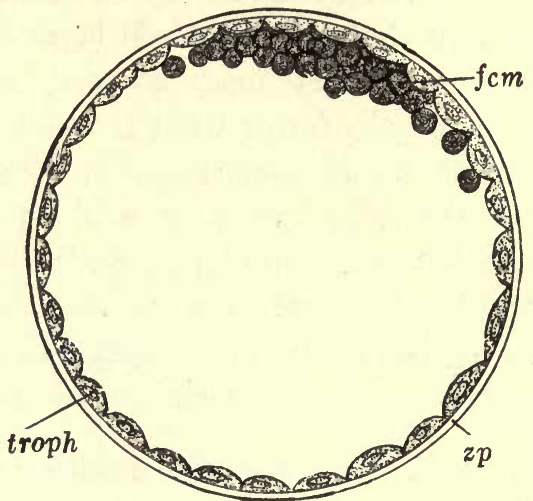

FIG. 149.-Section through the fully formed blastodermic vesicle of the rabbit. From Quain's Anatomy, after Van Beneden. fcm, Granular cells of the inner cell mass; troph, trophoblast cells; $z p$, zona pellucida.

velopment (third day within the uterus), when implantation commences. In the mouse the spherical vesicle is much smaller when implantation begins. In the Ungulates the vesicle becomes elongated and tapered at each end, and very large; in the sheep the twelve-days vesicle (Bonnet) reaches a length of more than $20 \mathrm{~cm}$., its diameter being only 1-2 mm. Ultimately the Ungulate vesicle may extend through the entire uterus, and may even be folded, so that a total length of $1 \mathrm{~m}$. may be reached. In all these cases the embryonic portion of the early vesicle is limited to a small, almost microscopic mass about in its middle.

\section{B. THE FORMATION OF THE EMBRYO AND THE EMBRYONIC}

\section{LAYERS}

We may now return to a stage where the small blastodermic vesicle is just established and consists of a single layer of 
subzonal cells, with the inner cell mass suspended from its inner surface like a hanging drop (Figs. 148, $C ; 149$ ). The first step in the differentiation of this structure consists in the rearrangement of the cells of the inner mass so that those bordering the cavity of the vesicle are formed into a definite, continuous layer, known as the embryonic endoderm (Figs. 152, 155). The relative time at which the endoderm becomes distinct may vary greatly. The endoderm cells multiply rapidly, and typically they spread distally over the entire inner surface of the subzonal layer, converting the blastodermic vesicle into a two-
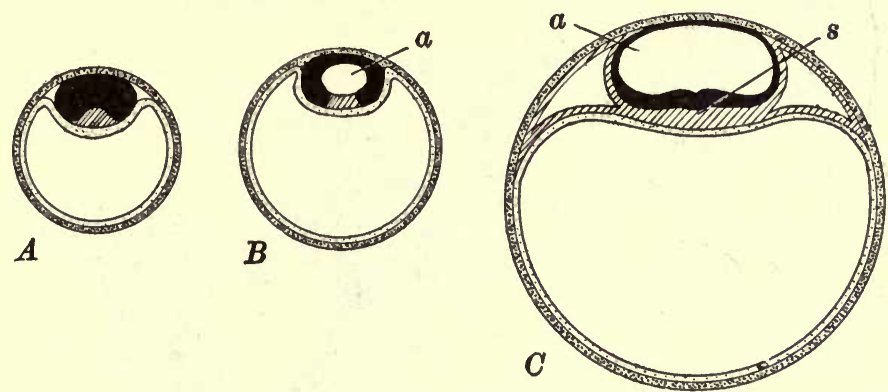

Fig. 150.-Diagrams of the formation of the amnion in the Insectivors. After Keibel. Black, embryonic ectoderm; heavy stipples, trophoblast; light stipples, endoderm; oblique ruling, mesoderm. $A$. Before the appearance of the amnionic cavity. Inner cell mass differentiated into embryonic ectoderm and mesoderm; endoderm extending completely around the wall of the vesicle. $B$. The amnionic cavity $(a)$ appearing in the ectoderm. C. Enlargement of the amnionic cavity. Mesoderm expanded and split into somatic and splanchnic layers, separated by the cœlom. $s$, Primitive streak.

layered structure, the gastrula (Figs. 150, 153, 155). In the Primates, however, the formation of the endoderm does not keep pace with the enlargement of the blastodermic vesicle, so that the endoderm forms a smaller second vesicle suspended below the inner cell mass (Fig. 161).

After the separation of the endoderm, the remainder of the inner cell mass is known as the embryonic ectoderm. In most instances this remains quite distinct from the original subzonal layer, which, in view of its future function of attaching the vesicle to the uterine mucosa, may now be termed the trophoblast (Hubrecht). The inner cell mass is now clearly differen- 
tiated into embryonic ectoderm and endoderm, but before we continue the later history of these layers, we must consider briefly two other matters, $(a)$ the relation between the ectoderm of the embryonic shield and the trophoblast cells
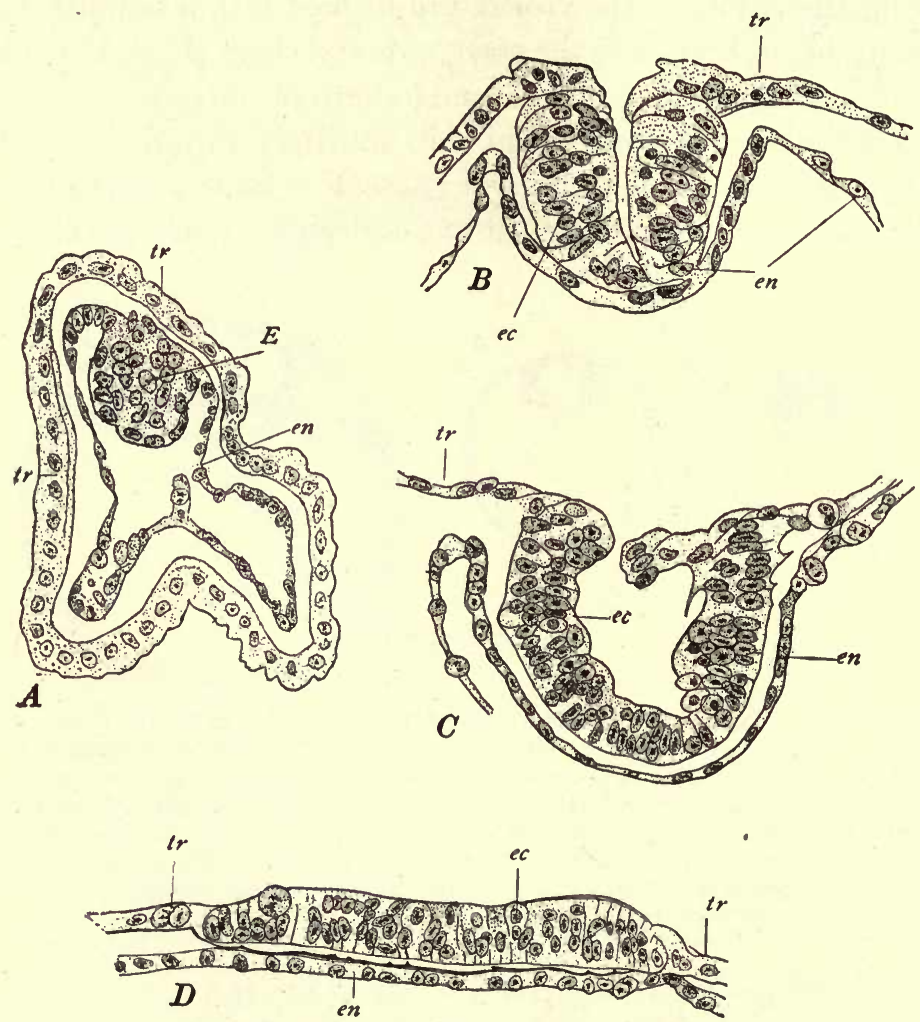

FIG. 151.- Sections through four stages in the early development of the Insectivor, Tupaija javanica. From Hubrecht. A. Blastodermic vesicle completely closed; endoderm still continuous with the embryonic ectoderm. $B, C$. Embryonic ectoderm split and folding out upon the surface of the vesicle, pushing away the trophoblast cells. D. Embryonic ectoderm forming a flat disc on the surface of the blastodermic vesicle. $E$. Inner cell mass ("ectodermal shield"); $e c$, embryonic ectoderm; en, endoderm; $t r$, trophoblast.

(subzonal layer), and (b) the establishment of an important cavity, the amnionic canity, which first appears about this time. There is a great deal of variation among the Mammals in the details of these relations, and we shall attempt to give only a very general statement of the more important conditions. 
We may make a preliminary distinction between those instances where the trophoblast is interrupted in a circumscribed area, just above the embryonic ectoderm, the embryonic disc then moving up and occupying this space, and thus acquiring a superficial position; and others where the trophoblast remains
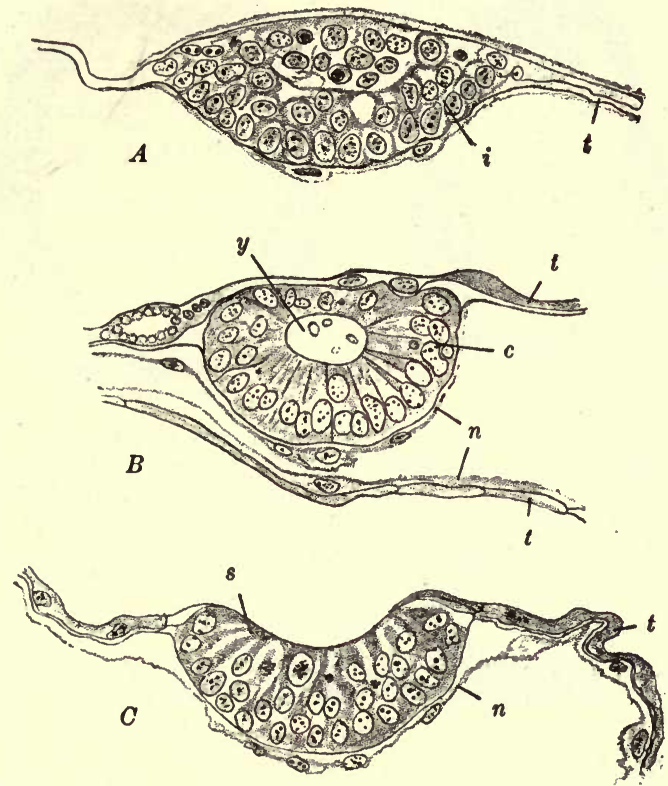

FIG. 152.-Three stages in the formation of the embryonic shield in the deer. After Keibel. A. Spaces appearing in the inner cell mass. $B$. Fully formed cavity (embryocyst) in the inner cell mass, which is still covered with a thin trophoblast. $C$. Embryocyst opened out upon the surface. $\quad c$, Ectoderm; $i$, inner cell mass; $n$, endoderm; $s$, embryonic shield; $t$, trophoblast; $y$, embryocyst.

continuous above the embryonic ectoderm, forming a complete enveloping layer around the endoderm and embryonic shield. The latter condition is known as the entypy of the germ.

Among the former instances we find again quite a variety of methods by which the embryonic layers acquire their superficial position. (1) In the Insectivor, Tupaija, the inner cell mass forms a cup-shaped structure which opens out upon the surface of the vesicle, pushing the trophoblast away (Fig. 151). (2) More commonly, as in most Ungulates, in Tarsius, and the 
opossum, the ectoderm moves up irregularly, gradually pushing away the trophoblast cells (Figs. 152, 153, 157). (3) In the rabbit, shrew, and probably in the dog, the trophoblast remains for a time continued as a very thin layer (Rauber's layer) over
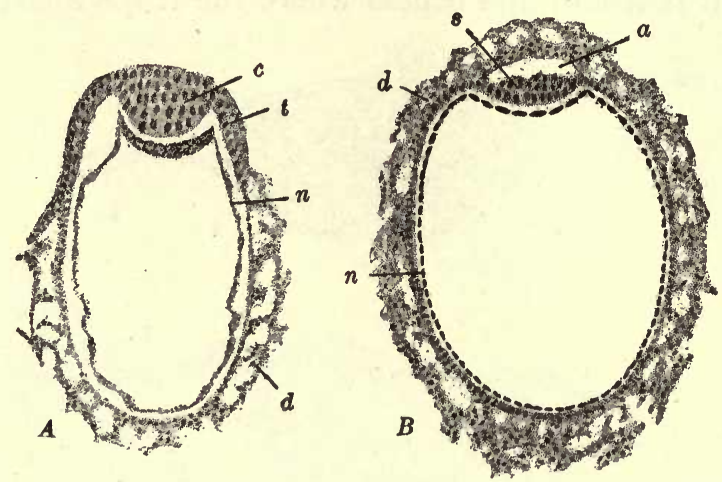

Fig. 153.-Transverse sections through the early blastodermic vesicle of the hedgehog, Erinaceus. After Hubrecht. A. Embryonic ectoderm forming a solid mass. Endoderm lining the entire trophoblast wall. Trophoblast becoming trophodermic. B. Amnionic cavity established. Trophoderm formed completely around the blastodermic vesicle. $a$, Amnionic cavity; $c$, ectoderm; $d$, trophoderm; $n$, endoderm; $s$, embryonic shield; $t$, trophoblast.
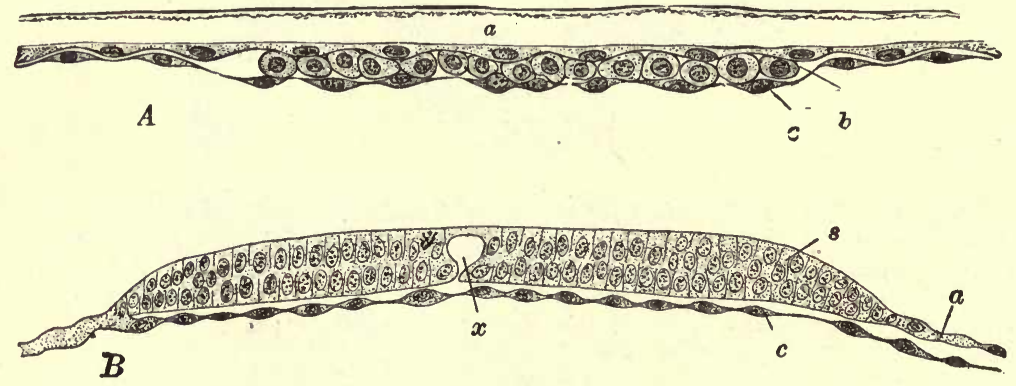

Fig. 154.-A. Section through part of the blastodermic vesicle of a six-day rabbit. From Quain's Anatomy, after Van Beneden. B. Transverse section through the embryonic shield of a dog of unknown age (between eleven and fifteen days). After Bonnet. $a$, Trophoblast (in $A$, Rauber's layer); $b$, embryonic ectoderm; $c$, endoderm; $s$, embryonic shield (embryonic ectoderm); $x$, space in embryonic shield.

the surface of the ectoderm of the embryonic region. Apparently the thin cells of the trophoblast finally disappear, leaving the ectoderm upon the surface, but it is possible that they mingle 
indistinguishably with the cells of the ectoderm.itself; in either case the trophoblast cells here disappear as such (Figs. 154, 157, $G, H)$.

In the second condition mentioned, where the trophoblast remains continuous above the embryonic layers (entypy), we find a still greater variety in the details of the relations, which may become very complicated. This general type of development is found in many Primates (probably in man), many Rodents including the mouse, rat, and guinea-pig, in the Chiroptera (Fig. 155), and in some Insectivora including the hedgehog (Erinaceus) Galeopithecus and Gymnura. In all these forms a space appears in the ectoderm, known as the amnionic cavity. This space may result from a definite splitting apart of two cell masses (e.g., hedgehog), or it may result from the gradual confluence of irregular spaces (e.g., bat, Fig. 155). It is important to bear in mind that in these instances of entypy, the true embryo develops only from the cells (ectoderm and endoderm) lying in the floor of the amnionic cavity.

Very often the trophoblast in this region becomes thickened, forming a trophoblastic knob ("träger"), which enlarges and pushes the embryonic shield down into the cavity of the blastodermic vesicle (rat and mouse, for example). An additional cavity, the false amnionic cuvity, may develop within this trophoblastic knob. This should not be confused with the true amnionic cavity, which here forms a completely closed vesicle, with ectodermal wall, entirely separate from the trophoblastic knob, and often of considerable size, even in these early stages (Fig. 156). A rather more special condition is found in the guinea-pig, where the small amnionic vesicle separates widely from the trophoblast, leaving a large space known as the interamnionic cavity between the true and the false amnionic cavities. The false amnionic cavity of the trophoblastic knob becomes very large (Fig. 156, B), while during these early stages the true amnionic cavity remains very small.

It is among these forms (e.g., rat, guinea-pig, etc.) that the phenomenon of the so-called "inversion of the germ layers" was described. It is now clear that no genuine inversion takes 

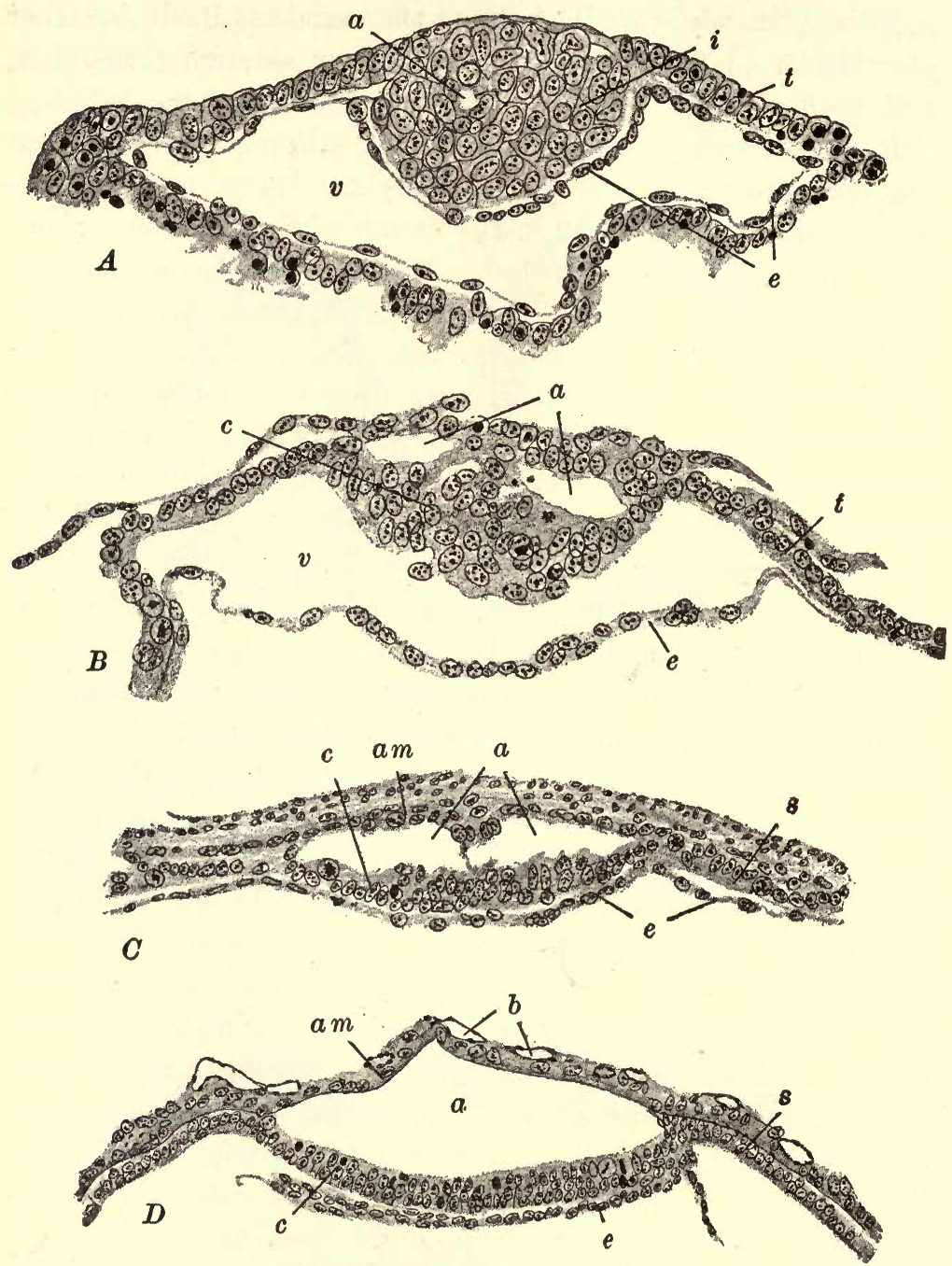

FIG. 155.- - Sections through the blastodermic vesicle, or its embryonic portion, of the bat, Vespertilio, showing the formation of the amnionic cavity. After Van Beneden (Brachet). A. Section passing through the inner cell mass showing the very beginning of the amnionic cavity. $B$. Amnionic cavity clearly indicated as irregular spaces. The endoderm is torn away from its normal position under the embryonic ectoderm. $C$. Thickened trophoblast now a syncytiotrophoblast. $D$. Amnionic cavity fully established. The endoderm is torn away from the wall of the vesicle at its ends. $a$, Amnionic cavity; $a m$, amnion; $b$, capillaries of the uterine wall; $c$, embryonic ectoderm; $e$, endoderm; $i$, inner cell mass; $s$, syncy tiotrophoblast; $t$, trophoblast; $v$, cavity of blastodermic vesicle. 
place, but the basis of the phrase is clear. The enlargement of the trophoblastic knob with its false amnionic cavity, pushes the embryonic ectoderm cells far down within the cavity of the blastodermic vesicle; this is especially marked in the guineapig. Then as the endoderm cells spread out over the inner surface of the embryonic ectoderm (Fig. 156) they may extend
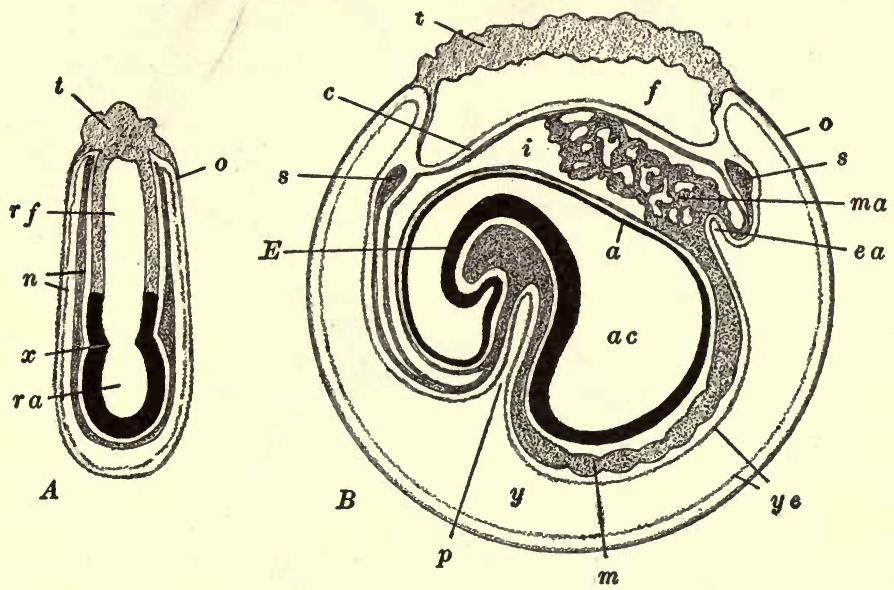

Fig. 156.-Diagrams of the relations of the cavities and layers in the rat, showing the "inversion" of the germ layers. After Selenka. Median sagittal sections. Embryo and amnion, black; ectodermal knob or "träger" in light tone; endoderm and mesoderm in darker tone. $A$. Early stage before the formation of the false amnionic cavity. $B$. Late stage showing false and true amnionic cavities and the interamnionic cavity. $a$, Amnion; $a c$, true amnionic cavity; $c$, chorion; $E$, embryo (anterior end); $e a$, endodermal rudiment of allantois; $f$, false amnionic cavity; $i$, interamnionic cavity; $m$, mesoderm; $m a$, mesoderm of allantois; $n$, endoderm; $o$, trophoblast (ectoderm); $p$, anterior intestinal portal; $r a$, rudiment of true amnionic cavity; $r f$, rudiment of false amnionic cavity; $s$, marginal sinus; $t$, "träger" (ectoderm); $y$, yolk-sac; ye, yolksac endoderm; $x$, amnionic folds.

over the walls of both the true and the false amnionic cavities, so that the embryonic ectoderm appears to lie within an endodermal sac (Fig. 156, A). When the embryo itself begins to be differentiated in the floor of the true amnionic cavity it is thus already surrounded by this endodermal layer, and the appearance of an inversion of the layers is produced. Consideration of the entire series of conditions mentioned above demonstrates the absence of a true inversion of the germ layers. 

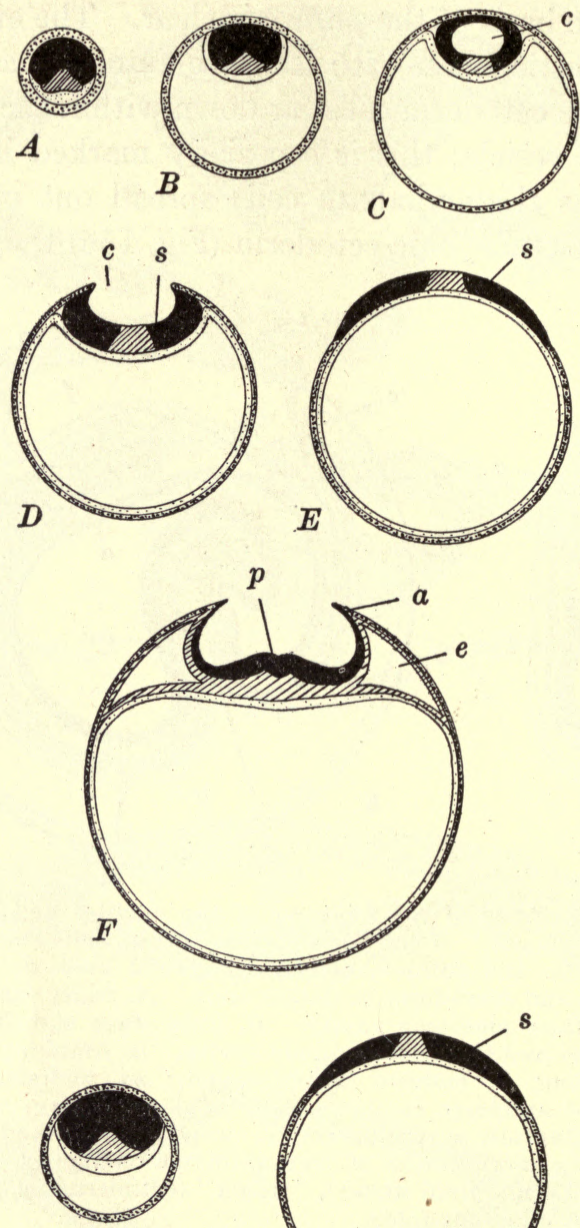

$\boldsymbol{G}$

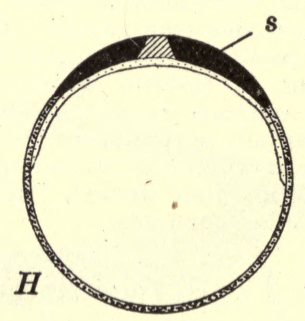

FIg. 157.-Diagrams of the formation of the amnion. After Keibel. $A-F$, in Ruminants, pig, and Mammals, with entypy of the germ. $G, H$, in the Carnivora. Black, embryonic ectoderm; heavy stipples, trophoblast; light stipples, endoderm; oblique shading, mesoderm. A. Morula. B. Early blastodermic vesicle; endoderm limited to embryonic region. $C$. Formation of cavity in the embryonic ectoderm (embryocyst). Extension of the endoderm. D. Embryocyst opening out; embryonic disc becoming superficial. Endoderm completely lining the vesicle. $E$. Embryonic dise or shield superficial in position (compare $H$ ). $\quad F$. Formation of amnionic folds, primitive streak, and exocœlom. G. Early carnivor blastodermic vesicle. $H$. Embryonic shield superficial in position (compare Fig. $154, B$.) A later stage would be identical with $F$. $a$, Amnionic folds; $c$. embryocyst; $e$, exocœlom; $p$, primitive streak; $s$, embryonic shield. 
An interesting condition, transitional between the two general classes described above, is found in the mole. Here spaces form between the ectoderm and the continuous trophoblast, which flow together as in the bat, forming a definite amnionic cavity. This cavity is then obliterated by the refusion of the ectoderm and trophoblast, resulting in a condition similar to that of the shrew and rabbit. Then the superficial cells of the trophoblast disappear, leaving the ectoderm on the surface of the blastodermic vesicle.

The formation of the amnionic cavity has not been mentioned in forms where the embryonic disc becomes superficial in position. In these instances the amnionic cavity is formed in a manner entirely different from that described above. Here a system of amnionic folds, much like those of the chick, appears just outside the embryonic region proper. These folds grow up over the embryo, establishing an amnionic cavity in which the embryo is enclosed (Figs. 157, 176). The history of the amnion will be taken up later, in connection with the other embryonic membranes.

We may consider now the processes leading to the formation of the definitive embryo. To give a comparative account of these processes as they occur in the whole group of Mammals would carry us far into details, as there is wide variation even within this single class, and we shall, therefore, describe these phenomena as they are found in such a form as the rabbit or dog, forms whose early history is comparatively well known.

Of the entire blastodermic vesicle, with its complicated. associated structures, the only truly embryonic portion is found in a restricted portion of the embryonic ectoderm and endoderm known as the embryonic shield (Figs. 151-155, 157, $158, A$ ). (We should note that in the rabbit the endoderm does not come to line the entire blastodermic vesicle until a relatively late stage, so that throughout early development only the upper portion of the vesicle is two-layered.) In the rabbit the embryonic shield first becomes visible about the fifth day, when the blastodermic vesicle is still spherical and only about $1.5 \mathrm{~mm}$. in diameter. By the seventh day the 

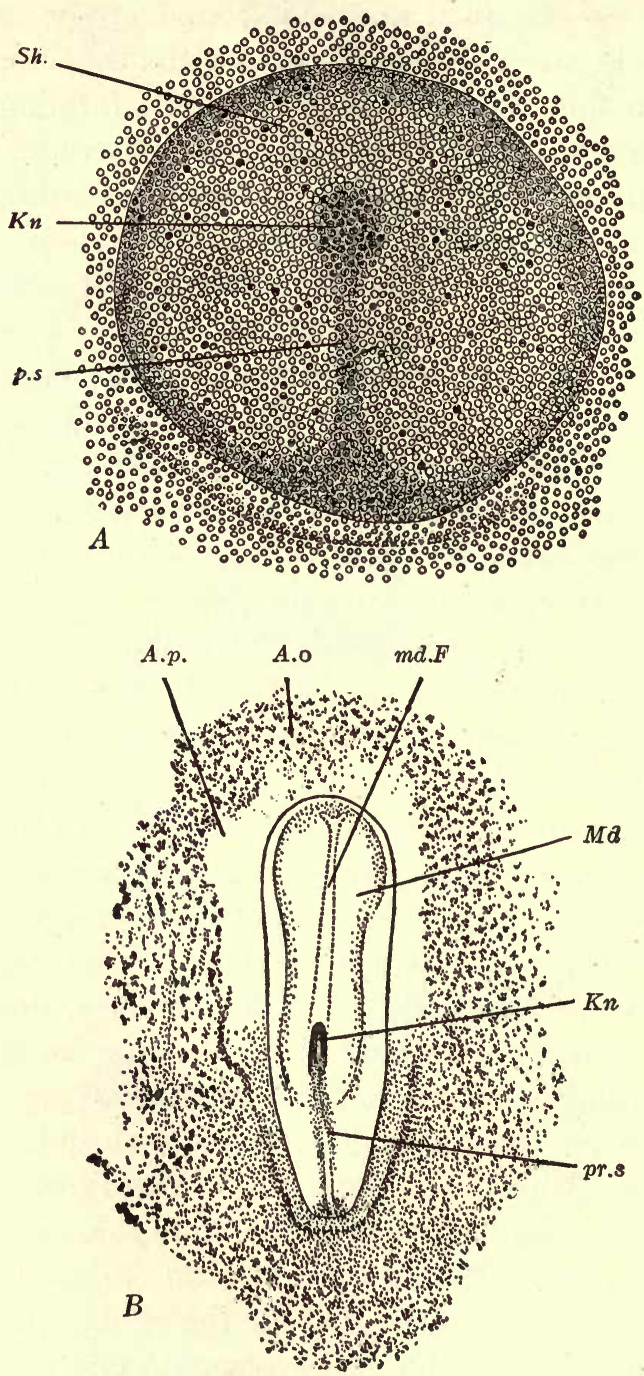

FIG. 158.-A. Surface view of embryonic shield of a dog of thirteen to fifteen days. B. Surface view of embryonic shield of dog showing medullary plate, etc. From Minot (Laboratory Text-Book of Embryology), A, after Bonnet. $A . o$. Area opaca; A.p., area pellucida; $K n$., Hensen's knot or node; $M d$., medullary plate; md.F., medullary furrow; $p s ., p r . s .$, primitive streak; Sh., embryonic shield. 
embryonic shield forms a well-marked oval thickening about $1.5 \times 1.0 \mathrm{~mm}$., the entire vesicle at this time measuring about $4.5 \times 3.5 \mathrm{~mm}$.

Sections through the embryonic shield show that it is formed largely by a circumscribed thickening of the embryonic ectoderm, three or four cells deep, beneath which the endoderm remains only one cell in thickness (Fig. 154). Over the surface of the shield the original trophoblast cells (Rauber's layer) are no longer distinguishable. Peripherally the shield passes into the thinner extra-embryonic ectoderm, or trophoblast of these forms.

The next phase of development is indicated (rabbit, about the end of the seventh day, dog, about thirteen to fifteen days) by the appearance of a slight opacity toward the middle of the embryonic shield. This is known as the primitive knot or Hensen's node. Usually the primitive knot is eccentric in position, toward what proves to be the anterior margin of the shield. At about this same time, or even before Hensen's node is distinctly visible, an opaque line appears across the shield, extending from the node to the posterior margin of the shield, where it joins an opaque crescent-shaped region (Fig. 158, A). This line is the primitive streak, and usually, along its middle can be seen the clearer primitive groove (Figs. 157, F; 159). A little later a less distinct opaque line may be seen extending a short distance forward from Hensen's node; this is the head process.

These superficial appearances in the embryonic shield may be understood only by the examination of sections. Sections across the primitive streak of the rabbit or mole, for instance, show that the opacity of the region is due to a very marked thickening of the ectoderm, with which is associated the formation of the middle layer or mesoderm. A section like that illustrated in Fig. 159, A, shows the primitive streak to be a region of rapid cell proliferation in the ectoderm. From the sides and inner surface of the primitive streak cells are given off which gradually take on the arrangement of a definite layer quite distinct from the ectoderm, and between the ectoderm and endoderm. This is the mesoderm which here, therefore, 
as in the chick, is in its origin more closely associated with the ectoderm than with endoderm. Sections through Hensen's node show that this is a region of thickened ectoderm and mesoderm, less clearly differentiated from one another than in the primitive streak region. The endoderm, however, is not fused with the other cells, as it is in the chick. And in front

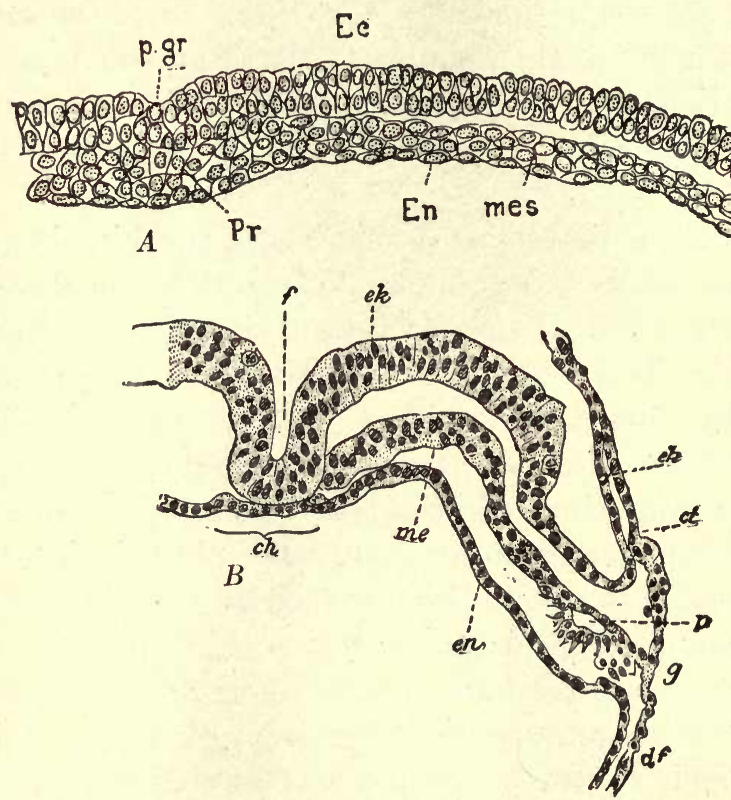

FIG. 159.-A. Transverse section through the primitive streak of the mole. $B$. Transverse section through a human embryo of $1.54 \mathrm{~mm}$. (Graf von Spee's Embryo Gle.) From Minot (Laboratory Text-book of Embryology, after Heape $(A)$, and Graf von Spee $(B)$. ch, Notochord; $c t$, somatic mesoderm; $d f$, splanchnic mesoderm; $E c, e k$, ectoderm; $e n, E n$, endoderm; $f$, dorsal furrow; $g$, junction of extra-embryonic somatic and splanchnic mesoderm; me, mes, mesoderm; $p$, rudiment of embryonic cœlom; $p . g r$., primitive groove; $P r$, primitive streak.

of Hensen's node, in the region of the head process, mesoderm is present, though entirely separate from the ectoderm, indicating its origin from the region of Hensen's node and not directly from the ectoderm of the head process region.

Later on conditions are found which make possible very close comparison between the primitive streak and associated structures of the Mammal with those of the chick. Within the head process, or in the region of Hensen's node, a rearrangement of 
cells produces a small cavity known as the notochordal canal. This varies greatly in extent. In such forms as the guinea-pig and bat, the canal is comparatively long, extending from Hensen's node, where it opens upon the surface by a definite per-
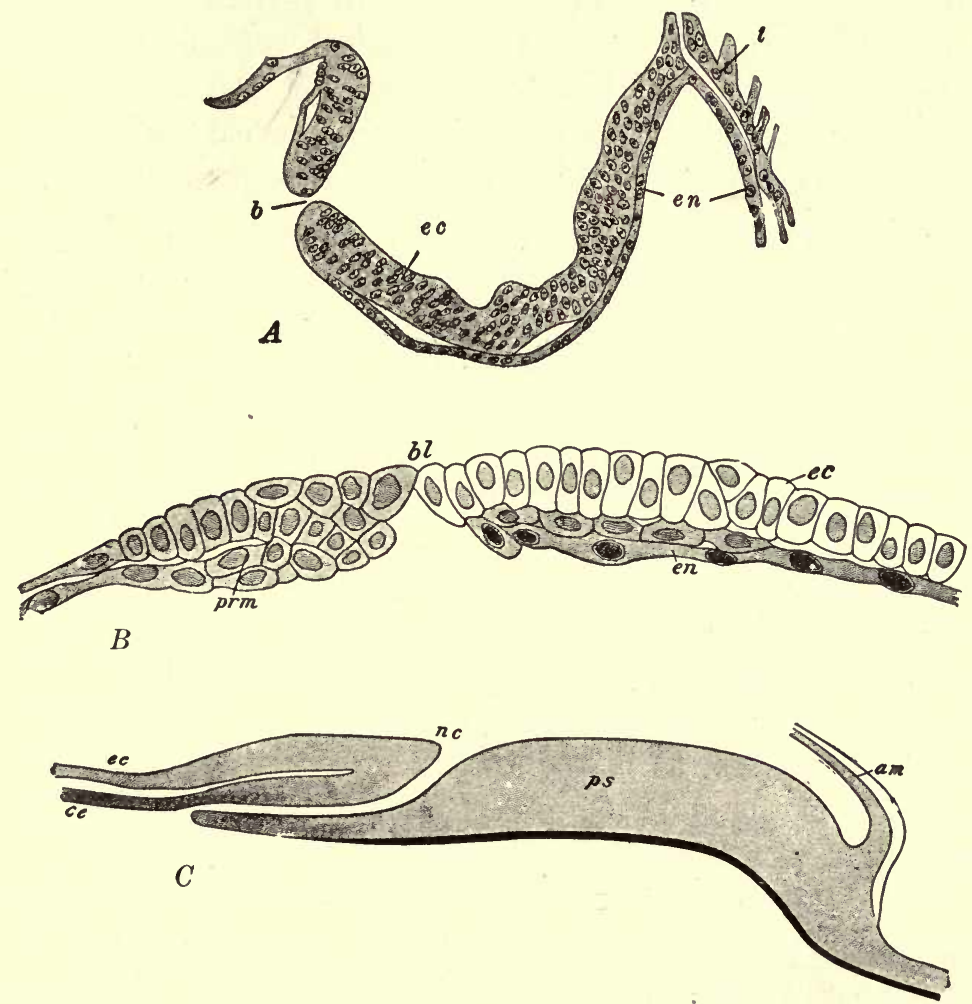

FIg. 160.-A. Sagittal section through the embryonic shield of the hedgehog, showing the transitory blastopore. After Hubrecht. B. Posterior part of a sagittal section through the embryonic disc of the mole. $C$. Diagram of a sagittal section through the embryonic disc of the mole. From McMurrich (Development of the Human Body), after Heape. am, Amnion; bl, blastopore; $c e$, chorda endoderm; $e c$, ectoderm; $e n$, endoderm; $n c$, neurenteric canal; prm, prostomial mesoderm; $p s$, primitive streak; $t$, trophoderm.

foration through the ectoderm, anteriorly and ventrally; finally either opening through the endoderm into the cavity of the endodermal vesicle (Fig. 160, C), or simply ending blindly 
among the cells of the head process. In other forms, such as the sheep, pig, or hedgehog, and in man, the notochordal canal is simply a vertical perforation through the embryonic layers, connecting the cavity of the endodermal vesicle directly with the outside (amnionic cavity) (Figs. 160, $A ; 161, D$ ). And in still other forms the canal may never quite perforate the layers of the shield (mole); or it may be reduced to a simple groove on the lower surface of the shield (rabbit, Fig. 160, B).

Sections through the notochordal canal reveal an arrangement of the germ layers which is practically that found around the more typical blastopore of the yolk-filled egg. In this region the layers are continuous with one another and the primitive groove may be deepened into a sort of "primitive pit" (compare chick). Altogether then we may recognize in the primitive streak of the Mammal the essential equivalent of the similarly named structure in the Sauropsida. The notochordal canal therefore becomes the modified equivalent of the blastoporal remains.

When the notochordal canal has the form of a simple perforation it may therefore correctly be termed the neurenteric canal, marking the posterior limit of the embryonic rudiment. The primitive streak, representing the modified and fused blastoporal lips, is here, as in the chick, a region from which the structures of the embryo are derived and differentiated in the antero-posterior direction, again much as in the chick.

All details regarding the formation of the embryonic layers and organs are to be omitted from the present account. It must suffice to say. that a typical medullary plate is formed, medullary folds appear and fuse, forming a neural tube (Figs. $158, B ; 159, B)$. A typical notochord is differentiated, running forward from the primitive knot. The mesodermal layer rapidly extends laterally from the axial region, finally passing widely beyond the embryonic region and taking a very important part in the development of the extra-embryonic structures. The mesodermal sheet is very early split into somatic and splanchnic layers by the appearance of a cœlomic cavity, both embryonic and extra-embryonic. 


\section{THE DEVELOPMENT OF THE EXTERNAL FORM OF THE HUMAN EMBRYO}

The earliest phases in the development of the human organism, up to the formation of the primitive streak, are not known at all, and it is not until the medullary plate stage is reached that the structure of the human embryo is fully known. Scarcely half a dozen embryos younger than this have been described. It is clear, however, that the processes leading to the formation of the embryonic layers, the amnionic cavity, etc., are in general similar to those found among the rat, mouse and bat. That is, the amnionic cavity is formed by delamination, between the continuous trophoblast and the embryonic ectoderm. The endodermal vesicle is much smaller than the ectodermal trophoblastic vesicle, leaving a wide space between the two layers (Fig. 161). When the mesoderm forms it extends rapidly through the extra-embryonic region of the blastodermic vesicle, one layer (somatic) applied to the inner surface of the trophoblast, the other (splanchnic) applied to the outer surface of the endodermal vesicle, so that the large cavity of the blastodermic vesicle becomes, in effect, an extra-embryonic colomic space. The layer of endoderm with the splanchnic mesoderm is at first the yolk-sac, although of course entirely yolkless, while the ectodermal trophoblast with the somatic mesoderm forms the chorion. (Further reference to the chorion, as well as to the amnion and the allantois, is deferred until a later section.)

The reader should note that students of mammalian development use the term "ovum" to designate any early stage in development. In this connection the "ovum" includes not only the embryo proper, but also all of the associated structures of the blastodermic vesicle.

As the embryo enlarges, its posterior end remains attached to the inner surface of the trophoblastic wall by a mass of mesoderm cells. This attachment is known as the body stalk or belly stalk. Sections through an early embryo would give, therefore, the appearance shown in Figs. $161, D ; 179, A, B$. 

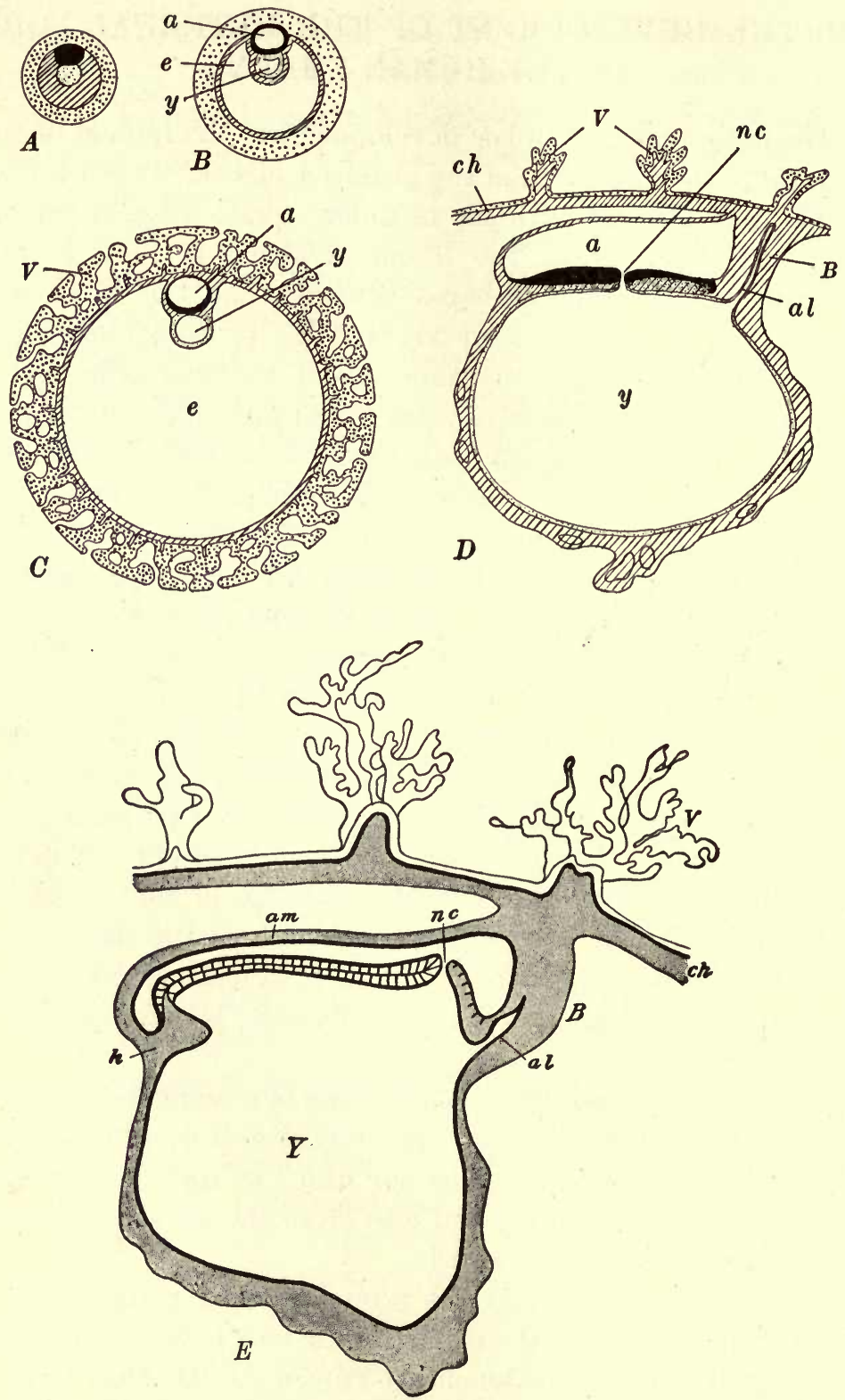

FIG. 161.-Diagrams of sagittal sections through the human blastodermic vesicle, showing the formation of the amnion and trophoderm. $A-D$, after Keibel and Elze. E, From McMurrich (Development of the Human Body), 
A slightly later stage is shown in sagittal section in Fig. 161, $E$. At this time the embryo (Graf Spee's embryo Gle) measures $1.54 \mathrm{~mm}$. in length by about $0.7 \mathrm{~mm}$. in greatest breadth, while the entire vesicle ("ovum") is approximately $10 \mathrm{~mm}$. in diameter. Its dorsal surface is occupied by the large neural plate, with a distinct neural groove. Toward the posterior end
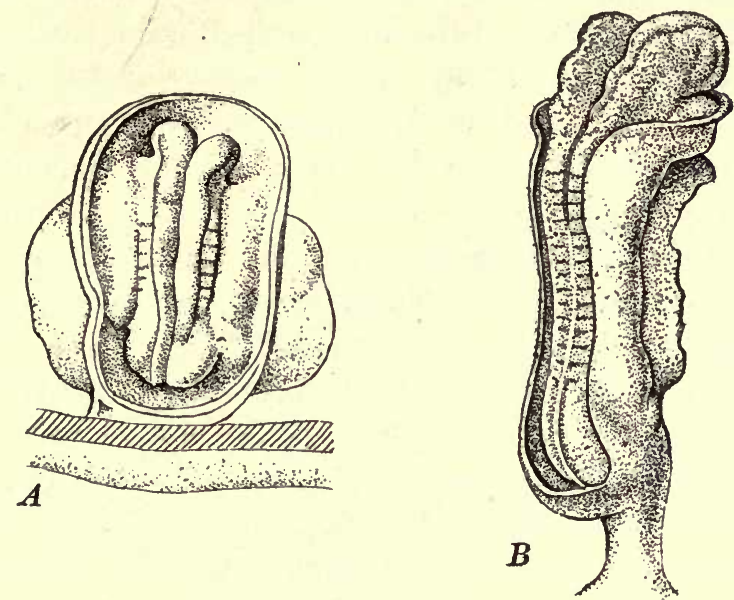

Fig. 162.-Young human embryos. After Keibel and Elze. $A$. Keibel and Elze's Embryo Klb, $\times 25$. B. Kollmann's Embryo Bulle, $\times 20$. For description, see text.

is the small neurenteric canal, behind which may be seen a short primitive streak and groove. A short thick body stalk attaches the embryo to the chorionic wall of the blastodermic vesicle. The wall of the ovoid yolk-sac, whose diameters are about equal to the length of the whole embryo, is already quite

after Graf von Spee. In all the figures the anterior end is toward the left Black, embryonic ectoderm; heavy stipples, trophoblast and trophoderm; light stipples, endoderm; oblique ruling, mesoderm. $A$. Hypothetical early stage; mesoderm a solid mass. $B$. Amnionic cavity and wide exocœlom established; endoderm limited to a small vesicle beneath the embryonic ectoderm. The exocœlom in reality contains scattered mesenchyme cells. C. Blastodermic vesicle enlarged and covered with trophodermic villi, into which the mesoderm is extending. Endodermic vesicle (yolk-sac) very small (stage of Peter's ovum). $D$. Embryonic portion only, of an older vesicle showing the neurenteric canal, primitive streak (in the plane of the section), and body stalk. The mesoderm of the yolk-sac is becoming vascular. $E$. Sagittal section through the human embryo of $1.54 \mathrm{~mm}$. (Graf von Spee's embryo Gle). a, Amnionic cavity; al, allantois; $a m$, amnion; $B$, body stalk; $c h$, chorion; $e$, exocœlom; $h$, heart region; $n c$, neurenteric canal; $V$, chorionic villi; $Y$, yolk-sac. 
vascular. The notochord is distinctly differentiated posteriorly, and the embryonic mesoderm is only incompletely separated into somatic and splanchnic layers (Fig. 159, B), although in the extra-embryonic region there is a very wide cœlom.

A sagittal section through this embryo shows that it is somewhat arched over the dorsal surface of the yolk-sac, and that an endodermal outgrowth, the rudiment of the allantois, is extending into the mesoderm of the body stalk (Fig. 161, $E$ ).

Shortly after this, in an embryo measuring $1.8 \times 0.9 \mathrm{~mm}$. (Keibel and Elze's embryo Klb.) the neural folds become very prominently elevated and the head and tail regions project slightly above the surface of the yolk-sac, as shown in Fig. 162, A. This figure shows also the persistent neurenteric canal, and the very short primitive streak. Five or six pairs of mesodermal somites are now present.

The head region now commences to enlarge rapidly although the neural groove is still open. In an embryo of $2.36 \mathrm{~mm}$. length (Kollmann's embryo, Bulle) illustrated in Fig. 162, B, the body is concavely arched toward the yolk-sac, while the head and tail regions show distinct downward flexures. The elementary divisions of the brain are already indicated, and the fore-brain is protecting downward from the anterior end of the neural axis. Though not shown in the figure the paired rudiment of the heart is present. About fifteen pairs of somites are visible externally.

As the embryo now begins to elongate rapidly it becomes clearly folded off from the extra-embryonic structures, and the opening of the yolk-sac out of the endodermal gut cavity of the embryo becomes relatively, though not actually, narrower. The yolk-sac thus appears to be attached to the embryo proper by a narrow stalk, the yolk stalk, the connection of which, with the embryo, is the yolk stalk umbilicus.

By the time the embryo reaches a length of $2.5 \mathrm{~mm}$. (Kollmann's embryo, $2.5 \mathrm{~mm}$., age given as thirteen to fourteen days, but probably much older) the high neural folds have begun to close together posteriorly (Fig. 163). The head region is considerably enlarged and extends downward in front of the heart, 
which is now very large and clearly differentiated into regions by the development of flexures.

The length of the embryo now affords a very unsatisfactory index of the age or degree of development on account of the considerable variability, and because of the bendings which appear in the longitudinal axis. Apparently, shortly after this time, the body becomes sharply bent downward into a U-form just opposite the umbilicus, producing what has been called the dorsal flexure. That this is entirely normal is, however, still open to question.

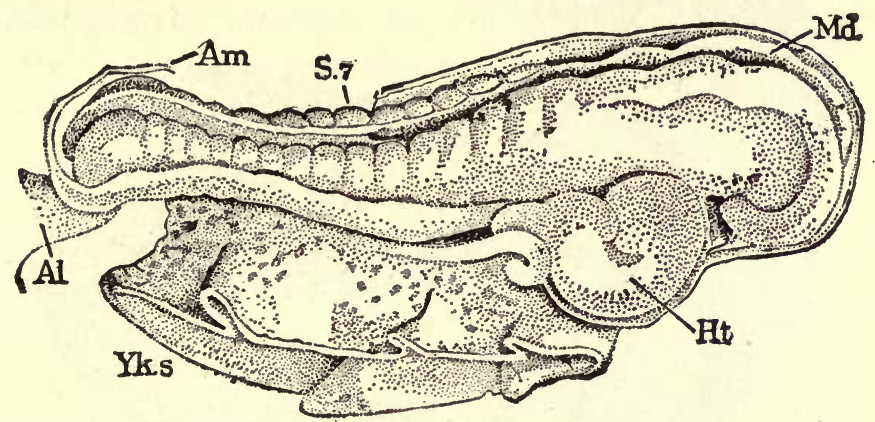

Fig. 163.-Human embryo of thirteen or fourteen days. From Minot (Laboratory Text-book of Embryology), after Kollmann. Al, Body stalk; $A m$, amnion; $H t$, heart; $M d$, medullary groove; $S_{7}$, seventh somite; $Y k s$, yolksac.

Figure 164 illustrates an embryo, enclosed in the amnion and with yolk-sac attached, whose length, in a straight line, is $2.6 \mathrm{~mm}$. and whose age was originally estimated at eighteen to twenty-one days, though very probably it was approximately one month (His's embryo, $M$ ). The entire blastodermic vesicle or chorionic vesicle ("ovum") still measures approximately $10 \mathrm{~mm}$. in diameter. This embryo shows many important advances. No trace of the dorsal flexure remains, while both the anterior and posterior extremities of the embryo are now bent downward and inward. The body is also slightly twisted so that the head lies toward the left, the tail toward the right (the direction of this twisting is not fixed, for in other embryos it may be in the opposite direction). The yolk-sac is some- 
what shrunken and elongated, and the yolk stalk is clearly distinguishable. Topographically the most anterior part of the embryo is formed by the mid-brain, beneath which the forebrain is now folded back toward the heart. The heart is very prominent and on the sides of the neck region three pairs of gill clefts are indicated, decreasing in size posteriorly. Four pairs of visceral arches (mandibular, hyoid, two branchial) are thus marked out, and the most anterior (mandibular) already

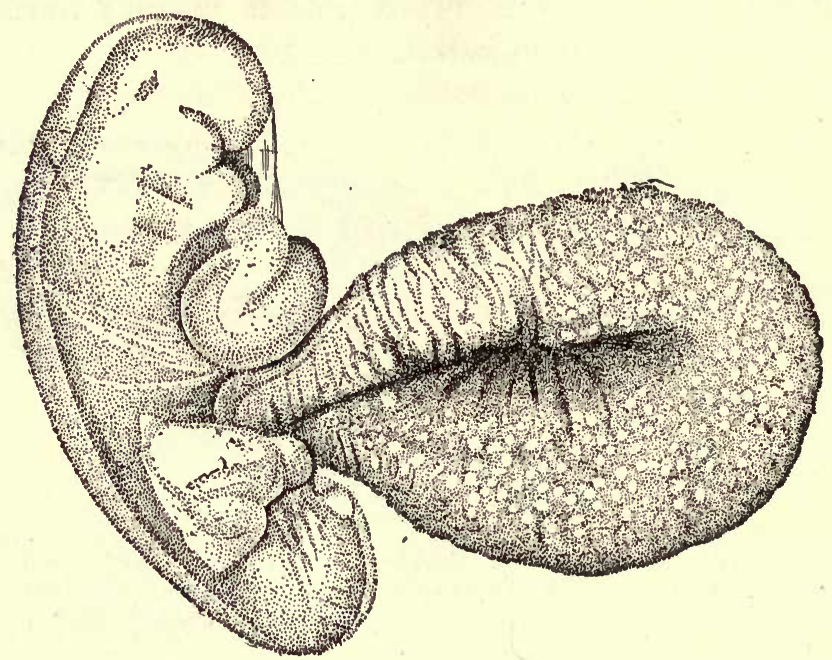

Fig. 164.-Human embryo of $2.6 \mathrm{~mm}$. From Minot (Laboratory Text-book of Embryology), after His. The embryo is enclosed in the amnion and shows the maxillary and mandibular processes, the rudiments of three gill clefts, and the large heart. The large yolk-sac extends ventrally, while posterior to its origin the root of the body stalk is shown turned dorsally.

shows signs of its transverse division into upper and lower portions, the maxillary and mandibular processes. Later a fourth cleft and fifth arch are indicated. Of course in the Mammal actual gill clefts are not present as perforations; the so-called clefts are vestigial structures and, excepting the first, merely form superficial grooves, opposite corresponding pockets out of the pharyngea! cavity. An anterior depression, the oral sinus (stomodæum) between the mandibular arches, marks the position of the future mouth, which is perforated very shortly after this time. 
Just a few words may be added concerning the internal structure of this embryo (Fig. 165). The slender notochord extends the entire length of the body and tail. Optic vesicles are distinct and the otocysts are entirely closed off below the surface. The expanded pharynx shows an anterior hypophysial outgrowth, in addition to the lateral branchial pockets. A small rudiment of the lung is indicated, and at the posterior end of the narrowed œsophagus the liver rudiment is well marked. Posterior to this the gut is open into the yolk-sac by way of the yolk stalk, and continuing posteriorly from this is the narrow intestine, which, near its extremity, sends a small allantoic outgrowth into the body stalk. Rudiments of the mesonephros (Wolffian body) and its duct are slightly indicated.

The vascular system is very well developed. Opening into the posterior end of the heart there are, the paired ductûs Cuvieri, formed by anterior and posterior cardinal veins, the paired vitelline or omphalo-

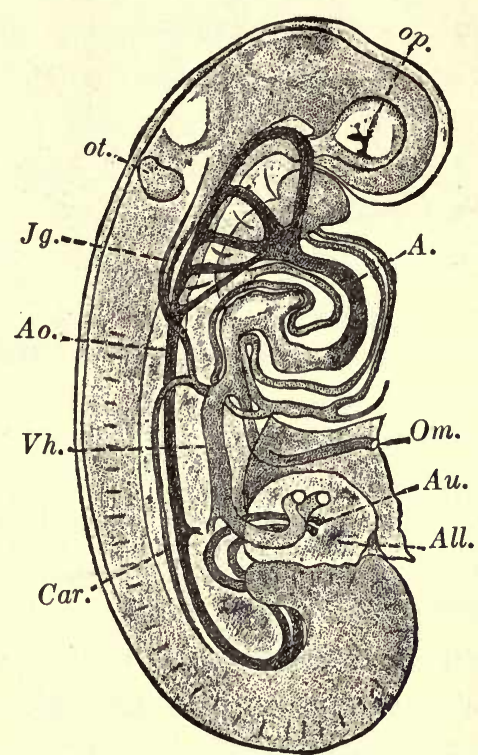

FIG. 165.-Reconstruction of a human embryo of $2.6 \mathrm{~mm}$. (See Fig. 164). From Minot (Laboratory Textbook of Embryology), after His. $A$, Aortic limb of heart. All, body stalk; $A o$, dorsal aorta; $A u$, umbilical arteries; $C a r$, posterior cardinal vein; $J g$, anterior cardinal vein (jugular vein). $\mathrm{Om}$, omphalomesenteric vein; $o p$, optic vesicle; ot, otocyst; $V h$, right umbilical vein.

mesenteric veins, coming from the yolk-sac by way of the yolk stalk, and the paired allantoic or umbilical veins coming from the allantoie region by way of the body stalk. Opening out of the anterior end of the heart is the ventral aorta, which immediately divides into right and left halves from each of which arise five aortic arches passing through the visceral arches to the dorsal side of the pharynx, 
where they reunite into the dorsal aorta. A small anterior carotid artery appears a little later. From the dorsal aorta is

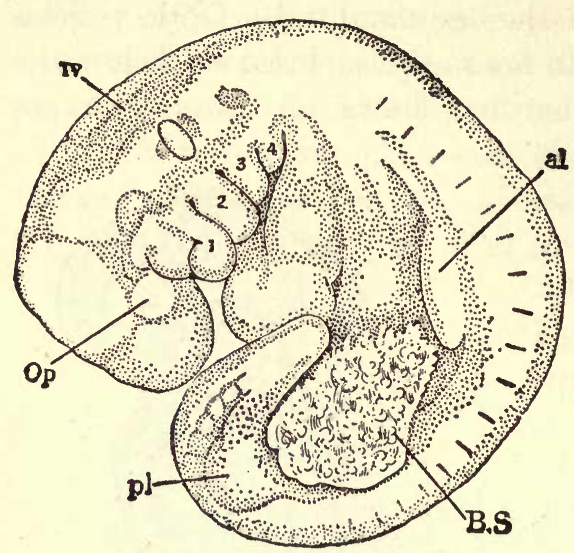

Fig. 166.-Human embryo of about twenty-three days $(4.0 \mathrm{~mm}$.). From Minot (Laboratory Text-book of Embryology), after His (Embryo $\alpha$ ). al, Fore-limb bud; $B S$, body stalk; $O p$, optic vesicle; $p l$, hindlimb bud; $I V$, fourth ventricle of brain; 1 , mandibular process; 2 , byoid arch; 3,4 , third and fourth visceral arches.

tail are almost brought in contact, and the entire embryo is almost circular in general outline (Fig. 166). The foreand hind-limb buds have appeared as low, extended elevations, and the abdominal region is becoming prominent on account of the growth of the liver and other viscera. The ventral body wall now becomes more completely closed together, and the tissues (somatopleural) at the base of the body stalk grow forward enclosing the root of the yolk stalk for a short distance, so that a given off a pair of small vitelline arteries supplying the yolk-sac, while posteriorly the dorsal aorta divides into a pair of umbilical or allantoic arteries, passing through the body stalk and supplying the allantois.

As the head now enlarges rapidly a wellmarked flexure (cervical flexure) appears just back of the gill cleft region, while through the entire body and tail a strongly marked curvature appears, so that head and

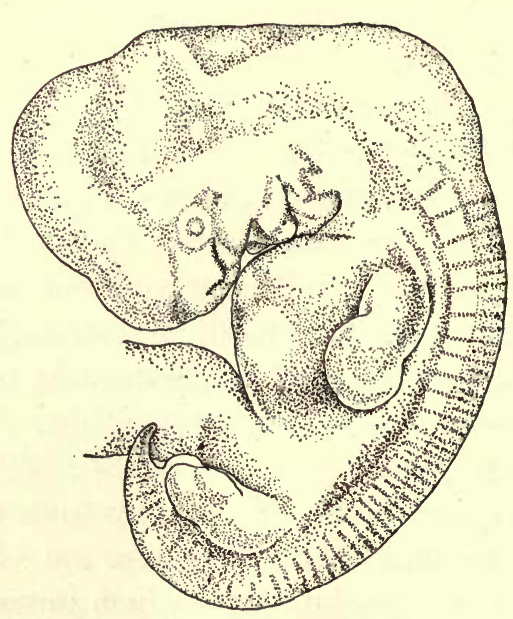

Fig. 167.-Human embryo of about 9.3 $\mathrm{mm}$. After Hochstetter, $\times 62 / 3$. For description see text. 
single stalk from the embryo now carries both the yolk stalk and the allantoic stalk. This is known as the umbilical stalk or cord, and its attachment to the embryo is the umbilicus. Ultimately the entire yolk stalk becomes enclosed in the umbilical cord, and the yolk-sac itself is surrounded by the tissues of the placenta as described below.

Up to this time the correlation between age and size of the embryo is very uncertain. According to the careful studies of Mall the ages of most of the early embryos have been underestimated, and it is very probable that the events thus far described have occupied about the first month of development. From this time on, however, the age of the human embryo is more certainly determined.

During the sixth week of development the embryo measures 9.0-10.0 $\mathrm{mm}$. in a straight line drawn from the apex of the mid-brain to the sacral flexure or rump ("crown-rump" length) (Fig. 167). The head, still the largest part of the embryo, is beginning to be elevated on account of the straightening out of the cervical flexure, and the whole body shows considerably less curvature than before. A lense has been formed opposite the small optic vesicle, and a pair of wellmarked olfactory pits has appeared on the under side of the head, in front of the maxillary processes. Both maxillary and mandibular processes are more prominent, while the posterior visceral arches and clefts have become sunk in a depression, the margins of which have nearly closed together forming a cavity below the surface known as the cervical sinus. The most anterior gill cleft (hyomandibular) is not included in this cervical sinus, but in part remains on the surface of the neck, as the rudiment of the external auditory meatus. The cervical sinus later disappears entirely, along with the posterior gill clefts.

The ventral body region is still protuberant, and anteriorly three elevations can often be observed, marking the underlying auricle, ventricle, and liver. The limbs are somewhat elongated, and the fore-limbs, which are always in a more advanced stage than the hind-limbs, show some indications of differentiation of the hand. The umbilical cord is elongating 
and the tail has reached nearly to the condition of its greatest devtiopment.

The further development of the general bodily topography may be sketched very briefly with the aid of the accompanying figures (Figs. 168, 169, 170). During the latter part of the second month (Fig. 169) the head continues to be elevated rapidly, and the body to straighten. The head is now at its

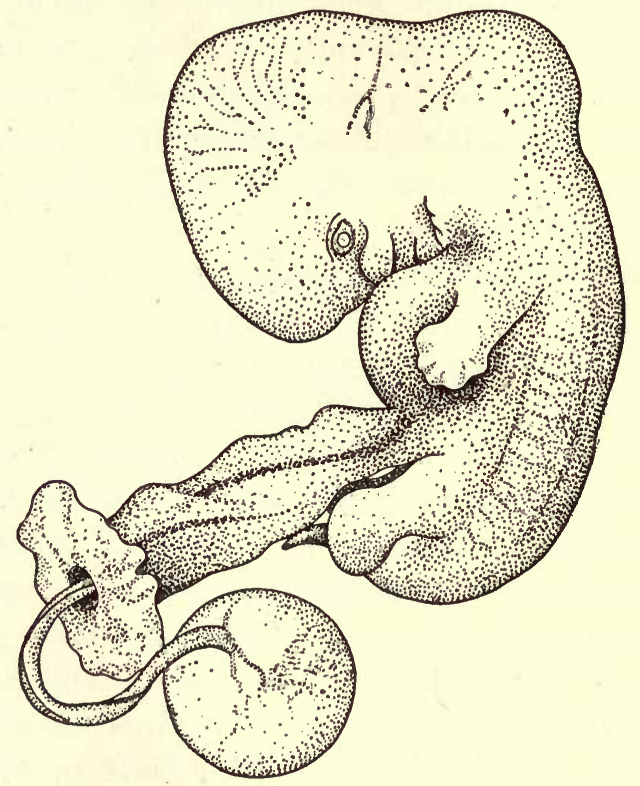

Fig. 168.-Human embryo of $14.5 \mathrm{~mm}$. (thirty-six days) showing thick umbilical cord, and yolk-sac at the end of the slender yolk stalk. After Minot. $\times$ 4.3. For description see text.

greatest relative size, constituting about 45 per cent. of the total weight of the embryo (Jackson). The pinna of the ear is formed from elevations of the first and second visceral arches around the external auditory meatus. The rudiments of the eye are fully established and the eyelids are formed. The ventral body wall still remains protuberant. The proximal end of the umbilical cord becomes considerably expanded, and into its extra-embryonic cœlomic cavity extend several coils of the embryonic intestine and even a portion of the liver. This characteristic extension of the intestine (intestinal hernia) 
reaches its maximum during the second month. The intestine is rapidly withdrawn later and at about nine or ten weeks is completely retracted into the embryonic body cavity. Beyond this expanded region the umbilical cord is considerably elongated and begins to show its characteristic spiral twisting. The yolk stalk is correspondingly elongated and now loses its endodermal cavity.

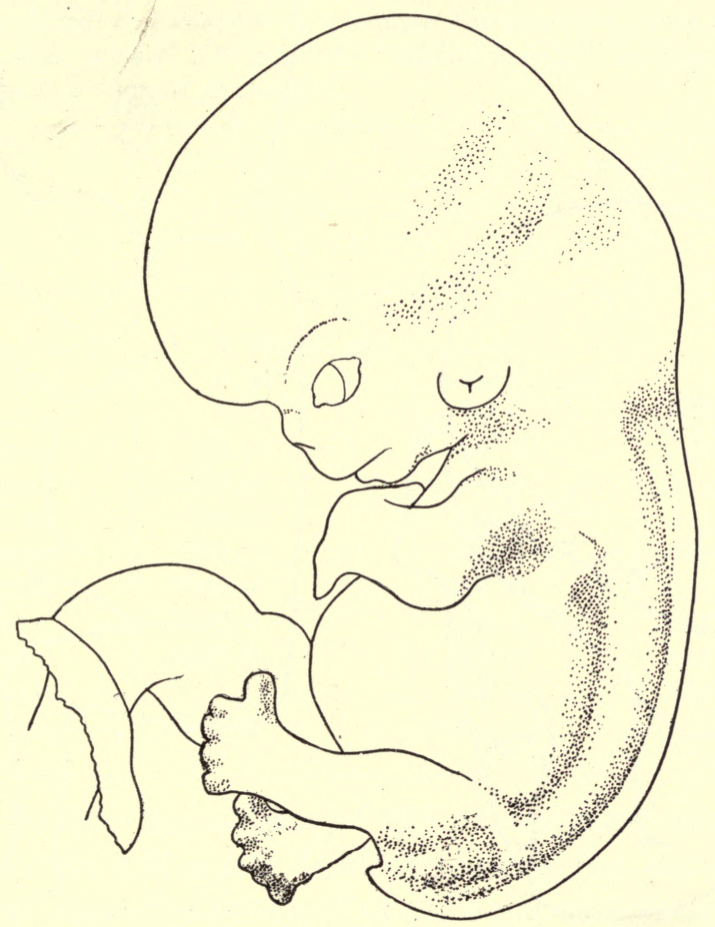

Fig. 169.-Human embryo of $22.8 \mathrm{~mm}$. (fifty-three days). After Minot, $\times 4$. For description see text.

The limbs grow rapidly during this month; they become differentiated into their three chief regions, and in the hand and foot the digits become clearly differentiated (Figs. 168, 169). By the end of this month the limbs project beyond the outlines of the body. The tail gradually recedes and by the time the embryo is two months old is scarcely visible externally. The structures of the facial region develop rapidly during this 
month (see below), and at its close the embryo acquires a distinctly "human" aspect and is generally known as a "fœetus". in distinction from the earlier "embryo."
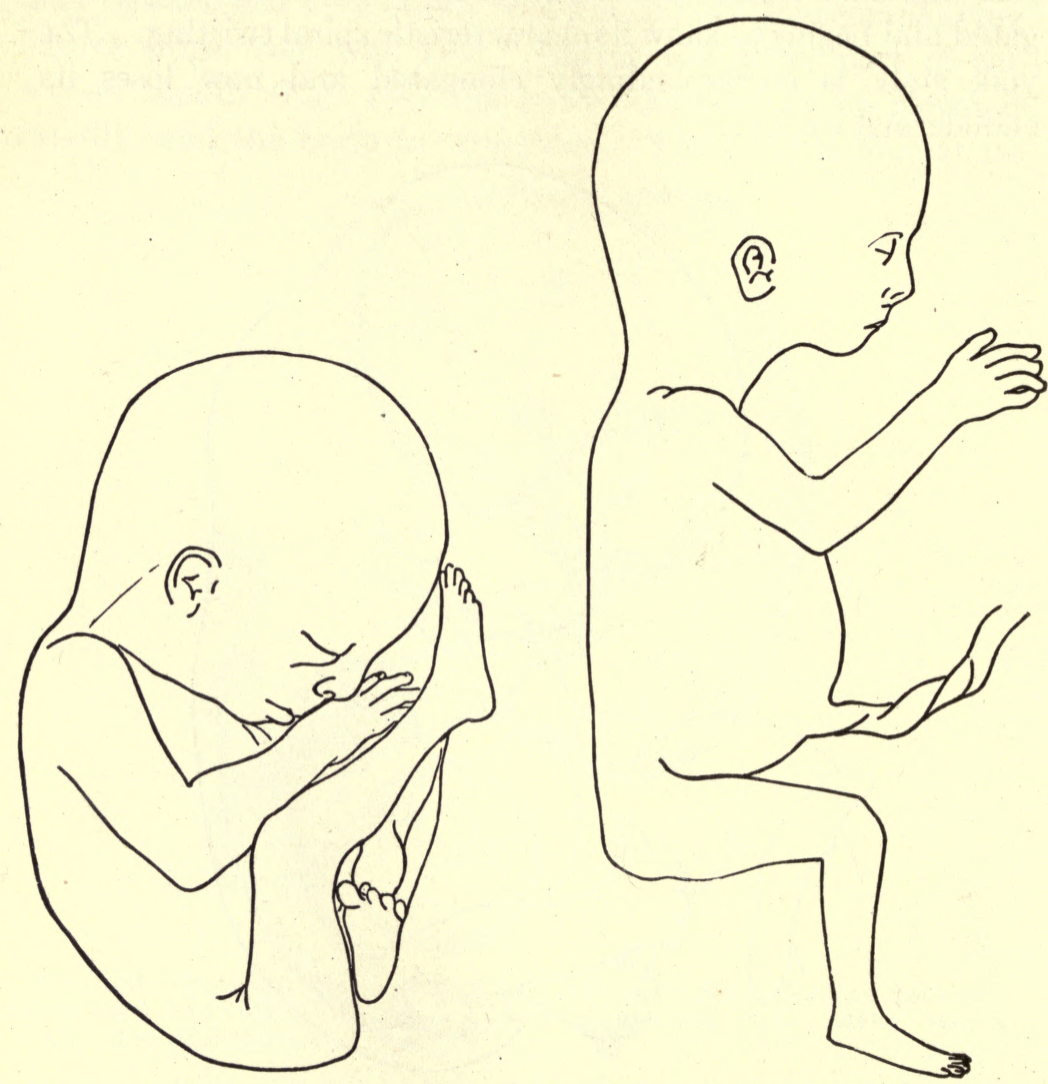

FIG. 170.-Outlines of human embryos of 106 to 110 days (118 to $120 \mathrm{~mm}$.). From Minot (Laboratory Text-book of Embryology). The figure to the left shows the most frequent position of the embryo in utero; that to the right shows the position assumed when removed from the embryonic membranes.

The external form changes now become relatively slower. The outlines of the head become more rounded; the facial characters are more fully established; the eyelids close together. The abdominal region recedes, the limbs become slender, elongated, and flexed. The fœetus is in a position of constraint within the uterus, as shown in Fig. 170 from Minot. 
The general changes in size and weight of the embryo and fœtus during the entire intra-uterine period are summarized in the accompanying table.

\section{TABLE SHOWING THE AVERAGE WEIGHT AND LENGTH OF THE HUMAN EMBRYO AND FETUS.}

Compiled from Jackson (weight) and Mall (length). (The column headed $\mathrm{CH}$ gives the length as measured in a straight line from the crown of the head to the heel; that marked CR gives the "sitting height," or length from the crown to rump or sacral flexure.)

\begin{tabular}{|c|c|c|c|}
\hline & \multirow{2}{*}{ Weight } & \multicolumn{2}{|c|}{ Length } \\
\hline & & $\mathrm{CH}$ & $\mathrm{CR}$ \\
\hline $\begin{array}{l}\text { Ovum (estimated). } \\
28 \text { days. } \\
56 \text { days. } \\
84 \text { days. } \\
112 \text { days. } \\
140 \text { days. } \\
168 \text { days. } \\
196 \text { days. } \\
224 \text { days. } \\
252 \text { days. } \\
270 \text { days. } \\
280 \text { days. }\end{array}$ & $\begin{array}{l}\quad 0.000004 \text { grm.. } \\
0.04^{*} \\
3.0 \\
36.0 \\
120.0 \\
330.0 \\
600.0 \\
1000.0 \\
1500.0 \\
2200.0 \\
\ldots \ldots \ldots \ldots \ldots . \\
3200.0\end{array}$ & $\begin{array}{r}2.5 \mathrm{~mm} . \\
30.0 \\
98.0 \\
180.0 \\
250.0 \\
315.0 \\
371.0 \\
425.0 \\
470.0 \\
500.0 \\
\ldots \ldots \ldots \ldots\end{array}$ & $\begin{array}{c}\ldots \ldots \ldots \ldots \\
2.5 \mathrm{~mm} . \\
25.0 \\
68.0 \\
121.0 \\
167.0 \\
210.0 \\
245.0 \\
284.0 \\
316.0 \\
336.0 \\
\ldots \ldots \ldots \ldots\end{array}$ \\
\hline
\end{tabular}

Before leaving the subject of the development of external form we should add a few details regarding the development of the facial characteristics and of the external genitalia.

\section{THE DEVELOPMENT OF THE FACE}

We may take as our starting point here, a stage of $2.6 \mathrm{~mm}$. (probably about thirty days) already described and figured (Fig. 164). At this time the first gill cleft is unreduced, the otocyst is not yet closed, and the optic vesicles are entirely lateral in position. The fore-brain region hangs down over the deep

* Age probably underestimated. 
oral sinus (stomodæum), the floor of which is still formed by the imperforate oral membrane. Within the next few days this membrane becomes perforated by the mouth opening. The oral sinus is bordered posteriorly by the mandibular processes, which do not quite meet in the mid-line, and antero-laterally by the maxillary processes, which are widely separated medially, the interval being occupied by the frontal process, a ridge over the surface of the fore-brain.
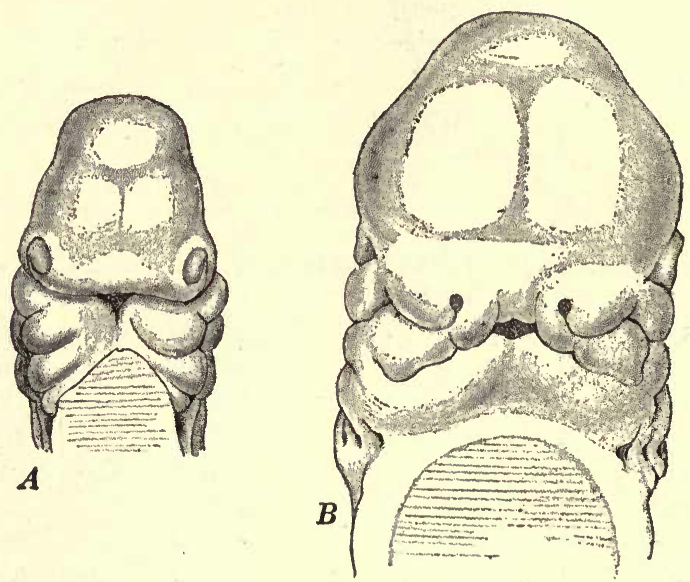

Fig. 171.-Early stages in the development of the head and face. After Rabl. A. Head of a human embryo of $8.3 \mathrm{~mm}$., seen from in front (ventrally). $B$. Head of human embryo of about $12 \mathrm{~mm}$., seen from in front. For explanation see text.

An important advance is to be seen in the development of the olfactory pits, which appear at the ends of the frontal process. Bordering the olfactory pits are inner or medial and outer or lateral elevations or olfactory processes (Fig. 171, $A$ ). The maxillary and mandibular processes are now closer together so that the opening of the oral sinus (now called the mouth) becomes a transversely elongated slit.

In the embryo of the fifth to sixth week (Fig. 171, $B$ ) the olfactory pits have deepened and have moved in toward the mid-line, thus separating the mouth from the fore-brain or forehead region. At the same time the medial and lateral olfactory processes become more prominent, and the former are 
uniting with the maxillary processes of the first visceral arch to form the rudiments of the upper jaw and lip. The mandibular processes are still separated medially by a groove. During the sixth week the eyes become visible from in front,
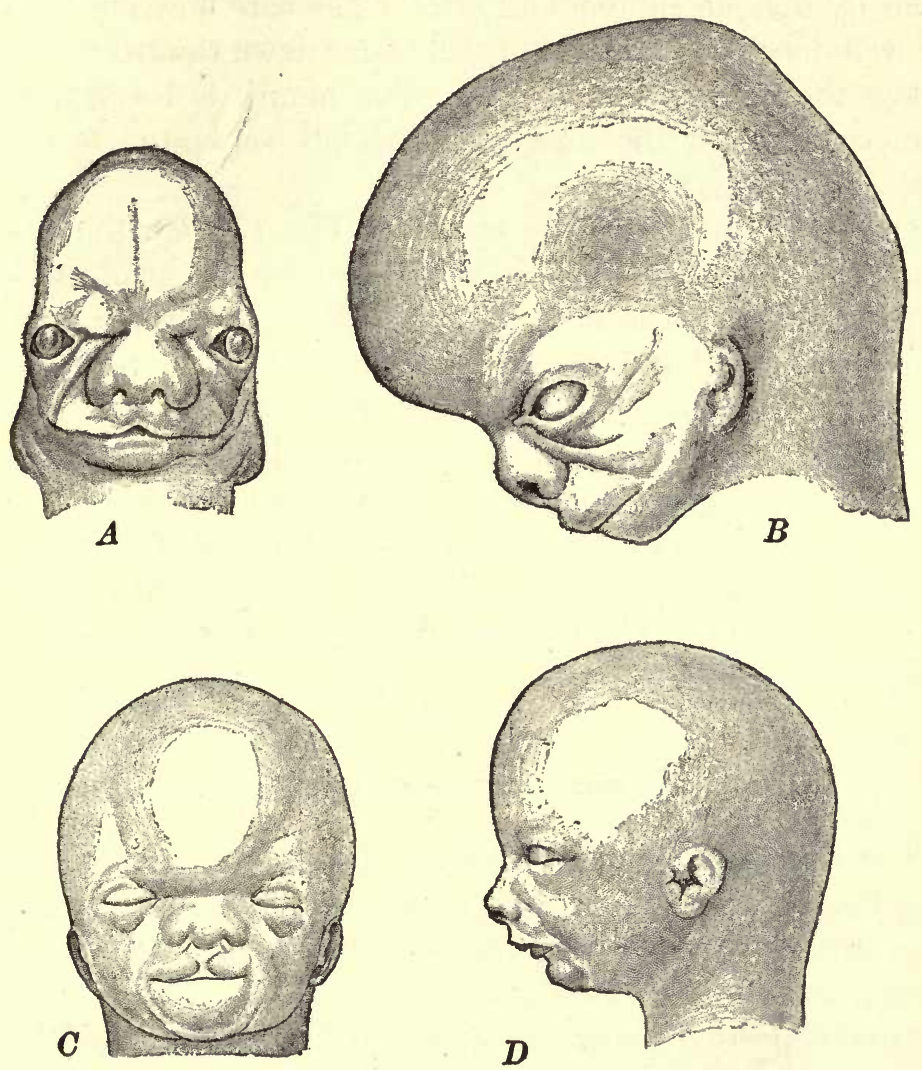

Fig. 172.-The development of the face of the human embryo. After Retzius. A. $18 \mathrm{~mm}$. embryo, $\times 4$. B. $25 \mathrm{~mm}$. embryo, $\times 4$. C. $42.5 \mathrm{~mm}$. embryo, $\times 2$. D. $117 \mathrm{~mm}$. embryo, $\times 4 / 5$. For explanation see text.

the upper lip begins to enlarge though still indented medially, the mandibular processes fuse completely forming the completed rudiment of the lower lip and jaw, and the chin appears.

The medial olfactory processes (globular processes) soon fuse together forming the nasal septum (Fig. 172, A), and the nose becomes slightly marked off from the forehead by a groove. 
The chin gradually enlarges, and the lips, now both complete medially, continue to enlarge (seventh week). At about eight weeks (Fig. 172, B) the eyelids are forming, and the eyes, now rapidly approaching one another, are separated from the forehead by oblique supraorbital folds. The ears now are marked by well-developed pinnæ, but still lie far down toward the neck, below the level of the mouth. The mouth is less extended transversely and the nose is completely separated from the forehead.

During the next week or ten days (Fig. 172, C), the eyelids close, the eyes move closer together, and the height of the forehead increases. The nose, though still very broad, begins to project slightly, and the external nares become temporarily closed by epidermal proliferations. The ear gains a somewhat higher position. The mouth is smaller, the lips thinner, and the lower jaw quite prominent. During the third month (Fig. $172, D)$, the pinna reaches nearly its adult position, the nose projects markedly, the lips, especially the upper, become thinner and protruded, and the essentials of the adult physiognomy are fairly established.

\section{THE DEVELOPMENT OF THE EXTERNAL GENITALIA}

The end of the gut posterior to the origin of the allantois (see below) forms the dilated cloaca, which is separated from the surface of the body by a thin portion of the body wall known as the cloacal membrane (Fig. 173, $A$ ). This membrane is later depressed below the surface of the body, at the bottom of a shallow depression (proctodæum). The cloacal cavity becomes divided into a ventral portion, the urinogenital sinus, receiving the openings of the excretory and reproductive ducts and the allantois, and the rectal portion. The cloacal membrane is correspondingly divided into the urinogenital membrane and the anal membrane, the two being separated by a narrow bridge of tissue forming the perineal rudiment.

In order to find the earliest traces of the external genitalia, we must go back to the embryo of the early part of the second 
month. Here we find a pair of ridges, either side of the cloacal membrane (Fig. 173, A), which gradually fuse and enlarge anteriorly, forming, toward the close of this month, a distinct cloacal tubercle. The urinogenital membrane is perforated
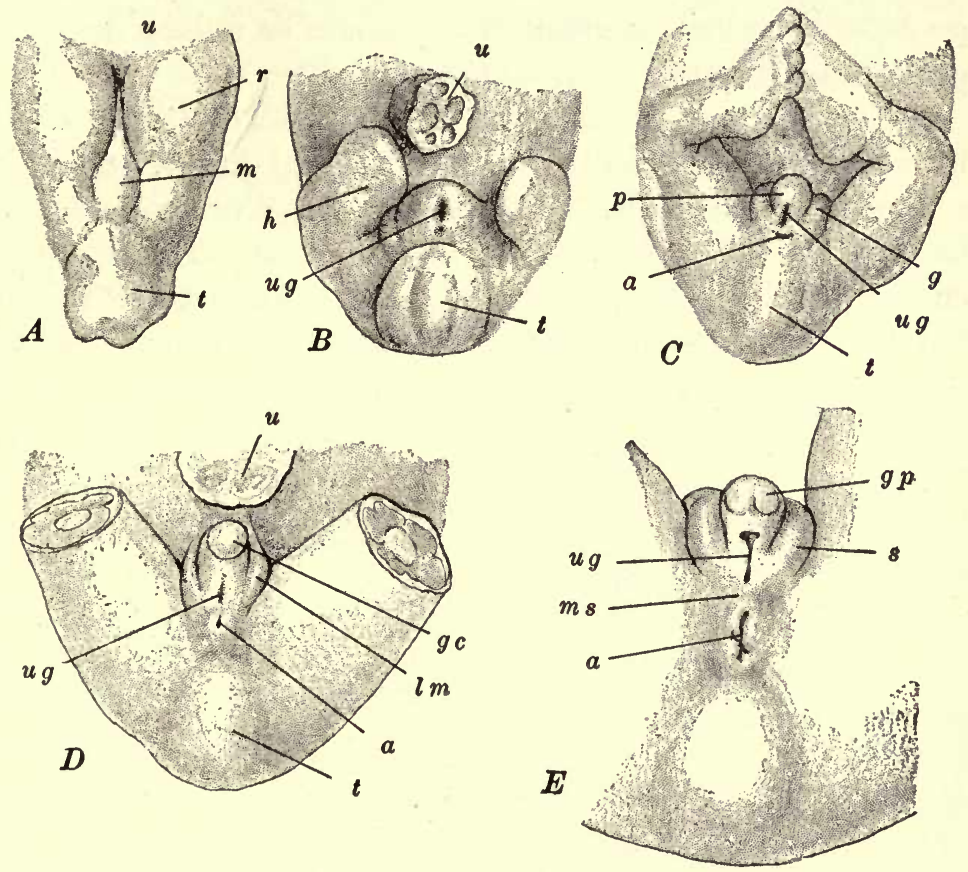

Fig. 173.-The development of the external genitalia. $A$, After Keibel. $B-E$, After Felix, from Meyer. $A$. Model of the cloacal region of a human embryo of $3 \mathrm{~mm}$. $B$. Ventral view of the caudal end of a human embryo of $18 \mathrm{~mm}$. C. Same of $28 \mathrm{~mm}$. Indifferent stage. D. Same of $32.5 \mathrm{~mm}$. Female. $E$. Same of three and one-half months. Male. $a$, Anal opening; $g$, genital ridge; $g c$, glans clitoridis; $g p$, glans penis; $h$, hind-limb; $l m$, labia majora; $m$, cloacal membrane; $m s$, median scrotal rudiment; $p$, phallus; $r$, cloacal ridge; $s$, scrotal ridge; $t$, coccygeal tubercle; $u$, umbilical cord (in $A$, umbilicus;) $u g$, urinogenital aperture.

about this time by the urinogenital aperture. (The time at which the anal opening is formed is quite variable, but usually is also toward the close of the second month.)

Upon the cloacal tubercle, and toward its posterior or anal side, there grows out quite rapidly a definitely circumscribed process called, at this stage, the phallus (Fig. 173, B). The 
remainder of the cloacal tubercle, at the base of the phallus and mostly anterior and lateral to it, is now known as the genital tubercle. The urinogenital aperture is continued forward upon the posterior (ventral) surface of the phallus as a narrow groove, the lateral margins of which are somewhat elevated as the genital folds, which gradually enlarge and so reduce the urinogenital aperture to a narrow elongated slit.

By the beginning of the third month (Fig. 173, $C$ ) the phallus has enlarged considerably, and its extremity has dilated as the rudiment of the glans. Lateral to each genital fold a second, larger ridge, the genital swelling, has appeared. This marks the end of the so-called indifferent period, during which there is but very slight external differentiation between the sexes. As a matter of fact, the sex of the individual is determined at the time of fertilization, and even during the latter part of this "indifferent period" the female embryo can be distinguished by the presence of a groove around the base of the phallus, which is lacking in the male.

The later development may be sketched very briefly. In the female, where the modifications are less extensive, the glans and the anterior (oral) portion of the phallus are transformed into the clitoris (Fig. 173, D), while the posterior (anal) portion of the phallus together with the lateral margins of the urinogenital aperture, become the labia minora. The labia majora are formed from the genital swelling and the genital tubercle (basal portion of the cloacal tubercle).

In the male (Fig. 173, E), the entire phallus is transformed into the penis, composed of the glans plus the shaft, the posterior (anal) portion of which is therefore equivalent to the labia minora. The anterior extension of the urinogenital aperture upon the male phallus is enclosed by the fusion of the genital folds and so added to the lower part of the urethra. The genital swellings in part fuse and are transformed into the scrotal sac, and in part disappear, to be replaced by other scrotal swellings which form the remainder of the scrotal sac. The essentials in the history of the external genitalia may be summarized as follows 


\begin{tabular}{|c|c|c|c|c|c|c|}
\hline \multicolumn{4}{|c|}{ Indifferent Period } & Female & \multicolumn{2}{|l|}{ Male } \\
\hline \multirow[t]{4}{*}{ Early } & \multirow[b]{3}{*}{$\begin{array}{l}\text { Genital } \\
\text { tubercle }\end{array}$} & \multirow{2}{*}{\multicolumn{2}{|c|}{$\begin{array}{c}\text { Late } \\
\text { Urinogenital aperture }\end{array}$}} & \multirow{3}{*}{$\left.\begin{array}{l}\text { Vestibule } \\
\text { Mons Veneris } \\
\text { Labia majora }\end{array}\right\}$} & \multirow{2}{*}{\multicolumn{2}{|c|}{ Terminal portion urethra }} \\
\hline & & & & & & \\
\hline & & $\begin{array}{l}\text { Genital } \\
\text { swelling }\end{array}$ & $\left\{\begin{array}{c}\text { Anterior } \\
\text { portion } \\
\text { Lateral } \\
\text { portions }\end{array}\right.$ & & $\left\{\begin{array}{l}\text { In part, portion of } \\
\text { scrotal sat } \\
\text { In part, replaced by } \\
\text { scrotal swellings }\end{array}\right.$ & $\left\{\begin{array}{c}\text { Scrotal } \\
\text { sac }\end{array}\right.$ \\
\hline & Phallus & $\begin{array}{l}\left\{\begin{array}{l}\text { Glans } \\
\text { Shaft }\end{array}\right. \\
\text { Genital fo }\end{array}$ & $\left\{\begin{array}{c}\text { Anterior } \\
\text { portion } \\
\text { Posterior } \\
\text { portion } \\
\text { ds }\end{array}\right\}$ & $\begin{array}{l}\text { Clitoris } \\
\text { Labia minora }\end{array}$ & $\left.\begin{array}{c}\text { Glans and anterior } \\
\text { portion shaft } \\
\begin{array}{c}\text { Posterior portion } \\
\text { shaft }\end{array}\end{array}\right\}$ & Penis \\
\hline
\end{tabular}

\section{THE EMBRYONIC MEMBRANES AND APPEND- AGES OF THE EUTHERIAN MAMMALS}

Scattered references have been made in the preceding pages to various details regarding the development of the amnion, the chorion, the allantois, and yolk-sac, and we must now give, possibly with some repetition, a more connected, though brief, account of the development of these structures. There is in general a remarkable similarity between the early history of the embryonic appendages of the Mammals and those of the Sauropsida, a similarity that is the more remarkable when the eggs of the two groups are compared. As mentioned in the introductory paragraphs of this chapter, these similarities are to be explained upon an historical basis, that of relationship through descent. But while there is, in the early stages, such close agreement between Mammal and Sauropsid, in these respects, during their later history, these mammalian membranes undergo profound changes in function associated with the intrauterine development of the embryo and the consequent substitution, as a source of nutritive substance, of the maternal tissues in place of the intra-oval yolk-mass or albuminous egg membranes of the Sauropsida.

In the chick the amnion serves, among other functions, to protect the embryo from drying and from the deforming pressure of the rigid shell; the yolk-sac contains a large part of the food substance for the developing embryo; the allantoic 
wall is the embryonic respiratory organ, while its cavity serves as an excretory reservoir; and the chorion (serosa) appears to have little, if any, physiological importance. In the Mammal this is all changed. The amnion is a membrane of secondary protective importance; the yolkless yolk-sac is a vestigial organ, often of little functional value; the allantois loses its respiratory and excretory significance and is usually concerned in relating the embryo to the source of its food supply; while the chorion, either as a whole or in part, becomes the chief organ concerned in the exchange of nutritive materials and excreted substances between the embryo and the maternal uterine circulation.

These characteristic relations of the mammalian embryonic membranes do not appear in this group in a fully established condition; there is, on the contrary, a long series of intermediate conditions, transitional in almost every respect, between the Sauropsid condition and that found in the highest Mammals, the Primates, where these relations are most highly developed. It is a familiar fact that in the lowest Mammals, the Prototheria (Monotremata or Ornithodelphia) including only the genera Ornithorhynchus, Echidna, and Proechidna, essentially Sauropsid conditions obtain here, as well as in so many morphological and physiological characteristics of the adults. Here the developing embryo has no organic relation with the mother, for the fully formed eggs are deposited outside the body of the parent, either in an integumentary fold (Echidna), or in a "nest" (Ornithorhynchus), where the young develop independently, enclosed within a tough parchment-like egg shell. Usually only a single egg is produced at one time in Echidna, while Ornithorhynchus normally produces two at a time. The eggs too are reptilian in character, much larger than any other mammalian egg, and yolk laden. As laid, they measure about 15-16.5 $\times 12-13 \mathrm{~mm}$. (in Echidna); the egg cell proper, as it leaves the ovary is, of course, smaller than this, but even so is much larger than the egg cell of the higher Mammals, being 3.0-4.0 mm. in diameter in Echidna, $2.5 \mathrm{~mm}$. in Ornithorhynchus.

Among the Metatheria (Didelphia or Marsupialia) many of the typical mammalian conditions are found. The ova, though 
commonly somewhat larger than in the higher Mammals, are sometimes of no greater size. The embryo has a brief intrauterine period of development, during which nutritive relations are established with the uterine wall by means of the surface of the chorion, which, however, retains its originally smooth surface and merely comes closely into contact with the vascular uterine epithelium, without acquiring a close organic union. The yolk-sac is very large in these forms and underlies nearly the entire chorion (serosa). Nutritive substances from the maternal circulation may thus pass, with some difficulty, through the chorion and the wall of the yolk-sac, into the blood of the latter.

Conditions suggestive of the higher Mammals are by no means lacking, however, for in Dasyurus (Hill) the yolk-sac in certain areas becomes very vascular and forms a close relation with the uterine wall. The ectoderm cells of the chorion, between the two, aid in establishing this relation between the maternal and the embryonic blood, a relation which is very different from the mere contact relation of the typical Metatheria. And in Perameles (Hill) the allantois takes up a similar relation with the uterine mucosa, sending into the latter well-developed vascular outgrowths (Fig. 174). We have here then, a condition that in many respecis resembles closely the relation found in many of the "placental" Mammals. Indeed, these structures are known as the "yolk-sac" and the "allantoic placenta" respectively.

Among the remaining Mammalian orders, or Eutheria (Monodelphia or Placentalia), there is the greatest diversity in the mode and degree of the relation between embryonic membranes and appendages, and the uterine wall, or, in other words, in the character of the placentation. In the simpler cases (pig, horse, and many others) the relation is much like that described in Perameles in its essentials, while in the more highly specialized instances (apes and man) the relation becomes very complex, involving considerable modification of what is regarded as the typical arrangement of the embryonic appendages. Between these two extremes there is the greatest variety of conditions 
of placentation, which frequently do not parallel the usual ordinal classification, so that even within a single order (e.g., Ungulata, Primates) there may be divergences which considerably exceed the range of the morphological traits upon which the orders are based.

Before attempting to describe any of the actual details of the structure and development of the placenta, we must give a

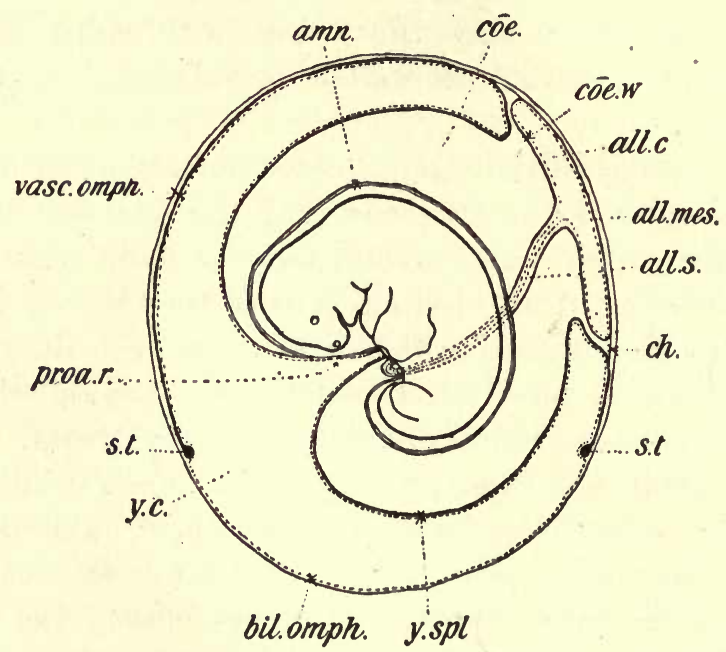

FIG. 174.-Diagram of the arrangement of the fœtal membranes in the Marsupial, Perameles. From Hill. The ectoderm is represented by a light continuous line, the endoderm by a dotted line, and the mesoderm by a heavy line. amn, Amnion; all. c., allantoic cavity; all. mes., allanto-chorionic mesenchyme; all. s., allantoic stalk; bil, omph.. ectodermal and endodermal wall of yolk-sac; $c h .$, margin of true chorion; cœ., exocœlom; cœ. w., inner wall of allantois; proa. $r$. , persisting remnant of proamnion; s. t., sinus terminalis; vasc. omph., three-layered portion of yolk-sac wall; $y$. c., cavity of yolk-sac; $y . s p l$., invaginated splanchnopleural wall of yolk-sac.

general outline of the ontogenetic history of the embryonic membranes and appendages. We may consider first the method by which the young embryo or "ovum" (blastodermic vesicle) effects its primary relation with the uterine wall. In the remaining pages it is understood that what is said is limited to the Eutheria. 


\section{Implantation}

Through the process of menstruation, or procestrous, its equivalent in the lower Mammals, the mucous membrane lining the uterus is kept in an active, wholly living condition, and as the ovum or blastodermic vesicle enters this cavity it almost immediately becomes attached or implanted upon the wall. It may become superficially attached to the wall of the main uterine cavity, so that as it grows it projects freely into the lumen of the uterus; this is known as central implantation and is found in the Ungulates and Carnivors, the lower Primates and some Rodents such as the rabbit. In other forms the vesicle may come to lie in a furrow or groove in the uterine wall, which is then closed off from the main cavity by the fusion of the margins of the furrow, enclosing the vesicle. This is eccentric implantation and is found in such forms as the mouse and some Insectivors. Or the vesicle may burrow into the substance of the mucous membrane lining the uterus, the mucosa then closing together over the point of entrance, as in man and some Rodents, such as the guinea pig and the gopher. This type of implantation is known as interstitial.

The structure primarily concerned in effecting this early connection between the vesicle and the uterine mucosa is the trophoblast (Hubrecht). We have already described the formation of this superficial layer of ectoderm cells which covers the entire blastodermic vesicle as this passes down the oviduct and enters the uterus (Figs. 154, A; 175). The trophoblast may remain for a short time the only component of the peripheral wall of the blastodermic vesicle. But extra-embryonic mesoderm usually forms very early around the wall of the vesicle, and the entire extra-embryonic wall may then be known as the chorion; the trophoblast may then be called the chorionic ectoderm.

It is convenient to distinguish two general types of behavior on the part of the trophoblast or chorionic ectoderm. In most instances of central implantation (e.g., pig, horse) it merely forms an adhesive layer which comes closely into 
contact, over practically its entire surface, with the uterine mucosa. In other instances of central implantation and in the eccentric and interstitial types, a part or even the whole of the trophoblast becomes highly specialized, physiologically, as the trophoderm (Minot), in which the cells proliferate rapidly forming a layer of considerable thickness (Figs. 175, 176). It is the function of the trophoderm to dissolve or digest the uterine mucosa, with which it is in contact. The trophoderm, probably through the action of specific enzymes, rapidly erodes the uterine wall, and the blastodermic vesicle becomes either partially or wholly embedded in the maternal tissue, so that the embryo bears a relation to the maternal organism which is quite that of an internal parasite.

In most cases a part of the trophoblast is thus specialized as trophoderm. In the rabbit, for example, the trophoderm forms a horse-shoe shaped area just around the embryonic rudiment, lateral and posterior to it; this region alone becomes embedded in the uterine tissue, while the remainder of the blastodermic vesicle, projecting into the lumen of the uterus, remains covered with the relatively unmodified trophoblast (chorion). In the spermophile (Rejsek) the trophoderm forms a thickened mass, in the wall of the vesicle, opposite the inner cell mass or embryonic rudiment. As the trophoderm erodes the mucosa, the vesicle is carried down and partially embedded in the uterine wall (Fig. 175). In forms like the hedgehog, apes, and man, the entire trophoblast becomes trophodermal (Figs. 153, 161). Here, then, the maternal tissues on all sides of the vesicle are eroded, and the "ovum" becomes completely embedded and surrounded by a mass of dissolved tissue.

The cells of the trophoderm very early begin to fuse together forming either small masses, known as multinuclear giant cells, or extensive protoplasmic masses known then as syncytia, or better, the syncytiotrophoderm (syncytiotrophoblast) (Figs. 175, 184). In the trophoblast, which is less intimately associated with the maternal tissues, the cell boundaries usually remain, and this is then distinguished as the cytotrophoblast. 
It is evident from this description that the trophoderm or syncytiotrophoderm, forms the boundary between the embryonic and the maternal tissues, and not only effects the implantation of the "ovum," but at the same time establishes the
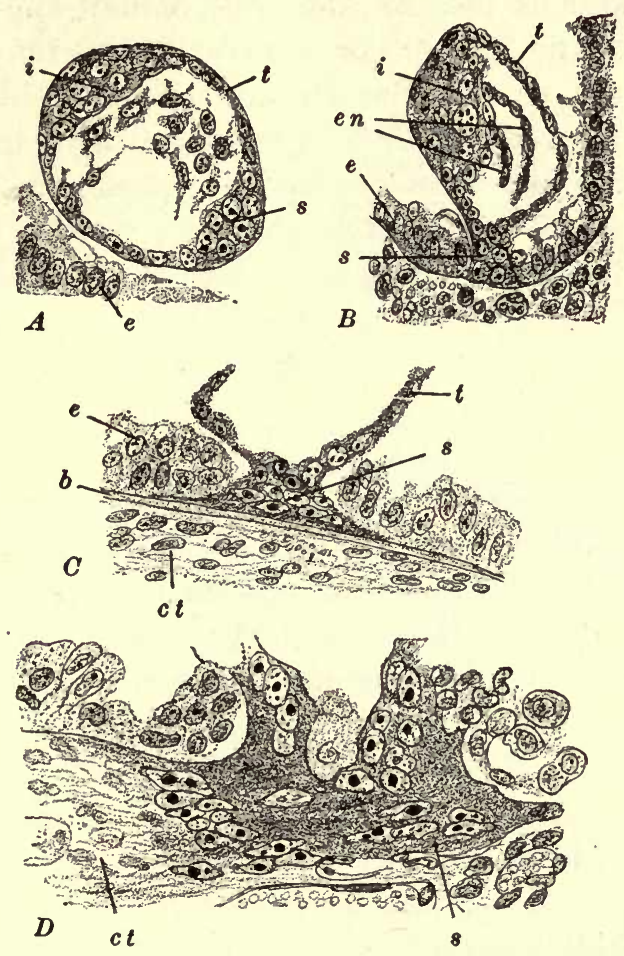

Fig. 175.-Early stages in the implantation of the blastodermic vesicle of the spermophile (Spermophilus citillus). After Rejsek. $A$. Unattached vesicle. $B$. Syncytiotrophoderm just penetrating the epithelium of the uterine mucous membrane. $C$. Syncytiotrophoderm extending along the basement membrane of the uterine epithelium. $D$. More highly magnified view of the syncytiotrophoderm after it has penetrated the kasement membrane and entered the connective tissue of the uterine mucosa. $b$, Basement membrane of uterine epithelium; $c t$, connective tissue of the uterine mucosa; $e$, epithelium of the uterine mucosa; en, endoderm; $i$, inner cell mass; $s$, syncytiotrophoderm; $t$, trophoblast.

primary element in the placenta. The trophoderm later becomes vascularized from the mesoderm of the chorion or allantois (yolk-sac in some cases), and acts as the chief absorp- 
tive or resorptive surface, taking in materials from the maternal tissues and blood.

The extent to which the trophoderm erodes the uterine lining varies greatly. Of course where no trophoderm is differentiated, little or no actual erosion occurs. And when the trophoderm is present, the erosion may affect only the epithelium of the mucosa, or it may involve the connectivetissue elements, or even the walls of the uterine blood-vessels. The degree of erosion has been suggested as a basis for the classification of the types of placentæ (Grosser), but this and many other facts regarding the later history of the trophoderm are better considered later, in connection with the placenta itself.

\section{The Amnion and Chorion}

Our description of the formation of these membranes may be very brief on account of their general similarity to those of the chick fully described in an earlier chapter (Fig. 178).

We must distinguish, at the very outset, between two general types of amnion formation found among the Mammals, a distinction that has already been noted above in describing the formation of the embryonic disc and its relation to the trophoblast. In the Carnivors, Ruminants, many Insectivors and some Rodents, such as the rabbit, the amnion is formed from a series of folds of the extra-embryonic somatopleure (wall of the blastodermic vesicle) much as in the chick. In other forms, such as the mouse, guinea-pig, bat, some Insectivors, and many Primates, including man, the amnionic cavity arises in situ (entypy), above the embryonic disc, through a splitting of the ectoderm or through the confluence of gradually enlarging spaces (Figs. 152, 153, 155).

The amnion and chorion of the rabbit may be described as a fair representative of the first type. Here, as in the chick, the mesoderm very early extends posteriorly and laterally from the embryo, but immediately anterior to it the wall of the blastodermic vesicle remains for a considerable period devoid 

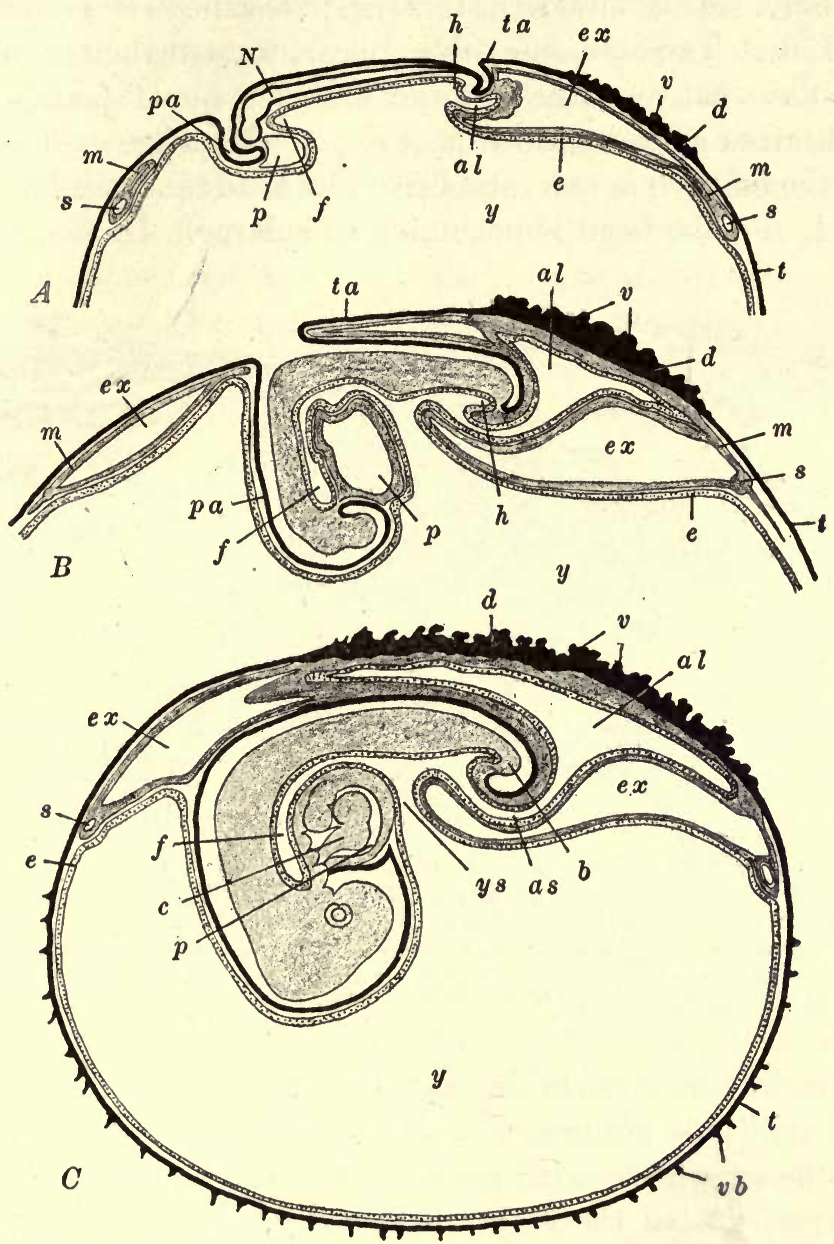

FIG. 176.-Diagrams of the formation of the embryonic membranes and appendages in the rabbit. After Van Beneden and Julin (partly after Marshall). Sagittal sections. $A$. At the end of the ninth day. $B$. Early the tenth day. $C$. At the end of the tenth day. Ectoderm black; endoderm dotted; mesoderm gray. $a l$, Allantois; $a s$, allantoic stalk; $b$, tail bud; $c$, heart; $d$, trophoderm; $e$, endoderm; $e x$, exocœlom; $f$, fore-gut; $h$, hind-gut; $m$, mesoderm; $N$, central nervous system; $p$, pericardial cavity; $p a$, proamnion; $s$, marginal sinus (sinus terminalis); $t$, trophoblast; $t a$, tail-fold of amnion; $v$, trophodermal villi; $v b$, trophoblastic villi; $y$, cavity of yolk-sac; $y s$, yolk-stalk. 
of mesoderm, and therefore composed of ectoderm and endoderm only. This mesoderm-free region is called the proamnion (Fig. 176). The amnionic folds appear, toward the end of the ninth day, between the embryo and the horse-shoe shaped implantation area described above. At this stage of development the embryo is well established, its head- and tail-folds are formed, and the head is beginning to enlarge. In the wall of

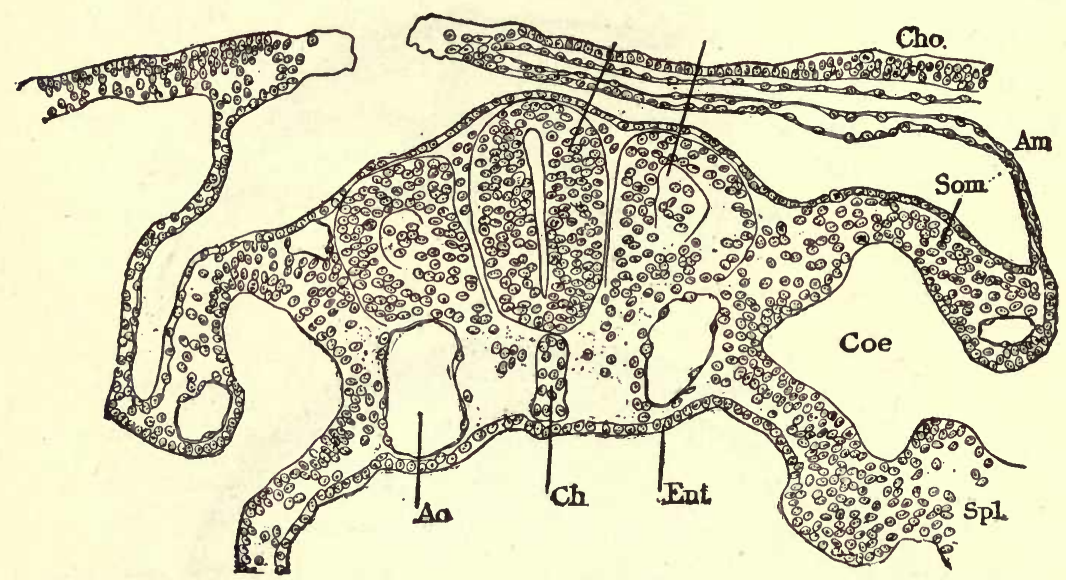

Fig. 177.-Transverse section through the rabbit embryo of eight days and two hours. From Minot (Laboratory Text-book of Embryology). Am, Amnion; Ao, lateral dorsal aorta; $C h$, notochord; Cho, chorion; $C a$, cœlom; Ent, endoderm; $M d$, medullary tube (nerve cord); Seg, myotome; Som. somatopleure; $S p l$, splanchnopleure.

the blastodermic vesicle the endoderm has extended nearly or quite completely around the inside of the vesicle (trophoblast), while the mesoderm with its exocœlom extends through only the upper third of the vesicle, which is nearly as far as it ever goes in the rabbit.

The tail-fold of the amnion appears first, composed of ectoderm and somatic mesoderm of the extra-embryonic region, and therefore containing an extension of the exocœlom; the mesodermal layer is unusually thick in the tail-fold. Laterally folds soon appear as anterior extensions of the extremities of the tail-fold. The head of the embryo rapidly enlarges, and as it sinks down into the proamnionic region this flows up to or 
above the level of the surface of the embryo forming the rudiment of the head-fold of the amnion, which is thus composed at first of the ectodermal and endodermal wall of this part of the vesicle. The posterior and lateral folds rapidly come together, close above the embryo, in the anterior direction (Fig. 177). Finally all four of the folds fuse together in front of the middle of the embryo. The region of their fusion, the seroamnionic connection, is a small knot, quite in contrast to the elongated seam of the chick; it should also be noted that the direction of the closure of the folds is just the reverse of what it is in the chick, for here the tail-fold, rather than the headfold, grows the more rapidly.

Following the complete fusion of the amnionic folds, occurs the separation of the inner and outer layers of the folds, thus establishing (1) an outer membrane, really an extension over the embryo of the wall of the blastodermic vesicle, known as the chorion; (2) an inner membrane, the amnion, separated from the embryo itself by (3) the amnionic cavity; and (4) an extension completely around the dorsal and lateral sides of the embryo, of the exocœlom (Fig. 176).

From the relation of these folds (Fig. 178), it is clear that the chorion is ectodermal superficially, lined with extra-embryonic somatic mesoderm, while the amnion is ectodermal internally with its somatic mesodermal layer turned away from the embryo. The exocœlom is of course entirely lined with mesoderm, while the amnionic cavity is wholly lined with ectoderm, embryonic and extra-embryonic. The amnion is a thin, semitransparent membrane, while the chorion is thicker and quite opaque. The amnion is non-vascular, while the chorion is, in the higher Mammals, often richly supplied with blood vessels.

As the embryo enlarges, the attachment of the amnion, and therefore the region where the amnionic ectoderm becomes continuous with the embryonic ectoderm, remains restricted to the region just around the origin of the yolk stalk and allantois or umbilicus (Fig. 176, B, C).

The proamnion finally becomes invaded by mesoderm, which has from a very early stage been present in front of the pro- 
amnion, and the entire amnion and chorion then have a mesodermal lining. In the rabbit the amnionic cavity remains relatively small, while the exocoelom expands so as to fill the cavity of the blastodermic vesicle, save for the space occupied by the yolk-sac and allantois (Fig. 180). The tail-fold appears extremely early in some forms, such as the mole (Talpa) and

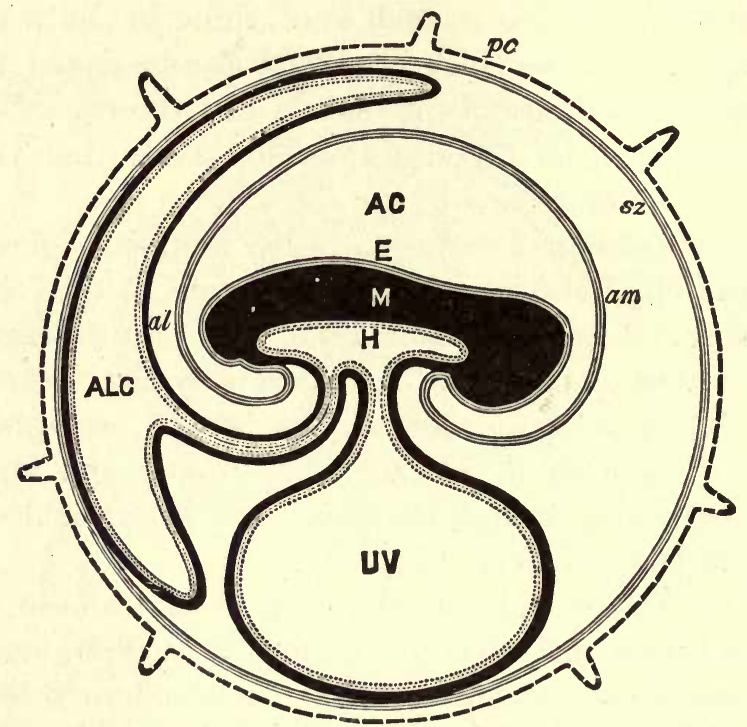

Fig. 178. - Diagram of the embryonic membranes and appendages of the Mammals in general. From Hertwig (Lehrbuch, etc.), after Turner. $A C$, Amnionic cavity; $a l$, allantois; $A L C$, allantoic eavity; $a m$. amnion; $E$, embryonic ectoderm; $H$, embryonic endoderm (the reference letter is placed in the gut cavity); $M$, embryonic mesoderm; $p c$, trophoblast or trophoderm with villi; $s z$, chorion; $U V$, yolk-sac.

in Tarsius, so that the point of the final closure of the amnionic folds is far forward over the head. The extent of the proamnion is also extremely variable; in some forms (e.g., Ruminants, bats, many Primates) it is nearly or quite absent, while in the opossum it forms nearly the entire amnion.

Turning to the second type, where the amnionic cavity is formed in situ, as a closed cavity from the very beginning, we may describe the human amnion as an example. The earliest stages in its formation have not been observed as yet, in man, 
but a relatively early condition has been illustrated in Fig. $161, D$. Here the embryo itself remains connected with the wall of the blastodermic vesicle by the body stalk, described above, and in this respect the human embryo is not a satisfactory example of this type of amnion formation. Usually this attachment represents the remains of a thickening in the wall of the vesicle ("träger," see above), to which is added later the definitive attachment of the allantois growing out from the embryo through the cavity of the vesicle to the inner surface of the chorion.

In the human vesicle, however, the amnionic cavity appears directly above the embryo, so that the embryonic disc itself forms its floor, while the body stalk bounds it posteriorly (Figs. 161, 179). At first the roof of the amnionic cavity is simply the trophoblast, but as the cavity enlarges it becomes partly free, below the trophoblast, so that its roof is a separate structure. The attachment of the amnion is around the margin of the embryonic disc (Fig. 162, A); its roof and sides are composed of a thin layer of ectoderm toward the embryo, outside of which is a thin mesodermal layer, for the extraembryonic mesoderm has already been formed throughout the entire vesicle. A proamnion is therefore lacking in forms whose amnion is developed in this manner.

As the embryo enlarges and extends in every direction the origin of the amnion appears to be carried ventrally, so that, as in the rabbit, it connects with the embryo just around the umbilicus. At the posterior end, however, it passes around the sides of the body stalk, to its posterior surface, so that this structure finally becomes wrapped in a part of the amnionic membrane (Fig. 179). In man the yolk stalk and sac, and the allantois, are also bound up in this body stalk or umbilical stalk (Fig. 179). The final disposition and character of the amnion and chorion are thus essentially the same as when formed from folds.

In man the amnionic cavity grows rapidly and by the third month becomes so large as completely to fill the cavity of the vesicle or "ovum." The exocœlom is thereby entirely obliter- 
ated, the amnion and chorion being brought into contact with one another over their mesodermal surfaces, and finally they may fuse together. The amnionic cavity is filled with a fluid known as the liquor amnii, now thought to be formed by the amnionic epithelium. It contains solids, mostly albumins, grape sugar, and urea, to the extent of about 1 per cent. The
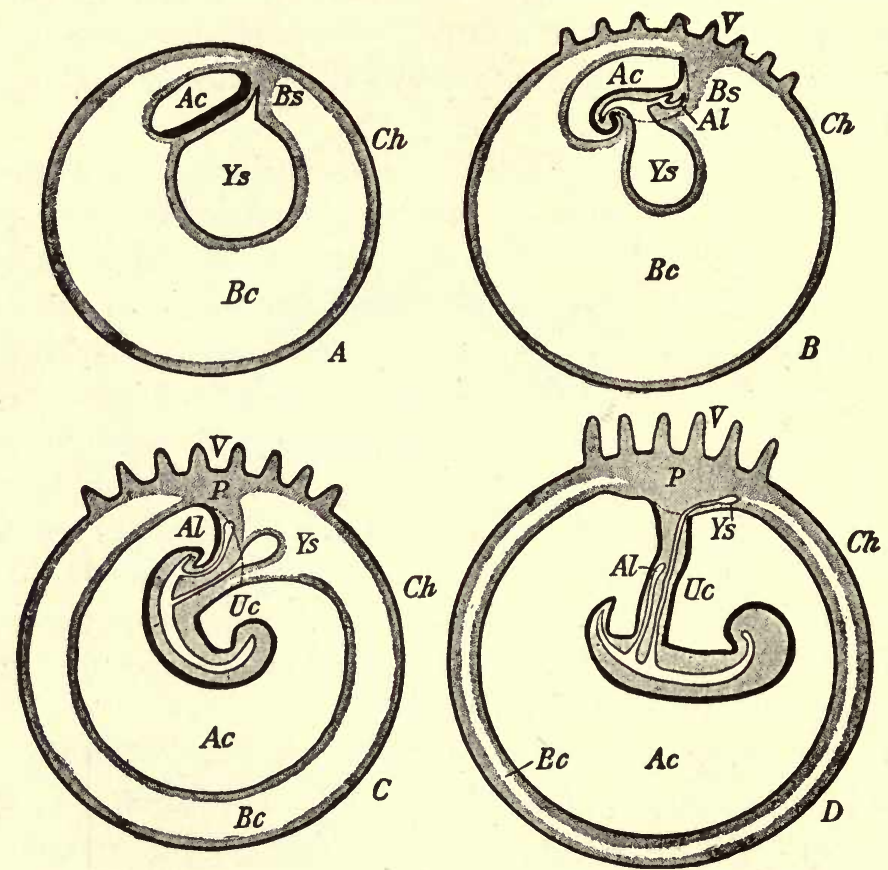

Fig. 179.-Diagrams illustrating the formation of the umbilical cord and the relations of the allantois and yolk-sac in the human embryo. From McMurrich (Development of the Human Body). The heavy black line represents the embryonic ectoderm; the dotted line marks the line of the transition of the body (embryonic) ectoderm into that of the amnion. Stippled areas, mesoderm. $A c$, Amnionic cavity; $A l$, allantois; $B c$, exocœlom; $B s$, body stalk; $C h$, chorion; $P$, placenta; $U c$, umbilical cord; $V$, chorionic (trophodermic) villi; $Y s$, yolk-sac.

amount of the liquor amnii in man varies considerably, but usually between 0.5 and 1.0 liter at the period of its maximum amount, which is some time before parturition. At parturition, of course, both the amnion and chorion normally are ruptured and the amnionic fluid escapes with or before the fotus.

The more important aspects of the chorion are those con- 
cerned with placentation and will be considered in connection with the development of the placenta.

\section{The Yolk-sac}

We may summarize what has already been said concerning the yolk-sac and add a few facts of importance, chiefly from the comparative standpoint. We are to think of the yolk-sac of the Mammals as typically occupying the chief part of the early blastodermic vesicle, its cavity opening widely into the embryonic gut by the broad yolk stalk, and its wall separated

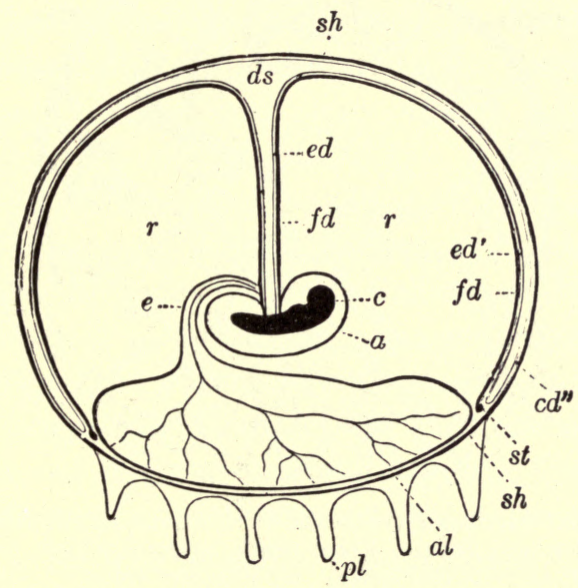

Fig. 180.-Diagrammatic section through the fully formed blastodermic vesicle of the rabbit, showing the reduced yolk-sac. From Hertwig (Lehrbuch, etc.) after Bischoff. $a$, Amnion; $a l$, allantois; $d s$, yolk-sac; $e$, embryo; $e d, e d^{\prime}, e d^{\prime \prime}$ yolk-sac endoderm; $f d$, vascular layer (mesoderm) of yolk-sac; $p l$, villi; $r$, exocœlom; st, sinus terminalis; $u$, allantoic stalk.

from the chorion by the exocœlom (Figs. 161, 179). The yolksac is thus a splanchnopleuric structure, in contrast to the somatopleuric amnion and chorion. This typical relation, however, is subject to varied and often profound modification. In the rabbit we have seen that the endodermal wall of the vesicle develops slowly, so that for a considerable early period the yolksac is incomplete ventrally. Finally the endoderm does form a complete sac, in contact with the chorionic ectoderm; the 
extra-embryonic mesoderm then develops very slowly pushing down between the ectoderm and endoderm, but limited to the upper half of the vesicle (Fig. 176). The yolk-sac in the rabbit is therefore strictly splanchnopleural only in its upper half; below this the chorionic ectoderm is in direct contact with the yolk-sac endoderm. When the exocœlom expands so considerably as it does in the rabbit, the yolk-sac is compressed and

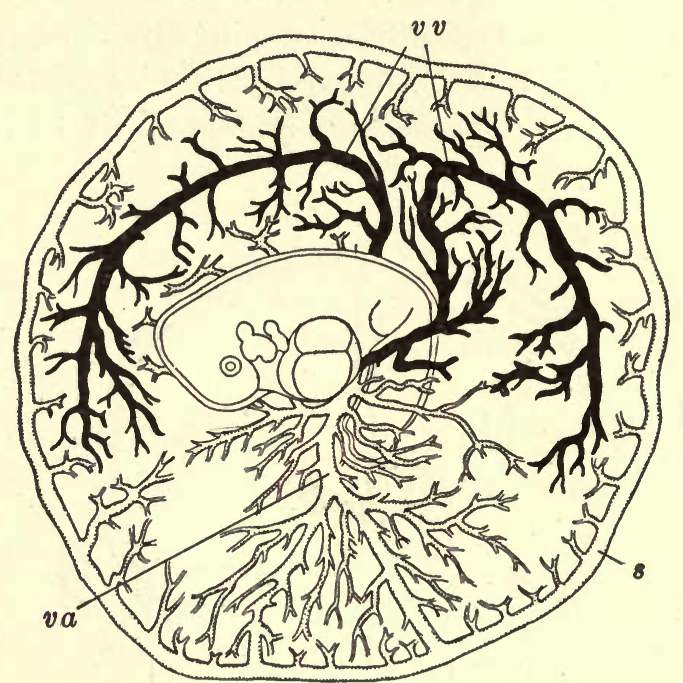

Fig. 181.-Area vasculosa (yolk-sac circulation) of an eleven-day rabbit. After Van Beneden and Julin. Veins black, arteries white. $s$, Marginal sinus (sinus terminalis); $v a$, vitelline artery; $v v$, vitelline veins.

reduced to an umbrella-shaped structure with a very narrow cavity, connected with the embryonic gut by a long narrow yolk stalk, the wall of which includes mesoderm as well as endoderm (Fig. 180).

As in the chick the mesodermal wall of the yolk-sac becomes very vascular. Its rich network of blood vessels is supplied by the vitelline or omphalomesenteric arteries arising from the dorsal aorta (Fig. 181). After spreading over the surface of the sac these collect into a well-marked sinus terminalis, which is a complete ring in the rabbit, and from which the blood is returned to the embryo through the vitelline or omphalomesenteric 
veins. These empty into the posterior end of the heart after penetrating the liver; later the extra-embryonic portions of these veins disappear, while their embryonic portions are transformed into the portal (hepatic portal) vein.

In the Carnivors the yolk-sac is very large and has a complete mesodermal investment which is unusually vascular. At first it is closely in contact with the chorion, and thus may have temporarily a placental relation, but soon it is in part separated from the chorion by the extension, between the two membranes, of the allantois which then assumes the definitive placental relation. The primarily placental character of the yolk-sac in the marsupial, Dasyurus, has been mentioned. In the mole, Talpa, a similar relation has been described, the vascular mesoderm of the upper part of the yolk-sac, with the endodermal wall of its lower part, coming into close organic relation with the chorion and uterine epithelium.

In many other Mammals the yolk-sac is from the beginning a relatively small organ (Tarsius, hedgehog, Primates), or it may early be reduced from an originally well-developed state (horse). In man, which may be described as in a general way representative of this type, the yolk-sac at all stages comes far short of filling the cavity (exocœlom) of the blastodermic vesicle. It grows slowly out into the exocœlom during the first month or more of development, its diameter about equal to the length of the embryo. Its wall is richly vascular even in its very early stages, the blood vessels producing a characteristic roughness of its outer (mesodermal) surface (Fig. 164). After reaching a size of about $11 \times 7 \mathrm{~mm}$. it begins to diminish in size. A yolk stalk becomes clearly differentiated and elongates rapidly, and we have already seen how it becomes enclosed proximally within the umbilical stalk or cord. Finally the entire yolk stalk is thus enclosed and, as the amnionic membrane expands, filling the entire cavity of the "ovum," wiping out the exocœlom, the yolk-sac itself disappears from view, embedded in the mesodermal tissues of the placental region (Fig. 179).

The greater part of the yolk stalk becomes a solid cord of endoderm during the latter part of the second month, and finally 
it disappears entirely; its proximal end may occasionally remain tubular as Meckel's diverticulum of the intestine. The yolksac remains as a very small vestige, ordinarily $5 \mathrm{~mm}$. or less in diameter, even up to the the time of parturition.

\section{The Allantois}

The allantois is no less variable among the Eutheria, both in its mode of development and in its definitive relations, than are the other embryonic appendages. And again there is little parallel between allantoic characters and the usual ordinal classification of the Eutheria. Among the Carnivora and the lower Primates (Lemurs) the allantois becomes very large and fills the exocolomic space, while, as an opposite condition, among the other Primates it forms not even a free vesicle, but remains as a vestigial structure, enclosed within the umbilical cord. Between these two extremes there is a great variety of conditions.

The earlier embryonic history of the allantois is considerably less variable than its later history and final relations. At first there is essential similarity to the condition already described in the chick, so that the earlier phases in its development need not be described here. Many of the later modifications of the avian type of allantois are correlated with the fact that among the Mammals the allantoic circulation functionally takes the place of the avian yolk-sac circulation, or in other words, the allantois takes an essential, though secondary, part in the formation of the embryonic placental structures, indeed it may even be limited to this relation, as in the rabbit. From the functional relations of the allantois, among the Mammalia, it is clear that its mesodermal structures, in particular its blood vessels, are its most important elements, and there is relatively slight variation in their arrangement.

As in describing the amnion we may consider two of the important types of allantoic formation and history, and then mention briefly a few comparative points of interest.

In the rabbit the allantois appears on the eighth day of development, as a small mass of mesoderm cells extending into the 
exocolom opposite the posterior end (primitive streak) of the embryo (Fig. 176, A). At its base the tail-fold of the amnion is just appearing, and there is already a slight indication of the evagination of the endoderm into it. By the ninth day the tail of the embryo has begun to grow out, and the allantoic rudiment is forced into a ventral position; the amnion therefore appears to arise posteriorly to the allantois, beneath the base of the tail. By this day the allantois has enlarged and its extremity has dilated forming a vesicle which extends freely into the exocœlom, while the narrower allantoic stalk is attached beneath the embryo, just back of the attachment of the yolk-sac, from the cavity of which the allantoic cavity is now clearly marked off.

As the allantois grows out it comes immediately into relation with the inner surface of the chorion, in the region where the chorionic ectoderm has become trophodermic (Fig. 176, B), and since the trophoderm is the beginning of the placental structure, the allantoic stalk thus becomes the direct pathway between the embryo and the placental region.

During the tenth to twelfth days the allantois expands rapidly beneath the chorionic trophoderm, its mesodermal wall thickens and in it a rich vascular network is developed (Fig. $176, C)$. Blood vessels have been present in the allantoic mesoderm from a very early period, and by the tenth day there are present a pair of umbilical arteries, and a pair of umbilical veins, having relations similar to those already described in the human embryo. It is through the allantoic blood vessels, therefore, that the embryonic circulation is related with the placental, and in the rabbit this appears to be the only important function of the whole allantoic structure.

In man the history of the allantois is a very different story. Here, as in all Primates, the primitive connection of the hinder end of the embryo with the chorion is never interrupted, and this connection, known as the body stalk (see above), composed of mesoderm, may be regarded as the modified equivalent of the allantoic stalk of such a form as the rabbit. Into this body stalk there early extends a small tubular outgrowth from the 
endodermal lining of the yolk-sac, from which at this time the hind-gut is not distinguishable (Fig. 161, D, E).

The essential relation thus established is never extensively altered. When the hind-gut forms, it is dorsal or posterodorsal to the opening of the allantoic canal, and as the body stalk elongates the allantoic canal continues to extend up through it, finally reaching the region of the chorionic mesoderm (Fig. 179). The allantois never expands into a free vesicle in the exocœlom, but remains as a vestigial structure, wholly embedded within the tissues of the body stalk, or of what is later the umbilical stalk (see above). The endodermally lined allantoic canal within the umbilical cord, remains present in this condition throughout the fotal period. As the ventral body wall of the embryo is formed it encloses the proximal portion of the allantoic stalk, and this becomes enlarged, forming the rudiment of the urino-genital sinus and the urinary bladder; between this and the body wall it is reduced to a solid strand of connective tissue called the urachus.

The blood vessels of the allantois (umbilical arteries and veins) remain typically developed here, in spite of the vestigial character of the endodermal portions of the allantois, and as in the rabbit these are the only functional elements of the whole allantoic structure. The vessels are very large and form a very rich network in the placental region, beyond the limits of which the chorion becomes almost non-vascular, although in earlier stages the entire chorion is vascular.

Only among the higher Primates is the allantois vestigial to such a degree; and it is not often that it has as limited an extent as in the rabbit. In other forms, such as the Lemurs, Carnivors, and Ungulates, the allantois extends completely around the inner surface of the chorionic vesicle; among the Ungulates this seems to be correlated with the simple type of placenta (see below). In such cases, and in some other instances where the allantois is more nearly limited to a definite placental region, a definite allantoic cavity is present, small and compressed in forms like the sheep and pig, or large and filling a large part of the cavity of the entire vesicle. 


\section{The Placenta}

Among the Eutherian Mammalia placentation may be defined as an intimate relation between a portion of the uterine mucosa and a part or the whole of the chorionic membrane (trophoblast) of the blastodermic vesicle. This relation may involve merely the close apposition of these two tissues, or their actual fusion. Further, in order that this relation may be effective in the nutrition of the embryo, which is in fact its whole raison d'être, the blood vessels of the allantois become closely associated with the related chorionic and maternal tissues.

All of the structures thus associated in effecting the nutritive, respiratory and excretory interchanges between embryo and maternal organism, may collectively be termed the placenta. It is thus immediately evident that the placenta is a complicated structure and one that is extremely variable, including as it does, these several elements, themselves individually variable.

Many of the essential facts regarding the establishment and the grosser morphological relations of the placenta have been mentioned in other connections. In the section on the impiantation of the "ovum" we have seen that the earliest step in placentation is to be found in the relation established between the chorionic ectoderm, whether trophoblast or trophoderm, and the uterine wall (Fig. 175). This is followed considerably later by the vascularization of the chorion thus related to maternal tissues, by the blood vessels of the yolk-sac, as in certain Marsupials (Fig. 174), or of the allantois, as in the Ungulates and rabbit (Fig. 176), or by the vessels of the chorionic mesoderm itself, as in man.

Upon the character and completeness of the relation between chorionic ectoderm and the uterine tissues depends the fundamental character or type of the placenta developed later. Among most of the Marsupial Mammalia the surface of the chorion retains its smooth surface and is ordinarily not vascularized, either by the yolk-sac or allantois, and since it forms 
only simple contact with the uterine wall, nutritive interchanges between embryo and parent are carried on only with some difficulty. These forms have been termed the achoria or aplacentalia, although strictly speaking these terms are misnomers, for a simple chorion is present and a placental relation does exist, although to a very limited degree.

All of the Eutherian Mammalia may then be termed choriata or placentalia, and it is characteristic of these forms that upon the outer surface of the chorion there develop elevations or papillæ known as the villi (Figs. 153, 161, 184).

The villi are the organs of primary importance in effecting the nourishment of the embryo, and the essential characters, as well as many of the minor characters of the placenta, depend upon the form, mode of distribution, and other characteristics of the villi.

The chief variations in the characteristics of the villi may be enumerated as follows: they may be trophoblastic or trophodermic in origin; they may be simple papillæ or complexly branching, dendritic outgrowths; they may develop very early in embryonic history or very late after a considerable period of intra-uterine life; they may be almost non-vascular or very highly vascularized from the embryonic circulation (umbilical arteries and veins); they may be distributed quite uniformly over the greater part of the chorionic surface or definitely grouped and restricted to certain areas; when restricted they may be grouped in definite patches or cotyledons, scattered over the chorion, like polka-dots, or they may be restricted to certain general zones or bands, or to single large circular areas, or arranged in still other ways; they may be in contact with the maternal mucous epithelium lining the uterine cavity, or with its connective tissue stroma, or with the endothelium of the uterine vessels, or actually bathed directly in the maternal blood stream.

Several classifications of placentæ have been formulated; based upon one or another of these conditions. While none of these represents a natural classification, we may outline certain of the more important, as a convenient way of stating the 
essential facts of placental arrangement. The earlier classifications, such as those of Owen, Huxley and Kölliker, based upon the type of villous distribution and the degree of intimacy between the villi and the uterine mucous membrane, may be summarized as follows, following Hertwig in the main:

I. Achoria. Chorion with few or no villi. Monotremes and Marsupials.

II. Choriata. Chorion with many villi.
A. With uniformly distributed villi, not intimately related Ruminants (e.g., horse, pig, camel, deer, etc.).
B. With villi localized in definite areas, and more or less closely related with maternal tissues. The true Placentalia.
1. Villi in numerous small patches or cotyledons. Cotyle- donary placenta. Ruminants.
2. Villi in a band or zone around the chorion. Zonary placenta. Carnivora.
3. Villi in a single large circular area. Discoid placenta. Insectivors, Bats, Rodents, higher Primates including man.

The terms deciduate and non-deciduate in the classification above require a word of explanation. In several groups of Mammals the epithelium of the mucous membrane lining the uterine cavity becomes greatly thickened during pregnancy, or preceding menstruation. This thickening during pregnancy is termed the decidua, and usually the relations of the villi to the decidua are such that the separation of the placenta at parturition involves a certain loss of maternal tissue (deciduata). In other forms, however, the uterine epithelium shows no such proliferation, and the chorionic villi, at parturition are simply withdrawn from the pits in the mucosa where they have been lodged, and no destruction of maternal tissues results (non-deciduata). An intermediate condition, known as contra-deciduate, is found in the mole where, although a decidua is formed, the placental tissues are not lost at parturition, but are absorbed by the walls of the uterus.

A more recent and more detailed classification of placental arrangement, again based upon the morphological arrangement of the villi, is that of Strahl, as follows: 
I. Mammalia ovipara. Monotremata.

II. Mammalia vivipara.

A. Achoria (Aplacentalia). Most Marsupialia (the exceptions are Perameles, Dasyurus, and perhaps Phascolarctos).

B. Choriata (Placentalia).

1. Semiplacentalia (Partial placenta). Chorionic and uterine structures in close apposition but not fused; simple separation at parturition.

a. Semiplacenta avillosa. Chorion without villi. Perameles, Dasyurus.

b. Semiplacenta diffusa. Simple villi uniformly distributed. Horse, pig, tapir, hippopotamus, camel, deer, whale, Manis, Lemurs.

c. Semiplacenta multiplex. Villi in groups or cotyledons. (Cotyledonary placenta.) Ruminants.

d. Semiplacenta zonaria. Villi in a zone or band around the chorion. Only in the Dugong (Halicore).

2. Placentalia vera (Complete placenta). More or less complete fusion of chorionic and uterine tissues, involving tissue destruction at parturition.

e. Placenta zonaria. Placental fusion in the form of a broad band or zone, completely around the chorion. Most Carnivora. In Hyrax and the elephant combined with cotyledons.

f. Placenta zono-discoidalis. Placental fusion in the form of an incomplete band or zone. Mustelidee (e.g., marten, weasel, ferret, etc.).

g. Placenta discoidalis. Placental fusion in the form of a circular disc, usually simple in outline, though sometimes lobed. Insectivors, bats, Rodents, Tarsius, apes, man.

Of the many other classifications of placental types we may mention only one, that of Grosser, based upon an entirely different relation, namely, the extent of the erosion of the maternal uterine tissues effected by the trophodermal cells of the chorion. From this point of view placentæ are arranged in four groups, as follows:

I. Placenta epitheliochorialis. All maternal tissues retained, uneroded. Chorionic epithelium (of the villi) in contact with the uterine epithelium. Pig. 
II. Placenta syndesmochorialis. Uterine epithelium nearly or wholly eroded. Chorionic epithelium in contact with the connective tissue of the uterine mucosa. Ruminants.

III. Placenta endotheliochorialis. Epithelium and connective tissue of the uterine mucosa eroded. Chorionic epithelium in contact with the endothelial walls of the uterine blood vessels. Carnivora.

IV. Placenta hæemochorialis. Uterine epithelium, connective tissue, and vascular endothelium eroded. Chorionic epithelium in contact with maternal blood stream. Man.

Variation in the extent of the erosion of the maternal tissues determines to some extent also the character of the nutritive substances absorbed or resorbed by the chorionic epithelium. The nutritive materials taken into the fotal circulation are of two classes. (A) Food substances already dissolved in the maternal blood. These, known as homotrophe, may pass by diffusion or by active resorption directly into the embryonic circulation. Obviously this process is easier, and this type of nutritive substance more important, in those placentæ where the chorionic epithelium is more closely related with the maternal blood (Placenta endotheliochorialis and Pl. hæmochorialis). (B) Products of the uterine mucous membrane including the secretion of the uterine glands, the products of the erosive or dissolving action of the trophoderm, and the resultant extravasated blood. This is known as the embryotrophe or pabulum. Frequently these materials undergo a sort of digestive process before their absorption into the embryonic circulation. In forms whose chorionic (villous) epithelium is less closely related to the maternal blood stream (Pl. epitheliochorialis, $\mathrm{Pl}$. syndesmochorialis) the embryotrophe is the more important source of nutrition. In the other types it may be of great importance during the early period of development, and gradually give place to the hæmotrophe as the relation between the villous and uterine blood streams becomes more intimate.

With these general facts regarding the variety of placental arrangement in mind, we may describe the development and structure of but a single type-the human placenta (Placenta discoidalis, hæmochorialis). (For a description of the placental 
relations in other forms, the student may be referred to Marshall's "Physiology of Reproduction.")

The very early stages in the history of the human ovum are not yet known, but from comparisons with other similar forms, and from the conditions of the youngest embryos known (see Peters, Bryce-Teacher) the characters of the early human blastodermic vesicle may be inferred with a high degree of probability. It is entirely probable that the entire trophoblastic surface of the vesicle becomes trophodermic (Fig. 161), and digests or erodes the uterine mucosa all around itself, when it becomes attached to the uterine wall after entering the uterine cavity. This attachment and implantation of the "ovum" usually takes place on the anterior (upper) wall of the cavity, between the openings of the oviducts, in what is known as the fundus of the uterus. Attachments to other parts of the wall are not infrequent, however, and do not affect the normality of development.

The "ovum" or vesicle apparently eats its way a short distance into the mucosa, which closes behind it, and by destroying the adjacent tissues becomes surrounded by a narrow space filled with fluid-extravasated blood and the products of erosion (embryotrophe). This space is the rudiment of what is later known as the intervillous cavity. The uterine cells in this region then begin to proliferate rapidly, so that as the blastodermic vesicle enlarges it remains covered with a distinct layer of the aterine mucosa; this, as we shall see, is the beginning of the decidua capsularis or reflexa. In some of the Rodents the injury of the mucosa, when combined with the presence of an internal secretion from the ovary (or corpus luteum) serves as the stimulus to this proliferation (L. Loeb).

The chorionic villi are formed very early and at first develop all over the surface of the vesicle. Some of the villi simply extend freely into the intervillous cavity, while others grow across it and reach the undisturbed tissues of the uterine mucosa, to which they become definitely attached as the fixation villi.

Before tracing further the history of the villi and the for- 
mation of the placenta, we must consider some facts regarding the general epithelial lining of the uterine cavity (Fig. 182). During pregnancy this epithelium is not sloughed off as it is during menstruation; on the contrary it thickens rapidly and considerably, over the entire wall of the uterine cavity forming the decidua. Its later history varies in different regions. That part of the decidua not directly related with the blastodermic vesicle, and therefore its greater part, is known as the decidua vera. The region covering the vesicle and separating it from the uterine cavity is the decidua capsularis or decidua reflexa; while the portion beneath the vesicle, between it and the muscular layer of the uterine wall, is the decidua basalis or decidua serotina.

As the decidua vera thickens, during the early phases of pregnancy, its vascularity increases and the uterine glands within it elongate, becoming branched and anastomosing, extending down into the deeper layers of the mucosa. 'Toward their openings upon the surface of the mucosa they dilate considerably and the connective tissue matrix in which they are embedded is correspondingly reduced, so that a superficial layer of the decidua may be distinguished, as the spongy layer. The deeper region is known as the compact layer; here the glands do not dilate and the decidual cells multiply and enlarge. The decidua vera reaches its maximum development during the second or third month of development, when it may be $6-10 \mathrm{~mm}$. in thickness; degenerative changes have already begun at this time, however.

At first the structure of the decidua capsularis is not markedly unlike that of the decidua vera, save where all its structure has been destroyed by the entering vesicle, but its glands soon atrophy. As the blastodermic vesicle rapidly enlarges the decidua capsularis becomes extended, and by the fifth month it is pushed out into contact with the decidua vera, and the original cavity of the uterus is obliterated. The decidua capsularis now becomes non-vascular, gradually thins out, and finally disappears completely, leaving the chorionic surface of the vesicle in contact with the decidua vera. But this too 


\section{OUTLINES OF CHORDATE DEVELOPMENT}

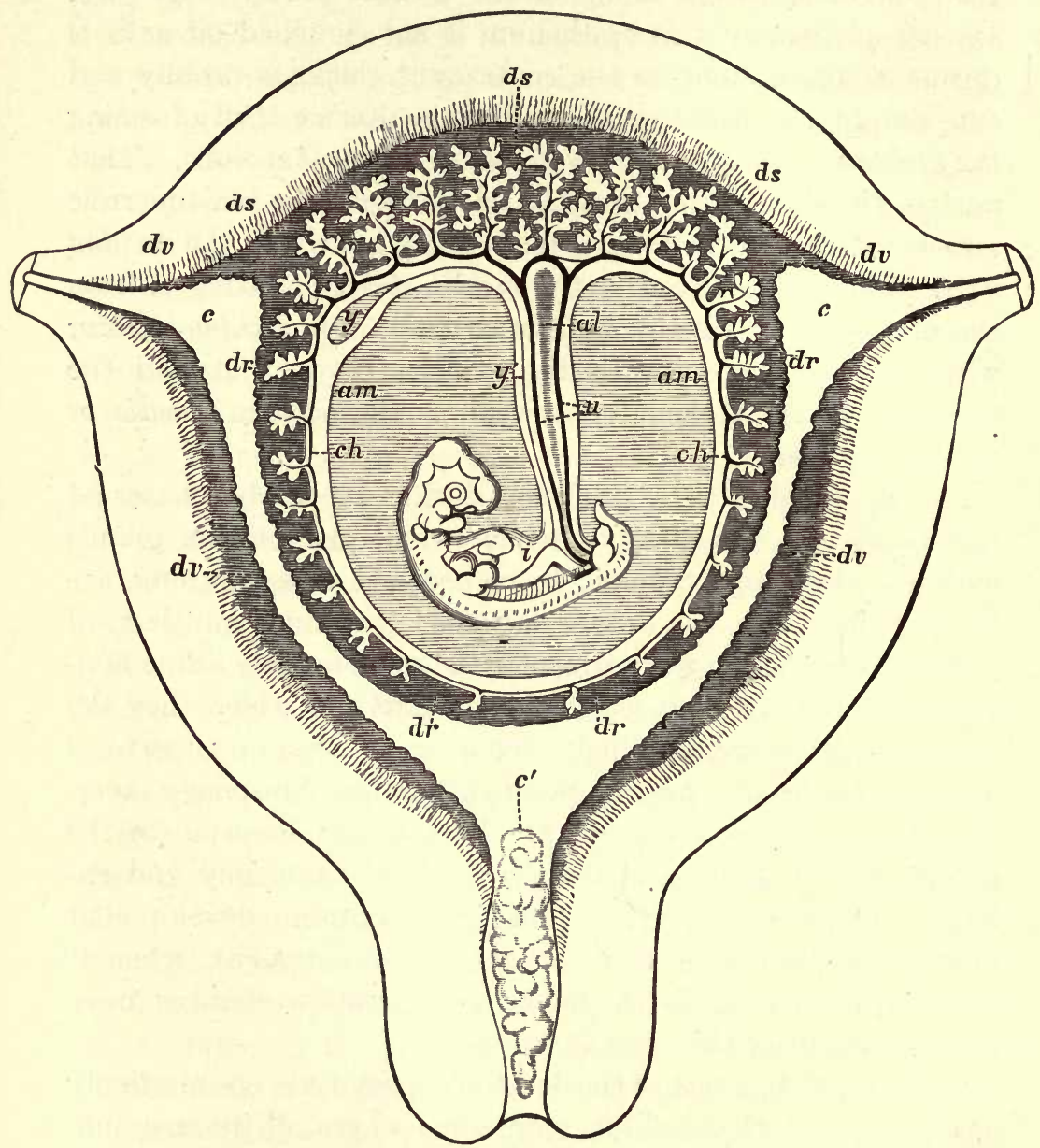

Fig. 182.-Diagrammatic section through the gravid human uterus and the embryo at the seventh or eighth week. From Quain's Anatomy, after Allen Thomson. al, Allantois; am, amnion; $c, c$, openings of the oviducts (Fallopian tubes) into the uterine cavity; $c^{\prime}$, cervix filled with mucous plug (the reference letters $c, c, c^{\prime}$, are placed in the cavity of the uterus; $c h$, chorion with vascular villi growing into the decidua capsularis and decidua basalis; in the decidua capsularis the villi are becoming atrophied (chorion læve); $d r$, decidua capsularis; $d s$, decidua basalis; $d v$, decidua vera; $i$, embryo; $u$, umbilical cord; $y$, yolk-sac; $\boldsymbol{y}^{\prime}$, yolk-stalk; 
has partly degenerated; it has become less vascular, its superficial epithelium and spongy layer have disappeared, and there remain only a part of its compact layer and the deeper portions of the uterine glands.

The early history of the decidua basalis is not essentially unlike that of the decidua vera, save that its glands disappear, but later, instead of exhibiting any phenomena of atrophy its

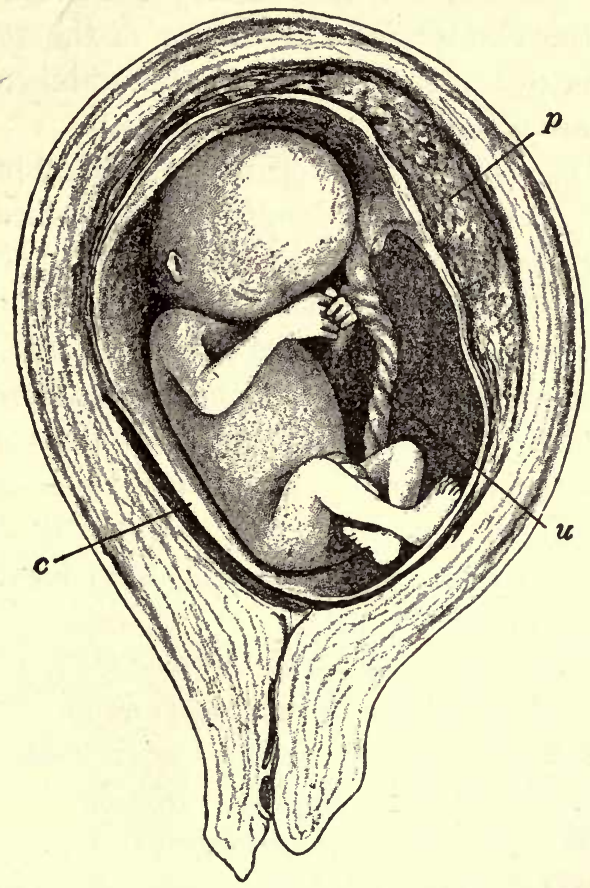

Fig. 183.-Human embryo of the fourth month in utero, showing the arrangement of the membranes and placenta. After Strahl. $c$, Chorion and amnion; $p$, placenta; $u$, umbilical cord.

importance increases; its capillaries dilate, its decidual interglandular cells continue to multiply, and it takes an essential part in the formation of the placenta, forming in fact the whole maternal portion of this organ. To understand the origin and structure of the placenta we must return to the early blastocyst, upon the surface of which the villi are forming. The villi are formed at first wholly of the ectodermal tropho- 
derm, and as we have seen, extend into or across the intervillous cavity from all surfaces of the blastodermic vesicle. As the vesicle enlarges the villi on the sides toward the decidua capsularis disappear along with the capsularis itself, and the smooth chorion thus left is the chorion love, which then comes into contact with the decidua vera on the opposite side of the uterus (Figs. 182, 183). The villi in relation with the decidua basalis alone remain, forming then the chorion frondosum; this part of the chorion is in the region of the attachment of the body stalk of the embryo, where the umbilical (allantoic) blood vessels are distributed.

The villi of the chorion frondosum enlarge and branch, many of them finally assuming a dendritic appearance, extending irregularly through the intervillous cavity (Fig. 184). While at first simply ectodermal, the mesoderm of the chorion soon pushes out into them and becomes extremely vascular. Even before this the superficial cells of the trophoderm (and villi) have fused into a syncytial layer known as the syncytiotrophoderm, which is lined internally, for a time, by a simple epithelium of the cells of Langhans, also apparently derived from the chorionic ectoderm. The vascular mesoderm then forms the core of the villus (Fig. 184).

As the villi grow out they continue to erode the substance of the decidua basalis, and the intervillous cavity is consequently enlarged and filled with maternal blood from the opened capillaries and vessels of the region. Finally the whole intervillous space is occupied by large vascular sinuses or lacunæ, and when the Langhans cells disappear, the embryonic and maternal blood streams are separated only by the endothelium of the villous capillaries and the syncytiotrophoderm covering them (placenta hæmochorialis). Of course the two blood streams are nowhere in direct communication.

The formation of the placenta has now been described. It is a structure combined from two distinct sources, a maternal portion, the decidua basalis, and an embryonic portion, the chorion frondosum; the two are separated by the intervillous, or as we may now say, intra-placental cavity, filled with maternal 

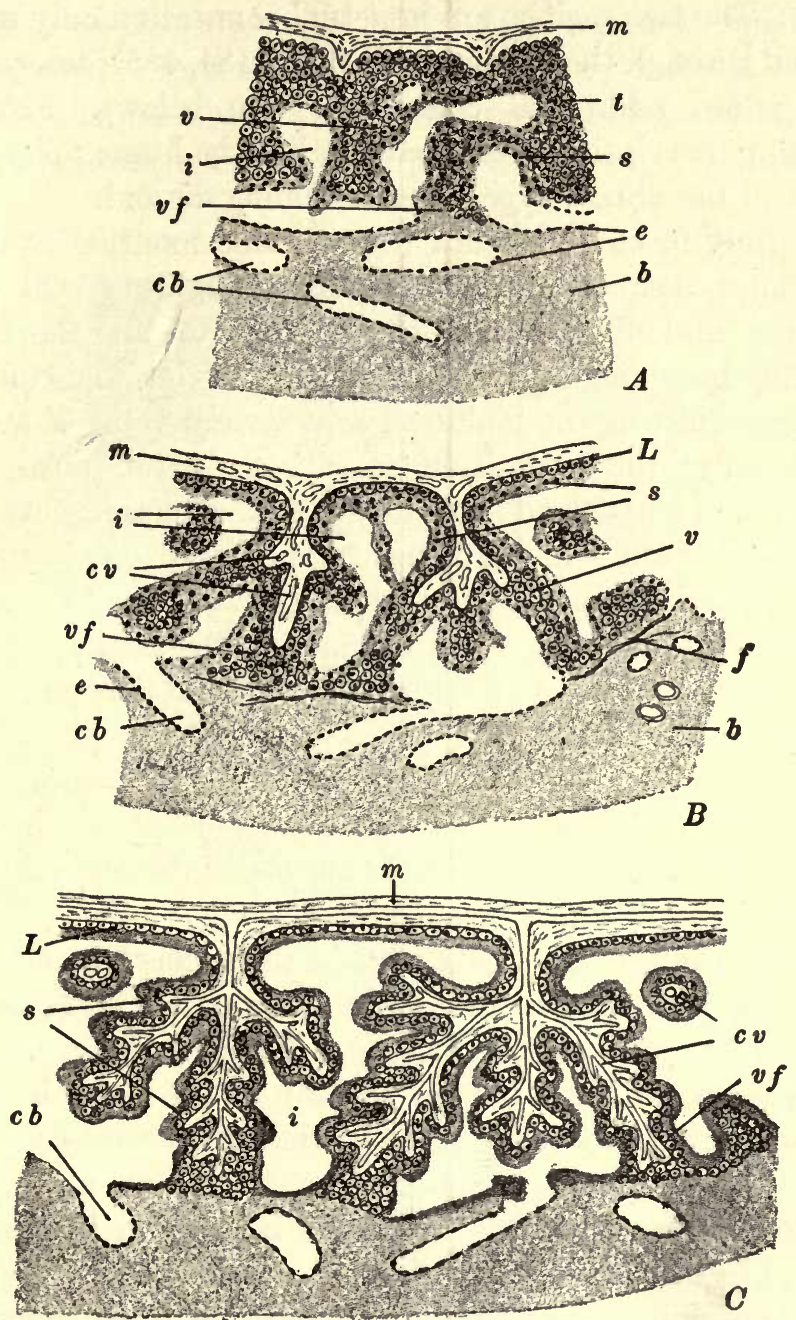

Fig. 184.-Diagrams illustrating the developmene of the villi in the human placenta. A. B, After Peters; $C$. after Bryce. $A$. Chorionic mesoderm just beginning to extend into the villi. $B$. Mesoderm invading the villi which are now branched. Layer of Langhans cells forming beneath the syncytiotrophoderm. C. Continued branching of the villi, all now covered only by the syncytiotrophoderm and the single layer of Langhans cells. $b$, Decidua basalis; $c b$, capillaries of the decidua basalis; $c v$, capillaries of the villi; $e$, endothelium of the maternal capillaries; $f$, fibrin deposited at the junction of the trophoderm and decidua basilis; $i$, intervillous cavity filled with maternal blood; $L$, Langhans cells; $m$, chorionic mesoderm; $s$, syncytiotrophoderm; $t$, trophoderm; $v$, villi; vf, fixation villi. 
blood. The two regions are in actual connection only marginally and through the fixation villi (Figs. 184, 185); later certain septa afford additional connection (see below). From the preceding description it is apparent that the human placenta is at first of the diffuse type, later becoming discoid.

The fully formed placenta (i.e., at nine months) is an oval or circular disc of irregular outline, roughly $16-20 \mathrm{~cm}$. in diameter, and about $3 \mathrm{~cm}$. in thickness toward the middle, gradually becoming thinner toward the margin. The umbilical cord transmitting the umbilical arteries and veins, is attached eccentrically; the cord itself measures, at full term, about $50-60 \mathrm{~cm}$. in length and has a diameter of something over $1 \mathrm{~cm}$. The villous surface of the placenta is marked out in irregular areas by partitions or septa, extending upward from the decidua basalis, some of which, when the placenta is in situ, attach to the chorion and so divide the intra-placental or intervillous cavity into chambers. These areas are known as the loculi, or sometimes as cotyledons, and they must not be confused with the groups of villi of the cotyledonary placenta of the Ruminants. The villi extend from the surface of the septa as well as from the general chorionic surface.

Examination of Fig. 185, illustrating diagrammatically a section through the placenta at its margin, will serve to make clear the structure of the placenta. The embryonic surface of the placenta, which is toward the top of the page, is seen to be covered with the thin amnion; that is, the exocœlom has been obliterated by the apposition of the amnion and chorion, and the cavity of the embryonic vesicle is therefore the amnionic cavity, filled with the amnionic fluid.

The chorion presents first a connective tissue membrane (close vertical ruling) beneath which are the vascular villi (horizontal ruling) which in reality form a dense spongy mass. The vessels extend through the complexly branched villi, both types of which are shown. Toward the close of gestation the fixation villi become very loosely attached to the decidua basalis, in preparation for the separation of the placenta at parturition. The villi are for the most part freely suspended 
in the intra-placental cavity filled with maternal blood (black in Fig. 185). Beneath this are the greatly reduced layers of the decidua basalis, traversed by the maternal blood vessels communicating with the intra-placental cavity. In the deeper
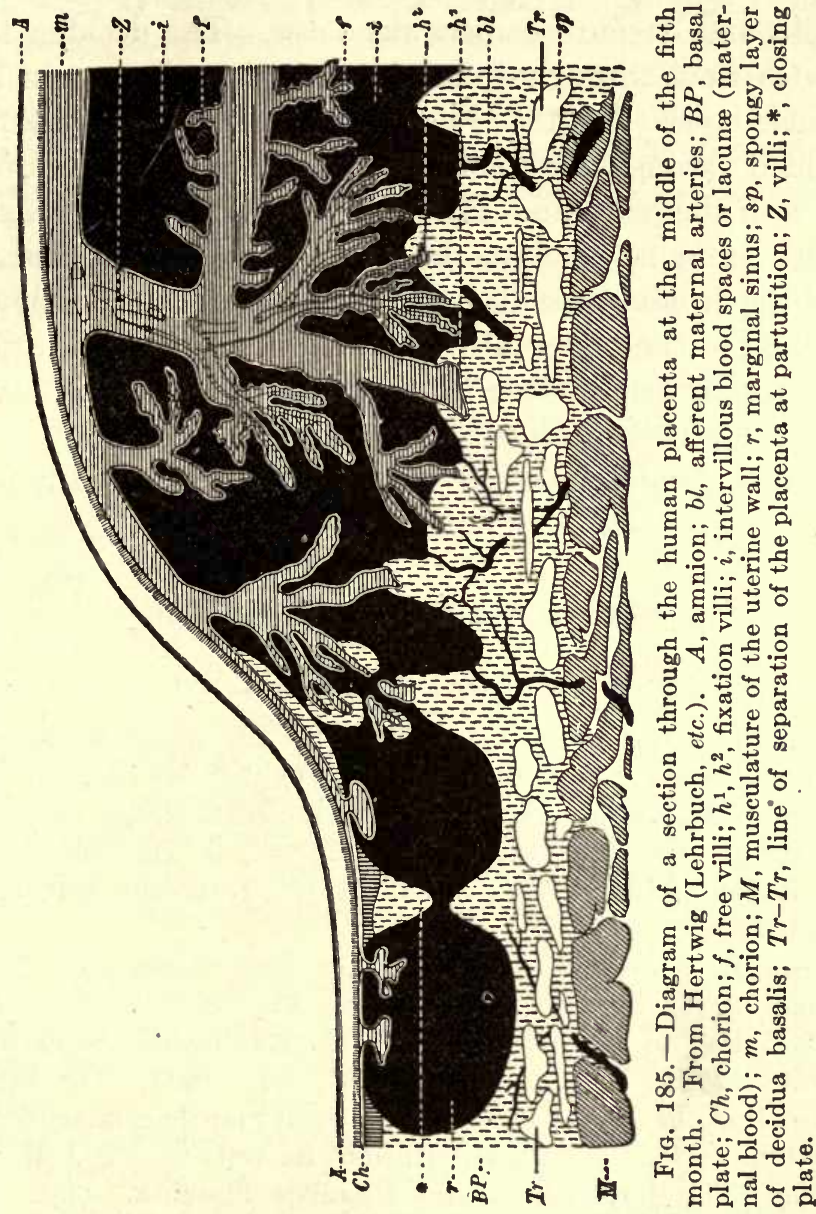

layer of the decidua basalis portions of the uterine glands remain present. Finally the decidua basalis rests upon the muscular layer of the uterine wall (oblique ruling).

Peripherally the irregular chambers of the intra-placental cavity are nearly or quite free from villi, and form the marginal 
sinus. This is incompletely separated from the remainder of the cavity by an irregular septum known as the closing plate.

At parturition, when the amnion has been ruptured and the amnionic fluid and fotus have been expelled by the contractions of the uterine musculature, the amnion itself and the placenta and deciduæ come away later. The decidua basalis separates first through the spongy region, just outside the glandular layer (Fig. 185, $T r-T r$ ). The decidua vera similarly is divided through the remains of its spongy layer and comes away with the placenta. The so-called "after-birth" therefore includes amnion, chorion, decidua vera, placenta, and a portion of the decidua basalis. The hemorrhage which follows this separation of the maternal tissues is diminished by the general contraction of the uterine walls. The entire uterine cavity is then lined with the deeper decidual layer containing vestiges of uterine glands, and from this tissue the decidua is formed anew.

\section{REFERENCES TO LITERATURE}

\section{CHAPTER VI}

This list contains but a very few of the important works bearing directly upon the topics of the chapter. For very full references to the literature of Mammalian development see especially the works in the following list, by O. Hertwig, Handbuch, etc., O. Hertwig, Lehrbuch, etc., F. Keibel and F. P. Mall, Handbuch, etc., Hubrecht, Marshall, and Minot, 1893.

Assheton, R., A Re-investigation into the Early Stages of the Development of the Rabbit. Q. J. M. S. 37. 1894. On the Causes which lead to the Attachment of the Mammalian Embryo to the Walls of the Uterus. Q. J. M. S. 37. 1894. The Primitive Streak of the Rabbit; the Causes which may determine its Shape, and the Part of the Embryo formed by its Activity. Q.J.M.S. 37. 1894. The Morphology of the Ungulate Placenta. Phil. Trans. Roy. Soc. 198. 1906. The Segmentation of the Ovum of the Sheep, with Observations on the Hypothesis of a Hypoblastic Origin for the Trophoblast. Q. J. M. S. 41. 1898. The Development of the Pig during the First Ten Days. Q. J. M. S. 41. 1898. Early Ontogenetic Phenomena in Mammals. Q. J. M. S. 54. 1909. 
VAn Beneden, E., Recherches sur l'embryologie des mammifères-La formation des feuillets chez le Lapin. Arch. Biol. 1. 1880. Recherches sur les premiers stades du développement du Murin (Vespertilio murinus). Anat. Anz. 16. 1899. (Brachet, ed.) Recherches sur l'embryologie des Mammifères. I. De la segmentation, de la formation de la cavité blastodermique et de l'embryon didermique chez le Murin. Arch. Biol. 26. 1911. II. De la ligne primitive, du prolongement céphalique de la notochorde et du mésoblaste chez la lapin et chez le murin. Arch. Biol. 27. 1912.

Van Beneden, E. and Julin, C., Observations sur la maturation, la fécondation et la segmentation de l'œuf chez les Cheiropteres. Arch. Biol. 1. 1880.

Bonnet, R., Beiträge zur Embryologie des Hundes. I. Anat. Hefte. 9. 1897. II. Anat. Hefte. 16. 1901. III. Anat. Hefte. 20. 1902.

Bryce, T. H. and Teacher, J. H., Contributions to the Study of the Early Development and Imbedding of the Human Ovum. I . An Early Ovum imbedded in the Decidua. Glasgow. 1908.

Burckhard, G., Die Implantation des Eies der Maus in die Uterusschleimhaut und die Umbildung derselben zur Decidua. Arch. mikr. Anat. 57. 1901.

Danies, J. F., Observations on the Period of Gestation in White Mice. Jour. Exp. Zool. 9. 1910.

Eternod, A.-C.-F., L'œuf humain. Implantation et gestation, trophoderm et placenta. Genèva. 1909.

Felix, W., See Keibel-Mall, Handbuch, etc.

Grosser, O., Vergleichende Anatomie und Entwicklungsgeschichte der Eihäute und der Placenta, mit besonderer Berücksichtigung des Menschen. Wien. 1909. See also Keibel-Mall, Handbuch, etc.

Guyer, M. F., Accessory Chromosomes in Man. Biol. Bull. 19. 1910.

Heape, W., The Development of the Mole (Talpa Europea). The Formation of the Germinal Layers, and Early Development of the Medullary Groove and Notochord. Q. J. M. S. 23. 1883. 27. 1887.

Hertwig, O., Lehrbuch der Entwickelungsgeschichte des Mensch und den Wirbeltiere. 9 Aufl. Jena. 1910. (Editor.) Handbuch der vergleichenden und experimentellen Entwickelungslehre der Wirbeltiere. Jena. 1906.

Hrll, J. P., The Placentation of Perameles. (Contributions to the Embryology of the Marsupialia.-I.) Q. J. M. S. 40. 1897. On the Fœtal Membranes, Placentation and Parturition of the Native Cat (Dasyurus viverrinus). Anat. Anz. 18. 1900.

His, W., Unsere Körperform und das physiologische Problem ihrer 
Entstehung. Leipzig. 1874. Anatomie menschlicher Embryonen. I-III. Leipzig. 1880-1885.

Hochstetter, F., Bilder der äusseren Körperform einiger menschlicher Embryonen aus den beiden ersten Monaten der Entwickelung. München. 1907.

Hubrecht, A. A. W., Early Ontogenetic Phenomena in Mammals and their Bearing on our Interpretation of the Phylogeny of the Vertebrates. Q. J. M. S. 53.1908.

JACKson, C. M., On the Prenatal Growth of the Human Body and the Relative Growth of the Various Organs and Parts. Amer. Jour. Anat. 9. 1909.

Keibel, F., Zur Entwickelungsgeschichte des Rehes. Verh. Anat. Gesell. Tübingen. 1899. Die Gastrulation und die Keimblattbildung der Wirbeltiere. Ergeb. Anat. u. Entw. 10. 1900 (1901). See also Hertwig's Handbuch, etc.

Keibel, F. and Elze, C., Normentafeln zur Entwicklungsgeschichte des Menschen. Jena. 1908.

Keibel, F. and Mall, F. P., (Editors and contributors). Handbuch der Entwicklungsgeschichte des Menschen. Leipzig. 1910, 1911. American Edition, Manual of Human Embryology. Philadelphia. 1910, 1912.

Kirkham, W. B., Maturation of the Egg of the White Mouse. Trans. Connecticut Acad. Arts and Sciences. 13. 1907. (See also Biol. Bull. 12. 1907.) Ovulation in Mammals, with Special Reference to the Mouse and Rat. Biol. Bull. 18.1910.

KöLliKer, A. von, Entwicklungsgeschichte des Menschen und der höheren Thiere. 2 Aufl. Leipzig. 1876, 1879. Grundriss der Entwicklungsgeschichte des Menschen und der höheren Thiere. 2 Aufl. Leipzig. 1884.

Kollmann, J., Die Körperform menschlicher normaler und pathologischer Embryonen. Arch. Anat. Physiol. Suppl. 1889. Handatlas der Entwickelungsgeschichte des Menschen. Jena. 1907.

Kolster, R., Weitere Beiträge zur Kenntnis der Embryotrophe. IV. Zur Kenntnis des Chorionepithels. Anat. Hefte. 40. 1909.

Lane-Claypon, J. E., On the Origin and Life-history of the Interstitial Cells of the Ovary of the Rabbit. Proc. Roy. Soc. B. 77. 1905.

Loeb, L., Beiträge zur Analyse des Gewebwachstums. III. Die Erzeugung von Deciduen in dem Uterus des Kaninchens. Arch. Entw.-Mech. 27. 1909. The Function of the Corpus Luteum, the Experimental Production of the Maternal Placenta, and the Mechanism of the Sexual Cycle in the Female Organism. Medical Record. 77. 1910.

Long, J. A., The Living Eggs of Rats and Mice, etc. Univ. of California Publications in Zool. 9. 1912. 
Long, J. A. and Mark, E. L., The Maturation of the Egg of the Mouse. Publ. Carnegie Institution. Washington. No. 142. 1911.

LoNGLey, W. H., The Maturation of the Egg and Ovulation in the Domestic Cat. Amer. Jour. Anat. 12. 1911.

McMurrich, J. P., The Development of the Human Body. 3rd. ed. Philadelphia. 1909.

MaLl, F. P., Early Human Embryos and the Mode of their Preservation. Bull. Johns Hopkins Hospital. 4. 1893. See also Keibel-Mall, Handbuch, etc.

Marshald, F. H. A., The Physiology of Reproduction. London. 1910. Melissinos, K., Die Entwickelung des Eies der Mäuse von den ersten Furchungs-Phænomen bis zur Festsetzung der Allantois an der Ectoplacentarplatte. Arch. Mikr. Anat. 70. 1907.

Minot, C. S., Human Embryology. New York. 1892. A Bibliography of Vertebrate Embryology. Mem. Boston Soc. Nat. Hist. 4. 1893. A Laboratory Text-Book of Embryology. 2nd. ed. Philadelphia. 1911.

NAGEL, W., Das menschliche Ei. Arch. mikr. Anat. 31. 1888.

Newman, H. H., The Ovum of the Nine-banded Armadillo. Growth of the Ovocytes, Maturation and Fertilization. Biol. Bull. 23. 1912.

Peters, H., Ueber die Einbettung des menschlichen Eies und das früheste bisher bekannte menschliche Placentationsstadium. Leipzig and Wien. 1899.

RABL, C., Die Entwickelung des Gesichtes. Das Gesicht der Säugethiere. Leipzig. 1902.

Rejsek, J., Anheftung (Implantation) des Säugetiereies an die Uteruswand, insbesondere des Eies von Spermophilus citillus. Arch. mikr. Anat. 63. 1904.

Retzius, G., Weitere Beiträge zur Kenntniss der Spermien des Menschen und einiger Säugethiere. Biol. Unters. N. F. 10. 1902. Zur Kenntnis der Entwicklung der Körperform des Menschen während der fötalen Lebensstufen. Biol. Unters. N. F. 11. 1904.

Robinson, A., Observations upon the Development of the Segmentation Cavity, the Archenteron, the Germinal Layers, and the Amnion in Mammals. Q. J. M. S. 33. 1892.

Rubaschkin, W., Ueber die Reifungs- und Befruchtungs-prozesse des Meerschweincheneies. Anat. Hefte. 29. 1905. Ueber die Urgeschlechtszellen bei Säugetieren. Anat. Hefte. 39. 1909.

Schoenfeld, H., Contribution à l'Etude de la Fixation de l'œuf des Mammifères dans la cavité uterine, et des premiers stades de la Placentation. Arch. Biol. 19. 1903.

Selenka, E., Studien über Entwickelungsgeschichte der Thiere. IV.

Das Opossum. 1887. V. 1. Beutelfuchs und Känguruhratte; zur 
Entstehungsgeschichte der Amnion der Kantjil (Tragulus javanicus) ; Affen Ost-Indiens. 1891. V. 2. Keimbildung des Kalong. Dottersack und Placenta des Kalang. 1892.

Sовотта, J.. Die Befruchtung und Furchung des Eies der Maus. Arch. mikr. Anat. 45. 1895. (For corrections, see Kirkham.) Die Furchung des Wirbeltiereies. Ergeb. Anat. u. Entw. 6. 1896 (1897). Die Bildung der Richtungskörper bei der Maus. Anat. Hefte. 35. 1907.

Soвotta, J. and Burckinard, G., Reifung und Befruchtung des Eies der weissen Ratte. Anat. Hefte. 42. 1910.

Spee, F. Graf von, Beobachtungen an einer menschlichen Keimscheibe mit offener Medullarrinne und Canalis neurentericus. Arch. Anat. Physiol. 1889. Neue Beobachtungen über sehr frühe Entwickelungsstufen des menschlichen Eies. Arch. Anat. Physiol. 1896. Die Implantation des Meerschweincheneies in die Uteruswand. Zeitschrift für Morphologie und Anthropologie. 3. 1901.

Strahl, H., See Hertwig's Handbuch, etc.

Turner, W., Lectures on the Comparative Anatomy of the Placenta. Edinburgh. 1876.

WidaкошісH, V., Ueber die erste Bildung der Körperform bei Entypie des Keimes. Zeit. wiss. Zool. 94. 1910.

Winiwarter, H. von, Recherches sur l'ovogenèse et l'organogenèse de l'ovaire des Mammifères (Lapin et Homme). Arch. Biol. 17. 1901. Nachtrag zu meiner Arbeit über die Oogenese der Saugetiere. Anat. Anz. 21. 1902. 


\section{INDEX}

(Pages 1-61 refer to Amphioxus:62-228 to the frog: 229-367 to the chick: 368-454 to the Mammal. Page numbers in heavy-face type refer to illustrations.

Accessory cleavage furrows, 244, 246 Achoria, 438, 439, 440

adolescence, 370

adrenal bodies, 206-207, 207 : 363364

after-birth, 450

age, frog, estimation of, 64, 127-128;

of human embryos, 403,407 air, chamber, 233, 234; - sacs, 332,335

albumen, 233, 234, 241, 288, 289; - formation, 241 ; - later history, $298 \mathrm{ff}$.; - sac, 288, $289,298 \mathrm{ff}$.

alimentary canal, 33: 158 ff.: 269, $326 \mathrm{ff}$., 335

allantois, 280, 287, 288, 289, 291, 295 ff., 296, 297, 335: 400, 402, 428, 430, 431, 434 ff.; — at hatching, 300 ; blood vessels of, 299 : 435,436 ; — functions of, 295 : 434 ; fusions of, 297-298; human, 400, 430, 435-436, 444; - later history, 430, 431, $434 \mathrm{ff}$.; - mammalian compared with avian, 435 ; relation with chorion, 430, 431, 435; stalk of, 287, 288, 289, 296, $297,297,348: 430,431$

Allen, 205

amnio-cardiac vesicles, 252, 263, 266, 267, 268, 272, 273

amnion, 280, 287, 288, 289, $292 \mathrm{ff}$., $296,310,351: 444,445$; cavity, 280, 287, 288, 289, 293, 294 : 385, 386, 388, 389, 390, 390, $391,391,392,393,399,400,424$, 444,445 ; - cavity, false, 389 , 391,391 ; cavity, formation in man, $428 \mathrm{ff}$., 430 ; - folds, 262, 283, 292 ff.: 391, 392, 393, $396,425,426$ ff., 426, 427 ; folds, closure of, 293, 296, 297 : $425,426,427$; - folds, head, 262, 292: 425, 427; - later history, $424 \mathrm{ff} ., 428,430$

Amphioxus, outline of life-history, 3 amplexus, 78

ampullæ, 150, 151: 322, 323

anal, membrane, 414, 415; opening, 415,415 ; $\longrightarrow$ plate, 280, 296, 296, 297, 334

annulus tympanicus, 214, 217

anterior, chamber (eye), 317, 321; - gut diverticula, 25, 32-33, 32, 35 ; intestinal portal, 261, 262, 266, 269, 272, 276, 278, 282, 291, 304, 345

anus, 35: 118, 169: 296: 415, 415 aorta, dorsal, 179, 182, 184, 184 : $273,275,276,278,281,291,294$, $329,341,342,342,348: 405$; lateral dorsal, 179, 180, 182 :

272, 291, 292, 341; ventral, 177: 273, 275, 278, 282

aortic arches, 180, 181, 181, 184 :

$283,285,291,329,338,340 \mathrm{ff}$., $341,342,348: 405,405$; carotid, 184, 184: 283, 341, 342 ;

- hyoid, 179; 183: 340, 341;

- mandibular, 179, 183: 275, 278 ; - pulmo-cutaneous, 184, 184 ; — pulmonary, 184: 342, 342 ; - systemic, 184, 184:

341,342

aperture, auriculo-ventricular, 338;

sinu-auricular, 337 ;
urinogenital, $415 \mathrm{ff}$., 415

Aplacentalia, 438, 440

appendicular skeleton, 221-222 aqueduct of Sylvius, 132: 307

arch, see aortic; - branchial, 120, 123, 160, 163, 164: 330: 404, 404, $406,407,408$; - hyoid, 65 , $122,160,160$ : 330 ; - mandibular, $122,160,160,163,164$, 216: 283, 285, 304, 330; pectoral, 221; - pelvic, 221; visceral, 120, 123, 160, 160, 163, 164 : 286, 328 ff.; 404 ff., 404, 406, 408; - visceral, cartilaginous, 211, $216 \mathrm{ff}$., 217, 218

archenteron, $15 \mathrm{ff}$., 16, 21 : 98, 101, 102, 106: 250 
(Amphioxus, pages 1-61; frog, 62-228; chick, 229-367; mammal, 368-454.)

area, opaca, 233, 248, 252, 275: 394 ;

- pellucida, 233, 247, 252, 255,

275: 394 ; - vasculosa, 252 , $255,256,256,275-277,277$, 291: 432; - vitellina, externa and interna, 252, 255, 275-277, 277

arterial system, $179 \mathrm{ff} ., 180,181$, 182, 184 : 291, 340 ff., 341, 348: 405

artery, allantoic, 297; see aorta; basilar, 182 ; - branchial, 40 : 179 ff., 180, 181, 181, 182; - carotid, external and internal, 180, 182, 183: 291, 329, $341,341,342,348$; caudal, 348 ; - cerebral, 182 ; - cœliac, 343, 348; - commissural, 182, 183; - cutaneous, 182, 185; — genital, 343; - intersomitic, 280; - lingual, 182, 183; - mesenteric, 343, 348; - of mandibular and hyoid arches, 179, 182, 183: 275, 278, 340,341 ; omphalomesenteric, 343: 432, 432; — palatine, 182 ; - pharyngeal, 182, 183; pulmonary, 182, 184, 184: 329, $338,339,341,342,348$; renal, 343 ; - sciatic, 343 , 348; - segmental, 280, 342; subclavian, 184: $341,342,343$, 348; - umbilical, 343, 348: 435,436 ; - vertebral, 184 ; - vitelline, $273,283,285,290$, 291, 294, 338, 343: 432, 432

articulation, jaw, 216

asymmetry, of Amphioxus larva, $49 \mathrm{fJ}$.

atrial cavity, 46 ff., 47, 48

atriopore, 47, 49

atrium, see atrial cavity, auricle auditory, capsule, $211 \mathrm{ff}$., 212, 214; - organ, rudiment, 121: 282, 283, 285, 321; - organ, later history, $149 \mathrm{ff}$., 150 : $322 \mathrm{ff}$., 322 ; - ossicles, 324-325; - pit, 282,283 ; - sac, 140,149 , 150: 321 ff., 322, 322, 329

auricle, 176, 177: 283, 285, 291, 310, $337,339,342,348,355$

auriculo-ventricular aperture, 338 axis, of chick embryo, 243

axons, of retinal cells, 320 ; - of spinal nerves, 311

von Baer, 299: 376

basal, ganglia, 306; - plate, of cranium, 211, 212, 213; of placenta, 449 basilar chamber, 150, 151

bat, amnion, 424; blastodermic vesicle, 390 ; cleavage, 381 ; notochordal canal, 397; placentation, 439, 440; trophoblast, 389

beak, 159: 328

beating, of heart, commencement of, 339

belly stalk, 399, 400, 429, 430, 446

between-brain, $129,131,133$ : 283, 303, 304, 306 ff., 310

Bidder's organ, 167

bile duct, 167: 333 ; common, 333

birth, 369

bladder, gall 166, 167, 167 : 333 ; - urinary, 170, 201

blastocœl, 14,16: 93, 94, 96, 98, 102 : $245,245,247,248$

blastoderm, 238, 239, 240, 242-243; - at 30 hours, 275 ; - at time of egg laying, 232,233 ; growth of, $247 \mathrm{ff} . ;-$ unincubated, 250

blastodermic vesicle, $382,383 \mathrm{ff}$., $384,385,386,390,399$; —_ growth of, 384 ; _ - human, 399

blastopore, 16, 18 ff.; 98, 101 ff., 102, 118, 119, 120: 249 ff., 249, 258, 258: 397-398; —_ closure of, 103, 120; - position of, 105,105

blastula, 12, 14, 16: 92, 93 ff., 94, 96, 247, 247, 248: 383; — symmetry of, 96

blood, cells, 256, 273; - corpuscles, 179: 350 ; - islands, 178, 179: 252, 255, 256, 271, 272, 277; - origin, $178-179,178$;

vessels, 46 : $271 \mathrm{ff}$., 273,277 ; vessels, allantoic, 280, 299: 435, 436 ; — - branchial, $179 \mathrm{ff}$., $180 ;--$ origin, 178-179; - rudiments in Amphioxus, 31 ; - yolk-sac, 290 , 291 : 432-433, 432; see also artery, vein

Blount, 246, 247

body, cavity, 124, 170, 173: $350 \mathrm{ff}$., 352; see also cœlom; - form of human embryo, $399 \mathrm{ff} \cdot ;$ Malpighian, 200, 201, 205: 356, $357,359,362$; - pineal, 130: 307 ; — pituitary, 132: 327; - post-branchial, 331,331 ; pseudothyroid, 164 ; — suprapericardial, 163,164 ; - ultimobranchial, 163, 164; - vitreous, 148: 320 ; - wall, 282 ; - 
(Amphioxus, pages 1-61; frog, 62-228; chick, 229-367: mammal, 368-454.)

body, Wolffian, $198 \mathrm{ff}$., 199, 200 : 335, 357, 358, 359, 360

bone, angular, 220; _ dentary, 217,220 ; - dermal, of skull, 214, 219-220; - exoccipital, 214, 215; - frontal, 219;

fronto-parietal, 214, 219;

lateral occipital, 215 ; - maxillary, 214, 219; _ nasal, 214, 219 ; $\longrightarrow$ orbito-sphenoid, 215 ; - palatine, 220; — parasphenoid, 219; — parietal, 219; proötic, 215 ; — - pterygoid, 214 , 220 ; quadrato-jugal, 214, 215,220 ; septo-nasal (intranasal), 219; - sphenethmoid, 215 ; squamosal, 220 ;

vomer, 220

Bonnet, 384

Bouin, 206

Bowman's capsule, 199, 200: 357

Brachet, 79, 86, 136, 179

brain, 25, 28: 118, 121, 166: 262, 266, 271, 278, 303 ff.; 402 ff. See also, flexure, nervous system, prosencephalon, etc.

branchial, arches, $120,123,160,160$, 163, 164: 330 : 404, 404, 406, 407, 408; — blood vessels, $179 \mathrm{ff}$., $180 ;-$ clefts, see gill, clefts; - _ grooves, 65, 122; - pouches, $41: 122,158,160,160,163,164$ : 269, 278, 328 ff., 329

branchiomeric nerves, 135

bronchi, 332

buccal cavity, 42,45: 327-328; cirri, 42, 44, 45

bulbus aortæ, 177, 178; - arteriosus, 176: 282, 285, 291, 304, $310,335,338,339,340$

bursa Fabricii, 334

Bryce-Teacher, 442

Cæcal process, 335

cæcum (cæca), 36, 42, 55; intestinal, 335.

camel, placentation, 439, 440

canal, central, 134: 280, 305, 308; -neurenteric, 16, 21, 25, 28:118, 119, 120: 259: 397, 398, 400, 401; — notochordal, 397-398, 397; 323

semicircular, 150, 151: 322,

capsule, auditory, $211 \mathrm{ff}$., 212, 214 ; — Bowman's, 199, 200: 357; - olfactory, $210,211,212,214$, 215 ; — ovarian, follicular, 376 ; - pronephric, 194, 197; sense, $210 \mathrm{ff}$.
Carnivora, allantois, 434, 436; amnion, 392, 424; implantation, 421; placentation, 439, 440, 441; uteri, 372; yolk-sac, 433

carotid, arch, 184, 184: 283, 341, 342 ; - gland, $163,163,182$, 184,184

cartilage, annular, 213, 217;

basibranchial, 217, 218, 218;

basihyoid, 218; - branchial, 217 ff., 217, 218; _ ceratobranchial, 217, 218, 219; ceratohyal, 217, 218, 218, 219; — hyoid, 217 ff., 217, 218; - infrarostral, 211, 212, 216, 217 ; - intervertebral, 209; - labial, 210, 211, 212, 215; mandibular, 164 ;

Meckel's, 211, 212, 216, 217, 220; - mento-Meckelian, $217 ;-$ mesotic, 212 ; occipital, 212 ; palato-quadrate, 164, 211, 211, 212, 214, 215, 216 ff.; parachordal, 210; - quadrate, 216 ; - suprarostral, 210, 211, 212, 215; - trabecular, 210, 211, 212, 213; — vertebral, 208209,208

cat, chromosomes, 377; fertilized ovum, 380 ; gestation, 369 ; maturation, 380 ; ovulation, 379

cavity, amnionic, see amnion;

body, 124, 170, 173: $350 \mathrm{ff}$;

buccal, 42, 45: 327-328; gonadial, 56, 57, 58; $\stackrel{-}{\longrightarrow}$ interamnionic, 389 , 391; - intervillous, 442, 447, 449; - intraplacental, 446, 449; - opercular, 161,163 ; - parietal, see exocolom; - pericardial, 166, $174,175,180,192: 262,267,275$, $310,350 \mathrm{ff}$., 351 ; - perigonadial, $56 ;-$ peritoneal, 352 ; - pleural, 351; — preoral, 25, 32, 33; — segmentation, $14,16: 93,94,96,99,102: 245$, 245, 247, 248; — subgerminal, $245,245,248$; _ tubo-tympanic, 152 ; - tympanic, 152 : 324,328

central, canal, 134: 280, 305, 308; cells, 244, 244, 245;

nervous system, see nervous system cerebellum, 129, 132, 133: 305, 307 cerebral, hemispheres, $129,131,133$ :

305, 306:- suture, 274, 276;

- vesicle, $25,35,38$

cervical sinus, 406, 407

cervix, uteri, 444

chalazæ, 233, 234, 241 
(Amphioxus, pages 1-61; frog, 62-228; chick, 229-367; mammal, 368-454.)

chamber, air, 233, 234; - anterior (eye), 317, 321; — basilar, 150, $151 ;-$ laryngeal, 165,166 ; posterior (eye), 146, 147: 317,318 ; - pronephric, 196,197 chiasma, optic, 129, 131, 133, 148: 306

chick, embryo at 30 hours, $275 \mathrm{ff}$.; formation of embryo, $251 f f$., 252, $256 f f$., 258, 260, of developmental history, 231; - position in egg, 243, 299

Chiroptera, see bat.

choanæ, 154, 155, 158: 325

chondrocranium, $210 \mathrm{ff}$.

chorda, see notochord.

Choriata, 438, 439, 440

chorion, 69: 287, 288, 289, $292 \mathrm{ff}$., $294,310,351: 399,400,421,438$, $439,444,445,449$; - ectoderm of, 421 ; frondosum, 446;

human, $399,400,444,445,449$; - læve, 444, 446; - later history, $424 \mathrm{ff}$., 426, 428, 430; - relation to allantois, 430,431 , 435 ; — villi of, $438 \mathrm{ff}$; see also villi.

choroid, fissure, 146, 146, 147: 310 , 318 ff., 318, 329; - knot, 149 ; plexus, of III ventricle, 130 133: 305,$307 ;-\longrightarrow$ of IV ventricle, $134: 305,308$

chromosomes, number in Mammals, 377

cicatrix (cicatrices), 7, 57, 58: 235, 240: 378

cilia, superficial, $26: 116$

ciliary process, 319

circulation, allantoic, 299: 435, 436; — yolk-sac, 290, 291: 432-433, 432

cirri, buccal, 42, 44, 45

clavicle, 221

cleavage, $10,11 f f ., 12: 80,91 f f$., 92: 243 ff., 244, 245: 380 ff., 382 ; - accessory, 244, 246; delaminating, 93, 94; - first, plane of, 11: $89 \mathrm{ff}$.; 243: 382 ; gastrular, 99, 100; - pores, 14 cleft, see gill.

clitoris, 415, 416, 417

cloaca, 169: 280, 296, 297, 334, 348 : 414

cloacal, membrane, 297: 414 ff., 415 ; —_ tubercle, $415,415,417$ closing plate, 449,450

club-shaped gland, 25, 32, 34, 35, 40,43

coccygeal tubercle, 415

cochlea, 150, 151: 322, 323 cochlear duct, 323

cœlogastrula, 17

cœlom, 31, 32, 47, 52 : 124: 256, 265, 266-267, 294: 398, 402; branchial, 49; - dorsal, 49; endostylar, 49

coitus, relation to ovulation, 379

collecting tubule, mesonephric, 357 , 358 ; — metanephric, 358 columella, 152: 213,215

commissure, anterior, 129, 133 : 305 ,

306 ; - habenular, 130, 133:

305; - pallial, 305 ;

posterior, 132, 133: 305 ;

spinal, 305 ; — tubercular, 133 common trunk, pronephric, 194

concrescence, 103: $257 \mathrm{ff}$., 258

condyles, occipital, 215

confluence, 103, 112: 257 ff., 258

contra-deciduata, 439

copula, 217, 218, 219

copulation path, 80,80

coracoid, 221

cord, sex, 204, 204, 205: 362, 362 ; spinal, $37: 117,118,134,134$ : 308 ; - sympathetic, 143,143 : 312-313, 312; - umbilical, 406, $407,408,430,444,445$

cornea, 147, 149: 317, 321

Corning, 136

cornu (cornua), hyoid, 218, 219;

— trabecular, 210, 211, 212, 215

corona radiata, $373,376,379,380$

corpora quadrigemina, 133;

striata, 306

corpuscles, blood, 179: 350

corpus luteum, 378

cotyledons, 438, 448

cow, gestation, 369

cranium, $210 \mathrm{ff}$.

crescent, gray, 85,85

crest, neural, 119, 124, 136, 137, 139,

142,194 : 265, 270, 309

cristær, auditory, 323

critical stage, 4,54

crop, 335

crown-rump length, 407, 411

crura cerebri, 132: 307

cup, optic, 145, 146, 147: $316 \mathrm{ff}$., 317, 318

cushion, endothelial, 338

cutis, 53; - - plate, 171-172, 171 : 352-353

Cuvierian sinus, see ductus Cuvieri

cytotrophoblast, $422 \mathrm{ff}$.

Daniel, 369

Dasyurus, embryonic membranes, 419 ; gestation, 370 ; placentation. $419,439,440$ 
(Amphioxus, pages 1-61; frog, 62-228; chick, 229-367; mammal, 368-454.)

decidua, 439, 443; — basalis, 443, $444,445,447,449,450$; — capsularis, $442,443,444 ;-$ compact layer, $443 ;-$ reflexa, 442,443 ; serotina, $443 ;-$ spongy layer, $443 ;-$ vera, $443,444,450$ deciduata, 439

"Deckschicht," 130

deer, cleavage, 381: embryocyst, 387: embryonic shield, 387; fertilization, 381; gestation, 369 ; ovum, 373; placentation, 439, 440 denticulate ligament, 49

dermal, bones, of skull, 214, 219-220; fold, 52

dermatome, 52, 52: 171-172, 171 : 294, 352-353

deutoplasm, 4, 5: 69, 74, 75: 230; see also yolk.

Didelphia, ova, 418-419; embryonic period, 419

Didelphys, blastodermic vesicle, 388 ; gestation, 370

diencephalon, 129, 131, 133 : 283, 303, 304, 306 ff., 310

digestive tract, see alimentary canal. discus proligerus, 375

diverticulum, gut, anterior, 25, 32-33, 32,35 ; —- Meckel's, 434

dog, cleavage, 381: embryo, 394 ; embryonic shield, 393, 394 ; formation of embryo, $393 \mathrm{ff}$.; gestation, 369 ; oögenesis, 375 ; ovary, 375 ; ovulation, 379 ; ovum, 373 ; trophoblast, 388,388

dorsal aorta, see aorta

dorsal, cœlomic canal, 49; thickening (brain), 129, 131

duct, bile, 167: 333; - cochlear, 323 ; - - mesonephric (Wolffian), 199, 199, 202 ff.; 265; 294, 355 ff., $357,358,359,360$; metanephric, 359,359; Müllerian, $202 f f$. , 203:335,360-361;- pancreatic, 168 ; pronephric (segmental), 193 ff., 194, 195, 196, 198;

reproductive, $202 \mathrm{ff}$.; $360 \mathrm{ff}$., thoracic, 191: 349 ; $\longrightarrow$ urinogenital, $202 \mathrm{f}$.

ductus, arteriosus, 341, 348;

Botalli, 185: 342; - choledochus, 167: 333; - Cuvieri, 185 ff., 192: 283, 285, 291, 310, $329,337,344,345,351$; endolymphaticus, 150, 151: 322 , 322 ; - venosus, 285, 291, 304, $333,337,344,345,348$

dugong, placentation, 440

duodenal loop, 168

duodenum, 335, 336
Ear, 149 ff., 150, $211 f f ., 212,214$ : 321 ff., 322; internal, 324; middle, 152-153: 324;

outer, 324 ; see also auditory.

Echidna, ovum, 418

ectoderm, 15, 16: $98 \mathrm{ff} ., 98,100$ : 247, 249 ff., 249, 253, 280: 385 ff., $386,391,395 \mathrm{ff}$.

egg, see ovum; - membranes, 4, Б, 8, 9, 10: 69, 80, 81, 82: 233, 234: $373,374,376,380,382$; membranes, functions, 82 ; tooth, 328

" egg," of fowl, $231 \mathrm{ff} ., 233,242$

elephant, gestation, 369 ; placentation, 440

Elliot, 172, 173

embryo, axis of, 243 ; - formation of, $251 \mathrm{ff}$., 252, $256 f f$., 258, 260 : $384 \mathrm{ff}$.; - position in "egg," 243,299 ; see also human.

embryocyst, 387, 392

embryonic, membranes, $286 \mathrm{ff}$., 287 , 288, 289: 417 ff., 425, 428, 430; see also amnion, chorion etc.; - comparison of mammalian and sauropsidan, 417;

functions, 290,295 : 417-418; shield, 387, 388, 392, 393 ff., 394

embryotrophe, 441,442

enamel organ, $220: 328$

endocardial septum, 266

endoderm, 15, 16: 98, 100, $102: 247$, 249 ff., 249, 253, 267 ff.; 385, 386, $390,391,391,392,395,396$; yolk-sac, 256, 272, 277, 289-290: $390,391,425,431$

endolymphatic, duct, 150, 151: 322 , 322; - sac, 152: 323

endostyle, $34,35,40,42,43$

endothelial cushion, 338

endothelium, 175-176, 175, 178, 187 :

256, 272, 274

enterocœl, 21, 24, 26, 29, 30

enterocœlic grooves, 110, 110, 114

enteroderm, 121, 122

enteron, 25, 29, 33 ff.: 122-123, 158 ff.: 326 ff.

entoderm, see endoderm.

entypy, 387, 389

ependyma, 37

ependymal cells, 134: 308

epiblast, see ectoderm.

epiboly, 15 ff.: 98, 99: 251

epicoracoid, 221

epididymis, 362,363

epipharyngeal groove, 44

epiphysis, 118, 118, 130, 131, 133 :

$285,304,305,307$ 
(Amphioxus, pages 1-61; frog, 62-228; chick, 229-367; mammal, 368-454.)

epithelioid bodies, 163, 163: 331

epithelium, acustic, 323 ; - follicular, 373, 374 ff., 375; minal, 361,362 ; - neuro-, 326 ; - olfactory, 326

Erinaceus, see hedgehog.

erosion, of uterine wall, 422,424 ; centæ, 440-441

ethmoid region, 215

Eustachian tube, 152: 324, 328

Eutheria, embryonic membranes, $419 \mathrm{ff}$.; placentation, $437 \mathrm{ff}$.

excretory system, 53: $192 \mathrm{ff} .: 355 \mathrm{ff}$. exocœlom, 262, 266, 267, 272, 278, $280,281,282,287,287,288,289$, $294: 427,428$; - human, 400

external, form, chick embryo, establishment of, $282 \mathrm{ff}$.;

human embryo, 399 ff., 400, 401 ff.; - genitalia, 414 ff., 415 ; _- gills, 65, 161

extra-embryonic cœlom, see exocœlom.

eye, 65, 121, 144 ff., 144, 145 : 316 ff., 317,318 ; see also optic; muscles, innervation, 315

Face, development in human embryo, $411 \mathrm{ff} ., 412,413$

Fallopian tube, 371, 372

false amnionic cavity, 389, 391, 391 fascia, 52

fat bodies, 71, 72, 205

feather papillæ, 286

Federow, 189

fenestra vestibuli, 213

ferret, ovulation, 379; placentation, 440

fertilization, 6, 9: 78, $79 \mathrm{ff} ., 80,85$ : 240-241:379-380,380;- meridian, 79,84

Field, 194

filtering organ, 162

fimbriated opening, 311; — during ovulation, 378

fissure, choroid, 146, 146, 147 : 310 , 318 ff., 318, 329; — tubal, 328; ventral, 135

fixation villi, $442,447,448,449$

flexures, of brain, 270, 283, 284: 404; - cervical, 284, 285, 308: 405, 406,406 ; — cranial, 284, 285, 304,308 ; — pontine, 308 ; ventral, 128, 130; — of head and tail, $402 \mathrm{ff}$.

folds, amnionic 262, 283, $292 \mathrm{ff}$.: 391, 392, 393, 396, 425, 426 ff., 426, 427; - - amnionic, closure of, $293,296,297: 425,426,427$; folds, genital, 415, 416, 417; medullary (neural), 16, 21, 22, 23 : 261, 266, 268, 269, 270, 278: 398 ; - mesoderm, 21, 24; - metapleural, 40, 47, 47, 48; itive, 253,254 ; - supraorbital, 413,414 ; - suspensory, 49 ; tail, 280, 293, 296, 296, 297 ff., 297 follicle, Graafian, 375,$376 ;$ ovarian, 56, 57: 206, 235, 236, 238, 239 : 373, 374, 375, 376, 378; ovarian rupture of, 378

follicular epithelium, 373, $374 \mathrm{ff}$., 375

fontanelle, basicranial, 211, 211, 212 ; - supracranial, 213

foramen (foramina), interventricular 340 ; - jugular, 211, 212, 212 ; - magnum, 212, 213; - of Monro, 131-132: 306; - ovale, 153

fore-brain, 129, 131, 131: 262, 271, 282, 302, $303 \mathrm{ff}$., 317

fore-gut, 122,159 ff.; $260,261,261$, 262, 268, 274, 276, 278, 326 ff., 329

fovea, 69,74

frog, age, at maturity, 68 ; - age, estimation of, $64,127-128$; "early embryo," 64, 65, 116, 120; — metamorphosis, $67-68 ;$ outline of life history, $63 \mathrm{ff}$.

frontal process, 412,412

fronto-nasal process, 325

fundus, eye, 146: $319 ;-$ uterus, 372,442

furrow, intersomitic, 263

Gall bladder, 166, 167, 167 : 333

ganglion (ganglia), acustico-facialis, $136,137,157: 282,283,310,329$; - basal, 306; _- ciliary, 316 ; —_ cranial, 309 ; _ crest, 136 , 137, 138; - facial, see acusticofacialis; - Gasserian, 140: 329; geniculate, 315,329 ; glossopharyngeal, 136, 137 ; habenular, 130; - jugulare, $314 ;$ — jugulare-nodosum, 309; - nodosum, 196; 314, 329; - ophthalmic, 138 ; — petrosum, 309, 329; - pneumogastric, 137, 137; - profundus, 315; - Remak's, 312; spinal, 135, 142, 143: 294, 309, 311 ; sympathetic, 144, 207, 207: 364 ; trigeminal, 136, 137, 138, 139, 140, 157 : 283, 285, 310,$315 ;-$ vagus, 137,137 , 157 
(Amphioxus, pages 1-61; frog, 62-228; chick, 229-367; mammal, 368-454.)

gastro-hepatic ligament, 335

gastrula, 16, 17, 19-20: 65, $99 \mathrm{ff}$. 98, 102, 104, 105: 249, 252, 257 ff.; 385 ; rotation of, 98,104 , 105 ff., 105

gastrular cleavage (groove), 98, 100. gastrulation, $15 \mathrm{ff}$., 16, 17 : $99 \mathrm{ff} ., 98$, 102, 106, 111 ff.: 248 ff., 249, 251,259 ; comparisons, Amphioxus, 113 ff.; - — frog, $111 \mathrm{ff}$; — chick, 259

genital, folds, 415, 416, 417 ; ridge, $415 ;-$ ridge (sex-cell), 204, 204, 205; — swelling, 415, 416,417 ; — tubercle, 415, 416, 417

genitalia, external, $414 f f$.

germ, cells, 4, 5, 6: 65, $68 \mathrm{ff}$., 70, 81, 85: 232 ff., 233 : $372 \mathrm{ff}, 373,383$; - formation of, $64,71 \mathrm{ff}$., 199, 205: 361, 362, 363: 375, disc, see blastoderm;

layers, $15 \mathrm{ff}$., 16, 21, $24 \mathrm{ff.:} 98 \mathrm{ff}$., 99, 102, $107 \mathrm{ff} ., 110,111 \mathrm{ff} .: 247$, 248 ff., 249, 252, 254, 254, 260, 264, 272, 280: 385, 385, 390, 391, 392, 395 ff., 396, 397, 398, 399; - layers, inversion of, 389,391 ; see also ectoderm, endoderm, mesoderm; - ring, 16, 18, 24: 94, $95,96,101,112$; wall, 247, 247, 248, 254, 256, 258, 264, 272 germinal, cells (nervous), 134: 308; $\longrightarrow$ epithelium, 361, 362

gestation, duration of, 369

gill, clefts (slits), 32, 35, 35, 39 ff., 40, 41, 42, 55: 160: 283, 286:404ff., 404 ;

primary, 32, 35, 39 ff., 40, 42 ;

- secondary, $39 \mathrm{ff} ., 40$;

— tertiary, 41, $55 ;$ -

vestiges of, 162, 163, 164: 330,331 ; $\frac{}{1}$ filaments, 162 ; plate, 65,$122 ;-$ pouches, 41 : $122,158,160,160,163,164: 269$,

278, 328 ff., 329 ; rakers, 180 gills, external and internal, 65, 161, 180,181

gizzard, 335, 336

gland, carotid, 163, 163, 182, 184, 184; - club-shaped, 25, 32, $34,35,40,43$; uterine, 443, 445

glans clitoridis, 415; - penis, $415,416,417$

glia cells, 134: 308

globular process, 413,413

glomerulus, 182, 184, 196-197, 196, $198,199,200$

glottis, 165,166 : 332 gonad, 6, 55 ff., 57 : 71-73, 72, 203 ff., 204, 205 : 335, 361 ff., 362. See also ovary, testis.

gonadial cavities, 56, 57, 58

gonocœl, 7, 56, 57

gonoducts, see ducts, reproductive.

gono-nephrostome, 56

Goodrich, 53

Graafian follicle, 375, 376

Graf von Spee's Embryo Gle, 400, 401 granulosa cells, 71:237, 239: 375, 376 gravitational plane, 86

gravity, as a factor in determining symmetry, $86 \mathrm{ff}$.

gray crescent, 85,85

groove, branchial, 65, 122;

enterocœlic, 110, 110, 114;

epipharyngeal, $44 ;$ hyperbranchial, 44; — interventricular, 338; - intestinal, 281, 281; medullary (neural), 65, 111, 117, 120: 263, 269: 401; — of Hatschek, 35, 40,45, 46; _ olfactory, 154; - pleural, 310, 351; primitive, $119,120: 252,252$, 253, 254, 263, 268: 395, 396, 398, 401

Grosser, 424, 440

guinea-pig, amnionic cavity, 389, 424; gestation, 369 ; implantation, 421; notochordal canal, 397 ; ovum 373 ; trophoblast, 389

gut, 25, 29, 33 ff.: 122, 158 ff.: 326 ff.: 402 f.; $-\frac{}{2 i v e r t i c u l a, ~ a n t e r i o r, ~}$ 25, 32-33, 32, 35; postanal, 170: 280, 296, 296, 334; preoral, 262, 304, 328, 329

Guyer, 377

Hæmotrophe, 441

Halicore, placentation, 440

Hall, 198

Harrison, 156

hatching, 26: 66: 299-300

Hatschek's groove, 35, 40, 45, 46; nephridium, $35,42,45,46$

head, cavity, 25, 32, 33; —_ fold, 252, 260, 260, 261, 262, 263, 278, 281 ; fold, of amnion, 262, 292: 425, 427; —_ kidney, 193; process, 252, 253, 259: 395

heart, 118, 125, 166, $174 f f ., 175,176$, 182: 262, 266, 273 ff., 276, 278, 286, 291, 304, 337 ff., 338, 342, 351: $402 \mathrm{ff} . ;-$ commencement of beating, 339 ; — lymph, 189, 191: $349-350$

heat, effect upon symmetry, 88; see also œstrus. 
(Amphioxus, pages 1-61; frog, 62-228; chick, 229-367; mammal, 368-454.)

hedgehog, blastodermic vesicle, 388 ; cleavage, 381 ; notochordal canal, 398 ; trophoblast, 389; trophoderm, 422 ; yolk-sac, 433

Held, 142

hemispheres, cerebral, 129, 131, 133 : 305, 306

Hensen's node (knot), 252, 253, 257, 260 : 394, 395, 396

hernia, intestinal, 408

Hertwig, 439

Hill, 303: 419

hind-brain, 129, 131, 132, 133 : 262 , $271,276,303$; - gut, 122,169 170: 279, 296, 296, 327, 334

hippopotamus, placentation, 440

His's Embryo M, 403, 404

horse, gestation, 369; implantation, 421 ; placentation, 439,440 ; yolksac, 433

\section{Hoyer, 190}

Hubrecht, 385, 421

human embryo, early development, 399, ff., 400, 401; - - external form, 399 , ff.;

face, 411, ff., 412, 413;

measurement of, 407 ;

- structure at $2.6 \mathrm{~mm} ., 405 \mathrm{ff}$., 405. See also man.

humor, vitreous, 148: 320

Huxley, 439

hydatid, 372

hyobranchial, apparatus, 217, 218, 218, 219; - pouch, 162

hyoid arch, 65, 122, 160, 160: 330 ; - cornu, 218, 219

hyomandibular pouch, $160,160,161$, 162: 324,330

hyperbranchial groove, 44

hypoblast, see endoderm.

hypobranchial plate, 217, 218, 218

hypochordal rod (hypochorda), 169, 194, 204

hypophysis, 118, 121, 130, 131, 132, 133, 166: 304, 305, 327, 329

Hyrax, placentation, 440

Ilium, 221

implantation, 378, $421 \mathrm{ff.;}$ central, eccentric, interstitial, 421 ;

- of human "ovum," 442

incubation, 231

incus, 325

indifferent period, genitalia, 416; gonad, 361-362

infundibulum (brain), 38, 39: 130, $130,131,133,145,160$ : 262, 282, $304,305,306$; - of oviduct, 203 : 235, 236, 360: 371, 372 ; during ovulation, 378 inner cell mass, 382, 383, 384, 385 , 386,390

Insectivora, amnion, 385, 424; blastodermic vesicle, $385,386,387$; cleavage, 381 ; germ layers; 385 ; implantation, 421; placentation, 440; trophoblast, 389

integumentary organs, 156

interamnionic cavity, 389,391

interauricular septum, 177: 338, 339

intermediate cell mass, 173, 193:265266, 265: 355

internasal plate (septum), 210, 211, 215

intersomitic furrow, 263

interventricular, foramen, 340 ; septum, 338, 340

intervillous cavity, 442, 447, 449

intestinal, cæca, 336; - groove, 281,281 ; - hernia, 408; portal, anterior, 261, 262, 266, 269, 272, 276, 278, 282, 291, 304, 345 ; posterior, 280

intestine, 122, 168-169: $335 \mathrm{ff}$., 335, 348

intra-placental cavity, 446, 449

invagination, $15 \mathrm{ff} .: 99 \mathrm{ff}$., 98, 107, $111 \mathrm{f}$.

inversion, of germ layers, 389, 391

involution, $15 \mathrm{ff} .: 249$

iris, 147, 149: 317, 319, 321

ischium, 221

isthmus (brain), 262, 283, 285, 304, $307 ;-$ (oviducal), 235, 236

iter, 307

Jackson, 408, 411

Jacobson's organ, 154, 155

jaws, 325, 327, 328: 412, 412, 413, 413 ; - articulation, 216 ; horny, 159, 166

jejunum, 329, 336

Jenkinson, 88, 89

junction, zone of, 247, 258, 277, 277

Keibel and Elze's Embryo, Klb., 401, 402

kidney, 359; - head, 193

Kirkham, 378

knot, choroid, 149; - Hensen's (primitive), 252, 253, 257, 260 : 394, 395, 396

Knower, 189

Kölliker, 439

Kollmann's Embryo, Bulle, 401, 402; $2.5 \mathrm{~mm} ., 402$

Labia, majora and minora, 415, 416, 417 
(Amphioxus, pages 1-61; frog, 62-228; chick, 229-367; mammal, 368-454.)

labyrinth, 152: 324; - bony, cartilaginous, membranous, 324

lactation, 370

lagena, 150, 151: 322,323

lamina terminalis, 129. 131: 270, 306

Langhans, cells of, 446,447

larva, Amphioxus at the critical stage, 4,54

laryngeal chamber, 165, 166

laryngo-tracheal groove, 332

larynx, 329, 332

latebra, 239, 243, 245

lateral, dorsal aorta, 179, 180, 182 : $272,291,292,341$; - folds, 281 , 281 ; _ _ $\quad$ of amnion, 293; - line nerve, $142,156,157$;

line organs, 141, 156, 157; mesocardia, 344, 350 ff., 351; _- plate, $31: 123-124,170,171$,

194: 263,278

Legros, 53

Lemurs, allantois, 434, 436; placentation, 440

length, of human embryos, 407, 411

lens, $146-148,146,147$ :283, 316, 317, $318,318,320-321$; — placode, 146

lenticular zone, 319

life history, Amphioxus, 3; chick, 231; - frog, $63 \mathrm{ff}$.; Mammal, $369 \mathrm{ff}$.

ligament, denticulate, 49 ; - gastro-hepatic, 335; - intervertebral, 209; — uterine, 372

Lillie, 277; quoted, 299-300, 352

limbs (and buds), 65, 128: 285, 286, $347: 406,406,409$

lips, 158, 159, 166: 413, 413

liquor, amnii, 430; - folliculi, 376

liver, $36,42,55$ : 118, 122, 159, 165 , 166, 167 : 304, 332-333, 335, 345, 348,351

lobe, olfactory, $129: 305$; — optic, 129, 132: 307

lobus olfactorius impar, 38

loculi, placental, 448

Loeb, L., 378, 442

lung, $67,165,167: 304,310,332$, 335,351

lutein, 378 ; — cells, 378

lymphatic, hearts, 189, 191: 349-350; — sacs, 191 ; 191, 190; - system, 189-191, $190 ;-$ tissue, 168

Maculæ, acustic, 323

Mall, 407, 411

malleus, 325

Malpighian bodies, 200, 201, 205: 356, $357,359,362$
Mammal, outline of life history, $369 \mathrm{ff}$.

man, allantois, $435 \mathrm{ff}$.; amnion formation, $428 \mathrm{ff}$., 430; amnionic cavity, $399,400,424,444,445$; blastodermic vesicle, 399,400 ; chorion, 444, 445, 446; chromosome number, 377; embryo, early, $399,400,401$; - development of external form, $399 \mathrm{ff}$.; - in utero, 445; exocœlom, 429, 433; germ layers, 396 ; gestation, 369 ; lutein, 378; mesoderm, 399, 400; notochordal canal, 398; ovary, 371 , 372 ; oöcyte, 373 ; placenta, $442 f f$., 444, 445; placentation, 439, 440, 441 ; proamnion, 429 ; reproductive system, female, 372 ; trophoblast, 389 ; trophoderm, 422; uterus, gravid, 444; yolk-sac, 433 ff.; yolk-stalk, 444, 445

mandibular arch, aortic, 179, 183: 275,$278 ;-1$ cartilaginous, 164, 216; - visceral, 122 , $160,160,163$ : 283, 285, 304, 330 process, $404,404,406,407$, 412,$412 ;-$ ridge, 158

Manis, placentation, 440

marginal, cells, 244, 245-246, 245 ; notch, 258 ; sinus, 252,252 , $255,290: 431,432,432,449,449$ margin of overgrowth, 247, 249, 258, 277, 277

Marshall, 378, 442; - quoted, $67-$ 68 ; — and Bles, 183, 201

Marsupialia, embryonic membranes, 419 ; gestation, 370,419 ; ova, $418-$ 419 ; placentation, $419,437,439$, 440

marten, placentation, 440

mass, inner cell, 382, 383, 384, 385, 386,390 ; intermediate cell, 173, 193: 265-266, 266, 355

maturation, $5,8,9: 73 \mathrm{ff}$., 75, $82 \mathrm{ff}$.; $240,340,341: 374,376-377$

Maurer, 180

maxillary process, 404, 406, 407, 412 , 412

measurement of human embryos, 407 , 411

meatus, 325

Meckel's diverticulum, 434. See also cartilage

medulla (oblongata), 117, 129: 308

medullary folds, 16, 21, 22, 23: 261, 266, 268, 269, 270, 278: 398; furrow (groove), 269: 394, 396, 398, $401 \mathrm{ff}$., 401 ; - plate, $21,22,23$ : $110-111,110,117,120,124,137$, 194: 252, 253, 260, 261, 263, 264, 
(Amphioxus, pages 1-61; frog, 62-238; chick, 229-367; mammal, 368-454.)

medullary furrow, 269; - tube, 270,278 . See also, neural.

membrana granulosa, 71: 237, 239: 375,376

membrane, anal, 414, 415; cloacal, 297: $414 f f ., 415$; embryonic, 286 ff., 287, 288, 289 , 290, 295: $417 \mathrm{f}$.; - of egg, 4, 5, 8, 9, 10: 69, 80, 81, 82: 233, 234: $373,374,376,380,382$; - - of egg, functions of, 82 ; - - of egg, swelling of, 81 ; - oral, 132,158 , 159, 164 : 261, 262, 269, 276, 282, $304,327: 412,412$; - perivitelline, 5,9 ; - pleuro-peritoneal, 352; —— shell, 233, 234, 241; —-tympanic, 152, 213: 325; urinogenital, 414 ff.: 415; vitelline, 4, 5, 8: $69: 232, \mathbf{2 3 3}, \mathbf{2 4 5}$, 247, $287: 374$

menstruation, 379,421

meridian, fertilization, 79,84

mesencephalon, 129, 131, 132, 133 : $262,270,271,283,303,304,305$, 307

mesenchyme, 138, 139, 140, 141, 171, 172: $264,309,353$

mesenteron, 25, 29: 118, 122 ff.: 334 ff.

mesentery, dorsal and ventral, 174, 204, 204: 335, 351

mesocardium, dorsal and ventral, 175, 176, 192: 272, 274; lateral, 344, $350 \mathrm{ff}$., 351

mesoderm, 21, 24, 25, $29 \mathrm{ff}$ : : 99, 102 , 107, ff., 123, 137, 170, 194: 252, 253 ff., 254, 256, 262 ff., 263, 272, 278,294 : 395, 396, 398, 399, 402; - bands, 24 ; cœlomic, 255 , 256 ; folds, 21,24 ; _ formation in frog compared with Amphioxus, 114 ff.: - - gastral, 24, 26: 114115: 259,262 ; lateral plate of, 31: 123-124, 170, 171, 194: 263, 278 ; - peristomial, 26: 114 115: 259,$262 ;-$ somatic and splanchnic, 31 : 124, 124, 170: 256, $265,266-267,272,277$; —— yolksac, 425,432

mesogastrium, 335

mesohepaticum, 187, 192

mesonephros, 193, $198 \mathrm{ff} ., 199,200$ : 335, 348, 355, 356 ff., 357, 359 ; - disappearance of, $358 ;$ duct of, 199, 199, $202 \mathrm{ff} .: 265,294$, $355 \mathrm{ff}$., 357, 358, 359, 360; tubules of, primary, secondary, tertiary, 294, 356-357, 357, 359, 362 ; — units, 198 ff., 199, 200; - vestiges of, 360 mesorchium, 202, 205: 363

mesovarium, 71, 202, 205: 235, 236, $361: 371,372$

metagastrula, 383

metamerism, 265

metamorphosis of tadpole, 67-68

metanephrogenous tissue, 359

metanephros, 355, $358 \mathrm{ff}$; - duct of, 359,359 ; — tubules of, 358359,359

metapleural folds, 40, 47, 47, 48

Metatheria, gestation, 419; ova, 418-419

metencephalon, 129, 132, 133 : 283, 303, 304, 307

micropyle, $9: 79: 374$

midl-brain, $129,131,132,133: 262$, $270,271,276,283,303,304,305$, $307 ;-$ gut, 167, 169: 327, 334

Minot, 422

mole, amnion cavity, ..93; amnion folds, 428; germ layers, 396 ; notochordal canal, 397, 398; primitive streak, 396 ; yolk-sac, 433

Monodelphia, e mbry onic membranes, 419 ff.

Monotremata, ova, 418; placentation, 439,440

Monro, foramina of, 131-132: 306 mons veneris, 417

morula, 382, 383

mouse, amnion folds, 424 ; blastodermic vesicle, 384 ; chromosomes, 377 ; cleavage, 381 ; false amnionic cavity, 389 ; gestation, 369 ; implantation, 421 ; lutein, 378 ; ovulation, 379; ovum, 373; polar bodies, 377, 378; sperm entrance, 380; Träger, 389; trophoblast, 389 mouth, $34,35,40,42,44,47: 65,118$, $122,128,158,166: 269,304,327$ : 404,412 ,f., 412

mucosa, uterine, 443

Müllerian duct, 202 ff., 203: 335, 360-361

muscle, fibrillæ, 172: 353 ; —— of eyeball, innervation, 315 ; — plate, 171, 171 : 352-353

musculature, ventral, 53

Mustelidæ, placentation, 440

myelencephalon, $117,129,132,133$,

134: 283, 285, 303, 304, 307

myocœl, 31, 48, $52: 124,125,170: 265$, 265

myosardinm, 266, 272

myocommata, 172

myotome, $31,48,51,52: 123,171$, 171,194 : 294, 352-353

Nares, 154, 155: 325-326 
(Amphioxus, pages 1-61; frog, 62-228; chicle, 229-367; mammal, 368-454.)

nasal septum, 413, 413

nephridia, 40, 53, 54

nephridium of Hatschek, $\mathbf{3 5}, \mathbf{4 2}, \mathbf{4 5}, 46$

nephrostome, mesonephric, 201:358; metanephric, 359 ; - pronephric, 194, 195, 195, 196, 198; common, 197, 199

nephrotome, $173,193: 265,265,355$, 356

nerve, branchiomeric, 135;

cranial, 135, 136 ff.: 313 , ff.; - abducent (vi), 142: 315; - auditory (viii), 140, 157:

$314 ;-1$ facial (vii), 140 , 141: 315 ; - $\longrightarrow$ glossopharyngeal (ix), 141: 314,329 ; - - hypoglossus (xii), 313; - lateral line, 142, 156, 157 ; — oculomotor (iii), 142: 315 ; — - olfactory (i), 316,326 ; — — optic (ii), 316, 320 ; - pneumogastric (x), 141: 314,$329 ;-$ spinal accessory (xi), 314, 329 ;

trigeminal (v), 138, 140: 285, 315; —— trochlear (iv), 142: 315 ; - vagus (x), 141: 314, 329 ; — - vagus, visceral branch, 142; - spinal, 135, 142143, 173: 311 ff., 312 ;

axons of, 311 ; roots of, 142, 143: 311, 312 ;

table of arrangement, 173

nervous system, central, 20-23, 21, 27-28, 37-39, 38 : 116 ff., 128 ff.: 269 ff., 302 ff., 302 ;

peripheral, $135 \mathrm{ff} . ; 311 \mathrm{ff}$;

- sympathetic, $143-144,143$ : 312-313, 313

"nests," of ovarian cells, 205, 206 : 237, 363: 374

neural, crest, 119, 124, 136, 137, 139, 142, $194: 265,270,309 ;-$ folds (ridges), 16, 21, 22, 23: 261, 266, 268, 269, 270, 278: 398; - folds, lateral, $65,111,117,120$;

folds, transverse, $65,99,111,117$; - groove, $65,111,117,120$ : 263, 269: 401 ; 1 plate, 21,22 : $65,110-111,110,117,124: 269$, 270,278 ; - sheath, 52 ; tube, 21, 27: 270, 274, 278: 398 neurenteric canal, 16, 21, 25, 28:118, 119,120 : $259: 397,398,400,401$ neuroblasts, 135: 308 neurocœl, 21, 27

neuro-epithelium, 326

neuromeres, 128: 271, 302-303, 302 neuropore, $22,25,32,35,38: 118,128$ : 270 node, Hensen's, 252, 253, 257, 260 : 394, 395, 396

non-deciduata, 439

notch, marginal, 258

notochord, 16, 21, 23, 25, 28-29: 121 ff., 208-210, 213: 260, 262, 264, 265, 266, 268, 268, 278, 304: 398, 402

notochordal, canal, 397-398, 397; — sheath, 52: 208, 208

notogenesis, $99 \mathrm{ff}$., 98, 106, $112 \mathrm{ff}$.

nuclei, of periblast, 246-247

nucleus, of Pander, 243, 247; volk, 74, 75 : 376

Nussbaum, 205

Occipital condyles, 215

œsophagus, 159, 166, 168, 169: 304, $329,332,335$

œstrus, 378 ; — relation to ovulation, 379

olfactory, capsule, 210, 211, 212, 214, 215 ; - epithelium, $326 ;-$ groove, 154 ; l- lobes, 129 : 305 ; - organ, 145, $153 \mathrm{ff}$., $154: 325$ ff.; - pit, 38, 38: 153, 154, 160 : 325 : 407, 412, 412; - placode, $131,153,154 ;-$ process, 412 , 412 ; - recess; 129

omosternum, 221

oöcytes, 74, 75: 237, 238

oögenesis, 7: $73 \mathrm{ff}$., 75: 237, 238 : $374 \mathrm{ff}$.

oögonia, $7: 237,363$

opercular cavity, 161, 163

operculum, 67, 161, 163, 180 ;

(auditory), 153, 213

optic, chiasma, 129, 131, 133, 148: 305,306 ; cup, 145, 146, 147, 148: 316 ff., 317, 318 ; lobes, 129, 132: 307; - recess, 130, 133, 148: 262, 303, 304, 305, 306; - stalks, 129, 144, 145, 147, 148, 160: 303, 316, 317, 318, 320; - thalami, $129,148: 307,320$; - vesicles, $144,145,147: 266$, $271,274,276,282,283,316 \mathrm{ff}$., $317,318,329$; - vesicle, anterior chamber, 317,321 ;

posterior chamber, $146,147: 310$, 317,318

opossum, blastodermic vesicle, 388 ; gestation, 370

oral; cirri, 44, 45 ; - hood, 42,44 , 45 ; membrane (plate), 132, 158, 159, 164: 261, 262, 269, 276, 282, 304, 327: 412, 412; sinus, 404,412 ; - sucker, 65 , $66,120,164,180$

ora serrata, 319 
(Amphioxus, pages 1-61; frog, 62-228; chick, 229-367; mammal, 368-454.)

organs, of lateral line, 141, 156, 157 Ornithodelphia, ova, 418

Ornithorhynchus, ova, 418

ossicles, auditory, 324-325

ostium (oviducal), 72, 73, 203: 235, $236,360: 371$; — during ovulation, 378

otocyst, $140,149,150: 283,285,322$, 322

ovary, $6,7,56 f f ., 57: 71,72: 235$, 236, 363: 371, 372; - follicular capsules of, $56,57: 206: 235,236$, 238, 239 : 373, 374, 375, 376, 378; — stroma of, 205: 361,362

overgrowth, margin of, 247, 249, 258, 277,277

oviduct, 72, 202, $203: 235,236,360$ ff.: 371,372

ovulation, 78: 240 ff.: 377-379; relation to coitus and parturition, 379

ovum, 4, 5, 6, 10, 17 : 65, 68 ff., 70 : 232 ff., 233: 372 ff., 373, 376; _ at time of laying, 70:232 ff., 233 ; - formation of, $236 \mathrm{ff}$.; - human, 373,373 ; - longevity of, 379 ; number formed, 78; - polarity of, 4,5 , $6,10,11,17: 69,70,83 ;$ production of, 236-237, 242; sizes, $373 ;-$ symmetry, 4, 6, $10,11,17: 70,80,83 \mathrm{ff} ., 87,88 \mathrm{ff}$.

"ovum," the mammalian blastodermic vesicle and ovum, 399

Owen, 439

\section{Pabulum, 441}

pancreas, 167, 167 : 333, 335, 345

pancreatic duct, 168

Pander, nucleus of, 243, 247

papillæ, acustic, $323 ;-$ feather 286

parachordæ, 210

paradidymis, 362

paraphysis, 131: 305, 306

parencephalon, 304

parietal cavity, see exocclom

paroöphoron, 363

parovarium, 363: 372,375

pars basilaris, 150, 151

parturition, 450 ; $\longrightarrow$ relation to ovulation, 379

Patterson, 241, 248

Pearl, 241; - and Curtis, 241

pecten, 320

pectoral and pelvic arches, 221

penetration path, $79,80,81,84$

penis, 415, 416, 417

Perameles, embryonic membranes, 419, 420; placentation, 419, 439 periblast, 243 ; - central, 245, 245 ; marginal, 244, 245, 245, 277; nucleation, 246-247

pericardial cavity, 166, 174, 175, 180, 192: 262, 267, 275, 310, $350 \mathrm{ff}$., 351

pericardio-peritoneal septum, 191192

perigonadial cavity, 56

perilymph, 152: 324

perineum, 414

peripharyngeal bands, 42,43

peripheral nervous system, $135 \mathrm{ff}$ : $311 \mathrm{ff}$.

peritoneal cavity, 352

perivitelline, membrane, 5,9 ; space, 4 , 5, 9, 10: 80, 81:374

Peters, 442

phæochrome tissue, 206-207, 207

phallus, $415 \mathrm{ff}$. 415

pharynx, 33:118, 159, 160: 262, 269, $272,278,304,328 f f ., 329$

Phascolarctos, placentation, 439

pig, allantois, 436; amnion, 392 ; gestation, 369; implantation, 421 ; lutein, 378; notochordal canal, 398 ; placentation, 439,440

pigeon, gastrulation, $248 \mathrm{ff}$; oöcyte, 239 ; nucleation of periblast, 246 247

pigment, of ovum, $65,69,80,85,85$; of blastula, 97

pineal body, 130: 307

pinna, 413, 414

pit, auditory, 282, 283; - olfactory, $38,38: 153,154,160: 325$ : $407,412,412$; - preoral, 25, 32 , 33,44 ff.; - primitive, 252, 253, 257, 260: 398

pituitary body, 132: 327

placenta, 391, 425, 430, $437 \mathrm{ff}$., $444,445,447,448$ ff., 449; basal plate of, 449 ; - classification, $438 \mathrm{ff}$; - cotyledonary, 439 ; - discoid, 439; - discoidalis, 440, $441 \mathrm{ff} . ;-$ endotheliochorialis, 440 ; - epitheliochorialis, 440-441; —_ hæmochorialis, $441 \mathrm{ff}$., 446; — human, 441 ff. 444, 445, 448 ff. 449 ; $\frac{}{\text { loculi }}$ of, $448 ;-$ partial, 440 ; syndesmochorialis, 441 ; _ zonaria, 440; - zonary, 439; - zono-discoidalis, 440

Placentalia, 438, 439, 440; — vera, 440 ; - embryonic membranes of, $419 \mathrm{ff}$.

placentation, defined, 437

placode, 138, 139: $310 ;$ auditory 139,$149 ; \div$ facial, $139 ;$ 
( 1 mphioxus, pages 1-61; frog, 62-228; chick, 229-367; mammal, 368-454.)

placode, glossopharyngeal, 329 ; lateral line, 156; lens, 146; olf actory, 131, 153, 154; - olf actory,

plane, first cleavage, 11: $89 \mathrm{ff}$.; 243 : $382 ;-$ gravitational, $86 ;-$ of symmetry of ovum, 4, $6,10,11$, $17: 70,80,83 \mathrm{ff} ., 87,88 \mathrm{ff}$.; - of blastula, 96

plate, anal, 280, 296, 296, 297, 334; - basal, placental, 449 ; basal, cranial, 211, 212, 213; closing, 449, 450; - cutis, 171-172, 171: 352-353; — gill, 65,122 ; - hypobranchial, 217, 218, 218 ; internasal, 210, 211, 215; lateral, 31: 123-124, 170, 171, 194: 263, 278; —_ medullary (neural), 21, 22: 65, 110-111, 110, 117, 124: 269, 270, 278;

parachordal, 210, 211; - primitive, 247, 252, 253, 254 ; __ sense $123 ;-$ velar, 162,$180 ;$
vertebral, $170,194: 263,278$

vertebral, 170, 194: 2
plectrum, 153, 213, 214

pleural, cavity, 351 ; 310,351

pleuro-peritoneal membrane, 352

plexus, choroid, of iii ventricle, 130 , 133: 305,307 ; — — of iv ventricle, $134: 305,308$; — - prevertebral, $312 ;$ splanchnic, 312

plug, yolk, 65, 99, 101, 102, 120

polar bodies, $5,8,10: 70,75,81,82$ : $340,341: 377,378,380$

polarity, of ovum, $4,5,6,10,11,17$ : 69, 70, 83: 232-233

polyspermy, 79: 241

pons Varolii, 307

pore, cleavage, 14

portal, intestinal, anterior, 261, 262 , 266, 269, 272, 276, 278, 282, 291, 304,345 ; see also vein.

position of embryo in "ovum," 243 ; change in, 284

postanal gut, 170:280, 296, 296, 334 postbranchial bodies, 331, 331

pouch, branchial (gill), 41, and see pouch, visceral; - hyobranchial, $162 ;-$ hyomandibular, 160, 160, 161, 162: 324, 330; Seessel's, 304, 328, 329; - visceral, 41: 122, 158, 160, 160, 163, 164: 269, 278, 328 ff., 329

preoral, cavity, 25, 32, 33; - gut, 262, 304, 328, 329; - pit 25, 32, $33,44 \mathrm{ff}$.

pressure, effect upon symmetry, 88
Primates, allantois, 434, 436; amnion formation, 424; endoderm formation, 385 ; implantation, 421 ; ovulation, 379 ; placentation, 439 , 440; proamnion, 428; trophoblast, 389 ; trophoderm, 422 ; uteri, 372 ; yolk-sac, 433

primitive, folds, 253, 254 ;

groove, 119, 120: 252, 252, 253, 254, 263, 268: $395,396,398,401$; - knot, 252, 253, 257, 260 : 394, 395,396 ; 200:398; - plate, 247, 252, 253, 254; - streak, 119, 120: 251 ff., 252, 254, 258, 260, 274 : 394, 395, 396, 398, 401; - streak, relation to embryo, $256 \mathrm{ff}$., 258, 260

proamnion, $252,255,260,262,263$, 263, 266, 274, 282, 292: 426 ff.

process, ascending, of palato-quadrate, $211,211,214$; — cæcal, 335 ; — ciliary, 219; — frontal, 412,412 ; _ fronto-nasal, 325 ; globular, 413, 413; - head, 252, 253, 259: 395; - mandibular and maxillary, 404, 404, 406, $407,412,412$; - olf actory, 412, 412; — transverse, 209

procoracoid, 221

proctodæum, 65, 118, 119, 120, 169 : 334: 414

Proechidna, ova, 418

pronephric, capsule, 194, 197; chamber, 196, 197; - duct, 160 , $193 \mathrm{ff.:} 355 \mathrm{ff} . ;$ see also duct, Wolffian; tubules, 160, 194, 195, 195, 196: 355

pronephros, 65, 125, $193 \mathrm{ff} ., 194,195$, 196, 199: $355 \mathrm{ff}$.; $\longrightarrow$ degeneration of, 197-198, 199

procestrus, 379

prosencephalon, 129, 131, 131 : 262, 271, 282, 303, 317

Prototheria, ova, 418

protovertebra, 51

proventriculus, 336

pseudothyroid body, 164

pubis, 221

pulmo-cutaneous arch, 184, 184

pulmo-enteric recess, 351

pulmonary, arch, 184: 342, 342 ;

tract, 331-332

pupil, 146: 318, 318

Rabbit, allantois, 425, 431, 434 ff.; amnion, $424 f f$., 425, 426; amnion cavity, 428,431 ; blastodermic vesicle, 382, 384, 384, 425 ; chorion, 425 , 426,431 ; cleavage, 381,382 ; embryonic shield, 393; endoderm, 393; 
(Amphioxus, pages 1-61; frog, 62-228; chick, 229-367; mammal, 368-454.)

rabbit, exocœlom,431, 432; formation of embryo, 393 ff.; gestation, 369; implantation, 421, 422; lutein, 378; morula, 382; notochordal canal, 398; ovary, 371; ovulation, 379 ; ovum, 373 ; proamnion, 425, 426 ; trophoblast, $388,388,425$; trophoderm, 422,425 ; villi, 425 ; yolk-sac, 431,431

"Räderorgan," 35, 40, 42, 45, 46

Radford, 191

ramus, communicans, $135,143,143$ : $312,312,313$; lateralis, $\mathrm{x}$ cranial nerve, 156

rat, false amnion cavity, 389,390 ; gestation, 369 ; inversion of germ layers, 389, 390; ovulation, 379 ; träger, 389,390 ; trophoblast, 389 , 390

Rauber's layer, 388, 388, 395

recessus, mammillaris, 133 ;

olfactorius, 129 ; - opticus, 130 , 133, 148: 262, 303, 304, 305, 306; - pulmo-entericus, 351

rectum, 118, 167, 170: $297,335,336$ regulation, in ovum, $86 \mathrm{ff}$.

Rejsek, 422

reproductiive, ducts, $202 \mathrm{ff.:} 360 \mathrm{ff}$.; - system (organs), $55 \mathrm{ff}$., $\mathbf{5 7}$ : $71 \mathrm{ff} ., 72,201 \mathrm{ff} ., 203,205: 234$ 236, 235, 359 ff.: 371-372

rete efferentia, 363

retinal, layer, 146, 146, 147, 148:317, 317,$318 ;$; - zone, 319

rhombencephalon, $129,131,132,133$ : 262, 271, 276, 303

ribs, 209

Riddle, 239

ridge, genital, 415 ; - mandibular, 158; - neural, 16, 21, 22, 23: 261, 266, 268, 269, 270, 278: 398; - sex-cell (genital), 204, 204, 205 ; - sub-atrial, 48,48

rod, hypochordal (subnotochordal), 169, 194, 204

Rodents, amnion, $424 \mathrm{ff}$.; decidua, 442 ; implantation; 421 ; placentation, 439,440 ; uteri, 372

roots, of spinal nerves, $135,142,143$ : 311,312

rotation, of gastrula, 98, 104, $105 \mathrm{ff}$., $105 ;-$ of ovum, $81,83,86,89$

Rückert, 273

Ruminants, amnion, 392, 424; placentation, 439, proamnion, 428

Sac, albumen, 288, 289, $298 \mathrm{ff}$.; endolymphatic, 152: 323 ; lymphoid, 191; — scrotal, 416, saccule, 150, 151: 322, 323

sacrum, 221

scapula, 221

sclerotome, 48, 52, 52, 53: 172, 208:

294, 353, 354

scrotal, sac, 416,417 ; - swellings, $415,416,417$

Seessel's pouch, 304, 328, 329

segmental, duct, 193, ff., 194, 195,

196, 198; _ - plate, 123,170

segmentation, 265; - cavity, 14 ,

$16: 93,94,96,98,102: 245,245$,

247, 248

semicircular canals, 150, 151: 322 , 323

semilunar valves, 339

seminal vesicles, $73: 202$

semiplacenta (semiplacentalia), 440 sense, capsules, $210 \mathrm{ff}$; — 123

septum (septa), 172; — endocardial, 266; - interauricular, $177: 338,339$; —_ internasal, 210 , 211, 215; — interventricular, 338, 340; — nasal, 413, 413; - pericardio-peritoneal, 191192 ; - transversum, 191-192: 351 ; — yolk-sac, 287, 288, 289, 290

sero-amnionic fusion, $287,288,289$, 294, $294: 427$

sex, cells, 204, 204; - cords, 204, 204, 205: 362, 362; - distinction of, $206: 362: 416$

sheath, notochordal, 52: 208, 208; - neural, 52

sheep, allantois, 436; blastodermic vesicle, 384 ; gestation, 369 ; lutein, 378; notochordal canal, 398

shell, 233, 234, 241; - membrane, $233,234,241$

shield, embryonic, 387, 388, 392, 393, ff., 394

Shore, 186

shrew, trophoblast, 388

sinu-auricular aperture, 337 ; valves, 339

sinus, cervical, 406, 407; —Cuvier i see ductus; — lymphoid, 190 191, 190 ; - superior, $150 ;-$ terminalis (marginal), 252, 252, 255, 290: 431, 432, 432, 449, 449 ; - urinogenital, $414 \mathrm{ff}$., 415, 416 ; — venosus, 177: 291, 304, 337, 339,351

sitting height, human embryo, 411 size, of human embryo, 407, 411 skeletogenous layer, 52

skeleton, 207 ff.: 354; dicular, 221-222 
(Amphioxus, pages 1-61; frog, 62-228; chick, 229-367; mammal, 368-454.)

skull, 164, 210, ff.: 354; — bony elements of, 215 ; cartilaginous, $210 \mathrm{ff}$.

solenocytes, 45,53

somatic stalk, 282

somatopleure, 174: 266-267, 280

somites, 21, 25, 25, $29 f f ., 30,32,51$ ff.: 124, 170 ff., 171: - 264 ff., 266, 268, 274, 278: 352 ff.: 401, $402 \mathrm{ff}$.; — number of, 51: 172: 264,352 ; — of bead, 264-265; - table of, 173

space, perivitelline, $4,5,9,10: 80$,

$81: 374$

spawning, 8, 58: 64, 71, $77 \mathrm{ff}$.

spermatogenesis, 77

spermatogonia, 7

spermatozoön, 5: 70, 71; - - longevity of,379;- supernumerary, 246

spermophile, implantation, 422, 423 spinal, bulb, 129: 308 ; — cord, 37 : $117,118,134,134: 308$; ganglia, 135, 142, 143 : 309, 311; nerves, $135,142-143,143$, 173: $311 \mathrm{ff} ., 312$; — nerves, axons of, 311

spiracle, $67,161: 315,330$

splanchnic stalk, $282,289,327,334$ splanchnocœl, 25, 31, 52 : 124,124, 170 splanchnopleure, 174: 266-267, 280 spleen, 191: 350

stalk, allantoic, 287, 288, 289, 296, $297,297,348: 430,431 ;-$ body (belly), 399, 400, 429, 430, 446 ; optic, $129,144,145,147$, 148, 160: 303, 316, 317, 318, 320; - somatic, 282;- splanchnic, $282,289,327,334$

stapes, 325

sternum, 221

stigma, follicular, 378

stomach, 168, 169: 332, 335, 351

stomach-intestine, 33

stomodæum, 158, 164, 166: 269,327 , 329 : $404,412,412$

Strahl, 439

stratum granulosum, 375, 376

streak, primitive, 119, 120: $251 \mathrm{ff}$., 252, 254, 258, 260, 274 : 394, 395, $396,398,401$; - - relation to embryo, $256 \mathrm{ff}$., 258,260 stroma, ovarian, 205: 361, 362 subatrial ridges, 48,48

subgerminal cavity, 245, 245, 248 subnotochordal rod, 169, 204 subzonal layer, 382, 383, 385, 385 sucker, oral, 65, 66, 120, 164, 180 supra-orbital folds, 413,414 suprapericardial body, 163, 164 suprascapula, 221

suspensory fold, 49

suture, cerebral, 274, 276

swelling, genital, 415, 416, 417

Sylvius, aqueduct of, 132: 307

symmetry, of blastula, $96 ;-$ of ovum, $4,6,10,11,17: 70,80,83$ ff., $87,88 f f$.

sympathetic, cords, 143, 143 : $312-$ 313,$313 ;-$ ganglia 144:207, 207: 364; - nervous system, 143-144, 143 : 312-313, 313

syncœlom, 32

syncytia, trophodermal, $422 \mathrm{ff}$.

syncytiotrophoblast, $390,422 \mathrm{ff}$., 423

syncy tiotrophoderm, 422 ff., 423

śynencephalon, 304

systemic arch, 184, 184: 341, 342

Tadpole, $63 \mathrm{ff}$; $\longrightarrow$ metamorphosis of, 67-68

tail, 65, 120, 120: 404, 406, 406, 408, 408, 409, 409; - bud, 279, 280, 283, 296, 296, 297 ; - fold, 280, 293, 296, 296, 297.ff., 297

Talpa, see mole.

tapir, placentation, 440

Tarsius, amnion folds, 428; embryonic shield, 387 ; placentation, 440 ; yolk-sac, 433

teeth, 220 ; — vestiges, 328

"teeth" (horny), 159, 166

telencephalon, 129, 133: 283, $303 \mathrm{ff}$., 304

temperature, of incubation, 231

tentacles, velar, 44, 45

terminal sinus, see sinus.

testis, 6: 72, 73, 77: 362

thalami, optic, 129, 148: 307,320

thoracic duct, 191: 349

thy mus, 162, 163: 330,331

thyroid body, 163, 165, 166: 304, 331, 331

tongue, 165: 332; — bar, 41, 42

torus transversus, 129, 131, 133:305

trabeculæ, 210, 211, 212, 213

trabecular cornu, 210, 211, 212, 215

trachea, 329

"Träger," 389, 391, 429

transverse process, 209

trophoblast, 382, 384, $385 \mathrm{ff}$., 385, $386,388,390,421,423$; — knob, 389

trophoderm, 397, 422, 447;

functions of, 422-423; - syncytium, $422 \mathrm{ff}$; - vascularization of, 423

truncus arteriosus, $177,178,184,185$ : 282, 338, 339 
(Amphioxus, pages 1-61; frog, 62-228; chick, 229-367; mammal, 368-451.)

trunk, common pronephric, 194 tubal fissure, 328

tube, Eustachian, 152: 324, 328; Fallopian, 371, 372; - medullary(neural), $21 ; 27: 270,274,278$ : 398

tubercle, cloacal, 415, 415, 417; coccygeal, 415; - genital, 415, 416,417

tuberculum posterius, $129,131,133$ : 304, 305, 306

tubo-tympanic cavity, 152

tubule, mesonephric, 294, 356-357, $357,359,362$; —— collecting, 357, 358; - inner and outer, 198-201, 199, 200 ;

metanephric, 358-359, 359;

- collecting, 358 ; - pronephric, 160, 194, 195, 195, 196

Tupaija, blastodermic vesicle, 386 , 387

turbinates, 326

tympanic, cavity, 152: 324,328 ; - membrane, 152, 213: 325

Ultimobranchial body, 163, 164

umbilical cord and stalk, 335 : 406, $407,408,430,444,445$

umbilicus, 287, 288, 289 : 407; yolk-sac, 287, 288, 288, 289; yolk-stalk, 402

Ungulates, allantois, 436; blastodermic vesicle, 384; embryonic shield, 387; implantation, 421; ovulation, 379; placentation, 439; uteri, 372

urachus, 436

ureter, 199, 202: 358

urethra, 416

urinogenital, aperture, $415 \mathrm{ff} ., 415$; - ducts, 202 ff.; — membrane, $414 \mathrm{ff} ., 415 ;-$ sinus, 414 ff., 415, 436; - system, 192 ff.: $354 \mathrm{ff}$.

urostyle, 209

uterus, 235, 236: 372, 372, 444 ;

bicornis, duplex, simplex, 372 ; - cervix of, 444 ; fundus of, 372,$442 ;-$ glands of, 443,445 ; - human, gravid, 444 ;

ligaments of, 372 ; $\longrightarrow$ mucosa, 443

"uterus" of frog, 72, 73

utricle, 150, 151: 322

Vagina, 235, 236: 372, 372

valves, semilunar, sinu-auricular, 339 valvula cerebelli, 133

Van Beneden, 383 vasa efferentia, 73, 202, 206: 362

vascular, area, see area; - lacunæ, 256,256 ; — system, $174 \mathrm{ff}$.: 271 ff.: 336 ff.: 405-406, 405 vas deferens, 202, 206: 360

vein, abdominal (anterior), of Amphibia, 189, 346; - allantoic, 297; - cardinal (anterior and posterior), 56, $57: 186 \mathrm{ff}$., 187, 196, 201: 285, 291, 294, 310, 329, 343 ff., 347, 348, 357, 358; cardinal, median, $186 \mathrm{ff}$., 187 ; —_ caudal, 186, $187: 348$;

caval, see postcaval, precaval; coccygeal, 349; - Cuvierian, see ductus Cuvieri; - femoral, 187 ; — hepatic, 182, 185: 345, 346 ; - hepatic portal, 185: $346: 433$; — hyoidean, 182,183 ; — iliac, 187, 188; — intersegmental (intersomitic), 189: 280, 344 ; - jugular (external, internal, superior, inferior), $186 \mathrm{ff}$. : 285, 329, 343, 348; — lateral, 189 ; - lateral, of Elasmobranchs, 346 ; - mesenteric, 346 , 348 ; - mesonephric (advehent, revehent), 188; omphalomesenteric, 185: 274, 276, 282, 290, 291, 337, 344 ff.: 432, 432 ; pelvic, 187 ; - postcaval, 166, 186 ff., 187 : 345, 347-348, 348; - precaval, 188; - pulmonary, $177,182,189: 339,348 ;-$ renal, 187, 188: 348,349 ; renal portal, 187, 188: 348, 349; — segmental, 186: 280; somatic, $344 ;$ — splanchnic, 344 subcardinal, 294, 348, 348, 358 ; — - subclavian, 348 ; subintestinal, 48, 52: 346 ; umbilical, 285, 297, 345, 346, 347, 348: 405, 405, 435, 436; - vertebral, 188; - vitelline, 176, 177, 185: 273, 278, 281, 282, 283, 285, 290, 291, 345-346, 345, 348: 405, $405,432,432$; — vitello-intestinal, 185

velar, plate, 162,180 ; — tentacles, 44,45

velum, 42, 44, 45; - medullare, 305 ; 1 transversum, 283, 304, 305,306

vena cava; see vein, postcaval.

venous system, $185 \mathrm{ff} .: 343 \mathrm{ff}$.

ventral, aorta, 177: 273, 275, 278, 282 ; - fissure, 135 ; - musculature, 53

ventricle (cardiac), 176, 177: 282, 283, 304, 335, 337 ff., 338; - 
(Amphioxus, pages 1-61; frog, 62-228; chick, 229-367; mammal, 368-454.)

ventricle (cerebral), 131, 132, 133 : 304, 305, 306, 307

vertebræ, 208-209: 354; — table of, 173

vertebral plate (mesoderm), 170, 194: 263,278

vesicle, amnio-cardiac, 252, 267, 273; blastodermic, $382383 \mathrm{ff}$., 384, 385, 386, 390, 399; cerebral, $25,35,38$; - mesonephric, $198 \mathrm{ff}$., 199, 200 ; optic, $144,145,147: 266,271,274$, 276, 282, 283, 303, 316 ff., 317, 318, 329 ; - seminal, 73: 202

villi, 400, $438 \mathrm{ff}$., 442, 444, $445 \mathrm{ff}$., 447, 448, 449; - fixation, 442 , $447,448,449$; — of human placenta, 442, 444, 445 ff., 449

visceral, arches, $120,123,160,160$, 163, 164 : 286, 328 ff.: 404 ff., 404, 406, 408; — cartilaginous, 211, 216 ff., 217, 218; — clefts, see gill clefts; - pouches, 122 , 158,160 , 160, 163, 164 : 269, 278, 328 ff., 329

vitelline membrane, 4, 5, 8: 69:232, 233, 245, 247, 287 : 374

vitellus, 232, 233

vitreous body, 148: 320

Wall, body, 282

weasel, placentation, 440

Weber, 177

weight, of human embryo, 411 whale, placentation, 440

Willey, quoted, 2, 50

Wolffian, body, $198 \mathrm{ff}$., 199, 200 : 335, 357, 358, 359, 360; - duct, 199, 199, $202 \mathrm{ff} .: 265,294,355$, $356 \mathrm{ff}, 357,359,360$

Xiphisternum, 221

Yolk, influence upon development, 230 ; — mass, later history, 289 ff.; white and yellow, 233, 233, 239, 239, — nucleus, 74, 75 : 376 ; — plug, 65, 99, 101, 102 , 120 ; - - sac, $286 \mathrm{ff}$., 287, 288, 289, 310, 348: 428, 430 ; - sac, circulation, 290, 291 : 432-433, 432 ; - - disappearance of, 292 , 300 ; — - endoderm, 256, 272 , 277, 289-290: 390, 391, 425, 431; - human, 399, 400, 402 ff., 444 ; - later history of, 292, 300: $431 \mathrm{ff}$; —— - mesoderm of, 425, 432; - — - septa of, 287, 288, 289; - - umbilicus, 287, 288, 288, 289; stalk, 282, 287, 288, 289, 289, 335, 348 : 402,408 ; — — human, 431, 433, 444 ; —— umbilicus, 402

Zona, pellucida, 373, 374, 376, 384 ; radiata, $239,239: 374,376$

Zone of junction, 247, 258, 277, 277 





\section{DAY USE \\ RETURN TO DESK FROM WHICH BORROWED \\ EARTH SCIENCES LIBRARY}

This book is due on the last date stamped below, or on the date to which renewed.

Renewed books are subject to immediate recall.

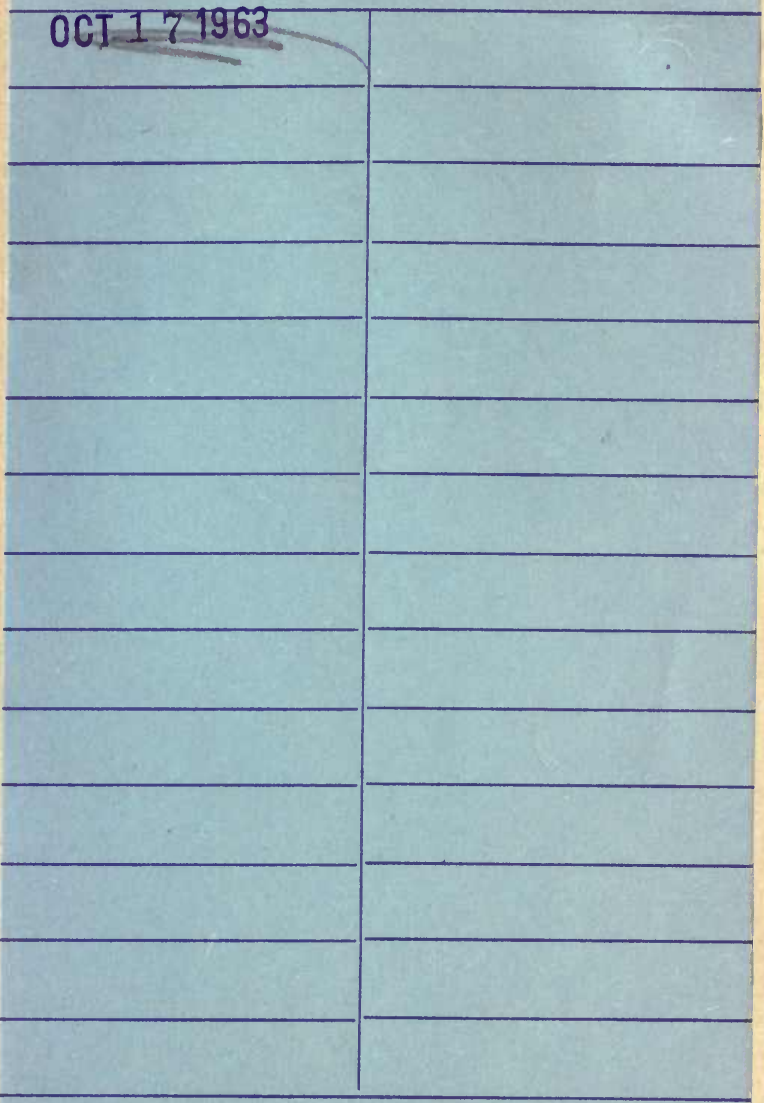

LD 21-50m-6,'60

(B1321810) 476

General Library

University of California

Berkeley 
$-841$

Ralph seri,j. 
